\title{
Potential Nonlinear Behaviour with VAD Use in Humans
}

\author{
by \\ Martin J. Conlon, B.Eng., M.Eng. \\ A thesis submitted to the Faculty of Graduate Studies and Research \\ in partial fulfillment of the requirements for the degree of

\section{Doctor of Philosophy} \\ Ottawa-Carleton Institute for Mechanical and Aerospace Engineering \\ Department of Mechanical and Aerospace Engineering \\ Carleton University \\ and \\ University of Ottawa Heart Institute \\ Ottawa, Ontario \\ Canada
}

(C) 2006 Martin J. Conlon 


$\begin{array}{ll}\begin{array}{l}\text { Library and } \\ \text { Archives Canada }\end{array} & \begin{array}{l}\text { Bibliothèque et } \\ \text { Archives Canada }\end{array} \\ \begin{array}{l}\text { Published Heritage } \\ \text { Branch }\end{array} & \begin{array}{l}\text { Direction du } \\ \text { Patrimoine de l'édition }\end{array} \\ \begin{array}{l}\text { 395 Wellington Street } \\ \text { Ottawa ON K1A ON4 }\end{array} & \begin{array}{l}\text { 395, rue Wellington } \\ \text { Ottawa ON K1A ON4 } \\ \text { Canada }\end{array}\end{array}$

Your file Votre référence ISBN: 978-0-494-16664-2 Our file Notre référence ISBN: 978-0-494-16664-2

NOTICE:

The author has granted a nonexclusive license allowing Library and Archives Canada to reproduce, publish, archive, preserve, conserve, communicate to the public by telecommunication or on the Internet, loan, distribute and sell theses worldwide, for commercial or noncommercial purposes, in microform, paper, electronic and/or any other formats.

The author retains copyright ownership and moral rights in this thesis. Neither the thesis nor substantial extracts from it may be printed or otherwise reproduced without the author's permission.
AVIS:

L'auteur a accordé une licence non exclusive permettant à la Bibliothèque et Archives Canada de reproduire, publier, archiver, sauvegarder, conserver, transmettre au public par télécommunication ou par l'Internet, prêter, distribuer et vendre des thèses partout dans le monde, à des fins commerciales ou autres, sur support microforme, papier, électronique et/ou autres formats.

L'auteur conserve la propriété du droit d'auteur et des droits moraux qui protège cette thèse. $\mathrm{Ni}$ la thèse ni des extraits substantiels de celle-ci ne doivent être imprimés ou autrement reproduits sans son autorisation.
In compliance with the Canadian

Privacy Act some supporting forms may have been removed from this thesis.

While these forms may be included in the document page count, their removal does not represent any loss of content from the thesis.
Conformément à la loi canadienne sur la protection de la vie privée, quelques formulaires secondaires ont été enlevés de cette thèse.

Bien que ces formulaires aient inclus dans la pagination, il n'y aura aucun contenu manquant.

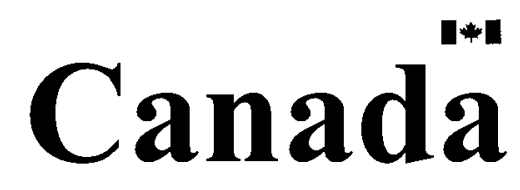


This Page Intentionally Left Blank 


\section{Abstract}

This work extends our understanding of the interaction between circulatory assist devices and either the human circulatory system or mock circulatory systems. The major results depend on both linear and nonlinear dynamic effects, and have significant impact on both the design of mock circulatory systems and our analysis of the human circulatory system.

Two complementary approaches were used, one theoretical and the other experimental. A comprehensive review of mock circulatory systems was performed. Multiple mathematical models were developed in order to reach the fundamental objective of gaining a better understanding of human circulatory system dynamics. These models are based on newly-developed analogies that equate the proximal arterial system to a nonlinear massspring-damper system, sometimes called an "impact oscillator" or "Fermi oscillator." This equivalence has implications for both VAD efficiency and stability, and also makes predictions about natural heart behaviour.

The experimental effort, using a specially designed mock circulatory system in varying configurations, was used to improve the confidence in the numerical predictions. Most existing mock circulatory systems were found to be a poor representation of the human arterial tree and an inadequate dynamic testbed for artificial heart development, although they are still useful for long-term reliability testing. Results from both mathematical simulation and experimental testing show that the addition of a second arterial compliance element to an existing mock circulatory system improves the dynamic representation, and the resultant arterial pressure waveforms more closely match those seen in the human body. Nonlinear aspects of the behaviour offer possible explanations for several cardiac conditions, and provide promising direction for future research. 


\section{Acknowledgments}

This manuscript is the result of a large amount of work that served to further increase my dependence on the bitter alkaloid $\mathrm{C}_{8} \mathrm{H}_{10} \mathrm{~N}_{4} \mathrm{O}_{2}$. The production would not have been possible without the guidance, understanding and support of many people, and I would like to express my gratitude to them.

Thanks must first go to Donald Russell, my thesis co-supervisor, for his counsel and reinforcement. He provided insight at key times and yet allowed the project to develop in a directed-random-walk fashion.

I would like to thank Tofy Mussivand, my thesis co-supervisor, for his support. One of the most enjoyable aspects of this work was the opportunity to finally move from the theoretical realm into the experimental, and the collaboration with the University of Ottawa Heart Institute went a long way towards that end.

Thanks go to Danyaal Raza for his help with the development of the experimental apparatus over the course of two summers. Jim Sliwka, Alex Proctor and Kevin Sangster provided invaluable design advice, and manufacturing assistance. I like to think I have become a decent machinist under their excellent tutelage.

Gary Clements was always available to answer my incidental experiment-related questions, and his experience was terribly useful. So too was the help provided by Steve Truttman, especially during the scramble that saw the experiment shift from the lab bench to the material test machine after a critical equipment failure. Calvin Rans also provided much-needed help at that juncture, schooling me on the MTS machinery and associated software. 
Ian Glasgow provided initial assistance with the Ottawa Heart Institute VAD (and mock circulatory system) while Kevin Holmes gave excellent emergency support during the final phases of the research. My sincere thanks to both of them.

I am also grateful to the Department of Mechanical and Aerospace Engineering's office staff (Christie, Marlene and Nancy). They did a good job of shielding me from the administrative headaches associated with completing a graduate degree, and were always pleasant and helpful.

I would like to think all of my friends for at least feigning interest in my work. Brian McAuliffe read the bulk of this manuscript, providing particularly pertinent feedback. And, in spite of appearances, our numerous coffee jaunts brought useful time to discuss our individual projects. Ali Mahallati provided equipment and support, and Jeremy Mitchell produced the shop drawings for the experimental apparatus.

Finally, I would like to thank my family, for continuing to put up with my odd hours, idiosyncrasies, and sometimes less-than-friendly disposition while still offering me muchneeded encouragement and support. 


\section{Contents}

Abstract

Acknowledgments $\quad$ iv

List of Figures $\quad$ xi

List of Tables $\quad$ xv

List of Symbols and Acronyms $\quad$ xvi

1 Introduction 1

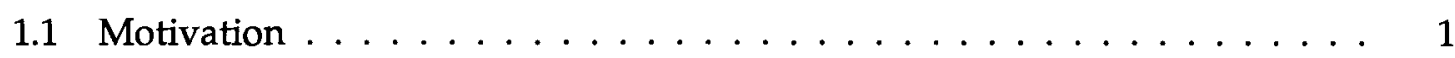

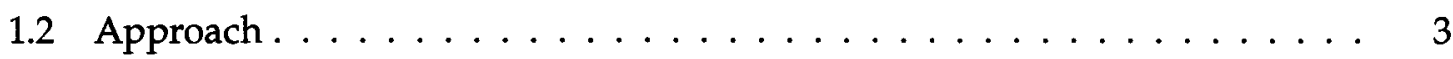

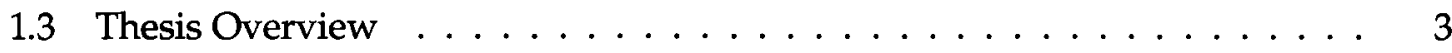

2 Background 6

2.1 Medical . . . . . . . . . . . . . . . . . . . . 6

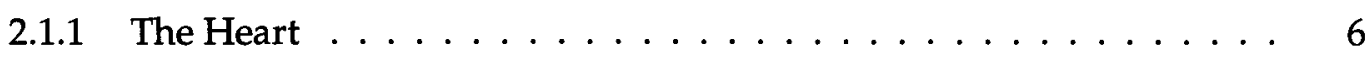

2.1 .2 Heart Disease . . . . . . . . . . . . . . . . . 9

2.1.3 The Cardiac Cycle . . . . . . . . . . . . . . . . . 10

2.1 .4 The Circulation . . . . . . . . . . . . . . . . . . 12

2.1 .5 VADs and TAHs . . . . . . . . . . . . . . . 14

2.2 Dynamics . . . . . . . . . . . . . . . . . . . . . 19

2.3 Bond Graphs . . . . . . . . . . . . . . . . . . . . 23

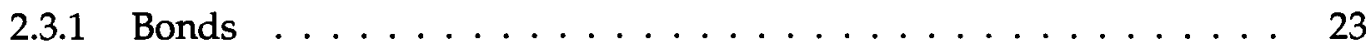

2.3 .2 Basic Elements . . . . . . . . . . . . . . . . 24

2.3 .3 Junctions . . . . . . . . . . . . . . . . . 25

2.3 .4 Articulation . . . . . . . . . . . . . . . 26

vi 
2.4 Hydraulics . . . . . . . . . . . . . . . . . . . 26

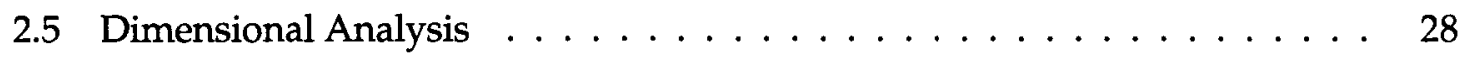

2.5.1 The Buckingham $\Pi$-Theorem $\ldots \ldots \ldots \ldots . \ldots \ldots$

2.5.2 Derivative Terms . . . . . . . . . . . . . . . . 35

2.6 Circulatory System Model . . . . . . . . . . . . . . . . . . . . . . . . . . . . . .

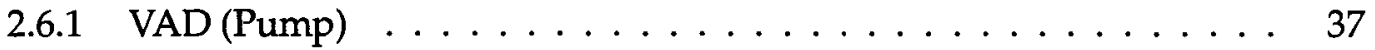

2.6 .2 Cannulae. . . . . . . . . . . . . . . . . . 39

2.6 .3 Valves ............................ 39

2.6 .4 Arteries (and Aorta) $\ldots \ldots \ldots \ldots$. . . . . . . . . . 40

2.6 .5 Peripheral Circulation . . . . . . . . . . . . . . 44

2.6 .6 Venous Segment . . . . . . . . . . . . . . . . 45

2.6 .7 Overall Model . . . . . . . . . . . . . . . . . . . . 48

3 Literature Review $\quad 52$

3.1 Circulatory System Dynamics . . . . . . . . . . . . . . . 52

3.1 .1 Modelling . . . . . . . . . . . . . . . 52

3.1 .2 Evidence of Chaos . . . . . . . . . . . . . . . 54

3.1.3 Evidence of Deterministic Behaviour . . . . . . . . . . . . . . . 57

3.2 Mock Circulatory Systems . . . . . . . . . . . . . . . . . 58

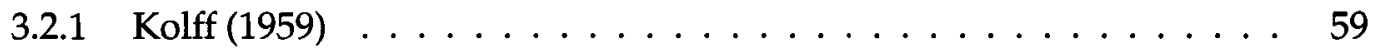

3.2 .2 Donovan $(1975) \ldots \ldots \ldots \ldots$. . . . . . . . . . . . . . . . . . . . . . . . . . . .

3.2.3 The Independent Tree . . . . . . . . . . . . . . . . . . 73

3.3 The Arterial Pressure Waveform . . . . . . . . . . . . . . . . . 77

3.4 Nonlinear Dynamic Systems . . . . . . . . . . . . . . . . . . . . 80

4 Theoretical $\quad 82$

4.1 Mathematical Model of the Arterial System . . . . . . . . . . . . . . . 83

4.1 .1 State-Space Formulation . . . . . . . . . . . . . . 86

4.1 .2 Characteristic Equation . . . . . . . . . . . . . 87

4.2 The Arterial Pressure Waveform . . . . . . . . . . . . . . . . . . . 92

4.3 Nonlinearities . . . . . . . . . . . . . . . . . . . . . . . 93

4.4 The Impact Oscillator Analogy $\ldots \ldots \ldots$. . . . . . . . . . . . . . 99

4.4 .1 Periodicity . . . . . . . . . . . . . . . . . . . . . 102

4.4 .2 Solid Wall Impacts . . . . . . . . . . . . . . . . . 105

4.4 .3 Soft Wall Impacts . . . . . . . . . . . . . . . . . . . . 111 
4.4.4 Accounting for Blood Flow . . . . . . . . . . . . . . . . 115

4.4.5 The Effect of VAD Pumping Modes . . . . . . . . . . . . . . 117

4.5 Theoretical Basis for Circulatory System Efficiency . . . . . . . . . . . . . . 118

5 Experimental $\quad 119$

5.1 UOHI Mock Circulatory System (Original Configuration) . . . . . . . . . . . 119

5.1 .1 Fixed-rate Tests . . . . . . . . . . . . . . . . . . . 122

5.1 .2 FFFE Tests . . . . . . . . . . . . . . . . . . 125

5.2 Addition of the Aortic Chamber . . . . . . . . . . . . . . . . . . 128

5.3 Chaotic Oscillations and the Loss of the VAD . . . . . . . . . . . . . . . 129

5.4 The Switch to the pVAD (and the MTS Machine) . . . . . . . . . . . . . . 132

5.4 .1 Validation of the PVAD . . . . . . . . . . . . . 135

5.5 Replacement of the Air-Water Arterial Chamber . . . . . . . . . . . . . 138

6 Results and Discussion $\quad 144$

6.1 Modelling the Human Circulatory System . . . . . . . . . . . . . . . . . . . 144

6.2 Analysis of the Arterial Pressure Wave . . . . . . . . . . . . . . . . . . 145

6.3 The Influence of Nonlinear Behaviour . . . . . . . . . . . . . . . . 146

6.3 .1 Physiological Changes . . . . . . . . . . . . . . . . . . 148

6.4 Experimental Findings . . . . . . . . . . . . . . . . . . . . . . . . 149

6.5 Correspondence Between Theory and Experiment . . . . . . . . . . . . 151

7 Conclusions and Recommendations for Future Work 153

7.1 Experimental Improvements . . . . . . . . . . . . . . . . . . 153

7.2 Theoretical Work . . . . . . . . . . . . . . . . . . . . 155

$\begin{array}{ll}\text { References } & 156\end{array}$

A Human Circulatory System Simulation $\quad 188$

Mathematica Simulation . . . . . . . . . . . . . . . . . . . . . . . . 189

B Linear Arterial System Models 205

Flow-in, flow-out with $\{$ IAorta, CArteries, QIn\} as repeating variables . . . . . . 206

Flow-in, pressure-out with $\{$ IAorta, CArteries, QIn\} as repeating variables . . . . 213

Flow-in, flow-out with $\{$ RAorta, IAorta, QIn\} as repeating variables . . . . . . . 221

Flow-in, pressure-out with $\{$ RAorta, IAorta, QIn\} as repeating variables . . . . . . 228 
Flow-in, flow-out with \{CArteries, RPeripheral, QIn\} as repeating variables . . . 236

Flow-in, pressure-out with \{CArteries, RPeripheral, QIn\} as repeating variables . 243

C University of Ottawa Heart Institute Mock Circulatory System 251

Mathematica Simulation . . . . . . . . . . . . . . . 252

D MTS Simulation $\quad 267$

Mathematica Simulation . . . . . . . . . . . . . . . . . . . 269

E Pressure Chamber Design $\quad 282$

E.1 Volume Sizing . . . . . . . . . . . . . . . . . . . . . 288

E.2 Pressure Sizing . . . . . . . . . . . . . . . . 288

E.3 Spring Sizing . . . . . . . . . . . . . . . . . . . . . . . . 289

E.3.1 Linear Aorta . . . . . . . . . . . . . . . . . . . . . . . . . . . . . 289

E.3.2 Nonlinear Aorta . . . . . . . . . . . . . . . . . . . 291

E.3.3 Arteries . . . . . . . . . . . . . . . . . . . 293

F Pressure Chamber Drawings 295

G Experimental Setup $\quad 306$

G.1 Valve Calibration . . . . . . . . . . . . . . . . . . 306

G.2 Pressure Transducer Calibration . . . . . . . . . . . . . . . . 306

G.3 Position Sensor Calibration . . . . . . . . . . . . . . . 307

G.4 Equipment Specifications . . . . . . . . . . . . . . . . 308

H Experimental Data

H.1 Fixed-rate VAD Tests . . . . . . . . . . . . . . . . . . . 313

H.2 FFFE VAD Tests . . . . . . . . . . . . . . . . . . . 315

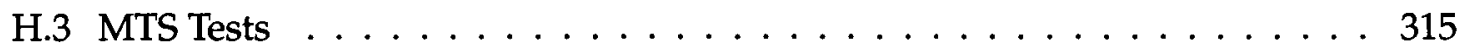

I Schematics 319

I.1 Transducer Excitation . . . . . . . . . . . . . . . . . . . . . . . . 319

I.2 Valve Mic . . . . . . . . . . . . . . . . . . . . . . . . . 321

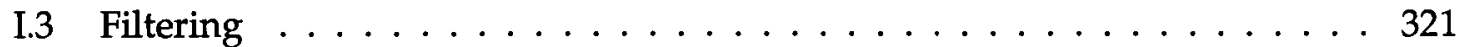

J XML Bond Graph Parsing 326

Mathematica Code . . . . . . . . . . . . . . . . . 327 
K Periodicity Determination $\quad 330$

L Mathematica Bugs $\quad 332$

L.1 NDSolve Memory Leaks . . . . . . . . . . . . . . . . . . . . . . . 332

L.2 Errors in the NumericalMath 'IntervalRoots Package . . . . . . . 333

Index 


\section{List of Figures}

2.1 Diagram of the heart (see, for example, Gray, 1858) . . . . . . . . . . 7

2.2 Cross-section of the heart (see, for example, Gray, 1858) . . . . . . . . 8

2.3 PQRST wave (see, for example, Tortora and Grabowski, 2000) . . . . . . . 10

2.4 Blood flow during atrial depolarization (P-wave) . . . . . . . . . . . . 11

2.5 Blood flow during ventricular depolarization (QRS-complex) . . . . . . . . 11

2.6 Blood vessel cross-section (see, for example, Tortora, 2000) . . . . . . . . 13

2.7 VAD illustration . . . . . . . . . . . . . . . . . . . . 16

2.8 Cannula illustration . . . . . . . . . . . . . . . . . . 17

2.9 Diagram of the heart with VAD cuff attached . . . . . . . . . . 18

2.10 Mass in a gravitational field . . . . . . . . . . . . . . . . . . . 19

2.11 Unforced mass-spring-damper system . . . . . . . . . . . . . . . 21

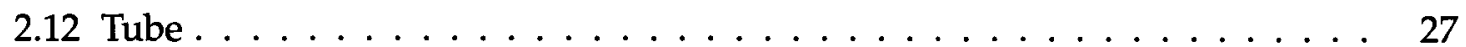

2.13 Mass in a gravitational field - reprint of Figure $2.10 \ldots \ldots \ldots$

2.14 Nondimensional solution to falling-mass problem (Case I) . . . . . . . . 31

2.15 Nondimensional solution to falling-mass problem (Case II) . . . . . . . . . . 32

2.16 Nondimensional solution to falling-mass problem (Case III) . . . . . . . . . 33

2.17 Nondimensional solution to falling-mass problem (Case III) using different

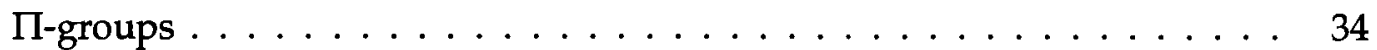

2.18 Unforced mass-spring-damper system - reprint of Figure $2.11 \ldots 35$

2.19 Two arterial system models, (a) SCM, (b) DCM . . . . . . . . . . . . . . . 40

2.20 Aortic pressure-volume data and curve fits . . . . . . . . . . . . 42

2.21 Aortic pressure waveform for a variety of ejection pressures . . . . . . . . 44

2.22 Two venous system models, (a) SCM, (b) DCM . . . . . . . . . . . . . . 46

2.23 Hydraulic schematic of the HCS model . . . . . . . . . . . . . . . . 49

2.24 Bond graph of the HCS model . . . . . . . . . . . . . . . 50

3.1 Kolff's (original) mock circulatory system . . . . . . . . . . . . . 59

xi 
3.2 Donovan mock circulatory system . . . . . . . . . . . . . . 66

3.3 Features of the aortic pressure waveform $\ldots \ldots \ldots \ldots$

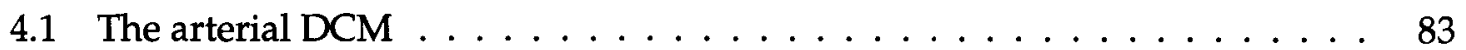

4.2 Arterial DCM bond graph with inlet flow source and outlet flow sink . . . 84

4.3 Arterial DCM bond graph with inlet flow source and outlet pressure sink . 84

4.4 General cubic polynomial equation . . . . . . . . . . . . . . . . . . 89

4.5 Contour plot of Equation (4.24) for $\Pi_{3}=20 \ldots \ldots \ldots \ldots$. . . . . . 91

4.6 Aortic pressure waveform for the SCM and DCM . . . . . . . . . . . 92

4.7 Various arterial element-BC configurations . . . . . . . . . . . . . . . 94

4.8 Forced mass-spring-damper system . . . . . . . . . . . . . . . . 95

4.9 Aortic pressure and flow waveforms . . . . . . . . . . . . . . . . 97

4.10 Impact oscillator behaviour for a variety of excitation frequencies . . . . . . 98

4.11 Forced mass-spring-damper systems (in a variety of configurations) . . . . . 101

4.12 Position versus time and velocity versus position for various forcing frequencies, demonstrating the concept of periodicity changes . . . . . . . . 103

4.13 Periodicity for oscillations of the impact oscillator (truncated at period-30) . 106

4.14 Periodicity for oscillations of the impact oscillator (truncated at period-30) by examining impact intervals . . . . . . . . . . . . . . . . . . . 107

4.15 Divergence of nearby trajectories (initial conditions $\left\{\Pi_{3}, \Pi_{3}^{\prime}\right\}=\{0 ., 0$.$\} and$ $\{0 ., 0.001\}, \Pi_{1}=10$. , and $\left.\Pi_{2}=9.7\right) \ldots \ldots \ldots \ldots . \ldots \ldots$

4.16 Divergence sequence for 1700 nearby trajectories as the system evolves in time over (b) 10 drive cycles, (c) 20 drive cycles, (d) 40 drive cycles, and (e) 80 drive cycles. . . . . . . . . . . . . . . . . . . . . . . . 110

4.17 The divergence of two nearby trajectories with respect to time . . . . . . . 111

$4.183 \mathrm{D}$ volume rendering of the periodicity as $\Pi_{1 \mid b}, \Pi_{1 \mid m}$, and $\Pi_{2}$ are varied . . 113

4.19 Periodicity for oscillations of the soft-wall oscillator with $\Pi_{1 \mid m}=10$ (truncated at period-30) . . . . . . . . . . . . . . . 114

4.20 Periodicity for oscillations of the soft-wall oscillator with $\Pi_{1 \mid m}=50$ (truncated at period-30) $\ldots \ldots \ldots \ldots \ldots$. . . . . . . . . . . 115

4.21 Chaotic oscillations in a system driven by a fixed-fill, full-eject input . . . . 117

5.1 Schematic diagram of the UOHI mock circulatory system . . . . . . . . . 120

5.2 Photo of the bench-top experimental setup in the original (UOHI) configuration . . . . . . . . . . . . . . . . . . . . 121 
5.3 Pressure waveform versus beat rate for fixed-rate VAD operation (maximum standard deviation is $3.51 \mathrm{mmHg}$; minimum standard deviation is

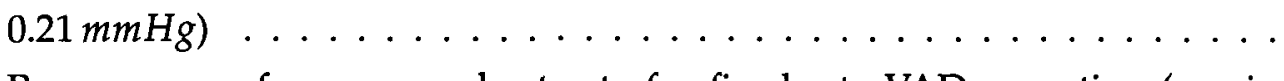

5.4 Pressure waveform versus beat rate for fixed-rate VAD operation (maximum standard deviation is $2.08 \mathrm{mmHg}$; minimum standard deviation is $0.26 \mathrm{mmHg}) \ldots \ldots \ldots \ldots \ldots \ldots \ldots . \ldots \ldots \ldots$

5.5 Effect of liquid sloshing on the arterial pressure waveform . . . . . . . 125

5.6 Arterial pressure waveform during FFFE-mode VAD operation . . . . . . 127

5.7 Theoretically predicted development of the dicrotic notch (in the aortic pressure waveform) with decreasing arterial compliance . . . . . . . . . . . 130

5.8 Schematic diagram of the UOHI mock circulatory system with the aortic chamber added . . . . . . . . . . . . . . . . . 131

5.9 The base of the pVAD chamber (with clamping plate attached) . . . . . . . 133

5.10 The final configuration of the MCS (with the aortic chamber and the new arterial chamber inline), connected to the PVAD and the MTS machine . . . 134

5.11 Subset of the first MTS run (maximum standard deviation is $1.27 \mathrm{~mm} \mathrm{Hg}$; minimum standard deviation is $0.25 \mathrm{mmHg}$ ) . . . . . . . . . . . . 135

5.12 The first MTS run (maximum standard deviation is $2.48 \mathrm{~mm} \mathrm{Hg}$; minimum standard deviation is $0.25 \mathrm{mmHg}$ ) . . . . . . . . . . . . . . . . 136

5.13 Simulation of the pVAD setup; unlike the experimental plots gray levels in this figure correspond to absolute pressure, rather than standard deviations 137

5.14 Schematic diagram of the new mock circulatory system . . . . . . . . . . 139

5.15 Development of the dicrotic notch (from the experimental data) . . . . . . 141

5.16 Comparison of worn and new diaphragms . . . . . . . . . . . . . 142

5.17 Simulated aortic pressure wave . . . . . . . . . . . . . . . . . 142

D.1 Bond graph representation of the MTS (and pVAD) setup . . . . . . . 268

E.1 Piston-cylinder assembly sealed using a rolling diaphragm . . . . . . . 283

E.2 Arterial pressure chamber . . . . . . . . . . . . . . . . . . 285

E.3 Closeup of arterial pressure chamber base . . . . . . . . . . . . 286

E.4 Closeup of arterial pressure chamber top . . . . . . . . . . . . 287

E.5 Aortic spring stiffness graph . . . . . . . . . . . . . . . . . 292

F.1 The assembled aortic pressure chamber (sitting in its cradle) . . . . . . 295 
G.1 Valve calibration curve for various voltage settings . . . . . . . . . . . . 307

G.2 Pressure transducer calibration curve . . . . . . . . . . . . . . . . 308

G.3 Cable-extension transducer calibration curve . . . . . . . . . . . . . 310

H.1 Experimental data for fixed-rate VAD operation; the beat rate was $60 \mathrm{bpm}$ and the peripheral valve voltage was set to $8 \mathrm{~V} \ldots \ldots \ldots \ldots$

H.2 Experimental data for fixed-rate VAD operation; the beat rate was $60 \mathrm{bpm}$ and the peripheral valve voltage was set to $6 \mathrm{~V} \ldots \ldots \ldots 314$

H.3 Experimental data for FFFE VAD operation; peripheral and inlet valve voltages were $4 \mathrm{~V}$ and $1 \mathrm{~V}$ (respectively) and the beat rate was approximately

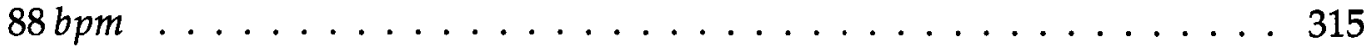

H.4 Experimental data for the first set of MTS experiments; peripheral valve

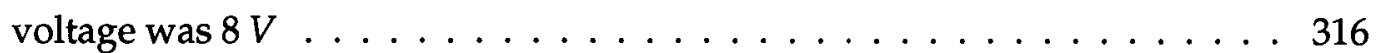

H.5 Experimental data for the final set of tests; the MTS beat rate was $60 \mathrm{bpm}$ and the compliance ratio $\left(C_{a r}: C_{a o}\right)$ is $3.3 \ldots \ldots . \ldots . \ldots . \ldots 317$

H.6 Experimental data for the final set of tests; the MTS beat rate was $60 \mathrm{bpm}$ and the compliance ratio $\left(C_{a r}: C_{a o}\right)$ is $2.7 \ldots \ldots \ldots \ldots$

I.1 Voltage regulator circuit; $10 \mathrm{~V}$ output, $15 \mathrm{~mA}$ maximum current . . . . . 320

I.2 Electret microphone circuit . . . . . . . . . . . . . . . . . . . . . . 322

I.3 Low-pass Bessel filter with $2 \mathrm{~Hz}$ corner frequency . . . . . . . . . . . . 324

I.4 Low-pass Bessel filter with $15 \mathrm{kHz}$ corner frequency . . . . . . . . . . . . 325 


\section{List of Tables}

2.1 Properties of vessels in the circulatory system . . . . . . . . . . . . . 15

2.2 Dimensions of the variables in Equation $(2.21) \ldots \ldots \ldots \ldots$

2.3 Dimensions of the variables in Equation $(2.33) \ldots \ldots \ldots$

5.1 FFFE beat rates for various peripheral and inflow valve settings . . . . . 128

E.1 Nominal parameters for the linear aortic chamber . . . . . . . . . . 290

E.2 Springs suitable for the linear aortic chamber. . . . . . . . . . . . . . . 290

E.3 Nominal parameters for the nonlinear aortic chamber . . . . . . . . . . . 291

E.4 Springs suitable for the nonlinear aortic chamber. . . . . . . . . . . . . 292

E.5 Nominal parameters for the linear arterial chamber . . . . . . . . . . . 293

E.6 Springs suitable for the linear arterial chamber. . . . . . . . . . . . . . . 294

G.1 Amplifier offset null drift . . . . . . . . . . . . . . . . . . . . . 309 


\section{List of Symbols and Acronyms}

$A \quad$ State matrix, first used in Equation 2.8, Page $22 \ldots \ldots \ldots \ldots \ldots \ldots[p \times p]$

a Acceleration vector, first used in Equation 2.1, Page $19 \ldots \ldots \ldots\left[\mathrm{kg} \cdot \mathrm{m} \cdot \mathrm{s}^{-2}\right]$

A Cross-sectional area, first used in Equation 2.17, Page 27..... $\left[\mathrm{cm}^{2}\right]$

$a(s) \quad$ Characteristic polynomial, first used in Equation 2.9, Page 22.

$a_{n} \quad$ Polynomial coefficient, first used in Equation 4.19, Page 90.

$a_{i j} \quad$ Power of fundamental unit " $i$ " for physical quantity " $j$ ", first used in Equation 2.19, Page 29.

AV Atrioventricular, first used on Page 7.

B Input matrix, first used in Equation 2.8, Page $22 \ldots \ldots \ldots \ldots \ldots[p \times r]$

$b \quad$ Damping, first used in Equation 2.5, Page $21 \ldots \ldots \ldots \ldots \ldots \ldots\left[\mathrm{kg} \cdot \mathrm{s}^{-1}\right]$

BC Boundary condition, first used on Page 93.

C Output matrix, first used in Equation 2.8, Page $22 \ldots \ldots \ldots \ldots \ldots[q \times p]$

C Compliance, first used in Equation 2.18, Page 27.......... $\left[\mathrm{cm}^{5} \cdot \mathrm{N}^{-1}\right]$

$C_{i} \quad$ Denotes constant of integration " $i$ ", first used in Equation 2.3, Page 20.

D Transmission matrix, first used in Equation 2.8, Page $22 \ldots \ldots \ldots[q \times r]$

$D \quad$ Diameter, first used in Equation 2.16, Page $27 \ldots \ldots \ldots \ldots \ldots[\mathrm{cm}]$

$d A \quad$ Differential area, first used in Equation 2.15, Page $27 \ldots \ldots \ldots .\left[\mathrm{cm}^{2}\right]$

DCM Dual-compliance model, first used on Page 41.

xvi 
ECG Electrocardiograph, first used on Page 10.

F Force vector, first used in Equation 2.1, Page $19 \ldots \ldots \ldots \ldots \ldots[N]$

$f \quad$ Generic vector function, first used in Equation 2.6, Page 21.

$F_{i} \quad$ Fundamental unit " $i$ ", first used in Equation 2.19, Page 29.

FFFE Full-fill, full-eject pumping mode, first used on Page 37.

$g \quad$ Generic vector function, first used in Equation 2.7, Page 21.

G(s) Transfer function matrix, first used in Equation 2.11, Page 22.

$g$ Standard acceleration due to gravity at the Earth's surface, first used in Equation 2.2,

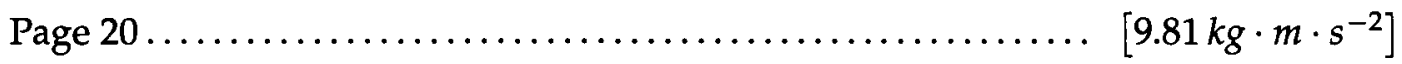

$\theta \quad$ Temperature (fundamental dimension), first used on Page 28.

$H \quad$ Container height, first used in Equation 5.1, Page $124 \ldots \ldots \ldots \ldots[f t]$

HCS Human circulatory system, first used on Page 36.

I Identity matrix, first used in Equation 2.9, Page 22.

$i \quad$ Index variable, first used on Page 29.

j Index variable, first used on Page 29.

$k \quad$ Stiffness, first used in Equation 2.5, Page $21 \ldots \ldots \ldots \ldots \ldots \ldots\left[\mathrm{kg} \cdot \mathrm{s}^{-2}\right]$

$L \quad$ Length, first used in Equation 2.16, Page $27 \ldots \ldots \ldots \ldots \ldots \ldots[\mathrm{cm}]$

$l \quad$ Length (fundamental dimension), first used on Page 28.

LHS Left-hand side, first used on Page 31.

LVAD Left ventricular assist device, first used on Page 3.

$m \quad$ Index variable, first used on Page 29.

$m \quad$ Mass (fundamental dimension), first used on Page 28.

$m \quad$ Mass, first used in Equation 2.1, Page 19............... [kg] 
MAE Mechanical and Aerospace Engineering (Department of), first used on Page 132.

MAP Mean arterial pressure, first used on Page 93.

MCS Mock circulatory system, first used on Page 3.

MTS Material Test Systems, Inc., first used on Page 129.

$\mu \quad$ Kinematic viscosity, first used in Equation 2.15, Page $27 \ldots \ldots \ldots\left[\mathrm{kg} \cdot \mathrm{cm}^{-1} \cdot \mathrm{s}^{-1}\right]$

n Index variable, first used on Page 29.

$\Delta P \quad$ Pressure drop, first used in Equation 2.16, Page 27......... $\left[N \cdot \mathrm{cm}^{-2}\right]$

$\Pi_{n} \quad$ Nondimensional group, first used on Page 29.

P Polynomial function, first used in Equation 4.19, Page 90.

$P \quad$ Pressure, first used in Equation 2.15, Page $27 \ldots \ldots \ldots \ldots \ldots \ldots\left[\mathrm{N} \cdot \mathrm{cm}^{-2}\right]$

$p \quad$ Momentum, first used in Equation 2.17, Page 27........... $\left[N \cdot \mathrm{s} \cdot \mathrm{cm}^{-2}\right]$

PDH Principle of Dimensional Homogeneity, first used on Page 28.

pVAD Pseudo-VAD, first used on Page 132.

$Q \quad$ Volume flow rate, first used in Equation 2.15, Page $27 \ldots \ldots \ldots\left[\mathrm{cm}^{3} \cdot \mathrm{s}^{-1}\right]$

$Q_{j} \quad$ Physical quantity " $j$ ", first used on Page 29.

$\rho \quad$ Density, first used in Equation 2.17, Page $27 \ldots \ldots \ldots \ldots \ldots \ldots\left[\mathrm{km} \cdot \mathrm{m} \cdot \mathrm{s}^{-2}\right]$

$R \quad$ Radius, first used in Equation 2.15, Page 27............ $[\mathrm{cm}]$

$r \quad$ Number of primary dimensions, first used on Page 29.

$r \quad$ Radial coordinate, first used in Equation 2.15, Page 27...... [ cm $]$

$s \quad$ Complex variable, first used in Equation 2.9, Page 22.

$s \quad$ Vertical position, first used in Equation 2.2, Page $20 \ldots \ldots \ldots \ldots[\mathrm{m}]$

$s_{0} \quad$ Initial position, first used in Equation 2.4, Page $20 \ldots \ldots \ldots \ldots[\mathrm{m}]$

SCM Single-compliance model, first used on Page 41. 
SI Système International d'Unités, first used on Page 28.

SL Semilunar (valve), first used on Page 8.

$\tau \quad$ Shear, first used on Page $\left.26 \ldots \ldots \ldots \ldots \ldots \ldots \ldots \ldots \ldots \ldots \ldots \ldots \ldots \ldots, \mathrm{kg}^{-1} \cdot \mathrm{s}^{-2}\right]$

$t \quad$ Time (fundamental dimension), first used on Page 28.

$t \quad$ Time, first used in Equation 2.2, Page 20.............. [s]

TAH Total artificial heart, first used on Page 1.

$U \quad$ Unit (dimension) matrix, first used in Equation 2.20, Page 29 .

$u \quad$ Input vector, first used in Equation 2.6, Page $21 \ldots \ldots \ldots \ldots \ldots[r \times 1]$

$u \quad$ Velocity, first used in Equation 2.15, Page 27............ $\left.\mathrm{cm} \cdot \mathrm{s}^{-1}\right]$

$U_{j} \quad$ Units for physical quantity " $j$ ", first used in Equation 2.19, Page 29.

UOHI University of Ottawa Heart Institute, first used on Page 119.

$V \quad$ Volume, first used in Equation 2.18, Page $27 \ldots \ldots \ldots \ldots \ldots \ldots\left[\mathrm{cm}^{3}\right]$

$v \quad$ Velocity, first used in Equation 2.12, Page 22............. $\left.m \cdot \mathrm{s}^{-1}\right]$

$v_{0} \quad$ Initial velocity, first used in Equation 2.4, Page $20 \ldots \ldots \ldots \ldots\left[\mathrm{m} \cdot \mathrm{s}^{-1}\right]$

$v_{n} \quad$ Generic variable, first used on Page 29.

VAD Ventricular assist device, first used on Page 2.

$\dot{x} \quad$ State vector derivative, first used in Equation 2.6, Page $21 \ldots \ldots[p \times 1]$

$x \quad$ State vector, first used in Equation 2.6, Page $21 \ldots \ldots \ldots \ldots \ldots[p \times 1]$

$x \quad$ Independent variable, first used in Equation 4.19, Page 90.

y Output vector, first used in Equation 2.7, Page 21.......... $[q \times 1]$

$\square_{a} \quad$ Subscript refers to artery (SCM), first used in Equation 2.40, Page 41.

$\square_{a 0} \quad$ Subscript refers to aorta (DCM), first used in Equation 2.41, Page 41.

$\square_{\text {incn }}$ Subscript refers to inlet cannula, first used in Equation 2.38, Page 39. 
$\square_{\text {otcn }}$ Subscript refers to outlet cannula, first used in Equation 2.39, Page 39.

$\square_{v n} \quad$ Subscript refers to veins, first used in Equation 2.44, Page 47. 


\section{Chapter 1}

\section{Introduction}

A

$T$ the outset, the stated goal of this research was "to develop a better understanding of the dynamic interaction between circulatory assist devices and the human circulatory system." Although the route proved different from the one originally envisioned, the aim was met, and several very interesting (unanticipated) results should provide a strong basis for future work.

\subsection{Motivation}

Heart disease remains the leading cause of death in North America, ${ }^{*}$ and many of the people who die of this illness each year would have been candidates for heart transplant. ${ }^{\dagger}$ Unfortunately, donor organs are few and the transplant waiting list is long; this organ shortage, coupled with the limitations of transplant, provides incentive to develop drug or mechanical alternatives to heart transplantation.

There are two main types of mechanical artificial heart: total artificial hearts, and ventricular assist devices (Kolff, 1990). Total artificial hearts (TAHs)are designed to move blood through the pulmonary loop as well as the systemic loop, and have two artificial ventricles. The blood flows into the TAH's right ventricle (via cannula) from the right atrium of the natural heart or from the vena cava, and the blood flows into the TAH's left ventricle from the left atrium of the natural heart. Such a complex mechanism is often unnecessary, as many cases of cardiovascular dysfunction involve a damaged left ventricle with a relatively healthy right ventricle. These cases can be treated by a ventricular assist device

\footnotetext{
"Statistics Canada, "Causes of Death 2002" (released 2004), and American Heart Association, "Heart Disease and Stroke Statistics" (2006 Update).

${ }^{+}$Estimates vary from tens of thousands to hundreds of thousands in North America alone.
} 
(VAD), which is designed to provide assistance to the natural heart. The blood flows into the VAD from the left ventricle of the natural heart, and its output cannula is attached to the aorta. The VAD energizes the blood by some means and ejects it into the aorta, assisting the natural heart by providing high-pressure blood flow into the circulatory system. Implant of a VAD (as opposed to a TAH) allows for the possibility of recovery; the natural heart is not removed when a VAD is installed.

Understanding the potential interaction between the VAD (or TAH) and the human circulatory system is important to engineers working on the devices as well as government regulatory agencies (among others) for a variety of reasons. From a control standpoint, a thorough understanding of the dynamics ensures predictability of the controller. Designing a controller for a complex system without an understanding of the system's subtle nuances is unwise. There are also VAD (or TAH) performance issues; the energy efficiency of the implanted device is an important concern, because external power is not always available, and there is a practical limit to the external power which might be safely transfered into the body. Understanding the interaction between the device and the circulatory system can serve to make it more efficient, reducing its power requirements.

VAD reliability (or, rather, predictability) is also a concern. While the concept of mechanical reliability is understood by most people, the idea of "predictability" is more abstract. In complex dynamic systems there are often so-called "basins of attraction" which can be thought of as local minima. If the system is sufficiently perturbed it can move to a different basin of attraction, one with a different local minimum, one where the behaviour might not be understood or controllable (Nayfeh and Balachandran, 1995). The aim of TAH or VAD development is to allow the patient to lead a "normal" life. This means that the device must reliably pump blood while the recipient pursues a variety of activities, some of which will noticeably perturb the system dynamics as the circulatory system parameters vary.

Chaotic behaviour would also be a worry. This catch-all description refers to behaviour which appears random but is in fact deterministic. The state trajectories lie on a strange attractor, often tracing a fairly closed path, but never returning to the same value. For all intents and purposes the beating of the artificial heart would become random. 


\subsection{Approach}

The work presented herein involved two complementary approaches, one theoretical and the other experimental. The fundamental objective was to gain a better understanding of circulatory system dynamics, and a mathematical treatment of the human circulatory system went a long way towards that end. However, as with any engineering project, artificial hearts are not designed in a theoretical vacuum, or without suitable prototyping. Besides the obvious reliability concerns, there are also installation issues that must be dealt with. (For example, will a certain type of connector prove difficult to manipulate during surgical implantation?)

Much total artificial heart and left ventricular assist device (LVAD) development work is performed in vitro, using so-called "mock circulatory systems." The mock circulatory system (MCS) provides short- and long-term performance and reliability data, which is key to the development process, however most MCS configurations allow for limited study of the actual circulation blood flow dynamics. A mathematical model of the human circulatory system (or even the MCS) complements this in vitro testing, as it permits the study of features and behaviours that would be impractical to investigate using a physical mock circulatory system. For example, model components can be added or removed, and their parameters varied with relative ease. Results for a wide range of situations can be found quickly by simulation thus identifying areas for productive experimental study.

\subsection{Thesis Overview}

This thesis presents the results of a study that has examined the dynamics of the human circulatory system as it relates to artificial heart development. The approach involved both theoretical and experimental aspects; artificial hearts are tested in vitro using mock circulatory systems which, although they provide decent reliability testbeds, do not, as we will show, accurately model the dynamics of the human circulatory system.

The theoretical and experimental components of the work are presented in separate chapters (Chapter 4 and Chapter 5, respectively) even though they were developed in tandem. There is some overlap between the two chapters, but each has a slightly different focus. Chapter 2 provides much of the requisite background material, including the 
development of the overall human circulatory system model, which forms the basis of all subsequent circulatory system simulations.

An overview of the literature related to the work is presented in Chapter 3; circulatory system dynamics, mock circulatory system development, and nonlinear dynamic systems are the three main foci. There are many circulatory system models in the literature and there is an ongoing debate as to whether or not the human circulatory system exhibits chaotic behaviour. Information on the human circulatory system is scattered among technical and non-technical journals. Empirical knowledge from a nursing journal (for example) can have significant influence when viewed in an engineering context.

Similarly, there are many mock circulatory systems described in the literature, and much of this development work seems to have been performed in apparent isolation. As we will see in Section 3.2, there are three distinct mock circulatory system branches, tracing back to 1959 when the first system was proposed. The lack of dissemination between these three projections may be a major contributing factor to the slow rate of development; many of the mock circulatory systems share common traits but (as will be seen in Section 4.2) few of them feature a representative arterial model.

Another portion of the literature review, Section 3.4, briefly outlines a nonlinear dynamic system known as an "impact oscillator." In Chapter 4 it is suggested that this class of behaviour is responsible for much of the nonlinear behaviour in the human circulatory system.

Chapter 4 details the theoretical work on the human circulatory system model, with some of the supporting calculations presented in appendices. The experimental work, which is outlined in Chapter 5, was performed in two stages, one using a ventricular assist device connected to a mock circulatory system, and the other using a different pump connected to the same mock circulatory system.

The experimental results support the theoretical predictions and have implications for both artificial heart and mock circulatory system design, as well as the understanding of human physiology. These implications are discussed in Chapter 6 which provides a 
general overview of the findings and their significance. Finally, Chapter 7 summarizes the conclusions from this work and suggests avenues for future work.

At the end of the thesis is an index which should help with cross-referencing and supplement the table of contents. 


\section{Chapter 2}

\section{Background}

$\mathbf{T}$ HS research straddles multiple disciplines, and might be of interest to people not necessarily versed in the requisite background material. Not all engineers are familiar with the workings of the human circulatory system, and not all medical professionals are comfortable with mathematical modelling or dynamics. In an effort to reach a wider audience, this chapter is included as an introduction to the applicable fields.

\subsection{Medical}

The next few sections will briefly outline some of the required circulatory system anatomy, including heart and blood vessel configuration. A basic understanding of the human circulatory system is necessary in order to appreciate the complexity of the body, and to gauge the fidelity of the mathematical modelling efforts (both in this work and in the literature at large). The cardiac cycle will be reviewed, as certain aspects of this sequence are used by researchers and medical personnel to analyze overall circulatory system behaviour. Finally, we will review some key concepts related to ventricular assist devices and total artificial hearts.

\subsubsection{The Heart}

It makes sense to begin a review of the human circulatory system at the central element, namely the heart. The heart, illustrated in Figure 2.1, is conical (or tapered) in shape, and enclosed in a protective membrane called the pericardium, which is not included in the figure. The heart is a hollow, muscular organ with the muscles arranged in spiral and circular bundles around the four internal chambers. The adult heart is approximately $12 \mathrm{~cm}$ long, and $9 \mathrm{~cm}$ in diameter at its widest part (Gray, 1858). Blood vessels (namely the coronary arteries and veins) are visible on the surface of the heart. 
Multiple vessels connect to the heart. The arteries* distribute blood pumped from the heart, with the aorta serving the body and the pulmonary artery feeding the lungs. The veins are responsible for the return of blood, with the superior vena cava ${ }^{\dagger}$ bringing blood from the head and anterior part of the body, the inferior vena cava returning blood from the lower part of the body, and the pulmonary veins retrieving oxygenated blood from the lungs. The blood vessels will be discussed in further detail after the treatment of the heart is complete.

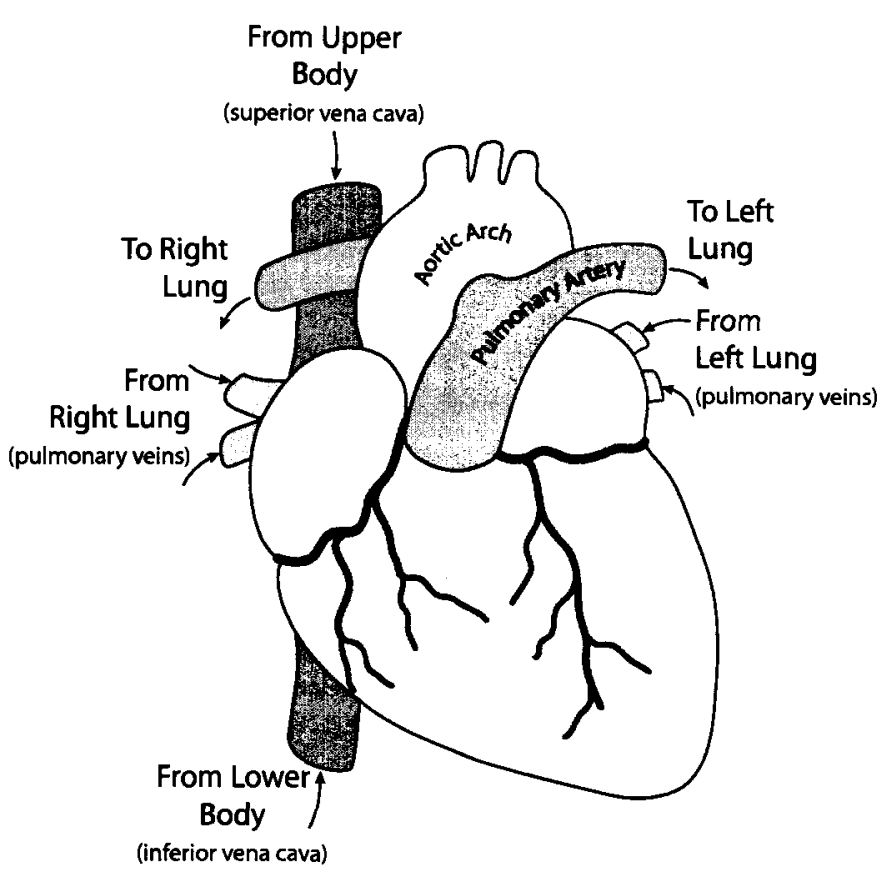

Figure 2.1: Diagram of the heart (see, for example, Gray, 1858)

A cross-section of the heart is illustrated in Figure 2.2. A longitudinal muscular wall divides the heart into two lateral halves, which are labelled "left" and "right" based on their relative positions in the chest cavity. Each half is divided in two by a transverse constriction. The upper vacuities are called the atria (or auricles) and the lower are called the ventricles. Each quadrant of the heart can be identified in this fashion (for example "right atrium" or "left ventricle").

The right atrium is slightly larger than the left, and its walls are slightly thinner. It has two openings to blood vessels where the superior and inferior venae cavae return deoxygenated blood from the upper and lower parts of the body (respectively). It also has valves to prevent regurgitation. The right atrium is separated from the right ventricle by a tricuspid valve. This valve, one of two atrioventricular (AV) valves in the heart, is

*From the Greek "arteria" meaning "windpipe"; the ancients believed they contained air (Gray, 1858).

tVena cava means literally "hollow vein" (etymology: New Latin) and "superior" is a medical coordinate direction (Merriam-Webster). 
composed of three trapezoidal membranes reinforced with fibrous tissue. These valve "leaves" are connected at their bases to the atrial-ventricular orifice, and along their sides to each other. There are fine tendons (not shown in Figure 2.2) which tie the valve leaves to the interior of the ventricle, and prevent them from reversing their orientation when the ventricle pressurizes.

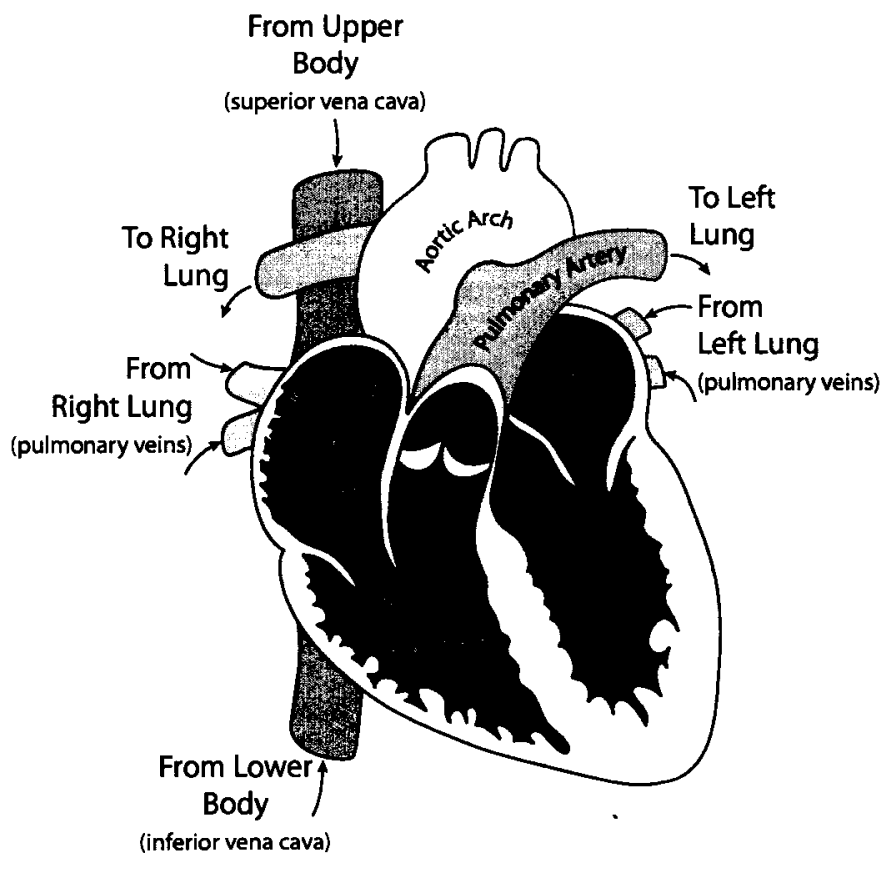

Figure 2.2: Cross-section of the heart (see, for example, Gray, 1858)
The right ventricle itself is nearly triangular in shape. Near its upper peak, beside and slightly in front of the atrial-ventricular orifice, it has an opening connecting to the pulmonary artery. The pulmonary semilunar (SL) valve, featuring three convex leaves, prevents regurgitation from the pulmonary artery into the right ventricle; it opens under a positive pressure gradient to allow blood ejection from the right ventricle into the pulmonary artery, but is normally closed as shown in the illustration.

The left atrium is smaller than the right atrium, but has a thicker wall. It also has four (rather than two) openings to blood vessels where the pulmonary veins return oxygenated blood from the lungs. Two of those openings are illustrated in Figure 2.2; the other two openings, connecting to the pulmonary veins labelled "from right lung" are obscured, but are situated on the right side of the chamber, or the left side of the illustration. The left atrium is separated from the left ventricle by an atrioventricular valve called the mitral valve. This valve, unlike the AV valve in the right side of the heart, has only two flexible flaps; it is bicuspid, not tricuspid. As with the other atrioventricular valve, there are fibrous tendons which maintain the cusps in their proper orientation. 
The left ventricle is similar to the right ventricle in shape and layout, but is slightly larger. Its walls are much thicker than the walls of the right ventricle, as might be expected; the left ventricle is responsible for ejecting high-pressure blood into the systemic side of the circulatory system. Near the top of the left ventricle (and obscured in Figure 2.2 by the pulmonary artery) is the opening to the aorta. As with the pulmonary artery, a semilunar valve prevents regurgitation.

\subsubsection{Heart Disease}

Heart disease remains the leading cause of death in North America.* Of the people dying of heart disease each year, approximately half would be candidates for heart transplant, because the degeneration of the heart occurs sufficiently slowly. ${ }^{+}$Treatable conditions include congestive heart failure and coronary heart disease. Congestive heart failure is a condition where the heart loses its pumping ability, normally due to prolonged high blood pressure, or heart attack. This leads to fluid accumulation in the body tissues, in particular the lungs and legs. Coronary heart disease involves a reduction in blood supply to the heart, through a narrowing of the coronary arteries due to atherosclerosis. $\ddagger$ The reduced nutrient supply weakens the heart muscle and can also lead to a heart attack.

Often a heart attack is the subitaneous event which leads to a diagnosis of heart disease. In this case, medical personnel have a variable period in which to intervene; some situations require attention within hours, some days and some weeks. Donor hearts (for transplantation) become available at a fraction of the required rate, and there is a strong impetus to develop drug or mechanical alternatives to heart transplantation.

The body's sympathetic response to a heart attack involves elevating the mean blood pressure, and instructing the renal system to retain water (Tortora and Grabowski, 2000). In the short-term this effect is beneficial, but over an extended period of time, the elevated blood pressure further damages the heart muscle.

\footnotetext{
*Statistics Canada, "Causes of Death 2002" (released 2004), and American Heart Association, "Heart Disease and Stroke Statistics" (2006 Update).

${ }^{+}$The other deaths occur suddenly; no intervention is possible.

$\ddagger$ Merriam-Webster: Degeneration of the inner coat of the arteries due to fatty plaque deposits.
} 
Diseased or damaged hearts often do not fail completely. In cases of congestive heart failure due to prolonged high blood pressure the left ventricle typically sustains more damage than the rest of the organ. This is because the left ventricle is responsible for the high-pressure systemic circulation, whereas the right half of the heart services the lower pressure pulmonary loop, and is less likely to be damaged by prolonged high blood pressure.

\subsubsection{The Cardiac Cycle}

The cardiac cycle is most often presented in the form of an electrocardiograph, or ECG.* An ECG is a graph of the electrical activity of the heart, and can show abnormal sinus rhythms ${ }^{\dagger}$ or detect heart muscle damage. A stylized version of a normal ECG is shown in Figure 2.3. Each peak corresponds to a certain portion of the regular cardiac cycle.

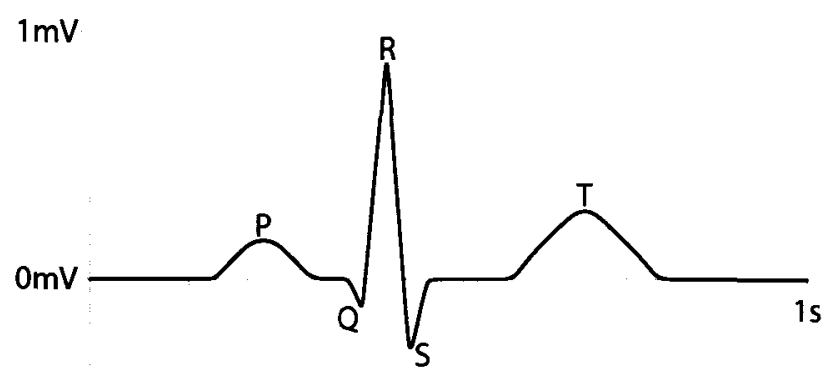

$-0.5 \mathrm{mV}$

Figure 2.3: PQRST wave (see, for example, Tortora and Grabowski, 2000)

The P-wave signals atrial depolarization, which is the phase of the cardiac cycle when the atria contract. This is illustrated in Figure 2.4. Note that during this portion of the cardiac cycle the semilunar valves are closed, preventing blood regurgitation from the arteries. Blood moves from the atria into the ventricles.

The next portion of the ECG graph is termed the QRS-complex, and corresponds to ventricular depolarization. It is illustrated in Figure 2.5. During this portion of the cycle, the semilunar valves are open, and the atrioventricular valves are closed. The fibrous tendons attached to the leaves of the $\mathrm{AV}$ valves prevent them from reversing into their respective atria.

\footnotetext{
*Also called EKG (from the original German Elektrokardiogramm).

tThe term "sinus rhythm" is used to refer to the normal, regular cardiac rhythm, which is stimulated by the sinoatrial node. (The heart's pacemaker.) See Merriam-Webster.
} 


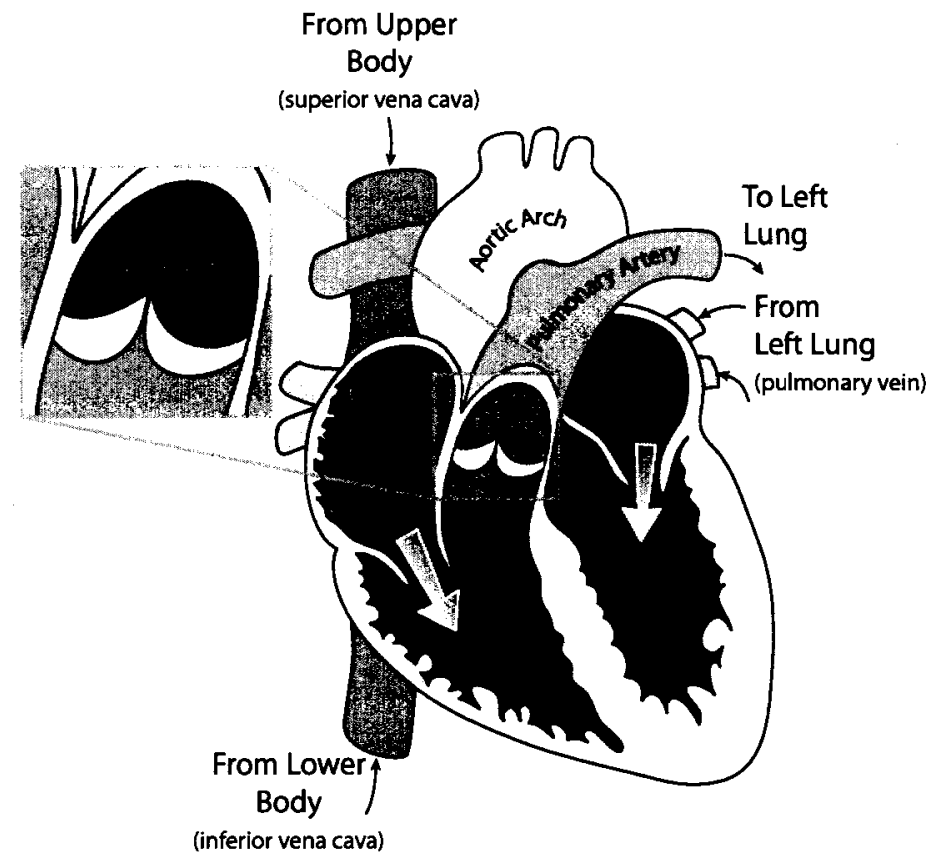

Figure 2.4: Blood flow during atrial depolarization (P-wave)

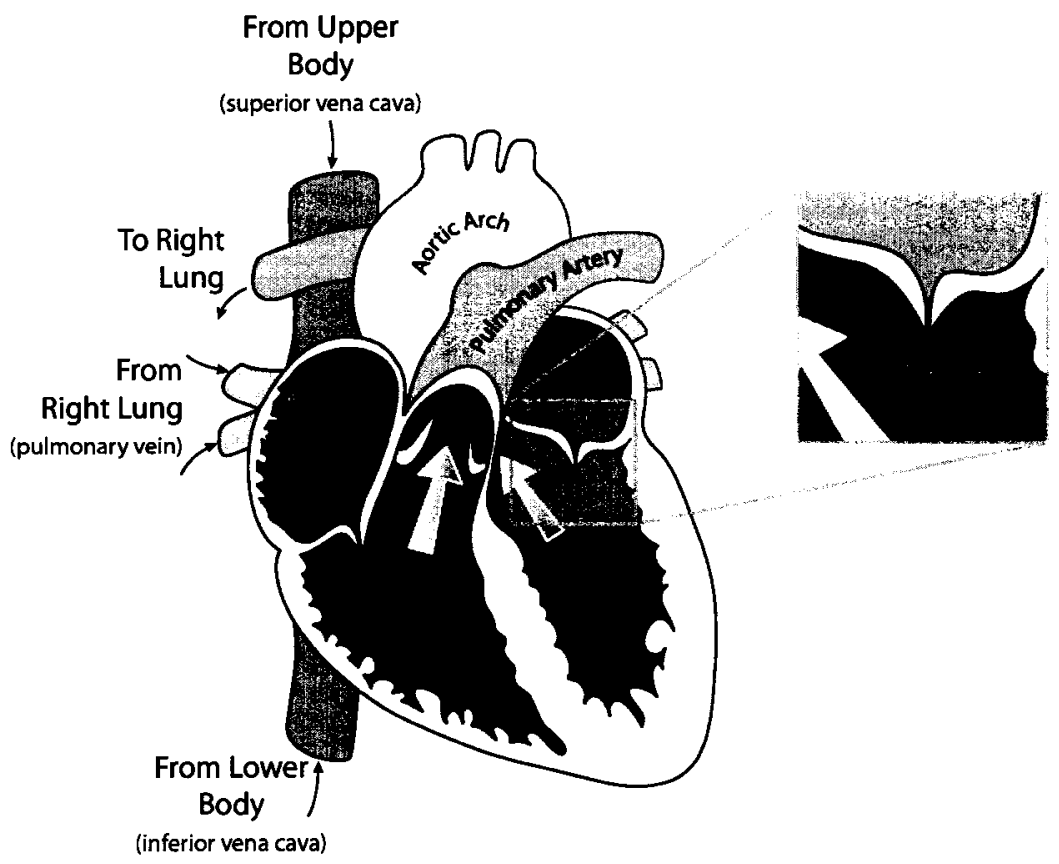

Figure 2.5: Blood flow during ventricular depolarization (QRS-complex) 
The phase of the cardiac cycle which corresponds to ventricular ejection is called systole, and the remainder of the cycle is called diastole. Referring back to Figure 2.3, systole spans from " $R$ " to " $T$ " and diastole from " $T$ " to " $R$ " in the next cardiac cycle. Often people speak of "R-R intervals"; the " $R$ " spike is the largest, most obvious feature on an ECG, and it is a convenient reference for describing variations in the sinus rhythm.

\subsubsection{The Circulation}

The vessels in the human circulatory system form two "loops" (Fung, 1997). The pulmonary loop* transports deoxygenated blood from the right ventricle to the lungs (via the pulmonary artery) and re-oxygenated blood from the lungs to the left atrium (via the pulmonary veins). The systemic loop ${ }^{\dagger}$ distributes high-pressure blood from the left ventricle to the body (via the aorta, associated arterial vessels and capillaries) and returns lowpressure, deoxygenated blood from the body to the right atrium (via the venules, veins and venae cavae).

A generic blood vessel cross-section (Tortora and Grabowski, 2000) is shown in Figure 2.6, and the following discussion will revolve around which of the identified layers are present (or missing) from the various vessels in the body.

We will begin with the vessels proximal to the heart, and follow the systemic circulation back around. The aorta and larger arteries consist of three layers (known as tunics), as illustrated in Figure 2.6. The internal layer has endothelial cells which are in contact with the blood ${ }^{\ddagger}$ and a reinforcing subendothelial layer. The medial layer consists of elastic fibres and smooth muscle, and is typically the thickest of the three layers. The ratios of elastic fibres and smooth muscle vary, as will be seen shortly. The external layer consists of elastic and collagenous fibres.

The very large arteries are known as the conducting arteries. These include the aorta, and the major arteries which branch off from the aorta. Their diameters are relatively large, normally $1-3 \mathrm{~cm}$, and their walls are thin relative to their diameters. $\S$ Their medial

\footnotetext{
*Also referred to as the pulmonary circulation, or lesser circulation.

${ }^{+}$Also referred to as the systemic circulation or greater circulation.

FThis is true with all of the vessels, including the capillaries.

$\S$ They are thin-walled vessels in the engineering sense.
} 


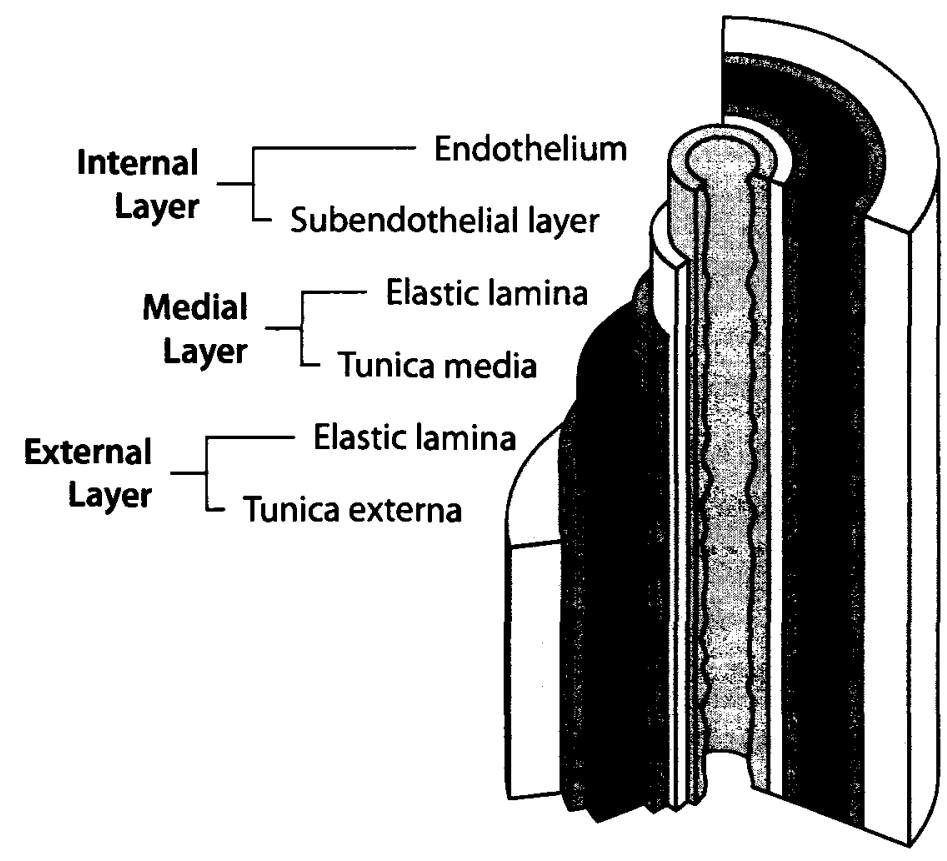

Figure 2.6: Blood vessel cross-section (see, for example, Tortora, 2000)

layers have a larger proportion of elastic fibres and less smooth muscle. The conducting arteries are responsible for absorbing the large changes in blood pressure (the heart ejects blood at a high pressure) and smoothing the blood pressure waveform while distributing it throughout the body. They have low flow resistance and limited vasoconstriction.*

The mid-sized arteries (with diameters $0.3-1 \mathrm{~cm}$ ) are called muscular or distributing arteries. They branch off from the conducting arteries and supply the organs and the muscles. Their medial layer has more smooth muscle and less elastic material. They are capable of vasodilation and vasoconstriction, regulating the blood flow to the various parts of the body.

The arterioles are the smallest of the arteries (with diameters $0.003-0.3 \mathrm{~cm}$ ) and control the flow of blood into the capillary beds. They have the same three tunic layers as the larger arteries, but the middle layer is composed of more smooth muscle than elastic fibres. They are also capable of dilation and constriction.

${ }^{*}$ Merriam-Webster: Narrowing of the lumen of blood vessels especially as a result of vasomotor action. 
The capillaries are microscopic vessels that are found near every cell in the body. They have only one layer, composed of endothelial cells, and are responsible for exchanging nutrients and waste between the blood and surrounding tissue. They have multiple openings to allow material to pass through their walls.

The deoxygenated blood moves through the venules into the veins. Venules are microscopic vessels (similar to capillaries). The veins are similar to the distributing arteries, but they have less elastic material and more smooth muscle in their medial and external layers. They also operate at a much lower pressure, and often have valves to prevent back-flow. (These valves are not illustrated in Figure 2.6; they would be in the inner lumen.)

The larger veins are called venae cavae. They operate at very low internal pressures, and (similar to the conducting arteries) have thin walls. The venae cavae are responsible for collecting the blood from the veins and delivering it into the right atrium of the heart.

Table 2.1 summarizes the estimates of vessel properties (such as cross sectional area, and length) available in the literature.

\subsubsection{VADs and TAHs}

As noted in Section 2.1.2 (Heart Disease), the heart transplant waiting list is long, and donor organs few. Researchers and doctors have been searching for a mechanical heart replacement for over a century, ${ }^{*}$ but only in the last 25 years have the technology and understanding begun to approach the required levels.

At first blush, the concept of an artificial heart is quite simple: the body needs a pump to circulate blood through both the pulmonary and systemic circulatory loops. This seemingly modest requirement is complicated by both the anatomy and the physiology of the body. The structural (anatomical) concerns are perhaps the most obvious: there is not a lot of free space inside the body, and an internal heart replacement is therefore restricted in terms of size. It is also restricted in terms of configuration; it needs to accept blood from the body's venous system(s), apply a motive force, and deliver pressurized blood into the arteries. As well, the device must be implantable, meaning that surgeons must be able to

\footnotetext{
${ }^{*}$ In the late 1800 s and early 1900 s there were already discussions on the topic.
} 


\begin{tabular}{|r||c|c|c|c|c|c|c|}
\hline & Aorta & Arteries & Arterioles & Capillaries & Venules & Veins & Venae cavae \\
\hline \hline Diameter $(\mathrm{cm})$ & $2.5^{a, b}$ & $0.4^{a}$ & $0.003^{a}$ & $0.0008^{a}$ & $0.002^{a}$ & $0.5^{a}$ & $3.0^{a}$ \\
\hline Length $(\mathrm{cm})$ & 20 & 30 & - & 30 & - & 30 & 50 \\
\hline Velocity $\left(\mathrm{cm} \cdot \mathrm{s}^{-1}\right)$ & $40^{a, c}$ & $10-\left\{40^{a}, 50^{c}\right\}$ & $0.1^{a}$ & $0.03^{a}$ & $0.3^{a}$ & $0.3-5^{a}$ & $5-20^{a, c}$ \\
\hline Volume $(L)$ & $0.15^{a}$ & $0.65^{a}$ & $0.15^{a}$ & $0.2^{a}$ & $0.15^{a}$ & $2.55^{a}$ & $0.5^{a}$ \\
\hline Pressure $(\mathrm{mmHg})$ & $80-120^{a, d}$ & $40-110^{c}$ & $25-40^{a, c}$ & $10-25^{d}$ & $8-12^{a}$ & $5-10^{a}$ & $2-5^{a, c}$ \\
\hline Resistance $\left(N \cdot \mathrm{s} \cdot \mathrm{cm}^{-5}\right)$ & 0.0053 & 0.0013 & 0.0036 & 0.0027 & 0.0012 & 0.00032 & 0.0004 \\
\hline Inertance $\left(N \cdot \mathrm{s}^{2} \cdot \mathrm{cm}^{-5}\right)$ & $2.4 \times 10^{-4}$ & $1.5 \times 10^{-5}$ & - & $1.2 \times 10^{-7}$ & - & $3.8 \times 10^{-6}$ & $6.3 \times 10^{-5}$ \\
\hline
\end{tabular}

${ }^{a}$ Tortora and Grabowski (2000)

${ }^{b}$ Gray (1858)

${ }^{c}$ Fung (1997)

${ }^{d}$ Guyton (1992)

Table 2.1: Properties of vessels in the circulatory system 
make the necessary connections without putting the patient's life at risk. The installation process cannot be overly complicated.

The physiological concerns are more subtle. The body rabidly attacks internal foreign objects, necessitating research in biocompatible materials (which must still possess the necessary mechanical properties). Blood itself is a non-Newtonian fluid with cells in liquid suspension. One of the blood's "jobs" is to clot to seal punctures, stopping blood loss and minimizing infection. A side effect of this behaviour is an excitability of sorts; blood will form clots on non-endothelial surfaces, and it will also form clots in other areas if its flowpatterns are sufficiently disturbed. Another more obscure requirement further complicates the engineering: the conducting arteries require a pulsatile blood flow, or their structure begins to deteriorate according to Maeta and Hori (1985). This requirement precludes any continuous-flow pump designs, which in turn complicates the device, as reciprocating mechanisms present an engineering challenge.

At the present time, there are two main categories of mechanical heart. The first, so-called total artificial hearts, replace the natural heart outright. They are responsible for circulating blood through both the pulmonary and systemic loops. The second variety are known as ventricular assist devices. These VADs supplement the pumping of the natural heart, helping the heart move blood through the systemic loop. At the outset artificial hearts were used as bridges to transplantation (while the patient waited for a donor organ) but a few have now been approved for long-term implant in patients.

A generalized VAD is illustrated in Figure 2.7. Blood flows into the inlet port, is energized in some fashion, and is ejected at high

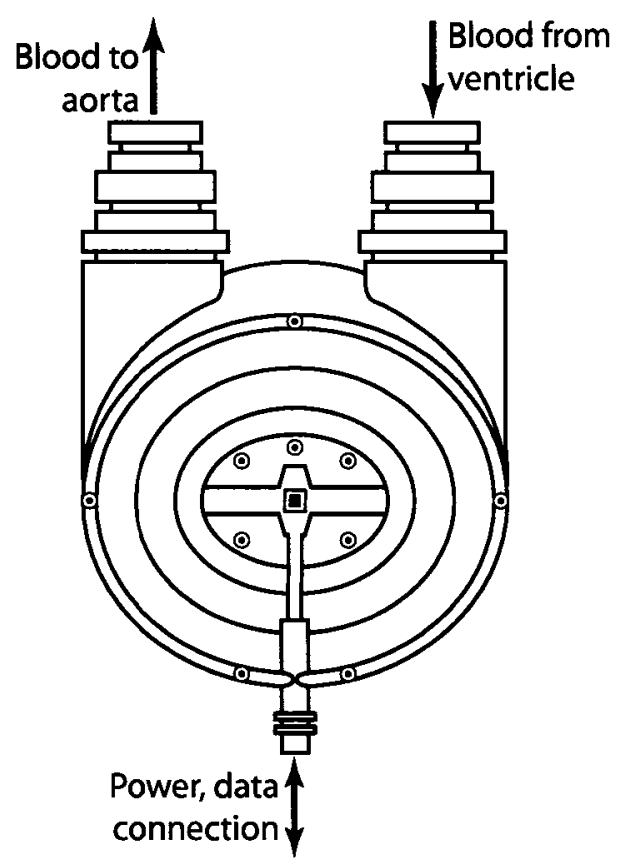
pressure through the outlet port. Some sort of

Figure 2.7: VAD illustration 
power cable provides the necessary electrical energy, and a data signal (to allow monitoring and control) is retrieved. A TAH would look similar, but would have four ports instead of two (i.e., two inlet ports and two outlet ports) and two separate chambers where the blood was admitted, pressurized and ejected. Also, two VADs (which individually represent "half a heart") can be coupled to produce a TAH setup, with one VAD circulating blood through the pulmonary loop and the other circulating it through the systemic loop. Obviously the VAD supplying the pulmonary loop would require modifications to filling and ejection pressures. Ports on the devices are normally oriented to facilitate installation in the body, with the inlet and outlet aligned with the appropriate blood vessels.

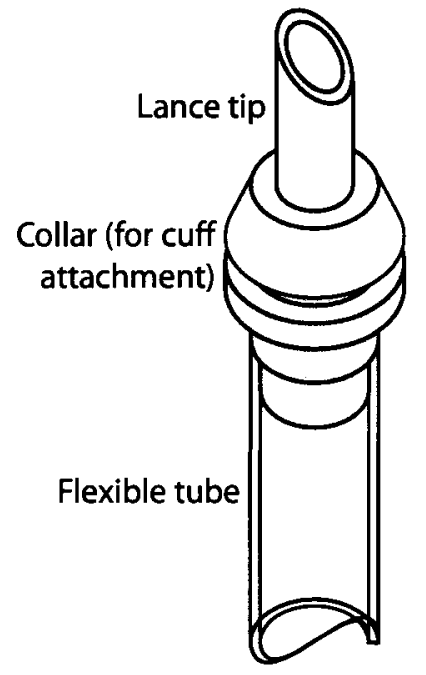

Figure 2.8: Cannula illustration

The actual pumping mechanisms vary. Each artificial heart or assist device has its own unique take on mechanisms to motivate blood flow. Common traits include smooth, simple flow paths and biocompatible materials throughout. Blood is typically contained in some sort of sac, or separated from the mechanical side of the device by some sort of membrane. Modern total artificial hearts and ventricular assist devices are implanted in the torso, along with a short-term power source and some sort of controller. This allows the recipient to forgo the normal external power source for brief periods of time. A connection to an external power source is made either by passing leads through a permanent hole in the skin or by some other means. Often there is an external controller which will let the patient dial in different activity levels, and allow doctors to collect operational data. External power can either be supplied through a wall outlet, or through external batteries, which are often worn in a vest or belt around the waist.

Inside the body, connections to the blood vessels are made using cannulae.* A typical cannula is illustrated in Figure 2.8. The stiff tip is often tapered, which makes it easier to pierce the tissue. Cuff material is attached to the collar and sutured to hold the cannula

\footnotetext{
${ }^{*}$ Cannulae are flexible tubes.
} 
in place. Flexible tubes carry blood from the inlet or outlet ports to or from the appropriate blood vessel. Although the tube in the illustration is smooth-walled, cannulae walls are often ridged. Some cannulae are made from very fine mesh-like material, which is reinforced with circumferential stiff plastic rings for rigidity. This type of cannula leaks initially, until the blood clots and fills in the holes in the mesh. The reinforcing rings give the inner and outer surfaces a ribbed texture, and a large circumferential stiffness. Any changes in geometry in a ribbed cannula would be confined to the axial direction.

During TAH installation, the natural heart is often removed from the chest cavity, and connections are made directly to the appropriate vessels. Recall that a total artificial heart delivers blood to both the pulmonary and systemic loops; it completely replaces the natural heart. With a ventricular assist device, on the other hand, the natural heart retains the responsibility of moving blood through the pulmonary loop but receives assistance for pumping the blood through the systemic loop. The inlet port of the VAD is connected to either the left ventricle (as shown in Figure 2.9) or the left atrium. The outlet port is connected to the aorta, typically at the aortic arch. Fig-

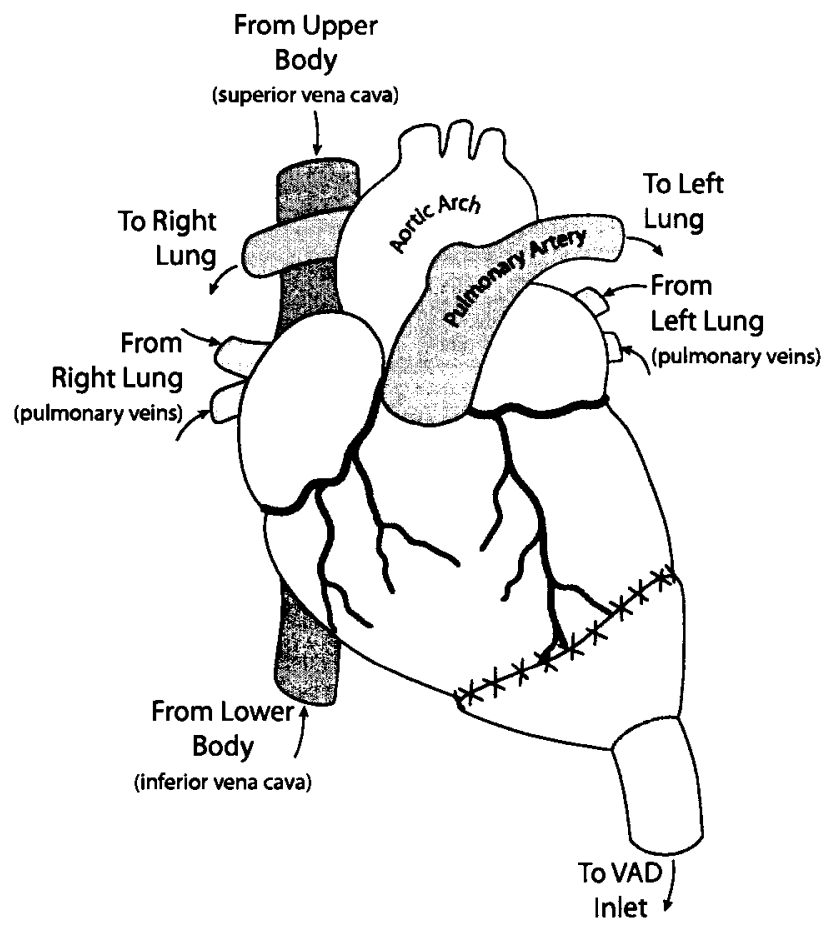

Figure 2.9: Diagram of the heart with VAD cuff attached ure 2.9 does not illustrate the outlet cannula connection to the aortic arch although the arch itself is labelled.

Whether or not the left ventricle is allowed to beat after VAD installation appears to be dependent on the particular device. Research has shown that an unloaded left ventricle can begin to recover from heart disease under VAD assistance. The recovery may never be 
complete, but this convalescent behaviour would favour the retention of full left ventricle function, even though it might complicate the VAD control dynamics.

\subsection{Dynamics}

In order to study the behaviour of physical systems, ${ }^{*}$ either analytically using mathematics or computationally using a computer, one needs a way of describing the system's behaviour in terms of equations. The foundation of this modelling process is Newton's Second Law, ${ }^{\dagger}$

$$
\Sigma \boldsymbol{F}=m \cdot \boldsymbol{a}
$$

which describes the relationship between forces acting on an object and the resultant motion of said object. It holds in all inertial ${ }^{\ddagger}$ frames of reference, and direct application results in a second order differential equation which can be integrated in time.

Figure 2.10 shows a simple mass in a gravitational field. For the purposes of this discussion, we will assume the mass is near the surface of the Earth and its weight is therefore $m \cdot g$ where $g=9.8 \mathrm{~m} \cdot \mathrm{s}^{-2}$, the gravitational acceleration near the Earth's surface. $\$$ This force of weight pulling in the direction of $g$ is the only force acting on the mass, therefore based on Equation (2.1) we would expect the mass to accelerate towards the ground at $9.8 \mathrm{~m} \cdot \mathrm{s}^{-2}$ (i.e., fall with an increasingly faster

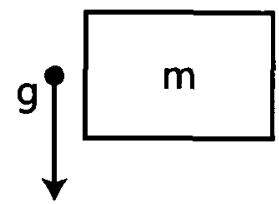

Figure 2.10: Mass in a gravitational field velocity). ${ }^{\text {I }}$

${ }^{*}$ A system is a combination of components which act together towards a specific objective. (Ogata, 1992.)

t The study of Newtonian mechanics, and its deficiencies, has led to many interesting developments including general relativity and quantum mechanics.

$\ddagger$ Non-accelerating.

\$This is determined from Newton's Law of Gravitation, $g \equiv G \frac{M_{F}}{R_{E}^{2}}$ where $G$ (Newton's Constant) is $66.73 \times$ $10^{-12} \mathrm{~m}^{3} \cdot \mathrm{kg}^{-1} \cdot \mathrm{s}^{-2}, M_{E}$ (the mass of the Earth) is $5.976 \times 10^{24} \mathrm{~kg}$, and $R_{E}$ (the radius of the Earth) is $6.378 \times$ $10^{6} \mathrm{~m}$.

II Newtonian mechanics there is no obvious connection between the gravitational mass of an object and the object's inertial mass. Einstein's General Theory of Relativity explained why these two masses were always observed to be numerically equal. 
Direct application of Equation (2.1) yields the following differential equation:*

$$
m \cdot g=m \cdot \frac{\mathrm{d}^{2} s}{\mathrm{~d} t}
$$

We can divide through by $m$ and solve the differential equation:

$$
s(t)=\frac{g \cdot t^{2}}{2}+t \cdot C_{1}+C_{2}
$$

Denoting the mass' initial position and velocity as $s_{0}$ and $v_{0}$ (respectively), we can solve for the constants of integration and arrive at the final form:

$$
s(t)=\frac{g \cdot t^{2}}{2}+t \cdot v_{0}+s_{0}
$$

This equation is simple and familiar; we will return to it in Section 2.5 (Dimensional Analysis).

A slightly more complicated system would be the mass-spring-damper system shown in Figure 2.11. Again the mass is in a gravitational field and has a gravitational force (weight), $m \cdot g$, pulling it downwards. The spring and the damper also apply forces to the mass. The spring supplies a force proportional to its compression or extension (relative to its unstretched length) and in the opposite sense. ${ }^{\dagger}$ The damper dissipates energy; it supplies a force proportional to the velocity of the mass but in the opposite sense. $\neq$

*The vertical coordinate direction is designated $s$, with down positive. This is to avoid confusing notation in an example a little further on.

${ }^{\dagger}$ In other words, a compressed spring always tries to decompress while a distended spring tries to contract.

IIn other words, when the mass is moving upwards the damper force is down; when the mass is moving downwards, the damper force is up. 


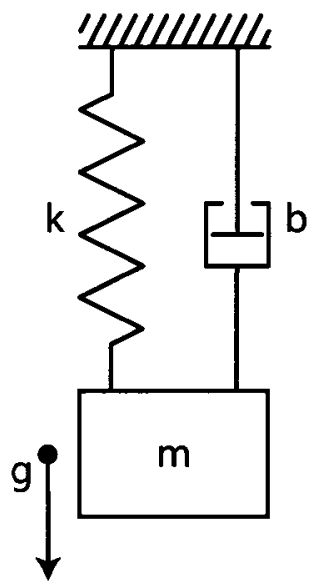

Figure 2.11: Unforced mass-spring-damper system

Applying Newton's Second Law directly, we arrive at the following differential equation:

$$
m \cdot \frac{\mathrm{d}^{2} s}{\mathrm{~d} t^{2}}+b \cdot \frac{\mathrm{d} s}{\mathrm{~d} t}+k \cdot s=m \cdot g
$$

Compared to Equation (2.2), Equation (2.5) is more difficult to solve analytically; it will be revisited in Chapter 4 when we take a closer look at the human arterial system.

In terms of computer simulation, the second order differential equations which result from a direct application of Newton's Second Law are not the most ideal formulation. Most discrete numerical integration schemes are only able to handle sets of simultaneous first order differential equations. For this reason, equations are often written in state space representation, ${ }^{*}$ as a vector equation.

$$
\dot{x}(t)=f(x, u, t)
$$

The p-dimensional vector function $f$ relates the system state $x$ and input $u$ to the first derivative of the state itself.

The output is written as:

$$
y(t)=g(x, u, t)
$$

The q-dimensional output is the result of a vector function $g$ operating on the system state and input. If the vector functions $f$ and $g$ do not explicitly involve time the system is said to be autonomous or time invariant; otherwise the system is nonautonomous. ${ }^{+}$

${ }^{*}$ Also referred to as state variable form.

${ }^{\dagger}$ The nonautonomous formulation is presented in Equation (2.6) and Equation (2.7), given that time is an explicit parameter in these two equations. 
If the system under study can be linearized, Equation (2.6) and Equation (2.7) can be written in matrix form, as follows:

$$
\begin{aligned}
& \dot{x}(t)=A \cdot x(t)+B \cdot u(t) \\
& y(t)=C \cdot x(t)+D \cdot u(t)
\end{aligned}
$$

Here $A$ is called the state matrix, $B$ is called the input matrix, $C$ is called the output matrix and $D$ is called the transmission matrix; $A$ is a square matrix, but the other three can be rectangular. All can be time-varying (for nonautonomous systems).

The characteristic polynomial* of the state matrix is:

$$
a(s)=\|s \cdot I-A\|
$$

The eigenvalues of the $\boldsymbol{A}$ matrix are the roots of the characteristic equation, or the solutions to:

$$
\|s \cdot I-A\|=0
$$

The transfer function of the system is defined as the ratio of the Laplace transform of the output to the Laplace transform of the input. The transfer function matrix, relating each system output to each system input is therefore:

$$
G(s)=D+\left(C \cdot(s \cdot I-A)^{-1}\right) \cdot B
$$

Returning to the mass-spring-damper system in Figure 2.11, we can write Equation (2.5) in state space form by substituting $v=\mathrm{ds} / \mathrm{dt}$. Then we have a system of two first-order differential equations:

$$
m \cdot \frac{\mathrm{d} v}{\mathrm{~d} t}+b \cdot v+k \cdot s=m \cdot g, \quad v=\frac{\mathrm{d} s}{\mathrm{~d} t}
$$

*A square matrix's characteristic polynomial encodes several of its important properties, most notably its eigenvalues, its determinant and its trace. $\lambda^{2}-\tau \cdot \lambda+\Delta$ where $\tau$ and $\Delta$ represent the trace and determinant (respectively) of the matrix $A$. 
In matrix form,

$$
\left(\begin{array}{c}
\dot{s} \\
\dot{v}
\end{array}\right)=\left(\begin{array}{rr}
0 & 1 \\
-\frac{k}{m} & -\frac{b}{m}
\end{array}\right) \cdot\left(\begin{array}{c}
s \\
v
\end{array}\right)+\left(\begin{array}{l}
0 \\
g
\end{array}\right)
$$

The output equation is given by

$$
y=\left(\begin{array}{ll}
1 & 0
\end{array}\right) \cdot\left(\begin{array}{l}
s \\
v
\end{array}\right)
$$

\subsection{Bond Graphs}

Bond graphs are a unique way of describing physical systems, simplifying discernment of the governing differential equations. A bond graph is a drawing in which the system's structure is readily visible; it consists of subsystems linked together by lines representing either signal or power flows, and is an excellent intermediate step in the system modelling process which sees real world phenomena distilled into a so-called ideal physical model through engineering decisions. (The ideal physical model is then rendered into mathematical equations.) By simplifying the discernment of the governing differential equations and amplifying the actual system structure, bond graphs aid the physical system modelling process. If desired, individual bond graph subsystems can be progressively refined into a myriad of smaller subsystems with their associated power or signal links.

\subsubsection{Bonds}

In a bond graph, the various subsystems are connected by directed* lines (called bonds) which represent either signal or power flows. These two types of links are differentiated by their respective arrowheads. The power bonds have half-arrowheads while the signal links ${ }^{\dagger}$ have full arrowheads. Signal bonds indicate an influence on the system from its environment with essentially zero power flow.

Power bonds, as their name implies, transfer power from one component or subsystem to another. They have associated with them two complementary variables called power variables whose product is the instantaneous power flowing along the link. In an electric circuit these would be voltage and current, in mechanical translation force and velocity,

*Each link has an arrowhead implying direction.

${ }^{\dagger}$ Signal links are also known as active bonds. 
in hydraulics pressure and volume flow rate, and so on. These variables are generalized as "effort" and "flow" variables, both of which are functions of time. The effort variable is written above or to the left of the link, and the flow variable is written below or to the right.

The power bonds have a short, perpendicular line (known as a causal stroke) at one end or the other. It indicates the direction of the effort signal.

\subsubsection{Basic Elements}

In a bond graph, the bonds connect to ports on the component elements. Each element can have single or multiple ports, and indeed they are often classified according to the number of ports they have. A small number of ports does not necessarily imply a simple subsystem. A wall outlet, for example, will present a single pair of effort and flow variables but the system on the other side of the outlet is very complex.

The simplest 1-port elements can either dissipate energy, store energy, or supply energy. The (normally) dissipative elements are known as resistors, and represented by an " $R$ " in a bond graph. Resistive elements have effort and flow variables that are related by a static function, which can be either linear or nonlinear. This is in fact the truer definition of a resistive bond graph element; sometimes R-elements are used to model a constitutive relation between effort and flow for a subsystem which can both supply and dissipate power, such as an electric motor. No matter the power direction, the effort and flow variables are always related by a static function.

There are two types of energy storing elements. The first is known as a capacitance and the second as an inertance (or inertia). A capacitance is a 1-port element (represented by a " $\mathrm{C}$ " in a bond graph) which has a static constitutive relation between an effort and a displacement. Physical examples would include a spring, a capacitor in an electrical circuit, or a hydraulic accumulator. These elements either store or inject energy without loss. Inertance elements are 1-port elements (represented by an " $\mathrm{I}$ " in a bond graph) which have a static constitutive relation between momentum and flow. Again, these elements store or inject energy without loss. In mechanical terms, a capacitance is connected with potential energy while an inertance is connected with kinetic energy. 
Energy-supplying 1-port elements are known as sources. Source elements can either supply effort or flow to a system. The wall outlet mentioned above supplies an $A C$ voltage to whatever is plugged in. Due to the sheer massiveness of the electrical grid, the voltage at the wall outlet is quite predictable for most levels of power demand. That is to say one can expect 120VAC (in North America) no matter the current draw (within reason, obviously). This sort of source would be represented as an effort source in a bond graph, and labelled "SE" short for "source of effort." The other source, known as a flow source, supplies a specified flow to the system, no matter the effort requirements. It is represented as "SF" in a bond graph, short for "source of flow." Obviously neither of these elements are particularly realistic; real devices are not sources, although they can often be approximated as sources over a certain range of parameters.*

There are two types of 2-port elements. For both elements power is conserved, meaning that the product of effort and flow on the input side equals the product of effort and flow on the output side. They differ in the way the inputs are transformed into the outputs. In a transformer (designated "TF" in a bond graph), the flow on the input side is scaled by the transformer modulus to produce the flow on the output side. To satisfy the laws of power conservation the effort at the input is divided by the transformer modulus to produce the effort at the output. In a gyrator (denoted as "GY" in a bond graph) the flow at the input side is scaled by the gyrator modulus to produce the effort at the output side, and the effort on the input side is divided by the gyrator modulus to produce the flow on the output side.

\subsubsection{Junctions}

There are two types of knots in bond graphs: zero-junctions and one-junctions. The former are known as flow junctions, ${ }^{\dagger}$ and the latter as effort junctions. ${ }^{\ddagger}$ Both junctions represent domain-independent generalizations of Kirchhoff's laws. The flow junction's symbol is a zero, with any number of bonds emanating from it. The efforts on all bonds of the 0-junction are the same, while the sum of all the flows vanishes, a generalization of Kirchoff's current law. The effort junction's symbol is a one, again with any number of

*The same can be said for any mathematical representation of a physical element; no spring is perfect, no resistor perfect, ...

${ }^{\dagger}$ Or common effort junctions.

$\ddagger$ Or common flow junctions. 
bonds stemming from it. The flows on all bonds of the 1-junction are the same, while the sum of the efforts vanishes, a generalization of Kirchoff's voltage law.

\subsubsection{Articulation}

The process of developing a bond graph model of a physical system, augmenting that archetype, and deriving the mathematical governing equations is too involved to present here. The reader is referred to the book by Karnopp et al. (1990) for further elaboration. It is hoped that the preceding (albeit brief) overview will allow readers to link the fully augmented bond graphs in this work with the corresponding differential equations. Variable names, numbering and subscripting are developed with this goal in mind.*

\subsection{Hydraulics}

For a first approximation, all vessels in the human circulatory system can be modelled as thin-walled, flexible tubes. Blood flow in much of the circulatory system is laminar, with a Reynolds number normally in the range between 2000-5000 (Fung, 1997). For many vessels the nominal cross sectional diameter is nearly constant over the length; most vessels branch before they taper too severely. The vessel cross-sectional diameter can be taken as constant in time, and the effect of gravity ignored if all vessel expansion occurs axially and the "patient" is lying down (respectively). If we are not being pedantic, the expansion or contraction of the vessels is equivalent to a change in blood density within our control volume, chosen as the blood vessel itself.

An idealized vessel cross-section is shown in Figure 2.12. The coordinates " $x$ " and " $r$ " designate the axial and radial directions, respectively. It can be shown that the shear varies linearly from the centreline $(\tau(0)=0)$ to the wall $\left(\tau(R)=\tau_{\max }\right)$ for both laminar and turbulent flows. If we assume no swirl flow ${ }^{\dagger}$ and the flow is fully developed, ${ }^{\ddagger} \tau=\mu \mathrm{du} / \mathrm{dr}$. Thus, laminar flow profile is parabolic with an axial velocity of $u=0$ at the wall (no slip condition) and $u=u_{\max }$ at the centreline.

*Appendix J presents a Mathematica routine which will generate state equations based on an XML bond graph description.

${ }^{\dagger}$ Circumferential variation in velocity.

¥The fluid velocity at a given radial distance does not change along the length of the vessel. 
By integrating the momentum differential equation for a fluid in cylindrical coordinates, and using the boundary condition at the centreline, we can find the shear distribution across the vessel, which in turn can be integrated using the boundary condition at the wall (no slip) to produce the exact solution of pressure drop for fully developed flow in a horizontal vessel:

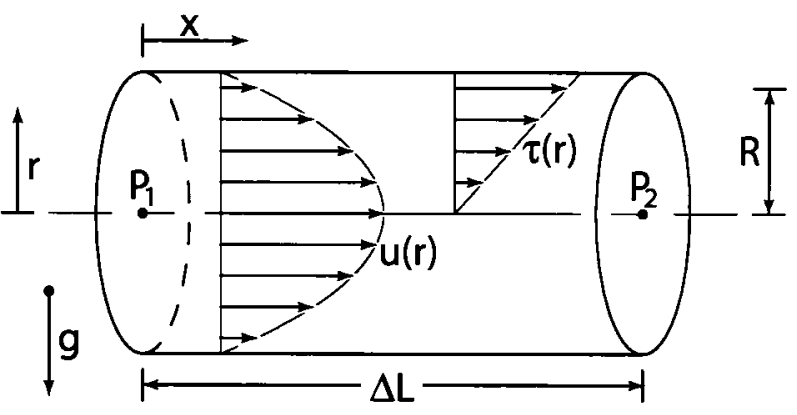

Figure 2.12: Tube

$$
\begin{aligned}
Q & =\int u d A \\
& =\int_{0}^{R} u_{\max }\left(1-\frac{r^{2}}{R^{2}}\right) 2 \pi r d r \\
& =\frac{\pi R^{4}}{8 \mu}\left(-\frac{d P}{d x}\right)
\end{aligned}
$$

From an energetic point of view, a vessel's flow resistance specifies the pressure drop across the segment as a function of flow rate:

$$
\begin{aligned}
\Delta P(Q) & =\frac{8 \mu}{\pi R^{4}} \cdot L \cdot Q \\
& =\frac{128 \mu}{\pi D^{4}} \cdot L \cdot Q
\end{aligned}
$$

The flow inertia in a segment of vessel describes the relationship between the fluid's momentum and flow rate:

$$
p(Q)=\frac{\rho \cdot L}{A} \cdot Q
$$

Similarly, a vessel's compliance (which is the inverse of stiffness) defines the change in volume for a given change in internal pressure:

$$
V(P)=C \cdot P
$$




\subsection{Dimensional Analysis}

Dimensional analysis is a process used to reduce the number of variables in a problem, in order to make it more tractable. It hinges on the Principle of Dimensional Homogeneity (PDH), which was apparently first stated by Fourier (Buckingham, 1914). When properly applied, dimensional analysis results in a generalized problem description featuring the smallest possible number of essential parameters.

The Principle of Dimensional Homogeneity states that all additive terms in an equation must have the same dimensions (i.e., you can't add apples and oranges). While this may seem self-evident, the implications are important; what follows from the PDH is that the units used to specify a dimension are irrelevant as long as they are internally consistent within a given problem description.

It is important to clarify some terminology at this point. The variables in a given problem, or set of equations represent a physical quantity. We would like to measure these quantities in a consistent set of units. In other words, we would like to quantify the variables in the problem using a set of fundamental or base units to measure the fundamental dimensions. The dimension of a physical quantity refers to the type of unit required to measure it. Common fundamental dimensions include mass $(m)$, length $(l)$, time $(t)$ and temperature $(\theta){ }^{*}$ The SI system of measurement uses the base units of kilogram $(\mathrm{kg})$, meter $(\mathrm{m})$, second $(s)$ and Kelvin $(K)^{\dagger}$ to gauge these fundamental dimensions.

\subsubsection{The Buckingham $\Pi$-Theorem}

The central theorem in dimensional analysis was set forth by Edgar Buckingham in a paper titled "On Physically Similar Systems" (Buckingham, 1914) and has since been labelled "Buckingham's $\Pi$-Theorem." The Greek character $\Pi$, which is the product symbol in mathematics, hints at the nature of the $\Pi$-theorem. The Buckingham $\Pi$-theorem states that:

If the solution to a problem depends on $n$ physical properties, the number of independent variables $v_{1}, v_{2}, \ldots, v_{n}$ can be reduced by the number of primary dimensions $r$ that appear in these physical properties by nondimensionalization

${ }^{*}$ Current, amount of substance, and luminous intensity would also be included here.

${ }^{\dagger}$ Ampere $(A)$, mole $(m o l)$, and candela $(c d)$ are the other base units. 
of the variables. The solution, or dependent parameters, will then be a function of these nondimensionalized independent parameters $\Pi_{1}, \Pi_{2}, \ldots, \Pi_{n-r}$.

The units for a physical quantity $Q_{j}$ can be written in terms of the fundamental units, $F_{i}$, as a product of powers:

$$
U_{j}=\prod_{i=1}^{m} F_{i}^{a_{i j}} \quad j=1, \cdots, n
$$

The set of all possible units is a vector space over the field of rational numbers, with the fundamental dimensions as the basis vectors. The specific unit describing each physical quantity is then the $m$-tuple of exponents needed for the fundamental units. The vector addition operation in this vector space is the multiplication of units, and the scalar multiplication operation is the raising of units to powers. We can assemble a dimension matrix $u$ from the specific units describing each physical quantity in a problem:

$$
U=\left(\begin{array}{ccc}
a_{11} & \cdots & a_{1 n} \\
\vdots & \ddots & \vdots \\
a_{m 1} & \cdots & a_{m n}
\end{array}\right)
$$

The key axiom of the Buckingham II-theorem is that the choice of fundamental units is arbitrary. In other words, the choice of basis vectors for our vector space is arbitrary; we are looking for the kernel (or null space) of the dimension matrix. The rank* of the dimension matrix determines the number of repeating variables which will appear in our nondimensional groups and therefore how many nondimensional groups can be formed by judiciously combining all of the variables in the problem.

The dimensional reduction which can by expected through application of the Buckingham $\Pi$-theorem is $r \leq m$, which limits the maximum reduction to the number of fundamental dimensions involved in the problem.

*The rank of a matrix is the dimension of the range of the matrix corresponding to the number of linearly independent columns of the matrix. It is the difference between the dimension of the range and the dimension of the null space. 


\section{Application of the П-Theorem to an Example Problem}

As an illustration of the Buckingham $\Pi$-Theorem, we will nondimensionalize the "falling mass" problem from Section 2.2 on Page 19; Figure 2.10 is reprinted at the right.*

Recall that the equation describing the motion of the falling mass is:

$$
s(t)=\frac{g \cdot t^{2}}{2}+t \cdot v_{0}+s_{0}
$$

We can check that this equation satisfies the Principle of Dimensional Homogeneity by comparing the dimensions of all the variables. These are enumerated in Table 2.2.

\begin{tabular}{|c|c|c|c|c|}
\hline$s$ & $t$ & $g$ & $v_{0}$ & $s_{0}$ \\
\hline \hline$\{1\}$ & $\{\mathrm{t}\}$ & $\left\{1 \cdot \mathrm{t}^{-2}\right\}$ & $\left\{1 \cdot \mathrm{t}^{-1}\right\}$ & $\{1\}$ \\
\hline
\end{tabular}

Table 2.2: Dimensions of the variables in Equation (2.21)

It is easy to show that each additive term in Equation (2.21) has units of length; the equation is homogeneous. Additionally, we can see from Table 2.2 that there are only two fundamental dimensions (length and time) present in the variables of this equation. Through application of Buckingham's $\Pi$-Theorem, we expect a maximum dimensional reduction of two. The unit matrix is:

$$
\boldsymbol{U}_{f . m a s s}=\left(\begin{array}{rrrrr}
1 & 0 & 1 & 1 & 1 \\
0 & 1 & -1 & -2 & 0
\end{array}\right)
$$

The rank of the unit matrix is indeed two, meaning that proper nondimensionalization will reduce this problem from one involving five variables to one involving three. If we were trying to render the problem graphically, a reduction of two dimensions (from five to three) would mean that the system was suddenly amenable to visualization. The corollary to this dimensional reduction is an abstraction of sorts; it is not always easy to understand what the nondimensional parameters actually represent physically. This makes sensible selection of the so-called repeating variables important.

${ }^{*}$ This example is also treated in detail in White (1994). 
Evaluation of the binomial coefficient $\left(\begin{array}{l}5 \\ 2\end{array}\right)$ shows that there are ten ways of selecting our repeating variables in this problem, however this "brute force" attempt leads to some outright difficulties or simply strange results. First of all, in order to be suitable basis vectors the repeating variables cannot form a $\Pi$-group on their own. This means (for instance) that we cannot simultaneously select $s$ and $s_{0}$ as repeating variables.* As well, Equation (2.21) describes the position of a mass with respect to time (as is evident from the LHS of the equation). It would not make sense to use either the independent or dependent variable (i.e., either $s$ or $t$ ) as repeating variables. Eliminating these two variables from the repeating variable pool leaves us with $\left(\begin{array}{l}3 \\ 2\end{array}\right)$ choices, any of which is valid. We will look at each of these three cases individually.

\section{Case I: Selecting $v_{0}$ and $s_{0}$ as repeat-}

ing variables If we wish to study (or emphasize) the effect of gravity on the falling mass, we select $v_{0}$ and $s_{0}$ as our repeating variables. In this case, our three nondimensional $\Pi$-groups are:

$$
\begin{gathered}
\Pi_{1}=\frac{s}{s_{0}} \quad \Pi_{2}=\frac{t \cdot v_{0}}{s_{0}} \\
\Pi_{3}=\frac{g \cdot s_{0}}{v_{0}^{2}}
\end{gathered}
$$

Substituting these nondimensional groups into Equation (2.21) and simplifying gives the following nondimensional equation:

$$
\Pi_{1}=1+\Pi_{2}+\frac{1}{2} \Pi_{2}^{2} \Pi_{3}
$$

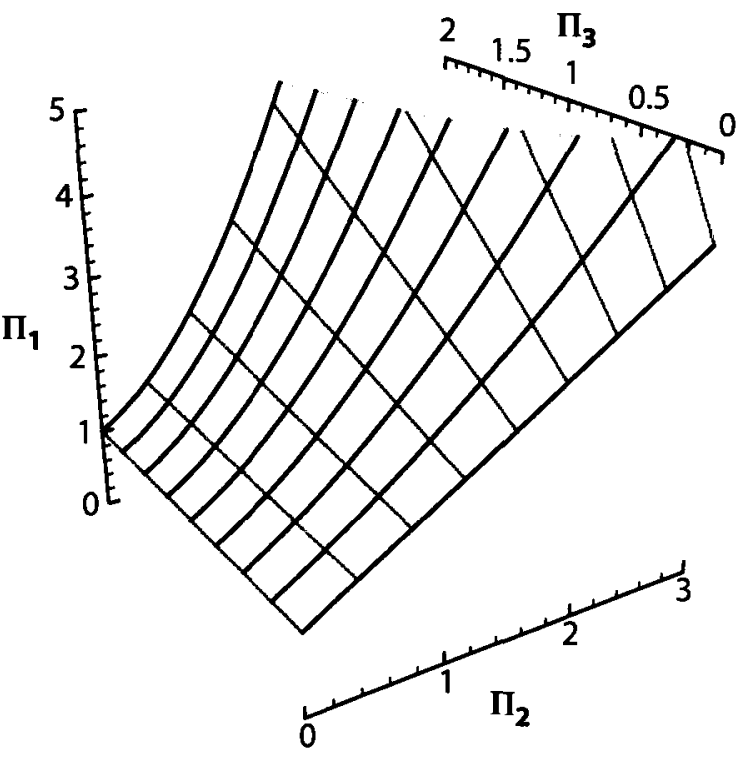

Figure 2.14: Nondimensional solution to falling-mass problem (Case I)

It is (hopefully) easy to see why this formulation emphasizes the effect of gravity; all of the nondimensional groups contain at least one of the repeating variables. Modulating the repeating variables has no net effect on the system because they are now scaling parameters.

\footnotetext{
${ }^{*}$ The ratio of $s$ and $s_{0}$ is dimensionless.
} 
A three-dimensional plot of Equation (2.24) is shown in Figure 2.14, illustrating the effect that changes in dimensionless time $\Pi_{2}$ and gravity $\Pi_{3}$ have on mass position. As would be expected, initial (dimensionless) position is the same* for all values of $\Pi_{3}$ at $\Pi_{2}=0$, and gravity increases the parabolic rate of fall for $\Pi_{2}>0$, but not the initial slope at $\Pi_{2}=0$.

\section{Case II: Selecting $v_{0}$ and $g$ as repeat-}

ing variables Similarly, if we wish to study the effect of initial position on the falling mass we would select $v_{0}$ and $g$ as our repeating variables, ensuring that the effects of both are hidden in the nondimensional groups, while the effects of all other variables remain obvious. Our three nondimensional $\Pi$ groups are then:

$$
\begin{gathered}
\Pi_{1}=\frac{s \cdot g}{v_{0}^{2}} \quad \Pi_{2}=\frac{t \cdot g}{v_{0}} \\
\Pi_{3}=\frac{s_{0} \cdot g}{v_{0}^{2}}
\end{gathered}
$$

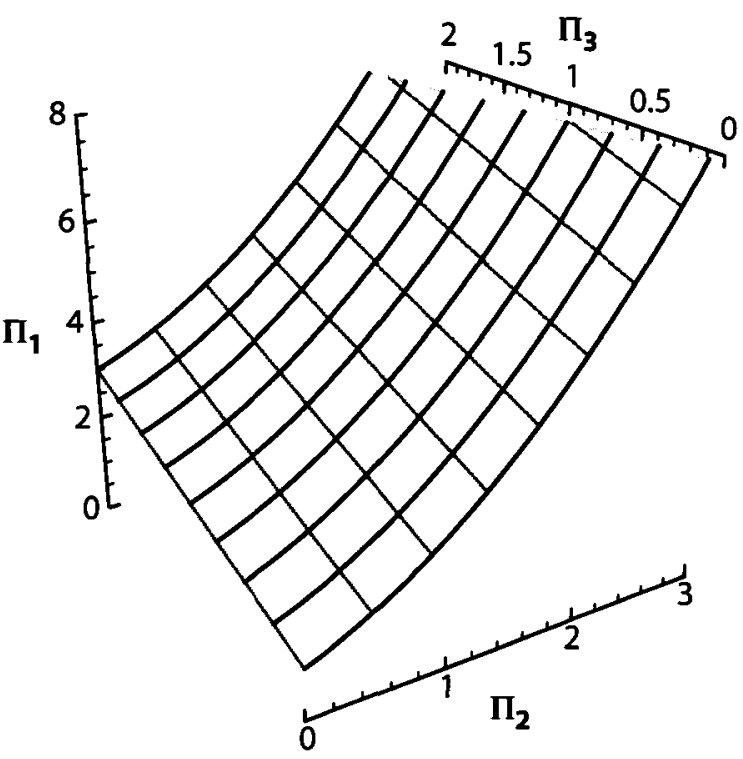

Figure 2.15: Nondimensional solution to falling-mass problem (Case II) variables into Equation (2.21) and simplifying results in:

$$
\Pi_{1}=\Pi_{2}+\frac{1}{2} \Pi_{2}^{2}+\Pi_{3}
$$

The three-dimensional plot of Equation (2.26) is shown in Figure 2.15. Unlike the parabolic scaling seen in Figure 2.14, changes in $\Pi_{3}$ (initial displacement) simply translate the trajectories upward without changing their shape. This is visible in the $\Pi_{1}-\Pi_{3}$ plane, where slices of the solution surface have a linear, positive slope.

Case III: Selecting $s_{0}$ and $g$ as repeating variables Finally, if we are interested in the effect of initial velocity on the falling mass, we would select $s_{0}$ and $g$ as our repeating variables.

${ }^{*}$ The position is $s=s_{0}$, or $\Pi_{1}=1$. 
As before, all of the nondimensional groups will contain at least one of the repeating variables; both initial position and gravity will become scaling parameters. For this formulation, the corresponding $\Pi$-groups are:

$$
\Pi_{1}=\frac{s}{s_{0}} \quad \Pi_{2}=\frac{t^{2} \cdot g}{s_{0}} \quad \Pi_{3}=\frac{v_{0}^{2}}{s_{0} \cdot g}
$$

Substituting these nondimensional groups into Equation (2.21) yields an equation which is quadratic in terms of nondimensional position, $\Pi_{1}$ :

$$
\Pi_{1}^{2}-\left(\Pi_{2}+2\right) \Pi_{1}+\frac{1}{4} \Pi_{2}^{2}+\left(1-\Pi_{3}\right) \Pi_{2}+1=0
$$

We can use the quadratic equation to solve for $\Pi_{1}$ :

$$
\Pi_{1}=\frac{1}{2}\left(2+\Pi_{2} \pm 2 \sqrt{\Pi_{2} \Pi_{3}}\right)
$$

A plot of Equation (2.29) is shown in

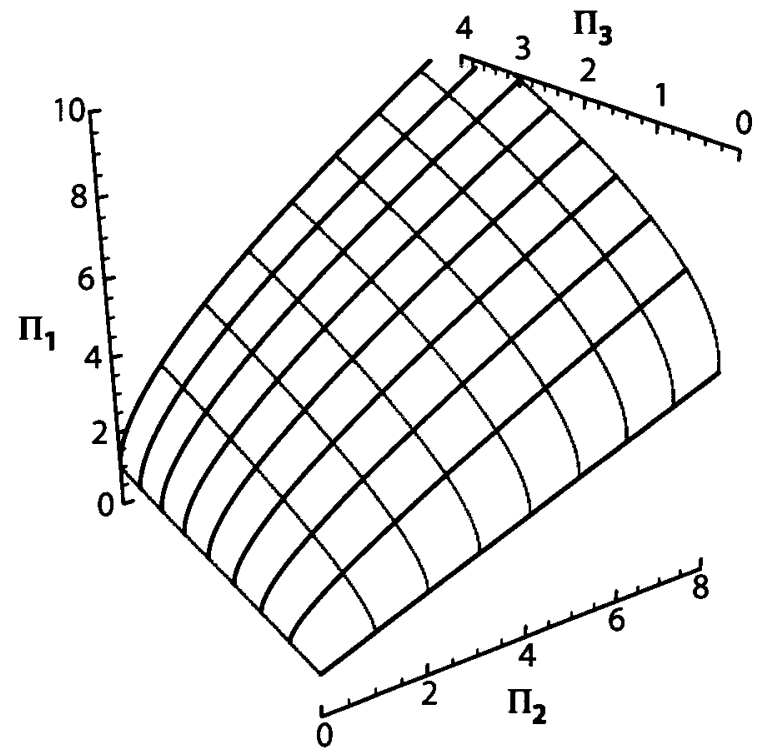

Figure 2.16: Nondimensional solution to falling-mass problem (Case III) Figure 2.16. Unlike the previous two plots, which were relatively easy to interpret, Figure 2.16 does not evince the expected trends. Varying either $\Pi_{2}$ or $\Pi_{3}$ changes the initial slope of the solution curve, and the surface shape in both directions is dominated by the effect of the square root, although the influence of the linear $\Pi_{2}$ term is clearly visible in any $\Pi_{1}-\Pi_{2}$ plane.

The bulk of the visual comprehension (or identification) problem associated with Figure 2.16 is due to the nondimensional time variable, $\Pi_{2}$. Sliced in the $\Pi_{1}-\Pi_{3}$ plane, the solution surface appears as expected; increasing initial velocity has a noticeable but diminishing effect on position for a given time slice. In the $\Pi_{1}-\Pi_{2}$ plane, however, the results are 
counter-intuitive. The mass appears to decelerate, with the slope of the solution surface decreasing as time increases.

To better understand the effects of initial velocity on the evolution of mass position in time, we can make use of an important principle of Buckingham's $\Pi$-Theorem: products of dimensionless groups are themselves dimensionless, and equally valid alternative problem formulations can be developed through mathematical manipulation of dimensionless groups.

An alternative set of $\Pi$-groups for "Case $\Pi 1$ " is as follows:

$$
\Pi_{1}=\frac{s}{s_{0}} \quad \Pi_{2}=t \cdot \sqrt{\frac{g}{s_{0}}} \quad \Pi_{3}=\frac{v_{0}^{2}}{s_{0} \cdot g}
$$

This set of $\Pi$-groups is similar to that given in Equation (2.27), but the nondimensional time variable is no longer quadratic in $t$. Substituting these into Equation (2.21) and simplifying results in:

$$
\Pi_{1}=\frac{1}{2}\left(2+\Pi_{2}^{2}+2 \cdot \Pi_{2} \sqrt{\Pi}_{3}\right)
$$

The effect of this subtle change is immediately visible in Figure 2.17; slices in any $\Pi_{1}-\Pi_{2}$ plane now show an accelerating rate of fall, as expected.

Much of the attractiveness of Buckingham's $\Pi$-Theorem stems from its ease of use; the nondimensionalization of complex problems is reduced to simple algebra. Besides illustrating the ap-

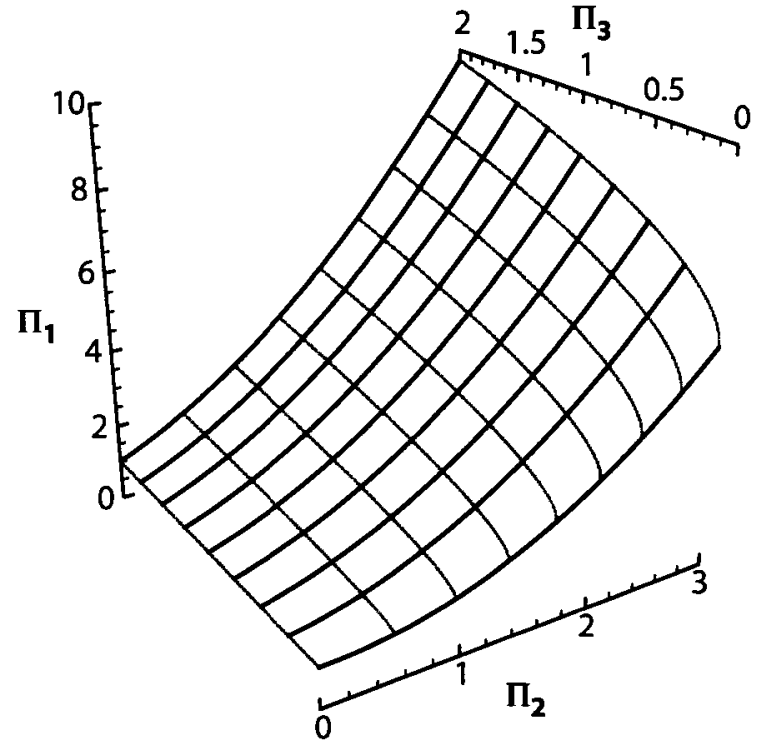

Figure 2.17: Nondimensional solution to falling-mass problem (Case III) using different ח-groups plication of the theorem (and its simplicity), the "falling mass" example also highlights some of the pitfalls. If nondimensionalization of a problem is to aid in its understanding, the resulting formulation must make 
both physical and intuitive sense. Careful selection of repeating variables and sensible manipulation of nondimensional groups ensures this.

\subsubsection{Derivative Terms}

As discussed in Section 2.2, the equations describing more complicated systems (such as the mass-spring-damper system) often involve derivative terms. Nondimensionalizing these equations involves the use of the Chain Rule from Calculus. Recall that

$$
\frac{\mathrm{d}}{\mathrm{d} r}=\frac{\mathrm{d} s}{\mathrm{~d} r} \cdot \frac{\mathrm{d}}{\mathrm{d} s}
$$

We can nondimensionalize the "mass-spring-damper" problem from Section 2.2 on Page 21; Figure 2.11 is reprinted at the right for convenience.

Recall, from Equation (2.5), that the equation describing the motion of this system is:

$$
m \cdot \frac{\mathrm{d}^{2} s}{\mathrm{~d} t^{2}}+b \cdot \frac{\mathrm{d} s}{\mathrm{~d} t}+k \cdot s=m \cdot g
$$

Equation (2.33) contains both first- and second-order derivatives with respect to time; we will need to express those terms using our nondimensional variables (once those have been decided upon).

We can check that this equation satisfies the Principle of Dimensional Homogeneity by comparing the dimensions of all the variables. These are enumerated in Table 2.3. Once again we have a homogeneous equation; in this case each additive term has dimensions mass-length per time squared, and we can see from Table 2.3 that there are three fundamental dimensions (mass, length and time) present in the variables of

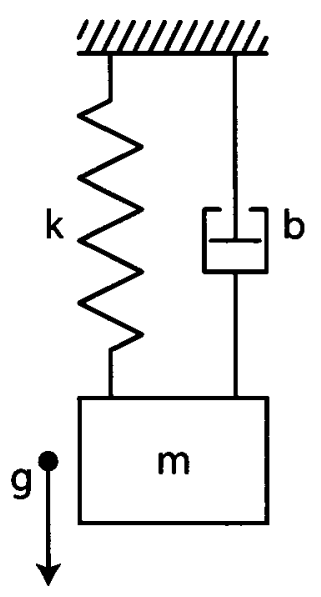

Figure 2.18: Unforced mass-spring-damper system - reprint of

Figure 2.11 this equation. We therefore hope for a dimensional reduction of three, through application of Buckingham's $\Pi$-Theorem. The unit matrix is:

$$
u_{m s d}=\left(\begin{array}{rrrrrr}
0 & 0 & 1 & 1 & 1 & 0 \\
1 & 0 & 0 & 0 & 0 & 1 \\
0 & 1 & 0 & -1 & -2 & -2
\end{array}\right)
$$




\begin{tabular}{|c|c|c|c|c|c|}
\hline$s$ & $t$ & $m$ & $b$ & $k$ & $g$ \\
\hline \hline$\{1\}$ & $\{\mathrm{t}\}$ & $\{\mathrm{m}\}$ & $\left\{\mathrm{m} \cdot \mathrm{t}^{-1}\right\}$ & $\left\{\mathrm{m} \cdot \mathrm{t}^{-2}\right\}$ & $\left\{1 \cdot \mathrm{t}^{-2}\right\}$ \\
\hline
\end{tabular}

Table 2.3: Dimensions of the variables in Equation (2.33)

The rank of this unit matrix is indeed three, implying that the problem can be reduced from the original six down to three variables through proper nondimensionalization. We must now choose our repeating variables, using the process outlined in Section 2.5.1. Eliminating the dependent and independent variables from our repeating variable pool leaves us with $\left(\begin{array}{l}4 \\ 3\end{array}\right)$ choices, only three of which are valid.* If we were seeking to emphasize the effect of spring stiffness (for example) on the system we would select $m, b$, and $g$ as our repeating variables. For this formulation, the corresponding $\Pi$-groups are:

$$
\Pi_{1}=\frac{s \cdot b^{2}}{g \cdot m^{2}} \quad \Pi_{2}=\frac{t \cdot b}{m} \quad \Pi_{3}=\frac{k \cdot m}{b^{2}}
$$

To eliminate the time derivative terms in favour of nondimensional groups, we apply the Chain Rule:

$$
\begin{aligned}
\frac{\mathrm{d}}{\mathrm{d} t} & =\frac{\mathrm{d} \Pi_{2}}{\mathrm{~d} t} \cdot \frac{\mathrm{d}}{\mathrm{d} \Pi_{2}} \\
& =\frac{b}{m} \cdot \frac{\mathrm{d}}{\mathrm{d} \Pi_{2}}
\end{aligned}
$$

And, finally, Equation (2.33) reduces to: ${ }^{+}$

$$
\Pi_{1}^{\prime \prime}+\Pi_{1}^{\prime}+\Pi_{3} \cdot \Pi_{1}=1
$$

\subsection{Circulatory System Model}

A mathematical lumped parameter model of the human circulatory system (HCS) has been developed (Conlon et al., 2006) to complement in vitro ${ }^{\ddagger}$ testing of ventricular assist devices. Components included in this model represent the major parts of the systemic

${ }^{*}$ Mass, damping and stiffness can form a nondimensional group.

${ }^{\dagger}$ Derivatives are taken with respect to $\Pi_{2}$.

$\ddagger$ Merriam-Webster: Outside the living body and in an artificial environment. 
HCS loop, with all component parameters based on physiological data available in the literature.

The circulatory system model was segmented into the arterial system, the peripheral system (subsuming the arterioles, venules and capillaries), and the venous system. The VAD model (featuring a simple piston and compliant blood sac configuration) was connected to the circulatory system model using two cannulae. Inlet and outlet valves were passive-type $^{*}$, with an adjustable bias pressure. ${ }^{\dagger}$

This basic model was refined by segmenting the arterial system into aortic- and arterysegments to study the effects of nonlinearities on the arterial pressure and flow waveforms, and the venous system into small- and large-veins to study the effects of vena cava collapse under low internal pressures (or applied suction).

Estimates of the various vessel properties (such as cross-sectional area and length) are available in the literature. Table 2.1 on Page 15 summarizes some of these from a variety of sources.

The various subunits will now be described in greater detail, beginning with the VAD, and following the circulation back to the heart.

\subsubsection{VAD (Pump)}

In this simulation, the blood is motivated by a simple pump, referred to throughout this work as a VAD (or "the VAD"). This pump is a simple piston-cylinder configuration with a compliant blood sac and is not based on any current VAD design. It features two passive flow valves which open or close based on the pressure gradient across the valve. Operating in "full-fill, full-eject" (FFFE) mode, the pump cycle (ejection and filling) functions as follows:

- At the onset of ejection, a constant, positive force is applied to the piston, increasing the internal pressure in the compliant blood chamber.

\footnotetext{
*The valves open and close based on the pressure gradient across them.

tIn this work the bias or offset pressure was set to zero.
} 
- The increase in internal pressure results in the closure of the inlet valve and opening of the outlet valve. Note that the valves are passive, and therefore do not necessarily open or close at the same instant.

- Blood is expelled from the pump (ejection) through the outlet valve until the internal blood volume reaches a preset minimum level ("full eject"), signalling the end of the ejection phase, and the beginning of filling.

- The piston force is reduced for VAD filling; this lowers the internal blood sac pressure, and reverses the pressure gradients across the two valves. The outlet valve closes and the inlet valve opens. (Again, not necessarily at the same time.)

- The piston regresses to its original position as blood flows into the pump (filling), and once the blood volume reaches a preset maximum level (the "full-fill" condition) the cycle repeats.

The pump can also operate in a "fixed-rate" mode, with preset time intervals for ejection and filling. In this case the pump would function as follows:

- At the onset of ejection, a constant, positive force is applied to the piston, increasing the internal pressure in the compliant blood chamber.

- The increase in internal pressure results in the closure of the inlet valve and opening of the outlet valve. As above, the valves are passive, and therefore do not necessarily open or close at the same instant.

- Blood is expelled from the pump (ejection) through the outlet valve for a fixed length of time. The ratio of this time to the total cycle length is referred to as the "ejection duty cycle."

- The piston force is reduced for VAD filling; this lowers the internal blood sac pressure, and reverses the pressure gradients across the two valves. The outlet valve closes and the inlet valve opens. (Again, not necessarily at the same time.)

- The piston regresses as blood flows into the pump (filling) for a fixed length of time. 


\subsubsection{Cannulae}

The pump connects to the HCS via two cannulae, modelled as thin, flexible tubes with limited compliance. Both cannulae were assumed to have the same internal diameter of $2 \mathrm{~cm}$ (which is reasonable for this type of cannula), and the lengths were chosen as $15 \mathrm{~cm}$ and $10 \mathrm{~cm}$ for the outlet and inlet cannula (respectively). The term "outlet cannula" refers to the cannula attached to the outflow side of the VAD.

Compliances for the two cannulae are based on results from Ogino et al. (1995) who measured the compliance of cannula made of several materials. We have chosen to base our values on their reported compliance of $0.0129 \mathrm{~cm}^{3} \cdot \mathrm{mm} \mathrm{Hg}^{-1}$ that corresponds to a "soft" cannula with a length of $11.5 \mathrm{~cm}$, tapering from a diameter of $2.2 \mathrm{~cm}$ to a diameter of $1.8 \mathrm{~cm}$. Scaling this value to the lengths used in the model, the compliance of the output cannula is $0.79 \mathrm{~N} \cdot \mathrm{cm}^{-5}$ and the compliance of the input cannula is $1.19 \mathrm{~N} \cdot \mathrm{cm}^{-5}$.

The flow inertances for the two cannulae were computed using the standard hydraulic equation for smooth tubes, Equation (2.17) on Page 27. The flow inertance for the outlet cannula is $1.1 \times 10^{-3} \mathrm{~N} \cdot \mathrm{s}^{2} \cdot \mathrm{cm}^{-5}$, and for the inlet cannula is $3.41 \times 10^{-5} \mathrm{~N} \cdot \mathrm{s}^{2} \cdot \mathrm{cm}^{-5}$. The inlet cannula resistance was calculated using Equation (2.16) on Page 27 with a blood density of $1070 \mathrm{~kg} \cdot \mathrm{m}^{-3}$ and a blood viscosity of $5 \times 10^{-3} \mathrm{~kg} \cdot \mathrm{m}^{-1} \cdot \mathrm{s}^{-1}$ :

$$
\Delta P_{\text {incn }}(Q)=1.3 \times 10^{-5} \cdot Q \quad\left[N \cdot \mathrm{cm}^{-2}\right]
$$

The resistance of the outlet cannula is difficult to calculate using basic hydraulic equations due to the complex nature of the flow. Instead it is based on adjusting the value to generate appropriate responses in the system. The value used is consistent with other similar values used in the literature (for example the aortic resistance used in Babbs et al., 1984):

$$
\Delta P_{o t c n}(Q)=0.0011 \cdot Q \quad\left[N \cdot \mathrm{cm}^{-2}\right]
$$

\subsubsection{Valves}

Flow in-to and out-of the pump is controlled by passive valves. The flow resistance through a valve depends on the valve state (open or closed), determined by the pressure 
gradient across the valve. Flow resistance through the valve was set at $8 \times 10^{-4} \mathrm{~N} \cdot \mathrm{s} \cdot \mathrm{cm}^{-5}$ when the valve was open, and $8 \times 10^{2} \mathrm{~N} \cdot \mathrm{s} \cdot \mathrm{cm}^{-5}$ when the valve was closed.

\subsubsection{Arteries (and Aorta)}

The pressure-volume relationship for the larger arterial vessels is nonlinear, but these nonlinearities can be neglected for a first approximation, and the entire arterial system treated as a single compliance. As will be seen in Chapter 3 , this is what is found in the Donovan mock circulatory system (MCS)* and many other MCSs. Studies such as Liu et al. (1986) comparing linear and nonlinear models of the arterial system have shown that a linear element tends to underestimate the end-diastolic pressure (which corresponds to the lowest arterial pressure during heart filling) and overestimate the peak systolic pressure (which corresponds to the maximum arterial pressure during ejection). The linear compliance model tends to behave well only about the mean arterial pressure; when the volume of blood is outside the nominal range, the pressures predicted by the linear model correlate poorly with those observed in the body.

Nevertheless, the entire arterial system (including the aorta) can be modelled as in Figure 2.19(a) using a single compliance element (Tan, 1996). The constitutive relationship

(a)

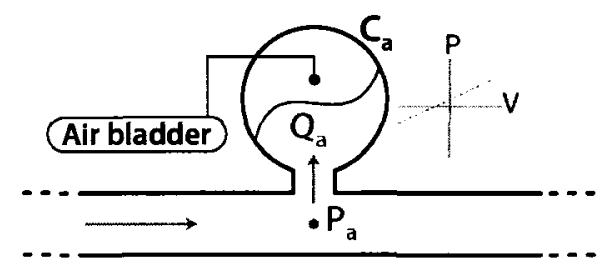

(b)

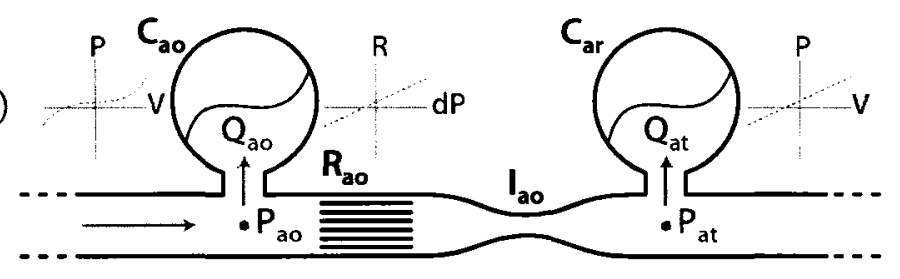

Figure 2.19: Two arterial system models, (a) SCM, (b) DCM

of this element relates the volume of the arterial system to changes in internal pressure.

${ }^{*}$ Section 3.2.2, Page 65. 
In the preliminary model, ${ }^{*}$ the compliance was assumed to be constant at a value of $100 \mathrm{~cm}^{5} \cdot N^{-1}$ as per the results presented in Laskey et al. (1990), which studied arterial pressure in ten patients. (That particular study found a mean compliance of $100 \mathrm{~cm}^{5} \cdot N^{-1}$ with a standard deviation of $2 \mathrm{~cm}^{5} \cdot N^{-1}$.) This value was later changed to $130 \mathrm{~cm}^{5} \cdot \mathrm{N}^{-1}$, to reflect newer data appearing in the literature (Stergiopulos et al., 1994). The pressure at nominal volume was chosen as $1.33 \mathrm{~N} \cdot \mathrm{cm}^{-2}(100 \mathrm{~mm} \mathrm{Hg})$ to match the pressures in the human arterial system (Fung, 1997) and the nominal volume was chosen as $700 \mathrm{~cm}^{3}$. This gives the following constitutive relation:

$$
P_{a}(V)=\frac{V}{130}-4.05 \quad\left[N \cdot \mathrm{cm}^{-2}\right]
$$

A more refined version of the arterial model was subsequently developed. This configuration, the so-called dual-compliance model (DCM), is illustrated in Figure 2.19(b) and consists of two compliance elements coupled with an inertial element. Once again this model was based on physiological data. The aortic compliance in the dual-compliance model was chosen to match data from the age group of 47-52 years given in King (1946). The data used by King were obtained during dissection of a human cadaver. Although tissue properties change post-mortem, this data was assumed to apply in vivo ${ }^{\dagger}$ and was used because no in vivo source of data was available. Tissues tend to lose their internal residual stress and elongation of the segment is possible unless careful experimental procedures are followed.

Three separate curves were fit to the experimental data, by minimizing the error (in the least squares sense) between the function(s) and the data points. These curve fits (and the data points) are plotted in Figure 2.20. The linear fit gave an aortic compliance of $29.3 \mathrm{~cm}^{5} \cdot \mathrm{N}^{-1}$, with an offset of $-1.94 \mathrm{~N} \cdot \mathrm{cm}^{-2}$ :

$$
\left.P_{a o}\right|_{\text {lin }}=\frac{V}{29.3}-1.94 \quad\left[N \cdot \mathrm{cm}^{-2}\right]
$$

*Referred to as the "single-compliance model" or "SCM."

'Merriam-Webster: In the living body of a plant or animal. 


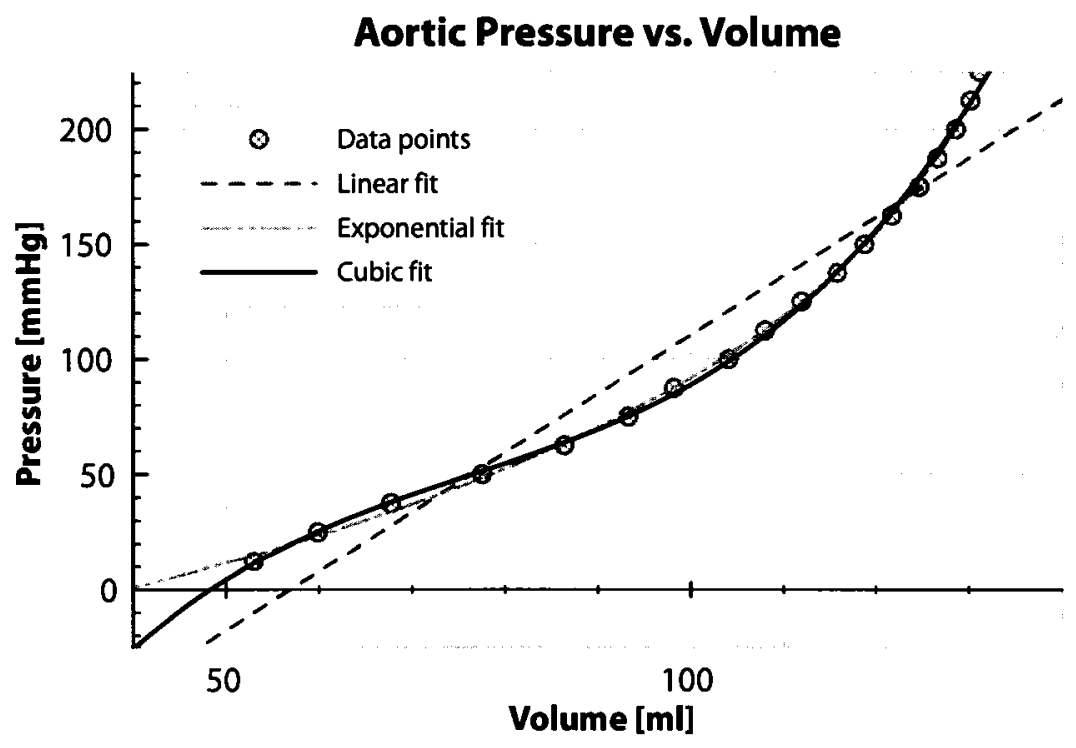

Figure 2.20: Aortic pressure-volume data and curve fits

The cubic aortic compliance function is:

$$
\left.P_{a o}\right|_{c u b}=7.66 \times 10^{-6} \cdot V^{3}-0.00175 \cdot V^{2}+0.151 \cdot V-4.08 \quad\left[N \cdot \mathrm{cm}^{-2}\right]
$$

The exponential aortic compliance function is:

$$
\left.P_{a o}\right|_{\text {exp }}=-1.43 \cdot \log (1.37-0.00943 \cdot V) \quad\left[N \cdot \mathrm{cm}^{-2}\right]
$$

In the dual-compliance model, the magnitude of the arterial compliance is dependent on the aortic compliance. The value of aortic compliance obtained by the linear leastsquares fit $\left(29.3 \mathrm{~cm}^{5} \cdot \mathrm{N}^{-1}\right)$ is subtracted from the total systemic arterial compliance of $130 \mathrm{~cm}^{5} \cdot N^{-1}$ to give an arterial compliance of $101 \mathrm{~cm}^{5} \cdot N^{-1}$, and the pressure at nominal volume is again taken to be $1.33 \mathrm{~N} \cdot \mathrm{cm}^{-2}$.

This ensures that the single- and dual-compliance systemic arterial models behave similarly when inserted in the overall HCS model.

The dual-compliance model was initially simulated without a flow resistance element in order to study the pressure oscillations which are produced due to the presence of the 
two compliances. These oscillations override the nominal pressure behaviour, changing the shape of the curve and distorting the maximum and minimum pressures in the aorta. This behaviour is undesirable, and the aortic resistance element was tuned to provide a smooth pressure waveform, and a more accurate flow rate waveform shape (as compared with the initial, single-compliance model).

The required flow resistance of $0.0011 \mathrm{~N} \cdot \mathrm{s} \cdot \mathrm{cm}^{-5}$ is substantially larger than the resistance which would be computed based on Equation (2.16), and the nominal aortic dimensions from Table 2.1, ignoring the non-Newtonian properties of the blood (i.e., treating it as water). With an aortic diameter of $2.5 \mathrm{~cm}$, and length of $20 \mathrm{~cm}$ (Tortora and Grabowski, 2000) we would expect a flow resistance of $1.0 \times 10^{-5} \mathrm{~N} \cdot \mathrm{s} \cdot \mathrm{cm}^{-5}$. The larger value may reflect effects of unsteady flow, changing flow geometry and losses from the stretching and relaxing of the vessel walls and is consistent with the literature (see for example Babbs et al., 1984).

Blood flow in the vessels proximal to the VAD* will likely be turbulent, as opposed to laminar; turbulent flow results in larger pressure losses, which would also justify the larger aortic resistance element.

Simulation of the aortic segment with the selected flow resistance yields pressure and flow behaviour similar to that found in the human circulatory system. The aortic pressure waveform for the dual-compliance model (and a number of different VAD ejection pressures) is shown in Figure 2.21.

The dual-compliance model includes an inertance element to dynamically decouple the states for the aortic and arterial segments. The inertance allows the volume in the aorta and the volume in the arterial segment (which excludes the aorta in the dual-compliance model) to vary independently. The value of inertance was calculated using Equation (2.17) based on the typical length and cross-sectional area estimates for the aorta. As above, the diameter was taken as $2.5 \mathrm{~cm}$ and the length as $20 \mathrm{~cm}$ (generally accepted values for the average diameter and length of the larger arterial vessels). Values of aortic inertance can vary from individual to individual. The aortic inertance that can be estimated based on Equation (2.17) using a blood density of $1070 \mathrm{~kg} \cdot \mathrm{m}^{-3}$ is $4.06 \times 10^{-5} \mathrm{~N} \cdot \mathrm{s}^{2} \cdot \mathrm{cm}^{-5}$ which

\footnotetext{
*The outlet cannula, and possibly the aorta.
} 


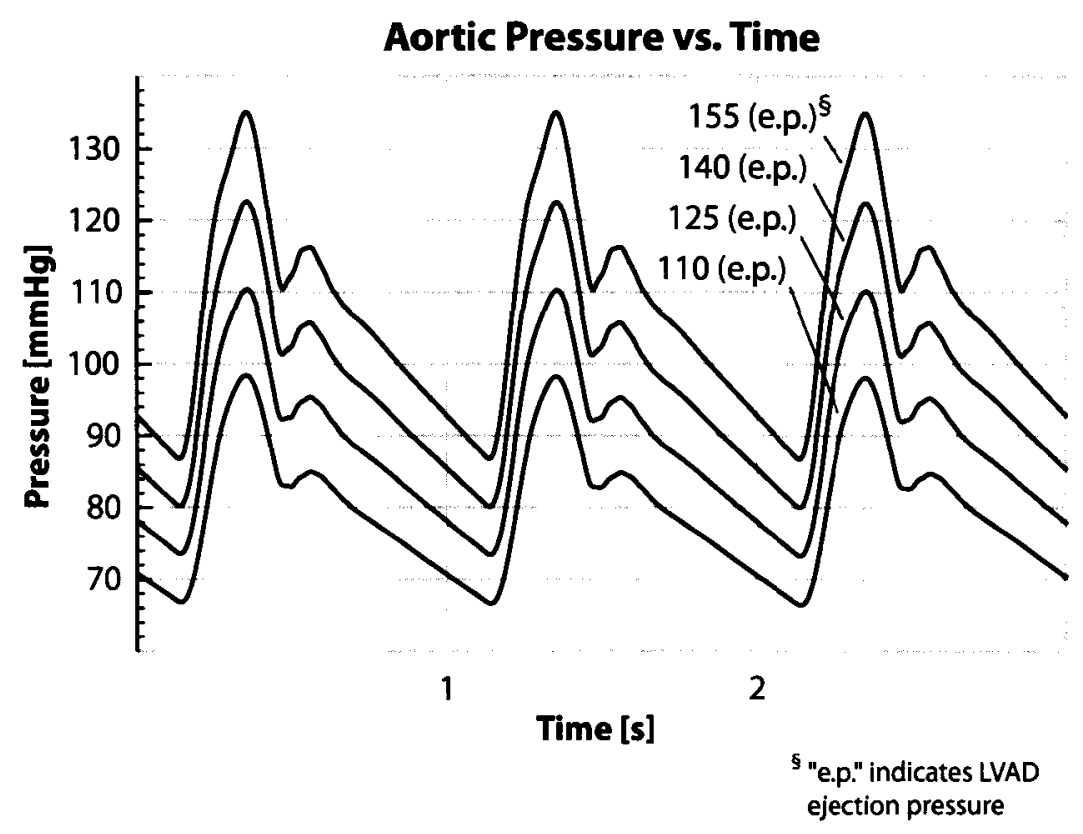

Figure 2.21: Aortic pressure waveform for a variety of ejection pressures

generally agrees with the values published in the literature (Fung, 1997). This value is used in the simulations.

\subsubsection{Peripheral Circulation}

The peripheral circulation model consists of an inertance element and a flow resistance element, representing all of the micro circulation in the body. The capillaries and the venules (which would be counted as part of the peripheral circulatory system) have an extremely small individual cross-sectional area, but a very large total cross-sectional area, meaning that they have a large flow inertia and significant flow resistance, but very small stiffness.

The peripheral flow inertance and resistance parameters are not directly available in the literature, because these individual lumps are modelling a complex, continuous system of vessels. The selected parameters were based on data from Tortora and Grabowski (2000), Fung (1997), Guyton (1992) and Tsach et al. (1989), giving a peripheral flow inertance of $0.0275 \mathrm{~N} \cdot \mathrm{s}^{2} \cdot \mathrm{cm}^{-5}$ and a peripheral flow resistance of $0.013 \mathrm{~N} \cdot \mathrm{s} \cdot \mathrm{cm}^{-5}$. 


\subsubsection{Venous Segment}

As with the aorta and arteries, the larger veins in the body such as the superior and inferior venae cavae can be modelled as thin-walled, flexible tubes. As such their pressurevolume constitutive relations are again nonlinear, but these nonlinear effects can be ignored for a first approximation. To obtain results that are more accurate, especially at off-nominal conditions, these nonlinearities must be taken into account.

The most important nonlinear venous behaviour, from a modelling perspective, is the tendency towards collapse at low internal pressures. The collapse of the larger veins (as with any thin-walled tube) dramatically increases resistance to blood flow. The venous system normally operates at low internal pressures $(2-5 \mathrm{~mm} \mathrm{Hg})$ and it is important to allow for the possibility of venous collapse, especially when modelling an implanted artificial heart which is able to apply suction to the venous system. In addition, the absence of the pulmonary loop in our model necessitates a direct connection between the VAD and the venous system.

As previously discussed in Section 2.1.4, the venous system is responsible for the return of blood to the heart. It consists of a series of blood vessels with increasingly larger diameters, namely the venules, the veins and the venae cavae. The venules are similar to the capillaries in that they are microscopic vessels, but unlike the capillaries, which branch off from the arteries, the venules join to return deoxygenated blood from the cells to the veins. Recall that the venules, like the capillaries, are included in the "peripheral circulation" lumped model. The veins are larger in diameter than the venules, but their mean crosssectional area is still quite small, and collapse of the veins is not a problem. In addition, the layers of elastic fibres, smooth muscle, and outer fibrous tissue mean that the veins are not thin-walled tubes in the engineering sense. The total cross sectional area for the veins is quite large $\left(80 \mathrm{~cm}^{2}\right.$, Fung, 1997) and nonlinearities in the pressure-volume relationship are negligible.

The venae cavae, on the other hand, are larger in diameter and qualify as thin-walled tubes. The average cross-sectional area is $7 \mathrm{~cm}^{2}$ (Fung, 1997) and nonlinear behaviour at both high and low internal pressures should be taken into consideration. 
For a first approximation, the entire venous system can be modelled using a single compliance element whose constitutive relation is the volume response of the venous system to changes in internal pressure, as illustrated in Figure 2.22(a). The compliance of the el-

(a)

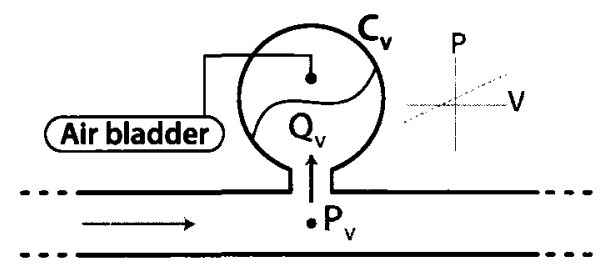

(b)

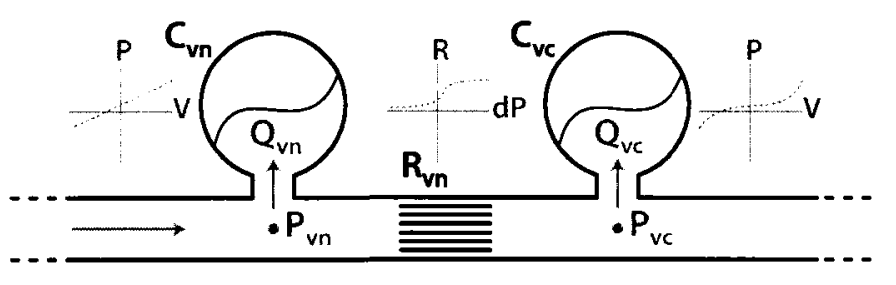

Figure 2.22: Two venous system models, (a) SCM, (b) DCM

ement was assumed to be constant at $3750 \mathrm{~cm}^{2} \cdot \mathrm{N}^{-1}$ and the pressure at nominal volume (i.e., $2500 \mathrm{~cm}^{3}$ ) was chosen as $20 \mathrm{mmHg}$. This pressure is slightly higher than would be found in the literature (Fung, 1997 or Tortora and Grabowski, 2000), but is necessary because in this HCS model, the venous pressure is solely responsible for filling the VAD. The VAD requires a pressure of approximately $5-7 \mathrm{~mm} \mathrm{Hg}$ (normally provided by the natural heart) in order to fill, while the typical venous pressure is only $2-5 \mathrm{mmHg}$. Decreasing the peripheral flow resistance in the systemic model results in a lower pressure drop across the segment, a higher average venous pressure, and sufficient filling pressure for the VAD. Other pressures and flow rates are not affected.

The venous system model was further refined. The resultant dual-compliance model, illustrated in Figure 2.22(b), is based on the realization (mentioned above) that due to the small individual diameters and large total cross-sectional area, nonlinear effects will be negligible in many of the smaller vessels in the venous system. (Specifically, the "intermediate" veins with less elastic fibre in their medial and external layers.) Only the larger venae cavae, which contain a relatively small percentage of the total venous blood volume, will exhibit noticeable nonlinearities. 
The venous blood volume was divided between the two compliance elements, with the first, linear, compliance containing (nominally) $2000 \mathrm{~cm}^{3}$ of the system's blood volume, and the second, nonlinear, compliance containing $600 \mathrm{~cm}^{3}$ of the system's blood volume. These volumes were determined based on the overall blood volume in our model and the approximate distribution of blood between the veins and the venae cavae shown in Table 2.1.

Splitting the venous system into two separate compliance elements has an added advantage: by inserting a nonlinear resistance element in between the two venous compliance elements, we can model the collapse of the larger veins at low internal pressures, and the corresponding increase in flow resistance. The constitutive relations for all three of these elements are inter-related. The linear compliance element is similar to that found in the single-compliance venous model. The pressure at nominal volume was chosen as $0.27 \mathrm{~N} \cdot \mathrm{cm}^{-2}(20 \mathrm{mmHg})$. The venous compliance was set to $2500 \mathrm{~cm}^{5} \cdot \mathrm{N}^{-1}$, which results in the following constitutive relation:

$$
P_{v n}(V)=\frac{V}{2500}-0.53 \quad\left[N \cdot \mathrm{cm}^{-2}\right]
$$

A piecewise linear curve (with three segments) was used to model the nonlinear vena cava compliance. Below a critical volume of $500 \mathrm{~cm}^{3}$ the vessel is deemed to have collapsed. The pressure at this critical volume was set to $0 \mathrm{~N} \cdot \mathrm{cm}^{-2}(0 \mathrm{mmHg})$. In the nominal range of volumes from $500 \mathrm{~cm}^{3}$ to $600 \mathrm{~cm}^{3}$ compliance of the vena cava was $500 \mathrm{~cm}^{5} \cdot \mathrm{N}^{-1}$ while at volumes below $500 \mathrm{~cm}^{3}$ and above $600 \mathrm{~cm}^{3}$ the compliance was $0.002 \mathrm{~cm}^{5} \cdot \mathrm{N}^{-1}$. The pressure at the nominal vena cava volume of $600 \mathrm{~cm}^{3}$ is $0.2 \mathrm{~N} \cdot \mathrm{cm}^{-2}(15 \mathrm{mmHg})$ which is (again) slightly higher than that commonly found in the literature.

The resistance function was chosen to allow a flow rate of $10 \mathrm{~cm}^{3} \cdot \mathrm{s}^{-1}$ when the vessel was collapsed, and $650 \mathrm{~cm}^{3} \cdot \mathrm{s}^{-1}$ when it was fully open (given a nominal pressure drop of $0.067 \mathrm{~N} \cdot \mathrm{cm}^{-2}$ ). The offset, or transition pressure difference, was chosen as $0.04 \mathrm{~N} \cdot \mathrm{cm}^{-2}$ based on the two compliance functions.

The flow resistance is a function of the pressure difference between the linear venous segment and the nonlinear vena cava segment. This has the effect of increasing venous 
flow resistance when the vena cava internal volume drops below the critical threshold level of $500 \mathrm{~cm}^{3}$ described above.

The ability of the veins to collapse is not as important in VAD simulation, because the VAD is isolated from the venous system by both the pulmonary loop and the natural heart. The feature becomes more important with TAH simulation, because those devices and the venous system are connected directly. Of course a VAD's "venous isolation" assumes the smaller, peripheral vessels provide sufficient dynamic decoupling between the arterial and venous systems. This is likely the case; as noted earlier, the peripheral vessels have extremely small individual diameters, but a very large total cross-sectional area. However, this has not been studied, and there may be something a VAD can do to cause a specific change in the arterial system, and induce venous collapse.

\subsubsection{Overall Model}

A hydraulic schematic of the overall circulatory system model is illustrated on the following page, in Figure 2.23. The major components are highlighted with gray boxes, and labelled accordingly. The arterial and venous models included in the circuit are the dual compliance models, with the corresponding single compliance models placed beside their respective dual-compliance model. Either arterial "lump" from Figure 2.19 can be inserted as the Arterial System and either venous model illustrated in Figure 2.22 can be inserted as the Venous System. (See Figure 2.23.) Various pressures of interest and model parameters are also annotated in Figure 2.23.

A fully augmented bond graph representation of the overall circulatory system model (using the arterial and venous dual-compliance segments) is shown in Figure 2.24. This bond graph can be used to derive Equations (2.45-2.57), the thirteen equations of state.

$$
\begin{aligned}
\dot{s}_{h t}(t)= & \frac{p_{h t}(t)}{I_{h t}} \\
\dot{p}_{h t}(t)= & F(t)-\frac{p_{h t}(t) \cdot R_{h t}}{I_{h t}}-A_{h t} \cdot P_{h t}\left(V_{h t}(t)\right) \\
\dot{V}_{h t}(t)= & \frac{p_{h t}(t) \cdot A_{h t}}{I_{h t}}+R_{\text {in }}\left(P_{\text {incn }}\left(V_{\text {incn }}(t)\right)-P_{h t}\left(V_{h t}(t)\right)\right) \\
& -R_{\text {otv }}\left(P_{h t}\left(V_{h t}(t)\right)-P_{\text {otcn }}\left(V_{\text {otcn }}(t)\right)\right)
\end{aligned}
$$




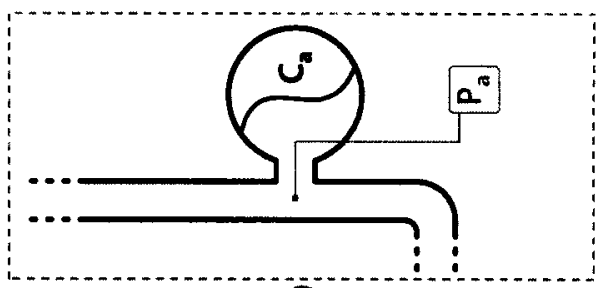

(;)

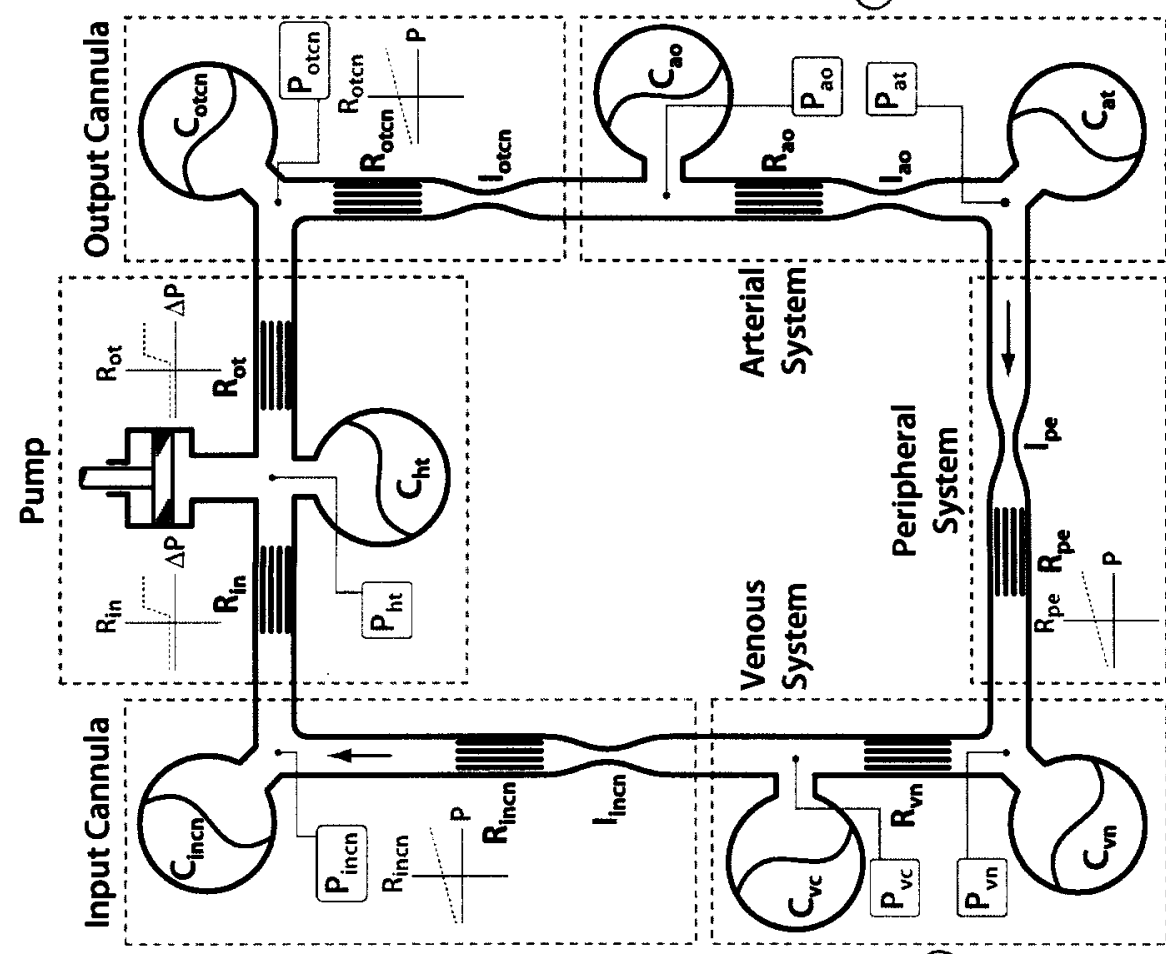

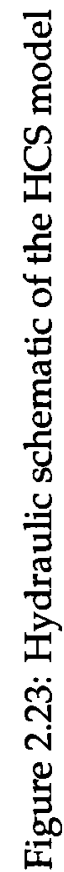

(2)

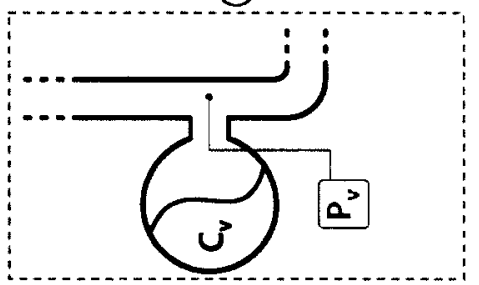




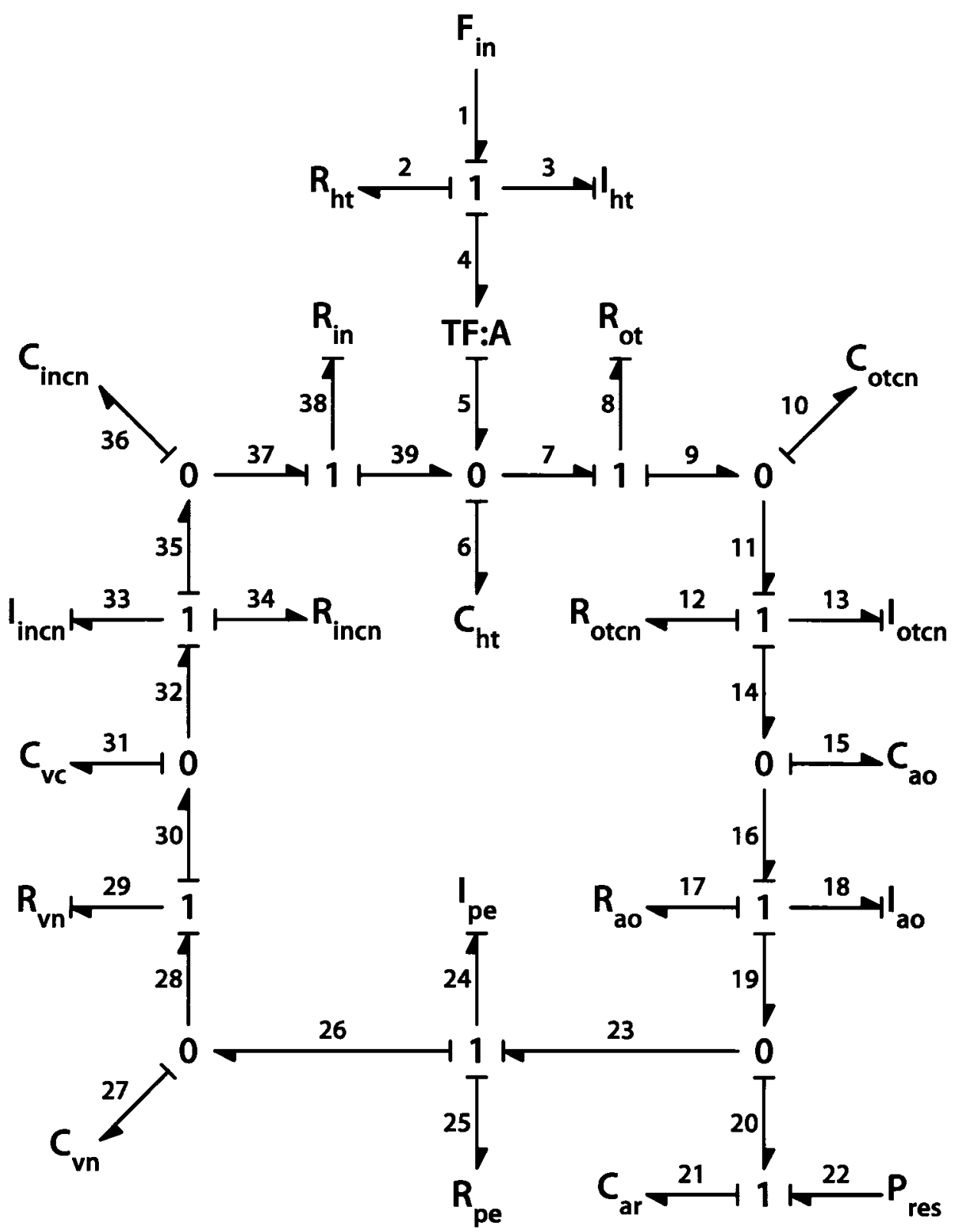

Figure 2.24: Bond graph of the HCS model 


$$
\begin{aligned}
\dot{V}_{o t c n}(t) & =R_{o t v}\left(P_{h t}\left(V_{h t}(t)\right)-P_{o t c n}\left(V_{o t c n}(t)\right)\right)-\frac{p_{o t c n}(t)}{I_{o t c n}} \\
\dot{p}_{o t c n}(t) & =P_{o t c n}\left(V_{o t c n}(t)\right)-\frac{p_{o t c n}(t) \cdot R_{o t c n}}{I_{o t c n}}-P_{a}\left(V_{a o}(t)\right) \\
\dot{V}_{a o}(t) & =\frac{p_{o t c n}(t)}{I_{o t c n}}-\frac{p_{a o}(t)}{I_{a o}} \\
\dot{p}_{a o}(t) & =P_{a o}\left(V_{a o}(t)\right)-\frac{p_{a o} \cdot R_{a o}}{I_{a o}}-P_{a r}\left(V_{a r}(t)\right)+P_{r e s}(t) \\
\dot{V}_{a r}(t) & =\frac{p_{a o}(t)}{I_{a o}}-\frac{p_{p e}(t)}{I_{p e}} \\
\dot{p}_{p e}(t) & =P_{a r}\left(V_{a r}(t)\right)-\frac{R_{p e} \cdot p_{p e}(t)}{I_{p e}}-P_{v n}\left(V_{v n}\right)-P_{r e s}(t) \\
\dot{V}_{v n}(t) & =\frac{p_{p e}(t)}{I_{p e}}-R_{v n}\left(P_{v n}\left(V_{v n}(t)\right)-P_{v c}\left(V_{v c}\right)\right) \\
\dot{V}_{v c}(t) & =R_{v n}\left(P_{v n}\left(V_{v n}(t)\right)-P_{v c}\left(V_{v c}\right)\right)-\frac{p_{i n c n}(t)}{I_{i n c n}} \\
\dot{p}_{i n c n}(t) & =P_{v c}\left(V_{v c}(t)\right)-\frac{p_{\text {incn }}(t) \cdot R_{\text {incn }}}{I_{\text {incn }}}-P_{\text {incn }}\left(V_{\text {incn }}(t)\right) \\
\dot{V}_{\text {incn }}(t) & =\frac{p_{i n c n}(t)}{I_{\text {incn }}}-R_{\text {in }}\left(P_{\text {incn }}\left(V_{i n c n}(t)\right)-P_{h t}\left(V_{h t}(t)\right)\right)
\end{aligned}
$$

These thirteen equations, along with their supporting constitutive relations, can be numerically integrated with respect to time to predict the behaviour of the cardiovascular system. 


\section{Chapter 3}

\section{Literature Review}

D UE to the breadth of the subject matter at hand, an exhaustive review of the available literature is impossible; what follows is a careful study and representative sample of previous work related to this thesis.

\subsection{Circulatory System Dynamics}

The study of circulatory system dynamics is an important aspect of artificial heart design. This research can be used to develop analogs of the human circulatory system, either in a computer simulation, or in an experimental lab setup. It can also reveal behaviour which is not intuitive, and which might otherwise be difficult to discover. For example, Kresh et al. (1990) showed that proper circulation (including blood movement through the pulmonary loop) can be maintained by the left ventricle alone. The reserve cardiac capacity is reduced, but proper circulation is still possible.

\subsubsection{Modelling}

Many mathematical models of the cardiovascular system have been developed, and they vary in complexity. A number of these models such as those of Melchior et al. (1992) or Croston et al. (1973) are extremely involved, and can only be simulated on analog computers, or using electrical networks. In fact, some models such as that of Tsitlik et al. (1992) are designed from the outset using equivalent electrical networks, as opposed to hydraulic elements. The complex models can be tedious to adjust due to the variation in circulatory parameters across humans; some people, for example, have arteries which are constricted by plaque buildup, and all people have arterial plaque to varying degrees. 
At the other end of the spectrum are the very simple circulatory system models. (Some of these models also use electrical circuits.) These models are typically designed with a very specific purpose in mind, such as studying the feasibility of intra-aortic balloon pumping (Kuklinski et al., 1984), but are also used to study artificial hearts ( $\mathrm{Xu}$ and $\mathrm{Fu}, 2000$, or Gardner et al., 1993).

As discussed in the Section 2.1.4 (The Circulation), the human circulatory system can be divided into two halves: the pulmonary loop, which moves low pressure blood through the lungs; and, the main loop, which moves high pressure blood throughout the body. Creating a mathematical model of the human circulatory system involves design trade-offs. For example, the main loop is of greater concern in VAD studies, because a left ventricular assist device is not connected to the pulmonary loop; the right side of the heart continues its normal function. A more detailed model of the left side of the circulatory system and a less detailed (or non-existent) model of the pulmonary loop is a good design trade-off because it allows a more detailed study of the main circulatory system while keeping the order at a manageable level. A lower-order mathematical system ensures efficient computer simulation, even when the system is nonlinear. (Linear simulations are relatively fast, regardless of the complexity.)

The model developed herein is thirteenth order and allows for nonlinearities in the arterial and venous systems, as well as modelling the aorta and the peripheral organs and veins. It does not model the pulmonary loop, but gives a thorough treatment of the left (high pressure) side of the human circulatory system.

Modelling considerations for the arterial system are described by Liu et al. (1986), Stergiopulos et al. (1995a), Stergiopulos et al. (1995b), Stergiopulos et al. (1994), Cappello et al. (1995), and Meara (1984). Similar investigations of the venous system are described by Katz et al. (1969), Croston et al. (1973) and Melchior et al. (1992). These two systems are the source of much of the nonlinearity in human circulatory system dynamics. The arterial system's pressure-volume relationship is highly nonlinear, and the large veins operate at low internal pressures, very close to collapse (and a corresponding large increase in flow resistance). 
The arterial behaviour is affected by chemicals in the body, as well as factors such as transmural pressure (Huang et al., 1998). Compliance estimates for the arterial and venous systems can be found in literature (Fung, 1997, or Gardner et al., 1993), and can be used in mathematical models, or the development of a mock circulatory loop. The venous system can be modelled as a flexible tube with low internal pressure, and as such can exhibit chaotic oscillations similar to those outlined by Jensen (1992).

The arterial system has similar properties (in terms of wall stiffnesses and diameters), but the arterial system operates at a much higher pressure, and collapse is not a concern. Nonlinearities in the arterial system stem from the pressure-volume relationship of the wall. Detailed treatments of the arteries can include upwards of eight segments, to allow for the variation in arterial diameter, and can be developed using the Navier-Stokes equations (Kuklinski et al., 1984), or using hydraulic principles (Ferrari et al., 2000). The traditional arterial model is known as the Windkessel model, but in many cases the threeelement Windkessel model has been superseded by more detailed, nonlinear treatments of the arterial system (Li et al., 1990).

Studies using nonlinear arterial models (in mathematical circulatory system simulations) have shown interesting behaviour, such as a temporal relationship between the compliance of the left ventricle of the heart and the compliance of the arteries; arterial elastance is dynamically coupled to the left ventricle during the ejection phase (Berger and $\mathrm{Li}, 1992)$. The smaller arteries can undergo significant changes in diameter in response to chemical stimuli, a behaviour known as vasomotion (Cavalcanti and Ursino, 1996). The human heart itself is very complicated, and difficult to model (Honerkamp, 1983).

\subsubsection{Evidence of Chaos}

Pressure oscillations in the arteries can be shown to be chaotic. This nonlinearity arises at the level of the vascular smooth muscle cells because, according to Griffith and Edwards (1994), oscillatory behaviour cannot be abolished by endothelial denudation. The fractal dimension of this behaviour is normally found to be between two and three, and varying arterial parameters produces no change in this dimension. Vasomotion or irregular oscillations observed in arterial micro-vessels exhibit chaotic behaviour with blood pressure increases (Cavalcanti and Ursino, 1996). 
To further facilitate the study of the origin of chaos in the human circulatory system, open loop analysis using an artificial heart was performed by Yambe et al. (1998). Low dimensional deterministic chaos was discovered by nonlinear mathematical analysis, suggesting the importance of blood vessel properties in the chaotic dynamics of the cardiovascular system. Also, coupling of the nonlinear information between blood vessels and sympathetic activity was suggested by analysis of mutual information. In other words, the central nervous system plays an important role through sympathetic activity, a finding supported by Osaka et al. (1998), who performed a mutual information study on heart rate, arterial blood pressure and nerve activity in artificial heart-implanted dogs.

Almog et al. (1999) performed experiments on blood pressure control and found a correlation dimension of six, and an embedding dimension of up to fourteen, an indication that the underlying system is extremely complex. The power spectrum of the heart rate exhibits power law $\left(1 / f^{n}\right)$ behaviour but studies such as Pilgram and Kaplan (1999) suggest non-stationary characteristics (i.e., $n>1$ ).

Studies of R-R intervals and systolic blood pressure, such as Fortrat et al. (1999), show that even slight movements affect the overall variability of $R-R$ intervals and the low frequency spectral components of heart rate, but do not affect heart rate or blood pressure. Dynamic aspects of $R-R$ intervals have been analyzed by means of linear and nonlinear measures. Cysarz et al. (2000) showed that entropies of binary sequences derived from ECG recordings reveal properties that cannot be found using the full information of R-R intervals.

In the investigation of heart rate dynamics (i.e., $R-R$ intervals), nonlinear models are better able to predict heart rate dynamics when compared with traditional linear models, such as the auto-regressive moving average. Igel and Wilkoff (1997) showed that the application of nonlinear techniques also improves computer recognition of tachyarrhythmia, which is useful for implantable defibrillators.

Studies have shown that nonlinear mechanisms may apply to the electrical behaviour of the heart muscle itself. Fractal anatomy can be seen in many aspects of the vascular system, and self-similar (fractal) scaling may underlie heart rate dynamics, as per Goldberger 
and West (1987b). Casaleggio et al. (1997) studied the Lyapunov exponents in heart rate variability signals, and found underlying nonlinear chaotic characteristics.

Eyal and Akselrod (2000) demonstrate a simple nonlinear beat-to-beat model of the human cardiovascular system that displays a bifurcation and sustained oscillations after an increase in sympathetic gain. Interestingly, further increase in gain does not result in another bifurcation. Also, introducing respiration into the model shows entrainment between respiration and beat interval.

A study of chaos in blood pressure control (Wagner et al., 1996) shows positive Lyapunov exponents and non-integer fractal dimensions in arterial blood pressure time series. Disruption of the baroreflex made the regulation less complex, and less sensitive to initial conditions, indicating that the feedback mechanism has an impact on the complexity of the system. Blood pressure control is therefore important in assessing cardiovascular risk.

A comparison of heart rate variability in heart transplant patients and a control group, performed by Guzzetti et al. (1996), shows nonlinear behaviour. Positive Lyapunov exponents suggest the trajectories diverge in state space, but the transplant patients showed a less complex and qualitatively different behaviour. The authors speculate that this may be due to the loss of neurocontrol of heart rate. This same reduction in complexity appears when a ventricular assist device is implanted, a very interesting parallel. Chemical blockage of autonomous neural activity also results in the reduction in the magnitude of the Lyapunov exponents, indicating a decrease in complexity.

It has been shown that left heart assistance creates a decrease in fractal dimension in the circulatory system dynamics. A decrease in fractal dimension normally indicates a reduction in the complexity of the system. This means that the addition of a ventricular assist device may actually reduce the complexity of the circulatory system. Or, in other words, "weaken the disorder of the system" (Yambe et al., 1997b). Other earlier studies by the same research group dispute this finding (Yambe et al., 1995), as the complexity of the circulatory system dynamics appears to increase in the presence of a ventricular assist device. Yambe et al. do not include a Lyapunov exponent analysis in their work, and the analysis of the complexity of the underlying dynamics is qualitative in the earlier paper. 
A decrease in fractal dimension has also been observed by Nitta et al. (1999) during studies of the chaotic dynamics of circulation with a vibrating flow pump. It was found that during left ventricular assistance with a vibrating flow pump, the fractal dimension of the hemodynamics was significantly decreased, and peripheral vascular resistance was significantly decreased. It is speculated that decreased sympathetic discharges may be the origin of the decreased fractal dimension.

Zwiener et al. (1996) produce a study of heart rate and blood pressure variability displaying both periodic and nonlinear properties. The authors hypothesize that heart rate fluctuations and mean arterial pressure fluctuations are the result of deterministic chaos. Although heart rate variability can be expressed using power spectra, aperiodic processes do not appear as distinct peaks, and are only described adequately using nonlinear methods. The same is true for diameter changes in small arteries, according to Wagner and Persson (1998).

Yambe et al. (1999) applied chaos and fractal theory to the volume of the left ventricle of the human heart, as opposed to the ECG, using acoustic analysis. As with the ECG, the left ventricular volume displays nonlinear behaviour. In addition, Prabhu (1999) found that load sensitivity of left ventricular relaxation exhibits a nonlinear biphasic response, which is more evident in failing hearts, but also present in normal, healthy hearts. Candidates for ventricular assist devices will normally have failing hearts, and a more complex load sensitivity in the left ventricle.

Additional routes to chaos may be introduced by the mechanical aspects of a ventricular assist device. For example, Gross et al. (1988) observed vortex shedding in bi-leaflet heart valve prostheses, and Lamson et al. (1991) studied cavitation in prosthetic heart valves. Gas bubble formation in mechanical heart valves has also been studied (Lin et al., 2000).

\subsubsection{Evidence of Deterministic Behaviour}

Nonlinear dynamics of blood flow studied by Lyapunov exponents produced contradictory results. Over a wide range of parameters, paired values of both the global and the local Lyapunov exponents were obtained, and at least one exponent equalled zero. This may be an indication of the deterministic nature and finite number of degrees of freedom of the 
cardiovascular system on the timescale of minutes, according to Bracic and Stefanovska (1998).

A study of heart rate variability by Kanters et al. (1994) concluded that there is no evidence of low-dimensional chaos in the time series of $R-R$ intervals, but that nonlinear determinism was present. In another study of R-R intervals by Le Pape et al. (1997), a lexical analysis seemed to indicate that the dynamics appear more random than deterministic, which contradicts this evidence (or at least confuses the issue).

\subsection{Mock Circulatory Systems}

The evolution of the modern mock circulatory system is difficult to delineate because the roots trace back to the 1950 s when artificial hearts were a somewhat fantastic research area and collaboration between distant (and autonomous) research groups was more difficult. Several varieties of mock circulatory system (MCS) have appeared, often at the same time, and often in isolation. It appears the researchers were unaware of the parallel work which was being carried out, and this segregation in research has continued to the present day. In other words, one can classify the mock circulatory system research into three distinct categories:

- Work derived from Kolff's (1959) research

- Work derived from Donovan's (1975) research, and

- Work performed independently (1974-), in apparent isolation

The dates highlighted above are deceptive; literature in the "Kolff tree" does not appear for many years after the initial published research. It is interesting that there is very little crossover between the three main "branches" of MCSs. Donovan does not cite Kolff, although several Donovan derivative works acknowledge the existence of Kolff's paper.

It is informative to follow the evolution of each of the three main trees from their roots to the present day. As noted above, the crossover between trees is minimal and examining the original works and their derivatives in isolation (as opposed to chronologically tracing the development of mock circulatory systems across all three trees) is informative. It should be noted that mock circulatory system research has been somewhat erratic, with large gaps in 
the literature where (apparently) no work was done. In fact, in each of the two trees with identifiable roots, after publication of the "root" paper, a period of at least ten years passes before a derivative work appears.

\subsubsection{Kolff (1959)}

Dr. Willem Kolff is perhaps bestknown as the inventor of the kidney dialysis machine, but he is also a pioneer in the area of artificial heart research. His 1959 paper outlines the requirements for an artificial heart, and a simple mock circulatory system intended to reduce animal experimentation to a minimum. The MCS is inelaborate, consisting of elevated reservoirs connected by tubing, illustrated in Figure 3.1. Kolff's MCS was designed to test artificial hearts, and had four reservoirs; Figure 3.1 shows one half of the setup, corresponding to the systemic loop, which might be used to test VADs.

The VAD (or the left ventricle of the artificial heart) ejects into a tube

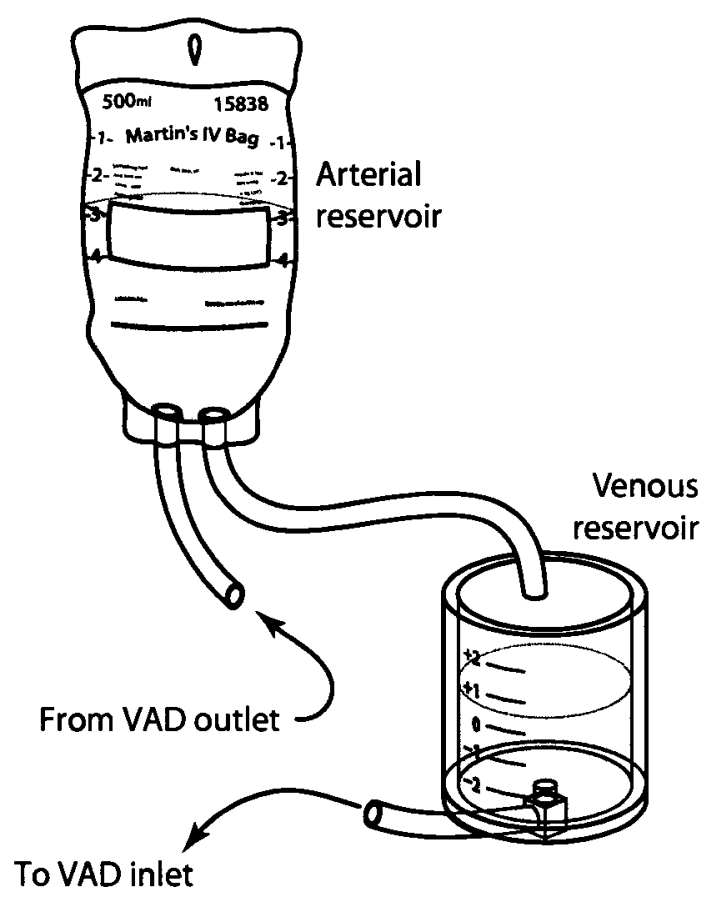
which rises vertically to the arterial

Figure 3.1: Kolff's (original) mock circulatory system reservoir, which might be as simple as an intravenous bag, open to atmosphere (to prevent formation of a siphon). The diastolic pressure in the base of the tube is determined by the height of the water column, i.e., the elevation of the reservoir. In order to overcome fluid inertia problems, Kolff suggests using distensible tubing or surrounding the tube's lowest part with an air chamber under pressure (not illustrated). 
From the arterial reservoir, the water flows under gravity to a lower, venous reservoir. Kolff instrumented his loop by placing a flow-meter in this tube, providing some measure of peripheral flow rate. In a TAH test loop, this venous reservoir would be fed to the right ventricle of the artificial heart, but in a VAD test loop it would be connected to the VAD inlet (as illustrated). Fill pressure for either the TAH or the VAD is set by the static fluid pressure in the venous reservoir.

Diastolic pressure can be changed by varying the heights of the fluid columns. Kolff decided against using tubing clamps or occlusions to affect flow resistance, since their resistance increases with increased flow, whereas the flow resistance provided by the tubing itself varies little with increased flow. Subsequent MCSs use sealed arterial pressure chambers (with trapped air), but the overall designs are still rather similar to Kolff's archetypal system.

\section{Mrava (1970)}

Mrava's work and derivatives comprise a relatively small portion of the literature, however Mrava was aware of Kolff's work (likely because Mrava was affiliated with the Cleveland Clinic, as was Kolff). Mrava's paper gives an excellent overview of the design considerations for MCSs.

The simulation requirements (based on physiology) are outlined in more general terms, without any numerical information, and material properties (including pressure-volume relationships) are discussed as well. Valving and working fluid are discussed to a lesser degree, and Mrava suggests that the working fluid might be replaced with actual blood at some point during artificial heart testing. It is unclear whether or not this practice is observed; the viscous properties of blood can be approximated by other working fluids, and blood does not have a very long shelf life outside of the body. As MCSs have found widespread use as a reliability test bed, it seems more practical to use a working fluid which is more stable than blood.

Mrava comments on the feasibility of mathematical models, citing early work by Otto Frank, who maintained that the behaviours of the various elements of the circulatory system were mathematical and exact, and could be separated and varied in order to form a complete study. Mrava disagrees with this point of view, reinforcing the importance of 
physical studies, and citing several sources to substantiate this claim. This might be one of the first and few papers which compares physical mock circulatory system models with their mathematical counterparts. Obviously this is a very early work, and computer modelling has advanced over the last thirty years.

It is unclear (from this paper) why Mrava favours physical models over mathematical ones. The main criticisms levelled include the working fluid, and the fact that mathematical models are normally based on discrete lumped parameters, a practice which continues to this day. The concern with respect to a Newtonian (in the mathematical models) versus non-Newtonian (in the physical models) working fluid is a valid one, and physical models would indeed allow a better treatment of the blood properties. Technically speaking blood itself could even be used as the working fluid, but (as noted above) the practicality of this is open to debate.

With regard to the lumped parameter models, Mrava's concern is unfounded. In fact the physical MCSs which are described (and favoured) by Mrava consist of discrete segments whose physical parameters are chosen to mimic the behaviour of a portion of the human circulatory system. In other words, the "ideal" mock circulatory system described in Mrava's paper is in fact a lumped parameter model, albeit a physical one, as opposed to a numerical one. Of course the parameter selection process for a physical lumped parameter model is no different than the parameter selection process for a mathematical lumped parameter model, and one could argue that the mathematical model involves one less layer of abstraction. In the physical model one must determine the properties of the segment of the human circulatory system and then apply those parameters to select an appropriate length, or diameter, or type of tubing (for example) which will best match the circulatory system. In a mathematical model the material selection process is removed; once the behaviour of the circulatory system component is established, it can be expressed in a mathematical relationship.

The advantages of a mathematical model (in light of Mrava's concerns) become even more pronounced with modern computers. The circulatory system could be discretized into finer and finer lumped components, which would make the numerical simulation more taxing in terms of simulation requirements, but theoretically more accurate if the lumped parameters closely matched the actual circulatory system. This virtually infinite 
discretization capability does not exist in the physical MCS, because of space and material concerns. (The human circulatory system contains $100,000 \mathrm{~km}$ of vessels.)

Mrava outlines three mock circulatory systems, beginning with Kolff's system. In this system the left ventricle of the artificial heart pumps the working fluid into an elevated reservoir, and the fluid then flows (under gravity) into a lower reservoir. Fluid from the lower reservoir is pumped by the right ventricle of the artificial heart into another elevated reservoir, and moves under gravity back to the original reservoir. All reservoirs are open to atmosphere, and the elevations are chosen to mimic the behaviours of the various segments of the human circulatory system. This loop is designed for total artificial heart testing, and the device is responsible for the movement of blood through both the body (represented by the first elevated reservoir) and the lungs (represented by the second elevated reservoir).

The second physical loop evaluated by Mrava is a more compact design by Rushmer. In this loop the artificial heart pumps fluid from a reservoir that is open to atmosphere into a pressurized chamber. From this pressurized chamber the fluid flows through multiple orifices, eventually exhausting into the reservoir which feeds the pump. With its two chambers, this MCS could be useful for evaluation of ventricular assist devices, but total artificial hearts would require two such loops, one modelling the peripheral flow in the body, and the other modelling the flow in the lungs.

The final MCS discussed by Mrava is the Hydrospace mock circulation system, which was designed by Hydrospace Research Corporation for the National Institute of Health. This loop is more elaborate than the preceding two, but few details are given in this paper. No references for this specific loop are given either, and both its construction and operation are referred to in the past tense, leading one to believe that its development and/or use has been discontinued for whatever reason.

Mrava's paper provides a good overview of the state-of-the-art in mock circulatory system design at the time of writing, as well as a nice treatment of the various design concerns involved in the construction of MCSs. It is odd that there are very few citations of this work. 


\section{Swanson (1977)}

Seven years after Mrava's paper appeared, Swanson cited it in two papers on the development of a mock circulatory system. The first paper, entitled "Cardiovascular System Simulation Requirements," focuses on modelling concerns with respect to mock circulatory system design. Swanson concludes that the single most important quantity is the proximal capacitance or compliance of the large vessels such as the aorta, but more detailed models would obviously involve more detailed studies of the individual contributions of the smaller vessels in the system.

The system Swanson develops differs from prior systems in the way that the circulation is treated. Prior work was based on lumped capacitance and resistance models, while Swanson treats the heart as a pump and the circulatory system as a distributed complianceinertance-resistance network. His first paper outlines the parameter determination procedure, and the second studies the design and performance of the MCS itself.

Swanson discusses the difficulty in developing a mock arterial system which matches the behaviour of the human arterial system. In particular, the pressure waveform in the human aorta and artery is difficult to reproduce using the common Windkessel (R-C-R) setup because of the way the pressure waves are reflected in the tapered, branching arteries in the human circulatory system.

These tapered, branching arteries which terminate in the capillaries are the determining factor for the pressure workload on the heart, according to Swanson, who develops his model accordingly. Swanson's circulatory system model matches the arterial impedance decrease at high heart rate, which is due to the interaction of the pressure waves and the nonlinear capacitance of the large arteries.

In his examination of the blood properties, Swanson discusses the differences between Newtonian and non-Newtonian fluids, and concludes that the most significant difference would be in turbulent behaviour of the blood or working fluid. In a healthy circulatory system there is minimal or no turbulence, with the only possible locations of turbulence being the heart chambers themselves or the aorta, because of the flow through the valves. Although it is not stated outright, it appears Swanson believes that neglecting 
non-Newtonian fluid effects is a defensible position. Based on the evidence given, this is reasonable.

Swanson declares the essential features of a circulatory system simulator to be:

- Variable stroke volume

- Variable pulse rate

- Controllable ejection fraction of beat period

- Correct flow and pressure pulses in the proximal vessels

- Access to pressure measurements across the valves and in the proximal vessels

- Direct and accurate measurement of regurgitant flow

- Direct measure of cardiac power and valve power consumption, and

- Test sections which are modular, model the actual geometry of the circulatory system, and are observable and photographable

Swanson's second paper (1977b), entitled “A Simple Cardiovascular System Simulator: Design and Performance" outlines the mock circulatory system based on these design concerns. This particular circuit includes a pump design which would not be suitable for implantation in the human body as an artificial heart or assist device. Although it is not specifically stated, Swanson's primary concern seems to be modelling the behaviour of the human circulatory system including the natural heart. It is not obvious what the goal of this line of development would be, unless the "pseudo-natural heart" pump could be replaced with an artificial heart without losing any of the circulatory system dynamics present in the mock circulatory test loop.

Swanson's original loop used a lumped parameter surge tank (similar to the loops discussed in the Mrava paper), but this configuration failed to give the proper pressure wave shape. Swanson's focus on instrumentation lends credibility to this result, suggesting that none of the MCSs discussed in the Mrava paper would adequately match the dynamics of the human circulatory system. This is an important revelation, as the "sealed pressure chamber, open reservoir" configuration exists in numerous MCS variants. Swanson's final 
loop design uses a distributed parameter system, in the form of a longer piece of tubing with several hose-clamps along its length. The hose clamps provided flow resistance, but also contributed to the flow turbulence. The argument is made that flow turbulence has a minimal effect on the system because it is overshadowed by arterial reactance.

The long length of tubing exhausts into a reservoir that is open to atmosphere, and this in turn feeds back into the pump. Obviously this loop models the left side of the circulatory system only; a second version would be needed to model the blood flow through the lungs.

\section{Scotten (1979)}

The final paper (to-date) to cite Mrava, and the final paper in the Mrava tree, by Scotten et al. (1979), is focused on the design of a MCS for testing artificial mitral valves, rather than artificial hearts. Even so, the developed loop is quite complicated, containing many of the features found in loops designed for artificial heart testing.

The mitral valve is the heart valve which controls the flow from the left atrium into the left ventricle, so Scotten's loop involves a model of the left side of the heart. The left atrium is treated as a reservoir open to atmosphere which empties into the left ventricle via the (artificial) mitral valve. The left ventricle itself is treated as a compliant chamber, and its expansion or contraction is driven by transmural pressure. The ventricle sits in a hydraulic fluid which can be pressurized or relaxed to simulate the cardiac cycle. The ventricle ejects the working fluid into a compliant tube, and the fluid moves through two adjustable Windkessel elements before returning to the left atrium reservoir.

The focus of this paper is on the flow through the artificial mitral valve, and very little discussion of the loop parameters is presented.

\subsubsection{Donovan (1975)}

Donovan's paper is perhaps the best received and most oft-cited work on mock circulatory systems. It presents detailed information on a mock circulatory system designed for the testing of total artificial hearts, which may explain its acceptance in the literature. (Many design and construction details are presented, along with some performance data; 
the paper is clearly focused on the design and construction of a mock circulatory system rather than its use or performance.)

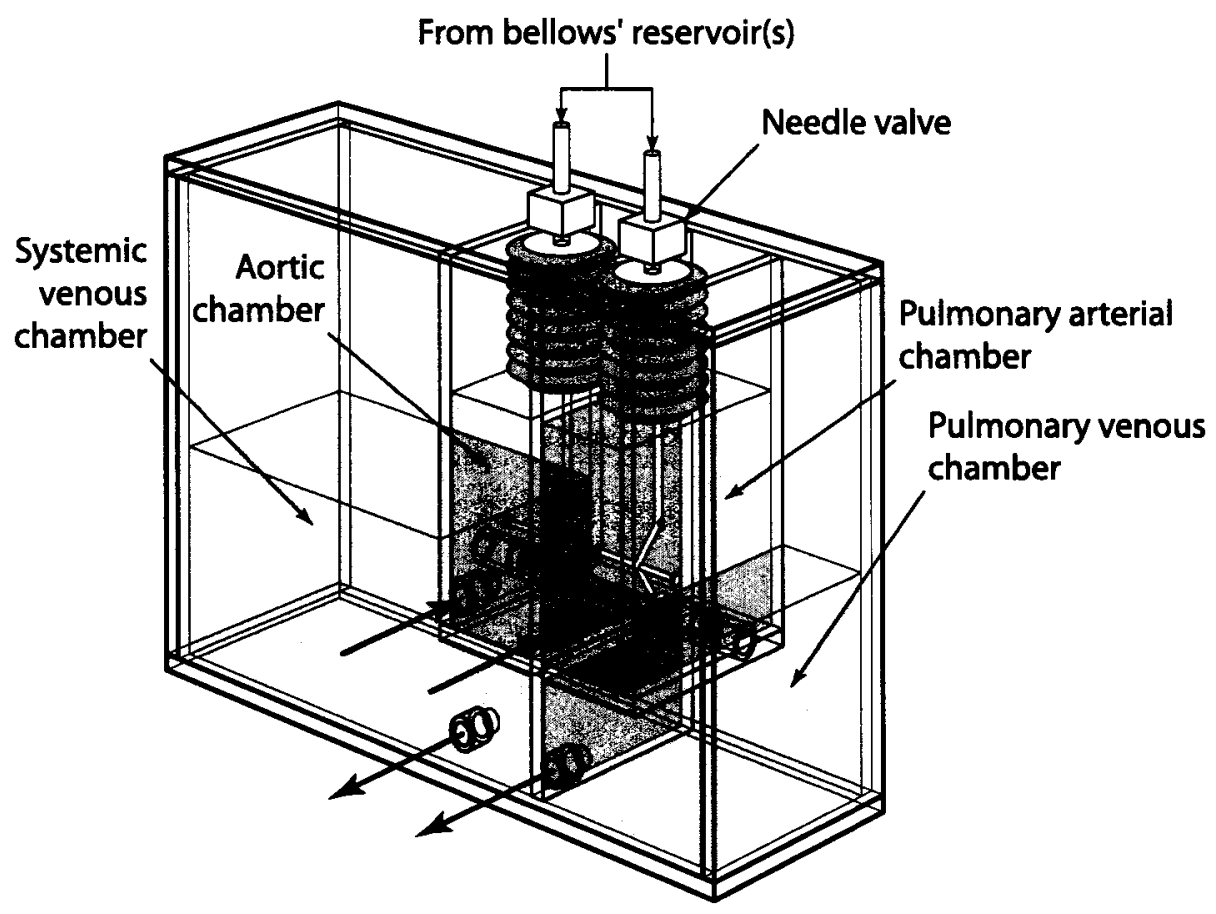

Figure 3.2: Donovan mock circulatory system

This particular mock circulatory system, illustrated in Figure 3.2, consists of four chambers, all closed to atmosphere. The chamber compliances are determined by the volume of trapped air, and the air pressure in each of the chambers can be varied. The flow resistances in the system are controlled by adjustable bellows which are linked to control valves. The bellows are filled with water and connected to reservoirs. The internal (water) pressure of the bellows can be adjusted by varying the height of the reservoirs, and the external pressure is simply the air pressure in the atrial chamber.

Fluid flows from the systemic venous reservoir (chamber) into the right ventricle of the artificial heart and is pressurized and ejected into the pulmonary arterial chamber. It flows through the pulmonary resistance valve into the pulmonary venous chamber. The pulmonary resistance is controlled by the aforementioned pulmonary bellows, which is filled 
with water internally, and located in the pulmonary arterial chamber. In other words, the pulmonary flow resistance is controlled by the height of the pulmonary water reservoir (which feeds the bellows) and the air pressure in the pulmonary arterial chamber.

Fluid then flows from the pulmonary venous chamber into the left ventricle of the artificial heart, where it is pressurized and ejected into the systemic arterial chamber. The flow between this chamber and the systemic venous chamber is through the systemic resistance valve, which is also controlled by a bellows mechanism. The systemic bellows is located in the systemic arterial chamber, and has a water reservoir separate from the pulmonary water reservoir.

The Donovan mock circulatory system allows one to vary four compliances, namely: the systemic venous compliance, the pulmonary arterial compliance, the pulmonary venous compliance, and the systemic arterial compliance. These are adjusted by changing the air pressure in each of the four chambers. In addition to the compliances, the two flow resistances can be changed by adjusting the spring constant of the bellows valves and the height of the bellows water reservoirs. Obviously the circulation pressure can also be varied.

The bellows-controlled resistances allow the system to adjust to changes in cardiac output. For example, if the artificial heart increases its output, the pressure increase in the respective arterial chamber compresses the bellows and decreases the flow resistance between the respective arterial and venous chambers. This has the effect of reducing the respective arterial pressure as more fluid moves into the respective venous chamber.

Although this loop was designed for testing total artificial hearts (and includes a pulmonary as well as systemic loop) it can also be modified for testing ventricular assist devices by eliminating or ignoring the pulmonary half of the loop.

\section{Arabia (1984)}

There is a nearly ten-year gap in the mock circulatory system literature following Donovan's paper. The reason for this is not clear. 
Arabia's paper, which is the next in the "Donovan Tree," details a MCS design focused on blood volume and pressure distribution, rather than solely on hydraulic impedancematching. The cardiac output is regulated by varying the systemic resistances and the mean circulatory pressure. (The systemic resistances affect the pressure-volume behaviour of the system.) Rather than using pressurized air chambers as compliance elements, Arabia's MCS design uses spring-loaded pistons with a rolling diaphragm seal. In this way the compliances can be varied by changing the spring constants, rather than changing air pressure.

The slope and offset of the venous compliance can be adjusted to simulate exercise or a reduction in activity level, but the authors feel that changes in mean circulatory pressure in the human body are due to variations in the venous compliance offset, not changes in the venous compliance (slope) itself. In the first prototype of the Arabia MCS, the venous compliance is assumed to be constant.

The arterial tree in the Arabia MCS is more complicated than the single-chamber in the Donovan loop; the Arabia aorta tree consists of four resistance-inertance-compliance (RLC) elements. These are tuned to match the hydraulic impedance an artificial heart would see in the human circulatory system as well as the total systemic compliance. The resistances in the Arabia mock circulatory loop are varied via control system, but the authors acknowledge that there is no data for the dynamic behaviour of either the resistances or the compliance elements. This restricts MCS experiments to steady-state conditions, as the dynamic behaviour of the circulatory system must be understood in order to model transient response. In this mock circulatory system, the resistances and compliances are adjusted by the operator based on the experimental conditions desired, and then held constant over the length of the experiment.

The authors also present a simplified mathematical analysis of the system, to illustrate the behaviour of the mock circulatory system, and how it should be controlled to produce a desired set of systemic conditions.

\section{Knierbein (1992)}

Once again there is a large gap in the literature after Arabia's work, until Knierbein introduces another, simplified, mock circulatory model. Knierbein's MCS is based on a 
three-element Windkessel model. The arterial compliance is modelled by a sealed (pressurized) air chamber, the peripheral resistance by an adjustable flow resistor (i.e., a throttle valve), and the venous system by an open return tube.

In this mock circulatory system, the artificial heart pumps fluid into the pressurized arterial chamber whose compliance can be adjusted by changing the volume of trapped air, as seen previously in the Donovan model. The fluid then passes through the throttle valve into the atrial chamber and is returned to the artificial heart. If a total artificial heart is to be tested, two MCSs can be configured with different compliances, one representing the behaviour of the pulmonary loop and the other representing the systemic behaviour.

The Knierbein MCS is one of the simpler configurations found in the literature, and much of the design emphasizes ease of construction, assembly and use, rather than matching the complicated physiological behaviour of the human circulatory system. That being said, the loop produces quite reasonable results in terms of arterial pressure and flow, for a variety of artificial hearts.

\section{Ferrari (1992)}

In stark contrast to the simple Knierbein mock circulatory system, the "Donovan Tree" also contains the most involved piece of work, by Ferrari. The development of this mock circulatory system is outlined in a series of papers, beginning in 1992 and continuing to present-day.

The development of the Ferrari MCS begins with a mathematical model of the human cardiovascular system, designed to assist in clinical training. The computer simulation is modular, and relatively simple in terms of its treatment of much of the circulatory system, with the exception of the ventricle behaviour. Ventricle behaviour (of the natural heart, not an artificial heart) is very complicated, and Ferrari et al. attempt to reasonably model the filling and ejection phases.

The remainder of the circulatory system is not treated in nearly as much detail. The arterial section can be configured as a simple Windkessel model, a modified Windkessel model, or a different (undetermined) model altogether. The venous system is a simple Windkessel model. 
The pulmonary loop is modelled in a similar fashion, with a somewhat involved ventricle model, an arterial section as described above, and a simple Windkessel venous segment.

Following the development of the mathematical model, Ferrari et al. (1994) construct a physical mock circulatory system that is based on the numerical computer simulation and designed to model the interaction of the left ventricle and a ventricular assist device with the circulatory system. This lumped parameter physical model closely matches the computer simulation in terms of design, however the first implementation omits the venous lumped parameter, which is found in the computer model.

In a physical MCS, the ventricle would ideally be treated using a variable elastance model which reproduces Starling's Law (Starling et al., 1987). Starling's Law relates the atrial pressure, stroke volume, end diastolic and end systolic volumes. This requires that the compliance of the ventricle be variable over the course of the mock loop operation, which is workable in a computer model but an extremely difficult condition to achieve in a physical system. Ferrari et al. overcome this obstacle by controlling ventricular volume instead of pressure in a fashion which complies with Starling's Law. This is achieved using a normalized ventricular volume waveform in a feedback control loop; atrial pressure is measured at the end of each cycle and used to update the volume waveform. The time delay of one cardiac cycle which is introduced by this method is found to be non-critical by Ferrari et al., who are interested in steady-state behaviour.

The left ventricle (feedback-controlled piston assembly) is connected to a pneumatic VAD which can be driven at a constant rate between 50 and $200 \mathrm{bpm}$. The VAD is connected to the arterial tree, which is a modified Windkessel model in that it includes a variable flow resistance element which is controlled by a computer. As mentioned above, the initial physical MCS developed by Ferrari et al. does not include the venous segment.

The second evolution of the Ferrari loop (1998) improves the arterial model and adds a simple venous segment to the system. This segment consists of a spring capacitor whose compliance is fixed. No other loop segments are changed noticeably, although the data acquisition system and instrumentation is improved in this iteration of the loop. 
The third evolution of the Ferrari loop (2000) involves the inclusion of a detailed physical model of the human systemic arterial tree which is designed based on the numerical computer simulation results obtained using the mathematical MCS model discussed above. The improved arterial model consists of a tapered tube with eight segments which represent parts of the human arterial tree, from the ascending to the abdominal aorta. Each segment has a different length, radius and thickness, but the same Young's Modulus (i.e., the same material). The entire assembly is cast in one continuous piece from silicone rubber.

Each segment in the arterial model can be modelled using an RLC configuration, with the compliance calculated based on the geometry and material properties. The model includes a resistive load for the peripheral circulation as well as Windkessel elements for larger sections of circulation such as the brain or limbs. To model this circulation the tapered tube branches after each segment, but these peripheral flow branches are merged into a single pipeline, rather than kept separate. According to Ferrari, this single pipeline simplification has limited effect on the impedance of the model.

With the addition of this new arterial segment, the Ferrari loop is quite an involved model of the proximal human circulatory system. The treatment of the venous segment remains simple, but some people argue that the venous behaviour has minimal impact on circulation.

Following the addition of the arterial segment, Ferrari et al. (2001) have focused on improving the ventricular model and testing under physiologic conditions (2002).

\section{Woodruff (1997)}

Woodruff $e$ al. identify a need for a mock circulatory system "of small size and weight that could function in a microgravity environment for the investigation of cardiovascular response to the weightlessness of space flight" and proceed to develop such a loop. The air-filled chambers which are used as compliance elements in the Donovan loop will not function properly in microgravity, so Woodruff et al. adapt a spring-loaded piston system found in the Penn State mock loop (similar to Arabia's system). The mock circulatory system developed has adjustable compliances (both slope and offset) and these adjustments can be made without the use of tools. The system is simple, consisting of one resistance 
element and two compliance elements, one compliance element representing the arterial compliance and the other the venous compliance. The results are found to match the Penn State MCS, but Woodruff's system has a smaller size and weight.

\section{Vermette (1998)}

Rather than modelling the entire human circulatory system (even in a lumped parameter fashion as many MCSs do), Vermette et al. focus on the flow conditions in one particular segment at any given time. This particular mock circulatory system is designed for material testing or prosthesis testing, and its small test section can reproduce the various flows found in the human circulatory system. These flow conditions range from the high pressure, pulsatile flow in the aorta to the low pressure, almost uniform flow in the venous system.

The system is designed to produce flows up to $6 \mathrm{lpm}$ through the test section, with a transmural pressure of up to $500 \mathrm{mmHg}$ and a pulsation frequency of $0-4 \mathrm{~Hz}$. No effort is made to model any of the various components of the circulatory system; in this particular loop the flow conditions in the human circulatory system must be known a priori in order to program the MCS controller.

Fluid temperature is also controlled in this MCS as it attempts to closely match the in vivo conditions which might be seen by the prosthetic component being tested. The test segment consists of two concentric tubes, one of which is filled with a stationary, temperature controlled "physiological" solution. The inner tube would be replaced with a cannula (for example) or have a valve inserted in it (for example) depending on the test being performed.

\section{Sharp (1999)}

This paper details the development of a simple mock circulatory system loop. As with many MCS designs, the focus is on matching the hydraulic impedance of the human circulatory system, which results in a very basic design. The physical model developed is based on numerical simulations, which attempted to optimize the number of elements in the MCS. Two-, three-, four- and five-element models were simulated, with all but the simplest model closely matching the human circulatory system impedance. Impedance 
modulus and phase were plotted and compared to measurements obtained from the human circulatory system.

The two-element resistance-compliance (RC, i.e., Windkessel) model's phase does not match the circulatory system behaviour at high frequencies, and the modulus diverges from the experimental measurements at high frequencies as well. One of the other models has phase problems at low frequencies, but the model is not identifiable from the included plot, and the authors do not discuss the distortion in the phase graph which occurs right in the operating range of the natural heart, at approximately $1 \mathrm{~Hz}$.

Based on the numerical results, the authors feel that the two-element RC model inaccurately reproduces the impedance behaviour of the human circulatory system, with the systolic flow peak occurring too early, and the magnitude of the flow being too large. The final configuration arrived at consists of a resistance, an air chamber compliance, and another resistance, all in series. All elements have a linear behaviour.

Results based on experimental tests show that the new resistance-compliance-resistance (RCR) model behaviour more closely matches the human circulatory system when compared to the Donovan MCS, or the Penn State MCS.

\section{Baloa (2001)}

Baloa et al. focus on the ventricular segment of mock circulatory systems, developing a new control strategy to model the Starling response of the natural heart. The elastance control loop calculates the desired chamber volume using a time-varying elastance function and the ventricular chamber volume. This is used as a reference signal for the pressure control loop. This approach is similar to Ferrari's, which uses normalized pressure-volume relationships for the ventricle.

\subsubsection{The Independent Tree}

In the literature there exist a number of papers that cannot be included in either of the major trees outlined above; they do not reference any of the above "root" papers, and many of them are not themselves referenced by other papers in the literature. They exist in relative isolation. 


\section{Wallner (1974)}

Wallner's paper predates Donovan's work, but isn't referenced by any subsequent papers. It briefly outlines the development of a mock circulatory system but few details are provided.*

Based on the schematic diagram included in the paper, the lumped elements include an aorta model, and arterial model, a peripheral resistance and a venous segment. Pressures and flow-rates are measured at several points in the system.

\section{Testa (1975)}

Testa et al. develop a mathematical model and a physical MCS in tandem. The system has three parallel branches for the systemic circulation, one of which represents the heart, one the brain, and the other the rest of the circulation. The particularly distinct aspect of Testa's mock circulatory loop is the natural heart model. The heart is included as nested chambers, and the working fluid perfuses through a bed of glass beads which are supposed to represent the myocardium. The actual ventricles themselves do not contract; they are merely chambers. The arterial and venous circulation are also represented using glass beads and the capillary circulation by cotton wool. It does not appear that there are any compliances in the system, although connections are made by surgical tubing (which is slightly compliant).

\section{Withington (1991)}

As part of the development of a ventricular assist device, Withington et al. utilize the National Heart Lung and Blood Institute MCS (which is a Donovan-style loop), and discuss problems discovered during certain phases of the pump cycle. These problems involve high and low pressures generated within the VAD which would not be seen in physiology. This is the situation outlined by Sharp and Dharmalingham in their studies on varying-element circulatory models. Sharp and Dharmalingham noted that the simple RC (Windkessel) configuration used by many MCSs does not correctly match the behaviour of the circulatory system, with the flow peak occurring too early, and having too large a magnitude. Sharp's paper is published eight years after this particular work, and would obviously not have been available to the authors.

\footnotetext{
*The paper is also in German, and my Deutsch is subpar.
} 


\section{Cochrane (1991)}

Cochrane's work modifies the Windkessel model to include valve-opening dynamics, including stenosis and regurgitation. This is accomplished using an antechamber located before the arterial compliance (air chamber). The antechamber has two valves, one representing the mitral valve and the other representing the aortic valve. The pump (which is a positive displacement piston) is fed from an elevated atrial chamber, through the mitral valve. The aortic valve separates the piston from the compliance and resistance elements. As the piston is withdrawn from the cylinder, the aortic valve closes and the mitral valve opens. In the absence of a positive-displacement pump, fluid from the atrial chamber would flow under gravity, but the piston in this loop would draw fluid into the cylinder. The mitral valve closes as the piston pressurizes the chamber, and the aortic valve opens.

This mock circulatory system can be used to study valve stenosis or valve regurgitation. To study stenosis, the mitral valve opening area and regurgitant area are fixed, and the aortic valve regurgitant area is also fixed. To study aortic valve regurgitation, the opening areas of both valves are fixed, and the regurgitant area of the aortic valve is varied.

In the regurgitation studies, the stroke volume was held constant, but in the natural system the heart would adapt to the regurgitation, increasing its output to supply sufficient blood flow to the body. Cochrane examines the excess work required by the pump in order to maintain the same flow rate in the presence of regurgitation.

The natural heart also adapts to aortic stenosis by increasing the ventricular pressure. Cochrane's loop can be used to study the maximum cardiac output (assuming a maximum ventricular pressure) for a given stenosis.

In addition to studying circulatory problems related to the heart valves and the aorta, Cochrane also presents results which show the variation of regurgitated volume with changes in peripheral resistance. As expected, the regurgitated volume increases with hypertension. (This also means the regurgitation would change as part of the response to exercise, or changes in external conditions.) 


\section{Tsitlik (1992)}

Tsitlik et al. develop an electrical, rather than hydraulic, model of the human circulation using nonlinear capacitors. Two modelling problems cited by the authors are: that electrical current must flow in closed loops, while there is no similar hydraulic constraint, and; that electrical capacitors behave differently than hydraulic compliance chambers. To overcome the second problem one would require a capacitance which the authors refer to as a Nonlinear Residual Charge Capacitor. Such a device does not exist, and that aspect of the developed model is purely mathematical. Electrical models of the various blood vessels and the left ventricular loading are discussed and criticized. The authors note that the flow through the electrical system in many popular models bears no relation to the actual hydraulic flow in a mock circulatory system. This is because the discharge paths for the capacitors are unrealistic, due to the necessary electrical connection of two nodes which are not connected in the hydraulic equivalent (a result of the first problem with electrical analogues noted above).

\section{Garrison (1994)}

Garrison et al. develop a MCS for the study of chemical additives in circulation and their effects on hemolysis. The resultant loop is made from biocompatible materials (unlike the previous loops discussed) and is obviously designed to be used with blood as the working fluid. The size of the loop is reduced in order to require minimal amounts of blood. (Other MCSs use a blood analogue normally composed of water and glycerin.)

The developed loop consists of an aortic compliance chamber, a clamp which provides peripheral resistance, and a venous compliance chamber. All corners are smooth, and flow from the connected VAD as well as aortic and venous pressure can be measured. Compliances are modelled using air pressure, but to avoid contact between blood and air the compliance chamber consists of two sacs in a Plexiglas box. The box is just large enough to contain one full and one empty sac, and one of the sacs is pressurized with air. The other sac is connected to the MCS. In this way blood flowing into the blood sac is maintained at a relatively constant pressure as the blood volume changes, without allowing the blood to come in contact with air. 
The resistance between the two compliance chambers results in a pressure drop, allowing the venous pressure to be much lower than the arterial pressure. Pressures and flows in the loop are consistent with those measured in the human circulatory system, but no comparison is made with other MCSs. This sort of a comparison would be straightforward and very advantageous, because there is a lot of literature on the various aspects of MCSs.

For the hemolysis study, the blood is first centrifuged and the amount of hemoglobin in the plasma is measured. The procedure was repeated after the experiment to measure the change in hemoglobin, which is related to the destruction of red blood cells (i.e., hemolysis). To ensure the shear stresses through the clamp were not causing hemolysis the loop was operated with and without a clamp and the effect on hemoglobin levels was measured. It was found that the clamp did not induce hemolysis.

\section{Chen (1997)}

Chen et al. develop an electrical model of the human circulatory system. It does not appear that they are aware of the work done by Tsitlik, and the problems with electrical analogues which Tsitlik mentions are not addressed by the authors. The mathematical (predicted) results obtained from the model are compared with several hydraulic models.

\subsection{The Arterial Pressure Waveform}

It seems reasonable that the blood vessels closest to the VAD will have the greatest influence on its operation. Therefore in order to ensure rigorous in vitro testing we need to match the arterial behaviour of the human circulatory system, both in our mock circulatory systems and our mathematical simulations.

The arterial pressure waveform has a distinct shape, illustrated in Figure 3.3, which shows aortic pressure over the course of three beats. Note that there is a slight delay between the onset of systole and the actual opening of the aortic valve. Once the valve is open aortic pressure rises rapidly, and then falls off at the end of systole. The closure of the aortic valve triggers a sort of elastic recoil, which presents itself as a notch in the pressure waveform - the so-called "dicrotic notch." This general waveform shape distorts as it travels down the arterial tree, but the main features such as the dicrotic notch persist 


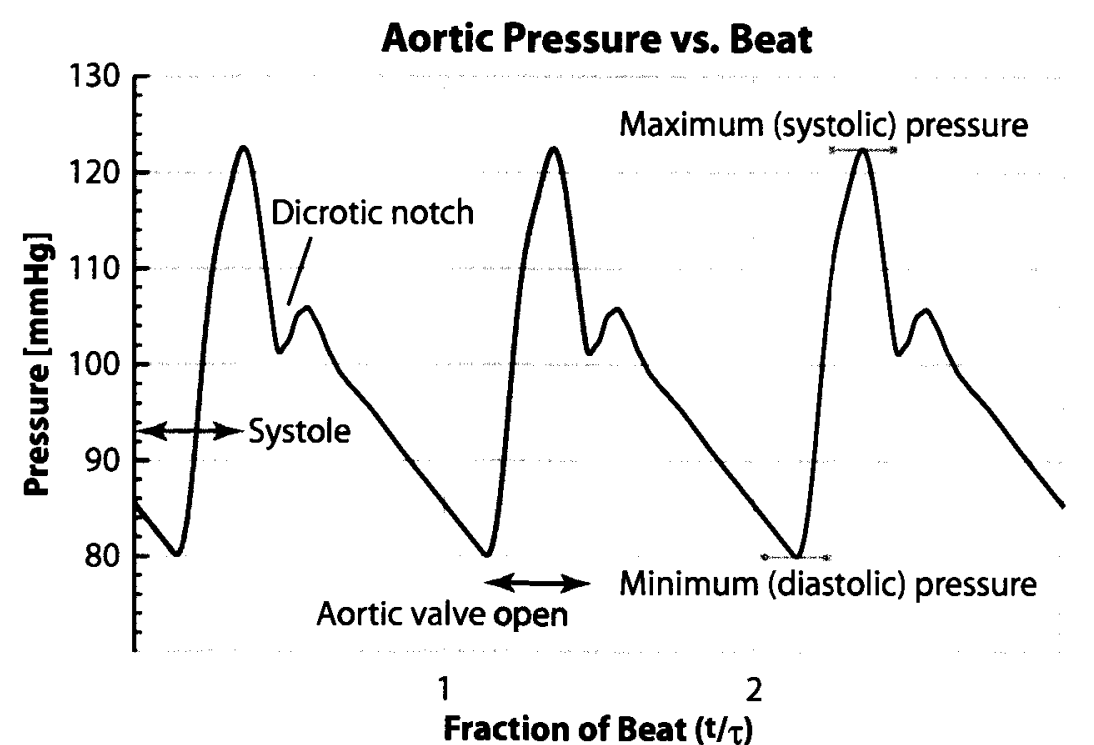

Figure 3.3: Features of the aortic pressure waveform

(Dahlgren et al., 1991). Although the dicrotic notch is triggered by the closure of the aortic valve, the arterial pressure is already dropping at that point; the end of systole and the corresponding systolic peak precede the valve closure. The elastic recoil is accompanied by a very slight reversal in flow, and affected by valve function (or dysfunction) as described by Fukuda et al. (1996). A study of the carotid* artery by Hasegawa et al. (1991) showed that the two carotid artery sounds are exactly coincident with the systolic pressure upstroke and dicrotic notch (respectively).

The various features of the aortic pressure waveform can be used to diagnose cardiovascular problems (McGhee and Bridges, 2002). In cases of low cardiac output, for example, the aortic pressure pulse is very distorted, and the dicrotic notch deepens, creating an Mshaped pressure wave and two distinct pulsations (Smith and Craige, 1986). Dawber et al. (1973) used the arterial pressure waveform to study heart disease, and Honda et al. (1995) use it to investigate arterial stenosis.

The aortic pressure waveform is mainly a function of ventricular output, aortic elastance, and aortic valve function. The actual dicrotic notch pressure (i.e., how "deeply" the notch

\footnotetext{
${ }^{*}$ The vessels supplying blood to the head.
} 
cuts into the pressure waveform) is related to mean arterial pressure and pulse pressure, but not heart rate or cardiovascular output or arterial compliance (Hebert et al., 1995). Note that Hebert's findings on cardiovascular output conflict with Smith's conclusions on the mechanism of the dicrotic pulse. These sorts of contradictions are present throughout the literature.

There are studies which assert that the dicrotic notch pressure is the mean arterial pressure, at least in the pulmonary system. This would be how the heart optimizes external power from the ventricle. Chemla et al. (1996) tested fifteen individuals, both at rest and during the Valsalva manoeuvre* and found a strong correlation $(r>0.98)$ between the dicrotic pressure and the mean arterial pressure. Hebert et al. (1995) contend that there is a slight difference between dicrotic pressure and mean arterial pressure, with the former overestimating the latter. Hebert notes that this discrepancy does not exist in children, suggesting that young people differ from adults in certain key arterial properties. King (1946) showed that the elastic response of the aorta changed with age, and we know that the geometric properties of the aorta change as people grow. These changes in vessel geometry may have subtle and unexpected impacts on cardiovascular performance.

Thyrault et al. (1998) study the correlation between dicrotic notch pressure and mean pulmonary arterial pressure in critically ill, mechanically ventilated patients and conclude that there is no statistical difference between the two pressures. The mechanical ventilation adds an interesting level of control to the experiment, because respiratory intervals would be consistent.

Schwid et al. (1987) study the arterial pressure waveform in the radial artery (the artery at the wrist where people typically take their pulse), and show that the resonant properties of the arterial system significantly alter the pressure waveform as it propagates from the

*The Valsalva manoeuvre is used to assess the reflex control of the cardiovascular system. The patient forcibly exhales against a closed throat (as if swallowing and exhaling simultaneously) and holds this for at least ten seconds. Aortic pressure increases, resulting in a slowed heart rate due to nervous system baroreceptor response. At the same time, the increased pressure in the chest cavity constricts venous return to the heart, resulting in reduced cardiac output. This leads to a subsequent fall in aortic pressure, and a reflex increase in heart rate. After several seconds the arterial pressure is reduced and the heart rate is higher. Once the patient starts breathing again the increased venous return causes a higher cardiac output and a corresponding increase in arterial pressure. Eventually both the heart rate and arterial pressure return to nominal levels. 
aorta to the radial artery. They conclude that the radial artery pressure peak is not a good measure of the end-systolic pressure, and that the radial arterial pressure waveform is mainly dependent on the vessel wall properties, not on the aortic valve or the peripheral resistance. The radial artery can be used to estimate mean and diastolic pressures but is unreliable for anything else.

\subsection{Nonlinear Dynamic Systems}

One of the stated goals at the outset of this research was to examine nonlinearities in the arterial system and their impact (if any) on circulatory dynamics. As outlined earlier, the literature displays evidence of potentially nonlinear and/or chaotic behaviour in the cardiovascular system. The author believes this behaviour may stem from the dynamics of the cardiovascular system itself, and in fact may be an integral part of the circulatory system design. Without the requisite understanding (i.e., in foresight) this section of the literature review will seem out of place, and perhaps prescient. It is included for completeness; its influence will be more apparent after the discussion in Chapter 4, Section 4.3. Needless to say, the connection between the arterial system and this class of oscillator was not always apparent to the author, and this portion of the literature review was done much later.

The difficulty in treating nonlinear systems is that they are (for the most part) mathematically intractable; ideally one tries to convert a system of equations into a normal form, where equivalence can be made between systems. Such problems are often treated numerically because there is no closed-form mathematical solution. Although it will not be immediately obvious why, the arterial tree can be related to a class of nonlinear systems known as "impact oscillators." This type of system involves a standard mass-spring-damper setup where the mass impacts a rigid, fixed stop, as outlined in Walker and Soule (1996). The elastic impact oscillator can display regular, chaotic, and unbounded behaviour depending on how it is driven (Lamba, 1995). Rather than a rigid wall impact, the oscillator can have a nonlinear elastic term and still display the same types of behaviour (Mickens, 2002 or Mickens, 2004).

The case for equivalence between these impact oscillators and the arterial system will be made in Chapter 4, Section 4.4. For a good introduction to the oscillators themselves, the 
reader is referred to Walker and Soule (1996), and Lamba (1995), both of whom treat the system in detail. Walker and Soule study the effect of impact position on the oscillations of a mass-spring system. Their investigation identifies both chaotic and periodic oscillatory behaviour, with each type of motion confined to its specific fractal basin of attraction. 


\section{Chapter 4}

\section{Theoretical}

G IVEN the intricacy of the human circulatory system, it is natural to assume that its interaction with an assist device would be complicated. There may be dynamic behaviour which would have an impact (either positive or negative) on artificial heart design, in terms of reliability, or efficiency, or both. Avoiding the detrimental regimes and taking advantage of the beneficial behaviour will be important to future devices.

There are some modelling decisions to be made; for a preliminary study it makes sense to focus our efforts on the proximal vessels, namely the arterial system. We want to capture any interesting dynamics, but we also want a tractable system, and simple equations. In order to allow for chaotic behaviour, our model must be at least second order.* The aorta model is itself second order, and we could choose to study it on its own, however including the additional arterial compliance element (from the DCM on Page 40) doesn't necessarily increase the order of the system. If we set the boundary conditions such that we control both the blood inflow (into the aorta) and outflow (out of the arteries), the arterial compliance element is not independent. On the other hand, setting the boundary conditions to provide an inflow source and an outlet back-pressure increases the order of the system.

Finding a representative arterial subsystem involves balancing the boundary conditions (and the difficulty in determining realistic boundary values) against the inclusion of additional model elements, and the more complicated equations which result. As an example: it makes sense to use volume flow rate as the inlet boundary condition; the literature contains this type of physiological data. The outlet boundary condition (and where to "break"

*Assuming we have a continuous-time system, and not a discrete-time map (Strogatz, 1994 or Nayfeh and Balachandran, 1995). 
the arterial system) is not as obvious. If we include only the aortic elements, and break the arterial system as in Figure 4.1, the outlet boundary condition is a back-pressure. Unfortunately, this pressure is not constant. For a first approximation, we might set the outlet boundary condition as the mean arterial pressure, however we know from simulation of the thirteenth-order system that the pressure fluctuations in the arteries are significant. These sorts of modelling concerns will be dealt with in the subsequent sections.

We will use Buckingham's $\Pi$-Theorem to nondimensionalize our models, and reduce the number of variables. The nondimensional groups depend on where we "break" the system (i.e., what components are included), and what we choose as boundary conditions. Recall that the repeating variables cannot form a $\Pi$-group amongst themselves.

We will deal with the linear dynam-

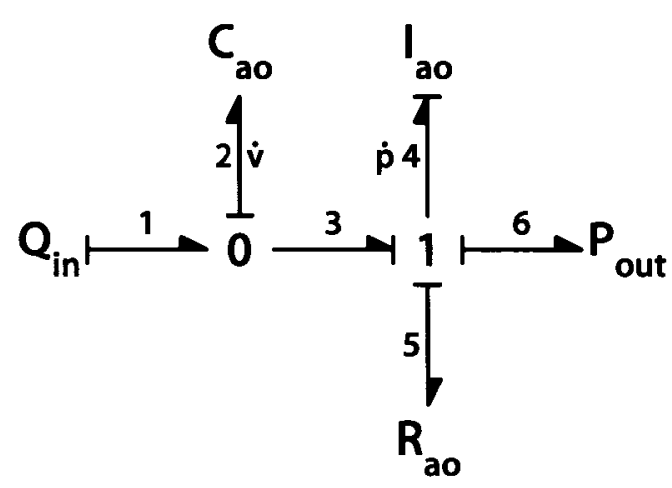

Figure 4.1: The arterial DCM ics of the arterial system first, and then examine nonlinear behaviour.

\subsection{Mathematical Model of the Arterial System}

We will examine two arterial system models. The choice of model depends on the desired boundary conditions; if we would rather control both the blood inflow and outflow, we can model the arterial system as in Figure 4.2. If, on the other hand, we would rather let the arterial system eject into a pressure sink, we can model the system as in Figure 4.3. Note that both Figure 4.2 and Figure 4.3 are subsets of the larger circulatory system model presented in Section 2.6, beginning on Page 36. In Section 2.6 this configuration was labelled the dual-compliance arterial model (DCM). In the DCM, the aortic constitutive relation is normally taken to be nonlinear, but this is not a requirement of the actual DCM itself; it is merely a better representation of the actual human circulatory system. For this study, we 


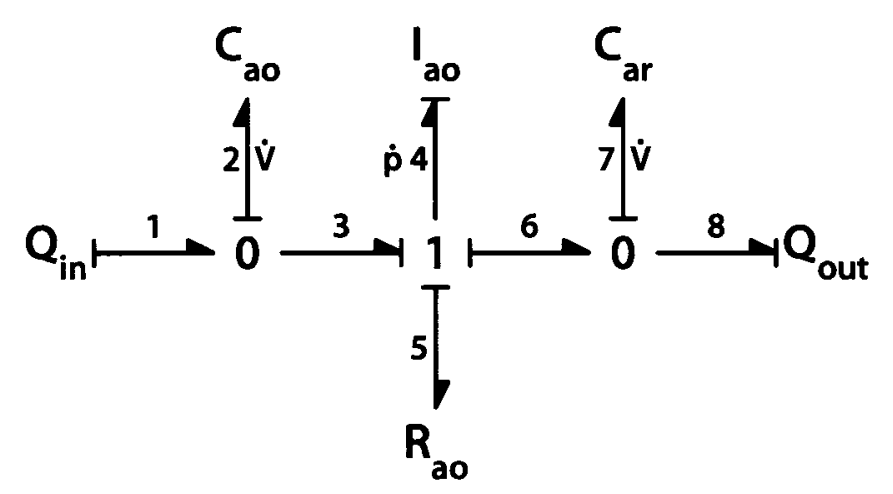

Figure 4.2: Arterial DCM bond graph with inlet flow source and outlet flow sink

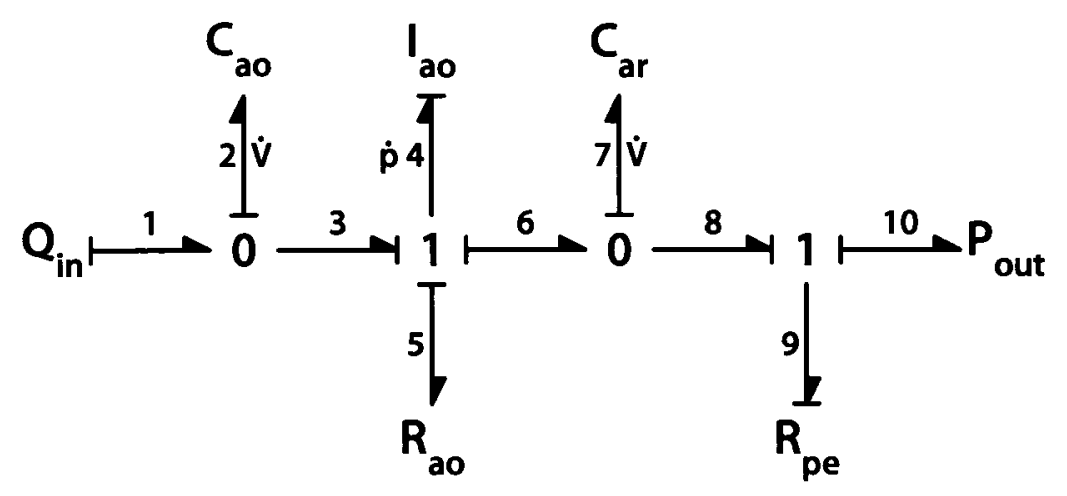

Figure 4.3: Arterial DCM bond graph with inlet flow source and outlet pressure sink

will treat the aortic compliance as linear, and examine the linear dynamics of the arterial system.

The systems in Figure 4.2 and Figure 4.3 differ in terms of both their boundary conditions and their order. Although it is not readily apparent from the bond graph, the flow-in, flowout system is only second-order; the two compliance volumes are not independent of each other. This is more readily seen from the equations. The differential equations describing 
the system in Figure 4.2 are:

$$
\begin{aligned}
& \dot{V}_{2}(t)=Q_{i n}(t)-\frac{p_{4}(t)}{I_{a o}} \\
& \dot{p}_{4}(t)=\frac{V_{2}(t)}{C_{a o}}-\frac{p_{4}(t)}{I_{a o}} \cdot R_{a 0}-\frac{V_{7}(t)}{C_{a r}} \\
& \dot{V}_{7}(t)=\frac{p_{4}(t)}{I_{a o}}-Q_{o u t}(t)
\end{aligned}
$$

Note that two of the eigenvalues are identical. This indicates a second-order system (there are only two independent modes) and will result in a state matrix of rank two, as we will see shortly. The advantage of this formulation is that a second-order system lends itself well to analysis. The third-order system (which results from Figure 4.3) is more complex. The equations describing this system are:

$$
\begin{aligned}
& \dot{V}_{2}(t)=Q_{i n}(t)-\frac{p_{4}(t)}{I_{a o}} \\
& \dot{p}_{4}(t)=\frac{V_{2}(t)}{C_{a o}}-\frac{p_{4}(t)}{I_{a 0}} \cdot R_{a o}-\frac{V_{7}(t)}{C_{a r}} \\
& \dot{V}_{7}(t)=\frac{p_{4}(t)}{I_{a 0}}-\frac{V_{7}(t)}{C_{a r} \cdot R_{p e}}+\frac{P_{o u t}(t)}{R_{p e}}
\end{aligned}
$$

These equations contain eleven variables (and some derivative terms). The unit matrix for the flow-in, flow-out configuration is

$$
\boldsymbol{U}_{q . \text { out }}=\left(\begin{array}{rrrrrrrrrrr}
1 & 1 & -1 & -1 & 1 & 0 & 0 & 0 & 1 & 0 & 0 \\
-4 & -4 & 4 & 4 & -4 & 3 & 3 & 3 & -1 & 3 & 0 \\
-1 & 0 & 2 & 2 & -1 & -1 & -1 & 0 & -1 & 0 & 1
\end{array}\right)
$$

and for the flow-in, pressure-out configuration is

$$
u_{p .0 u t}=\left(\begin{array}{rrrrrrrrrrr}
1 & 1 & -1 & -1 & 1 & 0 & 1 & 0 & 1 & 0 & 0 \\
-4 & -4 & 4 & 4 & -4 & 3 & -1 & 3 & -1 & 3 & 0 \\
-1 & 0 & 2 & 2 & -1 & -1 & -2 & 0 & -1 & 0 & 1
\end{array}\right)
$$

where the columns represent (in order): $R_{a 0}, I_{a 0}, C_{a 0}, C_{a r}, R_{p e}, Q_{i n},\left\{Q_{o u t}, P_{o u t}\right\}, V_{a o}, p_{a 0}, V_{a r}$, and $t$. The rows correspond to $m, l$, and $t$. 
The rank of either unit matrix is three, implying that a judicious choice of repeating variables will yield a dimensional reduction of three. We will select $R_{a 0}, I_{a 0}$ and $Q_{\text {in }}$ as our repeating variables. ${ }^{*}$ This produces the following eight nondimensional $\Pi$-groups:

$$
\begin{gathered}
\Pi_{1}=\frac{I_{a o}}{C_{a 0} \cdot R_{a o}^{2}} \quad \Pi_{2}=\frac{I_{a o}}{C_{a r} \cdot R_{a o}^{2}} \quad \Pi_{3}=\frac{R_{p e}}{R_{a 0}} \quad \Pi_{4}=\left\{\frac{Q_{o u t}}{Q_{i n}}, \frac{P_{o u t}}{Q_{i n} R_{a 0}}\right\} \\
\Pi_{5}=\frac{R_{a o} \cdot V_{a o}}{I_{a o} \cdot Q_{i n}} \quad \Pi_{6}=\frac{p_{a o}}{I_{a 0} \cdot Q_{i n}} \quad \Pi_{7}=\frac{R_{a o} \cdot V_{a r}}{I_{a 0} \cdot Q_{i n}} \quad \Pi_{8}=\frac{R_{a o} \cdot t}{I_{a 0}}
\end{gathered}
$$

Substituting these into the original differential equations, and applying the Chain Rule to the derivative terms gives the following nondimensional system for the flow-in, flow out configuration:

$$
\begin{aligned}
& \Pi_{5}^{\prime}\left(\Pi_{8}\right)=1 \cdot f\left(\Pi_{8}\right)-\Pi_{6}\left(\Pi_{8}\right) \\
& \Pi_{6}^{\prime}\left(\Pi_{8}\right)=\Pi_{1} \cdot \Pi_{5}\left(\Pi_{8}\right)-\Pi_{6}\left(\Pi_{8}\right)-\Pi_{2} \cdot \Pi_{7}\left(\Pi_{8}\right) \\
& \Pi_{7}^{\prime}\left(\Pi_{8}\right)=\Pi_{4} \cdot g\left(\Pi_{8}\right)+\Pi_{6}\left(\Pi_{8}\right)
\end{aligned}
$$

and for the flow-in, pressure-out configuration:

$$
\begin{aligned}
& \Pi_{5}^{\prime}\left(\Pi_{8}\right)=1 \cdot f\left(\Pi_{8}\right)-\Pi_{6}\left(\Pi_{8}\right) \\
& \Pi_{6}^{\prime}\left(\Pi_{8}\right)=\Pi_{1} \cdot \Pi_{5}\left(\Pi_{8}\right)-\Pi_{6}\left(\Pi_{8}\right)-\Pi_{2} \cdot \Pi_{7}\left(\Pi_{8}\right) \\
& \Pi_{7}^{\prime}\left(\Pi_{8}\right)=\frac{\Pi_{4} \cdot g\left(\Pi_{8}\right)}{\Pi_{3}}+\Pi_{6}\left(\Pi_{8}\right)-\frac{\Pi_{2} \cdot \Pi_{7}\left(\Pi_{8}\right)}{\Pi_{3}}
\end{aligned}
$$

where all derivatives are taken with respect to nondimensional time, $\Pi_{8}$, and $f$ and $g$ are the time course of the input and output functions (respectively), with nondimensional magnitude \pm 1 . For example, a periodic input to the system might be $f\left(\Pi_{8}\right) \equiv \sin \left(\Pi_{8}\right)$.

\subsubsection{State-Space Formulation}

Both systems are linear and we can write Equation (4.6) and Equation (4.7) in matrix form:

$$
\left(\begin{array}{l}
\Pi_{5}^{\prime} \\
\Pi_{6}^{\prime} \\
\Pi_{7}^{\prime}
\end{array}\right)=\left(\begin{array}{rrc}
0 & -1 & 0 \\
\Pi_{1} & -1 & -\Pi_{2} \\
0 & 1 & 0
\end{array}\right) \cdot\left(\begin{array}{l}
\Pi_{5} \\
\Pi_{6} \\
\Pi_{7}
\end{array}\right)+\left(\begin{array}{ll}
1 & 0 \\
0 & 0 \\
0 & 1
\end{array}\right) \cdot\left(\begin{array}{c}
1 \\
\Pi_{4}
\end{array}\right)
$$

*Appendix B presents three different choices of repeating variables. 
and

$$
\left(\begin{array}{c}
\Pi_{5}^{\prime} \\
\Pi_{6}^{\prime} \\
\Pi_{7}^{\prime}
\end{array}\right)=\left(\begin{array}{rrr}
0 & -1 & 0 \\
\Pi_{1} & -1 & -\Pi_{2} \\
0 & 1 & -\frac{\Pi_{2}}{\Pi_{3}}
\end{array}\right) \cdot\left(\begin{array}{l}
\Pi_{5} \\
\Pi_{6} \\
\Pi_{7}
\end{array}\right)+\left(\begin{array}{cc}
1 & 0 \\
0 & 0 \\
0 & \frac{1}{\Pi_{3}}
\end{array}\right) \cdot\left(\begin{array}{c}
1 \\
\Pi_{4}
\end{array}\right)
$$

As predicted, the flow-in, flow-out configuration has a state matrix of rank two whereas the flow-in, pressure-out has a state matrix of rank three. Both input vectors have a curious form, due to the choice of repeating variables. Recall that $Q_{i n}$, the flow in to the system, was chosen as a repeating variable; repeating variables become scaling parameters and their effect on the system is masked. This explains the unity input.

\subsubsection{Characteristic Equation}

The characteristic polynomials for the systems in Figures 4.2 and 4.3 can be computed as in Equation (2.9) on Page 22. They are (respectively):

$$
\begin{gathered}
s^{3}+s^{2}+s \cdot\left(\Pi_{1}+\Pi_{2}\right) \\
s^{3}+s^{2} \cdot\left(1+\frac{\Pi_{2}}{\Pi_{3}}\right)+s \cdot\left(\Pi_{1}+\Pi_{2}+\frac{\Pi_{2}}{\Pi_{3}}\right)+\frac{\Pi_{1} \cdot \Pi_{2}}{\Pi_{3}}
\end{gathered}
$$

As expected, the DCM model with inlet flow source and outlet flow sink has a characteristic equation of degree three, while the DCM model with the inlet flow source and outlet pressure sink results in a polynomial of degree two. Even though both configurations have three energy storing elements, the two compliance volumes are not independent if we control both the inflow and outflow; this reduces the order of the system.

As noted in Section 2.2, the characteristic equation for a given matrix encodes several of its important properties. The roots of the characteristic equation are the eigenvalues of the system, and these determine the stability of the system. Roots of polynomials can be either real or imaginary, with imaginary roots appearing as complex conjugates. Systems with positive real eigenvalues are unstable; negative real parts imply stability, and imaginary components indicate oscillatory behaviour.

The roots of Equation (4.10) can be found using the quadratic formula:

$$
\left\{\{s \rightarrow 0\},\left\{s \rightarrow \frac{-1-\sqrt{1-4 \cdot \Pi_{1}-4 \cdot \Pi_{2}}}{2}\right\},\left\{s \rightarrow \frac{-1+\sqrt{1-4 \cdot \Pi_{1}-4 \cdot \Pi_{2}}}{2}\right\}\right\}
$$


These roots always have negative real parts, so the system is always stable. This stability can be confirmed by constructing the Routh-Hurwitz array, as in Appendix B.

The discriminant (the term under the square root sign) determines whether or not the roots have imaginary parts. From the discriminant in Equation (4.12) we see that two of the roots appear as complex conjugates if

$$
\Pi_{1}+\Pi_{2}>\frac{1}{4}
$$

Recall that $\Pi_{1}$ and $\Pi_{2}$ are the nondimensional aortic and arterial compliance (respectively). We can use the circulatory system parameters presented in Section 2.6 to establish intervals for the nondimensional groups. ${ }^{*}$

$$
\begin{aligned}
C_{a 0} & =[20 ., 40 .] \\
C_{a r} & =130 .-C_{a o} \\
R_{a o} & =\left[2 . \times 10^{-4}, 0.0011\right] \\
I_{a o} & =[0.00003,0.00005] \\
R_{p e} & =[0.005,0.013]
\end{aligned}
$$

$$
\begin{aligned}
& \Pi_{1}=[0.62,62.5] \\
& \Pi_{2}=[0.23,13.9] \\
& \Pi_{3}=[4.5,65 .]
\end{aligned}
$$

We can see from these parameter intervals that the second-order DCM will always admit oscillations but, because the eigenvalues have negative real parts, the system is stable. Given that the standard equation is:

$$
s^{2}+2 \cdot \zeta \cdot \omega_{n} \cdot s+\omega_{n}^{2}
$$

${ }^{*}$ The square brackets in Equation (4.14) and Equation (4.15) are standard mathematical interval notation. [...] denotes a closed interval (which includes its endpoints), while (...) denotes an open interval (which does not include its endpoints). Units for Equation (4.14) are as follows: $C_{a 0}$ in $\mathrm{cm}^{5} \cdot N^{-1}, C_{a r}$ in $\mathrm{cm}^{5} \cdot N^{-1}, R_{a 0}$ in $N \cdot \mathrm{s} \cdot \mathrm{cm}^{-5}, I_{a 0}$ in $N \cdot \mathrm{s}^{2} \cdot \mathrm{cm}^{-5}$, and $R_{p e}$ in $N \cdot \mathrm{s} \cdot \mathrm{cm}^{-5}$. 
we can compare this with Equation (4.10) and say that

$$
\omega_{n}=\sqrt{\Pi_{1}+\Pi_{2}}
$$

and

$$
\zeta=\frac{1}{2 \cdot \sqrt{\Pi_{1}+\Pi_{2}}}
$$

which allows us to place bounds on the natural frequency and damping ratio for the system; the natural frequency is $[0.92,8.7]$ and the damping ratio is $[0.057,0.54]$.

We now turn our attention to the third-order model. While solving for the roots of a second-order equation is trivial, solving for the roots of a third-order polynomial is less so. We are particularly interested in oscillatory behaviour in the arterial system; using a variation on Cardano's Method*, we can develop a conditional test for arterial oscillations without solving for the actual roots of the characteristic equation.

\section{Solving the Cubic}

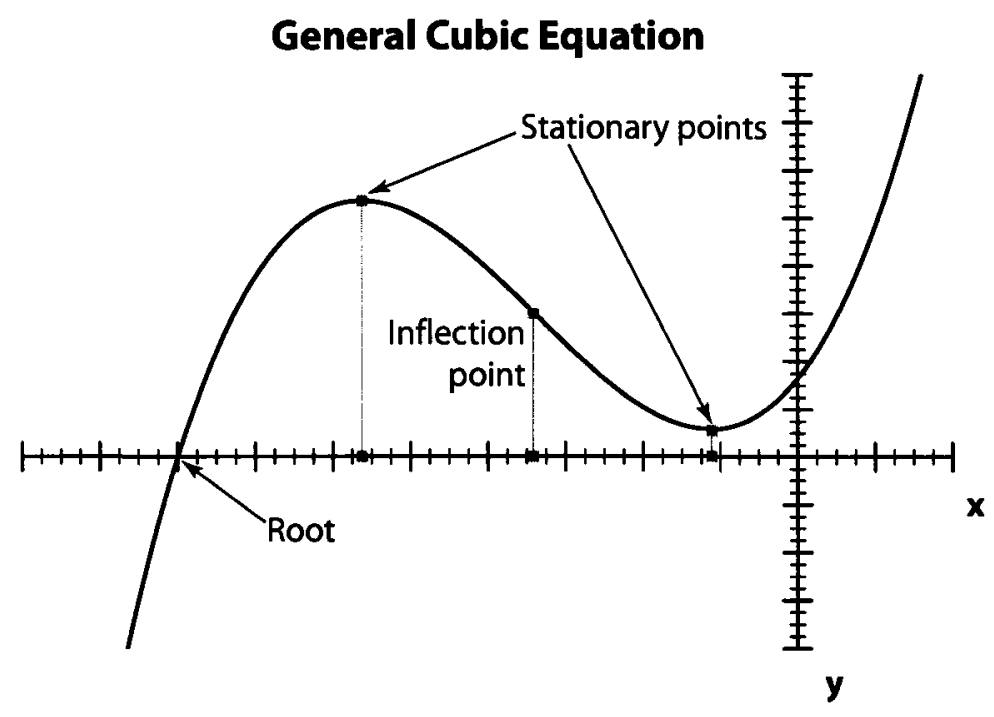

Figure 4.4: General cubic polynomial equation

*Cardano's Method is described (among other places) at Eric W. Weisstein's MathWorld, a Wolfram Web Resource (http://mathworld.wolfram.com/CubicFormula.html). 
Figure 4.4 shows a plot of a general cubic polynomial equation. A cubic polynomial* has either one or three stationary points where the first or second derivatives vanish; stationary points are either local minima or maxima, or inflection points. The cubic polynomial also has three roots, one or three of which can be real. The number of real roots depends on the shape of the curve, and whether or not the "wells" are deeper (or higher) than the inflection point's offset from the x-axis. The equation of a general cubic polynomial is given by Equation (4.19):

$$
P(x)=a_{0}+a_{1} x+a_{2} x^{2}+a_{3} x^{3}
$$

We can find the local extrema by taking the first derivative and solving the resultant quadratic:

$$
\begin{gathered}
P^{\prime}(x)=a_{1}+2 a_{2} x+3 a_{3} x^{2}=0 \\
x \rightarrow\left(\frac{-a_{2} \pm \sqrt{a_{2}^{2}-3 a_{3} a_{1}}}{3 a_{3}}\right)
\end{gathered}
$$

To find the discriminant of the general cubic, we first depress ${ }^{\dagger}$ it by a change of coordinates:

$$
\begin{aligned}
P^{\prime \prime}(x) & =2 a_{2}+6 a_{3} x=0 \\
x & \rightarrow\left(x-\frac{a_{2}}{3 a_{3}}\right)
\end{aligned}
$$

The $y$-value of the curve at the inflection point is then given by

$$
P\left(x-\frac{a_{2}}{3 a_{3}}\right)=\frac{2 a_{2}^{3}}{27 a_{3}^{2}}-\frac{a_{2} a_{1}}{3 a_{3}}+a_{0}
$$

and the difference between the extrema and the inflection point is given by

$$
\Delta y=P\left(\frac{-a_{2} \pm \sqrt{a_{2}^{2}-3 a_{3} a_{1}}}{3 a_{3}}\right)-P\left(x-\frac{a_{2}}{3 a_{3}}\right)
$$

For a general cubic, the inflection point may sit above or below the $x$-axis. Rather than having two dependent conditions for real roots, we note that the cubic is symmetric about

${ }^{*}$ Also referred to as a cubic.

${ }^{+}$A depressed cubic is one with no $x^{2}$ term. 
the inflection point, and each "well" is the same depth. We can then express the condition for three real roots as:

$$
a_{2}^{2} a_{1}^{2}-4 a_{2}^{3} a_{0}+18 a_{3} a_{2} a_{1} a_{0}-a_{3}\left(4 a_{1}^{3}+27 a_{3} a_{0}^{2}\right)>0
$$

Although Equation (4.24) is fairly complex, it pales in comparison to the expression for the actual roots of the cubic. Even still, it is better to examine the system numerically, as is done in Figure 4.5, which plots the value of Equation (4.24) over the intervals of the nondimensional parameters. Contour plotting routines are numerically sensitive, and the $x$ - and y-coordinates of Figure 4.5 are the reciprocals of $\Pi_{1}$ and $\Pi_{2}$ (respectively). Figure 4.5 is a representative "slice" of the parameter space corresponding to $\Pi_{3}=20$; the trends are consistent over the entire $\Pi_{3}$ interval. As with the second-order model, the third-order

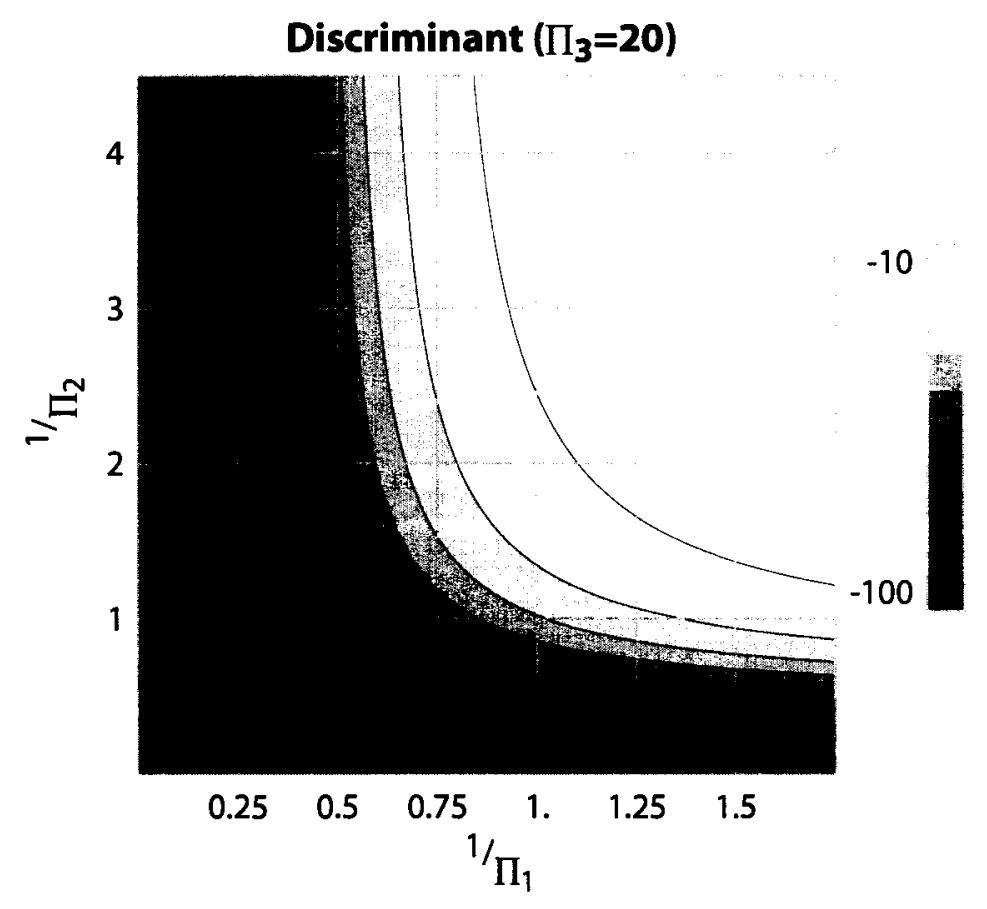

Figure 4.5: Contour plot of Equation (4.24) for $\Pi_{3}=20$

system has complex roots over the parameter range of interest. Smaller values of $\Pi_{1}$ and $\Pi_{2}$ (i.e., the upper right quadrant in Figure 4.5) reduce the magnitude of the discriminant but do not completely eliminate the complex conjugate roots. The arterial system will always permit oscillations. 


\subsection{The Arterial Pressure Waveform}

As mentioned earlier, in Section 2.6.4 (Page 40), and as seen in Section 3.2 (beginning on Page 58), most mock circulatory systems represent the arterial tree using a single, linear compliance element. Our modular mathematical model lets us examine the differences between the SCM and DCM when inserted in the overall human circulatory system. A plot of this simulated arterial (aortic) pressure waveform versus time is shown in Figure 4.6.

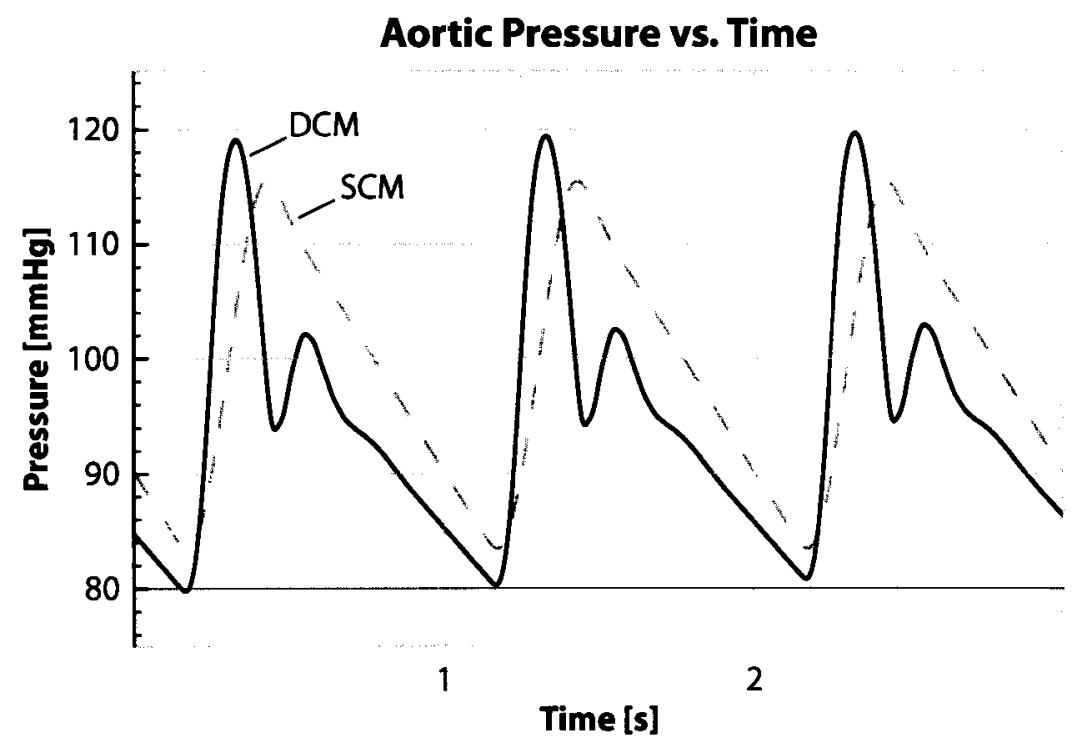

Figure 4.6: Aortic pressure waveform for the SCM and DCM

Figure 4.6 indicates that without the additional stiffer aortic element the SCM generates more of a sawtooth pressure waveform. This is not the type of pressure waveform which would be found in the larger arterial vessels of the human circulatory system; in the SCM, the opening and closing of the aortic valve do not have a pronounced effect on the arterial pressure, and there is no elastic recoil after systole. With the DCM, however, there is a dicrotic notch-like artifact in the pressure waveform even if the constitutive relations for both compliance elements are linear (as is the case in Figure 4.6). The simulation is consonant with the human circulatory system, in that the dicrotic pressure is very close to the mean arterial pressure. Recall that there is some controversy over whether or not these two pressures in the human circulatory system match exactly; in the mathematical simulation 
of the DCM (plotted in Figure 4.6) the mean arterial pressure (MAP) is $96 \mathrm{mmHg}$ and the dicrotic notch pressure is $95 \mathrm{mmHg}$. This falls within the statistical bounds identified by Hebert et al. (1995) or Chemla et al. (1996).

It is important for mock circulatory system designers to ask themselves what the goal of the apparatus is. If it is merely to act as a reliability testbed then a SCM-like setup will suffice. The pressures produced in the SCM are reasonable, and the waveform itself is somewhat similar to the physiological pressure wave. However, the SCM trace omits many of the more subtle features of the physiological arterial pressure wave: the SCM wave spans a more compressed pressure range, its ascending trace lags the physiologic waveform, and its descending trace lacks a dicrotic notch.

These missed subtleties become important if, for example, one uses a mock circulatory system to devise a new control strategy for artificial hearts. Or if one wants to make use of the arterial waveform properties to improve the efficiency of the device's pumping.

The more suitable results produced by the DCM suggest that the simple addition of another compliance chamber will allow a mock circulatory system to better match pressure behaviour found in the human arterial system. This is a minimal modification for most MCS loops, which generally consist of individual free-standing compliant chambers connected by tubes.

\subsection{Nonlinearities}

When examining nonlinear behaviour in the arterial system, as with the linear study, we are faced with modelling decisions involving both elements and boundary conditions. Some of the potential configurations are illustrated in Figure 4.7. As before, we would like the inlet boundary condition to be the volume flow rate; the heart injects a certain amount of blood into the aorta at each beat. The aorta expands to receive the blood (which is injected over a small fraction of the beat cycle) and then recoils to distribute the blood to the body. The outlet boundary condition is chosen based on where we truncate the arterial system model. If we include only the aortic elements, as in Figure 4.7 (a), the remainder of the arterial system appears as a pressure sink. Adding the arterial compliance, as in Figure 4.7 (b), changes the order of the system; in this case the outlet boundary condition 

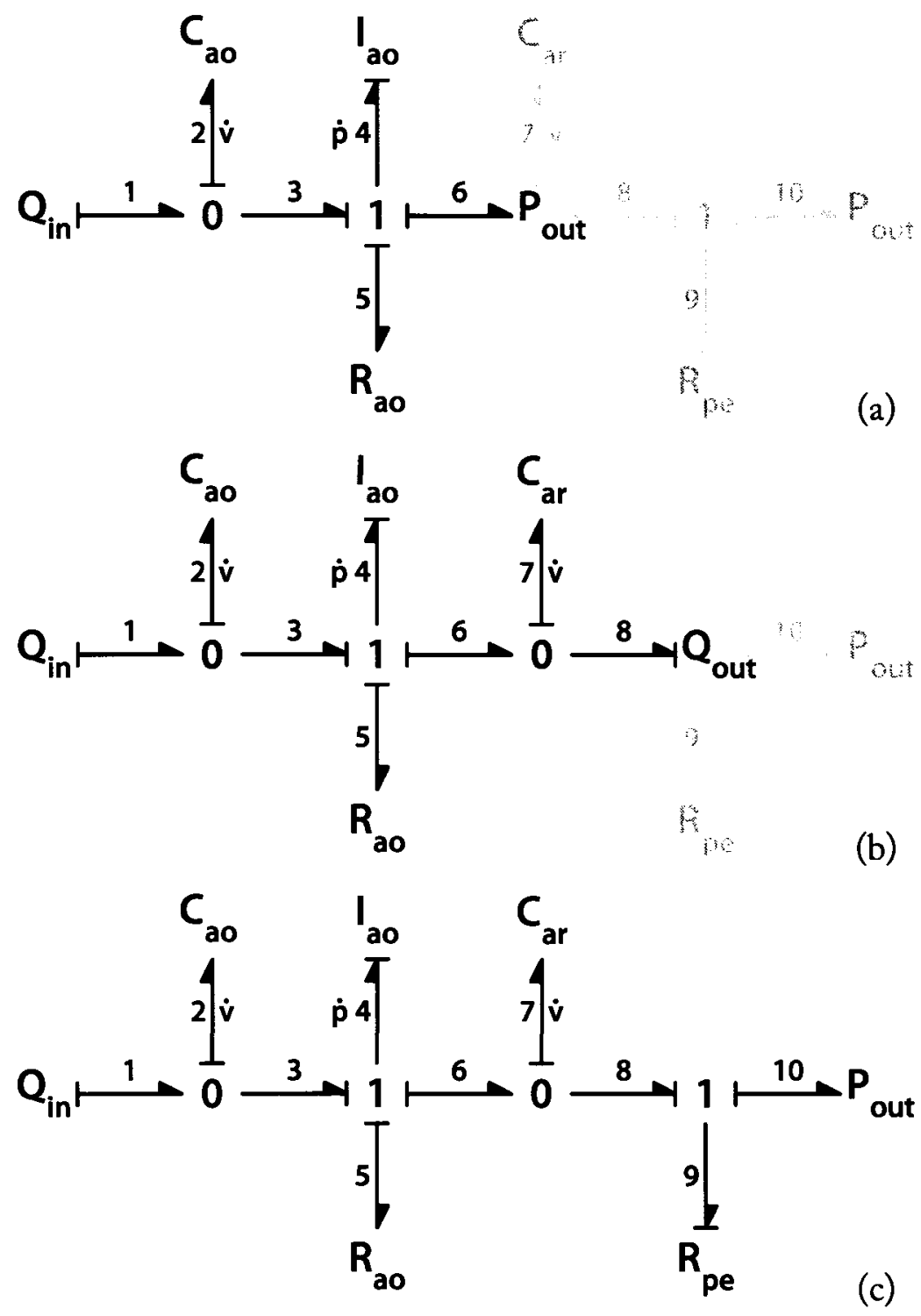

Figure 4.7: Various arterial element-BC configurations 
is the volume flow rate out of the arterial system, into the periphery. If, rather than a thirdorder system, we would prefer a second-order system, we can change the outlet boundary condition (in Figure 4.7 (b)) to the peripheral pressure. This changes the causality on bonds 7 and 8; the volume in the arterial compliance element is no longer an independent state. Finally, if we wish an outlet boundary condition of pressure rather than flow, and we do not want to change the causality on bond 7 (i.e., we want an independent arterial volume) then we can truncate the arterial system as in Figure 4.7 (c). In this case the outlet boundary condition is the venous pressure and the peripheral flow resistance element is included in the model. Note that the peripheral flow inertia is not included in the model; including the inertia would increase the order yet again.

Each of the arterial system configurations in Figure 4.7 is equivalent to a mass-spring-damper system (with varying complexity). In particular, if the outlet boundary condition is chosen to limit the order of the system, the arterial system model is dynamically equivalent to a massspring-damper system consisting of a single mass, single spring, and single damping element. In this system (unlike the systems we looked at in Chapter 2), the damping is proportional to the global velocity of the mass, rather than its relative velocity. ${ }^{*}$ This slightly modified configuration is illustrated in Figure 4.8; the mass-spring-damper equivalence will prove useful understanding the arterial system's dynamics.

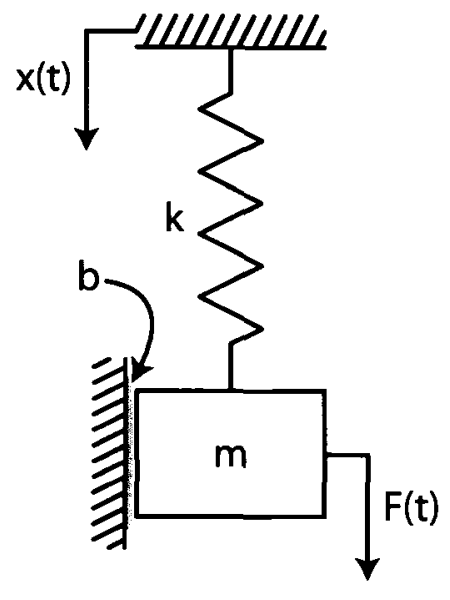

Figure 4.8: Forced mass-spring-damper system

Note that Figure 4.8 includes input forces and positions. The heart's blood injection (into the aorta) is the dynamic equivalent of moving the ceiling of the mass-spring-damper system. This input excites the mass-spring-damper system, producing oscillations. If the input is periodic, the response will have two components, one transient and the other periodic. The decaying solution is the unforced response and the periodic solution is the forced response of the system. In terms of differential equations, these are called the complimentary and particular so-

*In the mass-spring-damper system, if the ceiling is fixed there is no difference between global and relative velocities. 
lutions (respectively). The normal physiological aortic pressure and flow waveforms are shown in Figure 4.9.* For a first approximation, the inflow could be modelled as a halfsine wave. The integral of this waveform is the volume of blood which has been moved into the aorta or, in the mass-spring-damper analogy, the position of the ceiling. In other words, rather than oscillating about a fixed point, the ceiling in the mass-spring-damper system moves downwards in a periodic fashion. The reason for this is obvious from the circulatory system: the heart only injects blood into the aorta; it does not draw blood back into the ventricle. If the "ceiling" motion was about a fixed point, there would be no net circulation.

As we have seen previously, the aorta elastic response is nonlinear; the vessel stiffens as it distends. The injection of blood into the aorta expands the vessel, which is equivalent to stretching or compressing the spring in our mass-spring system. For reasons which will become more apparent shortly, we will equate the aortic expansion with a spring compression. In the mass-spring-damper system, this means that the ceiling moves down (blood is injected) and compresses the spring (expands the aorta). Assuming no further ceiling motion (blood injection), the spring then decompresses (the aorta recoils) as the mass moves downward (the blood moves into the rest of the circulatory system).

The compression of the spring (i.e., expansion of the aorta) is determined by both the volume of blood injected by the heart and the back-pressure from the arteries. This arterial back-pressure, which is not necessarily constant, appears as a force acting on our mass (in the mass-spring-damper system). The nonlinear (stiffening) behaviour of the aorta is dynamically equivalent to a nonlinear spring in our mass-spring-damper system, or representative of an elastic collision with a floor.

This analogy is important, because the second-order mass-spring-damper system with either input (ceiling motion or disturbing force) exhibits interesting nonlinear behaviour (including chaos) for a range of parameter values, as shown in Figure 4.10. Figure 4.10 displays the long-term behaviour of a mass-spring-damper system described by the following

${ }^{*}$ These waveforms come from computer simulation of the thirteenth-order circulatory system model, but agree well with what would be found in the body. 


\section{Aortic Pressure and Flow Rate vs. Time}

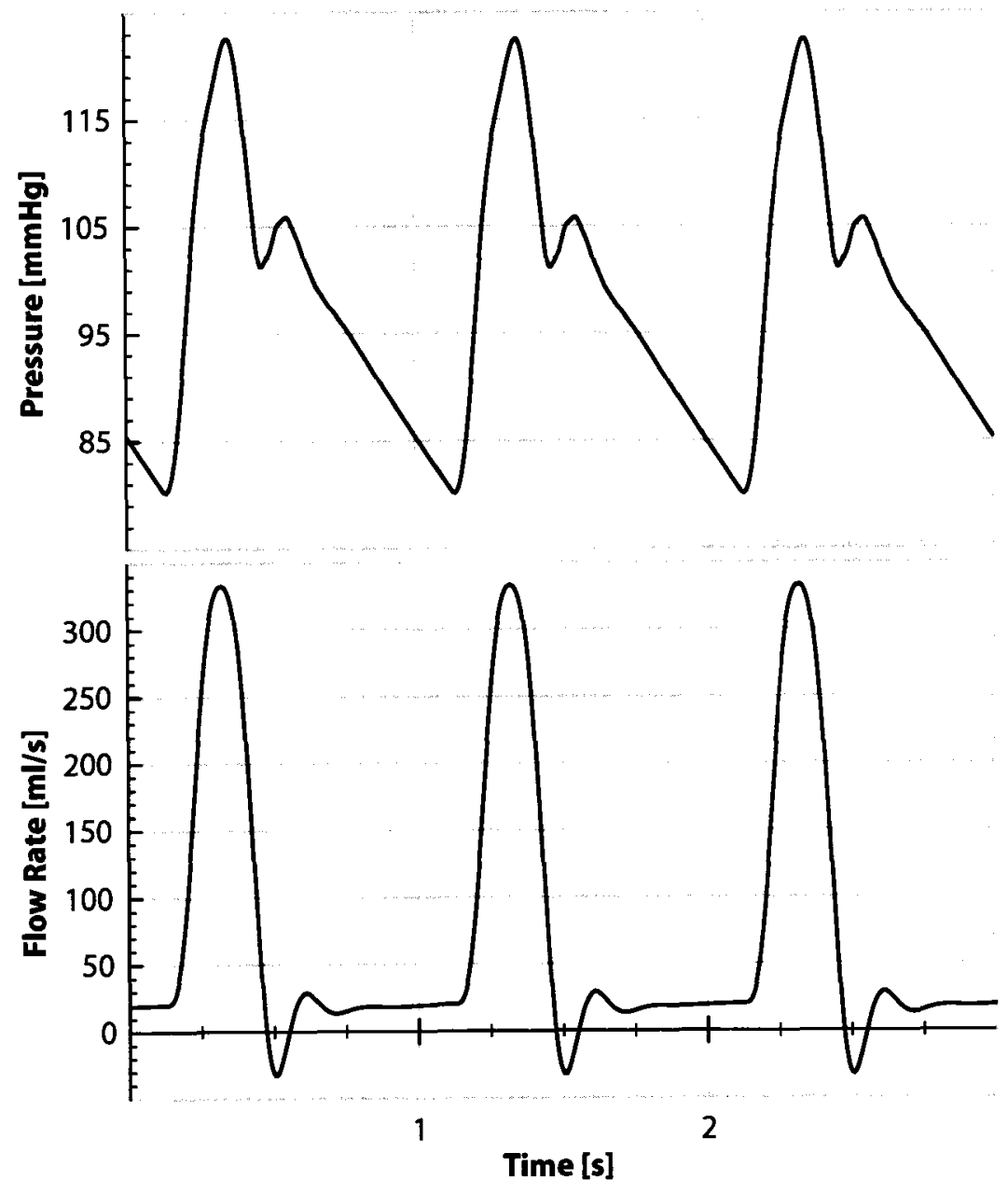

Figure 4.9: Aortic pressure and flow waveforms 


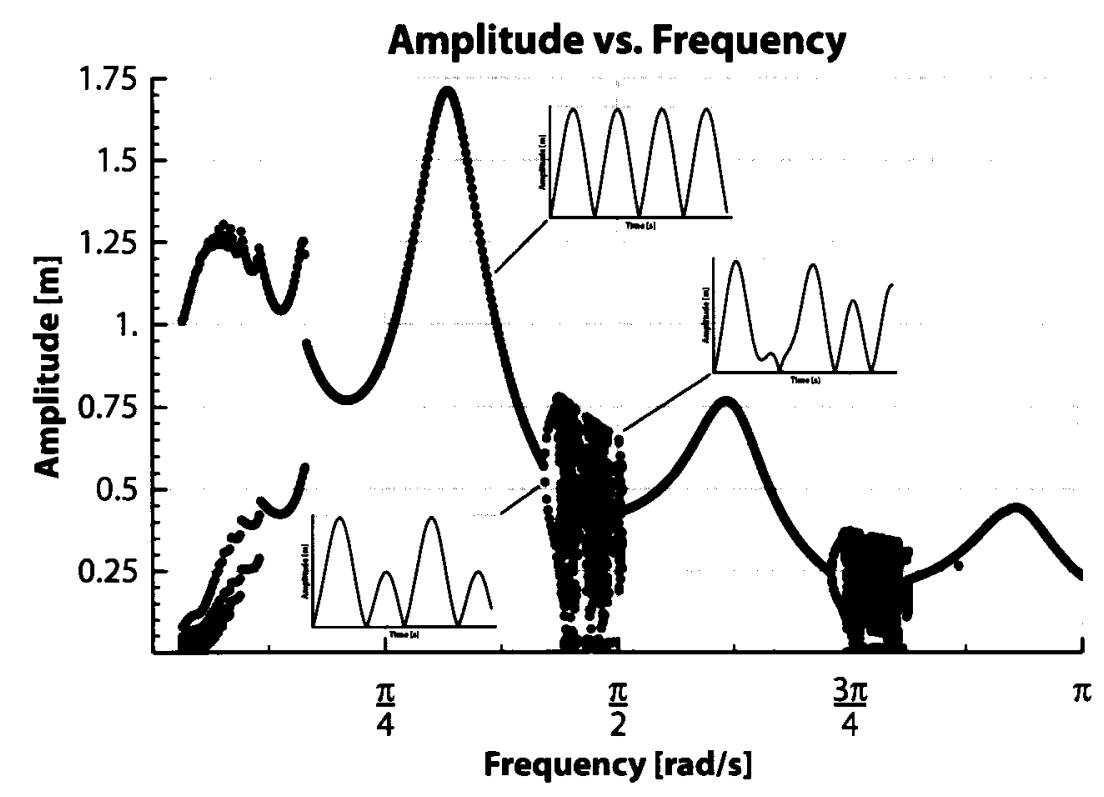

Figure 4.10: Impact oscillator behaviour for a variety of excitation frequencies

second-order differential equation:

$$
m \cdot \frac{\mathrm{d}^{2} s}{\mathrm{~d} t^{2}}+b \cdot \frac{\mathrm{d} s}{\mathrm{~d} t}+k \cdot s=F \cdot \cos (\omega \cdot t+\delta), \quad s>s_{f},
$$

where $s_{f}$ is the location of the rigid floor that the mass is reflected by and $s$ is the position of the mass.

The plot in Figure 4.10 was generated using parameters $m=4, k=1, b=1$ and $F=1$. The system was started at time $t=0$ with initial position $s=0$ and with zero velocity. It was integrated numerically until the mass passed through the floor, and then the integration was halted. A numerical root-finding method was used to determine the time, $t$, when the mass impacted the floor, and the integration was restarted, elastically reflecting the mass (i.e., inverting the velocity). This process was repeated for the first five hundred bounces, in order to eliminate any system transients. Of this set of five hundred bounces, only the last hundred were kept, and the maximum oscillation amplitude (over each bounce interval) was calculated. These maximum amplitudes were plotted versus the 
current driving frequency. The driving frequency was then incremented and the process was repeated.

Insets in Figure 4.10 highlight both areas of periodicity and areas of chaotic oscillations. Period-doubling bifurcations are visible enroute to chaos. Chaotic behaviour implies sensitive dependence on initial conditions, meaning that two trajectories which start out very close in phase-space will diverge exponentially over time. ${ }^{*}$ Numerical integration schemes introduce additional dynamics into the system being studied, and it is therefore important to ensure that any chaotic behaviour is owing to system dynamics, rather than some sort of interaction with the numerical integration scheme itself. To this end, the system was solved analytically, and the analytical solution used to confirm the bounce intervals. The numerical solutions in both MATLAB and Mathematica match the analytical solution with good accuracy both in terms of overall trend prediction and in terms of overall feature recognition or display. Both numerical simulations produce the same answers (even though each uses a different numerical integrator) and predict the same behaviours. ${ }^{\dagger}$

\subsection{The Impact Oscillator Analogy}

The mass-spring-floor system (with or without damping) described above is commonly called an "impact oscillator" (Lamba, 1995), "Fermi oscillator" (Lopac and Dananic, 1998), or "Bender bouncer" (Walker and Soule, 1996) and has been studied extensively in the literature. It can model everything from chatter in gear trains to the interaction of highenergy particles in the upper atmosphere. To facilitate comparison between these various systems, which exist at a variety of physical scales (macro, micro) and in a number of domains (particle physics, mechanics, fluid flow), it is helpful to nondimensionalize the differential equation. This nondimensionalization allows us to frame our arterial oscillator analogy in terms of systems which have already been looked at in the literature.

In addition to this, nondimensionalization gives a greater understanding of the system itself. As we will see shortly, the nondimensional parameter of interest is a function of mul-

\footnotetext{
*This is what makes long-term weather prediction so unreliable.

'Since the system is non-stiff, we use ode 45 in MATLAB, with different (tighter) error tolerances; AbsTol was set to $1 \times 10^{-9}$ and RelTol to $1 \times 10^{-6}$. In Mathematica, the second-order differential equation was integrated using NDSolve and the default AccuracyGoal, PrecisionGoal and WorkingPrecision.
} 
tiple physical properties; any of these individual properties can influence the behaviour of the system and encourage or eliminate chaotic behaviour. To-date this feature has been ignored in the "impact oscillator" literature, but it is important to us because many of the aortic properties vary over the course of a beat.

Once again we have a choice of repeating variables for our nondimensionalization. Concerns raised during the earlier discussion of repeating variable choices are still valid, and we will once again choose $R_{a 0}, I_{a 0}$, and $Q_{i n}$ as our repeating variables. This choice reduces the damping and inertia terms to scaling parameters, and masks the effect of the system input. (The latter is important because some models "shake the mass" instead of "moving the ceiling"; either is valid.) It allows us to investigate the effect of stiffness (or compliance) changes on the system response. Using the following nondimensional groups

$$
\Pi_{1}=\frac{I_{a o}}{C_{a o} \cdot R_{a o}^{2}} \quad \Pi_{2}=\frac{\omega \cdot I_{a o}}{R_{a o}} \quad \Pi_{3}=\frac{V_{a o} \cdot R_{a o}^{2}}{I_{a o} \cdot Q_{i n}} \quad \Pi_{4}=\frac{t \cdot R_{a o}}{I_{a o}}
$$

or

$$
\Pi_{1}=\frac{k \cdot m}{b^{2}} \quad \Pi_{2}=\frac{\omega \cdot m}{b} \quad \Pi_{3}=\frac{s \cdot b^{2}}{m \cdot F} \quad \Pi_{4}=\frac{t \cdot b}{m}
$$

Equation (4.25) becomes

$$
\Pi_{3}^{\prime \prime}+\Pi_{3}^{\prime}+\Pi_{1} \cdot \Pi_{3}=\cos \left(\Pi_{2} \cdot \Pi_{4}+\delta\right), \quad \Pi_{3}>\Pi_{f}
$$

where all derivatives are taken with respect to nondimensional time, $\Pi_{4}$, and $\Pi_{f}$ is the location of the rigid floor that the mass is reflected by.

In the arterial system, the stiffening behaviour (of the aorta) is not infinite as with a mass impacting a rigid floor; we need to investigate what happens with a soft-floor impact, where the mass is allowed to pass into the floor by some distance. This situation, which is mathematically equivalent to a nonlinear spring, is illustrated in Figure 4.11. The upper half of Figure 4.11 illustrates two configurations where a mass-spring-damper system can interact with a soft floor. In Figure 4.11 (a) the damping is proportional to the local velocity of the mass while in Figure 4.11 (b) the damping is proportional to the global velocity of the mass. The nonlinear equivalents of these two configurations are illustrated in the lower half of Figure 4.11. The spring response for the systems in Figure 4.11 (c) and Figure 4.11 (d) could be piecewise-linear, producing an exact (dynamic) match to the dual-spring systems in the top half of the figure. 

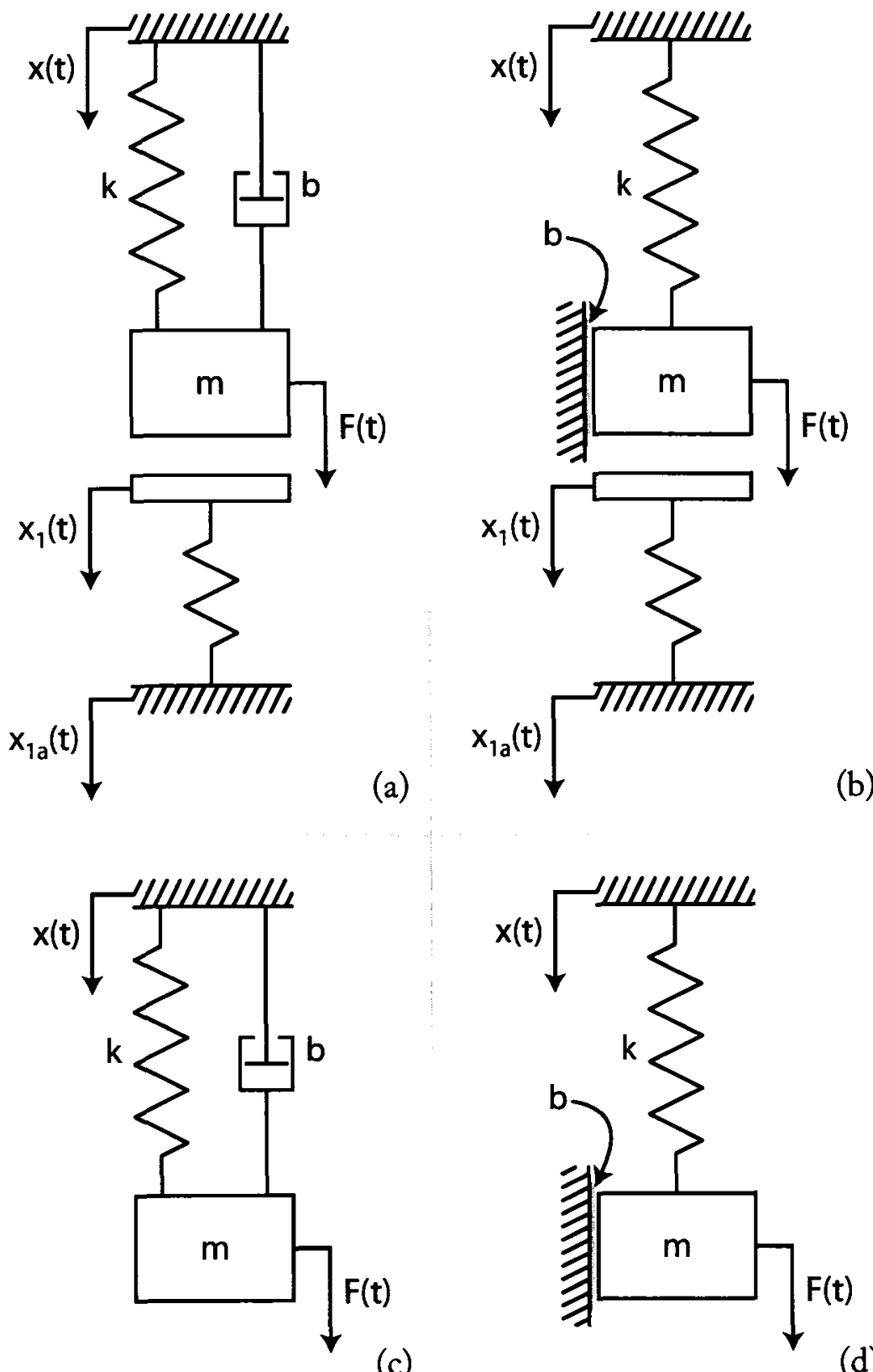

Figure 4.11: Forced mass-spring-damper systems (in a variety of configurations) 
Simulations (similar to the one which produced Figure 4.10) show that we still get period-doubling bifurcations for $\Pi_{1}$ (nondimensional stiffness) increases as low as $10 \times$, achievable with reasonable circulatory system parameter changes. Recall that $\Pi_{1}$ is a function of stiffness, inertia and damping; the impact of changes in vessel geometry (and the resultant changes in flow inertia and flow losses) should eventually be examined in more detail in order to determine their influence on $\Pi_{1}$. That sort of investigation, which is beyond the scope of this work, could employ nuclear magnetic resonance imaging to examine arterial vessel geometry non-invasively, and computational fluid dynamics to study flow losses.

\subsubsection{Periodicity}

A standard route to chaos is through period-doubling bifurcations as a parameter is varied. These bifurcations were seen in Figure 4.10 and highlighted in the insets, but it is important to understand what is meant by "period-doubling" and to establish the tools which will be used to analyze the response of the system. Periodicity implies repetition at intervals. A familiar example would be the function $\sin (x)$, which repeats every $2 \pi$. Oscillations are often categorized by their periodicity, and labelled "period- $n$ " based on how many undulations the response goes through before the pattern recurs. For example, a period-2 oscillation would appear as two separate wave functions overlaid on each other. Figure 4.12 illustrates this concept. The plots in Figure 4.12 were all generated from Equation (4.28) with $\Pi_{1}=4$ and $\Pi_{f}=0$. To produce the various rows, $\Pi_{2}$ was varied from 1.3 to 1.4 in non-uniform increments.* The left column presents the time trace of nondimensional position $\left(\Pi_{3}\right)$ after the initial transients have decayed, and the right column shows pseudo-phase plots of the system. ${ }^{\dagger}$ All plots are normalized based on the maximum amplitude of oscillation and maximum velocity to aid structural comparisons.

We see that with a driving frequency of $\Pi_{2}=1.3$, the response of the system is a simple, non-decaying waveform with a constant period. As the forcing frequency is increased, the system responds with increasing complexity. The response switches first to a period-2 oscillation, then period-4, and so on. Each of these responses is continuous as long as the driving frequency remains unchanged. In other words, after any startup transients have

\footnotetext{
${ }^{*}$ The rows (from top to bottom, in order) correspond to $\Pi_{2}=\{1.3,1.33,1.345,1.37,1.4\}$.

${ }^{\dagger}$ Pseudo-phase because the system is non-autonomous, and the resultant phase plot is therefore a twodimensional representation of a third-order system.
} 

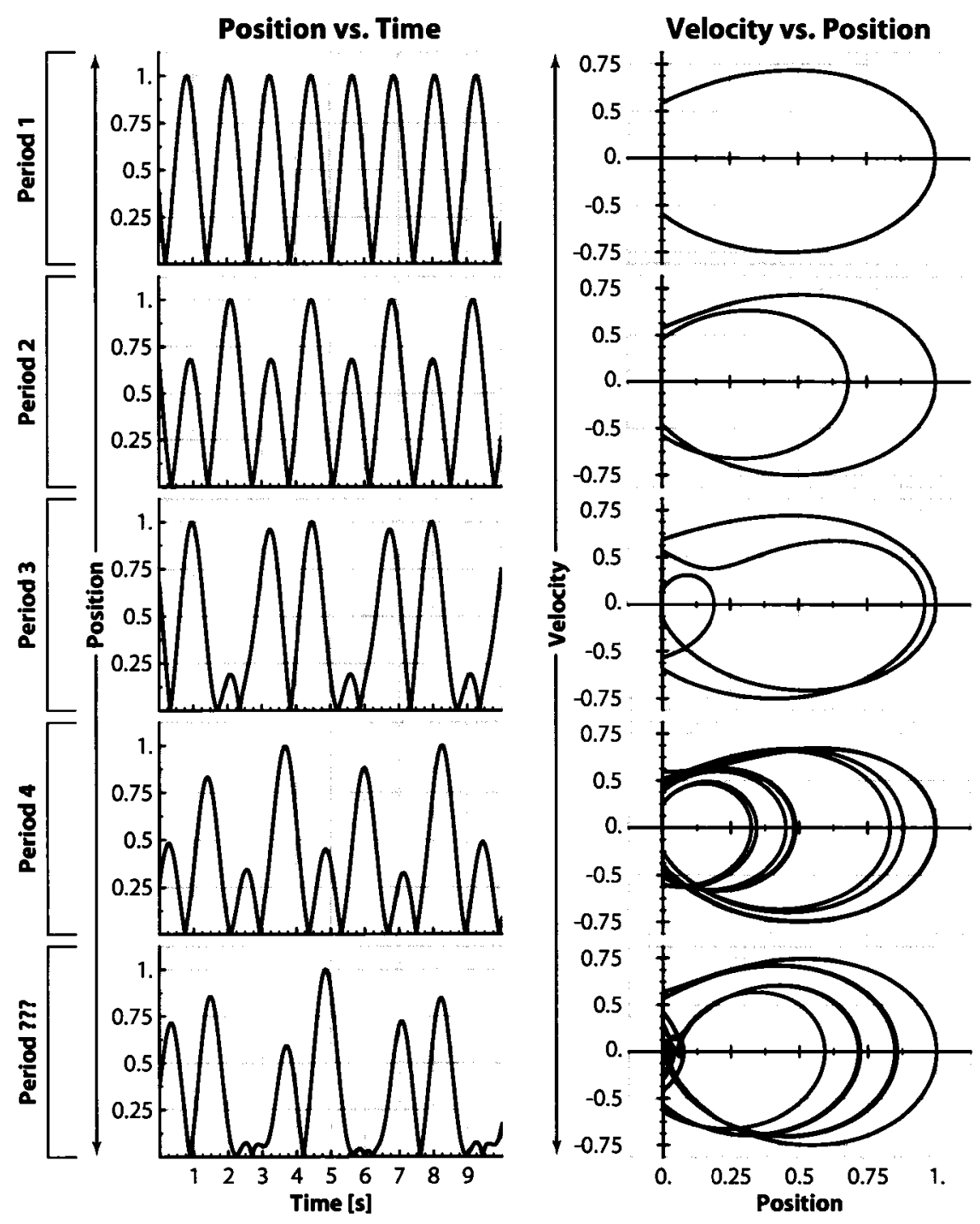

Figure 4.12: Position versus time and velocity versus position for various forcing frequencies, demonstrating the concept of periodicity changes 
waned, the system responds in a stable, predictable manner. The final row of Figure 4.12 shows a more complicated system response which is difficult to classify. If the pattern is still periodic, the periodicity is quite high; there is no repetition visible in the time interval show, and even if the interval is expanded the trace does not repeat.

The periodicity of a waveform is determined by the number of "loops" in the phase plot. For a non-chaotic oscillation the phase plot eventually repeats; we are interested in how many undulations manifest themselves before the pattern recurs. This is normally quite easy to determine by inspection of the phase plot, as evidenced by the graphs in the right column of Figure 4.12, but it is more difficult to automate on computer. One of the more reliable methods is to examine the peaks in an autocorrelation of the position trace. For longer time series, computing the traditional (statistical) autocorrelation becomes a CPU-intensive process. It is better to take the Fourier Transform of the data and do the calculations in the frequency domain; correlation is related to convolution, and is a simple multiplication in the frequency domain.*

We look for the second highest peak in the autocorrelation (the highest peak is at $n=1$ ), and treat that as the fundamental frequency of the waveform. The number of spikes which occur between the two highest peaks gives us the periodicity of the signal. This method of period determination begins to fail for chaotic oscillations, as it regularly reports a periodicity of three. A chaotic signal will show a large decrease in energy between the two peaks, however, and this information can be used to aid proper classification.

As an alternative method, if we have time series data for both the position and the velocity, we can numerically analyze the pseudo-phase plot, checking the position every time the velocity goes through zero. The number of unique positions (divided by two) gives us the periodicity. This method has difficulty with solid-wall impacts, especially those which result in complicated, small amplitude oscillations. In addition, it is not easily applied to non-numerical time series data because it requires noise free measures of both position and velocity.

${ }^{*}$ The Fourier Transform is periodic, and in order to produce a linear correlation (rather than a circular correlation) the time series must be the proper length. This entails either zero-padding the data to twice its original length, or using the overlap-add method to segment and correlate the series. 
A final option for determining periodicity is some sort of windowed analysis. We can divide the waveform into intervals, and then compare those intervals with each other, looking for repetition. This method also has its drawbacks; it is sensitive to the sampling rate and the frequency of the oscillations. Mathematica code for these methods of period determination is presented in Appendix $\mathrm{K}$.

\subsubsection{Solid Wall Impacts}

In order to strengthen the case for chaotic oscillations due to aortic parameter changes, we will work through the logical steps necessary to transform the "impact oscillator" system into a facsimile of the circulatory system, beginning with the nondimensional $\Pi$ groups and second order differential equation given in Equation (4.26) and Equation (4.28), respectively. As with the linear study, we can establish intervals for the nondimensional groups, based on the circulatory system parameters:*

$$
\begin{aligned}
C_{a 0} & =[20 ., 40 .] \\
R_{a 0} & =[0.0002,0.0011] \\
I_{a 0} & =[0.00003,0.0003] \\
\omega & =[4 ., 21 .]
\end{aligned}
$$

The interval for excitation frequency, $\omega$, is selected to cover the entire domain of human heart rate. Elite athletes can have resting heart rates as low as $40 \mathrm{bpm}$ and will reach just over $200 \mathrm{bpm}$ at peak exertion. Obviously "normal" people do not see either of these extrema. Also note that the interval for aortic flow inertia has been expanded compared to Equation (4.14); it is generally agreed (Olansen et al., 2000 or Lu et al., 2001) that the proximal aortic inertia is much larger than the distal aortic inertia. While the latter affects the blood flow modelled by Windkessel elements, the former is more important for the impact-oscillator analogy of heart ejection and aortic response. While proximal inertia values as high as $0.0007 \mathrm{~N} \cdot \mathrm{s} \cdot \mathrm{cm}^{-5}$ appear in the literature (Olansen et al., 2000), a more conservative maximum value of $0.0003 \mathrm{~N} \cdot \mathrm{s} \cdot \mathrm{cm}^{-5}$ was selected for this study. The lower limit for aortic inertia remains as in Equation (4.14).

\footnotetext{
*Once again the square brackets in Equation (4.29) are standard mathematical interval notation. [...] denotes a closed interval (which includes its endpoints), while (...) denotes an open interval (which does not include its endpoints). Units for Equation (4.29) are as follows: $C_{a o}$ in $\mathrm{cm}^{5} \cdot N^{-1}, R_{a o}$ in $N \cdot s \cdot \mathrm{cm}^{-5}, I_{a o}$ in $N \cdot \mathrm{s}^{2} \cdot \mathrm{cm}^{-5}$, and $\omega$ in $\mathrm{rads} \mathrm{s}^{-1}$.
} 
The intervals for nondimensional groups $\Pi_{1}$ and $\Pi_{2}$ are therefore:

$$
\begin{aligned}
& \Pi_{1}=[0.62,375 .] \\
& \Pi_{2}=[0.11,31.5]
\end{aligned}
$$

Figure 4.13 shows the periodicity of the impact oscillator response for a range of driving frequencies and $\Pi_{1}$ values. In Figure 4.13, oscillations greater than period-30 (including chaotic, non-repeating oscillations) are binned together, and show up as white regions.

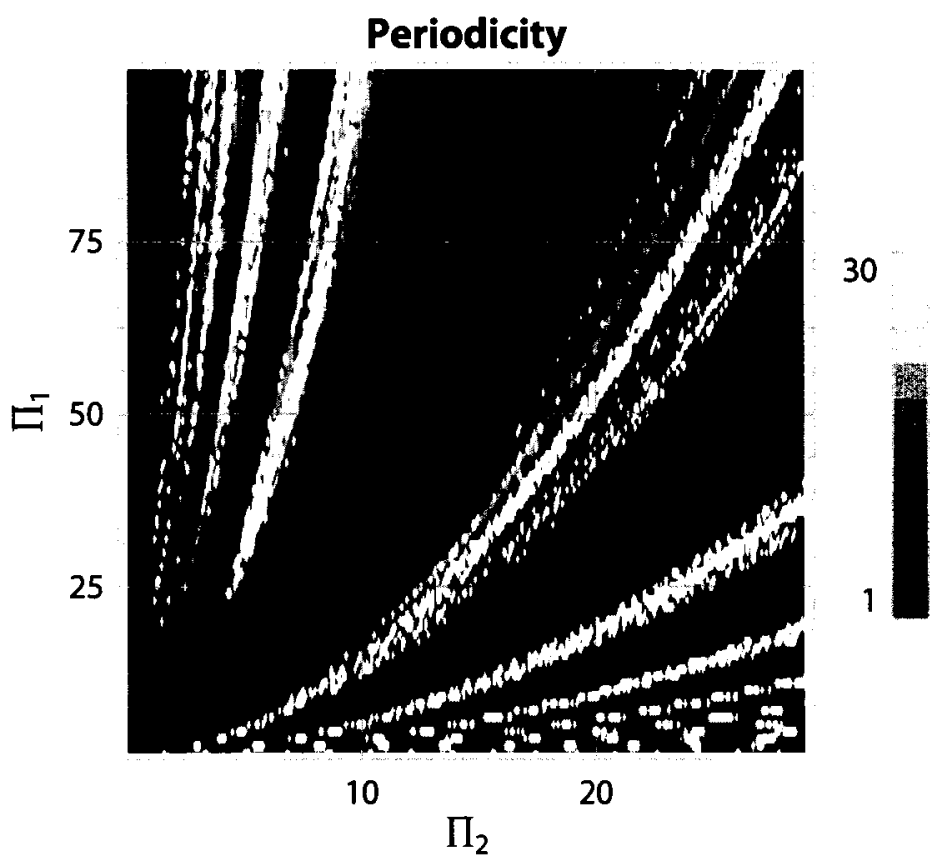

Figure 4.13: Periodicity for oscillations of the impact oscillator (truncated at period-30)

A different way to classify the motion of the impact oscillator is to look at the impact intervals, or the maximum position (or maximum velocity) between impacts. This was the strategy used to produce Figure 4.10. With numerical simulation of a linear solid wall impactor, the impact intervals are directly available and the maximum position or velocity between impacts is easily calculated because integration is normally halted after each impact and restarted with new initial conditions. This allows precise location of the impact point, and improved numerical accuracy. 
Position data (such as that which produced Figure 4.10) can be re-presented in a form similar to Figure 4.13. For each driving frequency and each $\Pi_{1}$, the system will respond with either a repeating pattern of oscillations or a chaotic trace. A period-1 oscillation will feature a single maximum bounce height or maximum velocity, facilitating classification; a series of identical-height bounces implies a period-1 oscillation. Discrepancies arise between this type of periodicity classification and those presented earlier; if an oscillation does not result in an impact with the floor, it is not added to the periodicity total, however the general trend and appearance will be the same, as seen in Figure 4.14. There is really no consensus as to what constitutes an oscillation, and how one should classify periodicity.

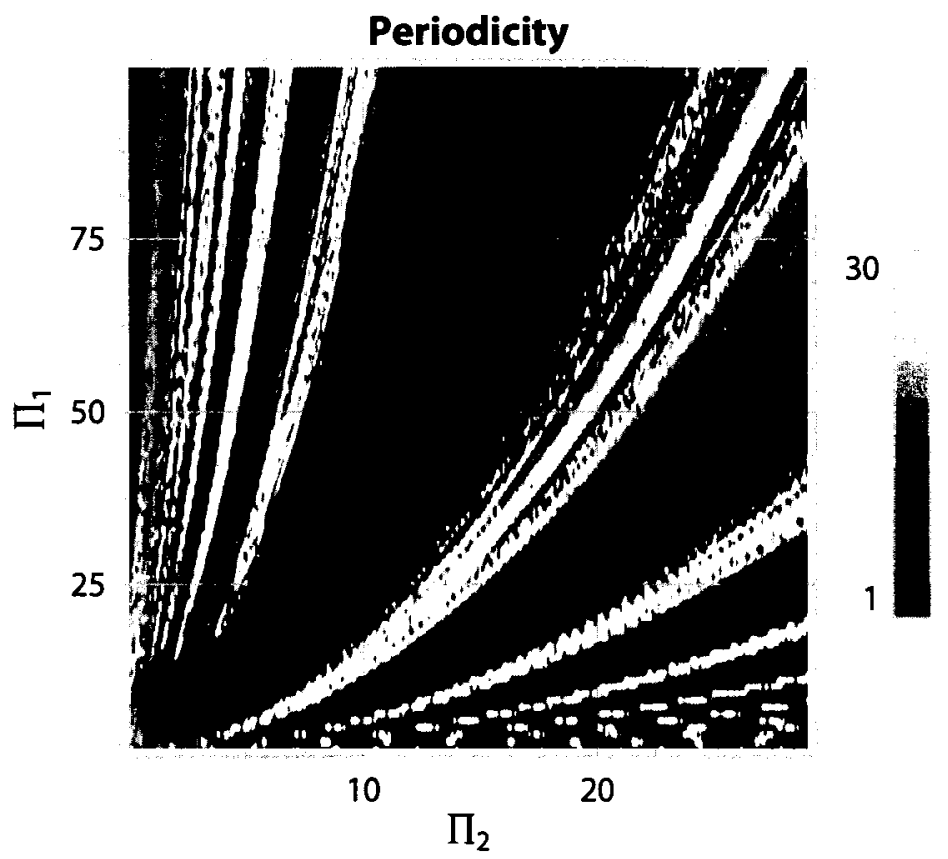

Figure 4.14: Periodicity for oscillations of the impact oscillator (truncated at period-30) by examining impact intervals

In the white regions of both Figure 4.13 and Figure 4.14 we expect the system response to be chaotic. Chaos is aperiodic long-term behaviour in a deterministic system that features sensitive dependence on initial conditions (Strogatz, 1994). There are trajectories which 
do not settle down to fixed points, periodic orbits, or quasi-periodic orbits as $t \rightarrow \infty$, and nearby trajectories separate exponentially fast. We can examine the latter effect by varying the system's initial conditions and observing the impact on the long-term behaviour.

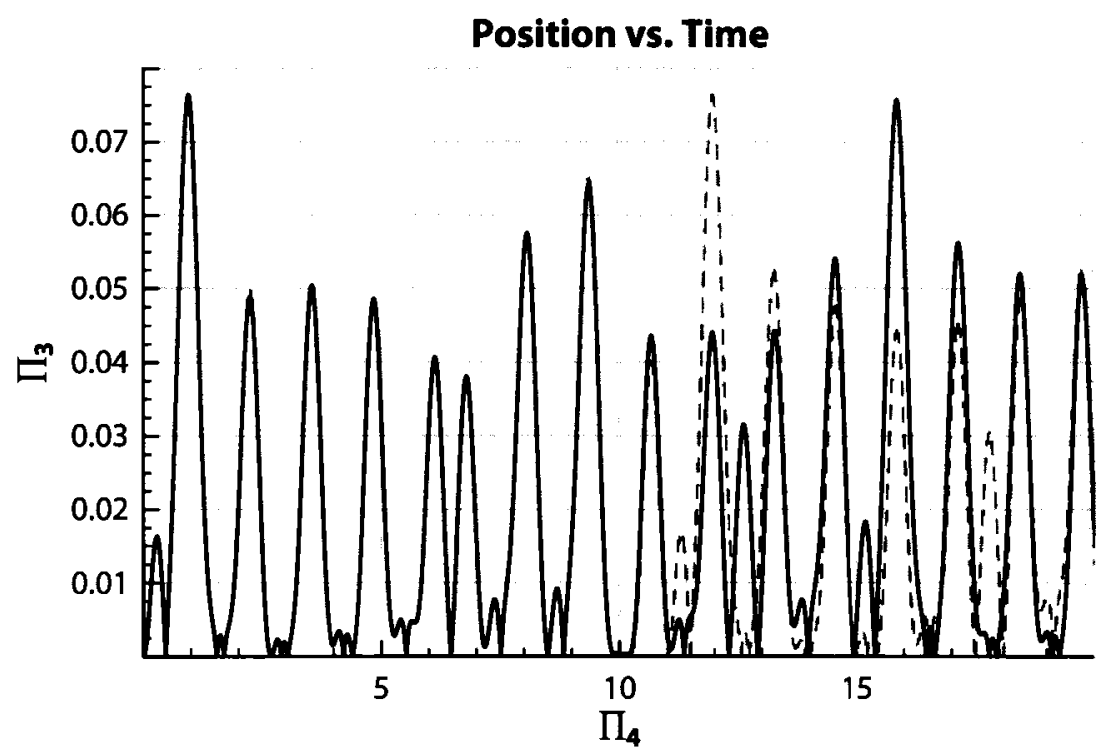

Figure 4.15: Divergence of nearby trajectories (initial conditions $\left\{\Pi_{3}, \Pi_{3}^{\prime}\right\}=\{0 ., 0$.$\} and$

$$
\left.\{0 ., 0.001\}, \Pi_{1}=10 . \text {, and } \Pi_{2}=9.7\right)
$$

Figure 4.15 demonstrates how nearby trajectories diverge in time. The system was started with slightly different initial conditions, $\{0 ., 0$.$\} and \{0 ., 0.001\}$ but with the same values of $\Pi_{1}$ and $\Pi_{2}$ (10. and 9.7, respectively). The trajectories are initially close together, well within the thickness of the line, and they move together until just past $\Pi_{4}=10$, when they begin to diverge.

If we want to study the divergence of these trajectories in phase space, we need a way of simplifying the pseudo-phase plot. Recall that the phase plot for a non-autonomous system self-intersects as the third order system is embedded in two-dimensional space. An elegant solution to this self-intersection problem is the Poincare section, produced by "strobing" the system at the same phase in each drive cycle and recording the trajectories as they pass through a plane perpendicular to the flow. 
Figure 4.16 records the migration of a series of 1700 points (initial conditions) as their trajectories evolve in time. The bundle of trajectories is initially very close together, and the initial conditions appear as a single, very tiny, dot located at the origin of the plot in Figure 4.16(a).* After 10 drive cycles, the trajectory bundle is still very compact, and still appears as a dot. An additional 10 drive cycles begins to shatter the bundle, which has now broken into two large bands, one smaller band, and a smattering of points, seen in Figure 4.16(c). This dispersion continues as the number of drive cycles increases, until (after only 40 forcing cycles) the trajectories appear as a scattershot across the intersection plane. In a chaotic system nearby trajectories diverge exponentially, and the rate of divergence is measured by the Lyapunov exponents.

We can estimate Lyapunov exponents by varying the initial conditions slightly and recording the divergence of nearby trajectories in time. A plot of $\ln |\delta|$ versus $t$ will show a linear trend, up until the system saturates. This saturation is the same effect seen in Figure 4.16(d) and (e). Simply put, there is a limit to the maximum possible divergence between two trajectories; each has to stay on the same attractor, confining them to the same region of $n$-dimensional space. Strange attractors are bounded sets which happen to have zero area (due to their fractal nature). Figure 4.17 shows the divergence of two nearby trajectories for the same parameters which produced Figure 4.15 and Figure $4.16, \Pi_{1}=10$ and $\Pi_{2}=9.7$. The system was started with initial conditions $\{0 ., 0$.$\} and \left\{0 ., 10 .{ }^{-6}\right\}$ and the evolution of these two trajectories was tracked over the interval $\Pi_{4}=[0 ., 100$.$] . Inte-$ gration in these sorts of systems must be carefully controlled; since the system is sensitive to initial conditions, it is also sensitive to numerical error during integration. In order for the initial disturbance (the variation in initial conditions) to propagate, it must be larger than the local truncation error of the integrator, otherwise it will be effectively lost. To propagate the small $\left(10^{-6}\right)$ deviation in initial conditions, we need to increase the absolute error goal and the number of significant figures used to do the calculation.

Although accuracy of the solution is a concern, there is the so-called "shadowing theory" (Hammel et al., 1988), which can be used to show that even if an approximate numerical solution with a particular initial condition may not remain close to the actual trajectory for that initial condition, the approximate solution does lie close to the actual trajectory for

${ }^{*}$ In each of the subplots of Figure 4.16 the dots are plotted over the strange attractor that the system unfolds on. It appears in light gray. 


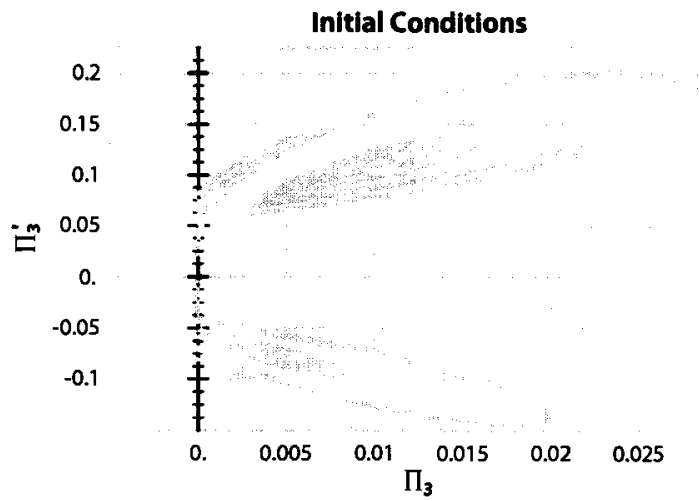

(a)

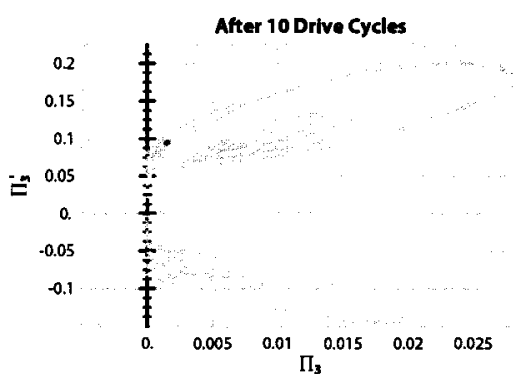

Atter 40 Drtve Cycles

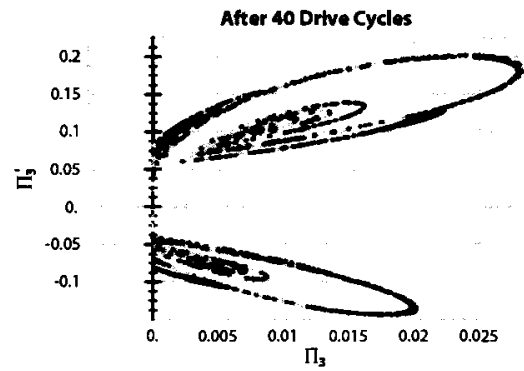

(b)

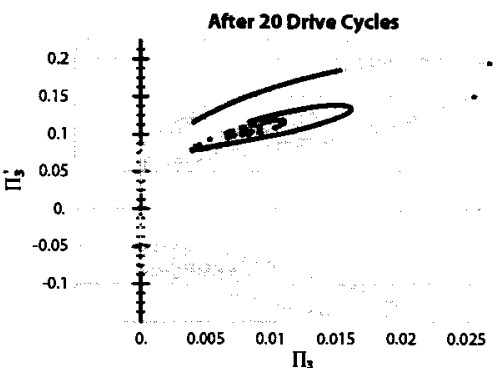

(c)

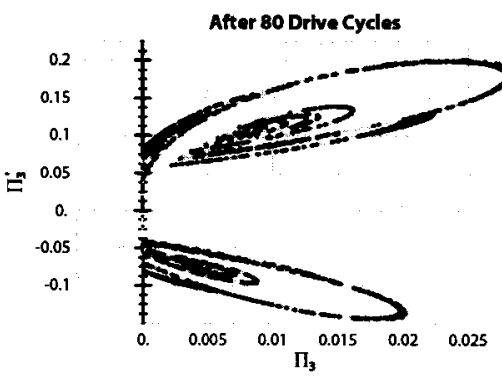

(e)

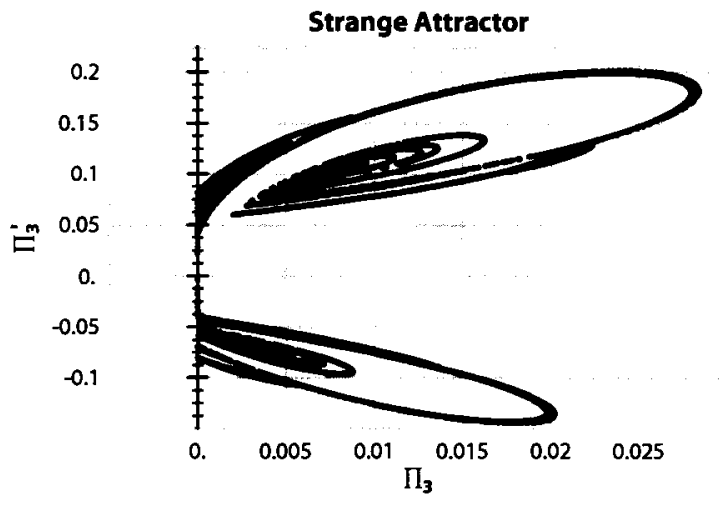

(f)

Figure 4.16: Divergence sequence for 1700 nearby trajectories as the system evolves in time over (b) 10 drive cycles, (c) 20 drive cycles, (d) 40 drive cycles, and (e) 80 drive cycles. 


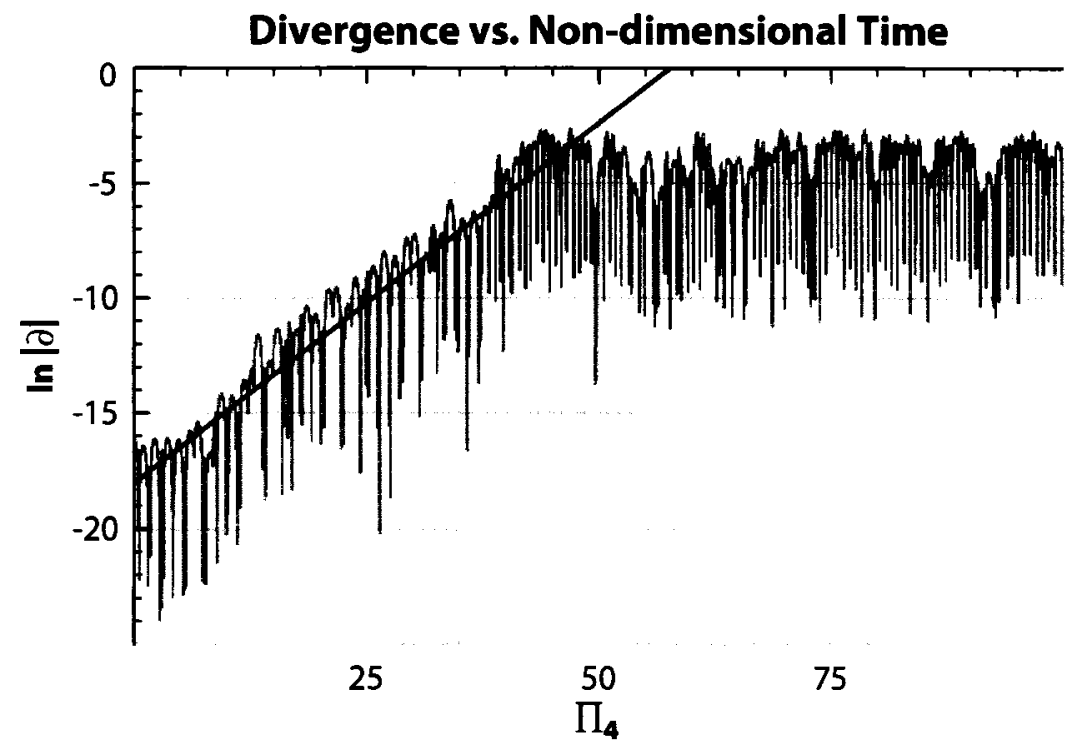

Figure 4.17: The divergence of two nearby trajectories with respect to time

some nearby initial condition. This justifies the use of numerical integrators on nonlinear systems; significant results are still valid.

Figure 4.17 includes a line of best fit (in the least-squares sense) for the pre-saturation linear portion of the divergence plot, up until the divergence is order one. Based on the slope of this line we can calculate a Lyapunov exponent of $\approx 0.4$, similar to that of the Heńon Map. Note that this is only one of the Lyapunov exponents for the system; divergence can happen in any of the dimensions. This spectrum of Lyapunov exponents is more difficult to estimate.

\subsubsection{Soft Wall Impacts}

As stated earlier, the stiffening behaviour of the aorta is not infinite; the next step in transforming the impact oscillator into the proximal arterial system involves replacing the hard wall with a softer spring. For this phase of the study the parameter intervals remain as in Equation (4.29). The intervals for the nondimensional groups are also unchanged, but we will modify our nomenclature slightly to deal with the piecewise change in spring stiffness upon impact with the wall. The baseline (nominal) spring stiffness is denoted 
$\Pi_{1 \mid b}$ and the stiffness multiplier (upon impact with the wall) is $\Pi_{1 \mid m}$. In other words, if the stiffness doubles upon impact, $\Pi_{1 \mid m}=2$.

Establishing the interval for $\Pi_{1 \mid m}$ is difficult. The nondimensional group $\Pi_{1}$ is dependent on a few different system parameters: inertia, stiffness and resistance. While these parameters are largely independent in a mass-spring-damper system, the opposite is true in a fluid system. Changes in vessel geometry which bear upon compliance (stiffness) also impact the fluid flow inertia and resistance. Aortic compliance can affect a five-fold increase in $\Pi_{1}$ on its own (King, 1946), without any influence from $I_{a 0}$ or $R_{a 0}$, both of which are modified by changes in vessel geometry. Note that $I_{a o}$ is antagonistic compared to $R_{a 0}$ and $C_{a 0}$; it appears in the numerator of the nondimensional $\Pi_{1}$ group. Also, $R_{a o}$ appears raised to the second power, increasing its impact on the nondimensional group.

To ensure coverage of a physiologically representative area of the parameter space, we will select the interval for $\Pi_{1 \mid m}$ as $[1 ., 100$.$] , giving the following domain:$

$$
\begin{aligned}
\Pi_{1 \mid b} & =[0.62,375 .] \\
\Pi_{1 \mid m} & =[1 ., 100 .] \\
\Pi_{2} & =[0.11,31.5]
\end{aligned}
$$

We have a three-dimensional parameter space to investigate, with each individual slice in the $\Pi_{1 \mid b}-\Pi_{2}$ plane appearing as in Figure 4.13 or Figure 4.14. Each of these contour plots takes a long time to generate, ${ }^{*}$ and the addition of a third dimension necessitates a coarser test grid. It also entails visualization problems, because we are interested in the periodicity of the system at any given point in this three dimensional space.

The parameter space was subdivided into 100 test planes in each of the three principal dimensions, producing periodicity data for one million parameter combinations. This periodicity data was exported to a POV-Ray ${ }^{\dagger}$ density file and used in a volume rendering, producing Figure 4.18. As before, areas of high periodicity appear lighter. Figure 4.18 displays the same trends seen in Figure 4.13 or Figure 4.14, with multiple regions displaying

\footnotetext{
${ }^{*}$ Figure 4.13 and 4.14 each require between 30 and $40 \mathrm{~h}$ of CPU time on an AMD64 3500+ machine.

tThe Persistence of Vision Raytracer (http://www. povray.org), a powerful freeware raytracing pro-
} gram. 


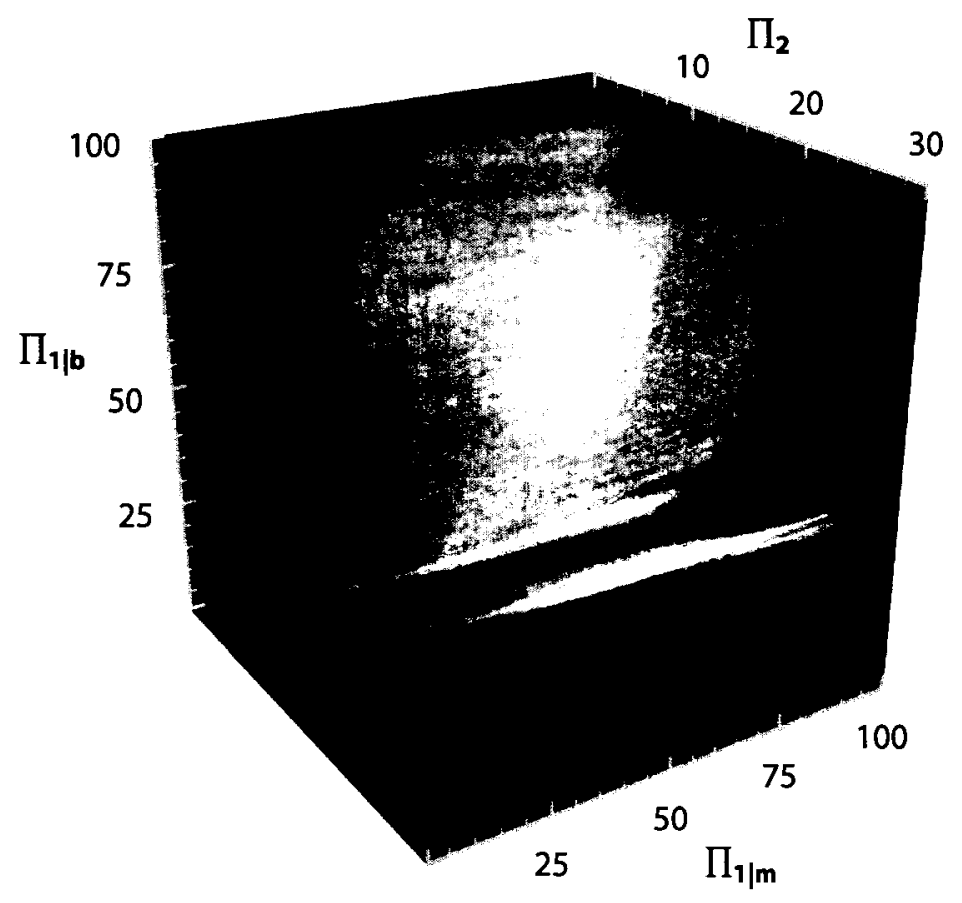

Figure 4.18: $3 \mathrm{D}$ volume rendering of the periodicity as $\Pi_{1 \mid b}, \Pi_{1 \mid m}$, and $\Pi_{2}$ are varied 
chaotic oscillations. After $\Pi_{1 \mid m}$ reaches a certain threshold the topology is nearly invariant in the $\Pi_{1 \mid m}$ direction. Low piecewise increases in stiffness do not produce chaotic oscillations, but we do see period doubling bifurcations for low values of $\Pi_{1 \mid m}$. As might be expected, periodicity increases as $\Pi_{1 \mid m}$ increases, and for values of $\Pi_{1 \mid m}>10$ we start to see very high periodicity, and chaotic oscillations.

Changes in $\Pi_{1 \mid b}$ and $\Pi_{2}$ have the same effect as in Figure 4.13 or Figure 4.14, with the solid wall impactor. This is to be expected, since the underlying dynamics of the system have not changed; the chaotic behaviour is due to the $\Pi_{1}$ nonlinearity, not any particular parameter value. If we wanted to create a solid wall impactor from this soft-wall system we could simply set $\Pi_{1 \mid m}=\infty$ and produce plots identical to Figure 4.13 or Figure 4.14.

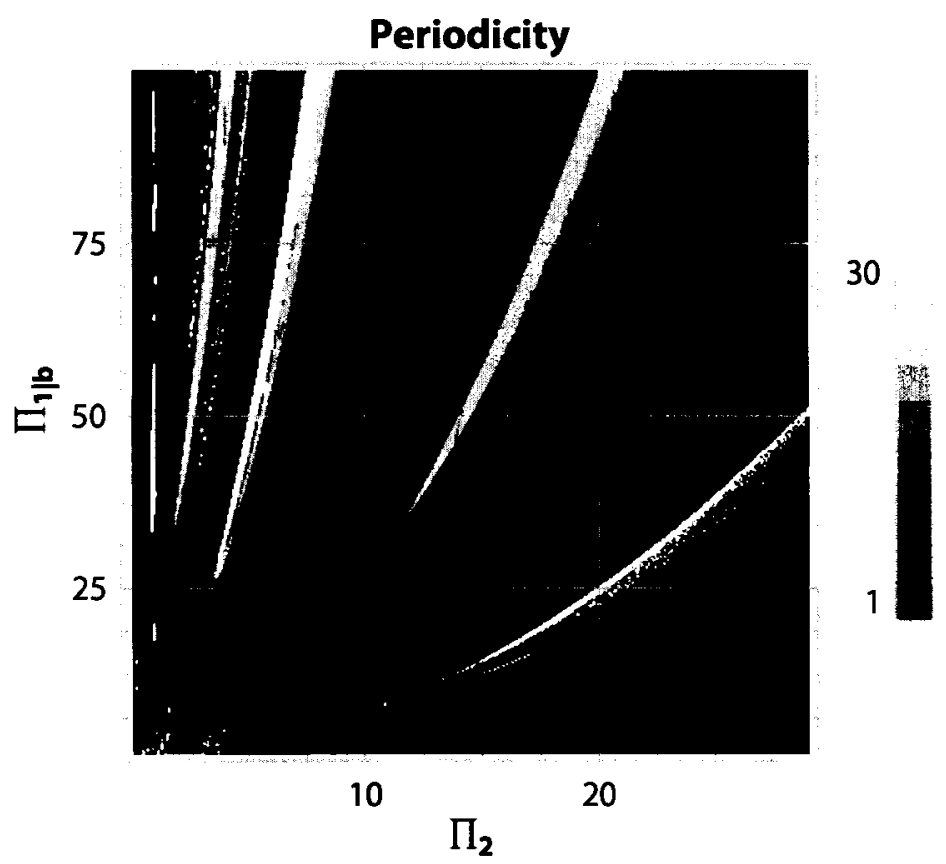

Figure 4.19: Periodicity for oscillations of the soft-wall oscillator with $\Pi_{1 \mid m}=10$ (truncated at period-30)

Figure 4.19 gives a high-resolution slice of the volume in Figure 4.18 in the $\Pi_{1 \mid b}-\Pi_{2}$ plane for $\Pi_{1 \mid m}=10$. Areas of higher periodicity are visible, and a few areas featuring chaotic 
oscillations have begun to appear. It is interesting that the regions which will eventually develop into areas of chaotic oscillation are already displaying complex behaviour. The trademark parabolic-like streaks begin to appear for very low values of $\Pi_{1 \mid m}$ and persist as $\Pi_{1 \mid m}$ is increased, although they shift location very slightly as that parameter is varied.

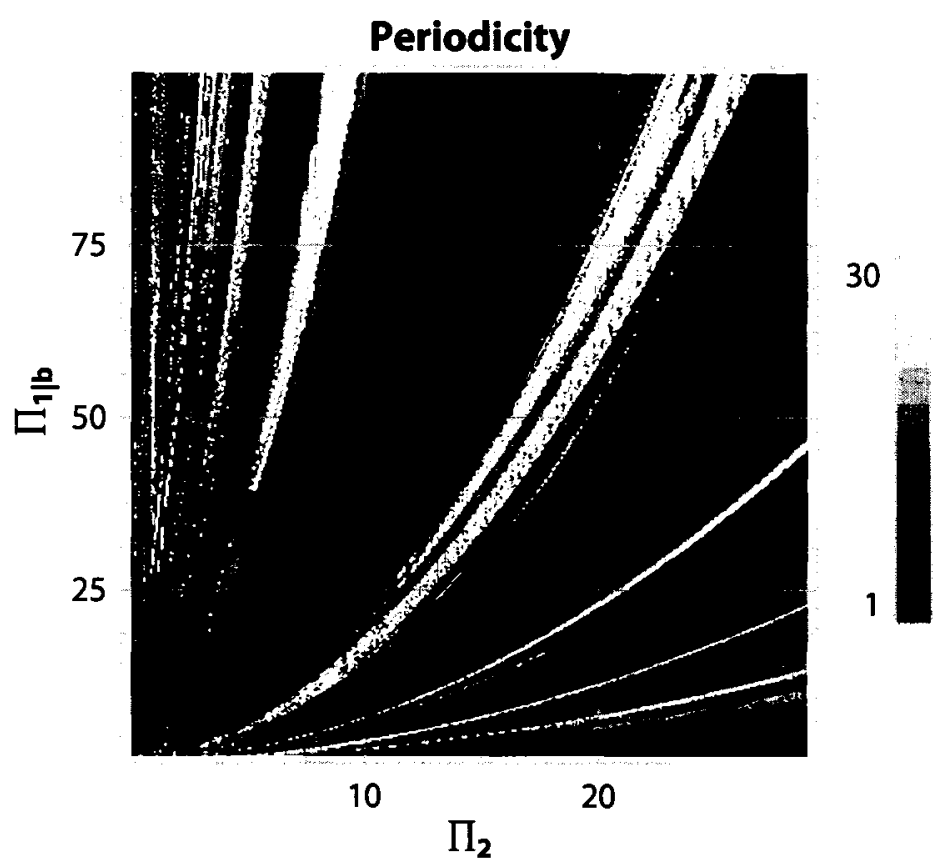

Figure 4.20: Periodicity for oscillations of the soft-wall oscillator with $\Pi_{1 \mid m}=50$

(truncated at period-30)

Figure 4.20 shows a slice for $\Pi_{1 \mid m}=50$; chaotic areas have now established themselves, and the plot appears very similar to the solid-wall impact plots of Figure 4.13 or Figure 4.14.

\subsubsection{Accounting for Blood Flow}

Recall from Figure 4.9 (on Page 97) that the aortic flow waveform is generally periodic, but not sinusoidal. The inflow portion of a beat (as the heart injects blood) could be modelled as a half-sine wave, but after the aortic valve closes there is minimal backflow. All of the oscillator systems in the previous sections have been driven by periodic, sinusoidal 
forcing functions. In order to make the final connection between these impact oscillator systems and the proximal arterial system, we need to either show that a waveform similar to the aortic flow waveform can induce chaotic behaviour in an oscillator system featuring a soft wall, or we need to show that the forcing function that drives the proximal arteries (and particularly the aorta) is nearly sinusoidal.

This is the reason why both system inputs have been included in each mass-springdamper system which has been treated thus far. The system in Figure 4.8 and each system in Figure 4.11 include(s) both a position input for the ceiling, allowing us to excite the mass by moving the ceiling, and a force input to the actual mass, allowing us to excite the system by shaking the mass. When we nondimensionalized the second order differential equation describing the mass-spring-damper system, we noted that an advantage of switching to the $\Pi$-groups was that it masked the source of the system input. Either moving the ceiling, or shaking the mass, or both moving the ceiling and shaking the mass are valid ways of driving the system. In the human circulatory system, the downstream arteries apply a backpressure to the aorta. In terms of dynamics, this backpressure looks like a disturbing force in our mass-spring-damper system where we inject blood by moving the ceiling downwards. The overall (net) influence on the arterial system appears as a periodic function with an additional positive linear term, which results in a net positive slope. The positive linear term is the average systemic blood flow rate, or the downward progress of the ceiling in any of the mass-spring-damper systems of Figure 4.11, and the spring compression determines the amount of blood in the aorta at any given time.

It is important to understand that none of the nonlinear behaviour outlined in this section (i.e., Section 4.4) is a result of the driving function itself. All of the features seen in Figure 4.10 or 4.20 (including the energy amplification and chaotic oscillations) are solely a result of the oscillator. This cannot be overstated.

In other words, impact is the only requirement for either our solid-wall or soft-wall oscillators, although the latter requires a sufficiently solid impact (i.e., a sufficiently high value of $\left.\Pi_{1 \mid m}\right)$. Walker and Soule (1996) treat a sinusoidally forced oscillator which impacts with a solid floor, but they study the influence of floor placement (relative to the system's equilibrium position) on the resultant dynamic response. This scenario is dynamically similar to ours. 


\subsubsection{The Effect of VAD Pumping Modes}

As outlined in Chapter 2 (Section 2.6), a typical VAD might operate in one of two pumping modes: "fixed-rate" or "full-fill, full-eject." In the absence of venous system dynamics (and the natural heart) these two modes are similar; the best approximation to the FFFE mode is a "fixed-fill, full-eject" hybrid cycle. In this operating mode the VAD would fill fully in a fixed rate of time, with the fill rate tuned to control the diastolic length of the beat. During systole it would pump until the internal blood volume reached the preset minimum level and the cycle would repeat.

For either of the two VAD operating modes, the system's equilibrium position would be shifted, and in order for impacts to occur (whether soft- or hard-walled) the rigid barrier must be placed at an appropriate location. Figure 4.21 shows the results of a fixed-fill,

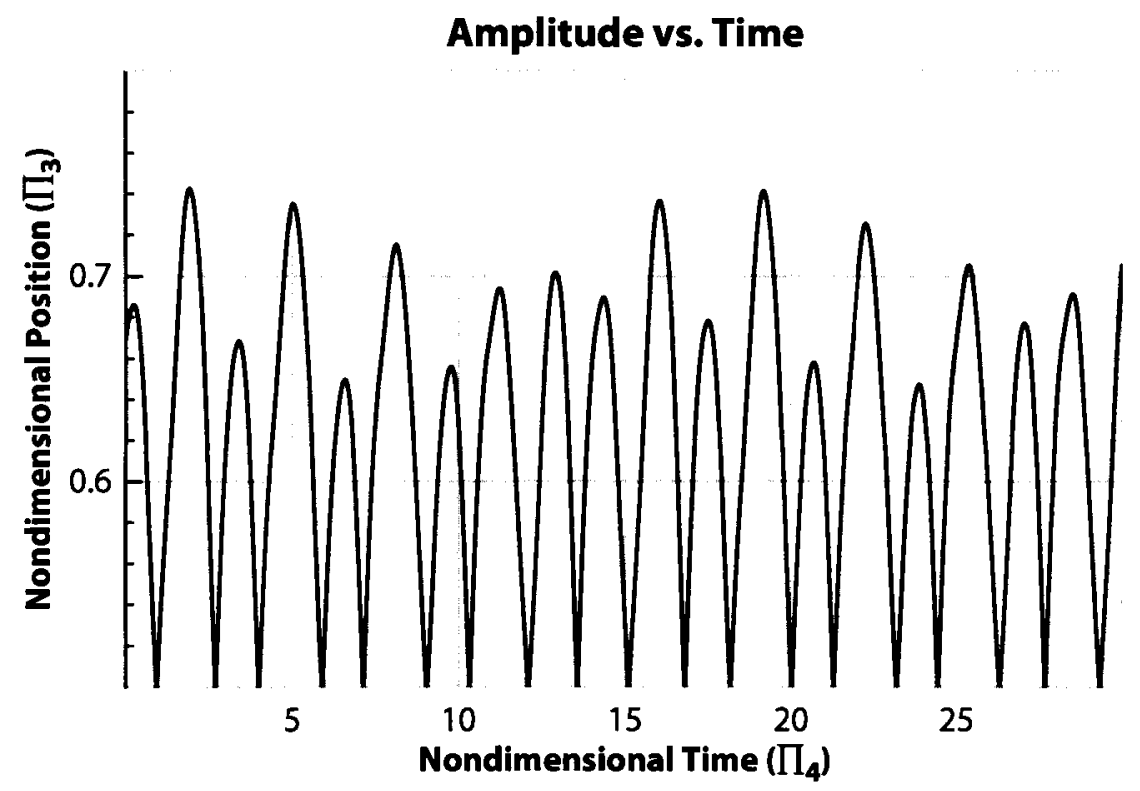

Figure 4.21: Chaotic oscillations in a system driven by a fixed-fill, full-eject input

full-eject simulation with the "floor position" set at $\Pi_{3}=0.5$. The oscillations are chaotic, as with the previous systems which were driven by sinusoidal inputs. In these systems (i.e., those driven by asymmetric inputs) the "floor position" plays a critical role in system stability, determining whether the response is periodic or chaotic, and for what length of time. In systems with asymmetric input functions the barrier impacts may be finite or 
infinite in number, depending on the position of the barrier, the forcing function, and the initial conditions of the system. Treating these various combinations in detail is beyond the scope of this work.

\subsection{Theoretical Basis for Circulatory System Efficiency}

Most engineers are familiar with the idea of mechanical resonance. When an oscillator is excited near its natural frequency it will respond with large-amplitude oscillations; it is easy to get an object to vibrate at its natural frequency with very little effort. There is an analogous feature in the impact-oscillator response: the amplitude peaks in Figure 4.10 (which are interspersed with areas of chaotic oscillation) are similar to resonant frequencies in a traditional oscillator.

We have seen that the "soft-wall" impacts produce responses similar to these "solidwall" impacts once the stiffness multiplier (dubbed $\Pi_{1 \mid m}$ ) reaches a certain threshold. Relatively small values of $\Pi_{1 \mid m}$ can produce period-doubling bifurcations and chaotic oscillations. The volumetric rendering in Figure 4.18 shows how the complexity of the system response varies with respect to $\Pi_{1 \mid b}$ and $\Pi_{1 \mid m}$. After a minimum threshold of approximately $\Pi_{1 \mid m}>10$ is exceeded the oscillator begins to display chaotic behaviour at certain forcing frequencies. The peaks in Figure 4.10 are much larger than the freely-oscillating amplitude of a similar (non-impacting) mass-spring-damper system with the largest peak being comparable to the resonant amplitude of a freely oscillating system - the maximum steady-state amplitude possible (Walker and Soule, 1996).

The nonlinear oscillator theory suggests that for each set of circulatory system parameters there is a particular beat rate which is optimal in terms of energetic efficiency. In between these amplitude peaks the nonlinear oscillator displays chaotic behaviour. This, in turn, suggests that there are certain beat rates which the natural heart will avoid. There is no obvious (continuous) way to move between parabolic bands in Figure 4.20 while avoiding chaotic regimes. It is also unclear if one would want to; the maximal oscillatory amplitude occurs at the first peak in Figure 4.10. Unless there is some advantage to running at a higher beat rate (for a given circulatory system state) the heart would confine itself to the wider parabolic band that is due to the first periodic peak. 


\title{
Chapter 5
}

\section{Experimental}

\begin{abstract}
1 s detailed in Chapter 3, most mock circulatory system designs use two compliance
chambers to model the whole of the human circulatory system. We saw in Section 4.2 that the single compliance arterial model (SCM) does a poor job of matching arterial pressure behaviour, and omits important features such as the dicrotic notch. Numerical simulations predict a sawtooth-like pressure waveform in the SCM; the addition of a second compliance element improves the physiological representation even if all constitutive relations are linear.
\end{abstract}

Due to the (anticipated) low signal levels, all DAQ channels were wired in differential mode, with $1 \mathrm{k} \Omega$ resistors to ground. This halves the number of channels available, but there are more than enough channels to take all of the required measurements.

\subsection{UOHI Mock Circulatory System (Original Configuration)}

In order to establish a baseline for mock circulatory system performance, the University of Ottawa Heart Institute (UOHI) MCS was tested in its original configuration for a number of VAD beat rates and valve settings. This particular mock circulatory system uses two cylindrical compliance chambers with identical diameters but different heights. Unlike the venous compliance chamber, which is open to atmosphere, the arterial compliance chamber is closed to atmosphere and the trapped, compressed air gives the chamber a nonlinear stiffness. Unfortunately, the arterial chamber's cross-sectional area is too large and this nonlinearity will not be felt over the course of a beat; much more than the typical stroke volume must be injected before the additional air compression becomes significant and the nonlinearity is felt. All calculations pertaining to the UOHI MCS are presented 
in Appendix $\mathrm{C}$, which also includes plots of the pressure-volume relation for the arterial chamber.

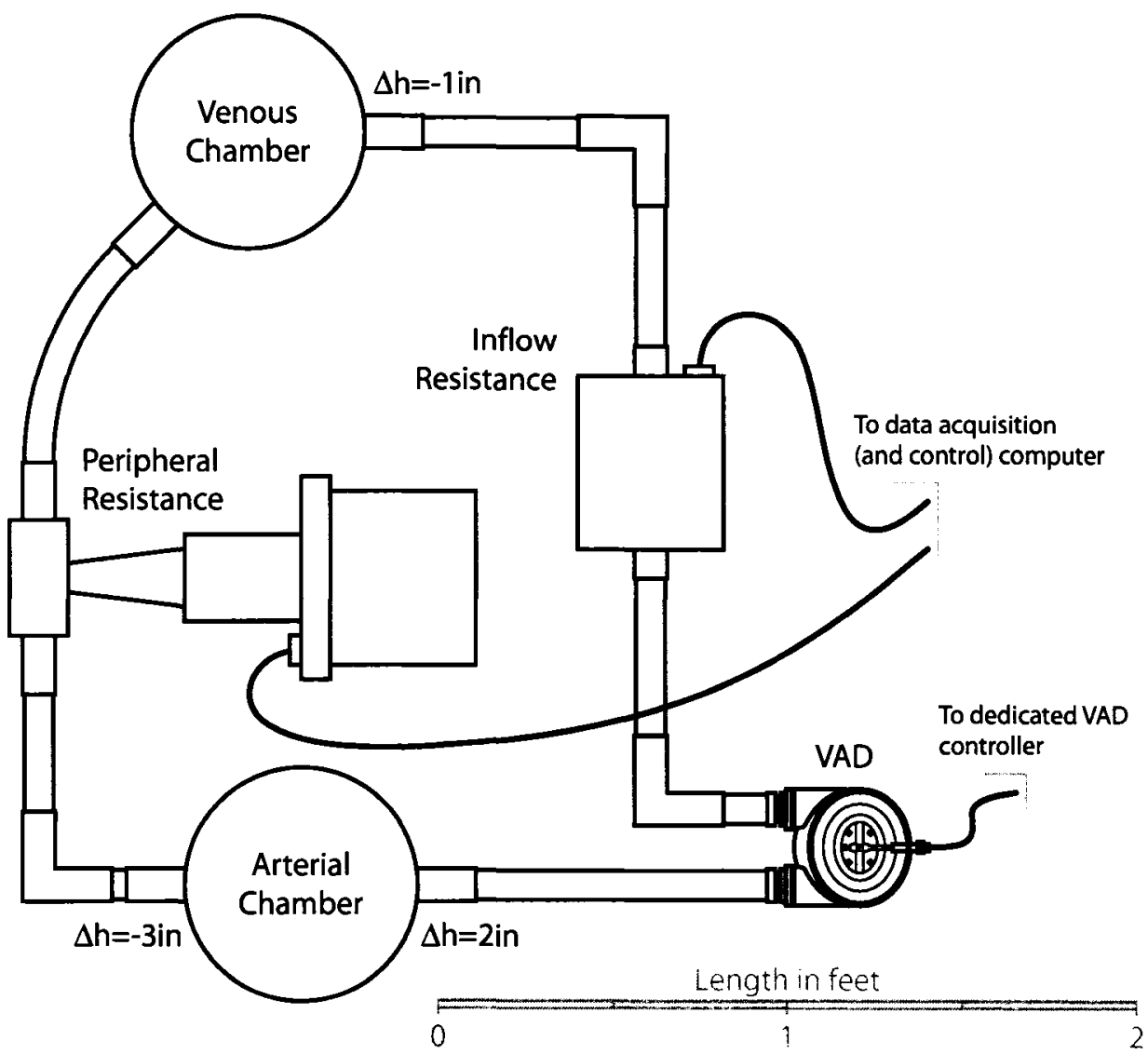

Figure 5.1: Schematic diagram of the UOHI mock circulatory system

A schematic of the UOHI mock circulatory system is illustrated in Figure 5.1.* The VAD pumps into the upper part of the arterial chamber through a 12 in long, 1 in internal diameter tube. The inlet centreline is elevated 2 in from the VAD outlet, and 6 in from the base of the chamber. The arterial chamber outlet is at a lower elevation than the inlet, with its centreline 3 in above the base of the chamber. The arterial and venous chambers are connected through tubing, and separated by a globe-type valve which is actuated using a servomechanism. Different fractional valve openings can be achieved by supplying differ-

*The components of the UOHI MCS were originally sized in Imperial Units. For some dimensions and calculations in this chapter we will break with our convention of using SI Units. 
ent DC voltages to the servo; lower voltages imply greater fractional openings. The valve calibration curve is presented as part of the "Experimental Setup" appendix, Appendix G.

The venous chamber is a static water column, with its top open to atmosphere (through a small opening). Its outlet is at a slightly lower elevation than its inlet, and it discharges through a ball-type valve designed to control the pre-load on the VAD, an important part of FFFE-mode operation. A photo of the experimental setup is shown in Figure 5.2.

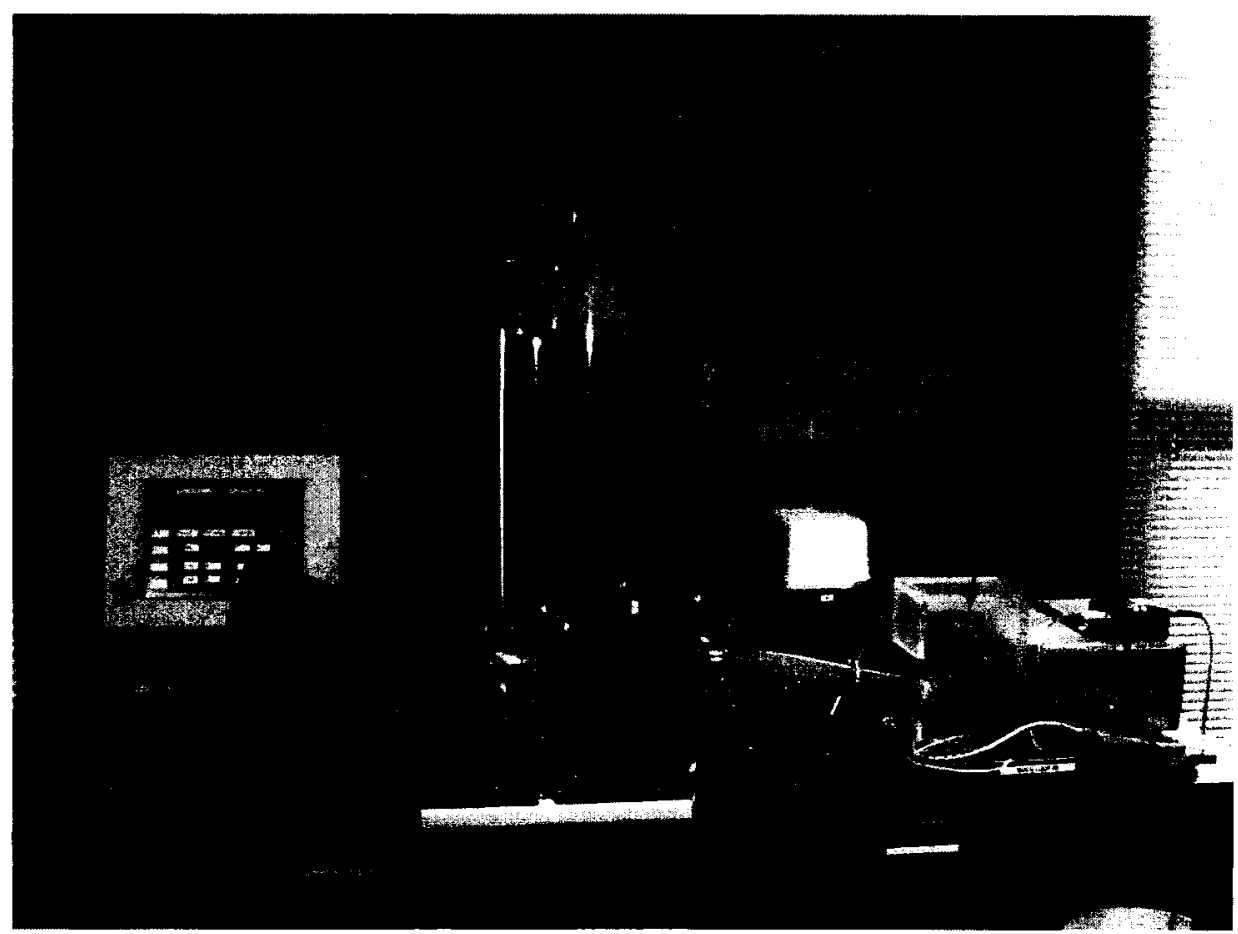

Figure 5.2: Photo of the bench-top experimental setup in the original (UOHI) configuration

A differential pressure transducer was used to record the difference between the arterial chamber pressure and the (nearly constant) venous chamber pressure. Relating this pressure trace to the VAD cycle requires a timing signal, but the VAD control computer and data acquisition computer are separate machines. Rather than interfacing the two machines, an amplified electret microphone* was used to record the VAD noise; valve action

\footnotetext{
${ }^{*}$ Schematics for all instrumentation circuitry are presented in Appendix I.
} 
can be extracted from this audio signal.

\subsubsection{Fixed-rate Tests}

For the preliminary tests, the VAD was run in fixed-rate mode with a range of beat rates and a constant peripheral resistance. The peripheral valve voltage was set to $8 \mathrm{~V}$ and the experimental procedure was as follows:

1. Data collection was started in LabVIEW

2. After approximately $5 s$ the VAD was started

3. Five full beats were marked and the 5 min countdown timer was started

4. When the countdown timer expired, the VAD was halted

5. $30 s$ later, data collection was stopped

The VAD controller allows integer beat-per-minute rate settings; the VAD was run at all (integer) beat rates between 50 and $70 \mathrm{bpm}$, and then in $5 \mathrm{bpm}$ increments between 70 and $110 \mathrm{bpm}$. Figure 5.3 shows the ensemble averaged pressure difference between the arterial and venous chambers for fixed-rate operation at a variety of beat rates between 50 and $70 \mathrm{bpm}$. The shading in Figure 5.3 corresponds to the measurement uncertainty, with lighter shades indicating narrow confidence intervals (and higher certainty).

At lower beat rates, sloshing in the arterial tank distorts the pressure waveform, varying the magnitude and temporal location of the maximum pressure peak. This distortion is recognizable in the darker areas of Figure 5.3.

For higher beat rates the VAD stroke volume is reduced, producing a lower peripheral flow rate and a lower peripheral pressure drop. This effect can be seen in Figure 5.3 as the pressure peak decreases with increasing beat rate.* After this behaviour was recognized, the peripheral resistance was lowered from 8 to $6 V$ (input DC voltage) and the test cases were re-run.

${ }^{*}$ The VAD control computer also registers stroke volume using the Hall-effect sensor output. 


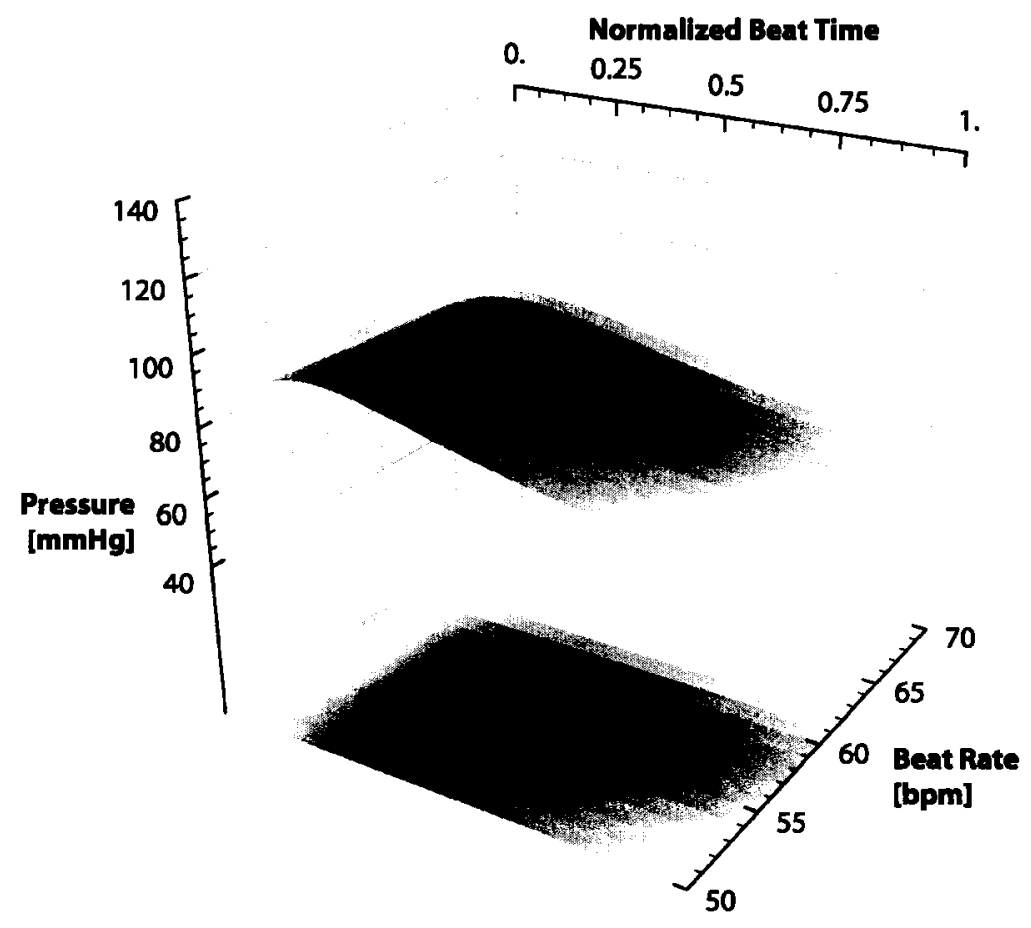

Figure 5.3: Pressure waveform versus beat rate for fixed-rate VAD operation (maximum standard deviation is $3.51 \mathrm{mmHg}$; minimum standard deviation is $0.21 \mathrm{mmHg}$ )

While this drop in peripheral resistance had the desired effect on the VAD stroke volume, it also emphasized the sloshing in the arterial tank and the corresponding distortion of the arterial pressure waveform. Figure 5.4 shows the ensemble averaged pressure difference between the arterial and venous chambers. Once again the shading in the figure corresponds to the uncertainty, with lighter shades indicating a narrower confidence interval. Compared with Figure 5.3, the lower peripheral resistance results in better certainty (i.e., a narrower confidence interval) and increasing peripheral pressure drop with increased beat rate, which implies a more constant stroke volume.

The major problem with the reduced peripheral flow resistance is the sloshing in the arterial chamber; the arterial pressure response is dominated by this surface wave oscillation. The fundamental period for a circular, cylindrical tank with rigid walls (Zeiny, 2000) 


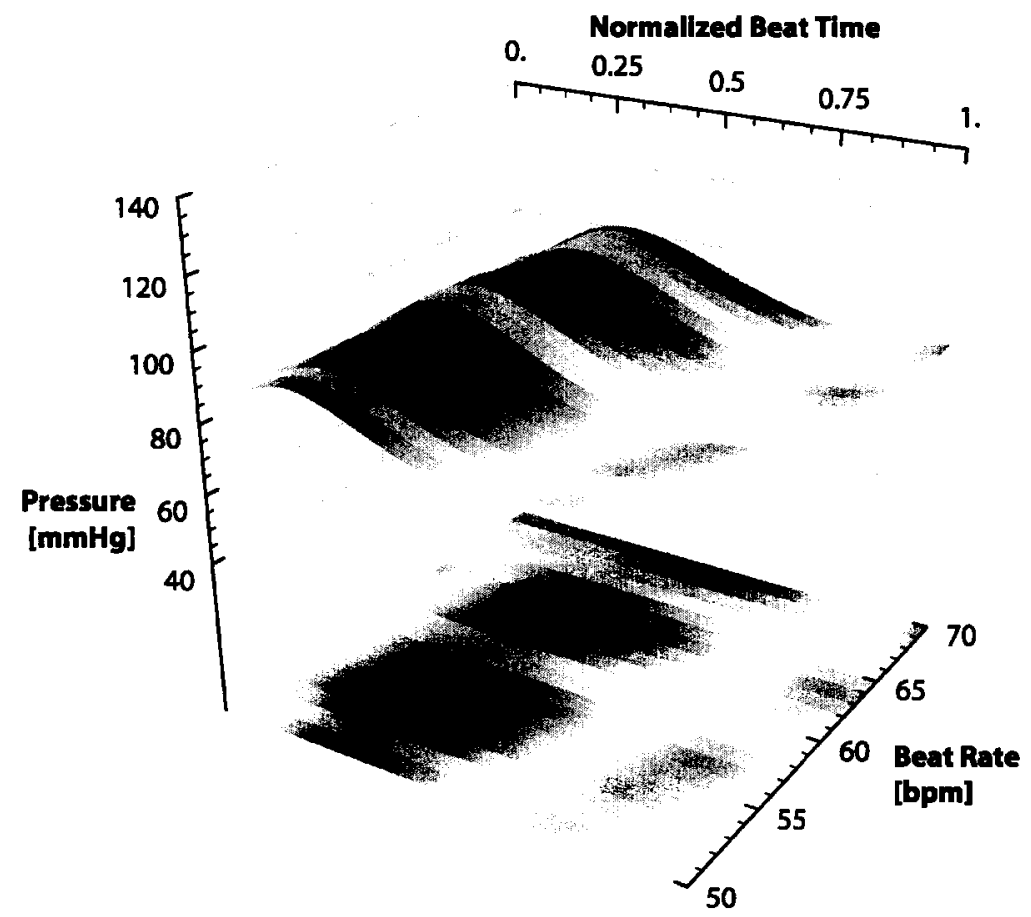

Figure 5.4: Pressure waveform versus beat rate for fixed-rate VAD operation (maximum standard deviation is $2.08 \mathrm{mmHg}$; minimum standard deviation is $0.26 \mathrm{mmHg}$ )

is:

$$
T=\frac{2 \cdot \pi}{\sqrt{\frac{1.84 \cdot g}{R} \cdot \tanh \left(\frac{1.84 \cdot H}{R}\right)}}
$$

where both $R$, the container radius, and $H$, the container height, are expressed in feet, and $g$, the gravitational constant is $32.17 \mathrm{ft} \cdot \mathrm{s}^{-2}$.

The UOHI arterial chamber has a natural period of oscillation of $0.472 s$, and when a line with this slope is overlaid on the pressure wave plot, the effect of sloshing is clearly visible. Figure 5.5 is a contour plot of the ensemble averaged arterial pressure waveform which corresponds to Figure 5.4, with lighter colours indicating areas of higher pressure. Trend lines illustrating the natural sloshing frequency have been overlaid on the plot, and there is strong agreement between the pressure behaviour of the arterial system and the natural sloshing frequency. This dominant pressure oscillation is not present at higher peripheral resistances. Based on these results, a peripheral resistance voltage of at least 


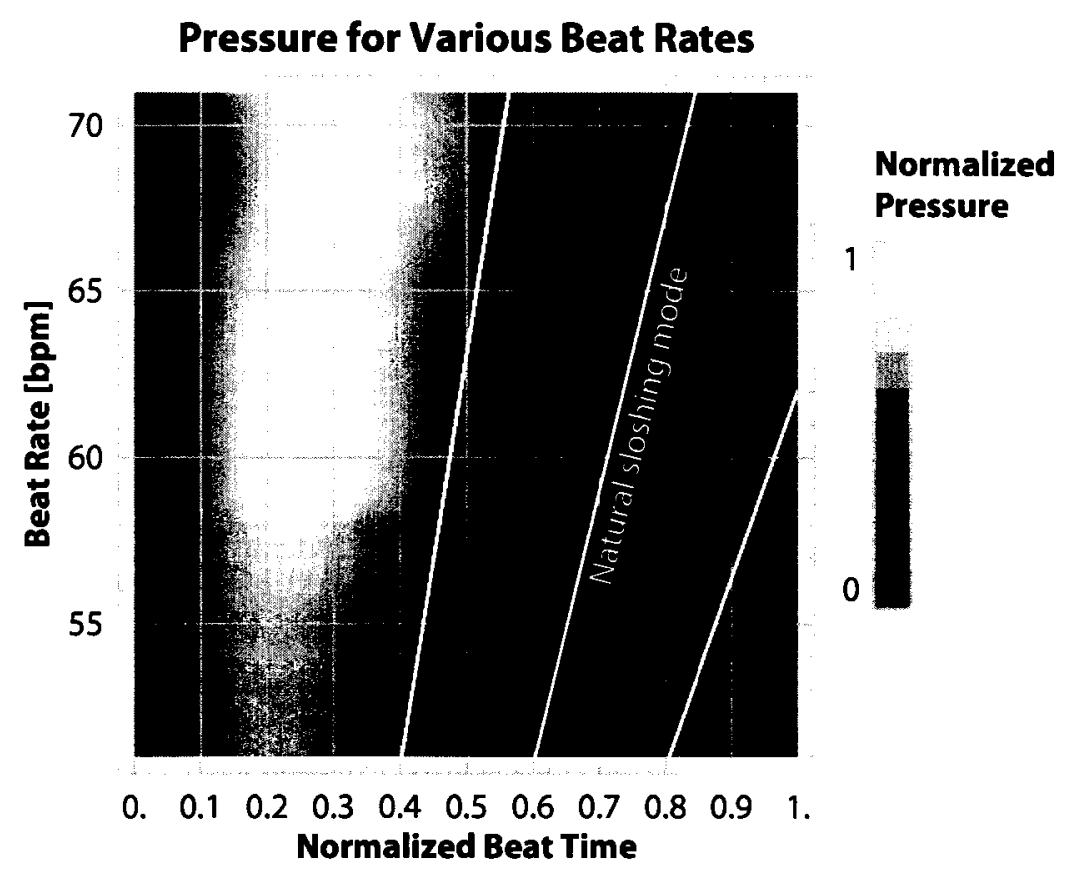

Figure 5.5: Effect of liquid sloshing on the arterial pressure waveform

$8 \mathrm{~V}$ is preferred, in spite of the consequent stroke volume decrease. It is also worthwhile to note that the resultant peripheral flow resistance agrees well with values found in the literature, and the value used in the mathematical simulation.

\subsubsection{FFFE Tests}

In full-fill, full-eject (FFFE) mode the peripheral and inflow valve settings directly influence VAD behaviour. The inflow valve, in particular, can alter the VAD cycle by affecting the preload pressure and hence the fill rate. In order to study the FFFE cycle, the VAD was run in FFFE mode for a variety of valve settings, and both valve voltages were varied independently over as much of their ranges as possible. At higher inflow and peripheral resistance settings the VAD will complain (alternately) "full-fill signal not changing" or "full-eject signal not changing" due to reduced inflow or outflow conditions (respectively). This set of experiments proceeded as follows:

1. Data collection was started in LabVIEW 
2. The valve settings were adjusted

3. Approximately $5 s$ after valve action ceased, the VAD was started in FFFE mode

4. Ten full beats were marked and the 5 min countdown timer was started

5. When the countdown timer expired, the VAD was halted

6. $30 \mathrm{~s}$ later, data collection was stopped

The inflow valve voltage was varied between 0 and $2 V$ (in integer increments) and the peripheral resistance valve voltage was varied between 0 and $8 \mathrm{~V}$ in $2 \mathrm{~V}$ increments. Figure 5.6 shows the ensemble averaged arterial pressure waveform (normalized by beat length) for each of these fifteen combination settings. The dashed lines in Figure 5.6 bound the $95 \%$ confidence interval for each of the ensemble averaged data sets. The narrow confidence intervals suggest a relatively steady-state beat rate and pressure waveform. The arterial pressure varies between a minimum of $85 \mathrm{~mm} \mathrm{Hg}$ and a maximum of $125 \mathrm{~mm} \mathrm{Hg}$ over the course of a beat, depending on valve settings. We can see from the various arterial pressures that the peripheral resistance valve has minimal effect at its lower settings; the mean arterial pressure does not change significantly until the peripheral resistance valve voltage is $8 \mathrm{~V}$, the "preferred" valve setting based on both physiological data and the fixed-rate tests outlined in the previous section.

Table 5.1 summarizes the VAD beat rates for the various FFFE tests; the $95 \%$ confidence interval is presented, along with the average beat rate. For each combination of peripheral and inflow valve settings, the beat rate is nearly constant, hovering around $90 \mathrm{bpm}$ for the two lower inflow valve voltages and around $70 \mathrm{bpm}$ for the higher inflow valve voltage. This reduced beat rate (for the higher inflow valve voltage) reflects the lower preload pressure and slower filling due to the higher occlusion of the inlet flow path. It also explains the lower mean arterial pressures seen in the rightmost column of Figure 5.6; a lower beat rate (with the same, constant, nominal stroke volume that FFFE mode produces) results in a reduced peripheral flow rate and a correspondingly lower peripheral pressure drop. 


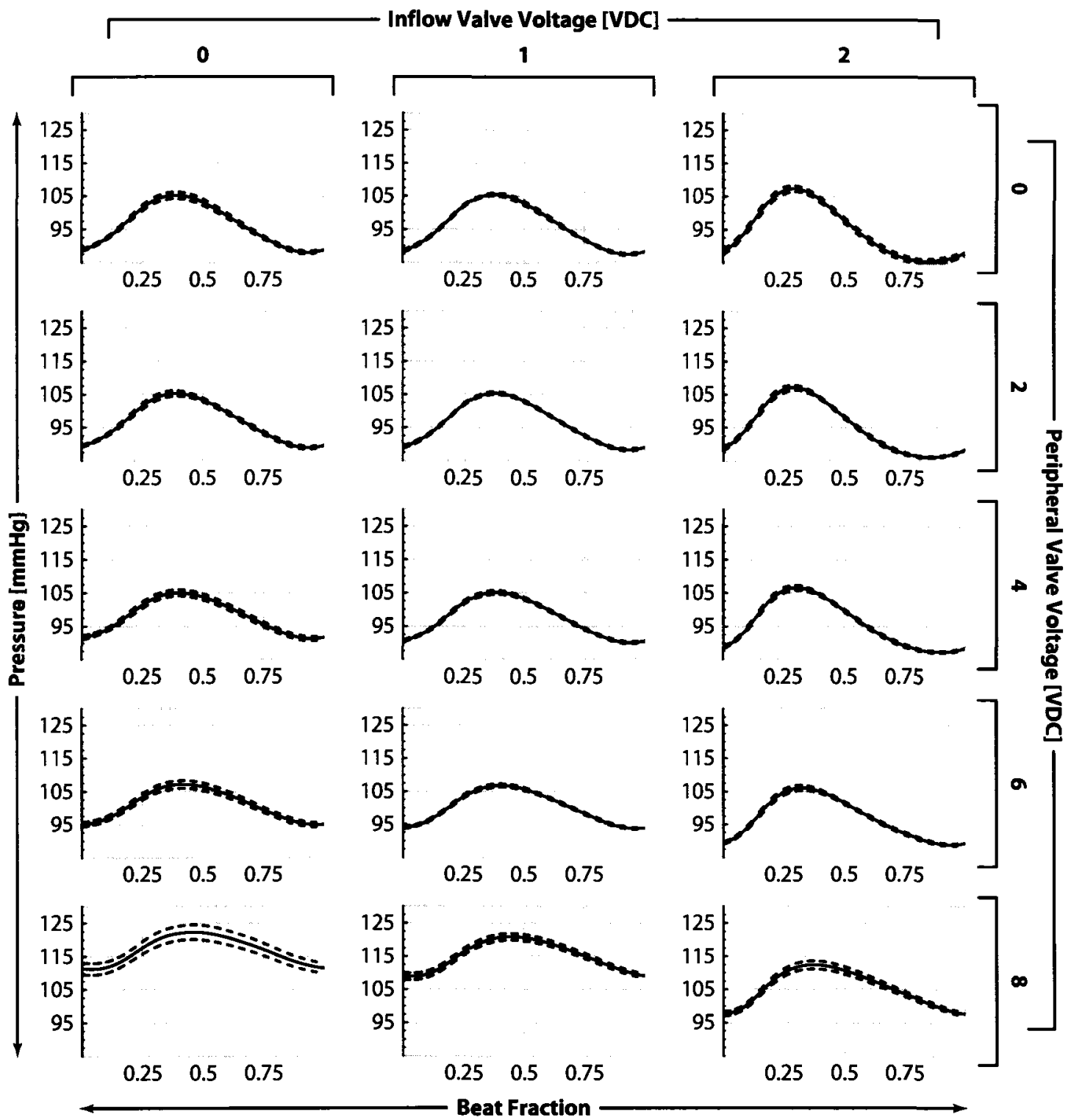

Figure 5.6: Arterial pressure waveform during FFFE-mode VAD operation 


\begin{tabular}{|c|c|c|c|c|}
\hline & \multicolumn{3}{|c|}{ Inflow Valve Voltage } \\
\hline & & 0 & 1 & 2 \\
\hline \multirow{10}{*}{ 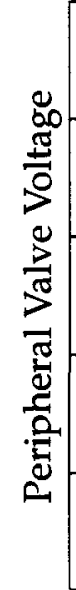 } & & {$[90.5618,91.3241]$} & {$[87.4953,88.1552]$} & {$[70.373,71.0881]$} \\
\hline & 0 & $\bar{\tau}=90.9414$ & $\bar{\tau}=87.824$ & $\bar{\tau}=70.7288$ \\
\hline & & {$[91.04,91.6478]$} & {$[87.882,88.0971]$} & {$[69.6737,70.2371]$} \\
\hline & 2 & $\bar{\tau}=91.3429$ & $\bar{\tau}=87.9894$ & $\bar{\tau}=69.9543$ \\
\hline & & {$[93.464,94.4448]$} & {$[88.1552,88.535]$} & {$[70.1651,70.6697]$} \\
\hline & 4 & $\bar{\tau}=93.9518$ & $\bar{\tau}=88.3447$ & $\bar{\tau}=70.4165$ \\
\hline & & {$[93.2027,93.9359]$} & {$[88.6754,88.9895]$} & {$[69.6492,70.2044]$} \\
\hline & 6 & $\bar{\tau}=93.5678$ & $\bar{\tau}=88.8322$ & $\bar{\tau}=69.9257$ \\
\hline & & {$[92.3934,93.0998]$} & {$[88.1393,88.3034]$} & {$[70.9338,71.7324]$} \\
\hline & 8 & $\bar{\tau}=92.7452$ & $\bar{\tau}=88.2213$ & $\bar{\tau}=71.3309$ \\
\hline
\end{tabular}

Table 5.1: FFFE beat rates for various peripheral and inflow valve settings

\subsection{Addition of the Aortic Chamber}

Recall (from Section 4.2) that the dual-compliance arterial model, with its separate aortic and arterial compliance elements, produced a more representative arterial pressure waveform. Even with purely linear constitutive relations the aortic pressure displays an artifact similar to the dicrotic notch, and the arterial pressure waveform develops a characteristic "knee" in the post-systolic phase of the curve.

The preliminary modification to the UOHI mock circulatory system was the inclusion of a separate aortic compliance chamber.* The existing air-water arterial chamber was retained, although calculations (presented in Appendix $C$ ) indicated that its nominal total compliance was too low to adequately model the arterial system. The stiffness could be increased by pressurizing the trapped air, but the placement of the inlet fitting ( 3 in from the top of the chamber) limits the amount of air which can be pre-charged into the cavity.

Although the stiffness of the UOHI arterial chamber is inadequate, it can give us interesting insight into the development of the arterial pressure waveform, and the dicrotic

${ }^{*}$ All of the additional compliance chambers are designed using rolling diaphragm seals, as detailed in Appendix E. The aortic chamber differs from the other chambers in that it has a much smaller total volume, and a correspondingly smaller cross-sectional area. 
notch in particular. Mathematical simulations of the arterial DCM show that a dicrotic artifact persists in the aortic pressure waveform, no matter the values of aortic and arterial compliance. If the arterial stiffness is low, the aortic pressure waveform mimics the flow waveform, as most of the pumped blood makes its way into the arterial chamber directly. The close of the outlet valve at the end of systole triggers a slight reversal in flow until the elastic recoil of the aorta moves the blood onwards into the arterial system. With increased arterial stiffness, as shown in Figure 5.7, the post-dicrotic leg of the aortic pressure waveform elevates; more blood stays in the aorta over the systolic fraction of the beat, and the aortic recoil becomes increasingly important as the main motivating force behind blood circulation.

A DCM with an arterial stiffness similar to that of the $\mathrm{UOHI}$ arterial (air-water) chamber will feature an aortic pressure waveform similar to the topmost plot in Figure 5.7. Between the UOHI arterial chamber, and the various springs available for the new arterial chamber (described in Appendix E) we are able to examine four different arterial compliance values, beginning with an arterial compliance of approximately $3000 \mathrm{~cm}^{5} \cdot \mathrm{N}^{-1}$.

The MCS was reconfigured as illustrated in Figure 5.8, with the aortic chamber inline between the VAD and the larger arterial chamber. However, before any data could be collected the VAD failed, prompting a switch to the MTS material test machine.

\subsection{Chaotic Oscillations and the Loss of the VAD}

For what proved to be the penultimate VAD run, the device was placed in FFFE mode and the peripheral resistance was set to a reasonably high value, with the peripheral valve voltage at $9 \mathrm{~V}$. This serves to increase the arterial pressures, however care was taken to ensure all pressures remained well within the nominal physiological range. The maximum aortic pressure during the run was $140 \mathrm{mmHg}$, which would qualify as mild hypertension.* With the VAD beating (in FFFE mode) at approximately $95 \mathrm{bpm}$, the arterial system began to display apparently random, aperiodic pressure oscillations. This is consistent with the prediction of chaotic behaviour by the numerical (impact oscillator) simulations. During a subsequent run, with the VAD set to $95 \mathrm{bpm}$ in fixed-rate mode, these oscillations

${ }^{*}$ Mild hypertension is normally defined as a systolic blood pressure of $[140 ., 159.] \mathrm{mmHg}$ and a diastolic blood pressure of $[90 ., 99.] \mathrm{mmHg}$. 


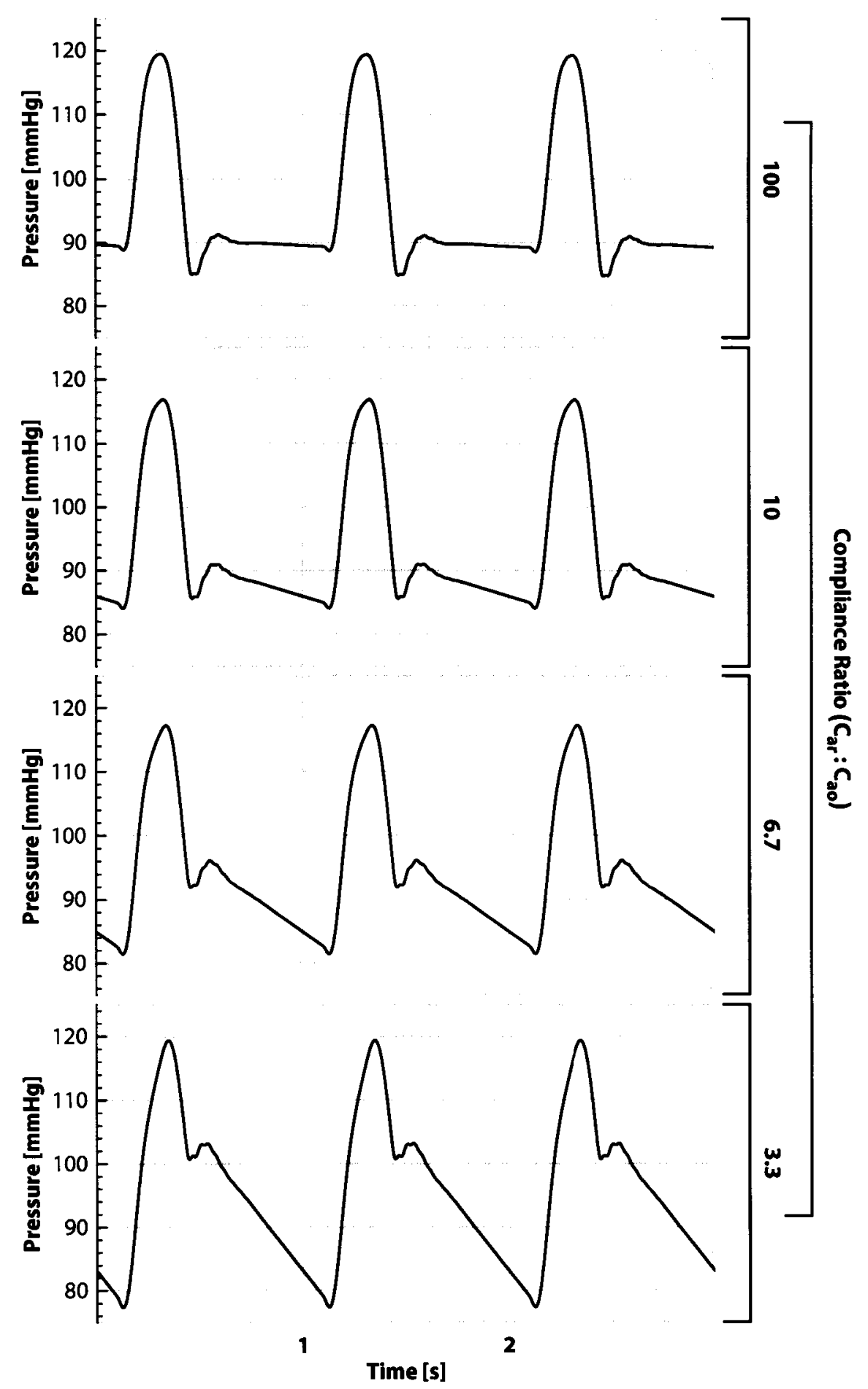

Figure 5.7: Theoretically predicted development of the dicrotic notch (in the aortic pressure waveform) with decreasing arterial compliance 


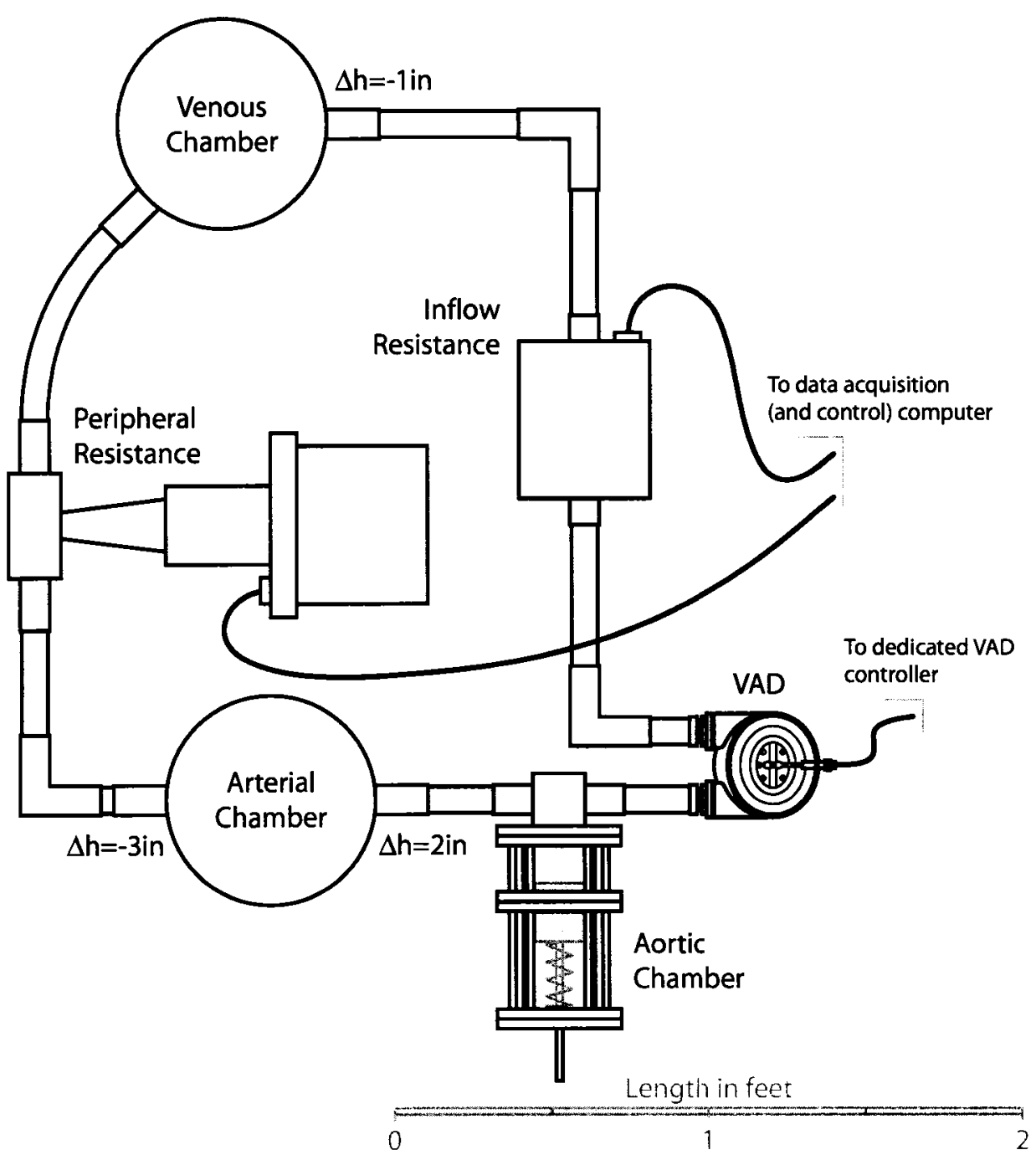

Figure 5.8: Schematic diagram of the UOHI mock circulatory system with the aortic chamber added 
were once again present. The data acquisition system was not active during either of these qualifying tests; the first was part of a group of runs performed to get a general feel for how the aortic chamber behaved in the MCS loop and the second was a demonstration for a colleague.

Unfortunately, a few hours after the final fixed-rate run (while the VAD was resting in "halt" mode) the main computer began registering errors (codes \#72 and \#73) as the external controller apparently lost communication with the device. The VAD was returned to the Ottawa Heart Institute for service, but no working replacement could be assembled from the various spare parts and devices at the UOHI.

During the initial experimental design stage this sort of eventuality was planned against and a contingency plan, albeit incomplete, was devised. Two identical arterial pressure chambers were built, with the idea that one of them could be used as a pump, or pseudoVAD (also referred to as "the pVAD"), in the event of VAD failure. None of the pumping details were finalized at that time, and the sudden VAD expiration resulted in a minor scramble. It was decided that the MAE Departmental MTS material test machine would be the new prime mover, and fittings were made to attach the piston rod and the base block of the chamber to the clamps on the MTS machine.

\subsection{The Switch to the pVAD (and the MTS Machine)}

The standard chamber base block was modified to accommodate the passive valves, and a steel clamping plate was added to the bottom. This plate allows the base to be held in the jaws of the material test machine. The setup is shown in Figure 5.9. Figure 5.10 shows the final configuration of the MCS attached to the pVAD (which is mounted in the jaws of the MTS machine). Note that the initial pVAD experiments were performed using the original MCS configuration (with the air-water arterial chamber); this particular photo was taken during the last set of experiments, when both the aortic and arterial chambers were inline.

The switch to the MTS has both advantages and disadvantages. On the positive side, the pumped volume is guaranteed repeatable and constant, as the machine's control system carefully monitors the piston position. There is also (virtually) no limit on ejection pressure as the machine can provide up to $100 \mathrm{kN}$ force. This combination gives good confidence 
that the commanded stroke is indeed the one produced and, due to the action of the passive valves, a sinusoidal piston motion produces a reasonable approximation to the normal VAD pumping. This operation mode is pre-programmed into the MTS controller; it is very easy to set the frequency and amplitude of oscillation and get an extremely accurate sinusoidal motion.

On the downside, the MCS is now much farther away from the pseudo-VAD, which changes $\Pi_{1}$ to a much less interesting value and creates a noticeable lag between valve closure and arterial pressure peak. The pump is also elevated with respect to the MCS, meaning that the pVAD must draw in water during diastole (due to reduced fill pressure). Rolling diaphragms are designed to run with positive internal pressure, and the suction requirement dramatically increases the wear on the pVAD chamber diaphragm. The inflow valve was left fully open to moderate this condition

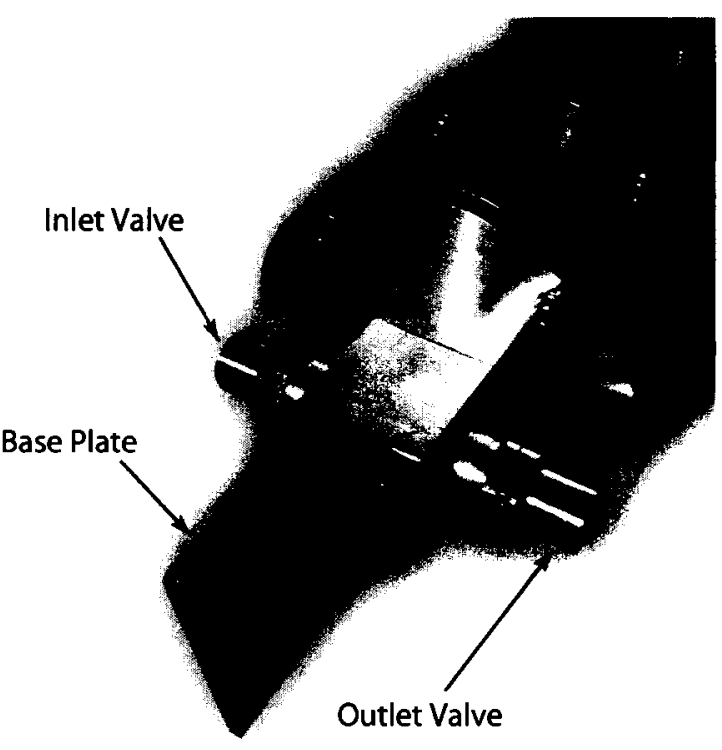
somewhat; direct connection between the venous system would be possible, but this type of connection would remove much of the isolation that exists between the inlet Figure 5.9: The base of the pVAD chamber (with clamping plate attached) side of the PVAD and the MCS itself.

The MTS system is much noisier than the VAD, and the valve microphone no longer works reliably; the microphone picks up too much of the broadband hydraulic noise, which is conveyed through connected tubing and fittings, and obscures the valve noise. Although this hydraulic noise has several dominant frequency components, the bulk of the signal energy is essentially white noise and bandpass filtering the valve recording did nothing to improve feature recognition. Finally, the load cell in the MTS machine is designed for much higher loads, and has a limited low-load resolution; force-feedback (and hence, FFFE-like tests) are not possible. 


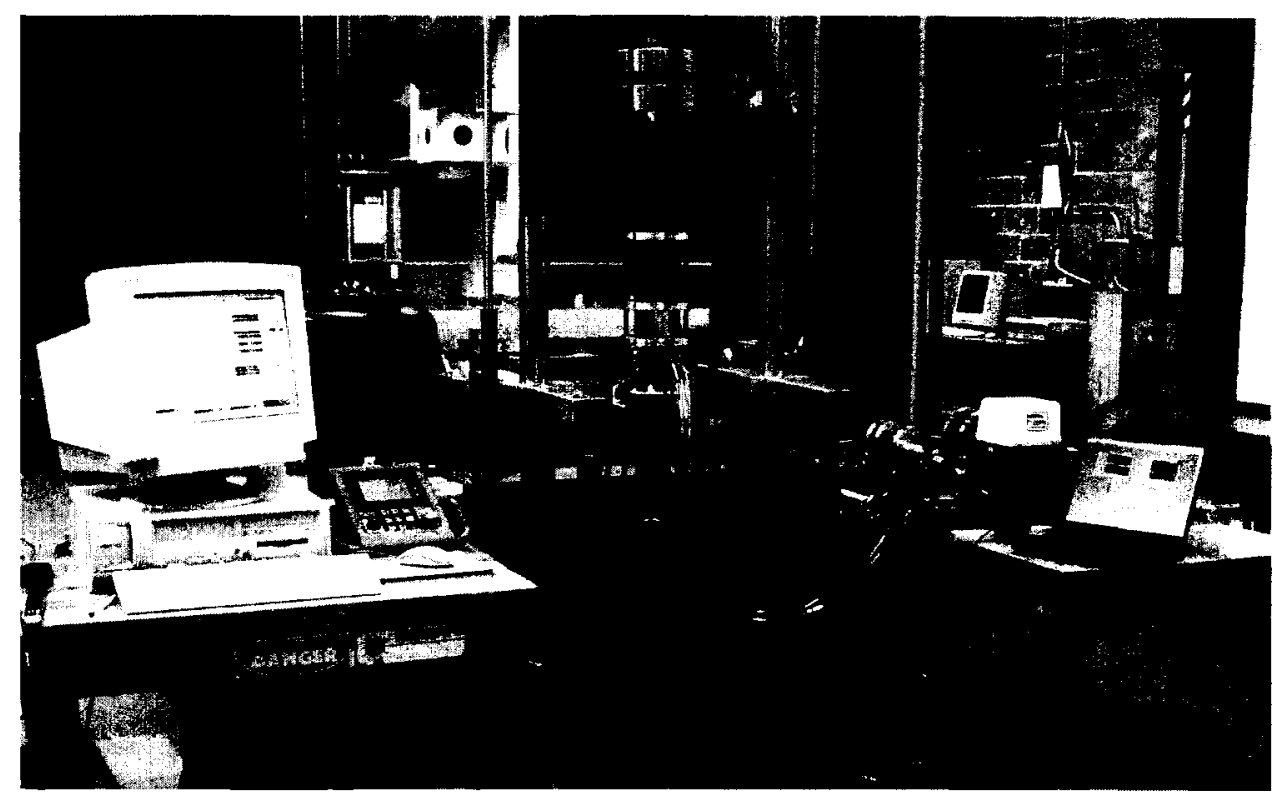

Figure 5.10: The final configuration of the MCS (with the aortic chamber and the new arterial chamber inline), connected to the PVAD and the MTS machine

In spite of these problems, the MTS setup provides a reasonable approximation to VAD operation and can be used to study MCS behaviour. The MTS action can be thought of as a sort of hybrid mode: the ejected volume is always constant, as with the FFFE mode of VAD operation, but the cycle time is also constant, as with the fixed-rate mode of VAD operation.* The pVAD test procedure was as follows:

1. The MTS cycle parameters (frequency, amplitude, number of cycles) were set in the MTS controller

2. Data collection was started in LabVIEW

3. After approximately $5 s$ the MTS was started

4. Approximately $30 s$ after the MTS cycling stopped, data collection was halted

*In fixed-rate mode the VAD stroke volume can change based on fill and ejection rates; in FFFE mode the VAD cycle time can change in response to these external factors. 


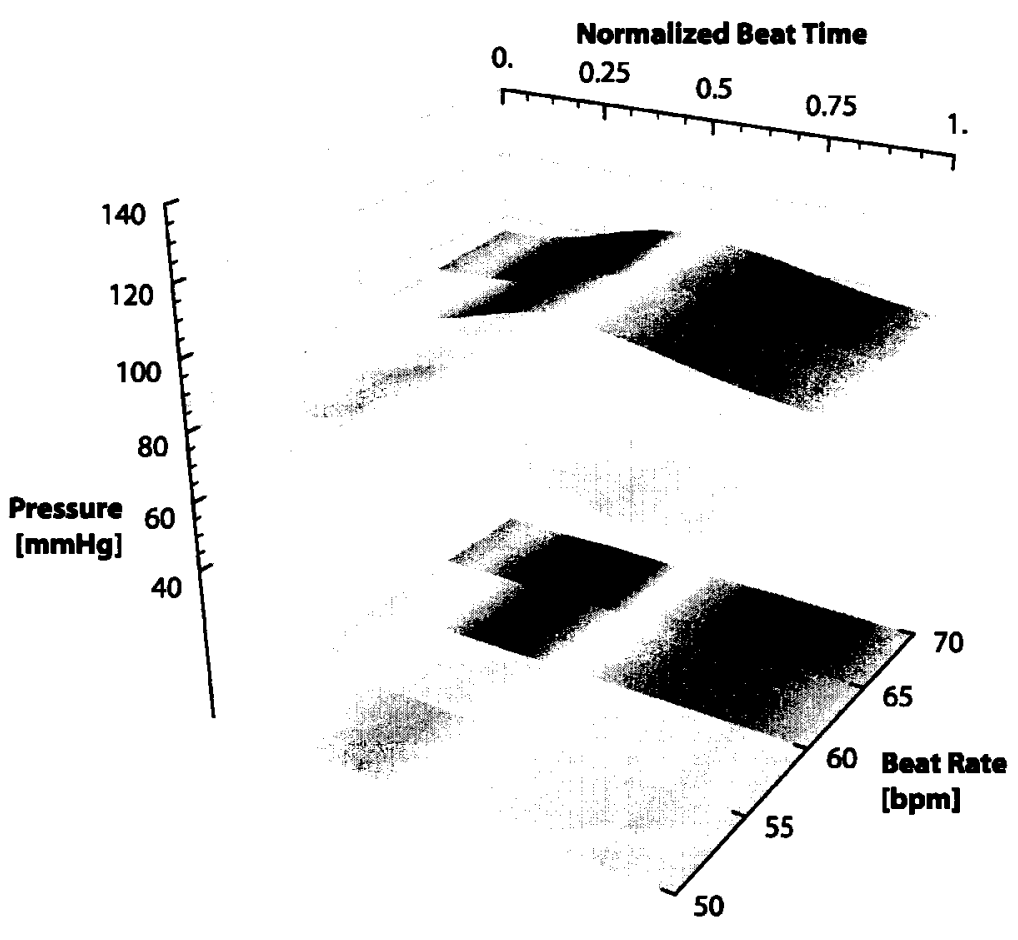

Figure 5.11: Subset of the first MTS run (maximum standard deviation is $1.27 \mathrm{~mm} \mathrm{Hg}$; minimum standard deviation is $0.25 \mathrm{mmHg}$ )

\subsubsection{Validation of the PVAD}

To qualify the pVAD, the MTS machine was used to duplicate the fixed-rate VAD tests. The mock circulatory system was assembled in the UOHI "Original Configuration" with a single (air-water) arterial chamber. The MTS machine was run at integer beat rates between 50 and $100 \mathrm{bpm}$, in $5 \mathrm{bpm}$ increments. Figure 5.11 shows a subset of the preliminary MTS tests, which compare well with the VAD results (Figure 5.3). We see that the MTS, which is position-controlled, maintains a constant stroke volume over the range of beat rates and therefore produces a higher peripheral flow rate (and correspondingly higher peripheral pressure drop) for higher beat rates. This serves to increase the mean arterial system pressure at higher beat rates. The $\mathrm{pVAD}$ produces a more repeatable arterial pressure waveform (as evidenced by the lower standard deviation in the results), and the arterial sloshing is less dominant, likely due to the longer tube connecting the PVAD to the MCS. Since the valve recording is no longer reliable and the beat demarcation must be 
done manually, the pressure peak in Figure 5.11 is centred on the beat interval. The diastolic and systolic beat fractions are the same, meaning that the pressure wave is symmetric rather than skewed towards the beginning of the diastolic portion of the beat interval (as was the case in Figure 5.3).

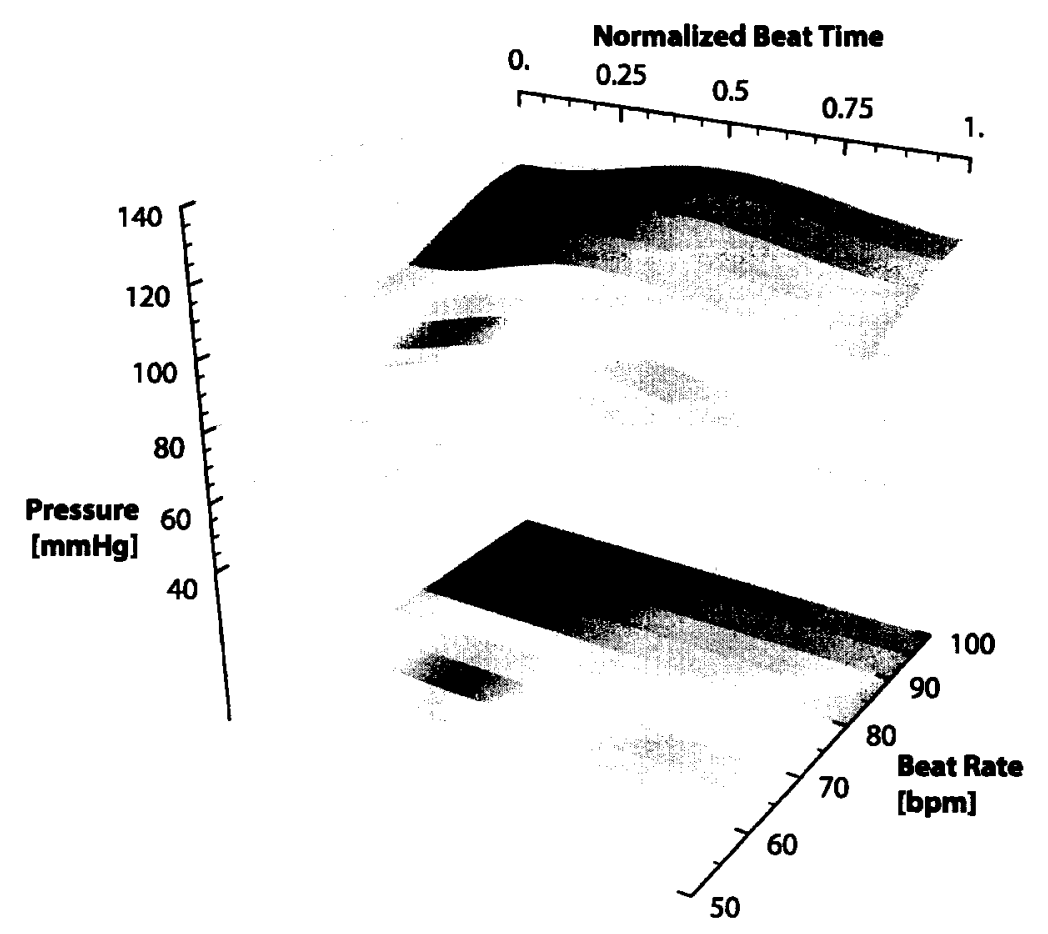

Figure 5.12: The first MTS run (maximum standard deviation is $2.48 \mathrm{~mm} \mathrm{Hg}$; minimum standard deviation is $0.25 \mathrm{mmHg}$ )

Figure 5.12 shows arterial pressure over the complete range of beat rates, between 50 and $100 \mathrm{bpm}$ in $5 \mathrm{bpm}$ increments. As mentioned above, the constant stroke volume produces a higher peripheral flow rate at faster beat frequencies. This increase is particularly noticeable at beat frequencies above $70 \mathrm{bpm}$. A mathematical simulation of the pVAD setup was also run; Figure 5.13 shows the results of the simulation, which are in good agreement with Figure 5.12. In this initial configuration, the simulated arterial pressure is repeatable over the beat interval, and the gray levels in Figure 5.13 correspond to absolute pressure, rather than standard deviations. The simulation under-predicts the arterial pressures at 


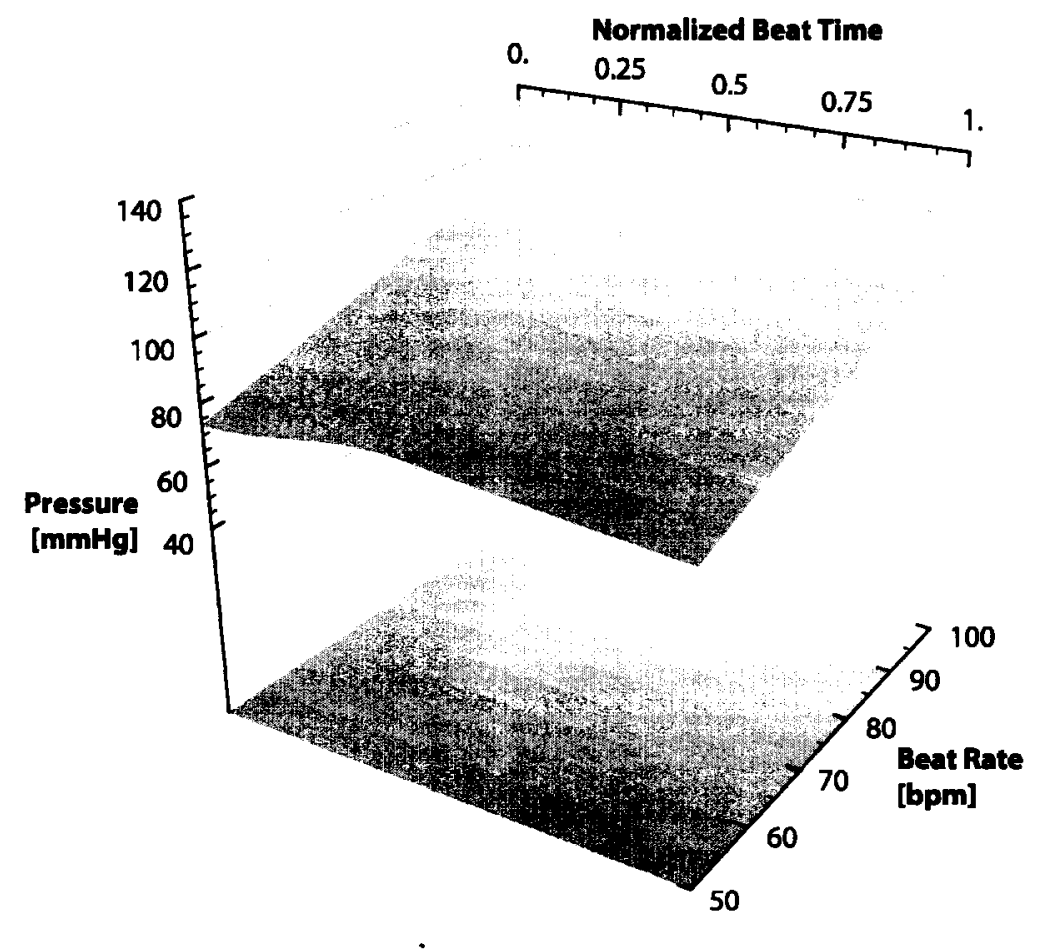

Figure 5.13: Simulation of the pVAD setup; unlike the experimental plots gray levels in this figure correspond to absolute pressure, rather than standard deviations 
low beat rates, and over-predicts the pressures (very slightly) at high beat rates, but this situation is more desirable than the converse.

We notice in both the experimental and the simulation results that the high compliance of the arterial chamber decreases the magnitude of the pressure wave; the pressure peak is only slightly higher than the base arterial pressure, a condition not normally seen in the human circulatory system. In a healthy circulatory system one might expect a pressure peak (during systole) of $120 \mathrm{mmHg}$ and a diastolic pressure of $80 \mathrm{mmHg}$. Rather than this $40 \mathrm{~mm} \mathrm{Hg}$ difference between systolic and diastolic pressures we see a maximum of $10 \mathrm{~mm} \mathrm{Hg}$ in the experimental data and $20 \mathrm{mmHg}$ in the numerical simulation results.

\subsection{Replacement of the Air-Water Arterial Chamber}

The final experimental phase, which will allow us to study the dicrotic notch development, involves replacing the air-water arterial chamber with a rolling diaphragm chamber. The new arterial chamber is similar to the aortic chamber in terms of design, but is larger overall.* The resultant MCS configuration is illustrated in Figure 5.14. While Figure 5.14 includes the VAD, all remaining experiments were carried out on the MTS material test machine using the pVAD as a pump.

The removal of the air-water arterial chamber impacts our ability to record pressures in the arterial system; recall that the arterial pressures were measured using a differential pressure transducer which had its positive pressure side attached to the air-water arterial chamber and its negative pressure side attached to the venous reservoir. In the remaining experiments arterial pressure must be inferred from piston position (and hence spring compression) in the two rolling diaphragm chambers. The aortic and arterial chamber were (alternately) instrumented with a Celesco cable-extension transducer. The calibration curve for the transducer is presented in Appendix G.

Due to the aortic chamber sizing, the aortic piston has a very small amplitude of motion under normal operating conditions. This leads to discretization problems with the DAQ card; the changes in piston position at the upper and lower limits of motion are close to the

\footnotetext{
*As mentioned previously, all of the additional compliance chambers are detailed in Appendix E.
} 


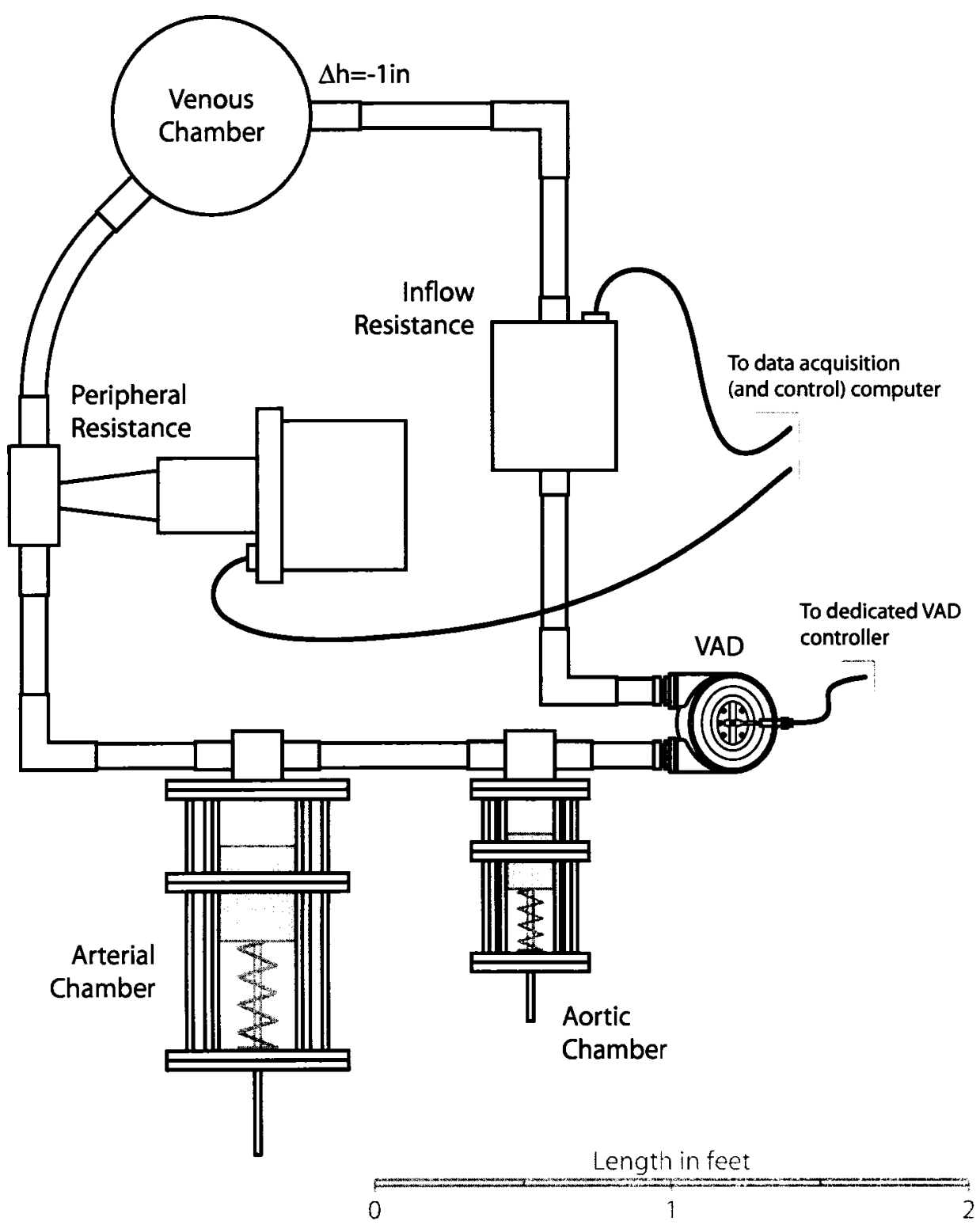

Figure 5.14: Schematic diagram of the new mock circulatory system 
precision of our 12-bit card. ${ }^{*}$ To work around any discretization artifacts the sampled aortic data was smoothed by taking a small-window moving average (of ten samples, which would span $0.01 \mathrm{~s}$ of time) before low-pass filtering to remove any instrumentation noise.

Figure 5.15 plots the normalized aortic pressure wave for three different arterial spring stiffnesses. Although the raw experimental data shows discretization artifacts, all of the expected waveform features are present; smoothing the raw data (and ensemble averaging the multiple beats) produces a very recognizable pressure trace which closely matches the numerical simulation results. The dicrotic notch is visible in all three plots of Figure 5.15 and its development (along with the corresponding changes in the post-systolic portion of the aortic pressure trace) matches what was predicted by numerical simulation. ${ }^{+}$The nominal aortic compliance is $30 \mathrm{~cm}^{5} \cdot N^{-1}$ and the nominal arterial compliance is $100 \mathrm{~cm}^{5} \cdot \mathrm{N}^{-1}$; Figure 5.15 brackets this nominal parameter ratio with two other instances. In all three cases, the main difference between the predicted and experimental results is the flattened top of the experimental waveform. The numerical results have a sharper peak, implying that something in the experimental setup is sapping energy from the system. Based on the suspicion that this energy sink may be due to frictional losses in the diaphragms, a viscous rolling term was added to the $\mathrm{UOHI}$ simulation.

While the rolling diaphragm manufacturers will describe the motion as frictionless, common sense dictates that there must be some losses in the diaphragm motion, whether due to the diaphragm itself or the linear support bearing which ensures that the piston runs true in the cylinder. Over the course of the experiments the aortic chamber diaphragm began wrinkling and binding due to excessive wear, and was replaced. Figure 5.16 compares the old (worn) diaphragm with the new one; the wear marks on the sidewall suggest losses in the rolling and unrolling of the elastomer diaphragm. (None of the cylinder components rub on each other, and the wear is not due to abrasion.)

Numerical simulation of the pVAD setup (with the two rolling diaphragm cylinders comprising the arterial side of the loop) shows that the pressure trace has a low amplitude, higher frequency oscillatory component. Analysis of the eigenvalues of the system

${ }^{*} \mathrm{~A} 12$-bit data acquisition card has (typically) 11 usable bits, and the accuracy is $\pm 0.5 \mathrm{LSB}$. For our $5 \mathrm{~V}$ this implies a $1.22 \mathrm{mV}$ resolution, which corresponds to roughly $0.3 \mathrm{~mm}$ of motion. Total arterial piston motion is only $4 \mathrm{~mm}$ peak-to-peak.

${ }^{\dagger}$ See Figure 5.7 on Page 130. 


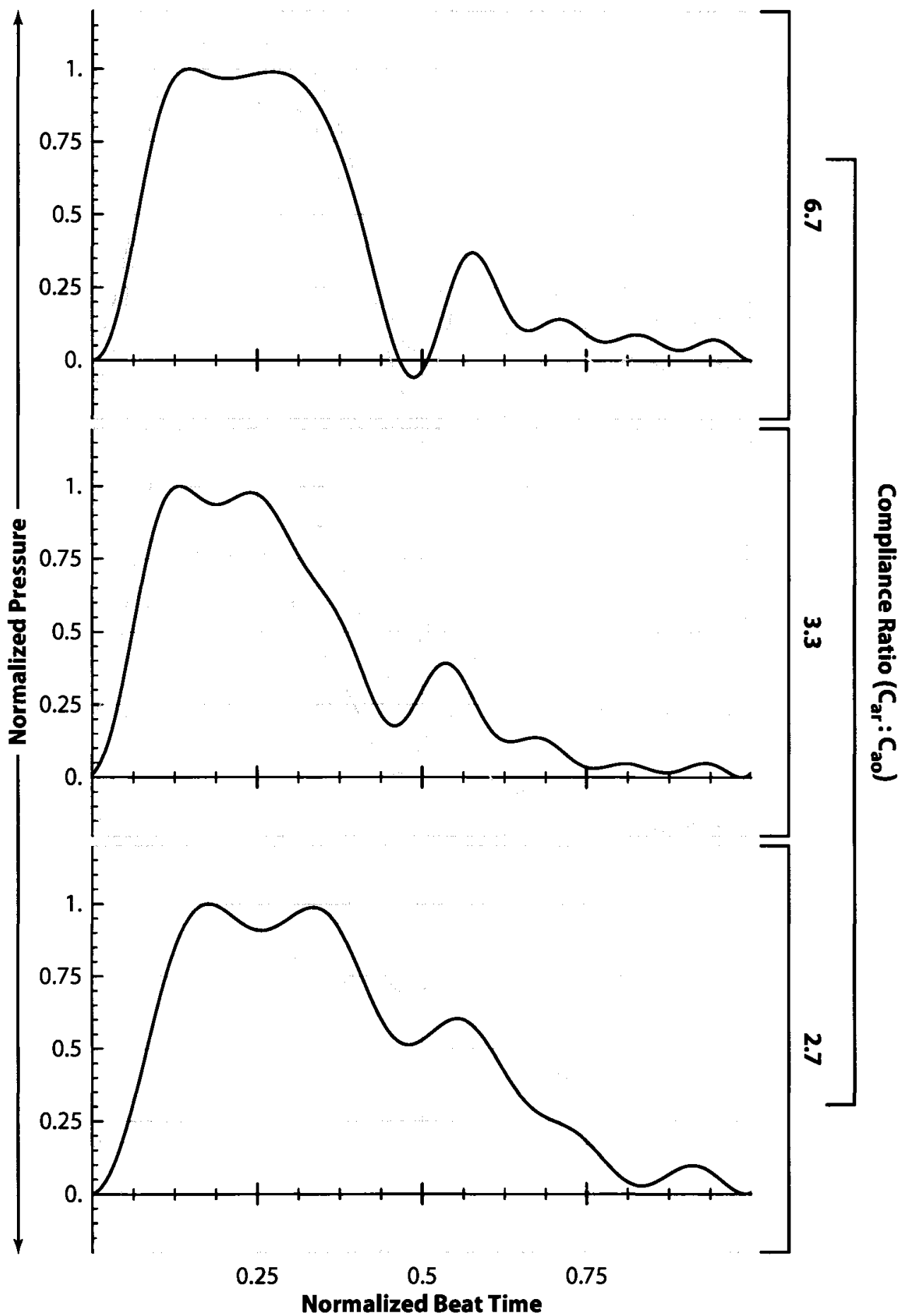

Figure 5.15: Development of the dicrotic notch (from the experimental data) 


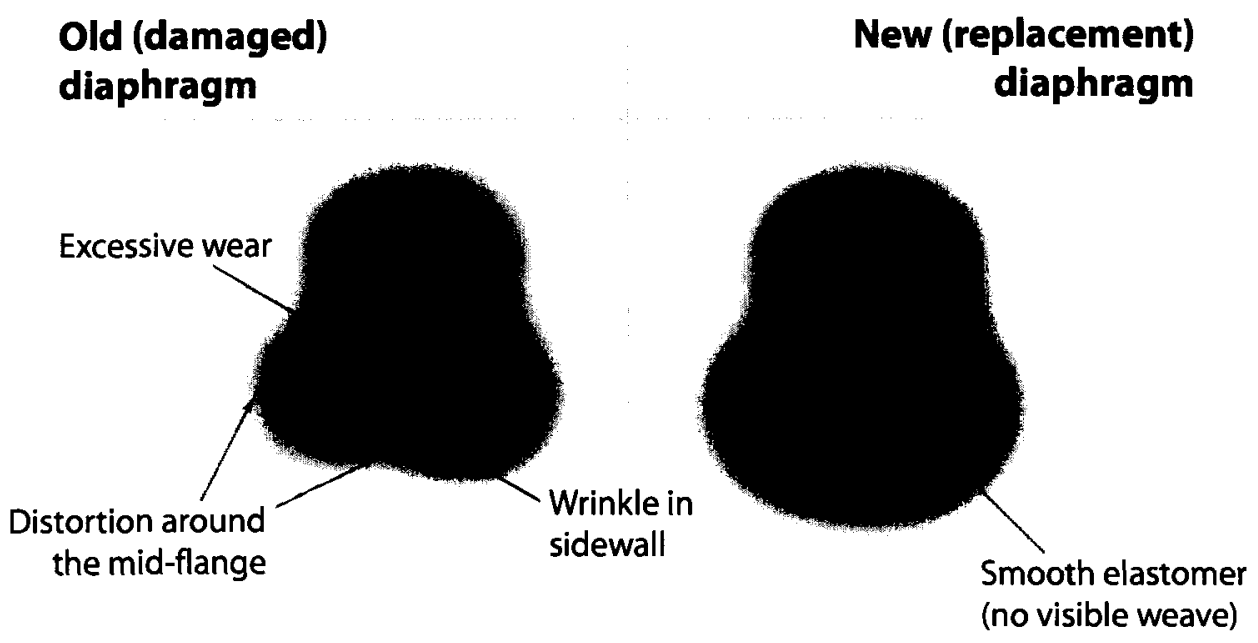

Figure 5.16: Comparison of worn and new diaphragms

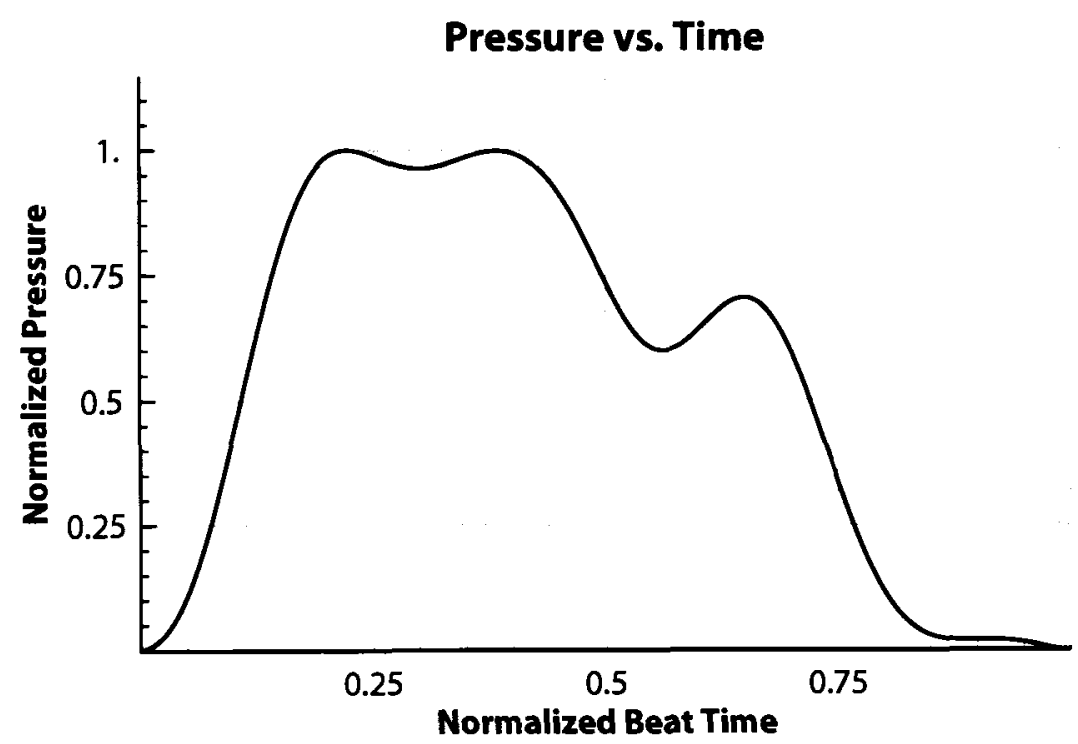

Figure 5.17: Simulated aortic pressure wave 
show that this oscillation is mainly attributable to the outlet tube; only two of the eigenvalues have imaginary parts, and although each eigenvalue is a function of multiple system parameters, the magnitude of the imaginary component is most affected by changes in the outlet tube dimensions. The oscillation is damped, with the damping affected by the outlet tube parameters and the coefficient of friction in the first (aortic) chamber. Figure 5.17 shows the simulated aortic pressure wave. The flattened top of the pressure trace would be sharper if the outlet tube were shorter (and had a higher natural frequency). The simulation predicts a maximum aortic pressure of $103 \mathrm{~mm} \mathrm{Hg}$ and minimum aortic pressure of $82 \mathrm{mmHg}$ at $60 \mathrm{bpm}$. This means that the (predicted) peak-to-peak motion of the aortic piston is on the order of $0.36 \mathrm{~cm}$, however in the experiment we see a lower amplitude of motion, only $0.1 \mathrm{~cm}$ peak-to-peak. This discrepancy may be due to the convolved portion of the rolling diaphragm seal; the experimental piston motion is calculated based on the "effective area" dimension provided by the manufacturer. 


\section{Chapter 6}

\section{Results and Discussion}

$\mathbf{W}$

HILE the preceding two chapters segregated parts of this work, classifying them as either "theoretical" or "experimental", it is important to look at the integrated findings and their impact on our understanding of human circulatory system (or mock circulatory system) behaviour.

\subsection{Modelling the Human Circulatory System}

Although the circulatory system is a continuous network of vessels which branch and taper, it can be successfully treated by lumped parameter models. These mathematical or experimental studies can highlight dynamic effects such as the interaction between the aortic valve closure and the dicrotic notch in the arterial pressure wave. The thirteenth order mathematical model presented in this work is a reasonable analysis of the human circulatory system; with minor changes it can also model existing mock circulatory systems.

Over a physiologically representative range of values the arterial system's characteristic equation has complex conjugate roots. The oscillatory frequencies predicted by the secondand third-order arterial models differ slightly, however no reasonable parameter values will make the eigenvalues real.

The mathematical study of the proximal arteries highlighted deficiencies in existing mock circulatory systems. Without the stiffer aortic compliance element a lumped parameter arterial model will produce a sawtooth pressure waveform. The opening and closing of the aortic valve do not have pronounced effects on the arterial pressure, and there is no dicrotic artifact in the resultant pressure wave. With the addition of this second (stiffer) 
compliance element the arterial pressure trace matches the human physiological waveform more closely, even if all of the constitutive relations are linear. In other words, nonlinearities in the arterial system are not necessarily responsible for the shape of the arterial pressure wave, nor is vessel branching a requirement. Even if pressure wave reflections (due to vessel bifurcations) are ultimately responsible for the dicrotic notch artifact, the idea that a dual-compliance arterial model can produce a dicrotic notch-like effect is an important realization, with implications for mock circulatory system design.

\subsection{Analysis of the Arterial Pressure Wave}

Both the theoretical and experimental results suggest that current mock circulatory systems are a poor representation of the human arterial tree. The pressure waveform produced in the single-compliance arterial model matches the pressure trace in the first (baseline) set of mock circulatory system experiments. This correspondence is most easily seen by comparing Figure 5.12 and Figure 5.13 (on Pages 136 and 137, respectively). Unfortunately, neither of these match the arterial pressure waveform found in the human circulatory system. This means that mock circulatory systems which feature a single arterial compliance chamber provide unrealistic test conditions for VAD prototyping; while the impedance load is reasonable, the dynamic effects are absent.

Most existing mock circulatory systems are modular, and adding additional compliance elements is relatively easy. In this work a second arterial compliance chamber was added to an existing mock circulatory system, improving its physiological representation. This modification, which was suggested and supported by the theoretical results, produces a much more realistic arterial pressure waveform. Although some of the literature suggests that the dicrotic notch is due to pressure wave reflection (because of branching in the arterial tree), this dynamic effect can be reproduced using two compliance elements in series. The relative compliance of the two serial chambers is an important parameter; the theoretical and experimental results suggest that a suitable ratio between arterial and aortic compliance $\left(C_{a r}: C_{a 0}\right)$ is approximately $3: 1$, as shown in Figure 5.15 (on Page 141).

The post-systolic portion of the arterial pressure trace is affected by mean arterial pressure, as well as the compliance ratio between the two arterial chambers. In the healthy human circulatory system peripheral flow resistance and cardiac rate change in response 
to the same stimuli, a feature which was necessarily absent in the experimental portion of this work. If we had a better understanding of this interaction, the peripheral valve in the UOHI MCS could be used to make changes to $R_{p e}$ over the course of an experimental run. (Or it could be set to an appropriate static value for an experimental run.) This would eliminate the unrealistically high pressures which were seen at higher beat rates in Figure 5.12 (on Page 136).

\subsection{The Influence of Nonlinear Behaviour}

One of the significant theoretical contributions of the current work is the demonstrated arterial mass-spring-damper equivalence. The impact-oscillator class of behaviour has implications for both artificial heart design as well as our understanding of human physiology.

The proximal arteries are dynamically equivalent to a mass-spring-damper system. The heart excites the system by injecting a volume of blood into the aorta, the equivalent of moving the ceiling in the mass-spring-damper system; this injection of blood expands the aorta, whose elastic response is nonlinear. Although there is some flow-through of blood, the systolic portion of a beat is violent enough that much of the injected blood stays in the aorta and, at the end of systole, after the aortic valve closes, the elastic recoil of the aorta moves blood into the rest of the arterial tree. The resultant pulsatile flow is recognizable even in the capillary beds.

The arterial back-pressure is responsible for maintaining an appropriate aortic blood volume at the end of diastole. This back-pressure, along with any change in ejected blood volume, determines whether or not the aortic nonlinearity affects the systolic portion of the beat. The control mechanism for this process would rely on baroreceptor feedback. It may ultimately be found that the Traube-Hering-Mayer wave (an approximately $0.1 \mathrm{~Hz}$ oscillation in the body) is responsible for this overall pressure regulation.

Nondimensionalization of the equivalent mass-spring-damper system allows us to see duplicated effort in the literature. For example, changing either the inertia or the stiffness term can have a similar effect on the system; in the nondimensionalized mass-springdamper equation both of these parameters present themselves in the same nondimensional 
group, $\Pi_{1 \mid b}{ }^{*}$ Changes to the nonlinearity and the forcing function have a similar (reciprocal) relationship. Solid-wall impacts of a mass-spring-damper system have already been shown to be chaotic, as have systems featuring nonlinear stiffness elements. The aortic nonlinearity can be treated in a piecewise fashion; rather than a continuous stiffening behaviour we can define some threshold at which $\Pi_{1}$ changes, and call the magnitude of this change $\Pi_{1 \mid m}$. These so-called "soft wall impacts" form the basis of our arterial system analogy.

We see from mathematical simulations that relatively small values of $\Pi_{1 \mid m}$ can produce period-doubling bifurcations and chaotic oscillations. The volumetric rendering in Figure 4.18 (on Page 113) shows how the complexity of the system response varies over a range of $\Pi_{1 \mid b}$ and $\Pi_{1 \mid m}$ values. After a minimum threshold level (of approximately $\left.\Pi_{1 \mid m}>10\right)$ is exceeded, the nondimensionalized nonlinear oscillator begins to display chaotic oscillations, and for values of $\Pi_{1 \mid m}>50$ there is little difference between soft-wall and solid-wall impacts.

The nonlinear oscillator theory suggests that there is a particular beat rate which is optimal in terms of energetic efficiency (Walker and Soule, 1996). At this particular frequency, which is not the natural frequency of the arterial system, the heart's pumping has the maximum effect on blood motion. This is most easily seen in the scatter plot of Figure 4.10 (on Page 98), or the darker parabolic bands of the periodicity contour plots. The peaks in Figure 4.10 are much larger than the freely-oscillating amplitude of a similar (nonimpacting) mass-spring-damper system, with the largest peak being comparable to the resonant amplitude in a freely-oscillating system - the maximum steady-state amplitude possible (Walker and Soule, 1996).

In between these amplitude peaks the nonlinear oscillator displays chaotic behaviour. This suggests that there are certain beat rates which the natural heart must either avoid or deal with through other systemic changes. Obviously these particular beat rates would differ from person to person; an artificial heart, which must remain stable, should be designed to identify and deal with chaotic behaviour. The influence of these potential chaotic oscillations on VAD cycling is unknown and it may be that the addition of a simple "always beat

${ }^{*}$ The reader is referred to Section 4.4 , beginning on Page 99, for a description of the nondimensional groups. 
at least once per second" control law would be sufficient to ensure VAD stability. There is no obvious (continuous) way to move between parabolic bands in Figure 4.20 (which appears on Page 115) while avoiding chaotic regimes. It is also unclear if one would want to; the maximal oscillatory amplitude occurs at the first peak in Figure 4.10. Unless there is some advantage to running at a higher beat rate (for a given circulatory system state) the heart would confine itself to the wider parabolic band that is due to the first periodic peak.

An artificial heart, in turn, could improve its efficiency by beating at the optimum frequency for a particular arterial system. Given the non-stationary nature of human circulatory system parameters, this "optimum frequency" will change continuously as the body reacts to its environment. Taking advantage of this efficiency increase may prove impossible for an artificial heart, but the potential payoff is such that it warrants further investigation.

\subsubsection{Physiological Changes}

The nonlinear oscillator analogy also gives interesting insight into normal human circulatory system behaviour, from birth through adulthood. By examining the changes in $\Pi_{1 \mid b}$ and $\Pi_{1 \mid m}$ we can make hypotheses about the effects of growth, aging, and disease. As an example, atherosclerosis, or hardening of the arteries, would most likely decrease the value of $\Pi_{1 \mid b}$ and increase the value of $\Pi_{1 \mid m}$. It would also decrease the value of $\Pi_{2}$ for a given beat rate. This suggests that there will be a strong correlation between atherosclerosis and tachycardia. In addition, people with hardened arteries will be more prone to cardiac arrhythmia due to the increase in $\Pi_{1 \mid m}$, which results in an increased likelihood of nonlinear behaviour.

Size has a similar impact on the nonlinear oscillator parameters. A small child will have a lower value of $\Pi_{1 \mid b}$, a higher value of $\Pi_{1 \mid m}$ and a nominal value of $\Pi_{2}$ due to their naturally higher heart rates. A newborn's baroreceptor system is underdeveloped and perhaps less able to deal with unusual changes in the arterial system. The inability to respond to (and control) chaotic oscillations in the proximal arterial system might ultimately be responsible for "sudden infant death syndrome" (SIDS, or crib death). If this is indeed the case, some sort of risk assessment could be devised based on the nonlinear oscillator theory. Current theories about SIDS revolve around respiration, which is closely tied to the 
cardiovascular function; Eyal and Akselrod (2000) found entrainment between respiration and beat interval.

\subsection{Experimental Findings}

As with most existing mock circulatory systems, the University of Ottawa Heart Institute MCS features two compliance chambers. The arterial compliance chamber is closed to atmosphere while the venous chamber (which is much taller than the arterial chamber) is open to atmosphere. The compressed air in the arterial chamber gives it a nonlinear stiffness, but its cross-sectional area is large enough that this nonlinearity would not be felt over the course of a beat. Moreover, the placement of the inlet fitting, which is near the top of the chamber, limits the amount of air which can be precharged into the chamber. This affects the maximum chamber compliance; the compliance of the UOHI arterial chamber is approximately $1300 \mathrm{~cm}^{5} \cdot N^{-1}$ (based on calculations presented in Appendix C), which is much higher than the nominal arterial system compliance of $130 \mathrm{~cm}^{5} \cdot N^{-1}$ found in the literature. Changing the diameter of this chamber to 2 in and keeping the same vertical dimensions would improve its compliance value; the new chamber compliance would be approximately $100 \mathrm{~cm}^{5} \cdot N^{-1}$.

During the fixed-rate set of experiments, especially at lower arterial system pressures (due to lower peripheral resistance values), the arterial pressure waveform was affected by liquid sloshing in the arterial tank, as shown in Figure 5.5 (on Page 125). Changing the cross-sectional area of the chamber as outlined above would also change this sloshing frequency to a more desirable value. The surface wave period in a $2 \mathrm{in}$ diameter tank is $0.236 \mathrm{~s}$, compared to the current value of $0.472 \mathrm{~s}$.

In full-fill, full-eject mode the arterial pressure wave is very repeatable, in spite of the poor characteristics of the arterial reservoir. This suggests that the FFFE mode of control is a very robust method; even though the resultant beating is essentially fixed rate, ${ }^{*}$ FFFE control allows the VAD to optimize its beat rate for a given set of systemic conditions.

After the addition of the aortic chamber, apparently random pressure oscillations were observed in the arterial system during both FFFE and fixed-rate VAD operation. The MTS

\footnotetext{
*See Table 5.1 on Page 128 for FFFE beat rates.
} 
material test machine setup was unable to duplicate this aperiodic behaviour due to the less ideal placement of the mock circulatory system (relative to the pVAD pump). In the MTS experiments, the pVAD was elevated relative to the loop, and connected by much longer outlet and inlet tubes which had to flex as the MTS cycled the chamber up and down. The pVAD itself proved to be a good representation of the VAD, based on the results of the "qualifying" experiments presented in Section 5.4.1 (beginning on Page 135), however the symmetric pVAD cycling is not ideal; VAD cycling is asymmetric, similar to the natural heart, with the systolic portion of the beat being shorter than the diastolic portion. This longer diastolic phase allows the aorta to recoil and move blood into the rest of the circulatory system.

The MTS cycle can be modified to more closely match the VAD, but the input waveform will require some discernment. Both the systolic and diastolic phases of the beat must have consistent volume because if the systolic and diastolic volumes do not match, the pVAD chamber will eventually reach its limit of motion. The rolling diaphragm in the pVAD chamber already suffers considerable wear, and discontinuous velocity changes only exacerbate this problem. The pumping waveform must therefore be smooth and fluid. During the preliminary MTS experiments a sawtooth output waveform was tested, and the rolling diaphragm wrinkled severely. At that time the diaphragm was new and highly resilient when compared with its current state. The current PVAD diaphragm has now seen just under 20000 cycles.

In spite of the pumping symmetry, the PVAD produces an arterial pressure wave which closely approximates the VAD output pressure. A more sensitive load cell would allow us to mimic the FFFE mode of VAD operation, but the current setup is limited to fixedrate, constant volume. This differs from the fixed-rate VAD mode, which is a fixed-rate but variable volume cycle, and impacts the arterial pressures at high beat rates (which produce correspondingly higher peripheral pressure drops, as seen in Figure 5.12 on Page 136).

The peripheral flow resistance in the UOHI MCS is controlled by a throttle valve whose properties limit its useful range; the flow resistance is too low over much of the valve range and most of the experiments were performed with the valve nearly fully closed. (The calibration curve for the peripheral valve is presented in Appendix G. The nominal 
peripheral resistance in the human circulatory system is $0.013 \mathrm{~N} \cdot \mathrm{s} \cdot \mathrm{cm}^{-5}$ or roughly $85 \%$ of the peripheral valve's maximum setting.)

Other than the pVAD, the aortic chamber diaphragm suffers the most wear and tear during normal use. The smaller chamber size (with correspondingly tighter tolerances and thinner elastomer in the diaphragm) has a negative impact on wear resistance. The aortic diaphragm was replaced once over the course of this work and there are currently three spares. The net aortic piston motion is of very small magnitude which does limit the stress on the diaphragm, but causes problems with position measurement. The linear transducer has an essentially infinite resolution, but the position changes at the upper and lower limits of motion are close to the precision of our 12-bit data acquisition card, leading to minor discretization problems.

If the aortic chamber is paired with an arterial chamber having the requisite compliance, the aortic pressure wave displays a dicrotic notch-like artifact, as seen in Figure 5.15 (on Page 141), and the arterial pressure wave displays the complementary "knee." These features, which closely match what is predicted by numerical simulation, reinforce the idea that a mock circulatory system should have a multi-component arterial system. Those chambers would not necessarily have to feature rolling diaphragms. While the rolling diaphragm chambers allow us to easily study the influence of compliance on the aortic and arterial pressure waveforms they are also lossy, costly, and rather unreliable. Now that we have a better understanding of the arterial system compliances (and more confidence in our numerical simulations) it might be feasible to switch back to appropriately sized air-water chambers.

\subsection{Correspondence Between Theory and Experiment}

In all portions of this work there is good agreement between the theoretical and experimental results. The pressure waveform predicted by the arterial single-compliance model, first shown in Figure 3.3 on Page 78 and then again (in a more expansive form) in Figure 5.13 on Page 137, agrees well with the experimental measurements. Experiments using both the $\mathrm{VAD}^{*}$ and the $\mathrm{PVAD}^{\dagger}$ produced pressure waveforms similar to those found by

\footnotetext{
${ }^{*}$ As in Figure 5.3 and Figure 5.4 on Pages 123 and 124, respectively.

${ }^{+}$As in Figure 5.11 and Figure 5.12 on Pages 135 and 136, respectively.
} 
numerical simulation of the arterial SCM. The addition of a second compliance element to the MCS produced a more physiologically correct arterial pressure waveform, once again in good agreement with the numerical results. The appearance of a dicrotic notch-like artifact was also predicted by the numerical work, and a similar influence of compliance ratio is found in both theoretical and experimental results. The experimental pressure traces, shown in Figure 5.15 (on Page 141) support the theoretical assertion that the ratio between arterial and aortic compliance should be approximately $3: 1$. Liquid sloshing in the airwater tank was unanticipated, but Figure 5.5 shows that the impact of the sloshing on the arterial pressure waveform can be accounted for numerically.

The MTS system (and the pVAD) behaved as expected based on the numerical results. Modifications to the mock circulatory system configuration would help eliminate some of pVAD's mechanical problems; rolling diaphragms are designed to operate under positive internal pressure, and elevating the MCS (relative to the pVAD) would help in this regard. Unfortunately, this would also raise the experiment to an uncomfortable level above the ground. The constant $\mathrm{pVAD}$ stroke volume produces a larger peripheral flow at high beat rates, and a correspondingly higher peripheral pressure drop. The resultant increase in arterial system pressure is seen in both the experimental and theoretical results (Figure 5.12 and Figure 5.13, respectively). 


\section{Chapter 7}

\section{Conclusions and Recommendations}

$\mathbf{T}$

HERE are two major contributions that arise from this work. The experimental results support the theoretical assertion that the addition of a second arterial chamber (as in the arterial dual-compliance model) would improve the physiological representation of many existing mock circulatory systems. This realization is the first important contribution of this work; even if the dicrotic artifact in the aortic pressure waveform is ultimately due to pressure wave reflections, the ability to reproduce it in vitro using a relatively simple setup has broad implications for artificial heart testing. Most mock circulatory system (MCS) designs are modular, and retrofitting them with another chamber would be relatively easy.

The second major contribution is the newly-developed analogy equating the proximal arterial system to a nonlinear mass-spring-damper system. This analogy has import for both VAD efficiency and stability, and also makes predictions about natural heart behaviour. The nonlinear aspects of this behaviour offer possible explanations for several cardiac conditions, and provide promising direction for future research. When viewed in the framework of the analogy, conditions like atherosclerosis, cardiac arrhythmia, and sudden infant death syndrome have alternative and compelling causes.

\subsection{Experimental Improvements}

The rolling diaphragm chamber design is perhaps not the best treatment of the aortic chamber; the very small volume changes (over the course of a beat) and the tighter tolerances mean more viscous friction, and more losses. It is, however, good for development work because it allows one to change the chamber compliance with relative ease. Nev- 
ertheless, a properly sized air-water chamber* might be a better solution for terrestrial applications. ${ }^{+}$

The PVAD and MTS system proved to be a good representation of an artificial heart, producing an arterial pressure wave similar to that seen in VAD experiments, but without a suitable load cell the MTS setup is limited to a constant volume, fixed-rate pumping mode. Outfitting the MTS machine with a more sensitive load cell would allow it to mimic both the fixed-rate and the full-fill, full-eject VAD pumping modes. (Recall from Chapter 5 that the fixed-rate VAD mode is not a constant volume mode; ejected volume depends on both fill rate and arterial pressure.)

There needs to be some way of synchronizing the DAQ measurements to the cycling of the MTS machine. The valve microphone used to monitor VAD cycling no longer works due to the broadband hydraulic noise which is transmitted through all of the tubes and fixtures connected to the MTS machine.

The PVAD chamber should be inverted in the MTS machine. This would mean that the piston (rather than the cylinder casing) would move up and down with each stroke, and would allow us to shorten the tubes connecting the PVAD to the MCS. The outlet and inlet tubes would no longer flex with each beat and the MCS could be moved closer to the pVAD, which would give a more desirable $\Pi_{1}$ value for the MTS tests. This reconfiguration would require some sort of a stand or table be built to support the mock circulatory system. The MTS machine has horizontal aluminum support channels clamped around the uprights, and some sort of extension could be built off the back of the machine to attach a table. The new table or stand would allow the MCS to be elevated relative to the pVAD, ensuring that the pVAD diaphragm maintained a positive internal pressure during cycling. This, in turn, would reduce the wear on the diaphragm. The elevated loop would bring initial setup problems, however. Normally the MCS is filled with water by way of the venous chamber, whose top is removable. The venous chamber is 26 in tall, and the outlet of the inverted PVAD (and the MCS) would be approximately $5 \mathrm{ft}$ above the ground. A step ladder would be required to fill the mock circulatory system with water.

\footnotetext{
${ }^{*}$ The aortic air-water chamber would need an appropriate cross-sectional area and a proper pre-charge of compressed air.

${ }^{\dagger}$ In the absence of gravity, an air-water chamber no longer works as a compliance element.
} 
The inverted pVAD and elevated loop would make it easier to bleed air from the PVAD chamber. In the current configuration the PVAD is more elevated than the arterial system, meaning that trapped air tends to move towards the pVAD. During setup air is bled from the inlet valve side of the PVAD (while it is clamped in the MTS machine). The pVAD is then de-clamped, lowered to an elevation below the arterial system, and inverted. The remaining air is driven out of the system by moving the piston quickly within the cylinder. The trapped air moves into the arterial system, and the pVAD is re-clamped in the MTS. Once pVAD cycling begins the air can be forced out of the arterial system (and into the venous chamber, which is open to atmosphere). Having the pVAD outlet and the MCS at similar elevations would simplify this process immensely.

\subsection{Theoretical Work}

On the theoretical side, we could use a computational fluid dynamic (CFD) analysis of aortic blood flow to refine our estimate of $\Pi_{1}$. In order to improve our estimate of the arterial system's $\Pi_{1}$ we need a better analysis of aortic flow losses and flow momentum than what is currently available in the literature; a CFD study could provide this. In addition, Magnetic Resonance Imaging (MRI) of the arterial system would allow the CFD model to be refined and permit us to study changes in vessel geometry over the course of a beat. Although the subject in the MRI machine would be resting rather than active, the Valsalva manoeuvre could be used to affect arterial pressures and the heart rate. All of these investigations would produce a better estimate of $\Pi_{1 \mid b}$ and $\Pi_{1 \mid m}$ and help to determine whether or not impact oscillator-type behaviour is a key influence in the operation of the human circulatory system.

A study of blood pressure versus heart rate (during both rest and exertion) would help to understand any changes in peripheral resistance which accompany changes in heart rate. This would provide some guidance for peripheral resistance changes in the mock circulatory system, which would allow the PVAD to operate at elevated beat rates without unnatural increases in arterial pressure (and the resultant distortion of the arterial pressure trace). 


\section{References}

Abel, F. L. (1984), "Influence of aortic compliance on coronary blood flow," Circulatory Shock, 12, pp. 265-276.

Ainsworth, M. (1997), Wavelets, multilevel methods, and elliptic PDEs, Numerical Mathematics and Scientific Computation, Clarendon Press; Oxford University Press, New York; Oxford.

Allen, G. S., Murray, K. D. and Olsen, D. B. (1996), "Control of the artificial heart," ASAIO Journal, 42, pp. 932-937.

Almog, Y., Oz, O. and Akselrod, S. (1999), "Correlation dimension estimation: can this nonlinear description contribute to the characterization of blood pressure control in rats?" IEEE Transactions on Biomedical Engeering, 46, pp. 535-547.

Altenhoff, G., Frank, P. M. and Moller, D. P. (1989), "A stress-dependent pulsatile cardiovascular model as the basis for regulating artificial hearts," Biomedizinische Technik, 34, pp. 101-106.

Altman, P. L. and Katz, D. D. (1971), Respiration and Circulation, Federation of American Societies for Experimental Biology, Bethesda, MD.

Andriessen, P., Koolen, A. M., Berendsen, R. C., Wijn, P. F., ten Broeke, E. D., Oei, S. G. and Blanco, C. E. (2003), “Cardiovascular fluctuations and transfer function analysis in stable preterm infants," Pediatric Research, 53, pp. 89-97.

Apel, J., Neudel, F. and Reul, H. (2001), "Computational fluid dynamics and experimental validation of a microaxial blood pump," ASAIO Journal, 47, pp. 552-558.

Arabia, M. and Akutsu, T. (1984), "A new test circulatory system for research in cardiovascular engineering," Annals of Biomedical Engineering, 12, pp. 29-48. 
Auslander, D. M., Lobdell, T. E. and Chong, D. (1973), "A large-scale model of the human cardiovascular system and its application to ballistocardiography," Bibliotheca Cardiologica, 32, pp. 15-21.

Avanzolini, G., Barbini, P. and Cappello, A. (1992), "Comparison of algorithms for tracking short-term changes in arterial circulation parameters," IEEE Transactions on Biomedical Engineering, 39, pp. 861-867.

Avanzolini, G., Barbini, P., Cappello, A., Cevenini, G., Moller, D., Pohl, V. and Sikora, T. (1989), "Electrical analogs for monitoring vascular properties in artificial heart studies," IEEE Transactions on Biomedical Engineering, 36, pp. 462-470.

Avanzolini, G., Belardinelli, E. and Passigato, R. (1972), “Numerical model of the peripheral arterial system," Bollettino della Società Italiana di Cardiologia, 17, pp. 159-178.

Avula, X. J. and Oestreicher, H. L. (1978), "Mathematical model of the cardiovascular system under acceleration stress," Aviation, Space, and Environmental Medicine, 49, pp. 279-286.

Awrejcewicz, J., Andrianov, I. V. and Manevich, L. I. (1998), Asymptotic approaches in nonlinear dynamics: new trends and applications, Springer Series in Synergetics, Springer, Berlin; New York.

Babbs, C. F., Ralston, S. H. and Geddes, L. A. (1984), "Theoretical advantages of abdominal counterpulsation in CPR as demonstrated in a simple electrical model of the circulation," Annals of Emergency Medicine, 13, pp. 660-671.

Bajanowski, T., Ortmann, C., Teige, K., Wedekind, H., Zack, F., Rose, I. and Brinkmann, B. (2003), "Pathological changes of the heart in sudden infant death," International Journal Of Legal Medicine, 117, pp. 193-203.

Baloa, L. A., Boston, J. R. and Antaki, J. F. (2001), “Elastance-based control of a mock circulatory system," Annals of Biomedical Engineering, 29, pp. 244-251.

Bapat, C. N. (1998), "Periodic motions of an impact oscillator," Journal of Sound and Vibration, 209, pp. 43-60.

Bassett, M. D., Winterbone, D. E. and Pearson, R. J. (2001), "Calculation of steady flow pressure loss coefficients for pipe junctions," Proceedings Of The Institution Of Me- 
chanical Engineers Part C - Journal Of Mechanical Engineering Science, 215, pp. 861881.

Bauernschmitt, R., Schulz, S., Schwarzhaupt, A., Kiencke, U., Vahl, C. F., Lange, R. and Hagl, S. (1999), "Simulation of arterial hemodynamics after partial prosthetic replacement of the aorta," Annals of Thoracic Surgery, 67, pp. 676-682.

Baur, G. M., Zupan, T. L., Gates, K. H. and Porter, J. M. (1983), "Blood flow in the common femoral artery - evaluation in a vascular laboratory," American Journal of Surgery, 145, pp. 585-588.

Bearnson, G. B., Olsen, D. B., Khanwilkar, P. S., Long, J. W., Allaire, P. E. and Maslen, E. H. (1996), "Pulsatile operation of a centrifugal ventricular assist device with magnetic bearings," ASAIO Journal, 42, pp. M620-M624.

Benedict, R. P. (1984), Fundamentals of temperature, pressure, and flow measurements, 3rd ed., Wiley, New York.

Berger, D. S. and Li, J. K. (1992), "Temporal relationship between left ventricular and arterial system elastances," IEEE Transactions on Biomedical Engineering, 39, pp. 404 410.

Berger, D. S., Li, J. K.-J. and Noordergraaf, A. (1995), "Arterial wave propagation phenomena, ventricular work, and power dissipation," Annals of Biomedical Engineering, 23, pp. 804-811.

Bertram, C. D., Timmer, J., Muller, T. G., Maiwald, T., Winterhalder, M. and Voss, H. U. (2004), "Aperiodic flow-induced oscillations of collapsible tubes: a critical reappraisal," Medical Engineering and Physics, 26, pp. 201-214.

Blazejczyk-Okolewska, B., Brindley, J., Czolczynski, K. and Kapitaniak, T. (2001), “Antiphase synchronization of chaos by noncontinuous coupling: two impacting oscillators," Chaos Solitons and Fractals, 12, pp. 1823-1826.

Boston, J. R., Antaki, J. F. and Simaan, M. A. (2003), "Hierarchical control of heart-assist devices," IEEE Robotics and Automation Magazine, 10, pp. 54-64.

Bourque, K., Gernes, D. B., Loree, H. M., Richardson, J. S., Poirier, V. L., Barletta, N., Fleischli, A., Foiera, G., Gempp, T. M., Schoeb, R., Litwak, K. N., Akimoto, T., Watach, 
M. J. and Litwak, P. (2001), “HeartMate III: pump design for a centrifugal LVAD with a magnetically levitated rotor," ASAIO Journal, 47, pp. 401-405.

Bowles, C. T., Shah, S. S., Nishimura, K., Clark, C., Cumming, D. V., Pattison, C. W., Pepper, J. R. and Yacoub, M. H. (1991), "Development of mock circulation models for the assessment of counterpulsation systems," Cardiovascular Research, 25, pp. 901-908.

Bracic, M. and Stefanovska, A. (1998), "Nonlinear dynamics of the blood flow studied by Lyapunov exponents," Bulletin of Mathematical Biology, 60, pp. 417-33.

Brown, K. A. and Ditchey, R. V. (1988), "Human right ventricular end-systolic pressurevolume relation defined by maximal elastance," Circulation, 78, pp. 81-91.

Buckingham, E. (1914), “On physically similar systems: illustrations of the use of dimensional equations," Physical Review, 4, pp. 345-76.

Burattini, R. and Natalucci, S. (1998), "Complex and frequency-dependent compliance of viscoelastic windkessel resolves contradictions in elastic windkessels," Medical Engineering and Physics, 20, pp. 502-514.

Byard, R. W. and Krous, H. F. (2003), "Sudden infant death syndrome: overview and update," Pediatric and Developmental Pathology, 6, pp. 112-127.

Cappello, A., Gnudi, G. and Lamberti, C. (1995), "Identification of the three-element windkessel model incorporating a pressure-dependent compliance," Annals of Biomedical Engineering, 23, pp. 164-177.

Carpio, A. (2004), "Nonlinear stability of oscillatory wave fronts in chains of coupled oscillators," http: //www. arxiv.org/pdf/nlin.PS/0404044.

Casaleggio, A., Cerutti, S. and Signorini, M. G. (1997), "Study of the Lyapunov exponents in heart rate variability signals," Methods of Information in Medicine, 36, pp. 274277.

Cavalcanti, S. and Ursino, M. (1996), "Chaotic oscillations in microvessel arterial networks," Annals of Biomedical Engineering, 24, pp. 37-47.

Chang, K. C. and Kuo, T. S. (1996), “Exponentially tapered T-tube model in the characterization of arterial non-uniformity," Journal of Theoretical Biology, 183, pp. 35-46. 
Chang, K. C., Tseng, Y. Z., Kuo, T. S. and Chen, H. I. (1995), "Impedance and wave reflection in arterial system: simulation with geometrically tapered T-tubes," Medical and Biological Engineering and Computing, 33, pp. 652-660.

Chate, H., Pikovsky, A. and Rudzick, O. (1999), "Forcing oscillatory media: phase kinks vs. synchronization," Physica D - Nonlinear Phenomena, 131, pp. 17-30.

Chemla, D., Hebert, J. L., Coirault, C., Salmeron, S., Zamani, K. and Lecarpentier, Y. (1996), "Matching dicrotic notch and mean pulmonary artery pressures: implications for effective arterial elastance," American Journal of Physiology, 271, pp. H1287-H1295.

Chen, C. W., Shau, Y. W. and Wu, C. P. (1997), “Analog transmission line model for simulation of systemic circulation," IEEE Transactions on Biomedical Engineering, 44, pp. 90-94.

Chevalier, P. A., Reed, J. H., Vandenberg, R. A. and Wood, E. H. (1978), "Effect of gravitational and inertial forces on vertical distribution of pulmonary blood flow," Aviation, Space, and Environmental Medicine, 49, pp. 768-778.

Chiu, C. C., Yeh, S. J. and Chen, C. H. (2000), "Self-organizing arterial pressure pulse classification using neural networks: theoretical considerations and clinical applicability," Computers in Biology and Medicine, 30, pp. 71-88.

Chon, K. H., Hoyer, D., Armoundas, A. A., Holstein-Rathlou, N. H. and Marsh, D. J. (1999), "Robust nonlinear autoregressive moving average model parameter estimation using stochastic recurrent artificial neural networks," Annals of Biomedical Engineering, 27, pp. 538-547.

Christini, D. J., Bennett, F. M., Lutchen, K. R., Ahmed, H. M., Hausdorff, J. M. and Oriol, N. (1995), "Application of linear and nonlinear time series modeling to heart rate dynamics analysis," IEEE Transactions on Biomedical Engineering, 42, pp. 411-415.

Cochrane, T. (1991), "Simple model of circulatory system dynamics including heart valve mechanics," Journal of Biomedical Engineering, 13, pp. 335-340.

Conlon, M. J. (2000), "Design and evaluation of a neural network-based controller for an artificial heart," Master's thesis, Carleton University. 
Conlon, M. J., Russell, D. L. and Mussivand, T. (2006), “Development of a mathematical model of the human circulatory system," Annals of Biomedical Engineering.

Coulam, C. M., Warner, H. R., Marshall, H. W. and Bassingthwaighte, J. B. (1967), “A steady-state transfer function analysis of portions of the circulatory system using indicator dilution techniques," Computers and Biomedical Research, 1, pp. 124-138.

Cox, E. A. and Mortell, M. P. (1992), "The evolution of nonlinear resonant oscillations in closed tubes: solution of the standard mapping," Chaos, 2, pp. 201-214.

Cox, E. A., Mortell, M. P. and Reck, S. (2002), "Nonlinear standing and resonantly forced oscillations in a tube with slowly changing length," SIAM Journal On Applied Mathematics, 62, pp. 965-989.

Croston, R. C., Rummel, J. A. and Kay, F. J. (1973), "Computer model of cardiovascular control system response to exercise," Journal of Dynamic Systems, Measurement, and Control, 95, pp. 301-307.

Cushman, R., Ferrer, S. and Hanssmann, H. (1999), "Singular reduction of axially symmetric perturbations of the isotropic harmonic oscillator," Nonlinearity, 12, pp. 389-410.

Cysarz, D., Bettermann, H. and van Leeuwen, P. (2000), "Entropies of short binary sequences in heart period dynamics," American Journal of Physiology - Heart and Circulatory Physiology, 278, pp. H2163-H2172.

Dahlgren, G., Veintemilla, F., Settergren, G. and Liska, J. (1991), "Left ventricular endsystolic pressure estimated from measurements in a peripheral artery," Journal of Cardiothoracic and Vascular Anesthesia, 5, pp. 551-553.

Darowski, M., de Lazzari, C., Ferrari, G., Clemente, F. and Guaragno, M. (2000), “Computer simulation of hemodynamic parameter changes by mechanical ventilation and biventricular circulatory support," Methods of Information in Medicine, 39, pp. 332338.

Dawber, T. R., Thomas, H. E. and McNamara, P. M. (1973), "Characteristics of the dicrotic notch of the arterial pulse wave in coronary heart disease," Angiology, 24, pp. 244255. 
Day, S. W., McDaniel, J. C., Wood, H. G., Allaire, P. E., Landrot, N. and Curtas, A. (2001), "Particle image velocimetry measurements of blood velocity in a continuous flow ventricular assist device," ASAIO Journal, 47, pp. 406-411.

de Lazzari, C., Darowski, M., Ferrari, G. and Clemente, F. (1998), "The influence of left ventricle assist device and ventilatory support on energy-related cardiovascular variables," Medical Engineering and Physics, 20, pp. 83-91.

de Lazzari, C., Darowski, M., Ferrari, G., Clemente, F. and Guaragno, M. (2000), “Computer simulation of haemodynamic parameters changes with left ventricle assist device and mechanical ventilation," Computers in Biology and Medicine, 30, pp. 55-69.

de Lazzari, C., Darowski, M., Ferrari, G., Clemente, F. and Guaragno, M. (2001), “Ventricular energetics during mechanical ventilation and intraaortic balloon pumping computer simulation," Journal of Medical Engineering and Technology, 25, pp. 103111.

de Lazzari, C., Darowski, M., Wolski, P., Ferrari, G., Tosti, G. and Pisanelli, D. M. (2005), "In vivo and simulation study of artificial ventilation effects on energetic variables in cardiosurgical patients," Methods of Information in Medicine, 44, pp. 98-105.

Desouzamachado, S., Rollins, R. W., Jacobs, D. T. and Hartman, J. L. (1990), "Studying chaotic systems using microcomputer simulations and Lyapunov exponents," American Journal of Physics, 58, pp. 321-329.

di Bernardo, M., Budd, C. J. and Champneys, A. R. (2001), “Normal form maps for grazing bifurcations in n-dimensional piecewise-smooth dynamical systems," Physica D - Nonlinear Phenomena, 160, pp. 222-254.

Donovan, F. M. (1975), “Design of a hydraulic analog of the circulatory system for evaluating artificial hearts," Biomaterials, Medical Devices, and Artificial Organs, 3, pp. 439 449.

Drzewiecki, G. M., Wasicko, M. J. and Li, J. K. (1991), "Diastolic mechanics and the origin of the third heart sound," Annals of Biomedical Engineering, 19, pp. 651-667.

Elanayar, S. and Shin, Y. C. (1994), "Radial basis function neural-network for approximation and estimation of nonlinear stochastic dynamic-systems," IEEE Transactions on Neural Networks, 5, pp. 594-603. 
Erickson, E. H., Fitzpatrick, E. L. and Stone, H. L. (1972), "Computer analysis of myocardial function in aerospace and biomedical research," American Journal of Veterinary Research, 33, pp. 243-248.

Eyal, S. and Akselrod, S. (2000), "Bifurcation in a simple model of the cardiovascular system," Methods of Information in Medicine, 39, pp. 118-121.

Ferrari, G., de Lazzari, C., Kozarski, M., Clemente, F., Gorczynska, K., Mimmo, R., Monnanni, E., Tosti, G. and Guaragno, M. (2002), "A hybrid mock circulatory system: testing a prototype under physiologic and pathological conditions," ASAIO Journal, 48, pp. 487-494.

Ferrari, G., de Lazzari, C., Mimmo, R., Ambrosi, D. and Tosti, G. (1994), "Mock circulatory system for in vitro reproduction of the left ventricle, the arterial tree and their interaction with a left ventricular assist device," Journal of Medical Engineering and Technology, 18, pp. 87-95.

Ferrari, G., de Lazzari, C., Mimmo, R., Tosti, G. and Ambrosi, D. (1992), “A modular numerical model of the cardiovascular system for studying and training in the field of cardiovascular physiopathology," Journal of Biomedical Engineering, 14, pp. 91-107.

Ferrari, G., de Lazzari, C., Mimmo, R., Tosti, G., Ambrosi, D. and Gorczynska, K. (1998), "A computer controlled mock circulatory system for mono- and biventricular assist device testing," International Journal of Artificial Organs, 21, pp. 26-36.

Ferrari, G., Kozarski, M., de Lazzari, C., Clemente, F., Merolli, M., Tosti, G., Guaragno, M., Mimmo, R., Ambrosi, D. and Glapinski, J. (2001), "A hybrid (numerical-physical) model of the left ventricle," International Journal of Artificial Organs, 24, pp. 456-462.

Ferrari, G., Nicoletti, A., de Lazzari, C., Clemente, F., Tosti, G., Guaragno, M., Mimmo, R., Ambrosi, D. and Gorczynska, K. (2000), "A physical model of the human systemic arterial tree," International Journal of Artificial Organs, 23, pp. 647-657.

Florian, J. (1996), Practical thermoforming: principles and applications, 2nd ed., M. Dekker, New York.

Fortrat, J. O., Formet, C., Frutoso, J. and Gharib, C. (1999), "Even slight movements disturb analysis of cardiovascular dynamics," American Journal of Physiology, 277, pp. H261-H267. 
Frasch, H. F., Kresh, J. Y. and Noordergraaf, A. (1996), "Two-port analysis of microcirculation: an extension of windkessel," American Journal of Physiology, 270, pp. H376H385.

Fruchter, G. and Ben-Haim, S. (1992), "Dynamic properties of cardiovascular systems," Mathematical Biosciences, 110, pp. 103-17.

Fu, M. H., Xu, L. Y., Medvedev, A., Smith, W. A. and Golding, L. A. R. (1997), "Design of a DSP controller for an innovative ventricular assist system," ASAIO Journal, 43, pp. M615-M619.

$\mathrm{Fu}, \mathrm{M}$. and $\mathrm{Xu}, \mathrm{L} .(2000)$, "Computer simulation of sensorless fuzzy control of a rotary blood pump to assure normal physiology," ASAIO Journal, 46, pp. 273-278.

Fukamachi, K., Harasaki, H., Massiello, A. L., Chen, J. F. and Kiraly, R. J. (1996), “In vitro evaluation of automatic control performance of a total artificial heart with changes in pump orientation," ASAIO Journal, 42, pp. M589-M592.

Fukuda, N., Oki, T., Iuchi, A., Tabata, T., Manabe, K., Sasaki, M., Yamada, H. and Ito, S. (1996), "Peculiar patterns of aortic regurgitation and carotid pulse due to dysfunction of a Medtronic Hall prosthetic valve: a case report," The Journal of Heart Valve Disease, 5, pp. 283-285.

Fung, Y. C. (1997), Biomechanics: circulation, 2nd ed., Springer, New York.

Gardner, J. F., Ignatoski, M., Tasch, U., Snyder, A. J. and Geselowitz, D. B. (1993), “Aortic pressure estimation with electro-mechanical circulatory assist devices," Journal of Biomechanical Engineering, 115, pp. 187-194.

Garrison, L. A., Frangos, J. A., Geselowitz, D. B., Lamson, T. C. and Tarbell, J. M. (1994), "A new mock circulatory loop and its application to the study of chemical additive and aortic pressure effects on hemolysis in the Penn State electric ventricular assist device," Artificial Organs, 18, pp. 397-407.

Geddes, L. A. and Roeder, R. (2002), "Controversy over the dicrotic notch and wave in the blood pressure record," IEEE Engineering in Medicine and Biology Magazine, 21, pp. 167-169. 
Gerhart, R. L., Horvath, D. J., Ochiai, Y., Krogulecki, A. Y. and Golding, L. A. R. (2002), "The effects of impact on the CorAide ventricular assist device," ASAIO Journal, 48, pp. $449-452$.

Gnudi, G. (1998), "New closed-form expressions for the estimation of arterial windkessel compliance," Computers in Biology and Medicine, 28, pp. 207-223.

Goldberger, A. L. (1996), "Non-linear dynamics for clinicians: chaos theory, fractals, and complexity at the bedside," Lancet, 347, pp. 1312-1314.

Goldberger, A. L. and West, B. J. (1987a), "Applications of nonlinear dynamics to clinical cardiology," Annals of the New York Academy of Science, 504, pp. 195-213.

Goldberger, A. L. and West, B. J. (1987b), "Fractals in physiology and medicine," The Yale Journal of Biology and Medicine, 60, pp. 421-435.

Gontier, C. and Toulemonde, C. (1997), "Approach to the periodic and chaotic behaviour of the impact oscillator by a continuation method," European Journal of Mechanics A - Solids, 16, pp. 141-163.

Graen, M. D., Ewert, D. L., Glower, J. S., Gray, L. A. and Koenig, S. C. (1999), “Electric analog model of the aortic valve for calculation of continuous beat-to-beat aortic flow using a pressure gradient," ASAIO Journal, 45, pp. 204-210.

Graen, M. D., Ewert, D. L., Glower, J. S., Gray, L. A. and Koenig, S. C. (2000), “Can a linear electrical analog model of a mechanical valve predict flow by using a pressure gradient?" ASAIO Journal, 46, pp. 563-568.

Gray, H. (1858), Gray's Anatomy: Descriptive and Surgical, John W. Parker and Son, London.

Green, J. F. and Miller, N. C. (1973), "A model describing the response of the circulatory system to acceleration stress," Annals of Biomedical Engineering, 1, pp. 455-467.

Griffith, T. M. and Edwards, D. H. (1994), “EDRF suppresses chaotic pressure oscillations in isolated resistance artery without influencing intrinsic complexity," American Journal of Physiology, 266, pp. H1786-H1800.

Gross, J. M., Shermer, C. D. and Hwang, N. H. (1988), "Vortex shedding in bileaflet heart valve prostheses," ASAIO Transactions, 34, pp. 845-850. 
Guckenheimer, J. and Holmes, P. (1983), Nonlinear oscillations, dynamical systems, and bifurcations of vector fields, Springer-Verlag, New York.

Guillemin, V. and Pollack, A. (1974), Differential topology, Prentice-Hall, Englewood Cliffs, NJ.

Guyton, A. C. (1992), Human physiology and mechanisms of disease, 5th ed., W. B. Saunders, Philadelphia, PA.

Guzzetti, S., Signorini, M. G., Cogliati, C., Mezzetti, S., Porta, A., Cerutti, S. and Malliani, A. (1996), "Non-linear dynamics and chaotic indices in heart rate variability of normal subjects and heart-transplanted patients," Cardiovascular Research, 31, pp. 441-446.

Haller, G. (1999), Chaos near resonance, Springer, New York.

Hamalainen, J. J. (1991), "Optimal arterial resistance for normal and failing heart," Proceedings of the 13th Annual International Conference of the IEEE Engineering in Medicine and Biology Society, Orlando, FL, 13, IEEE, Piscataway, NJ, pp. 2046-2048.

Hammel, D., Mollhoff, T., Soepawata, R., van Aken, H. and Scheld, H. H. (1997), "Mechanical myocardial support systems 1997: an overview of inta-aortic balloon counterpulsation to implantable left ventricular support systems," Anaesthesist, 46, pp. 408-418.

Hammel, S. M., Yorke, J. A. and Grebogi, C. (1988), “Numerical orbits of chaotic processes represent true orbits," Bulletin of the American Mathematical Society, 19, pp. 465-469.

Hansen, A. G. (1967), Fluid mechanics, Wiley, New York.

Harris, C. J. and Miles, J. F. (1980), Stability of linear systems: some aspects of kinematic similarity, 153, Academic Press, London; New York.

Harris, C. J. and Valenoca, J. M. E. (1983), The stability of input-output dynamical systems, 168, Academic Press, London; New York.

Hasegawa, M., Rodbard, D. and Kinoshita, Y. (1991), "Timing of the carotid arterial sounds in normal adult men: measurement of left ventricular ejection, pre-ejection period and pulse transmission time," Cardiology, 78, pp. 138-149.

Hebert, J. L., Lecarpentier, Y., Zamani, K., Coirault, C., Daccache, G. and Chemla, D. (1995), "Relation between aortic dicrotic notch pressure and mean aortic pressure in adults," American Journal of Cardiology, 76, pp. 301-306. 
Hendry, P. J., Masters, R. G., Keaney, M., Bourke, M., Mussivand, T. and Keon, W. J. (1996), "Evolution of an electrohydraulic ventricular assist device through in vivo testing," ASAIO Journal, 42, pp. M350-M354.

Hendry, P., Masters, R. G., Ibrahim, M., Bourke, M., Keaney, M., Kilborn, S., Keon, W. J. and Mussivand, T. (1999), "In vivo evaluation of an intrathoracic ventricular assist device," Asaio J, 45, pp. 123-126.

Hilborn, R. C. and Tufillaro, N. B. (1997), "Resource letter: Nonlinear dynamics," American Journal of Physics, 65, pp. 822-834.

Holdsworth, D. W., Norley, C. J., Frayne, R., Steinman, D. A. and Rutt, B. K. (1999), “Characterization of common carotid artery blood-flow waveforms in normal human subjects," Physiological Measurement, 20, pp. 219-240.

Holmes, P. (1979), "Non-Linear oscillator with a strange attractor," Philosophical Transactions of the Royal Society of London Series A - Mathematical, Physical, and Engineering Sciences, 292, pp. 419-448.

Honda, T., Hamada, M., Matsumoto, Y., Matsuoka, H. and Hiwada, K. (1995), "Noninvasive physiological assessment of vascular wall sclerosis using carotid pulse tracing and cine magnetic resonance," Angiology, 46, pp. 369-374.

Honerkamp, J. (1983), "The heart as a system of coupled nonlinear oscillators," Journal of Mathematical Biology, 18, pp. 69-88.

Hoyer, D., Kaplan, D., Schaaff, F. and Eiselt, M. (1998), “Determinism in bivariate cardiorespiratory phase-space sets," IEEE Engineering in Medicine and Biology Magazine, 17, pp. 26-31.

Hoyer, D., Schmidt, K., Bauer, R., Zwiener, U., Kohler, M., Luthke, B. and Eiselt, M. (1997), "Nonlinear analysis of heart rate and respiratory dynamics," IEEE Engineering in Medicine and Biology Magazine, 16, pp. 31-39.

Huang, Y., Jan, K. M., Rumschitzki, D. and Weinbaum, S. (1998), "Structural changes in rat aortic intima due to transmural pressure," Journal of Biomechanical Engineering, 120, pp. $476-483$. 
Hussain, S. and Subhash, E. (1998), “Dicrotic notch in females," Indian Journal of Physiology and Pharmacology, 42, pp. 421-422.

Igel, D. A. and Wilkoff, B. L. (1997), "Automated ventricular tachyarrhythmia recognition: a combination of cycle length and nonlinear dynamics measurements," Journal of Cardiovascular Electrophysiology, 8, pp. 388-397.

Iijima, T., Inamoto, T., Nogawa, M. and Takatani, S. (1997), "Control of centrifugal blood pump based on the motor current," Artificial Organs, 21, pp. 655-660.

Imachi, K., Chinzei, T., Abe, Y., Mabuchi, K., Imanishi, K., Yonezawa, T., Kouno, A., Ono, T., Atsumi, K. and Isoyama, T. (1991), "A new pulsatile total artificial heart using a single centrifugal pump," ASAIO Transactions, 37, pp. M242-M243.

James, K. (2000), PC interfacing and data acquisition: techniques for measurement, instrumentation and control, Newnes, Oxford; Boston.

Jaron, D., Moore, T. W. and He, P. (1985), "Control of intraaortic balloon pumping: theory and guidelines for clinical applications," Annals of Biomedical Engineering, 13, pp. 155-175.

Jelinek, J. (1972), "Hemodynamics of counterpulsation: the study of a lumped-parameter computer model," Journal of Biomechanics, 5, pp. 511-509.

Jensen, O. E. (1992), "Chaotic oscillations in a simple collapsible-tube model," Journal of Biomechanical Engineering, 114, pp. 55-59.

Jin, W. and Clark, C. (1994a), "Experimental investigation of the motions of the pumping diaphragm within a sac-type pneumatically driven ventricular assist device," Journal of Biomechanics, 27, pp. 43-55.

Jin, W. and Clark, C. (1994b), "Pressure development within a sac-type pneumatically driven ventricular assist device," Journal Of Biomechanics, 27, pp. 1319-1329.

Jordan, D. W. and Smith, P. (1987), Nonlinear ordinary differential equations, 2nd ed., Oxford Applied Mathematics and Computing Science Series, Clarendon Press; Oxford University Press, New York; Oxford. 
Kanters, J. K., Hojgaard, M. V., Agner, E. and Holstein-Rathlou, N. H. (1997), “Influence of forced respiration on nonlinear dynamics in heart rate variability," American Journal of Physiology, 272, pp. R1149-R1154.

Kanters, J. K., Holstein-Rathlou, N. H. and Agner, E. (1994), "Lack of evidence for lowdimensional chaos in heart rate variability," Journal of Cardiovascular Electrophysiology, 5, pp. 591-601.

Karnopp, D., Margolis, D. L. and Rosenberg, R. C. (1990), System dynamics: a unified approach, 2nd ed., Wiley, New York.

Katz, A. I., Chen, Y. and Moreno, A. H. (1969), "Flow through a collapsible tube - experimental analysis and mathematical model," Biophysical Journal, 9, pp. 1261-1279.

Kaufmann, R., Nix, C., Klein, M., Reul, H. and Rau, G. (1997), “The implantable fuzzy controlled Helmholtz-left ventricular assist device: first in vitro testing," Artificial Organs, 21, pp. 131-137.

Kawczyk-Krupka, A., Sieron, A. and Adamek, M. (1998), "Chaotic dynamics in medicine," Wiadomosci Lekarskie, 51, pp. 525-530.

Kenner, T. (1975), "The central arterial pulses - experiments on a hybrid model of the heart and the arterial system," European Journal of Physiology, 353, pp. 67-81.

Kesavan, S. K. and Yazdani, S. A. (1992), "Strain analysis of flexing blood pump diaphragms," Journal of Applied Biomaterials, 3, pp. 305-311.

Kim, S. H., Chang, B. C., Tack, G., Huh, J. M., Kang, M. S., Cho, B. K. and Park, Y. H. (1994), "In vitro sound spectral analysis of prosthetic heart valves by mock circulatory system," Yonsei Medical Journal, 35, pp. 271-278.

King, A. L. (1946), "Pressure-volume relation for cylindrical tubes with elastomeric walls - the human aorta," Journal of Applied Physics, 17, pp. 501-505.

Klopp, E. H. and Gott, V. L. (1975), "A simple model of the hemodynamic effects of a proximal coronary artery narrowing," Annals of Thoracic Surgery, 19, pp. 309-312.

Klute, G. K., Tasch, U. and Geselowitz, D. B. (1992), "An optimal controller for an electric ventricular-assist device: theory, implementation, and testing," IEEE Transactions on Biomedical Engineering, 39, pp. 394-403. 
Knierbein, B., Reul, H., Eilers, R., Lange, M., Kaufmann, R. and Rau, G. (1992), "Compact mock loops of the systemic and pulmonary circulation for blood pump testing," International Journal of Artificial Organs, 15, pp. 40-48.

Koenig, S. C., Pantalos, G. M., Gillars, K. J., Ewert, D. L., Litwak, K. N. and Etoch, S. W. (2004), "Hemodynamic and pressure-volume responses to continuous and pulsatile ventricular assist in an adult mock circulation," ASAIO Journal, 50, pp. 15-24.

Kolff, W. J. (1959), "Mock circulation to test pumps designed for permanent replacement of damaged hearts," Cleveland Clinic Quarterly, 26, pp. 223-226.

Kolff, W. J. (1990), "The invention of the artificial heart," International Journal of Artificial Organs, 13, pp. 396-403.

Konishi, H., Misawa, Y., Fuse, K. and Sohara, Y. (2001), "Pitfalls in the development of a rotary blood pump controller," ASAIO Journal, 47, pp. 397-400.

Kordas, M. (1968a), “Analysis of a simplified electrical analog of circulation,” Medical and Biological Engineering, 6, pp. 109-114.

Kordas, M. (1968b), "An electrical model of blood circulation," Medical and Biological Engineering, 6, pp. 449-451.

Kornet, L., Jansen, J. R., Nijenhuis, F. C., Langewouters, G. J. and Versprille, A. (1998), "The compliance of the porcine pulmonary artery depends on pressure and heart rate," Journal of Physiology, 512 Pt. 3, pp. 917-926.

Kozarski, M., Ferrari, G., Clemente, F., Gorczynska, K., de Lazzari, C., Darowski, M., Mimmo, R., Tosti, G. and Guaragno, M. (2003), "A hybrid mock circulatory system: development and testing of an electro-hydraulic impedance simulator," International Journal of Artificial Organs, 26, pp. 53-63.

Kramer, C., Gerhardt, H. J. and Bleifeld, W. (1974), "A model for closure of arterial vessels with special regard to the coronary arteries," Basic Research in Cardiology, 69, pp. 585-596.

Kresch, E. and Noordergraaf, A. (1969), "A mathematical model for the pressure-flow relationship in a segment of vein," IEEE Transactions on Biomedical Engineering, 16, pp. 296-307. 
Kresh, J. Y., Brockman, S. K. and Noordergraaf, A. (1990), "Theoretical and experimental analysis of right ventricular bypass and univentricular circulatory support," IEEE Transactions on Biomedical Engineering, 37, pp. 121-127.

Ku, C. C. and Lee, K. Y. (1995), "Diagonal recurrent neural networks for dynamic-systems control," IEEE Transactions on Neural Networks, 6, pp. 144-156.

Kuklinski, W. S., Jaron, D., Ohley, W. J. and Greenall, R. K. (1984), "The intraaortic balloon pump: a nonlinear digital computer model," Journal of Biomechanical Engineering, 106, pp. 220-228.

La Course, J. R., Mohanakrishnan, G. and Sivaprasad, K. (1986), "Simulations of arterial pressure pulses using a transmission line model," Journal of Biomechanics, 19, pp. 771-780.

Lamba, H. (1993), “Chaotic, regular and unbounded behaviour in the elastic impact oscillator," http: //www.arxiv.org/pdf/chao-dyn/9310004.

Lamba, H. (1995), "Chaotic, regular and unbounded behavior in the elastic impact oscillator," Physica D, 82, pp. 117-135.

Lamson, T. C., Geselowitz, D. B. and Tarbell, J. M. (1990), "Ventricular assist device volume compensation using a two phase fluid," ASAIO Transactions, 36, pp. M269-M273.

Lamson, T. C., Stinebring, D. R., Deutsch, S., Rosenberg, G. and Tarbell, J. M. (1991), "Realtime in vitro observation of cavitation in a prosthetic heart valve," ASAIO Transactions, 37, pp. M351-M353.

Lancaster, D. (1996), Don Lancaster's active-filter cookbook, 2nd ed., Newnes, Oxford; Boston.

Laskey, W. K., Parker, H. G., Ferrari, V. A., Kussmaul, W. G. and Noordergraaf, A. (1990), "Estimation of total systemic arterial compliance in humans," Journal of Applied Physiology, 69, pp. 112-119.

Le Pape, G., Giacomini, H., Swynghedauw, B. and Mansier, P. (1997), “A statistical analysis of sequences of cardiac interbeat intervals does not support the chaos hypothesis," Journal of Theoretical Biology, 184, pp. 123-131.

Lee, M., Ahn, J. M., Min, B. G., Lee, S. Y. and Park, C. H. (1996), “Total artificial heart using neural and fuzzy controller," Artificial Organs, 20, pp. 1220-1226. 
Lee, T. C., Huang, K. F., Hsiao, M. L., Tang, S. T. and Young, S. T. (2004), “Electrical lumped model for arterial vessel beds," Computer Methods and Programs in Biomedicine, 73, pp. 209-219.

Leefe, S. E. and Gentle, C. R. (1995), "A review of the in vitro evaluation of conduitmounted cardiac valve prostheses," Medical Engineering and Physics, 17, pp. 497506.

Lefevre, J. (1991), "A theoretical study of the relationships between fractal complexity and functional efficiency in the pulmonary arterial tree," Proceedings of the 13th Annual International Conference of the IEEE Engineering in Medicine and Biology Society, Orlando, $F L$, 13, IEEE, Piscataway, NJ, pp. 2198-2199.

Lefevre, J., Tavernier, A. and Durbaba, S. (1993), "The extended bond graph modelling and simulation method: a tool ideally suited to study cardiovascular energetics," Computational Biomedicine, edited by C. A. Brebbia, H. Power, R. D. Ciskowski, and K. D. Held, pp. 51-58, Southampton, pp. 51-58.

Leirner, A. A., Oshiro, M. S., Hayashida, S. A., Marques, E. F., Maizato, M. J., Stolf, N. A. and Jatene, A. D. (1994), "Design, manufacturing, and testing of a pulsatile ventricular assist device," Arquivos Brasileiros de Cardiologia, 63, pp. 239-245.

Levin, A. U. and Narendra, K. S. (1993), "Control of nonlinear dynamic-systems using neural networks - controllability and stabilization," IEEE Transactions on Neural Networks, 4, pp. 192-206.

Li, G. R., Ma, W. G. and Zhu, X. D. (2001), "Development of a new left ventricular assist device: the dynamic aortic valve," ASAIO Journal, 47, pp. 257-260.

Li, J. K., Cui, T. and Drzewiecki, G. M. (1990), "A nonlinear model of the arterial system incorporating a pressure-dependent compliance," IEEE Transactions on Biomedical Engineering, 37, pp. 673-678.

Lin, H. Y., Bianccucci, B. A., Deutsch, S., Fontaine, A. A. and Tarbell, J. M. (2000), "Observation and quantification of gas bubble formation on a mechanical heart valve," Journal of Biomechanical Engineering, 122, pp. 304-309. 
Lin Wang, Y.-Y., Jan, M.-Y., Shyu, C.-S., Chiang, C.-A. and Wang, W.-K. (2004), "The natural frequencies of the arterial system and their relation to the heart rate," IEEE Transactions on Biomedical Engineering, 51, pp. 193-195.

Liu, Z., Brin, K. P. and Yin, F. C. (1986), "Estimation of total arterial compliance: an improved method and evaluation of current methods," American Journal of Physiology, 251, pp. H588-H600.

Loebe, M., Koster, A., Sanger, S., Potapov, E. V., Kuppe, H., Noon, G. P. and Hetzer, R. (2001), "Inflammatory response after implantation of a left ventricular assist device: comparison between the axial flow MicroMed DeBakey VAD and the pulsatile Novacor device," ASAIO Journal, 47, pp. 272-274.

Lopac, V. and Dananic, V. (1998), "Energy conservation and chaos in the gravitationally driven Fermi oscillator," American Journal of Physics, 66, pp. 892-902.

Lu, K., Clark, J. W., Ghorbel, F. H., Ware, D. L. and Bidani, A. (2001), "A human cardiopulmonary system model applied to the analysis of the Valsalva maneuver," American Journal of Physiology - Heart and Circulatory Physiology, 281, pp. H2661-H2679.

Maeta, H. and Hori, M. (1985), "Effects of a lack of aortic "Windkessel" properties on the left ventricle," Japanese Circulation Journal, 49, pp. 232-237.

Mandarino, W. A., Griffith, B. P., Kormos, R. L., Ristas, J. M., Armitage, J. M., Hardesty, R. L. and Borovetz, H. S. (1990), "Novacor left ventricular assist filling and ejection in the presence of device complications," ASAIO Transactions, 36, pp. M387-M389.

Mandeville, J. B., Marota, J. J., Ayata, C., Zaharchuk, G., Moskowitz, M. A., Rosen, B. R. and Weisskoff, R. M. (1999), "Evidence of a cerebrovascular postarteriole windkessel with delayed compliance," Journal of Cerebral Blood Flow and Metabolism, 19, pp. 679689.

Martens, J. (1990), “A new total implantable artificial heart," Artificial Organs, 14, pp. 284289.

Martini, L. J. (1984), Practical Seal Design, M. Dekker, New York.

Mass, H. J., Gean, J. T. and Gwirtz, P. A. (1987), “Computer analysis of cardiovascular parameters," Computers in Biology and Medicine, 17, pp. 75-84. 
McClain, L. C., Wright, L. D., Bose, R. K., Spratt, J. A. and Maier, G. W. (1998), “Afterload sensitivity of nonlinear end-systolic pressure-volume relation vs preload recruitable stroke work in conscious dogs," Journal of Surgical Research, 75, pp. 6-17.

McCullagh, W. H., Covell, J. W. and Ross, J. (1972), "Left ventricular dilatation and diastolic compliance changes during chronic volume overloading," Circulation, 45, pp. 943951.

McGhee, B. H. and Bridges, E. J. (2002), "Monitoring arterial blood pressure: what you may not know," Critical Care Nurse, 22, pp. 60-4, 66-70, 73.

McRae, L. P. and Kartchner, M. M. (1984), "Pressure and volume measurements from the eye for detecting possible arterial obstruction," Annals of Biomedical Engineering, 12, pp. 63-78.

McVeigh, G. E., Hamilton, P. K. and Morgan, D. R. (2002), “Evaluation of mechanical arterial properties: clinical, experimental and therapeutic aspects," Clinical Science, 102, pp. 51-67.

Meador, S. A. (1986), "Computer simulation of cardiopulmonary resuscitation: computer analysis of a simple electrical model of the circulation," Resuscitation, 13, pp. 145-157.

Meara, L. A. (1984), "Pole-zero extraction by nonlinear regression of discrete-time arterial blood-flow waveforms," Medical and Biological Engineering and Computing, 22, pp. 281-284.

Mehta, V., Thompson, C., Mulpur, A. and Chandra, K. (1996), "Chaotic motion in an oscillatory boundary layer," Chaos, 6, pp. 601-616.

Melchior, F. M., Srinivasan, R. S. and Charles, J. B. (1992), “Mathematical modeling of human cardiovascular system for simulation of orthostatic response," American Journal of Physiology, 262, pp. H1920-H1933.

Menon, V. (1998), “Fuzzy logic controller for an artificial heart," Master's thesis, Carleton University.

Mickens, R. E. (2000), "Generalized harmonic oscillators," Journal of Sound and Vibration, 236, pp. 730-732. 
Mickens, R. E. (2001), "Mathematical and numerical study of the Duffing-harmonic oscillator," Journal of Sound and Vibration, 244, pp. 563-567.

Mickens, R. E. (2002), "Analysis of non-linear oscillators having non-polynomial elastic terms," Journal of Sound and Vibration, 255, pp. 789-792.

Mickens, R. E. (2004), "Quadratic non-linear oscillators," Journal of Sound and Vibration, 270, pp. $427-432$.

Mickens, R. E. and Semwogerere, D. (1997), "A phase space analysis of a non-linear oscillator equation," Journal of Sound and Vibration, 204, pp. 556-559.

Min, F. B. M. (1993), "The heart as a pump: the program "CARDIO"," Biomedical Modeling and Simulation on a PC, edited by R. P. van Wijk van Brievingh and D. P. F. Moller, pp. 157-172, Springer-Verlag, New York, pp. 157-172.

Mitsui, N., Fukunaga, S., Koura, Y., Morita, S., Hotei, H., Sueshiro, M., Sueda, T. and Matsuura, Y. (1996), "Numerical simulation of nonpulsatile left ventricular bypass," Artificial Organs, 20, pp. 632-636.

Moon, F. C. (1992), Chaotic and fractal dynamics: an introduction for applied scientists and engineers, Wiley, New York.

Mrava, G. L. (1970), "Mock circulation systems for artificial hearts," Advances in Biomedical Engineering and Medical Physics, 3, pp. 115-130.

Mukhopadhyay, S. and Narendra, K. S. (1993), "Disturbance rejection in nonlinear-systems using neural networks," IEEE Transactions on Neural Networks, 4, pp. 63-72.

Murawski, R. K. (2003), “Complex behaviour from a simple physical system: a numerical investigation," http://www.arxiv.org/pdf/nlin.CD/0303065.

Murray, W. B. and Foster, P. A. (1996), “The peripheral pulse wave: information overlooked," Journal of Clinical Monitoring, 12, pp. 365-377.

Mussivand, T., Hendry, P. J., Masters, R. G., Holmes, K. S., Hum, A. and Keon, W. J. (1996), "A remotely controlled and powered artificial heart pump," Artificial Organs, 20, pp. 1314-1319. 
Mussivand, T., Hendry, P. J., Masters, R. G. and Keon, W. J. (1999), “Development of a ventricular assist device for out-of-hospital use," Journal of Heart and Lung Transplantation, 18, pp. 166-171.

Myers, L. J. and Capper, W. L. (2001), “Analytical solution for pulsatile axial flow velocity waveforms in curved elastic tubes," IEEE Transactions on Biomedical Engineering, 48, pp. 864-873.

Nakata, K., Ohashi, Y., Muller, J., Gleuck, J. and Nose, Y. (1998), "Assessment of the total artificial heart (TAH) pulsatility: the coalition between pulsatility and ejection time in TAH in vitro study," Annals of Thoracic and Cardiovascular Surgery, 4, pp. 275-279.

Naszlady, A. and Kiss, L. (1970), "Some hemodynamic problems studied by means of a circulatory analog computer model," Polskie Archiwum Medycyny Wewnetrznej, 4, pp. $459-465$.

Nayfeh, A. H. and Balachandran, B. (1995), Applied nonlinear dynamics: analytical, computational, and experimental methods, Wiley Series in Nonlinear Science, Wiley, New York.

Negroni, J. A., Lascano, E. C. and Pichel, R. H. (1988), "A computer study of the relation between chamber mechanical properties and mean pressure-mean flow of the left ventricle," Circulation Research, 62, pp. 1121-1133.

Nickalls, R. W. D. (1993), "A new approach to solving the cubic: Cardano's solution revealed," The Mathematical Gazette, 77, pp. 354-359.

Nitta, S., Yambe, T., Kobayashi, S., Hashimoto, H., Yoshizawa, M., Mastuki, H., Tabayashi, K. and Takeda, H. (1999), "Chaotic dynamics in circulation with Tohoku University vibrating flow pump," Artificial Organs, 23, pp. 119-123.

Nose, Y., Tretbar, L. L., SenGupta, A., Topaz, S. R. and Kolff, W. J. (1965), "An artificial heart inside the chest," Journal of Thoracic and Cardiovascular Surgery, 50, pp. 792-799.

Offerdahl, C. D., Schaub, J. D., Koenig, S. C., Swope, R. D. and Ewert, D. L. (1996), “Development of an in-vitro circulatory system with known resistance and capacitance," Biomedical Sciences Instrumentation, 32, pp. 183-188.

O'Flynn, M. and Moriarty, E. (1987), Linear systems: time domain and transform analysis, Harper and Row, New York. 
Ogata, K. (1992), System Dynamics, 2nd ed., Prentice-Hall, Englewood Cliffs, NJ.

Ogino, H., Klangsuk, N., Jin, W., Bowles, C. T. and Yacoub, M. H. (1995), "Influence of the compliance of the pump housing and cannulas of a paracorporeal pneumatic ventricular assist device on transient pressure characteristics," Artificial Organs, 19, pp. 525534.

Olansen, J. B., Clark, J. W., Khoury, D., Ghorbel, F. and Bidani, A. (2000), “A closedloop model of the canine cardiovascular system that includes ventricular interaction," Computers and Biomedical Research, 33, pp. 260-295.

O'Leary, D. S., Pantalos, G. M. and Sharp, M. K. (1999), "Feedback control of mean aortic pressure in a dynamic model of the cardiovascular system," ASAIO Journal, 45, pp. 587-594.

Om, K. S., Ahn, J. M. and Min, B. G. (1999), "Static state hemodynamic variables estimation model for the moving-actuator type total artificial heart: Part I - cardiac output estimation," International Journal of Artificial Organs, 22, pp. 21-26.

Om, K. S., Jo, Y. H. and Min, B. G. (1999), "Static state hemodynamic variables estimation model for the moving-actuator type total artificial heart: Part II - Aortic pressure estimation," International Journal of Artificial Organs, 22, pp. 108-112.

on Fluid Meters, A. R. C. (1959), Fluid meters; their theory and application. Report of ASME Research Committee on Fluid Meters, 5th ed., American Society of Mechanical Engineers, New York.

Osaka, M., Yambe, T., Saitoh, H., Yoshizawa, M., Itoh, T., Nitta, S., Kishida, H. and Hayakawa, H. (1998), "Mutual information discloses relationship between hemodynamic variables in artificial heart-implanted dogs," American Journal of Physiology, 275, pp. H1419-H1433.

Oshiro, M. S., Hayashida, S. A., Maizato, M. J., Marques, E. F., Stolf, N. A., Jatene, A. D. and Leirner, A. A. (1995), "Design, manufacturing, and testing of a paracorporeal pulsatile ventricular assist device: Sao Paulo Heart Institute VAD," Artificial Organs, 19, pp. 274-279.

Othmer, H. G. and Xie, M. (1999), "Subharmonic resonance and chaos in forced excitable systems," Journal of Mathematical Biology, 39, pp. 139-171. 
Pantalos, G. M., Koenig, S. C., Gillars, K. J. and Ewert, D. L. (2002), "Mock circulatory system for testing cardiovascular devices," Proceedings of the 2002 IEEE Engineering in Medicine and Biology 24th Annual Conference and the 2002 Fall Meeting of the Biomedical Engineering Society (BMES/EMBS), 2, IEEE, Houston, TX, pp. 1597-1598.

Pantalos, G. M., Koenig, S. C., Gillars, K. J., Giridharan, G. A. and Ewert, D. L. (2004), "Characterization of an adult mock circulation for testing cardiac support devices," ASAIO Journal, 50, pp. 37-46.

Paynter, H. M. (1972), "The dynamics and control of eulerian turbomachines," Journal of Dynamic Systems, Measurement, and Control, 2, pp. 198-205.

Perlini, S., Solda, P. L., Piepoli, M., Calciati, A., Paro, M., Marchetti, G., Meno, F., Finardi, G. and Bernardi, L. (1991), "Time course of pressure and flow in ascending aorta during ejection," International Journal of Cardiology, 30, pp. 169-179.

Peskin, C. S., Kresch, E. and Noordergraaf, A. (1970), "Comment on "a mathematical model for the pressure-flow relationship in a segment of vein"," IEEE Transactions on Biomedical Engineering, 17, pp. 270-271.

Piiroinen, P. T., Virgin, L. N. and Champneys, A. R. (2004), "Chaos and period-adding: experimental and numerical verification of the grazing bifurcation," Journal of Nonlinear Science, 14, pp. 383-404.

Pilgram, B. and Kaplan, D. T. (1999), "Nonstationarity and 1/f noise characteristics in heart rate," American Journal of Physiology, 276, pp. R1-9.

Pirkle, J. C. and Gann, D. S. (1975), "Restitution of blood volume after hemorrhage: mathematical description," American Journal of Physiology, 228, pp. 821-827.

Pohl, M., Wendt, M. O., Werner, S., Koch, B. and Lerche, D. (1996), "In vitro testing of artificial heart valves: comparison between Newtonian and non-Newtonian fluids," Artificial Organs, 20, pp. 37-46.

Popel, A. S., Regirer, S. A. and Usick, P. I. (1974), "A continuum model of blood flow," Biorheology, 11, pp. 427-437.

Poullis, M. (1999), "Simulation of arterial hemodynamics after prosthetic replacement of the aorta," Annals of Thoracic Surgery, 68, pp. 1441-1442. 
Prabhu, S. D. (1999), "Load sensitivity of left ventricular relaxation in normal and failing hearts: evidence of a nonlinear biphasic response," Cardiovascular Research, 43, pp. 354-363.

Puskorius, G. V. and Feldkamp, L. A. (1994), "Neurocontrol of nonlinear dynamicalsystems with Kalman filter trained recurrent networks," IEEE Transactions on Neural Networks, 5, pp. 279-297.

Qassem, W., Jarrah, M. and Othman, M. (1998), "Heart response to horizontal impulse," Journal of Medical Engineering and Technology, 22, pp. 82-90.

Qiu, Y. and Tarbell, J. M. (1996), "Computational simulation of flow in the end-to-end anastomosis of a rigid graft and a compliant artery," ASAIO Journal, 42, pp. M702M709.

Rideout, V. C. (1991), Mathematical and computer modeling of physiological systems, Prentice Hall Biophysics and Bioengineering Series, Prentice Hall, Englewood Cliffs, NJ.

Ripoll, J. J. G. and Perez-Garcia, V. M. (1999), "Barrier resonances in Bose-Einstein condensation," Physical Review A, 59, pp. 2220-2231.

Robicsek, F., Thubrikar, M. J., Fokin, A., Tripp, H. F. and Fowler, B. (1999), “Pressure traps in femoro-popliteal reversed vein grafts - are valves culprits?" Journal of Cardiovascular Surgery, 40, pp. 683-689.

Rosenberg, G., Landis, D. L., Phillips, W. M., Stallsmith, J. and Pierce, W. S. (1978), “Determining arterial pressure, left atrial pressure and cardiac output from the left pneumatic drive line of the total artificial heart," ASAIO Transactions, 24, pp. 341-344.

Rosenson, R. S., McCormick, A. and Uretz, E. F. (1996), "Distribution of blood viscosity values and biochemical correlates in healthy adults," Clinical Chemistry, 42, pp. 11891195.

Rushmer, R. F. (1976), Cardiovascular Dynamics, 4th ed., Saunders, Philadelphia.

Saba, P. S., Ganau, A., Devereux, R. B., Pini, R., Pickering, T. G. and Roman, M. J. (1999), "Impact of arterial elastance as a measure of vascular load on left ventricular geometry in hypertension," Journal of Hypertension, 17, pp. 1007-1015. 
Sahar, T., Shalev, A. Y. and Porges, S. W. (2001), "Vagal modulation of responses to mental challenge in posttraumatic stress disorder," Biological Psychiatry, 49, pp. 637-643.

Sato, T., Yamashiro, S. M., Vega, D. and Grodins, F. S. (1974), "Parameter sensitivity analysis of a network model of systemic circulatory mechanics," Annals of Biomedical Engineering, 2, pp. 289-306.

Schima, H., Honigschnabel, J., Trubel, W. and Thoma, H. (1990), "Computer simulation of the circulatory system during support with a rotary blood pump," ASAIO Transactions, 36, pp. M252-M254.

Schwid, H. A., Taylor, L. A. and Smith, N. T. (1987), "Computer model analysis of the radial artery pressure waveform," Journal of Clinical Monitoring, 3, pp. 220-228.

Scotten, L. N., Walker, D. K. and Brownlee, R. T. (1979), "Construction and evaluation of a hydromechanical simulation facility for the assessment of mitral valve prostheses," Journal of Medical Engineering and Technology, 3, pp. 11-18.

Sedivy, R., Thurner, S., Kastner, J. and Maurer, G. (2000), “Nonlinear dynamics, chaos theory and wavelet analysis of the heart," Wiener Klinische Wochenschrift, 112, pp. 177183.

Segers, P., Stergiopulos, N., Schreuder, J. J., Westerhof, B. E. and Westerhof, N. (2000), "Left ventricular wall stress normalization in chronic pressure-overloaded heart: a mathematical model study," American Journal of Physiology - Heart and Circulatory Physiology, 279, pp. H1120-H1127.

Segers, P., Stergiopulos, N. and Westerhof, N. (2002), "Relation of effective arterial elastance to arterial system properties," American Journal of Physiology - Heart and Circulatory Physiology, 282, pp. H1041-H1046.

Segers, P., Stergiopulos, N., Westerhof, N., Wouters, P., Kolh, P. and Verdonck, P. (2003), "Systemic and pulmonary hemodynamics assessed with a lumped-parameter heartarterial interaction model," Journal of Engineering Mathematics, 47, pp. 185-199.

Shahcheraghi, N., Dwyer, H. A., Cheer, A. Y., Barakat, A. I. and Rutaganira, T. (2002), "Unsteady and three-dimensional simulation of blood flow in the human aortic arch," Journal of Biomechanical Engineering, 124, pp. 378-387. 
Shampine, L. F. and Reichelt, M. W. (1997), "The MATLAB ODE suite," SIAM Journal on Scientific Computing, 18, pp. 1-22.

Shannon, C. E. (1948), "A mathematical theory of communication," Bell System Technical Journal, 27, pp. 379-423.

Sharp, M. K. and Dharmalingham, R. K. (1999), "Development of a hydraulic model of the human systemic circulation," ASAIO Journal, 45, pp. 535-540.

Sharp, M. K., Pantalos, G. M., Minich, L., Tani, L. Y., McGough, E. C. and Hawkins, J. A. (2000), "Aortic input impedance in infants and children," Journal of Applied Physiology, 88, pp. 2227-2239.

Sipkema, P., Westerhof, N. and Randall, O. S. (1980), "The arterial system characterised in the time domain," Cardiovascular Research, 14, pp. 270-279.

Smith, D. and Craige, E. (1986), "Mechanism of the dicrotic pulse," British Heart Journal, 56, pp. 531-534.

Sneckenberger, D. S., Stinebring, D. R., Deutsch, S., Geselowitz, D. B. and Tarbell, J. M. (1996), "Mitral heart valve cavitation in an artificial heart environment," The Journal of Heart Valve Disease, 5, pp. 216-27.

Sneyd, J., ed. (2002), An introduction to mathematical modeling in physiology, cell biology, and immunology: American Mathematical Society, Short Course, January 8-9, 2001, New Orleans, LA, American Mathematical Society, Providence, RI.

Stamatis, N., Parthimos, D. and Griffith, T. M. (1999), "Forecasting chaotic cardiovascular time series with an adaptive slope multilayer perceptron neural network," IEEE Transactions on Biomedical Engineering, 46, pp. 1441-1453.

Starling, M. R., Walsh, R. A., Dell'Italia, L. J., Mancini, G. B., Lasher, J. C. and Lancaster, J. L. (1987), "The relationship of various measures of end-systole to left ventricular maximum time-varying elastance in man," Circulation, 76, pp. 32-43.

Stergiopulos, N., Meister, J. J. and Westerhof, N. (1994), "Simple and accurate way for estimating total and segmental arterial compliance: the pulse pressure method," Annals of Biomedical Engineering, 22, pp. 392-397. 
Stergiopulos, N., Meister, J. J. and Westerhof, N. (1995a), "Evaluation of methods for estimation of total arterial compliance," American Journal of Physiology, 268, pp. H1540H1548.

Stergiopulos, N., Meister, J. J. and Westerhof, N. (1995b), "Scatter in input impedance spectrum may result from the elastic nonlinearity of the arterial wall," American Journal of Physiology, 269, pp. H1490-H1495.

Stergiopulos, N., Westerhof, B. E. and Westerhof, N. (1999), "Total arterial inertance as the fourth element of the windkessel model," American Journal of Physiology, 276, pp. H81-H88.

Stremel, R. W., Bernauer, E. M., Harter, L. W., Schultz, R. A. and Walters, R. F. (1975), "Modeling static and dynamic human cardiovascular responses to exercise," Computer Programs in Biomedicine, 4, pp. 246-252.

Strogatz, S. H. (1994), Nonlinear Dynamics and Chaos: with applications to physics, biology, chemistry, and engineering, Studies in Nonlinearity, Addison-Wesley, Reading, MA.

Swanson, W. M. and Clark, R. E. (1977a), "Cardiovascular system simulation requirements," Journal of Bioengineering, 1, pp. 121-133.

Swanson, W. M. and Clark, R. E. (1977b), "A simple cardiovascular system simulator: design and performance," Journal of Bioengineering, 1, pp. 135-145.

Szemplianska-Stupnicka, W. and Troger, H. (1991), Engineering Applications of Dynamics of Chaos, Springer-Verlag, Wien; New York.

Szucs, B. and Monos, E. (1970), "Circulatory system analysis by a stochastic method using an analogue correlator," International Journal of Bio-Medical Computing, 1, pp. 87102.

Takagaki, M., Rottenberg, D., McCarthy, P. M., Smedira, N. G., Dessoffy, R., Al-Ahmadi, M., Shoshani, D. and Fukamachi, K. (2001), "A novel miniature ventricular assist device for hemodynamic support," ASAIO Journal, 47, pp. 412-416.

Tan, S. (1996), "Development of a dynamic model of a ventricular assist device for investigation of control systems," Master's thesis, Carleton University. 
Testa, H. J., Pullan, B. R. and Rowlands, D. J. (1975), “A computer model and a mechanical model of the circulation and their use in the evaluation of indices of myocardial blood flow," European Journal of Intensive Care Medicine, 1, pp. 175-183.

Thiry, P. S. and Roberge, F. A. (1976), “Analogues et modèles de l'écoulement arteriel systèmique," Revue Canadienne de Biologie, 35, pp. 217-238.

Thompson, D. R., Cmolik, B. L., Cheever, E. A., Geha, A. S. and George, D. T. (1997), "Optimizing muscle-to-cardiac timing for aortomyoplasty," Biomedical Sciences Instrumentation, 33, pp. 486-490.

Thyrault, M., Teboul, J. L., Richard, C., Coirault, C., Lecarpentier, Y. and Chemla, D. (1998), "Relation between dicrotic notch and mean pulmonary artery pressure studied by using a Swan-Ganz catheter in critically ill patients," Intensive Care Medicine, 24, pp. 77-80.

Torizuka, K. (1968), "Functional tests of the circulatory system," Japanese Journal of Clinical Pathology, 16, pp. 630-634.

Tortora, G. J. and Grabowski, S. R. (2000), Principles of Anatomy and Physiology, 9th ed., Benjamin Cummings, San Francisco, CA.

Toulemonde, C. and Gontier, C. (1998), "Sticking motions of impact oscillators," European Journal of Mechanics A - Solids, 17, pp. 339-366.

Tozeren, A. (1984), "Elastic properties of arteries and their influence on the cardiovascular system," Journal of Biomechanical Engineering, 106, pp. 182-185.

Tsach, U., Geselowitz, D. B., Sinha, A. and Hsu, H. K. (1989), "A novel output feedback pusher plate controller for the Penn State electric ventricular assist device," Journal of Dynamic Systems, Measurement, and Control, 111, pp. 69-74.

Tsitlik, J. E., Halperin, H. R., Popel, A. S., Shoukas, A. A., Yin, F. C. and Westerhof, N. (1992), "Modeling the circulation with three-terminal electrical networks containing special nonlinear capacitors," Annals of Biomedical Engineering, 20, pp. 595-616.

Tsukiya, T., Taenaka, Y., Tatsumi, E. and Takano, H. (2001), "Performance of a newly developed implantable centrifugal blood pump," ASAIO Journal, 47, pp. 559-562. 
Tufillaro, N. B. (1994), "Braid analysis of a bouncing ball," Physical Review E, 50, pp. 45094522 .

Valente, A. X., McClamroch, N. H. and Mezic, I. (2005), “Hybrid dynamics of two coupled oscillators that can impact a fixed stop," http://www. arxiv.org/pdf/nlin.CD/ 0309060 .

Vandenberghe, S., Segers, P., Meyns, B. and Verdonck, P. (2001), “Hydrodynamic characterisation of ventricular assist devices," International Journal of Artificial Organs, 24, pp. $470-477$.

Venkatesan, A. and Lakshmanan, M. (1998a), "Different routes to chaos via strange nonchaotic attractor in a quasiperiodically forced system," http://www.arxiv.org/ pdf/chao-dyn/9806008.

Venkatesan, A. and Lakshmanan, M. (1998b), "Different routes to chaos via strange nonchaotic attractors in a quasiperiodically forced system," Physical Review E, 58, pp. 3008-3016.

Vermette, P., Thibault, J. and Laroche, G. (1998), "A continuous and pulsatile flow circulation system for evaluation of cardiovascular devices," Artificial Organs, 22, pp. 746752.

Vetter, R., Celka, P., Vesin, J. M., Thonet, G., Pruvot, E., Fromer, M., Scherrer, U. and Bernardi, L. (1998), "Subband modeling of the human cardiovascular system: new insights into cardiovascular regulation," Annals of Biomedical Engineering, 26, pp. 293-307.

Vidyasagar, M. (1978), Nonlinear Systems Analysis, Prentice-Hall, Englewood Cliffs, NJ.

Vlasov, G. (2003), "Chaos as a basis of new principle for detecting the gravitational waves," http://www.arxiv.org/pdf/chao-dyn/9904010.

Wagner, C. D., Nafz, B. and Persson, P. B. (1996), "Chaos in blood pressure control," Cardiovascular Research, 31, pp. 380-387.

Wagner, C. D. and Persson, P. B. (1998), "Chaos in the cardiovascular system: an update," Cardiovascular Research, 40, pp. 257-264. 
Walker, J. S. and Soule, T. (1996), "Chaos in a simple impact oscillator: the Bender bouncer," American Journal of Physics, 64, pp. 397-409.

Wallner, F. (1974), "Development of a circulation mock-up," Langenbecks Archiv für Chirurgie, 335, pp. 61-65.

Wemple, R. M. (1972), "Origin of the dicrotic notch and wave," Biomedical Sciences Instrumentation, 9, pp. 45-7.

Westerhof, N. and Elzinga, G. (1991), "Normalized input impedance and arterial decay time over heart period are independent of animal size," American Journal of Physiology, 261, pp. R126-R133.

Westerhof, N. and Stergiopulos, N. (1996), "Role of input impedance on ventricular efficiency," Proceedings of the 1996 18th Annual International Conference of the IEEE Engineering in Medicine and Biology Society, Part 3 (of 5), Oct 31-Nov 3, Amsterdam, Netherlands, 3, IEEE, Piscataway, NJ, pp. 1349-1350.

White, F. M. (1994), Fluid Mechanics, 3rd ed., McGraw-Hill, New York.

Wieting, D. W., Akers, W. W., Feola, M. and Kennedy, J. H. (1971), “Analysis of a variable volume intraortic balloon pump in a mock circulatory system," Journal of Biomechanics, 4 , pp. $37-44$.

Winder, S. (2002), Analog and Digital Filter Design, 2nd ed., EDN series for design engineers, Newnes, Amsterdam; Boston; London.

Withington, P. S., Graham, T. R., Meikle, R. J., Allan, A., Marrinan, M. T. and Lewis, C. T. (1991), "In vitro evaluation of an implantable left ventricular assist device," Journal of Medical Engineering and Technology, 15, pp. 68-71.

Wolf, L. and Clinch, J. M. (1972), "Mock circulatory system for intra-aortic balloon testing," IEEE Transactions on Biomedical Engineering, 19, pp. 38-46.

Wolner, E., Thoma, H., Deutsch, M., Eckersberger, F., Fasching, W., Horcher, E., Losert, U., Stellwag, F., Stohr, H., Unger, F., Weisskirchner, R. and Polzer, K. (1979), "The research programme "Artificial Heart" at the 2nd Department of Surgery, University of Vienna: a ten year review," Wiener Klinische Wochenschrift, 91, pp. 74-81. 
Woodruff, S. J., Sharp, M. K. and Pantalos, G. M. (1997), "Compact compliance chamber design for the study of cardiac performance in microgravity," ASAIO Journal, 43, pp. 316-320.

Wu, Z. J., Wang, Y. and Hwang, N. H. (1994), “Occluder closing behavior: a key factor in mechanical heart valve cavitation," Journal of Heart Valve Disease, 3 Suppl 1, pp. S25-S34.

Xu, L. and Fu, M. (2000), "Computer modeling of interactions of an electric motor, circulatory system, and rotary blood pump," ASAIO Journal, 46, pp. 604-611.

Yambe, T., Hashimoto, H., Kobayashi, S., Sonobe, T., Naganuma, S., Nanka, S. S., Matsuki, H., Yoshizawa, M., Tabayashi, K., Takayasu, H., Takeda, H. and Nitta, S. (1997a), "Development and evaluation of totally implantable ventricular assist system using a vibrating flow pump and transcutaneous energy transmission system with amorphous fibers," Heart Vessels, Suppl 12, pp. 41-43.

Yambe, T., Kobayashi, S., Sonobe, T., Naganuma, S., Nanka, S., Hashimoto, H., Yoshizawa, M., Tabayashi, K., Takayasu, H., Takeda, H. and Nitta, S. (1997b), "Nonlinear mathematical analysis of the hemodynamic parameters during left ventricular assistance with oscillated blood flow," Artificial Organs, 21, pp. 625-629.

Yambe, T., Nanka, S., Kobayashi, S., Tanaka, A., Owada, N., Yoshizawa, M., Abe, K., Tabayashi, K., Takeda, H., Nishihira, T. and Nitta, S. (1999), "Detection of the cardiac function by fractal dimension analysis," Artificial Organs, 23, pp. 751-756.

Yambe, T., Nanka, S., Kobayashi, S., Tanaka, A., Yoshizawa, M., Abe, K., Tabayashi, K., Takeda, H. and Nitta, S. (1998), "Origin of chaos in the circulation: open loop analysis with an artificial heart," ASAIO Journal, 44, pp. M700-M703.

Yambe, T., Nanka, S., Sonobe, T., Naganuma, S., Kobayashi, S., Akiho, H., Kakinuma, Y., Mitsuoka, M., Chiba, S. and Ohsawa, N. (1995), "Chaotic behavior of hemodynamics with ventricular assist system," International Journal of Artificial Organs, 18, pp. 1721.

Yambe, T., Nitta, S., Sonobe, T., Naganuma, S., Kobayashi, S., Haga, Y., Tanaka, M., Fukuju, T., Miura, M. and Sato, N. (1993), "Identification of the deterministic chaos in cardiovascular dynamics by the use of the non-linear mathematics," The Science Reports of the Research Institutes, Tohoku University (Series C-Medicine), 39, pp. 1-5. 
Yearwood, T. L. and Chandran, K. B. (1982), "Physiological pulsatile flow experiments in a model of the human aortic arch," Journal of Biomechanics, 15, pp. 683-704.

Ying, H. and Sheppard, L. C. (1994), "Regulating mean arterial pressure in postsurgical cardiac patients," IEEE Engineering in Medicine and Biology, 13, pp. 671-677.

Yoshigi, M., Knott, G. D. and Keller, B. B. (2000), "Lumped parameter estimation for the embryonic chick vascular system: a time-domain approach using MLAB," Computer Methods and Programs in Biomedicine, 63, pp. 29-41.

Yoshikawa, M., Nakata, K., Ohtsuka, G., Takano, T., Glueck, J., Fujisawa, A., Makinouchi, K., Yokokawa, M. and Nose, Y. (1999), "Feasibility of a tiny Gyro centrifugal pump as an implantable ventricular assist device," Artificial Organs, 23, pp. 774-779.

Yoshizawa, M., Takeda, H., Miura, M., Yambe, T., Katahira, Y. and Nitta, S. (1993), "Realtime cardiac output estimation of the circulatory system under left ventricular assistance," IEEE Transactions on Biomedical Engineering, 40, pp. 266-275.

Yu, Y.-C., Boston, J. R., Simaan, M. A. and Antaki, J. F. (2002), "Minimally invasive estimation of systemic vascular parameters for artificial heart control," Control Engineering Practice, 10, pp. 277-285.

Yu, Y. C., Boston, J. R., Simaan, M. A., Miller, P. J. and Antaki, J. F. (2001), “Pressure-volume relationship of a pulsatile blood pump for ventricular assist device development," ASAIO Journal, 47, pp. 293-301.

Zeiny, A. (2000), "Nonlinear time-dependent seismic response of unanchored liquid storage tanks," Ph.D. thesis, University of California, Irvine.

Zwiener, U., Hoyer, D., Bauer, R., Luthke, B., Walter, B., Schmidt, K., Hallmeyer, S., Kratzsch, B. and Eiselt, M. (1996), "Deterministic-chaotic and periodic properties of heart rate and arterial pressure fluctuations and their mediation in piglets," Cardiovascular Research, 31, pp. 455-465. 


\title{
Appendix A
}

\section{Human Circulatory System Simulation}

\begin{abstract}
ECTION 2.6 presented a mathematical model of the human circulatory system. To preD dict the behaviour of the cardiovascular system, Equations (2.45-2.57), along with their supporting constitutive relations, can be numerically integrated with respect to time.
\end{abstract}

Software packages such as MATLAB or Mathematica are well suited to this task because their numerous integration routines are well vetted. Coding and debugging a fast and efficient numerical routine is a formidable task. This appendix presents the Mathematica worksheet for the human circulatory system model described in Section 2.6. Both the arterial and venous models are the so-called "dual-compliance models" outlined in Chapter 2. Rather than using discontinuous constitutive functions for the VAD valves and the venous resistance, the simulation employs sigmoidal curves to smooth the transition between fully-open and fully-closed. This change adds some computational overhead to the simulation (as the constitutive equations for the valves and venous resistance are more complex) but improves the realism; valves do not instantaneously switch from fully-open to fully-closed (or vice versa) and tubes do not instantaneously collapse or swell.

The MATLAB version of this simulation is included (in abbreviated form) as an appendix to Conlon et al. (2006). 


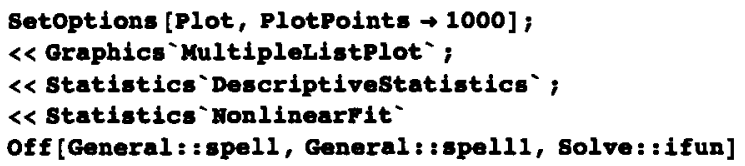

To convert from $\mathrm{mmHg}$ to $\frac{\mathrm{N}}{\mathrm{cm}^{2}}$, take the density of Mercury, $13579.04 \frac{\mathrm{kg}}{\mathrm{m}^{3}}$, and multiply it by the gravitational constant, $9.81 \frac{\mathrm{m}}{\mathrm{s}^{2}}$, and the height, $\mathrm{mm}$. Multiply the result by $\frac{1 \mathrm{~cm}}{10 \mathrm{~mm}}$ and $\left(\frac{1 \mathrm{~m}}{100 \mathrm{~cm}}\right)^{3}$ for unit conversion.

gravity $=9.81$

density Hg $=13579.04$

Hg2W $=$ densityHg $*$ gravity $*(1 / 10) *(1 / 100) \wedge 3$

H2Hg = $1 / \mathrm{Hg} 2 \mathrm{~N}$

Area $=19.27$

Fluid parameters for blood... density in $\frac{\mathrm{kg}}{\mathrm{m}^{3}}$ and viscosity in $\frac{\mathrm{kg}}{\mathrm{ms}}$.

densityH20 $=1070$

viscosityH2O = $5 * 10^{\wedge}-3$;

Pipe volume; substitute length and diameter to return volume with units $\mathrm{cm}^{2}$

PipeVolume $=$ Length $* P i *$ Diameter ${ }^{\wedge} 2 / 4 ;$

Pipe flow inertia function; substitute length and diameter to return flow inertia with units $\frac{\mathrm{Ns}}{\mathrm{cm}^{5}}$

PipeflowInertia $=$ densityn20*(1/100)^4*Length $/(P i / 4 *$ Diameter^2);

Pipe flow resistance function; substitute length and diameter to return flow resistance with units $\frac{\mathrm{N} s}{\mathrm{am}^{\mathrm{s}}}$

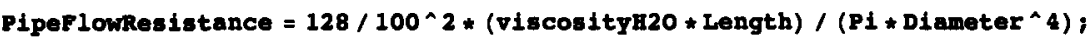


This is the desired blood volume flow rate at the periphery (in $\frac{\mathrm{m}^{3}}{5}$ )

DesiredPeripheralflownate $=80$

Length of the arotic segment

AortaLength $=20$;

Cross-sectional area of the aortic segment

Aortacsec $=P i / 4 * 2.5^{\wedge} 2$

This is to deal with the abnormally small cross-sectional area of $2.5 \mathrm{~cm}^{2}$ in the King paper

CrossSectionalscale $=$ MortaCSec $/ 2.5$

Our nominal aorta volume, just under $100 \mathrm{~cm}^{3}$

Aortavolume $=$ MortaLength $*$ AortaCSec;

Our nominal arterial system volume

Totalarterialvolume $=700$

Aortic pressure-volume data from King's paper

MortapvData $=\{\{1.35,12.5\},\{1.525,25\},\{1.725,37.5\}$

$\{1.975,50\},\{2.2,62.5\},\{2.375,75\},\{2.5,87.5\},\{2.65,100\}$,

$\{2.75,112.5\},\{2.85,125\},\{2.95,137.5\},\{3.025,150\},\{3.1,162.5\}$,

$\{3.175,175\},\{3.225,187.5\},\{3.275,200\},\{3.315,212.5\},\{3.34,225\}\}$;

Scale it by the length of the segment; adjust the cross-sectional area

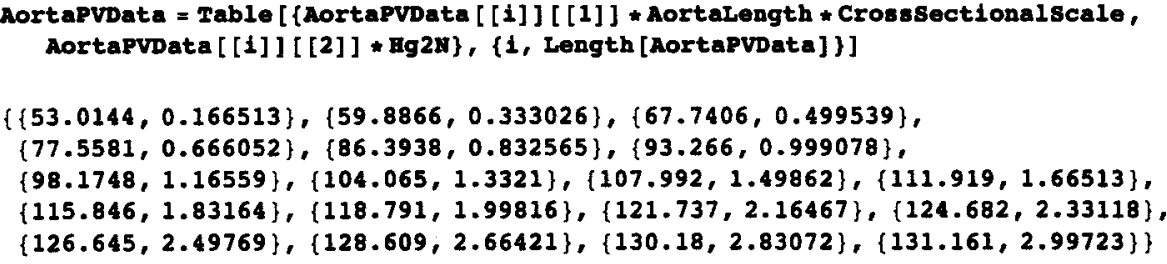


Linear curve fit for the aortic PV data

LinearCurve = Fit [AortaPVData , $\{1, \mathrm{v}], \mathrm{v}]$

$-1.94226+0.0341474 \mathrm{~V}$

Cubic curve fit for the aortic PV data

CubicCurve = Fit $\left[\right.$ Aortapvata , $\left.\left\{1, v, v^{\wedge} 2, v^{\wedge} 3\right\}, v\right]$

$-4.07967+0.151211 v-0.00175213 v^{2}+7.66459 \times 10^{-6} v^{3}$

Exponential curve fit for the aortic PV data

MonlinearFit [Table [ \{AortapVData [ [1] ] [ [2] ], AortapvData [ [1]] [ [1]]\},

$\{1$, Length [AortapVData $]\}], \mathbf{A}-\mathbf{B} * \operatorname{Exp}[-\mathrm{C} \star \mathrm{P}], \mathbf{P},\{\mathrm{A}, \mathrm{B}, \mathrm{C}\}$, MaxIterations $\rightarrow 1000]$;

Exponentialcurve $=\operatorname{simplify}[801 v e[=v, P][[1]][[1]][[2]]]$

$-1.42789 \log [1.36808-0.00943739 \mathrm{v}]$

This is the valve flow resistance function; it accepts a pressure (deita) input in $\frac{\mathrm{N}}{\mathrm{cm}^{2}}$ and returns a flow in $\frac{\mathrm{m}}{\mathrm{s}}$

$\mathbf{R}\left[\mathbf{x} \mathbf{x}_{-}\right]:=$

Module $\left[\{\right.$ RMin, RMax, slope, Offset $\}, \operatorname{RMin}=8 . * 10^{\wedge}-4 ;$ RMax $=1.25 * 10^{\wedge} 3 ;$ slope $=100$;

offset $=0 . ; \mathrm{x}[\mathrm{xx}$ *(RMin $+(\operatorname{RMax}-\operatorname{RMin}) /(1+\operatorname{Exp}[-2 * \operatorname{slope} *(x x+0$ ffset $)]))]]$; 
Plot $[R[t],\{t,-1,1\}]$

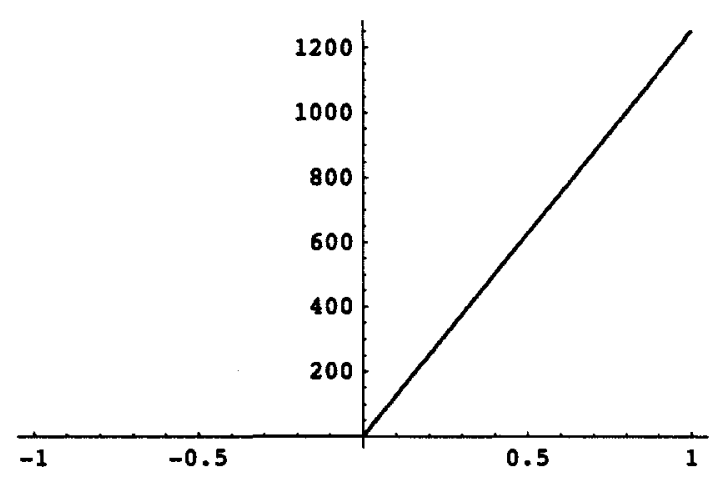

This is the venous flow resistance function; it accepts a pressure (delta) input in $\mathrm{mmHg}$ and returns a flow rate in $\frac{\mathrm{ml}}{\mathrm{s}}$

RVeins [xx_] : =

Module [ $\{\operatorname{Rmin}, \operatorname{Rmax}$, slope, Offset $], \operatorname{Amin}=10 / 0.067 ; \operatorname{Rmax}=650 / 0.067 ;$ slope $=10$;

offset $=0.4 ; x[x x *(\operatorname{Rmin}+(\operatorname{Rmax}-\operatorname{Rmin}) /(1+\operatorname{Exp}[2 * 81$ ope $*(x x-0 f f \operatorname{set})]))]]$; 
Plot $[R V e i n s[x],\{x,-2,2\}]$

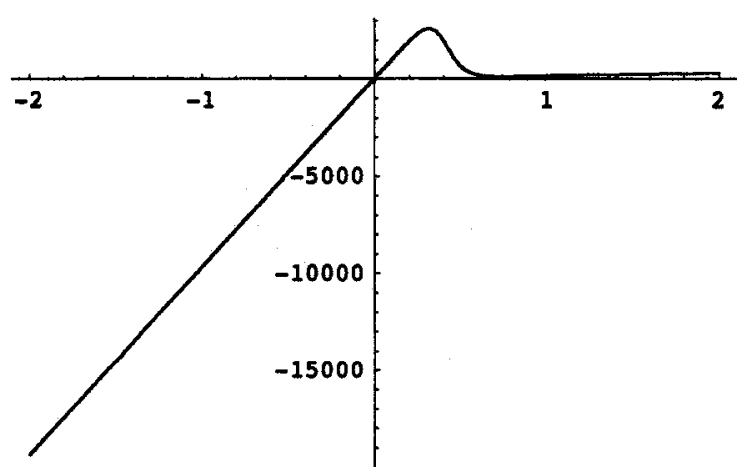

This is the VAD chamber's constitutive relation; it accepts a volume input in $\mathrm{ml}$ and returns a pressure in $\frac{\mathrm{N}}{\mathrm{cm}^{2}}$

PHeart [xx_] : = Module [ $\{$ Mominalv, HominalP, Compliance, Offset $]$, HominalV $=46.0$; HominalP $=10 *$ Hg2H; Compliance $=135$;

offset $=(-$ Hominalv $/$ Compliance + HominalP $) ; w[x x /$ Compliance + Offset $]$;

This is the outlet cannula's constitutive relation; it accepts a volume input in $\mathrm{ml}$ and returns a pressure in $\frac{\mathrm{N}}{\mathrm{cm}^{2}}$

POutletCannula $\left[x x_{\text {_ }}\right]:=$ Kodule $[$ Hominalv, MominalP, Compliance, offset $\}$, Mominalv =

(Pipevolume $/$. Diameter $\rightarrow 2 /$. Length $\rightarrow 15)$; WominalP $=110 *$ Hg2H; Conpliance $=0.79$;

offset $=(-$ Hominalv / Compliance + HominalP $) ; \mathrm{w}[x \mathbf{x} /$ Compliance + Offset $]$;

This is the aorta chamber's constitutive relation; it accepts a volume input in $\mathrm{ml}$ and returns a pressure in $\frac{\mathrm{N}}{\mathrm{cm}^{2}}$

PAorta [xx_] : = A [LinearCurve $/, v \rightarrow x x]$;

This is the arteries' constitutive relation; it accepts a volume input in $\mathrm{ml}$ and returns a pressure in $\frac{\mathrm{N}}{\mathrm{cm}^{2}}$

Parteries [xx_] : = Module [ $\{$ hominalv, Hominalp, Compliance, offset $\}$, NominalV = TotalArterialVolume - AortaVolume; MominalP = $100 *$ Bg2N ; Compliance $=130-$ Solve $[1==$ LinearCurve $[[2]], v][[1]][[1]][$ [2] ] ;

Offset $=(-$ MominalV / Compliance + HoninalP $) ; \boldsymbol{H}[(x \mathrm{x} /$ Compliance + Offset $)]]$;

This is the venous chamber's constitutive relation; it accepts a volume input in $\mathrm{ml}$ and returns a pressure in $\frac{\mathrm{N}}{\mathrm{cm}^{2}}$

PVeins [xx] : = Module $[$ HominalV, Mominalp, Compliance, Offset $\}$, Nominalv $=2000$; MoninalP $=20 * \mathrm{Hg} 2 \mathrm{H}$; Compliance $=2500$;

Offset $=(-$ TominalV $/$ Compliance + Hominalp $) ;$ i $[(x x /$ Compliance + Offset $)]]$; 
This is the vena cava' sconstitutive relation; it accepts a volume input in $\mathrm{ml}$ and returns a pressure in $\frac{\mathrm{N}}{\mathrm{cm}^{2}}$

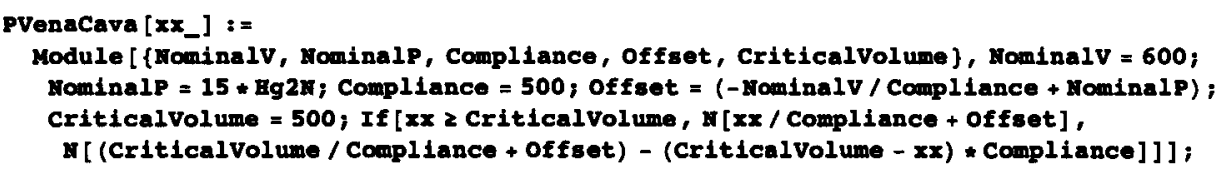

This is the inlet cannula's constitutive relation; it accepts a volume input in $\mathrm{ml}$ and returns a pressure in $\frac{\mathrm{N}}{\mathrm{cm}^{2}}$

PInletCannula [xx_] : = Module [ $\{$ Mominalv, Mominalp, Compliance, offset $\}$, Mominalv =

(PipeVolume /. Diameter $\rightarrow 2 /$. Length $\rightarrow 10$ ); NominalP $=15 *$ Hg2N; Compliance $=1.19$; offset $=(-$ Hominalv / Compliance + MominalP $) ; M[x x /$ Compliance + Offset $]$;

This is the fixed rate controller function; ejection duty cycle of 0.35

Force [xx_? Mumbere] : = Module [ $\{$ Minforce, Maxiorce, systole, Diastole $\}$, MinForce $=2.5 ;$ MaxForce $=37.5 ;$ systole $=0.35 ;$ Diastole $=0.65$; If [Mod [xx, systole + Dlastole] < Systole, MinForce + MaxForce, Minforce] ] ; 
Plot $[$ Force $[t],\{t, 0,2\}]$

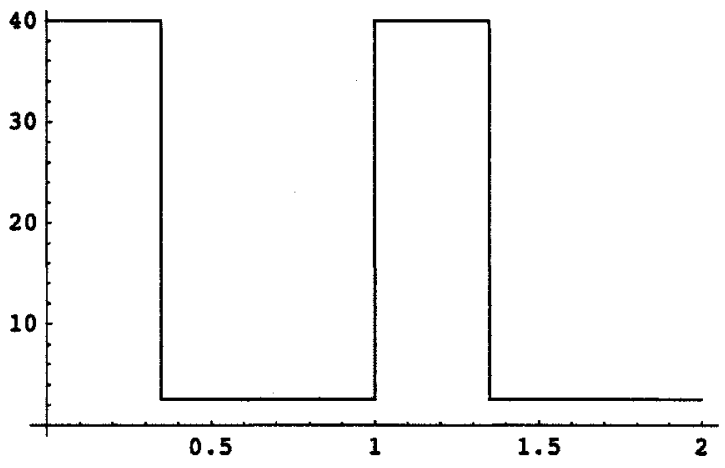

This is the FFFE controller

IASY STOLE = False; PREVIOUSTIME $=0$;

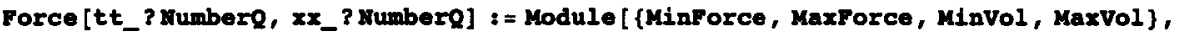
Minforce $=0,1$ MaxForce $=40 ;$ Minvol $=20$.; MaxVol $=100$.,

If [IMSYSTOLB = True, If [ ( $x \times \leq \mathrm{MInVO})$ i\& (PREVIOUSTIME < $t$ ) ,

IMSYstoLE = False; PREVIOUSTIA $=t t$; Kinforce, kaxporce + Minforce $]$,

If $[(x x \geq$ KaxVol) \& \& (PREVIOUSTINE < t $)$, IMsYsToLE = True;

PREVIOUSTIu $=t t$, MaxForce + MinForce, Minforce $]]]$

This is the residual beating function; currently zero

PResidualBeating [xx_] : =

Hodule [ Magnitude, Frequency , Magnitude $=0 ;$ Frequency $=0 ; 0]$;

Piston inertia

IPiston $=0.025$

Outlet cannula inertia

IOut letCannula $=$ PiperlowInertia $/$. Diameter $\rightarrow 2 /$. Length $\rightarrow 15$;

Reproduced with permission of the copyright owner. Further reproduction prohibited without permission. 
Aorta inertance

IAorta = PiperlowInertia /. Diameter $\rightarrow 2.5 /$. Length $\rightarrow 20$;

Peripheral inertia

IPeripheral $=0.0275$

Inlet cannula inertia

IInletCannula $=$ Piper lowinertia $/$. Diameter $\rightarrow 2 /$. Length $\rightarrow 10$;

Piston resistance (friction)

RPiston $=0.25$

Outlet cannula resistance

RoutletCannula $=0.0011$

Aorta resistance

RAorta $=0.0011$

Peripheral resistance

RPeripheral $=0.013$;

Inlet cannula resistance

RInletCannula $=$ PiperlowResistance $/$. Diameter $\rightarrow 2 /$. Length $\rightarrow 10$;

Piston position; not an independent state.

EQ1 = sPiston $[t]=$ pPiston $[t] /$ IPiston

Reproduced with permission of the copyright owner. Further reproduction prohibited without permission. 
Piston momentum for fixed rate mode

EQ2a $=$ pPiston ${ }^{\prime}[t]=$ Force $[t]-$ pPiston [t] / IPiston * RPiston - Area $*$ PHeart [VEeart [t]];

Piston momentum for FFFE mode

EQ2b = pPiston ' $[t]=$ Force $[t$, vHeart $[t]+100-\operatorname{sPiston}[t] *$ Area $]-$

pPiston [t] / IPiston * RPiston - Area * PHeart [VHeart [ $t$ ] ]

VAD compliance volume.

EQ3 $=$ vHeart ' $[t]=$ pPiston $[t] /$ IPiston $*$ Area +

R[PInletCannula [VInletCannula [t]] - PHeart [VHeart [t] ] ] -

$R$ [PHeart [VHeart [t]] - POutletCannula [VoutletCannula [t] ]

Outlet cannula volume.

E04 = voutletCannula ' $[t]=\mathbf{R}[$ PHeart [VHeart [t] ] - POutletCannula [vout letCannula [t] ] ] poutletCannula [t] / IoutletCannula;

Outlet cannula momentum.

BQ5 = poutletCannula $[t]=$ POutletCannula [VOutletCannula $[t]]-$

pout letCannula [t] / IOutletCannula * RoutletCannula - phorta [VAorta [t] ];

Aorta volume.

BQ6 = VAorta $[t]=$ poutletCannula $[t] /$ IOutletCannula - pAorta $[t] /$ IAorta

Aorta momentum.

$\mathbf{B Q 7}=$ pAorta $[t]=$ PAorta $[$ VAorta $[t]]$ -

pAorta [t] / IAorta * RAorta - PArteries [VArteries [t] ] + PResidualBeating [ $t$;

Reproduced with permission of the copyright owner. Further reproduction prohibited without permission. 
Arterial volume.

EQ8 = VArteries ' $[t]$ = pAorta $[t]$ / IAorta - pPeripheral $[t]$ / IPeripheral

Peripheral momentum

zo9 = pPeripheral $\cdot[t]=$

PArteries [VArteries [t]] - pperipheral [t] / IPeripheral * RPeripheral -

pVeins [VVeins [t] ] - pResidualBeating [t];

Venous volume

EQ10 = VVeins ' $[t]=$

pPeripheral [t] / IPeripheral - RVeins [PVeins [VVeins [t]] - PVenaCava[VVenaCava [t]]];

Vena cava volume

E011 = VVenaCava ' $[t]==$ RVeins [PVeins [VVeins [t]] - PVenaCava [VVenaCava [t] ] ] pInletCannula [t] / IInletcannula;

Inlet cannula momentum

Eq12 = pInletCannula ' $[t]$ = PVenaCava [VVenaCava [t] ] -

pInletCannula [t] / IInletCannula * RInletCannula - PInletCannula [vinletcannula [t]];

Inlet cannula volume

EQ13 = vinletCannula $[t]$ = pInletCannula $[t] /$ IInletCannula -

R [PInletCannula [VIn] etCannula [t] ] - PHeart [VHeart [t] ] ]

tStart $=0 . ;$ tstop $=30 . ;$ pstart $=27 . ;$ pstop $=30 . ;$

Reproduced with permission of the copyright owner. Further reproduction prohibited without permission. 


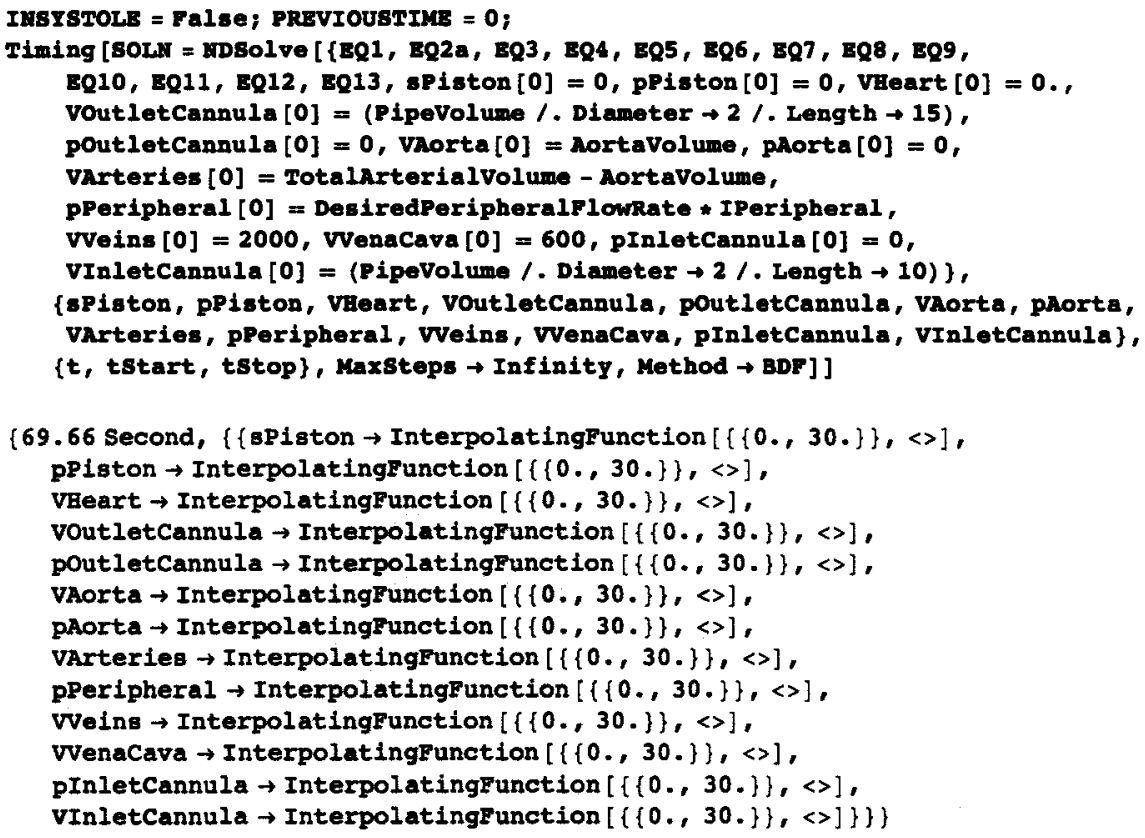

$\{69.66$ second, $\{$ sPiston $\rightarrow$ InterpolatingFunction $[\{\{0 ., 30\}\},.<>]$, pPiston $\rightarrow$ InterpolatingFunction $[\{\{0 ., 30\}\},.<>]$, VHeart $\rightarrow$ InterpolatingFunction $[\{\{0 ., 30\}\},.<>]$, voutletCannula $\rightarrow$ InterpolatingFunction $[\{\{0 ., 30\}\},.<>]$ poutletCannula $\rightarrow$ InterpolatingFunction $[\{\{0 ., 30\}\},.<>]$, vAorta $\rightarrow$ InterpolatingFunction $[\{\{0,30\}\},.<]$, pAorta $\rightarrow$ InterpolatingFunction $[\{\{0,30\}\},.\langle>]$, varteries $\rightarrow$ InterpolatingFunction $[\{\{0 ., 30\}\},.\langle>]$, pPeripheral $\rightarrow$ InterpolatingFunction $[\{\{0 ., 30\}\},.<]$, Weins $\rightarrow$ InterpolatingFunction $[\{\{0 ., 30\}\},.<>]$, Vvenacava $\rightarrow$ InterpolatingFunction $[\{\{0 ., 30\}\},.\langle>]$, pInletCannula $\rightarrow$ InterpolatingFunction $[\{(0 ., 30]\},.\langle>]$, vInletcannula $\rightarrow$ InterpolatingFunction $[(\{0 ., 30\}\},.<]\}\})$ 
Outlet valve flow rate versus time

Plot [R [PHeart [VHeart [t] ] - PoutletCannula [VoutletCannula [t] ] ] / sow, $\{t$, pstart, pstop\}, PlotRange $\rightarrow$ Al1];

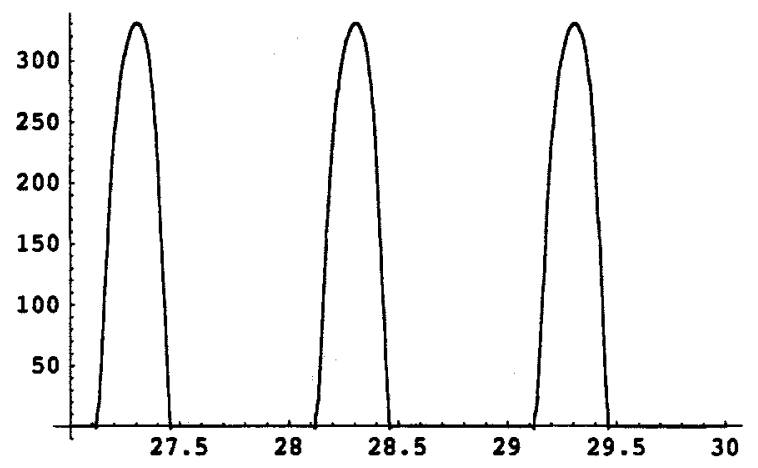

Find mean flow rate

Mean [Table [R [PHeart [VHeart [ $t]$ ] - POut letCannula [VOut letCannula [t] ] ] /. sou,

$\{t$, pstart, pstop, 0.001\}]]

$\{75.0865\}$ 
Aortic pressure versus time

Plot [PAorta [VAorta [t] ] * K2Bg /. soLN, \{t, pstart, pstop $\}$;

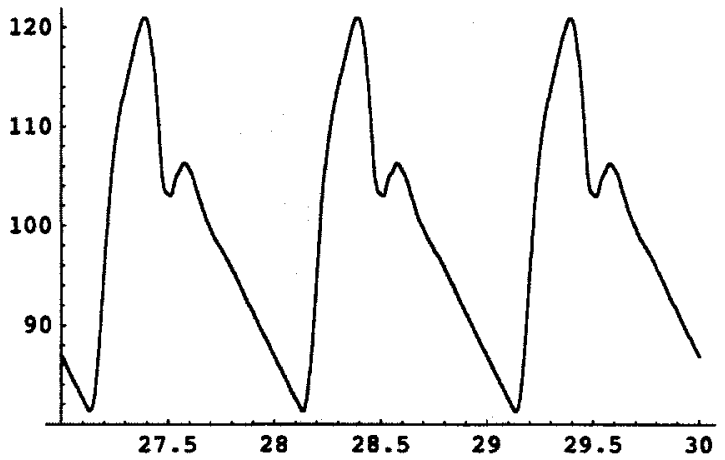

Check mean aortic pressure

Mean [Table [PAoxta [VAorta $[t]] *$ N2Hg /. soLk, \{t, pstart, pstop, 0.01\}] ]

$\{99.3443\}$

Arterial pressure versus time

Plot [ (PArteries [VArteries [t] ] / soum) $\star$ k2Bg, $\{t$, pstart, pstop $\}$;

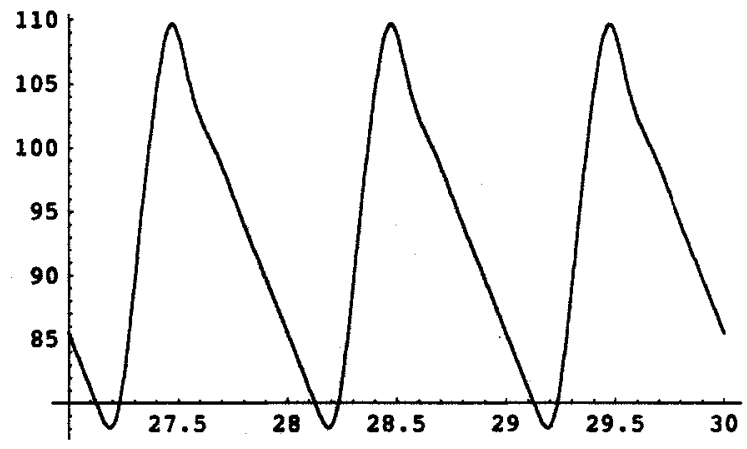


Peripheral flow rate versus time

Plot [ (pPeripheral [t] / IPeripheral) /. sowg, \{t, pstart, pstop\}];

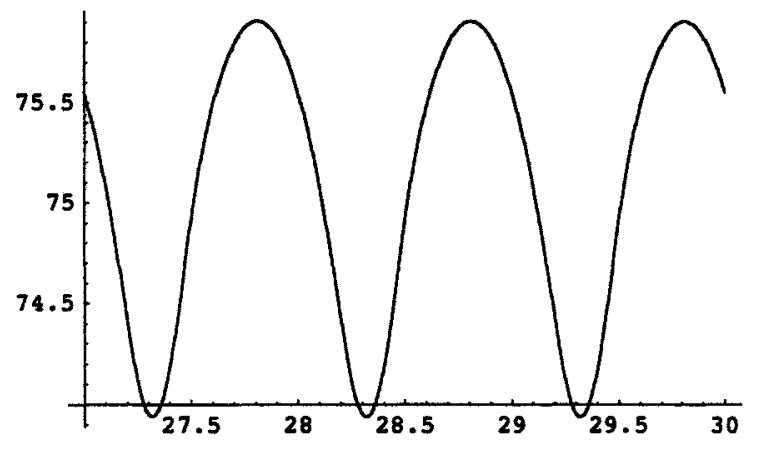

Check mean peripheral flow rate

Mean [Table [ (pPeripheral [t] / IPeripheral /. sows) [ [1]], (t, pstart, pstop, 0.001\}]]

75.1115 


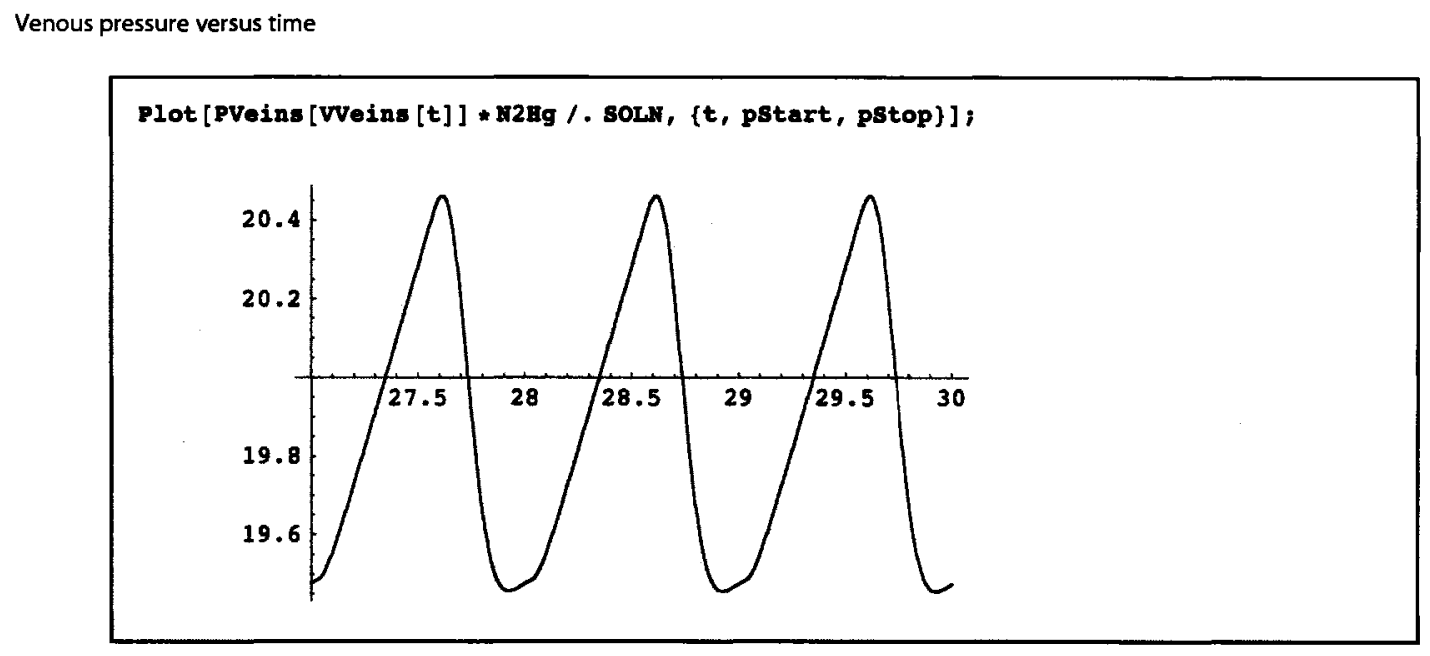

Vena cava pressure versus time

Plot [PVenaCava [VVenaCava [t]] * \$2Hg /. soun, $\{t$, pstart, pstop $]$;

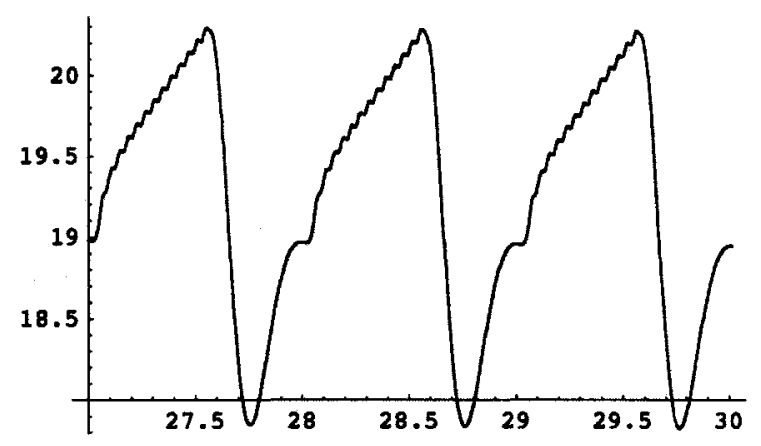


Inlet valve flow rate versus time

Plot [R [PInletCannula [VInletCannula [t] ] - PHeart [VHeart [ $t]$ ] ] . soLs, $\{t$, pstart, pstop\}, PlotRange $\rightarrow$ al1] ;

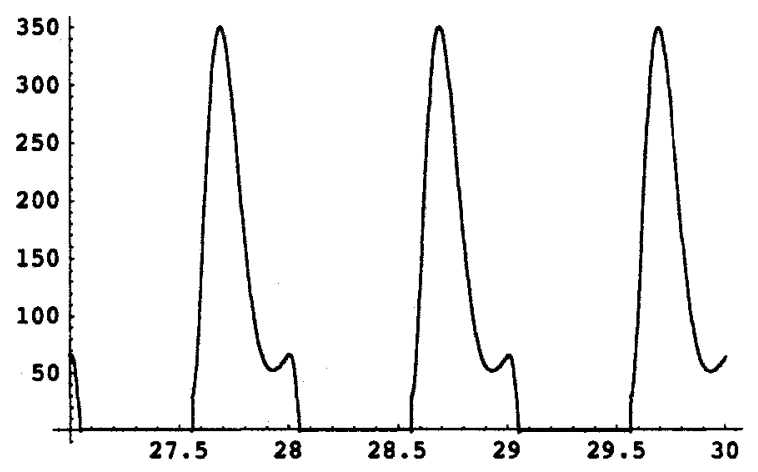

Find mean flow rate

Mean [Table [R [PInletCannula [VInletCannula [t]] - PHeart [VHeart [t]]] /. sous,

[t, pstart, pstop, 0.001\}]]

$\{75.0949\}$ 


\section{Appendix B}

\section{Linear Arterial System Models}

$\mathbf{T}$

HIS appendix presents the Mathematica worksheet calculations for the three different nondimensional models of the arterial system, with two different boundary conditions. In all three nondimensionalizations the flow-in, flow-out configuration is second order, due to the coupling between the two compliance volumes. This is readily seen in either the characteristic equation, or the differential equations describing the system.

No matter the nondimensionalization the arterial system allows oscillations for normal physiological parameters. Oscillations are bounded, indicating that the system is stable for the parameter intervals of interest, a result that is quite comforting! 


\section{Flow-in, flow-out with \{ IAorta, CArteries, QIn\} as repeating variables}

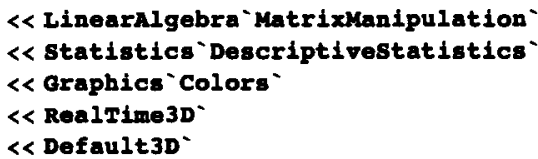

These are the $\{m\},\{L\}$ and $\{t\}$ units of all the variables in the flow-in-flow-out formulation. The columns represent (in order): RAorta, IAorta, CAorta, CArteries, RPeripheral, QIn, QOut and s.

$$
\begin{aligned}
& \text { Matrixform [FuldUnitkatrix = } \\
& \quad\{\{1,1,-1,-1,1,0,0,0\},\{-4,-4,4,4,-4,3,3,0\},\{-1,0,2,2,-1,-1,-1,-1\}\}] \\
& \left(\begin{array}{cccccccc}
1 & 1 & -1 & -1 & 1 & 0 & 0 & 0 \\
-4 & -4 & 4 & 4 & -4 & 3 & 3 & 0 \\
-1 & 0 & 2 & 2 & -1 & -1 & -1 & -1
\end{array}\right)
\end{aligned}
$$

Select repeating variables of IAorta, CArteries, QIn (these form the columns of "RepeatingVariabIeUnitMatrix"; the rows are $\{m\},\{L\}$ and $\{t\}\}$

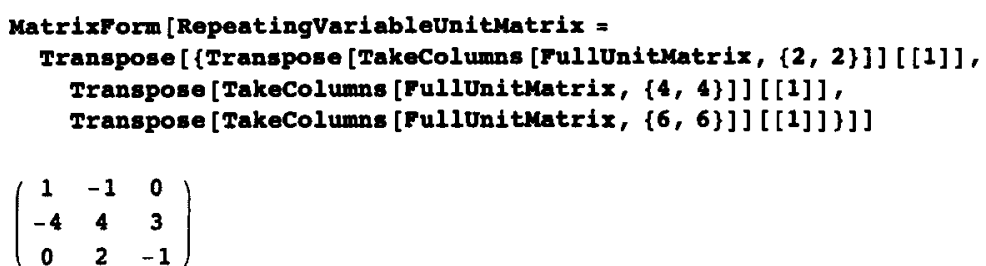

Buckingham's $\Pi$-Theorem... solve for the exponents

\{a [1], a [2], a [3]\} = Linearsolve [RepeatingVariableUnitMatrix -1 * Transpose [TakeColumns [Fullunityatrix, $\{1,1\}]][1]]]$; $\{a[4], a[5], a[6]\}=$ LinearSolve [RepeatingVariableUnitMatrix, -1 * Transpose [TakeColunns [FullUnitKatrix, \{3, 3\}] ] [ [1] ] ] ; $\{a[7], a[8], a[9]\}=$ Linearsolve [RepeatingVariableunitMatrix - 1* Transpose [TakeColums [FuIlUnitNatrix, \{5,5\}]] [ [1]]] ;

$\{a[10], a[11], a[12]\}=$ Linearsolve [RepeatingVariableUnitMatrix, -1 * Transpose [TakeColums [FullUnitkatrix, $\{7,7\}]][[1]]]$;

$\{a[13], a[14], a[15]\}=$ LinearSolve [RepeatingvariableUnityatrix -1 * Transpose [TakeColunns [FullUnitkatrix, $\{8,8\}]$ ] [ [1] ] ] ; 
First $\Pi$ group (RAorta plus repeating variables)

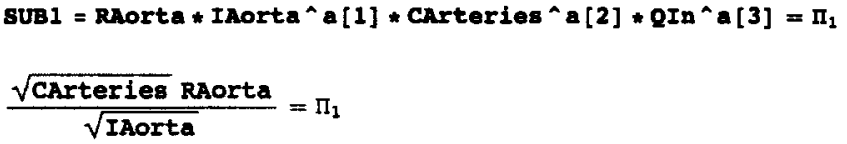

Second $\Pi$ group (CAorta plus repeating variables)

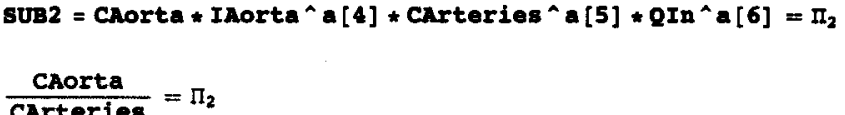

Third $\Pi$ group (RPeripheral plus repeating variables)

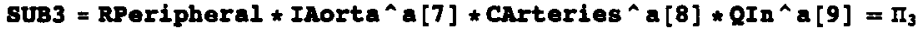

Fourth $\Pi$ group (@out plus repeating variables)

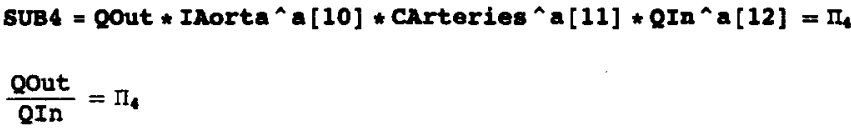

Fifth $\Pi$ group (s plus repeating variables)

suB5 $=8$ * Inorta^a [13] *CArteries^a [14] *QIn^a[15] $=\Pi_{5}$

$\sqrt{\text { carteries }} \sqrt{\text { IAorta }}:=\Pi_{5}$

This is where we form the transfer function matrix (based on the differential equations).

$\mathbf{A A}=$

$\{\{0,-1 /$ IAorta, 0$\},\{1 /$ CAorta, -RAorta/IAorta, $-1 /$ CArteries $\},(0,1 /$ IAorta, 0 $\}$; $\mathrm{BB}=\{\{1,0\},\{0,0\},\{0,1\}\}$;

CC = Identitykatrix $[3]$

$D D=$ Table $[0,\{i, 1,3\},\{j, 1,2\}]$; 
This is the characteristic equation

CharacteristicEquation $=$ Collect $[\operatorname{Det}[\mathrm{s}$ *IdentityMatrix $[3]-\mathrm{AA}], \mathrm{s}]$

$$
\left(\frac{1}{\text { CAOrta Inorta }}+\frac{1}{\text { CArteries IAOrta }}\right) 8+\frac{\text { RAOrta } s^{2}}{\text { IAOrta }}+8^{3}
$$

Nondimensionalize it

simplify [Eliminate [ \{CharacteristicEquation $=0$, sUB1, sUB2, sUB3, suB4, suB5], [RAorta, IAorta, CAorta, CArteries, RPeripheral, QIn, gout, s\}]]

$\Pi_{5}\left(1+\Pi_{2}\left(1+\Pi_{1} \Pi_{5}+\Pi_{5}^{2}\right)\right)=0$

xpCharacteristicEquation $=\left(\operatorname{Collect}\left[*, \Pi_{5}\right]\right) \approx 10$ Expand $/ e$ \&

$\left(1+\Pi_{2}\right) \Pi_{5}+\Pi_{2} \Pi_{2} \Pi_{5}^{2}+\Pi_{2} \Pi_{5}^{3}=0$

Check to see if there are any parameter ranges which might make the arterial system unstable (Routh-Hurwitz)

RouthHurwitzPrep [x_, var_] : = Module [

$\{\mathrm{cl}\}$,

cl = Reverse [CoefficientList [x, var] ]

If $[\mathrm{cl}[$ [Length $[\mathrm{cl}]]]=0, \mathrm{cl}=\operatorname{Most}[\mathrm{cl}]]$;

If [Oddg [Length [cl]], cl = Join [cl, $\{0\}]]$;

[Take $[c 1,\{1$, Length $[c 1], 2\}]$, Take $[c 1,\{2$, Length $[c 1], 2\}]\}$

]; 


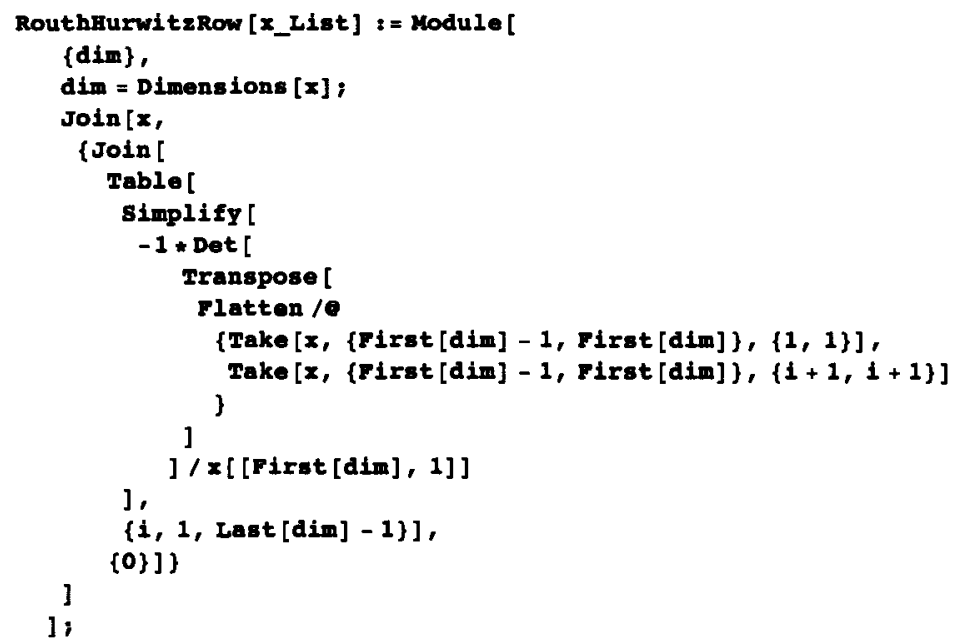

Solve the characteristic equation (easy, since it's a simple quadratic)

Solve [NDCharacteristiozquation, $\Pi_{5}$ ]

$\left\{\left\{\Pi_{5} \rightarrow 0\right\},\left\{\Pi_{5} \rightarrow \frac{-\Pi_{1} \Pi_{2}-\sqrt{\Pi_{2}} \sqrt{-4-4 \Pi_{2}+\Pi_{1}^{2} \Pi_{2}}}{2 \Pi_{2}}\right\},\left\{\Pi_{5} \rightarrow \frac{-\Pi_{1} \Pi_{2}+\sqrt{\Pi_{2}} \sqrt{-4-4 \Pi_{2}+\Pi_{1}^{2} \Pi_{2}}}{2 \Pi_{2}}\right\}\right\}$

This is the condition which precludes pressure oscillations in the arterial system

Contourfunc $=$ Coefficient [HDCharacteristicEquation $\left.[[1]], \pi_{5}, 2\right] \wedge 2$ -

(4* Coefficient [upcharacteristicsquation [ $\left.[1]], \Pi_{5}, 3\right]$ *

Coefficient [oDCharacteristicsquation $\left.[[1]], \Pi_{5}, 1\right]$ )

$\Pi_{1}^{2} \Pi_{2}^{2}-4 \Pi_{2}\left(1+\Pi_{2}\right)$ 


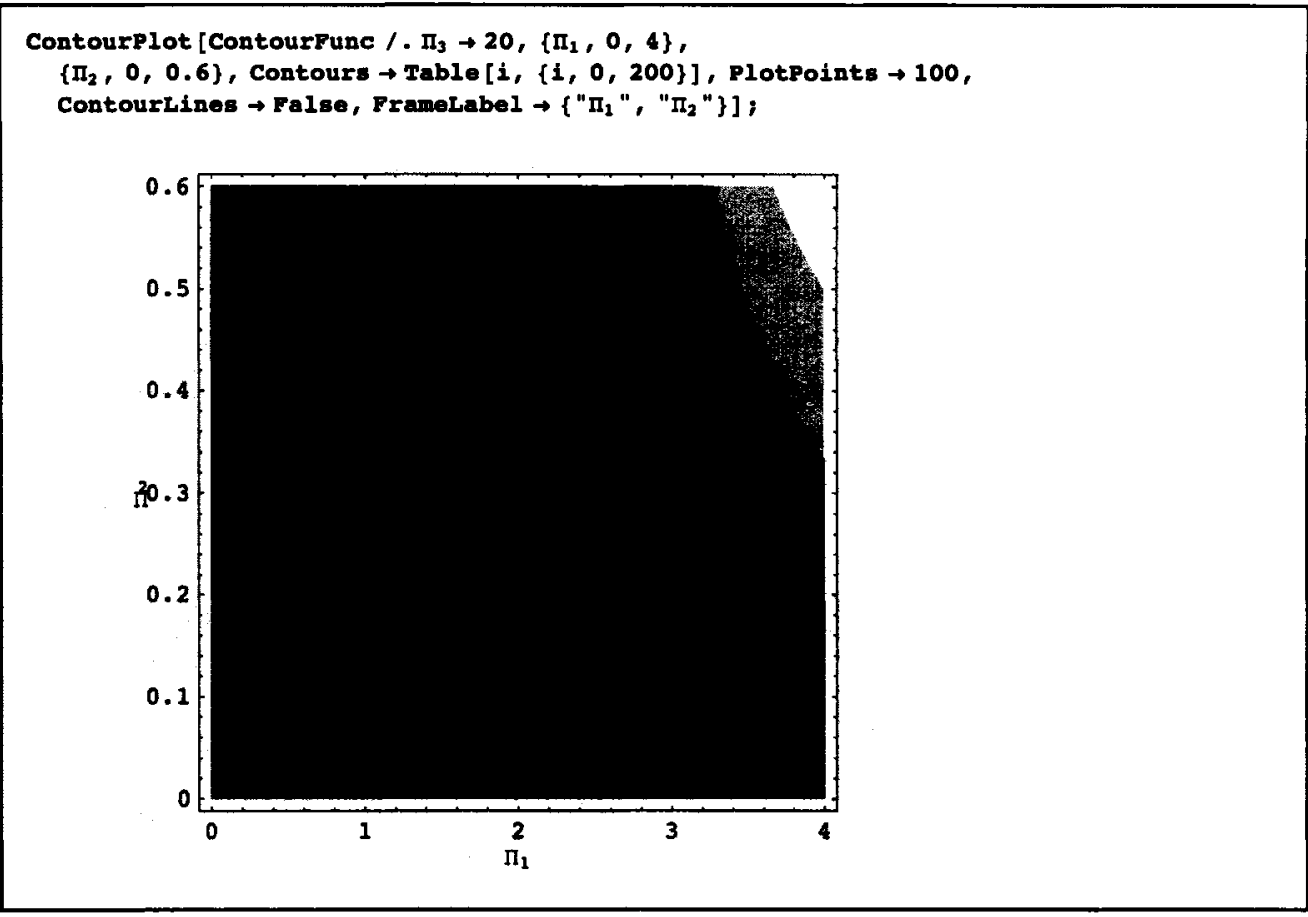

Contourfunc /. $\left\{\Pi_{1} \rightarrow 2, \Pi_{2} \rightarrow 0.3, \Pi_{3} \rightarrow 20\right\}$

$-1.2$ 
ContourPlot [Contourfunc $/ . \Pi_{3} \rightarrow 20,\left\{\Pi_{1}, 0,4\right\}$,

$\left\{\mathrm{I}_{2}, 0,0.6\right\}$, Contours $\rightarrow 25$, PlotPoints $\rightarrow 100$, Contourshading $\rightarrow$ False,

Contourstyle $\rightarrow\{$ ReBColor $[0.5,0.5,1]$.$\left.\} , Framelabel \rightarrow\left\{" \Pi_{1} ", " \Pi_{2} "\right\}\right]$;

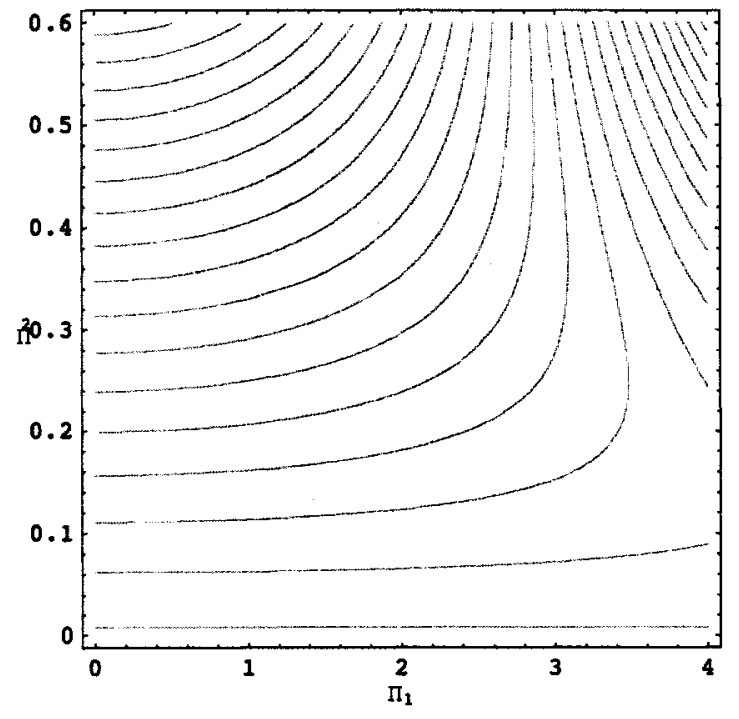


Show $[\{88,8\}]$

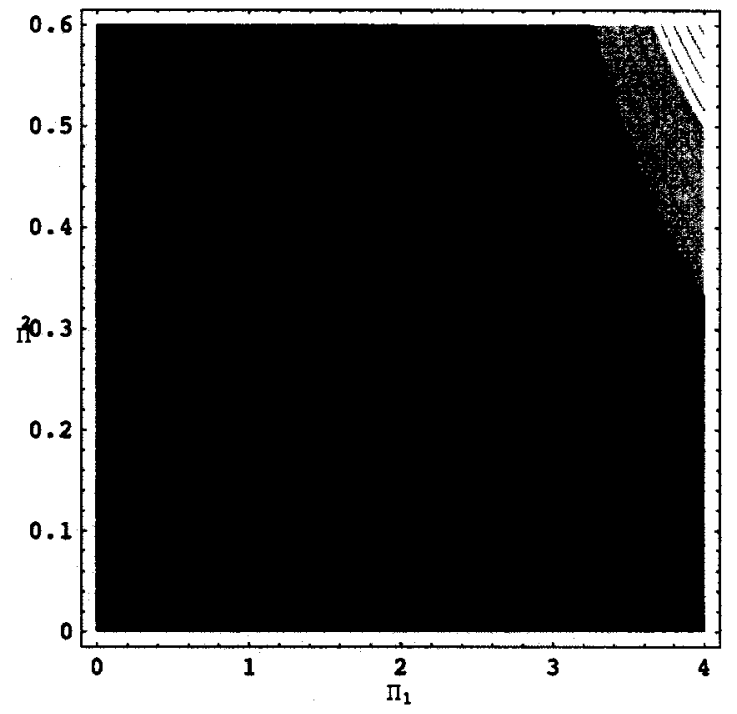

Check the fixed points; form differential equations

AA. $\{\{\nabla 2\},\{p 4\},\{\nabla 7\}\}+B B \cdot\{Q I n * f[t]$, QOut $* g[t]\}$

$\left\{\left(-\frac{\mathrm{p} 4}{\text { IAOrta }}+\right.\right.$ oIn $\left.f[t]\right\},\left\{-\frac{\text { p4 RAOrta }}{\text { IAorta }}+\frac{v 2}{\text { CAorta }}-\frac{v 7}{\text { CArteries }}\right\},\left\{\frac{\text { p4 }}{\text { IAorta }}+\right.$ Qout $\left.\left.g[t]\right\}\right\}$

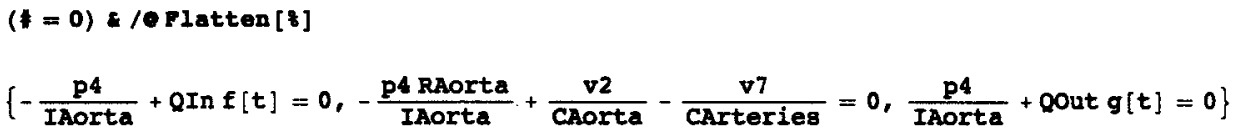

( ) 
Flow-in, pressure-out with \{IAorta, CArteries, QIn\} as repeating variables

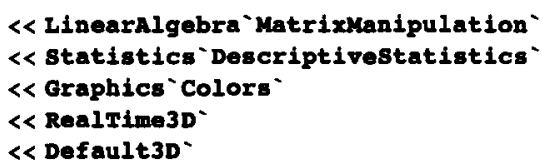

These are the $\{m\},\{L\}$ and $\{t\}$ units of all the variables in the flow-in-flow-out formulation. The columns represent (in order): RAorta, IAorta, CAorta, CArteries, RPeripheral, QIn, POut and s.

$$
\begin{aligned}
& \text { MatrixForm [FullUnitKatrix }=\{\{1,1,-1,-1,1,0,1,0\} \text {, } \\
& \{-4,-4,4,4,-4,3,-1,0\},\{-1,0,2,2,-1,-1,-2,-1\}\}] \\
& \left(\begin{array}{cccccccc}
1 & 1 & -1 & -1 & 1 & 0 & 1 & 0 \\
-4 & -4 & 4 & 4 & -4 & 3 & -1 & 0
\end{array}\right) \\
& \left(\begin{array}{llllllll}
-1 & 0 & 2 & 2 & -1 & -1 & -2 & -1
\end{array}\right)
\end{aligned}
$$

Select repeating variables of IAorta, CArteries, QIn (these form the columns of "RepeatingVariableunitMatrix"; the rows are $\{m\},\{L\}$ and $\{t\})$

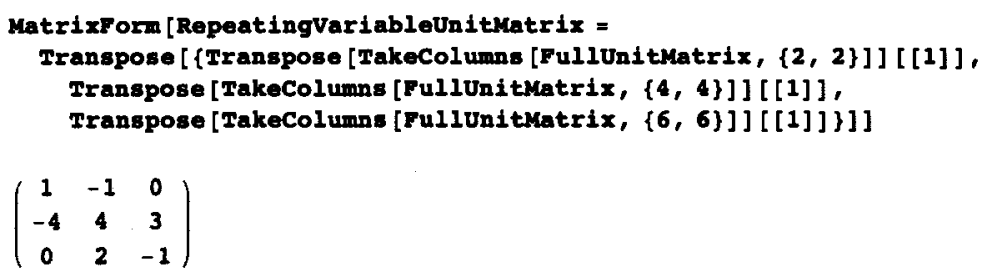

Buckingham's II-Theorem... solve for the exponents

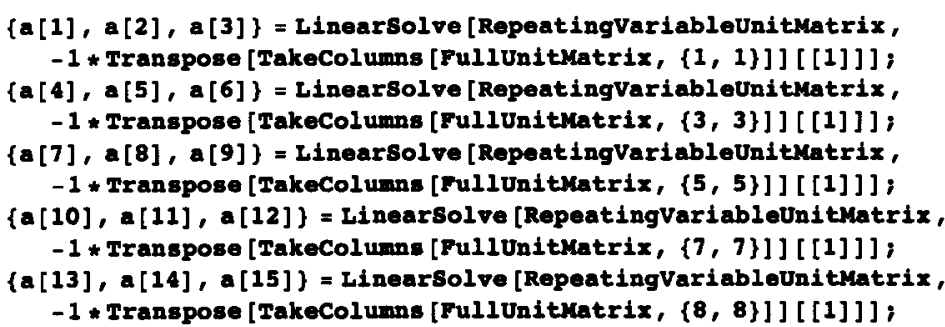


First $\Pi$ group (RAorta plus repeating variables)

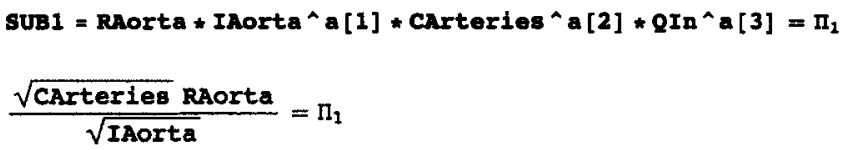

Second $\Pi$ group (CAorta plus repeating variables)

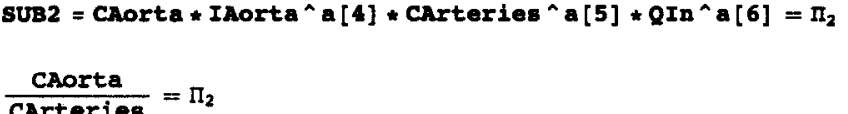

Third $\Pi$ group (RPeripheral plus repeating variables)

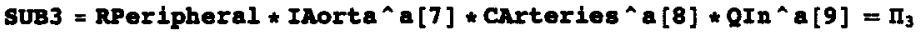

$\frac{\sqrt{\text { CArteries }} \text { RPeripheral }}{\sqrt{\text { IAorta }}}=\mathrm{n}_{3}$ $\sqrt{\text { IAorta }}$

Fourth $\Pi$ group (POut plus repeating variables)

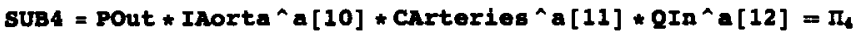

$\frac{\sqrt{\text { Carteries }} \text { Pout }}{\sqrt{\text { IAtra }}}=\pi_{4}$

$\sqrt{\text { IAorta }}$ QIn

Fifth $\Pi$ group (s plus repeating variables)

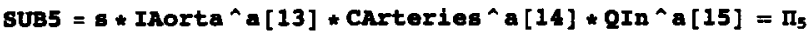

$\sqrt{\text { CArteries }} \sqrt{\text { IAorta }} \mathbf{s}=\Pi_{5}$

This is where we form the transfer function matrix (based on the differential equations).

AA $=\{\{0,-1 /$ IAorta, 0$\},\{1 /$ CAorta, -RAorta / IAorta, - $1 /$ CArteries $\}$,

$\{0,1 /$ IAorta, $-1 /$ (CArteries * RPeripheral) $\})$;

$\mathbf{B B}=\{\{1,0\},\{0,0\},\{0,1 /$ RPeripheral $\}\}$

CC = Identitykatrix [3];

$D D=T a b l e[0,\{1,1,3\},\{j, 1,2\}]$;

Reproduced with permission of the copyright owner. Further reproduction prohibited without permission. 
This is the characteristic equation

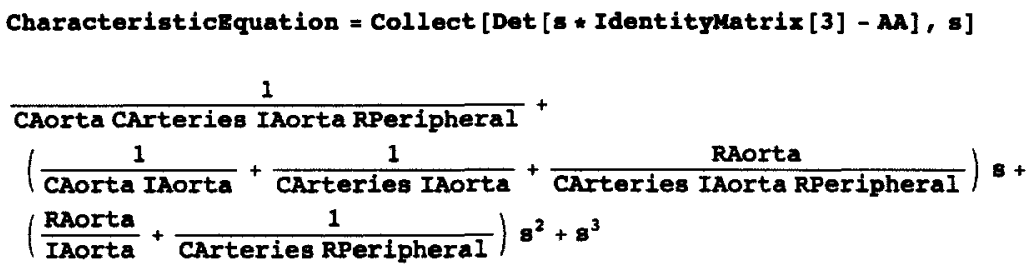

Nondimensionalize it

Simplify [E1 iminate [ CharacteristicBquation $=0$, sUB1, sUB2, SUB3, sUB4, sUB5], [RAorta, IAorta, CAorta, CArteries, Rperipheral, QIn, POut] ]]

$\Pi_{5}\left(\Pi_{2} \Pi_{5}+\Pi_{1} \Pi_{2}\left(1+\Pi_{3} \Pi_{5}\right)+\Pi_{3}\left(1+\Pi_{2}\left(1+\Pi_{5}^{2}\right)\right)\right)=-1$

WDCharacteristicEquation $=\left(\operatorname{Collect}\left[\$, \Pi_{5}\right]\right) \& / e$ Expand $/ \theta$

$\left(\Pi_{1} \Pi_{2}+\Pi_{3}+\Pi_{2} \Pi_{3}\right) \Pi_{5}+\left(\Pi_{2}+\Pi_{1} \Pi_{2} \Pi_{3}\right) \Pi_{5}^{2}+\Pi_{2} \Pi_{3} \Pi_{5}^{3}=-1$

Check to see if there are any parameter ranges which might make the arterial system unstable (Routh-Hurwitz)

RouthHurwitzPrep [x_, var_] : = Module [

$\{c 1\}$,

cl = Reverse [CoefficientList $[x$, var $]]$;

If [cl [ [Length [Cl]]] = o, cl = Most [cl]];

If [OddQ [Length [cl]], cl = Join $[c 1,\{0\}]]$;

\{Take $[c 1,\{1$, Length $[c 1], 2\}]$, Take $[c 1,\{2$, Length $[c 1], 2\}]\}$

];

Reproduced with permission of the copyright owner. Further reproduction prohibited without permission. 


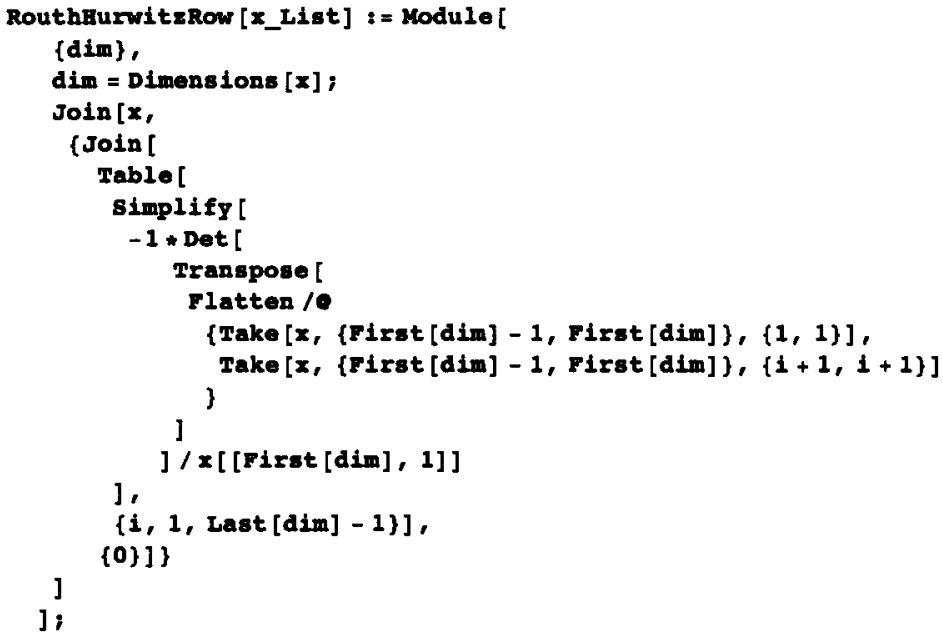

Solve the characteristic equation (not so easy; it's a cubic)... First, depress the cubic by making a substitution to eliminate the quadratic term

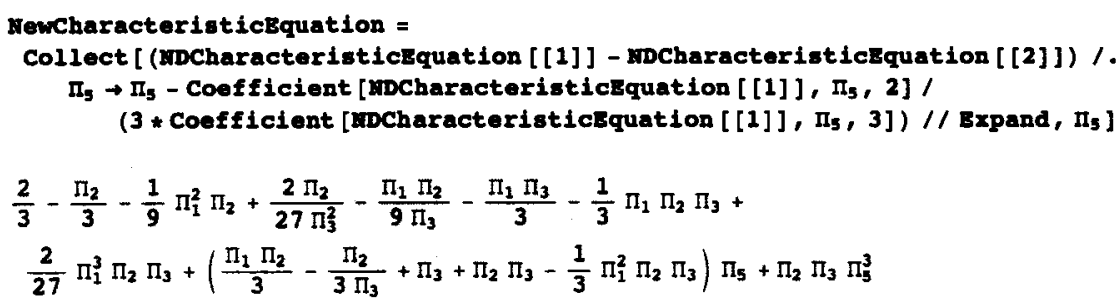


Now, blow it up into a degree-6 polynomial

$$
\begin{aligned}
& \text { RationalCharacteristicEquation = NewCharacteristicEquation } / \Pi_{5} \rightarrow(\rho+v / \rho) / / \text { Expand; } \\
& \text { Degreesixpolynomial = Collect [Expand [RationalCharacteristicEquation } \left.\left.* \rho^{\wedge} 3\right], \rho\right] \\
& \rho^{6} \Pi_{2} \Pi_{3}+v^{3} \Pi_{2} \Pi_{3}+\rho^{4}\left(\frac{\Pi_{1} \Pi_{2}}{3}-\frac{\Pi_{2}}{3 \Pi_{3}}+\Pi_{3}+\Pi_{2} \Pi_{3}+3 v \Pi_{2} \Pi_{3}-\frac{1}{3} \Pi_{1}^{2} \Pi_{2} \Pi_{3}\right)+ \\
& \rho^{2}\left(\frac{1}{3} v \Pi_{1} \Pi_{2}-\frac{v \Pi_{2}}{3 \Pi_{3}}+v \Pi_{3}+v \Pi_{2} \Pi_{3}+3 v^{2} \Pi_{2} \Pi_{3}-\frac{1}{3} v \Pi_{1}^{2} \Pi_{2} \Pi_{3}\right)+ \\
& \rho^{3}\left(\frac{2}{3}-\frac{\Pi_{2}}{3}-\frac{1}{9} \Pi_{1}^{2} \Pi_{2}+\frac{2 \Pi_{2}}{27 \Pi_{3}^{2}}-\frac{\Pi_{1} \Pi_{2}}{9 \Pi_{3}}-\frac{\Pi_{1} \Pi_{3}}{3}-\frac{1}{3} \Pi_{1} \Pi_{2} \Pi_{3}+\frac{2}{27} \Pi_{1}^{3} \Pi_{2} \Pi_{3}\right)
\end{aligned}
$$

Find the constant which eliminates the quartic term

Substitution $=$ Solve $[$ Coefficient $[$ DegreesixPolynomial, 0,4$]=0, v][[1,1]]$

$v \rightarrow \frac{\Pi_{2}-\Pi_{1} \Pi_{2} \Pi_{3}-3 \Pi_{3}^{2}-3 \Pi_{2} \Pi_{3}^{2}+\Pi_{1}^{2} \Pi_{2} \Pi_{3}^{2}}{9 \Pi_{2} \Pi_{3}^{2}}$

Check to make sure it eliminates the quadratic term as well

simplify [ (Coefficient [Degreesixpolynomial, 0,2$] /$ substitution) $=0$ ]

True

Turn it into a quadratic using this substitution

Quadratic = Expand [Degreesixpolynomial /. substitution ];

Collect [Quadratic, o]

$-\frac{2 \Pi_{1}}{27}+\frac{2 \Pi_{1}^{3}}{81}-\frac{\Pi_{1}}{27 \Pi_{2}}-\frac{\Pi_{1} \Pi_{2}}{27}+\frac{2}{81} \Pi_{1}^{3} \Pi_{2}-\frac{1}{243} \Pi_{1}^{5} \Pi_{2}+\frac{\Pi_{2}}{729 \Pi_{3}^{5}}-\frac{\Pi_{1} \Pi_{2}}{243 \Pi_{3}^{4}}-$

$\frac{1}{81 \pi_{3}^{3}}-\frac{\Pi_{2}}{81 \Pi_{3}^{3}}+\frac{2 \Pi_{1}^{2} \Pi_{2}}{243 \Pi_{3}^{3}}+\frac{2 \Pi_{1}}{81 \pi_{3}^{2}}+\frac{2 \Pi_{1} \Pi_{2}}{81 \Pi_{3}^{2}}-\frac{7 \Pi_{1}^{3} \Pi_{2}}{729 \Pi_{3}^{2}}+\frac{2}{27 \Pi_{3}}-\frac{\Pi_{1}^{2}}{27 \Pi_{3}}+$

$\frac{1}{27 \Pi_{2} \Pi_{3}}+\frac{\Pi_{2}}{27 \Pi_{3}}-\frac{\Pi_{1}^{2} \Pi_{2}}{27 \Pi_{3}}+\frac{2 \Pi_{1}^{4} \Pi_{2}}{243 \Pi_{3}}-\frac{\Pi_{3}}{9}+\frac{2}{27} \Pi_{1}^{2} \Pi_{3}-\frac{1}{81} \Pi_{1}^{4} \Pi_{3}-\frac{\Pi_{3}}{27 \Pi_{2}^{2}}-$

$\frac{\Pi_{3}}{9 \Pi_{2}}+\frac{\Pi_{1}^{2} \Pi_{3}}{27 \Pi_{2}}-\frac{\Pi_{2} \Pi_{3}}{27}+\rho^{6} \Pi_{2} \Pi_{3}+\frac{1}{27} \Pi_{1}^{2} \Pi_{2} \Pi_{3}-\frac{1}{81} \Pi_{1}^{4} \Pi_{2} \Pi_{3}+\frac{1}{729} \Pi_{1}^{6} \Pi_{2} \Pi_{3}+$

$\rho^{3}\left(\frac{2}{3}-\frac{\Pi_{2}}{3}-\frac{1}{9} \Pi_{1}^{2} \Pi_{2}+\frac{2 \Pi_{2}}{27 \Pi_{3}^{2}}-\frac{\Pi_{1} \Pi_{2}}{9 \Pi_{3}}-\frac{\Pi_{1} \Pi_{3}}{3}-\frac{1}{3} \Pi_{1} \Pi_{2} \Pi_{3}+\frac{2}{27} \Pi_{1}^{3} \Pi_{2} \Pi_{3}\right)$ 
This is the condition which precludes pressure oscillations in the arterial system

simplify [ (Coefficient [Quadratic, $\rho, 3] \wedge 2-4 *$ Coefficient [Quadratic, $\rho, 6]$ * Coefficlent [Quadratic, 0,0$])>0,\left\{\Pi_{1}>0, \Pi_{2}>0, \Pi_{3}>0\right\}$ ]

$4 \Pi_{3}^{4}+\Pi_{2} \Pi_{3}^{2}\left(8-8 \Pi_{1} \Pi_{3}+12 \Pi_{3}^{2}-\Pi_{1}^{2} \Pi_{3}^{2}\right)+$

$2 \Pi_{2}^{2}\left(2-10 \Pi_{3}^{2}+\Pi_{1}^{3} \Pi_{3}^{3}+6 \Pi_{3}^{4}+\Pi_{1} \Pi_{3}\left(-4+\Pi_{3}^{2}\right)+\Pi_{1}^{2}\left(\Pi_{3}^{2}-\Pi_{3}^{4}\right)\right)>$

$\Pi_{2}^{3}\left(\Pi_{1}+\Pi_{3}\right)^{2}\left(1-2 \Pi_{1} \Pi_{3}-4 \Pi_{3}^{2}+\Pi_{1}^{2} \Pi_{3}^{2}\right)$

Contourfunc $=[[2]]-[[1]]$

$-4 \Pi_{3}^{4}-\Pi_{2} \Pi_{3}^{2}\left(8-8 \Pi_{1} \Pi_{3}+12 \Pi_{3}^{2}-\Pi_{1}^{2} \Pi_{3}^{2}\right)+\Pi_{2}^{3}\left(\Pi_{1}+\Pi_{3}\right)^{2}\left(1-2 \Pi_{1} \Pi_{3}-4 \Pi_{3}^{2}+\Pi_{1}^{2} \Pi_{3}^{2}\right)-$

$2 \Pi_{2}^{2}\left(2-10 \Pi_{3}^{2}+\Pi_{1}^{3} \Pi_{3}^{3}+6 \Pi_{3}^{4}+\Pi_{1} \Pi_{3}\left(-4+\Pi_{3}^{2}\right)+\Pi_{1}^{2}\left(\Pi_{3}^{2}-\Pi_{3}^{4}\right)\right)$
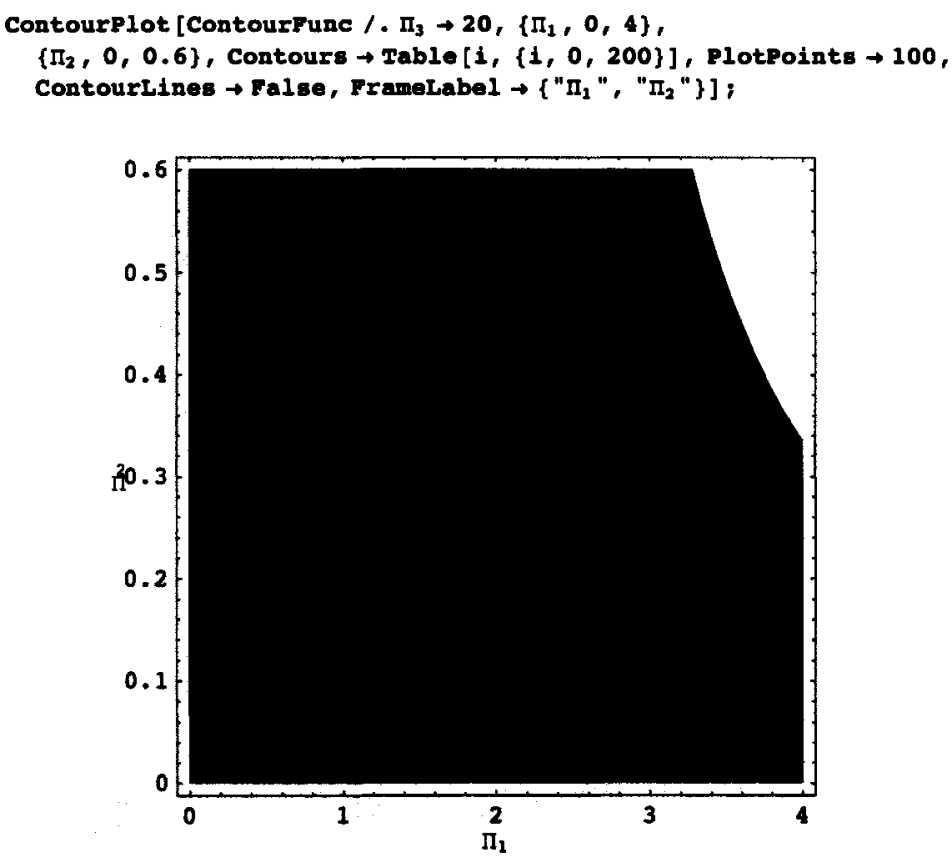

Contourfunc $/ .\left\{\Pi_{1} \rightarrow 2, \Pi_{2} \rightarrow 0.3, \Pi_{3} \rightarrow 20\right\}$

$-1.05913 \times 10^{6}$ 
ContourPlot [Contourfunc $/, \Pi_{3} \rightarrow 20,\left\{\Pi_{1}, 0,4\right\}$,

$\left\{\Pi_{2}, 0,0.6\right\}$, Contours $\rightarrow 25$, Plotpolnts $\rightarrow 100$, Contourshading $\rightarrow$ False,

Contourstyle $\rightarrow\left\{\right.$ ReBColor $[0.5,0.5,1.1\}$, Framelabel $\left.\rightarrow\left\{" \pi_{1} ", " \Pi_{2} "\right\}\right]$;

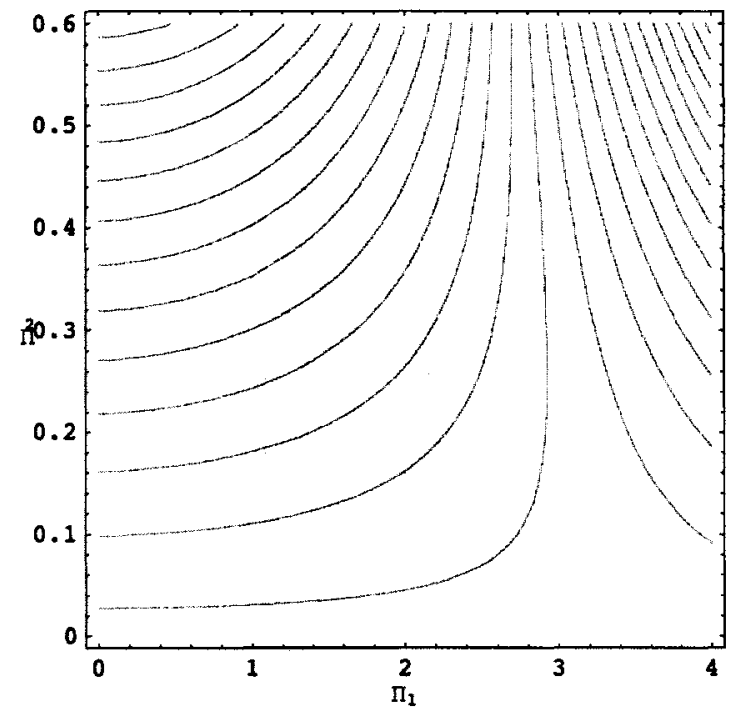


Show $[\{8 z, z\}]$;

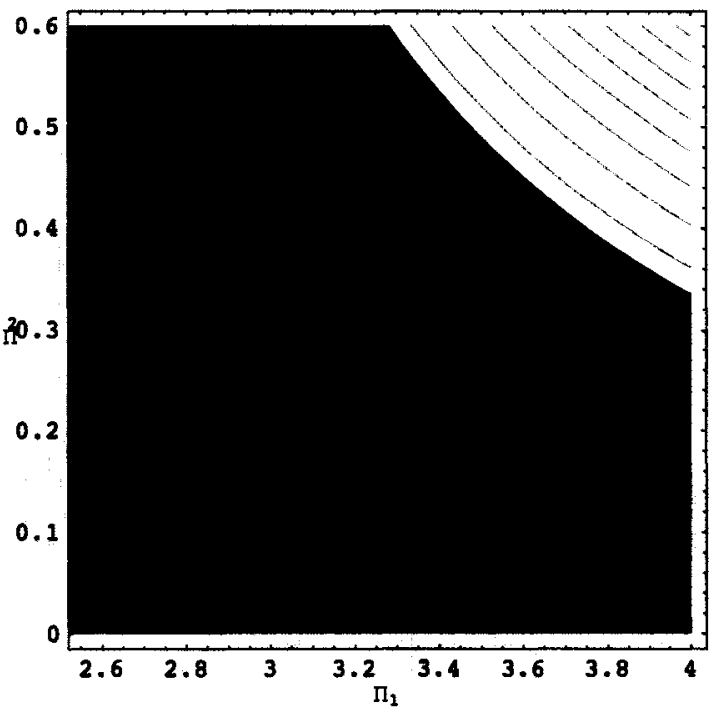

Check the fixed points; form differential equations

AA. $\{\{\nabla 2\},\{p 4\},\{\nabla 7\}\}+B B \cdot\{Q I n * f[t]$, POut $* g[t]\}$

$\left\{\left\{-\frac{p^{4}}{\text { IAorta }}+\right.\right.$ QIn $\left.f[t]\right\},\left\{-\frac{\text { p4 RAorta }}{\text { IAorta }}+\frac{\mathbf{v} 2}{\text { CAorta }}-\frac{v 7}{\text { CArteries }}\right\}$, $\left.\left\{\frac{\text { p4 }}{\text { IAorta }}-\frac{\mathbf{v 7}}{\text { CArteries RPeripheral }}+\frac{\text { POut g }[t]}{\text { RPeripheral }}\right\}\right\}$

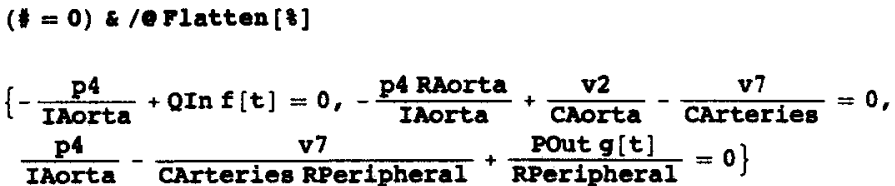

$\{$ [v2 $\rightarrow$ CAOrta OIn RAOrta $f[t]+$ CAOrta QIn RPeripheral $f[t]+$ CAorta POut $g[t]$ $v 7 \rightarrow$ CArteries QIn RPeriphera] $f[t]+$ CArteries POut $g[t], p 4 \rightarrow$ IAOrta $\operatorname{QIn} f[t]\}\}$ 
Flow-in, flow-out with \{RAorta, IAorta, QIn\} as repeating variables

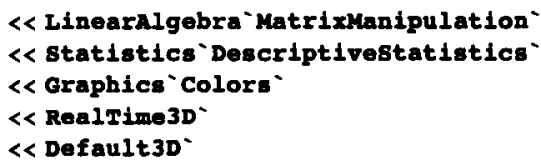

These are the $\{m\},\{L\}$ and $\{t\}$ units of all the variables in the flow-in-flow-out formulation. The columns represent (in order): RAorta, IAorta, CAOrta, CArteries, RPeripheral, QIn, QOut and s.

Matrixform [Fullunituatrix =

$\{\{1,1,-1,-1,1,0,0,0\},\{-4,-4,4,4,-4,3,3,0\},\{-1,0,2,2,-1,-1,-1,-1\}\}$

$$
\left(\begin{array}{cccccccc}
1 & 1 & -1 & -1 & 1 & 0 & 0 & 0 \\
-4 & -4 & 4 & 4 & -4 & 3 & 3 & 0 \\
-1 & 0 & 2 & 2 & -1 & -1 & -1 & -1
\end{array}\right)
$$

Select repeating variables of RAorta, IAorta, $Q$ In (these form the columns of "RepeatingVariableUnitMatrix"; the rows are $\{m\},\{L\}$ and $\{t\})$

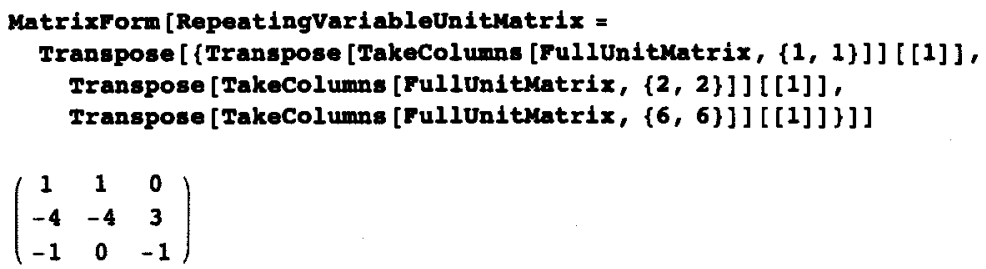

Buckingham's П-Theorem... solve for the exponents

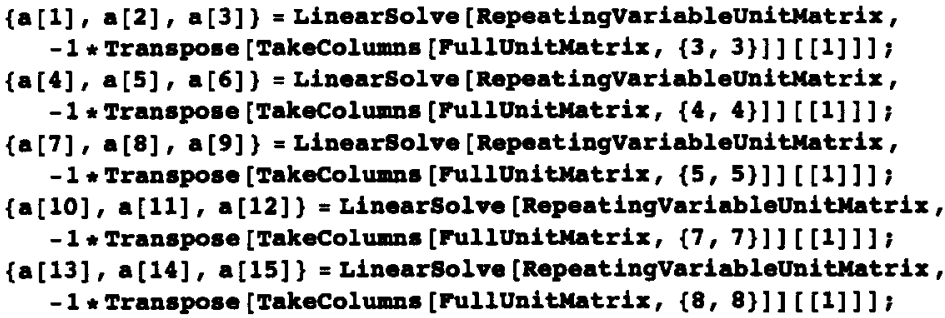


First II group (CAorta plus repeating variables)

SUB1 $=$ CAOrta * RAOrta^a[1]*IAOrta^a[2]*QIn^a[3] $=\Pi_{1}$

$\frac{\text { CAorta RAorta }}{2}=\mathrm{I}_{1}$

Second $\Pi$ group (CArteries plus repeating variables)

SUB2 $=$ CArteries * RAorta^a [4] *IAorta^a[5]*QIn^a[6] $=\Pi_{2}$

CArteries RAorta ${ }^{2}=\Pi_{2}$

IAOrta

Third $\Pi$ group (RPeripheral plus repeating variables)

SUB3 $=$ RPeripheral * RAorta`a $[7] *$ IAorta^a[8]*QIn^a[9] $=\Pi_{3}$

RPeripheral $=\Pi_{3}$

RAorta

Fourth $\Pi$ group (QOut plus repeating variables)

suBA $=$ cout $*$ RAorta^a $[10] *$ IAorta^a $[11] * Q I n \wedge a[12]=\Pi_{4}$

$\frac{\text { Qout }}{\text { OIn }}=n_{1}$

Fifth $\Pi$ group (s plus repeating variables)

sUB5 $=g$ * RAOrta^a[13]*IAOrta^a[14]*QIn^a[15] $=\Pi_{5}$

$\frac{\text { IAorta } \mathrm{s}}{\text { RAOrta }}=\Pi_{5}$

This is where we form the transfer function matrix (based on the differential equations).

$\mathbf{A A}=$

$\{\{0,-1 /$ IAorta, 0$\},\{1 /$ CAorta, -RAorta / IAorta, -1/CArteries $\},\{0,1 /$ IAorta, 0 $\}$

$B B=\{\{1,0\},\{0,0\},\{0,1\}\} ;$

CC = IdentityMatrix [3]

DD $=$ Table $[0,\{1,1,3\},\{\}, 1,2\}] ;$

Reproduced with permission of the copyright owner. Further reproduction prohibited without permission. 
RouthHurwitzPrep [MDCharacteristicEquation [ [1] ], $\Pi_{5}$ ]

$\left\{\left\{\Pi_{1} \Pi_{2}, \Pi_{1}+\Pi_{2}\right\},\left\{\Pi_{1} \Pi_{2}, 0\right\}\right\}$

Hest [RouthHurwitzRow, s, 1] // MatrixPorm

$\left(\begin{array}{cc}\Pi_{1} \Pi_{2} & \Pi_{1}+\Pi_{2} \\ \Pi_{1} \Pi_{2} & 0 \\ \Pi_{1}+\Pi_{2} & 0\end{array}\right)$

Solve the characteristic equation (easy, since it's a simple quadratic)

Solve [ADCharacteristicEquation, $\left.\Pi_{5}\right]$

$\left\{\left\{\Pi_{5} \rightarrow 0\right\},\left\{\Pi_{5} \rightarrow \frac{-\Pi_{1} \Pi_{2}-\sqrt{\Pi_{1}} \sqrt{\Pi_{2}} \sqrt{-4 \Pi_{1}-4 \Pi_{2}+\Pi_{1} \Pi_{2}}}{2 \Pi_{1} \Pi_{2}}\right\}\right.$,

$\left.\left\{\Pi_{5} \rightarrow \frac{-\Pi_{1} \Pi_{2}+\sqrt{\Pi_{1}} \sqrt{\Pi_{2}} \sqrt{-4 \Pi_{1}-4 \Pi_{2}+\Pi_{1} \Pi_{2}}}{2 \Pi_{1} \Pi_{2}}\right\}\right\}$

This is the condition which precludes pressure oscillations in the arterial system

Contourfunc $=$ Coefficient [BDCharacteristicEquation $\left.[1]], \Pi_{5}, 2\right] \wedge 2-$

(4* Coefficient [NDCharacteristictquation $\left.[[1]], \Pi_{5}, 3\right]$ *

Coefficient [NDCharacteristicsquation $\left.[1]], \Pi_{5}, 1\right]$ )

$\Pi_{1}^{2} \Pi_{2}^{2}-4 \Pi_{1} \Pi_{2}\left(\Pi_{2}+\Pi_{2}\right)$ 

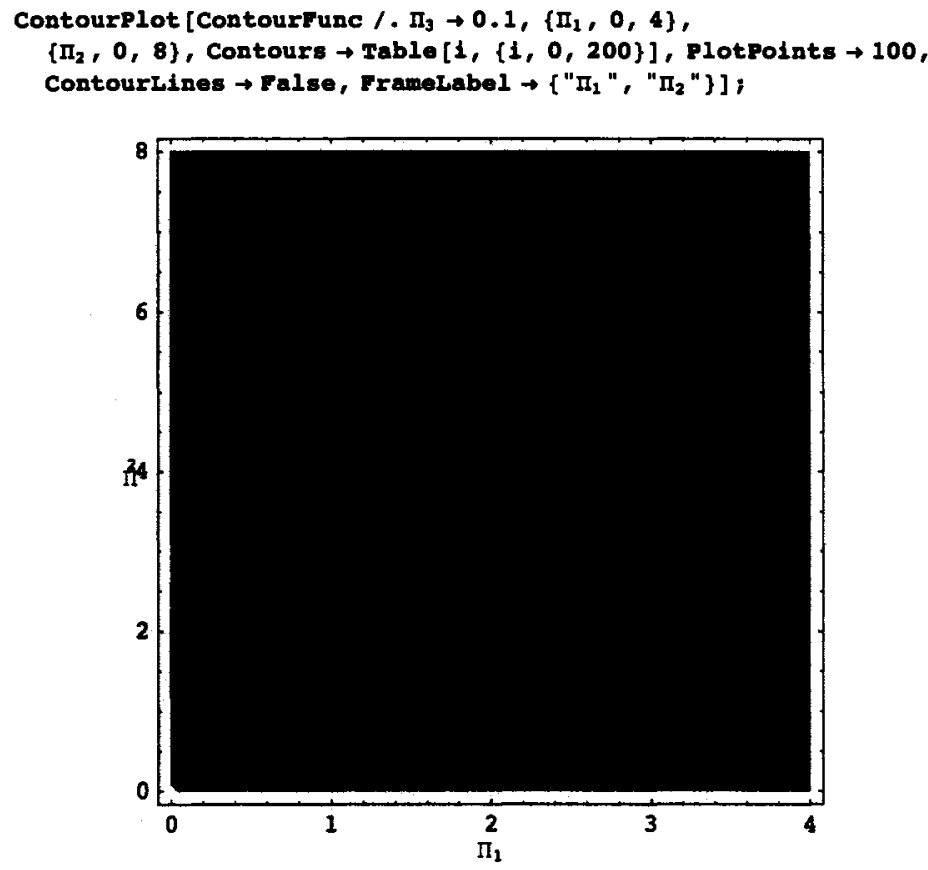

Contourfune $/ .\left\{\Pi_{1} \rightarrow 2, \Pi_{2} \rightarrow 4, \Pi_{3} \rightarrow 0.1\right\}$

$-128$ 
ContourPlot [ContourFunc $/ . \Pi_{3} \rightarrow 0.1,\left\{\Pi_{1}, 0,4\right\}$,

$\left\{\Pi_{2}, 0,8\right\}$, Contours $\rightarrow 25$, PlotPoints $\rightarrow 100$, Contourshading $\rightarrow$ False,

Contourstyle $\rightarrow\{$ RGBColor $[0.5,0.5,1]\}$, Framelabel $\left.\rightarrow\left\{" \Pi_{1} ", " \Pi_{2} "\right\}\right]$;

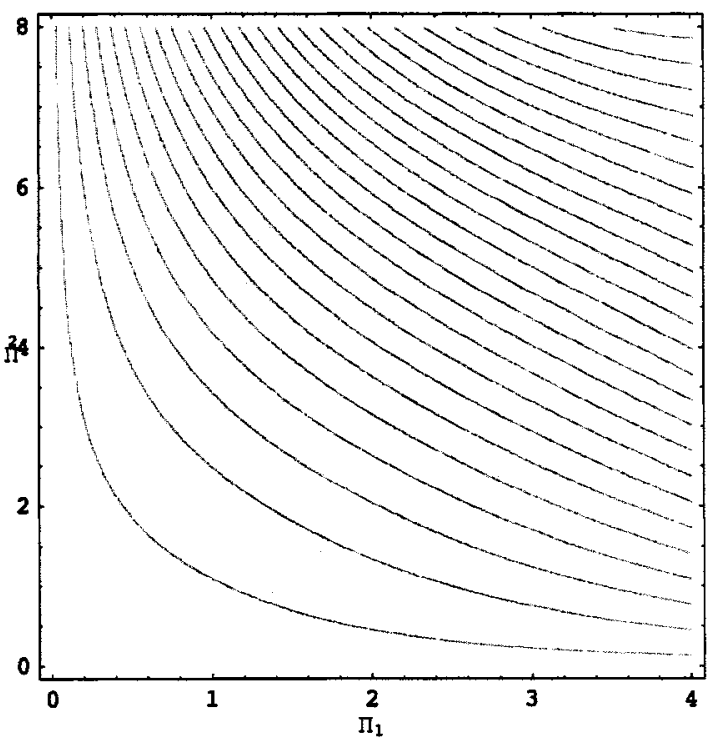




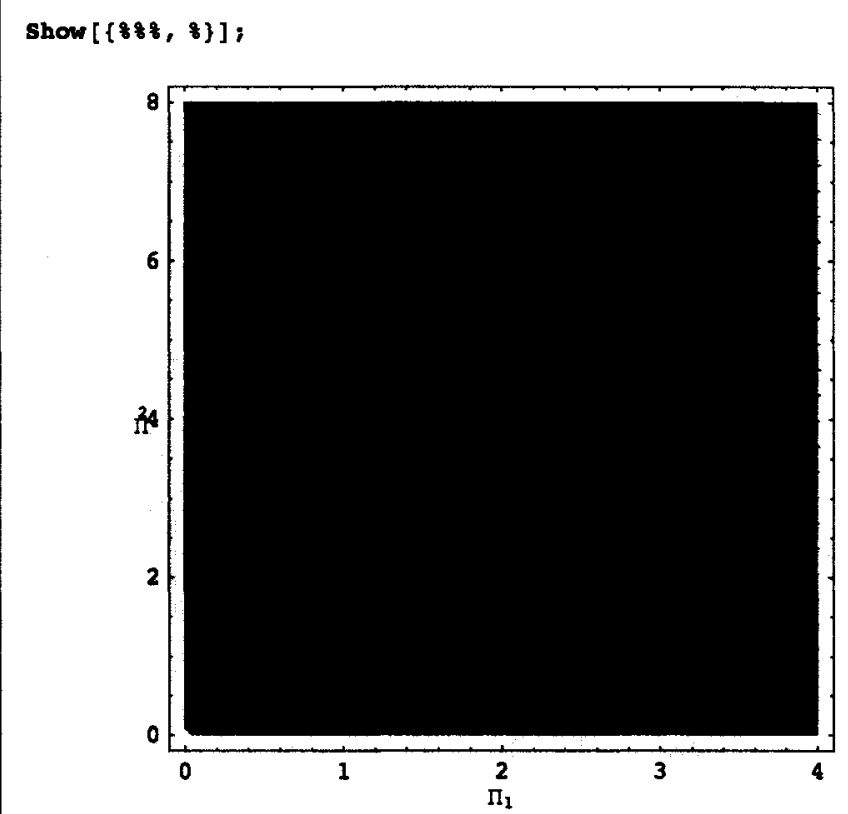

Check the fixed points; form differential equations

AR. $\{\{\nabla 2\},\{p 4\},\{v 7\}\}+B B .\{$ QIn $* f[t]$, Pout $* g[t]\}$

$\left\{\left\{-\frac{\text { p4 }}{\text { IAorta }}+\right.\right.$ QIn $\left.f[t]\right\},\left\{-\frac{\text { p4 RAorta }}{\text { IAorta }}+\frac{v 2}{\text { CAorta }}-\frac{v 7}{\text { CArteries }}\right\},\left\{\frac{\text { p4 }}{\text { IAorta }}+\right.$ Pout g[t] $\left.\}\right\}$

$(*=0) \&$ Platten $[z]$

$\left\{-\frac{p 4}{\text { IAorta }}+\operatorname{QIn} f[t]=0,-\frac{\text { p4 RAorta }}{\text { IAorta }}+\frac{v 2}{\text { CAorta }}-\frac{v 7}{\text { CArteries }}=0, \frac{p 4}{\text { IAorta }}+\right.$ Pout $\left.g[t]=0\right\}$

Solve $[8,\{\nabla 2, p 4, \nabla 7\}]$

() 
Flow-in, pressure-out with $\{$ RAorta, IAorta, $\mathrm{QIn}\}$ as repeating variables

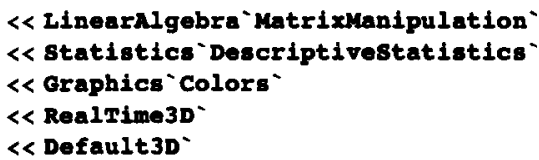

These are the $\{m\},\{L\}$ and $\{t\}$ units of all the variables in the flow-in-flow-out formulation. The columns represent (in order): RAorta, IAorta, CAorta, CArteries, RPeripheral, QIn, POut and s.

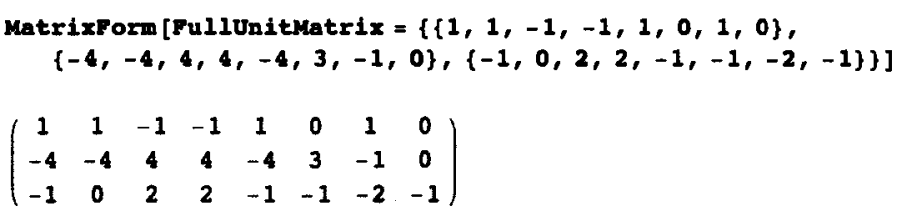

Select repeating variables of RAorta, IAorta, QIn (these form the columns of "RepeatingVariableUnitMatrix"; the rows are $\{m\},\{L\}$ and $\{t\})$

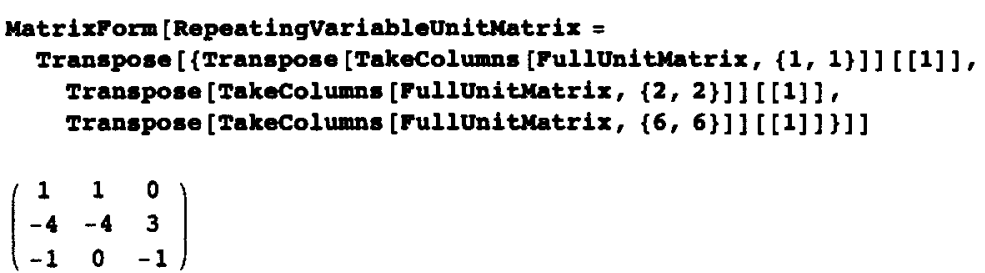

Buckingham's $\Pi$-Theorem... solve for the exponents

$\{a[1], a[2], a[3]\}=$ Linearsolve [RepeatingVariableUnitMatrix, -1 * Transpose [TakeColumns [FullUnitkatrix, $\{3,3\}]][[1]]]$;

$\{a[4], a[5], a[6]\}=$ LinearSolve [RepeatingVariableUnitMatrix, -1 * Transpose [TakeColumns [FullUnitMatrix, \{4, 4\}]] [1]]]; $\{a[7], \mathbf{a}[8], \mathbf{a}[9]\}=$ Linearsolve [Repeatingvariableunitkatrix, -1 * Transpose [TakeColumns [FullUnitKatrix, $\{5,5\}]][[1]]]$;

$\{a[10], a[11], a[12]\}=$ Linearsolve [RepeatingvariableUnitMatrix, -1 * Transpose [TakeColums [FullUnitkatrix, $\{7,7\}]$ ] [ [1]]];

$\{\mathbf{a}[13], \mathbf{a}[14], \mathbf{a}[15]\}=$ Linearsolve [RepeatingVariableUnitMatrix, -1 * Transpose [TakeColume [Ful1UnitMatrix, $\{8,8\}]][[1]]]$; 
First II group (CAorta plus repeating variables)

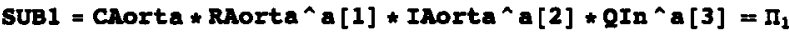

$$
\frac{\text { CAorta RAOrta }}{\text { IAOrta }}=\Pi_{1}
$$

Second $\Pi$ group (CArteries plus repeating variables)

suB2 = CArteries * RAorta^a[4]*IAorta^a[5]*QIn^a[6] $=\Pi_{2}$

CArteries RAorta ${ }^{2}=\Pi_{2}$

IAOIta

Third $\Pi$ group (RPeripheral plus repeating variables)

SUB3 $=$ RPeripheral * Rhorta^a $[7] *$ Inorta^a $[8] * \operatorname{QIn}^{\wedge} \mathbf{a}[9]=\Pi_{3}$

RPeripheral $=\Pi_{3}$ RAorta

Fourth $\Pi$ group (Pout plus repeating variables)

BUB4 $=$ POut * RAOrta^a $[10] \star$ IAorta $^{\wedge} \mathbf{a}[11] * \operatorname{QIn}^{\wedge} \mathbf{a}[12]=\Pi_{4}$

Pout

$\frac{\text { PIn RAorta }}{\text { QIn }}=\Pi_{4}$

Fifth $\Pi$ group ( $s$ plus repeating variables)

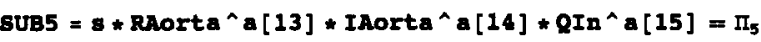

$\frac{\text { IAorta } 8}{\text { Raorta }}=\Pi_{5}$

This is where we form the transfer function matrix (based on the differential equations).

$\mathbf{M A}=\{\{0,-1 /$ Inorta, 0$\},\{1 /$ Chorta, -RAorta / IAorta, - $1 /$ CArteries $\}$,

$\{0,1 /$ IAorta, $-1 /$ (CArteriea * RPeripheral) $\}\}$;

$B B=\{\{1,0\},\{0,0\},\{0,1 /$ RPeripheral $\}\}$;

CC = Identityuatrix [3]

DD = Table $[0,\{1,1,3\},\{j, 1,2\}]$; 
This is the characteristic equation

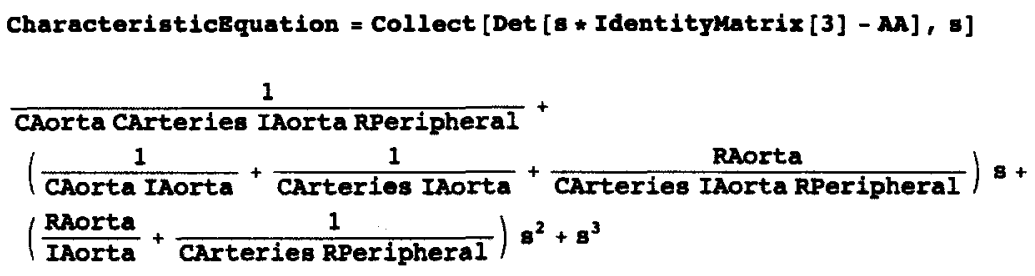

Check to see if there are any parameter ranges which might make the arterial system unstable (Routh-Hurwitz)

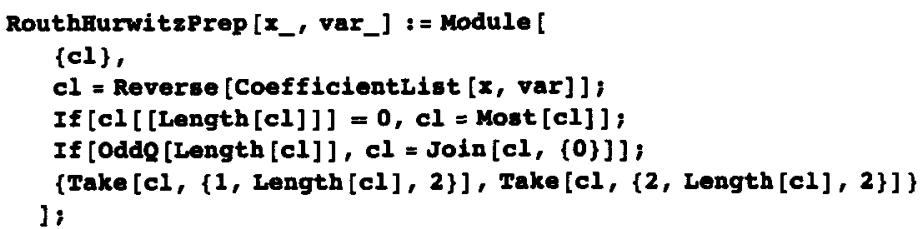




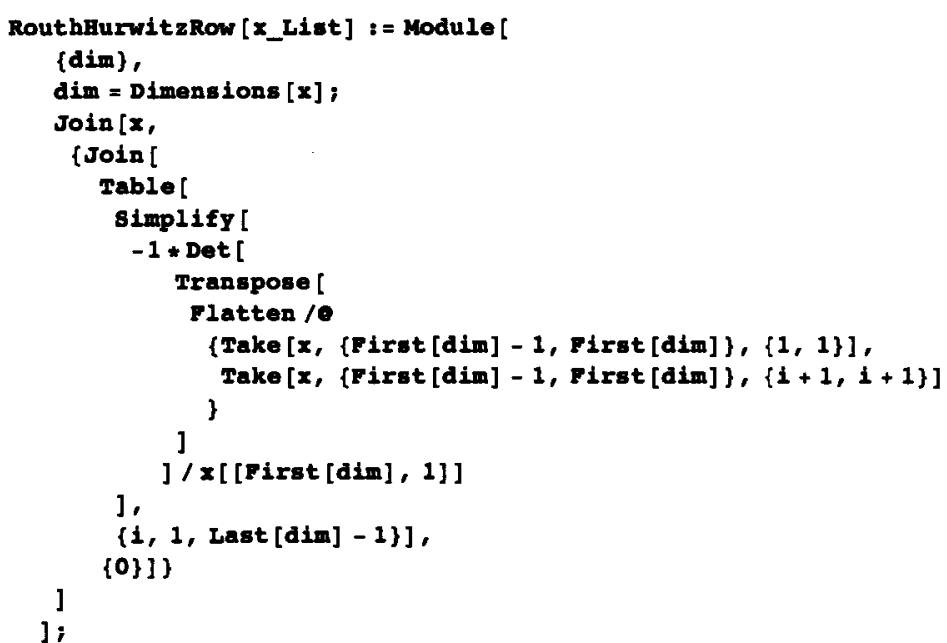

Solve the characteristic equation (not so easy; it's a cubic)... First, depress the cubic by making a substitution to eliminate the quadratic term

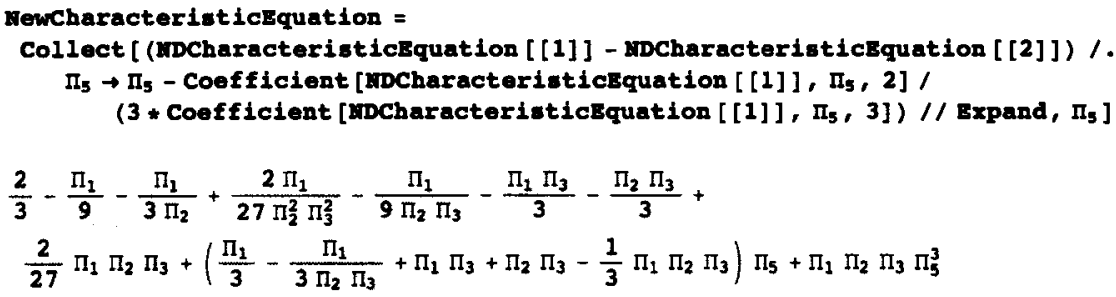


Now, blow it up into a degree-6 polynomial

$$
\begin{aligned}
& \text { RationalCharacteristicEquation = MewCharacteristicEquation } / \Pi_{3} \rightarrow(\rho+v / \rho) / / \text { Expand; } \\
& \text { Degreesixpolynomial = Collect [Expand [RationalCharacteristicEquation * } \left.\left.\rho^{\wedge} 3\right], \rho\right] \\
& \rho^{6} \Pi_{1} \Pi_{2} \Pi_{3}+v^{3} \Pi_{1} \Pi_{2} \Pi_{3}+ \\
& \rho^{3}\left(\frac{2}{3}-\frac{\Pi_{1}}{9}-\frac{\Pi_{1}}{3 \Pi_{2}}+\frac{2 \Pi_{1}}{27 \Pi_{2}^{2} \Pi_{3}^{2}}-\frac{\Pi_{1}}{9 \Pi_{2} \Pi_{3}}-\frac{\Pi_{1} \Pi_{3}}{3}-\frac{\Pi_{2} \Pi_{3}}{3}+\frac{2}{27} \Pi_{1} \Pi_{2} \Pi_{3}\right)+ \\
& \rho^{4}\left(\frac{\Pi_{1}}{3}-\frac{\Pi_{1}}{3 \Pi_{2} \Pi_{3}}+\Pi_{1} \Pi_{3}+\Pi_{2} \Pi_{3}-\frac{1}{3} \Pi_{1} \Pi_{2} \Pi_{3}+3 v \Pi_{1} \Pi_{2} \Pi_{3}\right)+ \\
& \rho^{2}\left(\frac{v \Pi_{1}}{3}-\frac{v \Pi_{1}}{3 \Pi_{2} \Pi_{3}}+v \Pi_{1} \Pi_{3}+v \Pi_{2} \Pi_{3}-\frac{1}{3} v \Pi_{2} \Pi_{2} \Pi_{3}+3 v^{2} \Pi_{1} \Pi_{2} \Pi_{3}\right)
\end{aligned}
$$

Find the constant which eliminates the quartic term

Substitution $=$ Solve $[$ Coefficient $[$ Degreesixpolynomial, $p, 4]=0, v][[1,1]]$

$u \rightarrow \frac{\Pi_{1}-\Pi_{1} \Pi_{2} \Pi_{3}-3 \Pi_{1} \Pi_{2} \Pi_{3}^{2}-3 \Pi_{2}^{2} \Pi_{3}^{2}+\Pi_{1} \Pi_{2}^{2} \Pi_{3}^{2}}{9 \Pi_{1} \Pi_{2}^{2} \Pi_{3}^{2}}$

$$
9 \Pi_{1} \Pi_{2}^{2} \Pi_{3}^{2}
$$

Check to make sure it eliminates the quadratic term as well

simplify [ (Coefficient [Degreesixpolynonial, $\rho, 2] /$ substitution) $=0$ ]

True

Turn it into a quadratic using this substitution

Quadratic = Bxpand [DegreesixPolynomial /. substitution ] ;

Collect [Quadratic, $\rho$ ]

$\frac{2}{81}-\frac{1}{27 \Pi_{1}}-\frac{\Pi_{1}}{243}-\frac{\Pi_{1}}{27 \Pi_{2}^{2}}-\frac{2}{27 \Pi_{2}}+\frac{2 \Pi_{1}}{81 \Pi_{2}}+\frac{\Pi_{2}}{729 \Pi_{2}^{5} \Pi_{3}^{5}}-\frac{\Pi_{1}}{243 \Pi_{2}^{4} \Pi_{3}^{4}}-$

$\frac{\Pi_{1}}{81 \Pi_{2}^{4} \Pi_{3}^{3}}-\frac{1}{81 \Pi_{2}^{3} \Pi_{3}^{3}}+\frac{2 \Pi_{1}}{243 \Pi_{2}^{3} \Pi_{3}^{3}}+\frac{2 \Pi_{1}}{81 \Pi_{2}^{3} \Pi_{3}^{2}}+\frac{2}{81 \Pi_{2}^{2} \Pi_{3}^{2}}-\frac{7 \Pi_{1}}{729 \Pi_{2}^{2} \Pi_{3}^{2}}+\frac{\Pi_{1}}{27 \Pi_{2}^{3} \Pi_{3}}+$

$\frac{2}{27 \Pi_{2}^{2} \Pi_{3}}-\frac{\Pi_{1}}{27 \Pi_{2}^{2} \Pi_{3}}-\frac{1}{27 \Pi_{2} \Pi_{3}}+\frac{1}{27 \Pi_{1} \Pi_{2} \Pi_{3}}+\frac{2 \Pi_{1}}{243 \Pi_{2} \Pi_{3}}+\frac{2 \Pi_{3}}{27}-\frac{\Pi_{3}}{9 \Pi_{1}}-\frac{\Pi_{1} \Pi_{3}}{81}-$

$\frac{\Pi_{1} \Pi_{3}}{27 \Pi_{2}^{2}}-\frac{\Pi_{3}}{9 \Pi_{2}}+\frac{\Pi_{1} \Pi_{3}}{27 \Pi_{2}}-\frac{\Pi_{2} \Pi_{3}}{81}-\frac{\Pi_{2} \Pi_{3}}{27 \Pi_{1}^{2}}+\frac{\Pi_{2} \Pi_{3}}{27 \Pi_{1}}+\frac{1}{729} \Pi_{1} \Pi_{2} \Pi_{3}+\rho^{6} \Pi_{1} \Pi_{2} \Pi_{3}+$

$\rho^{3}\left(\frac{2}{3}-\frac{\Pi_{1}}{9}-\frac{\Pi_{1}}{3 \Pi_{2}}+\frac{2 \Pi_{1}}{27 \Pi_{2}^{2} \Pi_{3}^{2}}-\frac{\Pi_{1}}{9 \Pi_{2} \Pi_{3}}-\frac{\Pi_{1} \Pi_{3}}{3}-\frac{\Pi_{2} \Pi_{3}}{3}+\frac{2}{27} \Pi_{1} \Pi_{2} \Pi_{3}\right)$ 
This is the codition which precludes pressure oscillations in the arterial system

simplify [Coefficient [Quadratic, 0,3$]^{\wedge} 2-4 *$ Coefficient [Quadratic, 0,6$]$ * Coefficient [Quadratic, $\rho, 0]>0,\left\{\Pi_{1}>0, \Pi_{2}>0, \Pi_{3}>0\right\}$ ]

$4 \Pi_{2}^{4} \Pi_{3}^{4}>\Pi_{1}\left(\Pi_{2}^{2} \Pi_{3}^{2}\left(-8+4 \Pi_{2}\left(2-3 \Pi_{3}\right) \Pi_{3}+\Pi_{2}^{2} \Pi_{3}^{2}\right)+\Pi_{1}^{2}\left(1+\Pi_{3}\right)^{2}\left(1+\Pi_{2}^{2} \Pi_{3}^{2}-2 \Pi_{2} \Pi_{3}\left(1+2 \Pi_{3}\right)\right)+\right.$ $\left.2 \Pi_{1}\left(-2+\Pi_{2}^{3}\left(-1+\Pi_{3}\right) \Pi_{3}^{3}+2 \Pi_{2} \Pi_{3}\left(2+5 \Pi_{3}\right)-\Pi_{2}^{2} \Pi_{3}^{2}\left(1+\Pi_{3}+6 \Pi_{3}^{2}\right)\right)\right)$

ContourFunc $=z[[2]]-8[1]]$

$-4 \Pi_{2}^{4} \Pi_{3}^{4}+\Pi_{1}\left(\Pi_{2}^{2} \Pi_{3}^{2}\left(-8+4 \Pi_{2}\left(2-3 \Pi_{3}\right) \Pi_{3}+\Pi_{2}^{2} \Pi_{3}^{2}\right)+\Pi_{1}^{2}\left(1+\Pi_{3}\right)^{2}\left(1+\Pi_{2}^{2} \Pi_{3}^{2}-2 \Pi_{2} \Pi_{3}\left(1+2 \Pi_{3}\right)\right)+\right.$ $\left.2 \Pi_{1}\left(-2+\Pi_{2}^{3}\left(-1+\Pi_{3}\right) \Pi_{3}^{3}+2 \Pi_{2} \Pi_{3}\left(2+5 \Pi_{3}\right)-\Pi_{2}^{2} \Pi_{3}^{2}\left(1+\Pi_{3}+6 \Pi_{3}^{2}\right)\right)\right)$

Contourplot [Contourfunc $/ . \Pi_{3} \rightarrow 0.1,\left\{\Pi_{1}, 0,4\right\}$,

$\left\{\Pi_{2}, 0,8\right\}$, Contours $\rightarrow$ Table $[i,\{i, 0,200\}]$, PlotPoints $\rightarrow 100$,

ContourLines $\rightarrow$ False, FrameLabel $\left.\rightarrow\left\{" \Pi_{1} ", " \Pi_{2} "\right\}\right]$;

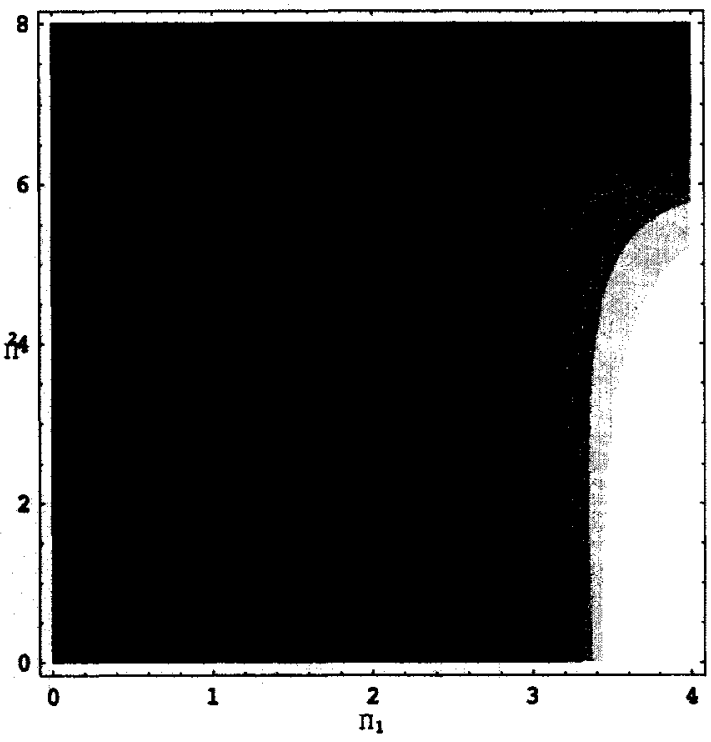

Contourfunc /. $\left\{\Pi_{1} \rightarrow 2, \Pi_{2} \rightarrow 4, \Pi_{3} \rightarrow 0.1\right\}$

$-1.7504$ 


\begin{abstract}
Contourplot [Contourpunc $/ . \Pi_{3} \rightarrow 0.1,\left\{\Pi_{1}, 0,4\right\}$
$\left\{\Pi_{2}, 0,8\right\}$, Contours $\rightarrow 25$, plotpoints $\rightarrow 100$, Contourshading $\rightarrow$ False,
\end{abstract}

Contourstyle $\rightarrow\{$ RGBColor $[0.5,0.5,1]$.$\left.\} , FrameLabel \rightarrow\left\{" \Pi_{1} ", " \Pi_{2} "\right\}\right]$;

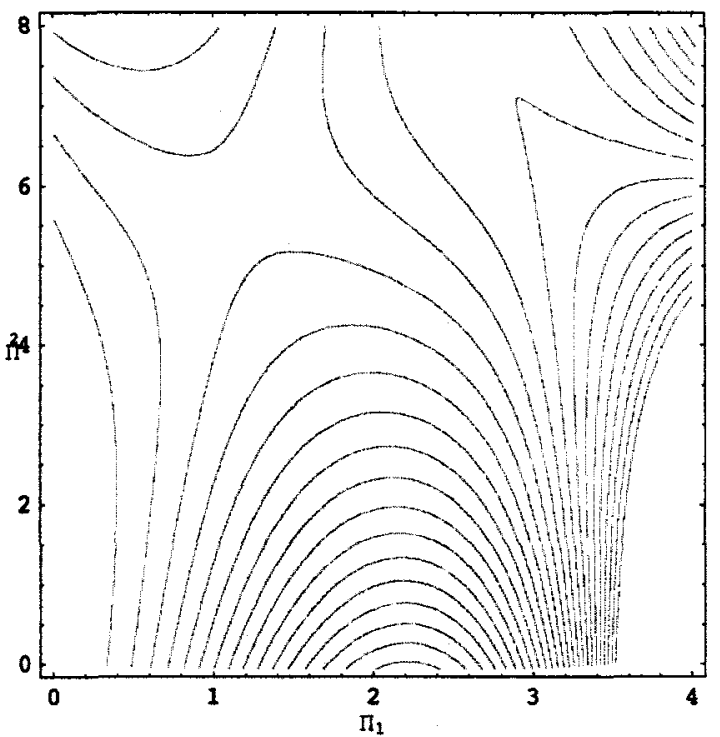

Reproduced with permission of the copyright owner. Further reproduction prohibited without permission. 


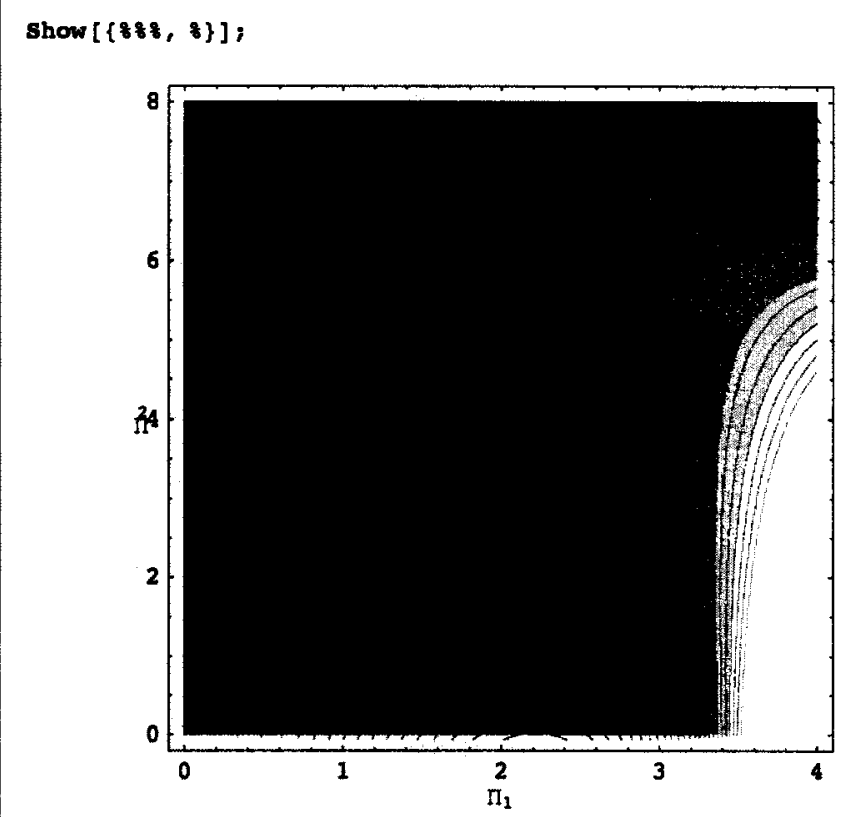

Check the fixed points; form differential equations

AA. $\{\{v 2\},\{P 4\},\{v 7\}\}+B B \cdot\{Q I n * f[t]$, POut $* g[t]\}$

$\left\{\left\{-\frac{\text { p4 }}{\text { IAorta }}+\right.\right.$ QIn $\left.f[t]\right\},\left\{-\frac{\text { P4 RAorta }}{\text { IAorta }}+\frac{\text { v2 }}{\text { CAorta }}-\frac{v 7}{\text { CArteries }}\right\}$, $\left\{\frac{p_{4}}{\text { IAorta }}-\frac{v 7}{\text { Carteries RPeripheral }}+\frac{\text { POut g }[t]}{\text { RPeripheral }}\right\}$

$(*=0)$ \& /e Flatten [8]

$\left\{-\frac{p 4}{\text { IAorta }}+Q \operatorname{Qn} f[t]=0,-\frac{\text { p4 RAorta }}{\text { IAorta }}+\frac{v 2}{\text { CAorta }}-\frac{v 7}{\text { CArteries }}=0\right.$.

$\left.\frac{\text { p4 }}{\text { IAorta }}-\frac{v 7}{\text { CArteries RPeripheral }}+\frac{\text { POut g[t] }}{\text { RPeripheral }}=0\right\}$

Solve $[8,\{v 2, p 4, \nabla 7\}]$

$\{\{v 2 \rightarrow$ CAOrta QIn RAOrta $f[t]+$ CAOrta QIn RPeripheral $f[t]+$ CAOrta POut g[t],

$v 7 \rightarrow$ CArteries QIn RPeripheral $f[t]+$ CArteries POut $g[t]$, p4 $\rightarrow$ IAorta QIn $f[t]\}\}$ 
Flow-in, flow-out with \{CArteries, RPeripheral, QIn\} as repeating variables

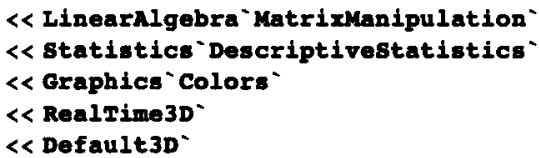

These are the $\{m\},\{L\}$ and $\{t\}$ units of all the variables in the flow-in-flow-out formulation. The columns represent (in order): RAorta, IAorta, CAorta, CArteries, RPeripheral, QIn, QOut and s.

$$
\begin{aligned}
& \text { Matrixform [FullunitKatrix = } \\
& \qquad\{1,1,-1,-1,1,0,0,0\},\{-4,-4,4,4,-4,3,3,0\},\{-1,0,2,2,-1,-1,-1,-1\}\}] \\
& \left(\begin{array}{cccccccc}
1 & 1 & -1 & -1 & 1 & 0 & 0 & 0 \\
-4 & -4 & 4 & 4 & -4 & 3 & 3 & 0 \\
-1 & 0 & 2 & 2 & -1 & -1 & -1 & -1
\end{array}\right)
\end{aligned}
$$

Select repeating variables of CArteries, RPeripheral, QIn (these form the columns of "RepeatingVariableUnitMatrix"; the rows are $\{m\},\{L\}$ and $\{t\})$

$$
\begin{aligned}
& \text { MatrixForm [RepeatingVariableUnitkatrix }= \\
& \text { Transpose [ Transpose [TakeColunns [FullUnitMatrix, }\{4,4\}]][[1]], \\
& \text { Transpose [TakeColumns [FullUnitMatrix, }\{5,5\}]][[1]], \\
& \text { Transpose [TakeColumns [FullUnitMatrix, }(6,6\}]][1]]\}]] \\
& \left(\begin{array}{ccc}
-1 & 1 & 0 \\
4 & -4 & 3 \\
2 & -1 & -1
\end{array}\right)
\end{aligned}
$$

Buckingham's ח-Theorem... solve for the exponents

$$
\begin{aligned}
& \{a[1], a[2], a[3]\}=\text { Linearsolve [RepeatingvariableunitMatrix, } \\
& -1 \text { * Transpose [TakeColumns [FullUnitkatrix, }\{1,1\}]][[1]]] \text {; } \\
& \text { \{a[4], a[5], a [6] \} = LinearSolve [RepeatingVariableUnitMatrix, } \\
& \text {-1 * Transpose [TakeColuans [FullUnitMatrix, }\{2,2\}]][[1]]] \text {; } \\
& \{\mathbf{a}[7], \mathbf{a}[8], \mathbf{a}[9]\}=\text { Linearsolve [RepeatingVariableunituatrix, } \\
& \text {-1 * Transpose [TakeColuans [FullunitMatrix, }\{3,3\}]][[1]]] \text {; } \\
& \{a[10], a[11], a[12]\}=\text { Linearsolve [RepeatingvariableUnitKatrix, } \\
& -1 \text { * Transpose [TakeColuans [FullUnitMatrix, }\{7,7\}] \text { ] [1]]]; } \\
& \text { \{a [13], a [14], a [15]\} = Iinearsolve [RepeatingvariableUnitMatrix, } \\
& -1 \text { * Transpose [TakeColumns [FullUnitMatrix, }\{8,8\}]][[1]]] \text {; }
\end{aligned}
$$


First $\Pi$ group (RAorta plus repeating variables)

sUB1 $=$ RAorta $*$ CArteries $^{\wedge}[1] *$ RPeripheral $^{\wedge} \mathbf{a}[2] * \operatorname{QIn}^{\wedge} \mathbf{a}[3]=\Pi_{1}$

RAorta

$\frac{\text { RAorta }}{\text { RPeripheral }}=\Pi_{1}$

Second $\Pi$ group (IAorta plus repeating variables)

suB2 = IAorta *CArteries^a [4]* RPeripheral^a [5] * QIa^a[6] $=\Pi_{2}$

IAorta

CArteriea RPeripheral ${ }^{2}=\Pi_{2}$

Third II group (CAorta plus repeating variables)

suB3 = CAorta * CArteries^a [7] * RPeripheral`a [8]*QIn^a $[9]=\Pi_{3}$

CAorta

$\frac{\text { CAorta }}{\text { CArteries }}=\Pi_{3}$

Fourth II group (QOut plus repeating variables)

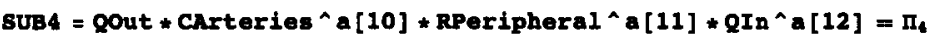

$\frac{\text { Qout }}{\text { oIn }}=\pi_{4}$

Fifth $\Pi$ group (s plus repeating variables)

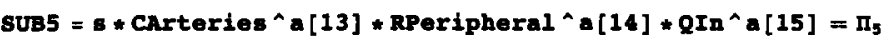

CArteries RPeripheral $8=\Pi_{5}$

This is where we form the transfer function matrix (based on the differential equations).

$\mathbf{A}=$

$\{\{0,-1 /$ IAorta, 0$\},\{1 /$ CAorta, -Rhorta / IAorta, $-1 /$ CArteries $\},\{0,1 /$ Inorta, 0 $\}$;

$\mathrm{BB}=\{\{1,0\},\{0,0\},\{0,1\}\} ;$

CC = IdentityMatrix [3];

$D D=$ Table $[0,\{1,1,3\},\{\}, 1,2\}]$; 
This is the characteristic equation

CharacteristicEquation = Collect $[\operatorname{Det}[\mathrm{s} *$ IdentityMatrix $[3]-\mathrm{AA}], 8]$

$$
\left(\frac{1}{\text { CAOrta IAorta }}+\frac{1}{\text { CArteries IAorta }}\right) s+\frac{\text { RAorta } s^{2}}{\text { IAorta }}+8^{3}
$$

Nondimensionalize it

simplify [Eliminate [ CharacteristicEquation $=0$, SUB1, sUB2, sUB3, sUB4, sUB5], [RAorta, IAorta, CAorta, CArteries, RPeripheral, QIn, QOut, 8\}]]

$\Pi_{5}\left(1+\Pi_{3}\left(1+\Pi_{1} \Pi_{5}+\Pi_{2} \Pi_{5}^{2}\right)\right)=0$

MDCharacteristicEquation $=\left(\operatorname{Collect}\left[*, \Pi_{5}\right]\right) \& / 0$ Expand $/ Q$ \&

$\left(1+\Pi_{3}\right) \Pi_{5}+\Pi_{1} \Pi_{3} \Pi_{5}^{2}+\Pi_{2} \Pi_{3} \Pi_{5}^{3}=0$

Check to see if there are any parameter ranges which might make the arterial system unstable (Routh-Hurwitz)

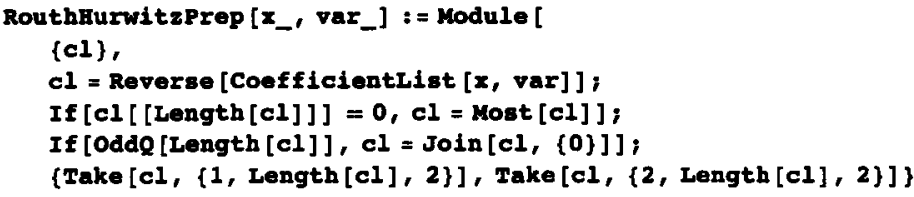




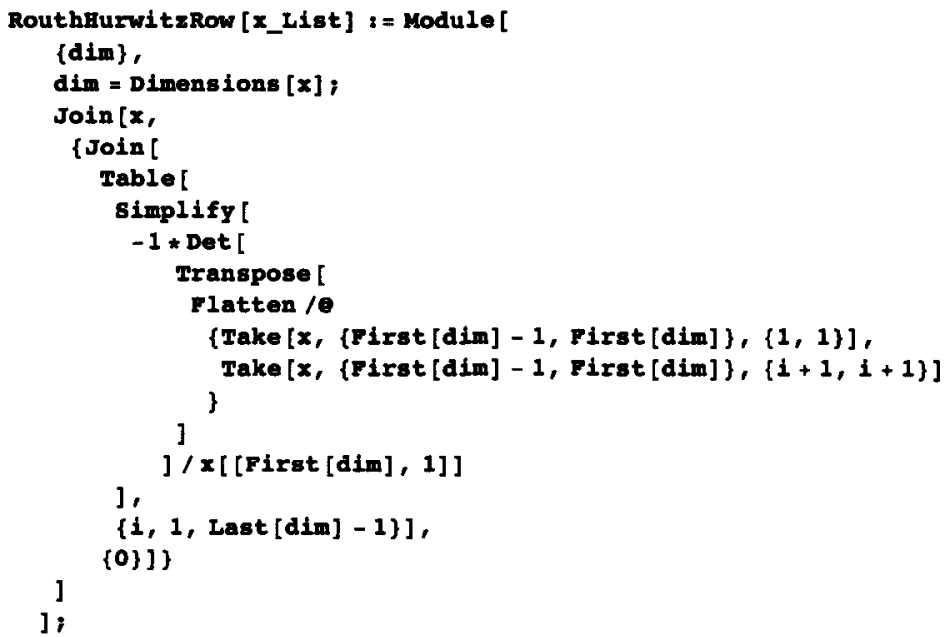

Solve the characteristic equation (easy, since it's a simple quadratic)

$$
\begin{aligned}
& \text { Solve [HDCharacteristicEquation, } \Pi_{5} \text { ] } \\
& \left\{\left\{\Pi_{5} \rightarrow 0\right\},\left\{\Pi_{5} \rightarrow \frac{-\Pi_{1} \Pi_{3}-\sqrt{\Pi_{3}} \sqrt{-4 \Pi_{2}+\Pi_{1}^{2} \Pi_{3}-4 \Pi_{2} \Pi_{3}}}{2 \Pi_{2} \Pi_{3}}\right\},\right. \\
& \left.\left\{\Pi_{5} \rightarrow \frac{-\Pi_{1} \Pi_{3}+\sqrt{\Pi_{3}} \sqrt{-4 \Pi_{2}+\Pi_{1}^{2} \Pi_{3}-4 \Pi_{2} \Pi_{3}}}{2 \Pi_{2} \Pi_{3}}\right\}\right\}
\end{aligned}
$$


This is the condition which precludes pressure oscillations in the arterial system

Contourfunc $=$ Coefficient $\left[\right.$ mpcharacteristicsquation $\left.[[1]], \Pi_{5}, 2\right] \wedge 2-$

(4* Coefficlent [MDCharacteristicEquation $\left.[1]], \Pi_{5}, 3\right]$ *

Coeficient [EDCharacteristicrquation $\left.\left.[[1]], \Pi_{5}, 1\right]\right)$

$\Pi_{1}^{2} \Pi_{3}^{2}-4 \Pi_{2} \Pi_{3}\left(1+\Pi_{3}\right)$

Contourplot [Contourfunc $/ . \Pi_{3} \rightarrow 30 / 100,\left\{\Pi_{1}, 0,0.2\right\}$,

$\left\{\Pi_{2}, 0,0.02\right\}$, Contours $\rightarrow$ Table $[1,\{1,0,0.1,0.01\}]$

PlotPoints $\rightarrow 100$, ContourLines $\rightarrow$ False, FrameLabel $\rightarrow\left\{" \Pi_{1} ", " \Pi_{2}\right.$ " $\}$;

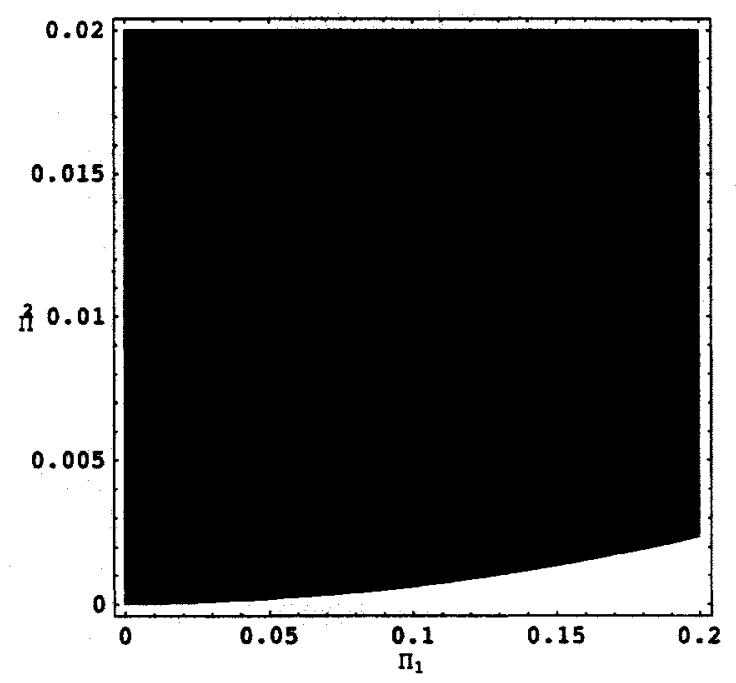

Contourfune $/ .\left\{\Pi_{1} \rightarrow 0.1, \Pi_{2} \rightarrow 0.01, \Pi_{3} \rightarrow 30 / 100\right\}$

$-0.0147$ 
Contourplot [Contourfunc $/ . \Pi_{3} \rightarrow 30 / 100,\left\{\Pi_{1}, 0,0.2\right]$,

$\left\{\Pi_{2}, 0,0.02\right\}$, Contours $\rightarrow 25$, PlotPoints $\rightarrow 100$, Contourshading $\rightarrow$ False,

Contourstyle $\rightarrow\{$ RGBColor $[0.5,0.5,1]$.$\left.\} , Framelabel \rightarrow\left\{" \Pi_{1} ", " \Pi_{2} "\right\}\right]$;

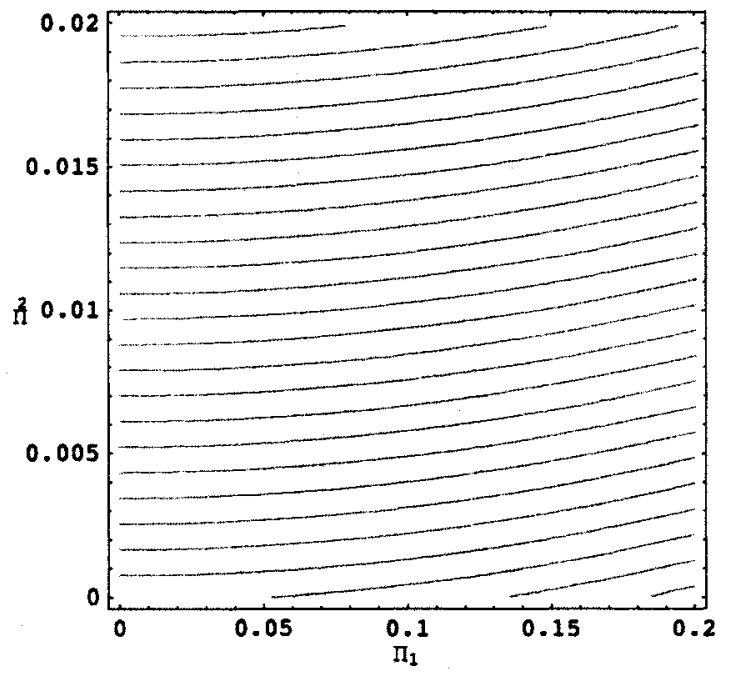




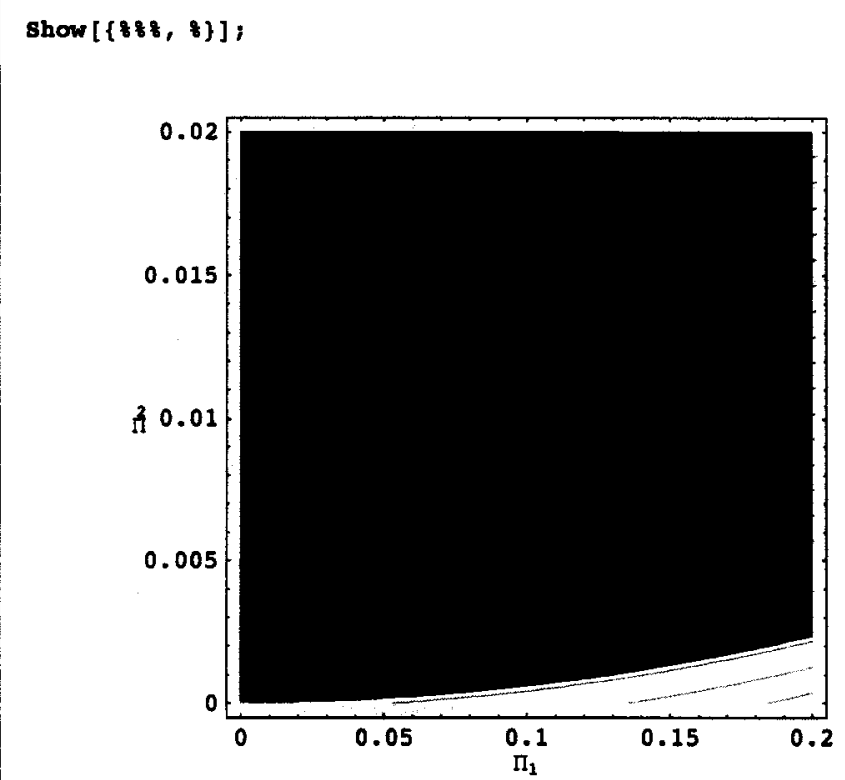

Check the fixed points; form differential equations

AA. $\{\{\nabla 2\},\{p 4\},\{\nabla 7\}\}+B B \cdot\{Q \operatorname{In} * f[t]$, QOut $* g[t]\}$

$\left\{\left\{-\frac{\text { p4 }}{\text { IAorta }}+\right.\right.$ oIn $\left.f[t]\right\},\left\{-\frac{\text { p4 RAorta }}{\text { IAorta }}+\frac{\text { v2 }}{\text { CAorta }}-\frac{v 7}{\text { CArterieg }}\right\},\left\{\frac{\text { p4 }}{\text { IAorta }}+\right.$ QOut $\left.\left.g[t]\right\}\right\}$

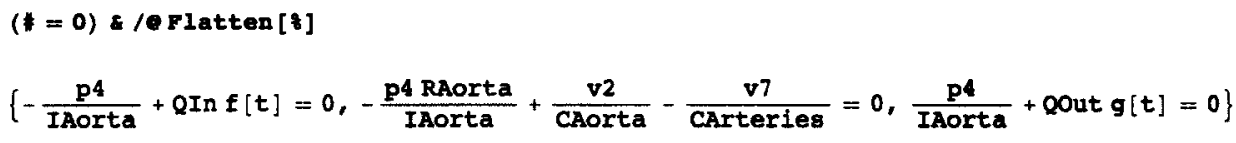

\{\} 
Flow-in, pressure-out with \{CArteries, RPeripheral, QIn\} as repeating variables

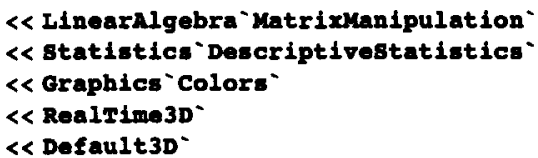

These are the $\{m\},\{L\}$ and $\{t\}$ units of all the variables in the flow-in-flow-out formulation. The columns represent (in order): RAorta, IAorta, CAorta, CArteries, RPeripheral, QIn, POut and s.

$$
\begin{aligned}
& \text { MatrixForm [FullunitMatr } 1 x=\{\{1,1,-1,-1,1,0,1,0\}, \\
& \{-4,-4,4,4,-4,3,-1,0\},\{-1,0,2,2,-1,-1,-2,-1\}\}] \\
& \left(\begin{array}{cccccccc}
1 & 1 & -1 & -1 & 1 & 0 & 1 & 0 \\
-4 & -4 & 4 & 4 & -4 & 3 & -1 & 0 \\
-1 & 0 & 2 & 2 & -1 & -1 & -2 & -1
\end{array}\right)
\end{aligned}
$$

Select repeating variables of CArteries, RPeripheral, QIn (these form the columns of "RepeatingVariableunitMatrix"; the rows are $\{m\},\{L\}$ and $\{t\})$

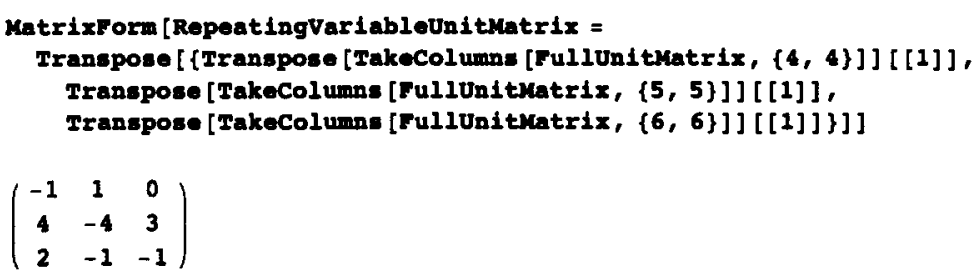

Buckingham's $\Pi$-Theorem... solve for the exponents

\{a [1], a [2], a [3]\} = Linearsolve [Repeat IngVariableUnitMatrix, - 1* Transpose [TakeColumns [FullUnitkatrix, $\{1,1\}]][[1]]]$;

$\{a[4], a[5], a[6]\}=$ Linearsolve [RepeatingVariableunitMatrix, - 1 * Transpose [Takecolumns [Fullunitkatrix, $\{2,2\}]$ [ [1]]];

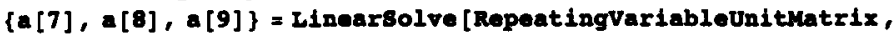
- 1 * Transpose [TakeColumns [Ful]Unitkatrix, $\{3,3\}]$ ] [ [1] ] ];

$\{a[10], a[11], a[12]\}=$ Iinearsolve [RepeatingVariableunituatrix , -1 * Transpose [TakeColumns [FulIUnitkatrix, $\{7,7\}]][[1]]]$;

\{a [13], a [14], a [15]\} = Linearsolve [Repeatingvariableunityatrix , -1 * Transpose [Takecolume [Fullunithatrix, $\{8,8\}]$ ] [1]]]; 
First $\Pi$ group (RAorta plus repeating variables)

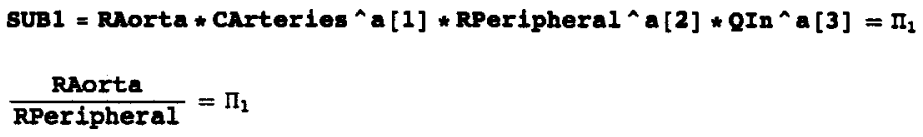

Second $\Pi$ group (IAorta plus repeating variables)

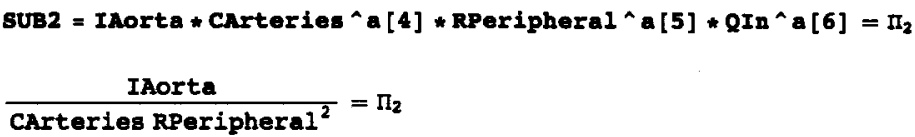

Third $\Pi$ group (CAorta plus repeating variables)

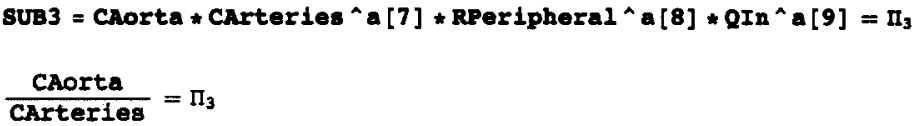

Fourth $\Pi$ group (Pout plus repeating variables)

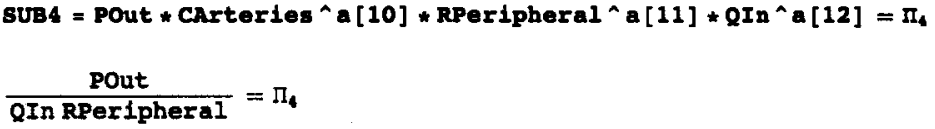

Fifth $\Pi$ group (s plus repeating variables)

sUB5 $=8 *$ CArteries^a $[13] *$ RPeripheral`a $[14] \star Q I_{n} \wedge a[15]=\Pi_{5}$

CArteries RPeripheral $8=\Pi_{5}$

This is where we form the transfer function matrix (based on the differential equations).

$\mathbf{A A}=\{\{0,-1 /$ IAorta, 0$\},\{1 /$ CAorta, - RAorta / IAorta, - $1 /$ CArteries $\}$,

$\{0,1 /$ Inorta, $-1 /$ (CArteries $\star$ RPeripheral) $\}\}$;

$B B=\{\{1,0\},\{0,0\},\{0,1 /$ RPeripheral $\}\}$;

CC = IdentityMatrix [3],

DD $=$ Table $[0,\{1,1,3\},\{\}, 1,2\}]$; 
This is the characteristic equation

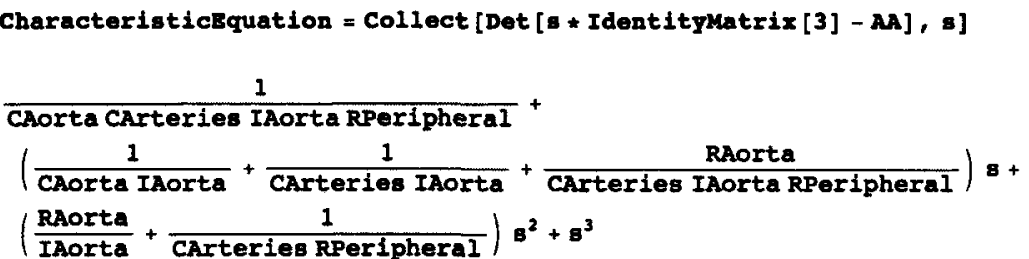

Nondimensionalize it

simplify [Eliminate [ \{CharacteristicEquation $=0$, sUB1, sUB2, sUB3, sUB4, sUB5], [RAorta, IAorta, CAorta, CArteries, RPeripheral, QIn, POut\}]]

$\Pi_{5}\left(1+\Pi_{3}\left(1+\Pi_{1}\left(1+\Pi_{5}\right)+\Pi_{2} \Pi_{5}\left(1+\Pi_{5}\right)\right)\right)=-1$

IDCharacteristicEquation $=\left(\operatorname{Collect}\left[f, \Pi_{5}\right]\right) \& / e$ Expand $/ e$

$\left(1+\Pi_{3}+\Pi_{1} \Pi_{3}\right) \Pi_{5}+\left(\Pi_{1} \Pi_{3}+\Pi_{2} \Pi_{3}\right) \Pi_{5}^{2}+\Pi_{2} \Pi_{3} \Pi_{5}^{3}=-1$

Check to see if there are any parameter ranges which might make the arterial system unstable (Routh-Hurwitz)

RouthHurwitzPrep [x_, var_] : = Module [

$\{c l\}$,

cl = Reverse $[$ CoefficlentList $[x, \operatorname{var}]]$;

If $[\mathrm{cl}[$ [Length $[\mathrm{Cl}]]]=0, \mathrm{cl}=\operatorname{Most}[\mathrm{cl}]]$,

If [oddo [Length [cl] ], cl = Joln [cl, $\{0\}]]$;

\{Take $[C 1,\{1$, Length $[c 1], 2\}]$, Take $[c 1,\{2$, Length $[c 1], 2\}]\}$

$1 ;$ 


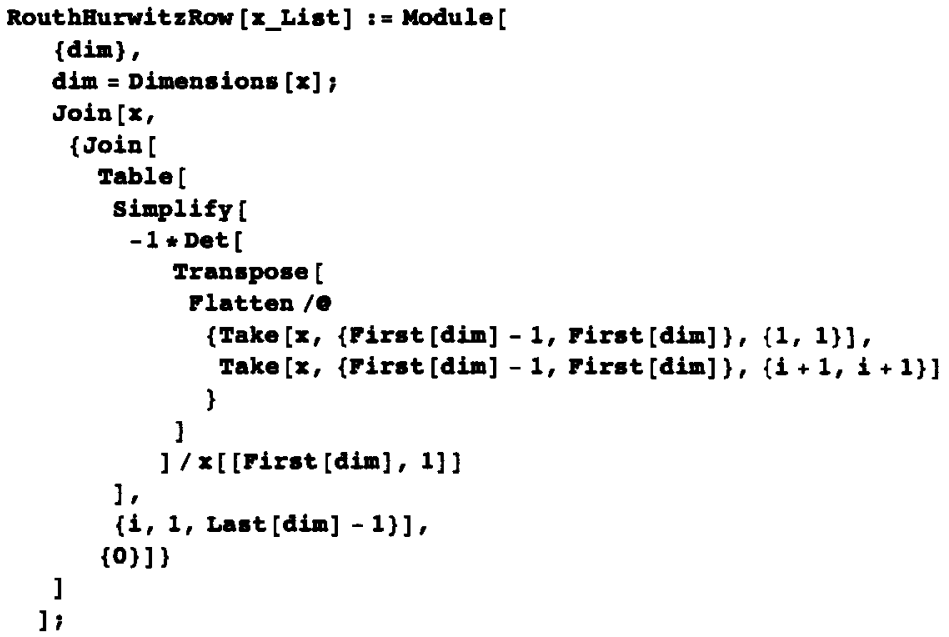

Solve the characteristic equation (not so easy; it's a cubic)... First, depress the cubic by making a substitution to eliminate the quadratic term

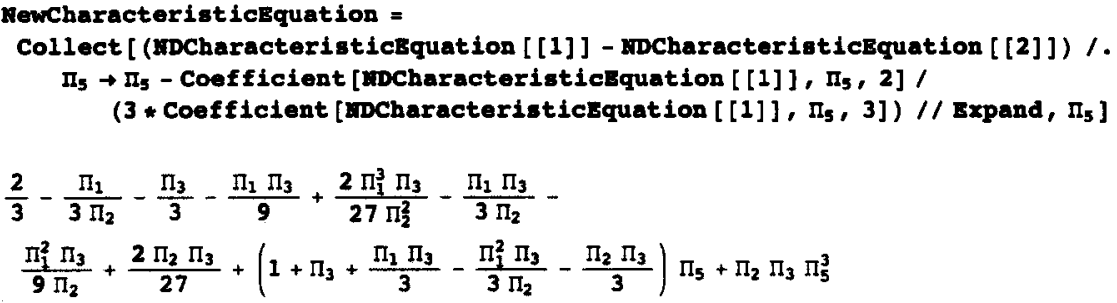


Now, blow it up into a degree- 6 polynomial

$$
\begin{aligned}
& \text { Rational CharacteristicEquation = MewCharacteristicEquation } / \Pi_{5} \rightarrow(\rho+v / \rho) / / \text { Expand; } \\
& \text { Degreesixpolynomial = Collect [Expand [RationalCharacteristicEquation } * \rho^{\wedge} 3 \text { ], } \rho \text { ] } \\
& \rho^{6} \Pi_{2} \Pi_{3}+v^{3} \Pi_{2} \Pi_{3}+\rho^{3}\left(\frac{2}{3}-\frac{\Pi_{1}}{3 \Pi_{2}}-\frac{\Pi_{3}}{3}-\frac{\Pi_{1} \Pi_{3}}{9}+\frac{2 \Pi_{1}^{3} \Pi_{3}}{27 \Pi_{2}^{2}}-\frac{\Pi_{1} \Pi_{3}}{3 \Pi_{2}}-\frac{\Pi_{1}^{2} \Pi_{3}}{9 \Pi_{2}}+\frac{2 \Pi_{2} \Pi_{3}}{27}\right)+ \\
& \rho^{4}\left(1+\Pi_{3}+\frac{\Pi_{1} \Pi_{3}}{3}-\frac{\Pi_{1}^{2} \Pi_{3}}{3 \Pi_{2}}-\frac{\Pi_{2} \Pi_{3}}{3}+3 v \Pi_{2} \Pi_{3}\right)+ \\
& \rho^{2}\left(v+v \Pi_{3}+\frac{1}{3} v \Pi_{1} \Pi_{3}-\frac{v \Pi_{1}^{2} \Pi_{3}}{3 \Pi_{2}}-\frac{1}{3} v \Pi_{2} \Pi_{3}+3 v^{2} \Pi_{2} \Pi_{3}\right)
\end{aligned}
$$

Find the constant which eliminates the quartic term

Substitution $=$ Solve $[$ Coefficient $[$ DegreesixPolynomial, $\rho, 4]=0, v][[1,1]]$

$u \rightarrow-3 \Pi_{2}+\Pi_{1}^{2} \Pi_{3}-3 \Pi_{2} \Pi_{3}-\Pi_{1} \Pi_{2} \Pi_{3}+\Pi_{2}^{2} \Pi_{3}$

$9 \Pi_{2}^{2} \Pi_{3}$

Check to make sure it eliminates the quadratic term as well

Simplify [ (Coefficient [Degreesixpolynomial, $\rho, 2] /$ substitution) $=0$ ]

True

Turn it into a quadratic using this substitution

Quadratic = Expand [DegreesixPolynomial /. substitution ];

Collect [Quadratic, $\rho$ ]

$-\frac{1}{81}-\frac{\Pi_{1}^{4}}{81 \Pi_{2}^{4}}+\frac{2 \Pi_{1}^{2}}{27 \Pi_{2}^{3}}+\frac{2 \Pi_{1}^{3}}{81 \Pi_{2}^{3}}-\frac{1}{9 \Pi_{2}^{2}}-\frac{2 \Pi_{1}}{27 \Pi_{2}^{2}}-\frac{\Pi_{1}^{2}}{27 \Pi_{2}^{2}}+\frac{2}{27 \Pi_{2}}+\frac{2 \Pi_{1}}{81 \Pi_{2}}-\frac{1}{27 \Pi_{2}^{2} \Pi_{3}^{2}}+\frac{\Pi_{1}^{2}}{27 \Pi_{2}^{3} \Pi_{3}}-$

$\frac{1}{9 \Pi_{2}^{2} \Pi_{3}}-\frac{\Pi_{1}}{27 \Pi_{2}^{2} \Pi_{3}}+\frac{1}{27 \Pi_{2} \Pi_{3}}-\frac{\Pi_{3}}{81}-\frac{\Pi_{1} \Pi_{3}}{243}+\frac{\Pi_{1}^{6} \Pi_{3}}{729 \Pi_{2}^{5}}-\frac{\Pi_{1}^{4} \Pi_{3}}{81 \Pi_{2}^{4}}-\frac{\Pi_{1}^{5} \Pi_{3}}{243 \Pi_{2}^{4}}+\frac{\Pi_{1}^{2} \Pi_{3}}{27 \Pi_{2}^{3}}+$

$\frac{2 \Pi_{1}^{3} \Pi_{3}}{81 \Pi_{2}^{3}}+\frac{2 \Pi_{1}^{4} \Pi_{3}}{243 \Pi_{2}^{3}}-\frac{\Pi_{3}}{27 \Pi_{2}^{2}}-\frac{\Pi_{1} \Pi_{3}}{27 \Pi_{2}^{2}}-\frac{\Pi_{1}^{2} \Pi_{3}}{27 \Pi_{2}^{2}}-\frac{7 \Pi_{1}^{3} \Pi_{3}}{729 \Pi_{2}^{2}}+\frac{\Pi_{3}}{27 \Pi_{2}}+\frac{2 \Pi_{1} \Pi_{3}}{81 \Pi_{2}}+\frac{2 \Pi_{1}^{2} \Pi_{3}}{243 \Pi_{2}}+$

$\frac{\Pi_{2} \Pi_{3}}{729}+\rho^{6} \Pi_{2} \Pi_{3}+\rho^{3}\left(\frac{2}{3}-\frac{\Pi_{1}}{3 \Pi_{2}}-\frac{\Pi_{3}}{3}-\frac{\Pi_{1} \Pi_{3}}{9}+\frac{2 \Pi_{1}^{3} \Pi_{3}}{27 \Pi_{2}^{2}}-\frac{\Pi_{1} \Pi_{3}}{3 \Pi_{2}}-\frac{\Pi_{1}^{2} \Pi_{3}}{9 \Pi_{2}}+\frac{2 \Pi_{2} \Pi_{3}}{27}\right)$ 
This is the condition which precludes pressure oscillations in the arterial system

simplify [Coeficient [Quadratic, 0,3$]^{\wedge} 2$ -

$(4 *$ Coefficient [Quadratic, 0,6$] *$ Coefficient $[Q u a d r a t i c, 0,0])>$

$\left.0,\left\{\Pi_{1}>0, \Pi_{2}>0, \Pi_{3}>0\right\}\right\}$

$2 \Pi_{2}\left(2+\left(6-4 \Pi_{1}\right) \Pi_{3}+\left(6+\Pi_{1}+\Pi_{1}^{2}+2 \Pi_{2}^{2}\right) \Pi_{3}^{2}+\left(1+\Pi_{1}\right)^{2}\left(2+\Pi_{1}\right) \Pi_{3}^{3}\right)>$

$\Pi_{3}\left(\Pi_{1}^{2}\left(1-2\left(-1+\Pi_{1}\right) \Pi_{3}+\left(1+\Pi_{1}\right)^{2} \Pi_{3}^{2}\right)+\Pi_{2}^{2}\left(-8+4\left(5+2 \Pi_{1}\right) \Pi_{3}+\left(1+\Pi_{1}\right)^{2} \Pi_{3}^{2}\right)\right)$

Contourfune $=8[[2]]-8[1]]$

$-2 \Pi_{2}\left(2+\left(6-4 \Pi_{1}\right) \Pi_{3}+\left(6+\Pi_{1}+\Pi_{1}^{2}+2 \Pi_{2}^{2}\right) \Pi_{3}^{2}+\left(1+\Pi_{1}\right)^{2}\left(2+\Pi_{1}\right) \Pi_{3}^{3}\right)+$

$\Pi_{3}\left(\Pi_{1}^{2}\left(1-2\left(-1+\Pi_{1}\right) \Pi_{3}+\left(1+\Pi_{1}\right)^{2} \Pi_{3}^{2}\right)+\Pi_{2}^{2}\left(-8+4\left(5+2 \Pi_{1}\right) \Pi_{3}+\left(1+\Pi_{1}\right)^{2} \Pi_{3}^{2}\right)\right)$
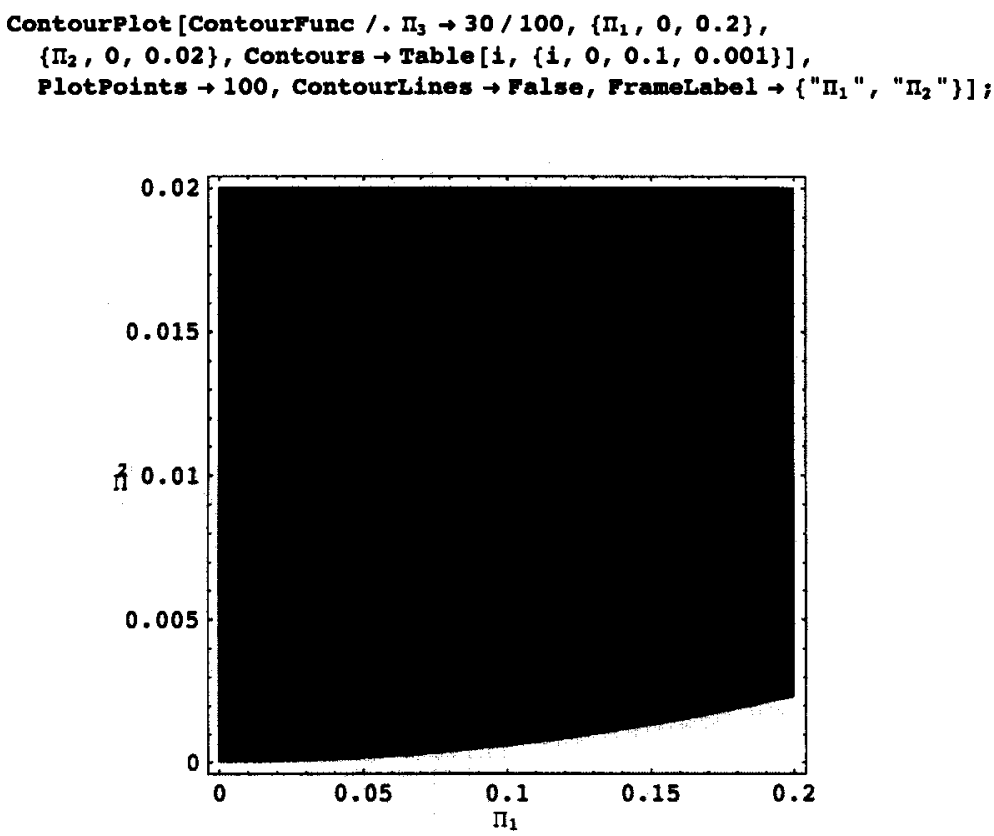

ContourFunc /. $\left\{\Pi_{1} \rightarrow 0 ., \Pi_{2} \rightarrow 0.01, \Pi_{3} \rightarrow 30 / 100\right\}$

$-0.0879377$ 
Contourplot [Contourrune $/ . \pi_{3} \rightarrow 30 / 100,\left\{\Pi_{1}, 0,0.2\right\}$,

$\left\{\Pi_{2}, 0,0.02\right\}$, Contours $\rightarrow 25$, Plotpoints $\rightarrow 100$, Contourshading $\rightarrow$ ralse,

Contourstyle $\rightarrow\{$ RGBColor $[0.5,0.5,1]\}$, Framelabel $\left.\rightarrow\left\{" n_{2} ", " n_{2} "\right\}\right]$;

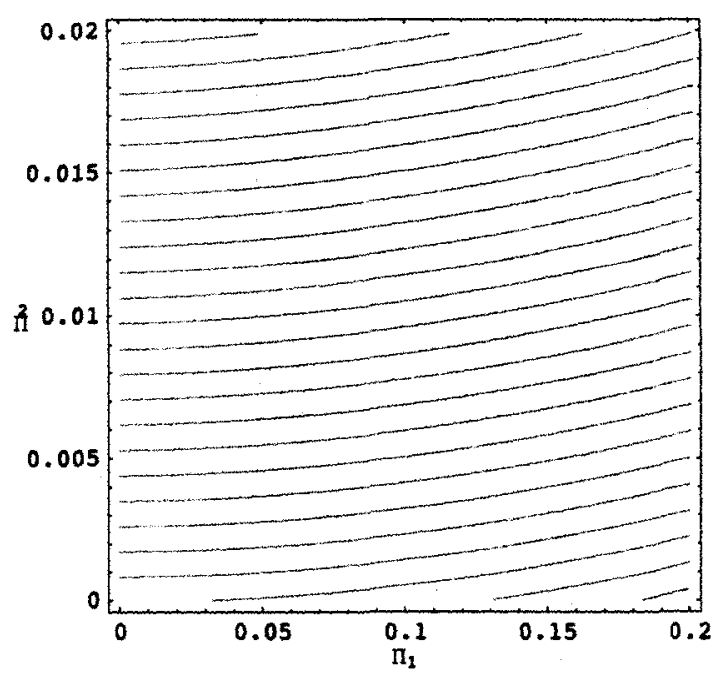

Reproduced with permission of the copyright owner. Further reproduction prohibited without permission. 


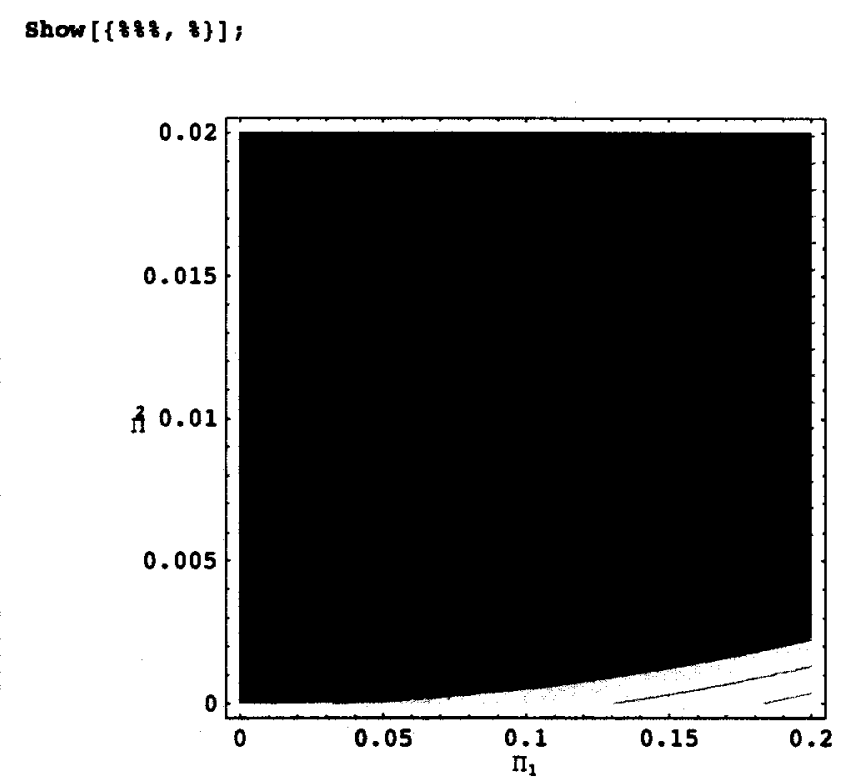

Check the fixed points; form differential equations

$$
\begin{aligned}
& \text { AA. }\{\{\nabla 2\},\{p 4\},\{\nabla 7\}\}+B B \cdot\{Q I n * f[t], \text { QOut } * g[t]\} \\
& \left\{\left\{-\frac{p 4}{\text { IAorta }}+Q I n f[t]\right\},\left\{-\frac{p 4 \text { RAorta }}{\text { IAOrta }}+\frac{v 2}{\text { CAOrta }}-\frac{v 7}{\text { CArteries }}\right\},\right. \\
& \left\{\frac{\text { p4 }}{\text { IAorta }}-\frac{\text { QOut } g[t]}{\text { CArteries RPeripheral }}+\frac{\text { RPeripheral }}{\text { Rat }}\right\}
\end{aligned}
$$

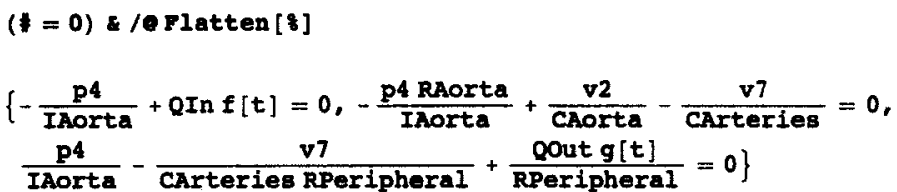

\{ (v2 $\rightarrow$ CAOrta QIn RAOrta $f[t]+$ CAOrta QIn RPeripheral $f[t]+$ CAOrta QOut $g[t]$ $v 7 \rightarrow$ CArteries QIn RPeripheral $f[t]+$ CArteries QOut $g[t], p 4 \rightarrow$ IAorta $Q I n f[t]\}\}$ 


\section{Appendix C}

\section{UOHI Mock Circulatory System}

$\mathbf{T}$

HE University of Ottawa Heart Institute mock circulatory system features two cylindrical compliance chambers with identical diameters but different heights. Unlike the venous compliance chamber, which is open to atmosphere, the arterial compliance chamber is closed to atmosphere and the trapped, compressed air gives the chamber a nonlinear stiffness. Unfortunately, the arterial chamber's cross-sectional area is too large and this nonlinearity will not be felt over the course of a beat; much more than the typical stroke volume must be injected before the additional air compression becomes significant and the nonlinearity is felt.

The VAD pumps into the upper part of the arterial chamber through a 12 in long, 1 in internal diameter tube. The inlet centreline is elevated 2 in from the VAD outlet, and 6 in from the base of the chamber. The arterial chamber outlet is at a lower elevation than the inlet, with its centreline 3 in above the base of the chamber. The arterial and venous chambers are connected through tubing, and separated by a globe-type valve which is actuated using a servomechanism. Different fractional valve openings can be achieved by supplying different DC voltages to the servo; lower voltages imply greater fractional openings.

The venous chamber is a static water column, with its top open to atmosphere (through a small opening). Its outlet is at a slightly lower elevation than its inlet, and it discharges through a ball-type valve designed to control the pre-load on the VAD, an important part of FFFE-mode operation. 


\section{University of Ottawa Heart Institute Mock Circulatory System Simulation}

Setoptions [Plot, PlotPoints $\rightarrow$ 1000]

To convert from $\mathrm{mmHg}$ to $\frac{\mathrm{N}}{\mathrm{cm}^{2}}$, take the density of Mercury, $13579.04 \frac{\mathrm{kg}}{\mathrm{m}^{3}}$, and multiply it by the gravitational constant, $9.81 \frac{\mathrm{m}}{\mathrm{s}^{2}}$, and the height, $\mathrm{mm}$. Multiply the result by $\frac{1 \mathrm{~cm}}{10 \mathrm{~mm}}$ and $\left(\frac{1 \mathrm{~m}}{100 \mathrm{~cm}}\right)^{3}$ for unit conversion.

gravity $=9.81$

densityig $=13579.04$

Hg2 $=$ density

T2Eg = $1 /$ Bg2N;

Area $=19.27$

Fluid parameters for blood; density in $\frac{\mathrm{kg}}{\mathrm{m}^{3}}$ and viscosity in $\frac{\mathrm{kg}}{\mathrm{ms}}$.

density $20=998$.

viscosity $20=1 . * 10^{\wedge}-3$

Reynold's number; substitute for kinematic viscosity

$v * d / v / .\{v \rightarrow \mu / \rho\}$

$\frac{d v \rho}{\mu}$

Use friction factor (from Moody chart, laminar flow) to compute head loss in tube

$$
\begin{aligned}
& v^{\wedge} 2 /(2 * g) *(f * I / d) / .(f \rightarrow 64 / \text { t }\} \\
& \frac{32 L v \mu}{d^{2} g \rho}
\end{aligned}
$$


Substitute for velocity (flow rate by cross-sectional area)

$$
\begin{aligned}
& 8 / \cdot\{\nabla \rightarrow Q / A\} \\
& \frac{32 \perp Q \mu}{A d^{2} g \rho}
\end{aligned}
$$

Substitute for cross-sectional area to get pipe-flow head loss equation

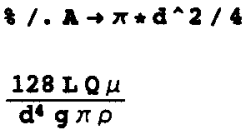

Pipe-flow equation (head loss multiplied by specific weight; change units from meters to centimeters)

Pipeflowresistance $=\rho * g * \nabla / 100 \wedge 2 / . \mu \rightarrow v i s c o s i t y H 20$

$4.07437 \times 10^{-6} \mathrm{~L} \mathrm{Q}$ $d^{4}$

Form equation based on loss coefficient, $k$

$$
\begin{aligned}
& \rho * v \wedge 2 / 2 * x \\
& \frac{1}{2} v^{2} x \rho
\end{aligned}
$$

Substitute for velocity (flow rate by cross-sectional area)

$$
\begin{aligned}
& 8 / . \nabla \rightarrow Q / A \\
& \frac{Q^{2} K \rho}{2 A^{2}}
\end{aligned}
$$

Substitute for cross-sectional area to get fitting head loss equation

$$
\begin{aligned}
& / \mathrm{A} \rightarrow \pi * \mathrm{~d}^{\wedge} 2 / 4 \\
& \frac{8 \mathrm{Q}^{2} K D}{\mathrm{~d}^{4} \pi^{2}}
\end{aligned}
$$


Fitting loss equation (first-order Taylor series expansion about mean flow rate; change units from meters to centimeters)

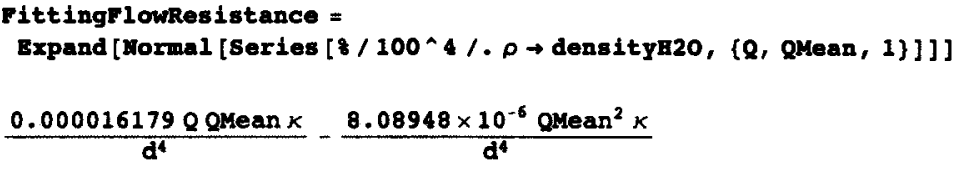

Plot both the original and linearized functions to make sure they look right

Plot $\left[\left\{8 Q^{\wedge} 2 * x *\right.\right.$ densityz20 $/\left(d^{\wedge} 4 * \pi^{\wedge} 2 * 100^{\wedge} 4\right) / .\{x \rightarrow 3.45, d \rightarrow 2.54\}$, FittingFlowResistance $/ .\{x \rightarrow 3.45, d \rightarrow 2.54$, QMan $\rightarrow 80\}\}$,

$\{0,60,240\}$, PlotRange $\rightarrow\{$ A11, $\{0$, Automat $1 C\}\}]$;

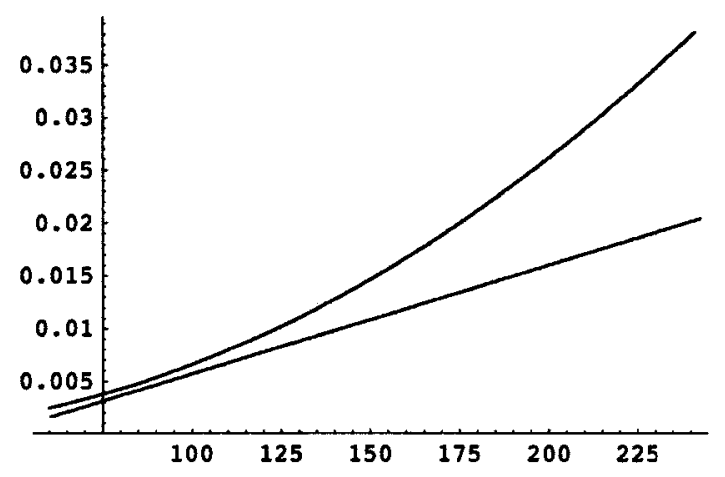

Pressure losses across the fully-open VAD outlet valve

FittingflowResistance $/ .\{x \rightarrow 2.45, d \rightarrow 2.54$, QMean $\rightarrow 80\}$

$-0.00304742+0.00007618540$

Pressure losses in the first segment of tubing (including fittings) between the VAD and the arterial pressure chamber

(Pipeplowresistance $/ .\{L \rightarrow 12 * 2.54, d \rightarrow 2.54\})+$

(FittingFlownesistance $/ .\{x \rightarrow 1, d \rightarrow 2.54$, QMean $\rightarrow 80\}$ )

$-0.00124384+0.0000340797 Q$ 
This is the Goff-Gratch equation for the SVP of air over liquid water

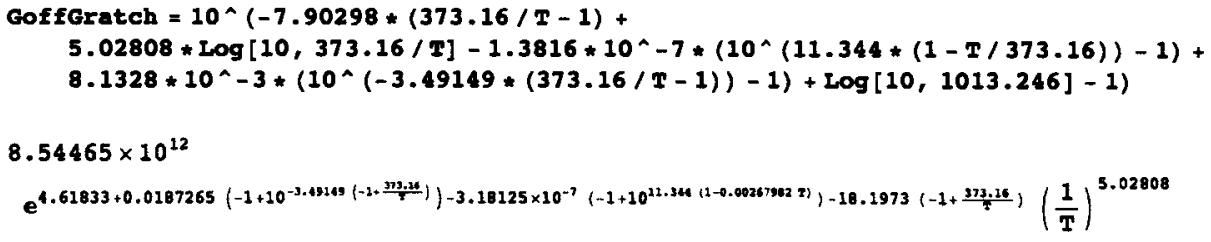

Plot it in the temperature range of interest

Plot [GoffGratch, $\{1,15+273,30+273\}]$;

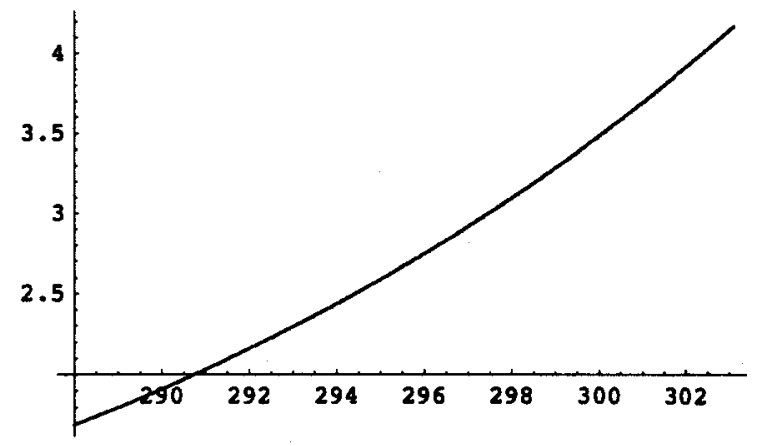

Calculate the air density in the aortic chamber using the Goff-Gratch equation for the partial pressure of the water vapour (at room temperature). The pressure at the surface of the water is $15.5 \mathrm{in} \mathrm{H} 2 \mathrm{O}$ or $3861 \mathrm{~Pa}$.

$3861 /(287.05 * 296.15)+($ GoffGratch $/ . T \rightarrow(23+273)) * 10^{\wedge} 3 /(461.495 * 296.15)$

0.0657699

Compute the static pressure head due to the trapped air (in $\mathrm{Pa}$ )

$8 * \operatorname{gravity} * 1.5 * 25.4 * 10^{\wedge}-3$

0.0245822

Treat the trapped air as an ideal gas

AoConst $=3861 * P i * 1.5 * 8 \wedge 2 / 4 * 2.54^{\wedge} 3 / 100^{\wedge} 2$

477.048

Reproduced with permission of the copyright owner. Further reproduction prohibited without permission. 
Cylinder cross-sectional area (in $\mathrm{cm}^{\wedge} 2$ )

CSecArea $=P i * 8 \wedge 2 / 4 * 2.54^{\wedge} 2$

324.293

Calculate the total volume in the aortic cylinder

Aovolume $=9 *$ Csecarea $* 2.54$

7413.33

This is the pressure at any (vertical) location in the aortic chamber (units $N / \mathrm{cm}^{\wedge} 2$ ) for a given volume of water

AOEQ $=$ simplify $\left[\right.$ AoConst $/($ Aovolume $-v)+\rho * g *(V /$ CsecArea $-h) / 100^{\wedge} 3 /$

$\{\rho \rightarrow$ densityH2o, g $\rightarrow$ gravity $\}]$

$-0.00979038 h+\frac{477.048}{7413.33-1 . v}+0.0000301899 v$

Here is the inlet pressure (for a given volume of water)

AOEQ $/ . \mathrm{h} \rightarrow 15.24$

$-0.149205+\frac{477.048}{7413.33-1 . v}+0.0000301899 v$

Plot $[$ AOEQ $/ . h \rightarrow 15.24,\{v, 15.24 *$ Csecarea, $17.78 *$ Csecarea $\}]$;

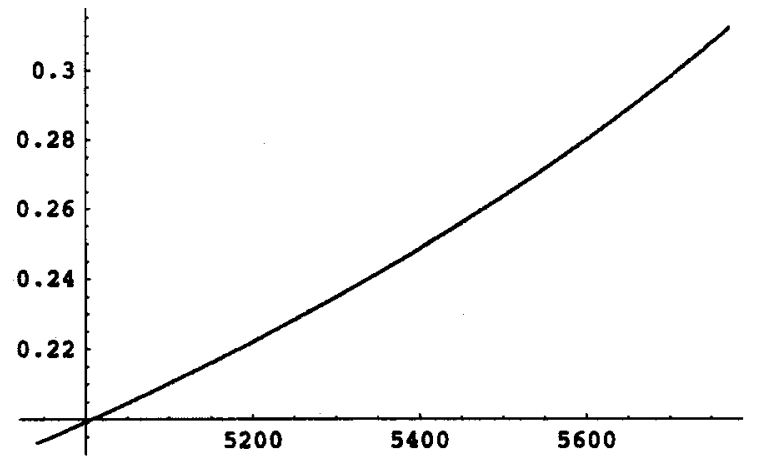

Reproduced with permission of the copyright owner. Further reproduction prohibited without permission. 
Pressure losses in the next segment of tubing (including fittings) between the arterial chamber and the peripheral resistance valve

(PipeFlowResistance $/ .\{\mathrm{L} \rightarrow 11.5 * 2.54, \mathrm{~d} \rightarrow 2.54\})$

(FittingFlowResistance $/ .\{x \rightarrow 5.72, d \rightarrow 2.54$, QMean $\rightarrow 80\}$ )

$-0.00711479+0.000180729 Q$

Pressure losses in the curved section of tubing (including fittings) between the peripheral resistance valve and the venous chamber

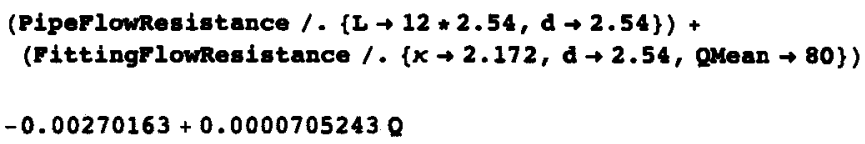

The venous chamber is open to atmosphere, therefore the compliance is a function of the cross-sectional area

VnEQ $=$ Expand $\left[\rho * g *(V /\right.$ CsecArea $-3 * 2.54) / 100^{\wedge} 3 / .\{\rho \rightarrow$ densityH2o, g $\rightarrow$ gravity $\left.\}\right]$

$-0.0746027+0.0000301899 v$

Pressure losses in the segment of tubing (including fittings) between the venous chamber and the inflow control valve

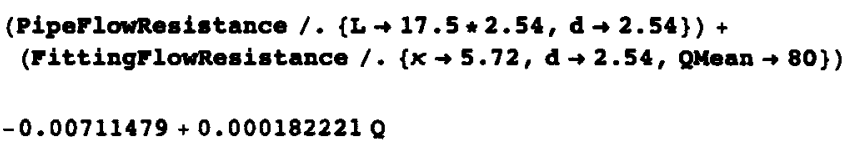

Pressure losses in the segment of tubing (including fittings) between the inflow control valve and the VAD inlet valve

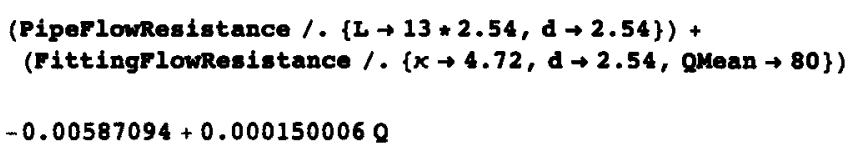

Pressure losses across the fully-open VAD inlet valve

FittingFlowResistance $/ .\{x \rightarrow 2.45, d \rightarrow 2.54$, QMean $\rightarrow 80\}$

$-0.00304742+0.0000761854 Q$ 
This is the value of Rot (open valve)

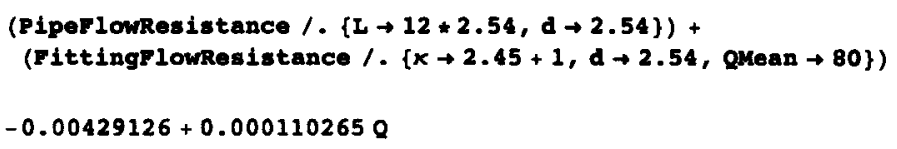

This is the value of Rpe, silver valve taken as 0.013 nominal

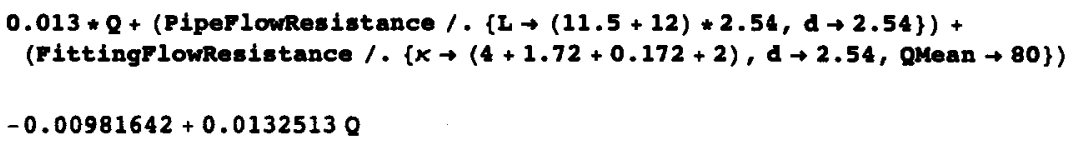

This is the value of Rin (open valve) with yellow valve taken as 0.01

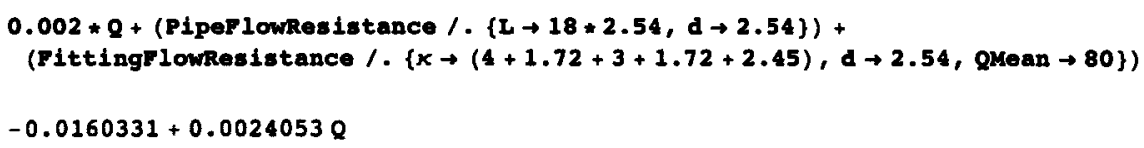

RInletValveMin $=$ Coefficient $[\%, Q, 1]$

0.0024053

This is the outlet valve flow resistance function; it accepts a pressure (delta) input in $\frac{\mathrm{N}}{\mathrm{cm}^{2}}$ and returns a flow in $\frac{\mathrm{ml}}{\mathrm{s}}$

ROutletValve $\left[x_{-}\right]:=I f[x>0, x /$ RoutletValveMin, $x /(8 . * 10 \wedge 2)]$

This is the inlet valve flow resistance function; it accepts a pressure (delta) input in $\frac{\mathrm{N}}{\mathrm{cm}^{2}}$ and returns a flow in $\frac{\mathrm{m}}{\mathrm{s}}$

RInletValve [x_] : = If $\left[x>0, x /\right.$ RInletValveMin, $\left.x /\left(8 . \star 10^{\wedge} 2\right)\right]$ 
Piston area

Area $=19.27$

Piston inertia

IPiston $=0.03$

Piston resistance (friction)

RPiston $=0.37$

This is the pumping force function; currently fixed rate.

Force [xx_] : = Kodule [ $\{$ Hinforce, MaxForce, Systole, Diastole $\}$

Minforce $=0.5 ;$ MaxForce $=39.5 ;$ systole $=0.3 ;$ Diastole $=0.7$;

If [Mod [xx, systole + Diastole] < systole, Minforce + Haxporce, Minforce] ]

Plot $[$ Force $[t],\{t, 0,2\}]$;

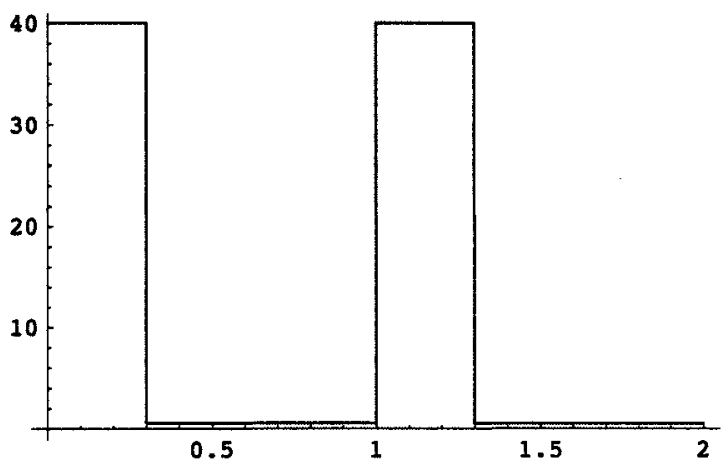

IMSYSTOLE $=$ False; PREVIOUSTIME $=0$

Force [tt_? Numbere, xx_? MumberQ] : = Module [ $($ MinForce, MaxForce, MinVol, MaxVol $\}$, Kinforce $=0 . ;$ MaxForce $=40 . ; \mathrm{KinVol}=20 . ; \mathrm{MaxVol}=100$.;

If [IRSYSTOLE = True, If $[(x x \leq$ MinVol) (PREVIOUSTIME $<t t)$

InSYSTOLE = False; PREVIOUSTIME = tt; MinForce, MaxForce + Minforce ,

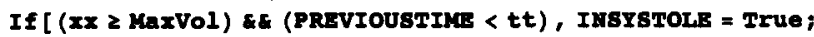

PREVIOUSTIME $=t t ;$ MaxForce + Minforce, MinForce $]]$ 
This is the VAD chamber's constitutive relation; it accepts a volume input in $\mathrm{ml}$ and returns a pressure in $\frac{\mathrm{N}}{\mathrm{cm}^{2}}$

PHeart [xx_] : = Module [ $\{$ Mominalv, Mominalp, Compliance, Offset $\}$,

Hominalv $=46.0$; NominalP $=10 *$ Hg2N; Compliance $=135$;

Offset $=$ (-Hominalv / Compliance + Hominalp); $\mathrm{H}[\mathrm{xx} /$ Compliance + Offset $]$;

This is the aortic chamber's constitutive relation; it accepts a volume input in $\mathrm{ml}$ and returns a pressure $\ln \frac{\mathrm{N}}{\mathrm{cm}^{2}}$

PAOrta $\left[x x_{-}\right]:=$AOEQ $/ .\{h \rightarrow 15.24, v \rightarrow x x\}$

This is the venous chamber's constitutive relation; It accepts a volume input in $\mathrm{ml}$ and returns a pressure in $\frac{\mathrm{N}}{\mathrm{cm}^{2}}$

PVeins $\left[x x_{-}\right]:=\operatorname{Varg} / . \mathrm{v} \rightarrow \mathrm{xx}$

Piston position; not an independent state.

EQ1 = sPiston ' $[t]=$ pPiston [t] / IPiston;

Piston momentum (use this for fixed-rate controller)

EQ2 $=$ pPiston ' $[t]=$ Force $[t]-$ pPiston [t] / IPIston * RPiston - PHeart [VHeart $[t]]$ * Area;

Piston momentum (use this for FFFE controller)

EQ2 = pPiston ' $[t]=$ Force $[t$, VHeart $[t]+100-8 P i s t o n[t] *$ Area $]$ -

pPiston [t] / IPiston * RPiston - PHeart [VHeart [t] ] * Area;

VAD compliance volume

EQ3 = VHeart ' [t] = pPiston [t] / IPiston * Area + RInletValve [PVeins [VVeins [t] ] +

density $120 *$ gravity $* 1 * 2.54 / 100^{\wedge} 3$ - PHeart [VHeart [t]] ] - ROutletValve [

PHeart [VHeart [t] ] - densityH20 $*$ gravity $* 2 * 2.54 / 100^{\wedge} 3$ - PAorta [VAorta [t]] ]

Aorta volume

sQ4 = VAorta ' $[t]=$ ROutletvalve [

pHeart [VHeart [ $t]$ ] - densityH20*gravity $* 2 * 2.54 / 100 \wedge 3$ - pAorta [VAorta [t] ] ] -

(PAorta [VAorta [t] ] + densityH20 *gravity *3*2.54/100^3-PVeins [VVeins [t] ]) /

RPeripheral; 
Venous volume

EQ5 = VVeins ' $[t]=$

(PAorta [VAorta [t] ] + densityH20* gravity *3*2.54/100^3-PVeins [VVeins [t] ]) /

RPeripheral - RInletvalve [

PVeins [VVeins [t] ] + densityH20 * gravity *1*2.54/100^3 - PHeart [VHeart [ $t$ ] ] ;

tstart $=0 ;$ tstop $=120 ;$ pstart $=110 ;$ pstop $=120 ;$

IAsYstoL: = False; PREVIOUSTIM: $=0$;

Timing [sOLF = EDsolve [ [EQ1, EQ2, EQ3, E04, EQ5, sPiston [0] $=0$, pPiston [0] $=0$,

vieart $[0]=0$, vaorta $[0]=$ CsecArea $* 7.5 * 2.54$, veins $[0]=$ csecArea $* 23 * 2.54\}$,

\{spiston, ppiston, VHeart, vaorta, Vveins\}, \{t, tstart, tstop\},

Maxsteps $\rightarrow$ Infinity, Maxstepsize $\rightarrow 0.001]$

$\{20.69$ Second, $\{\{8$ Piston $\rightarrow$ InterpolatingFunction $[\{[0,, 120\}\},.<>]$, pPiston $\rightarrow$ InterpolatingFunction $[\{\{0,120\}\},.<]$,

VHeart $\rightarrow$ InterpolatingFunction $\{\{\{0, .120\}\},.<>\}$

VAorta $\rightarrow$ InterpolatingFunction $\{\{\{0 ., 120\}\},.<>]$,

vveins $\rightarrow$ InterpolatingFunction $[\{\{0 ., 120\}\},.<>\}\}\}$

Piston position versus time

Plot [sPiston [t] /. sous, \{t, pstart, tstop $\}]$

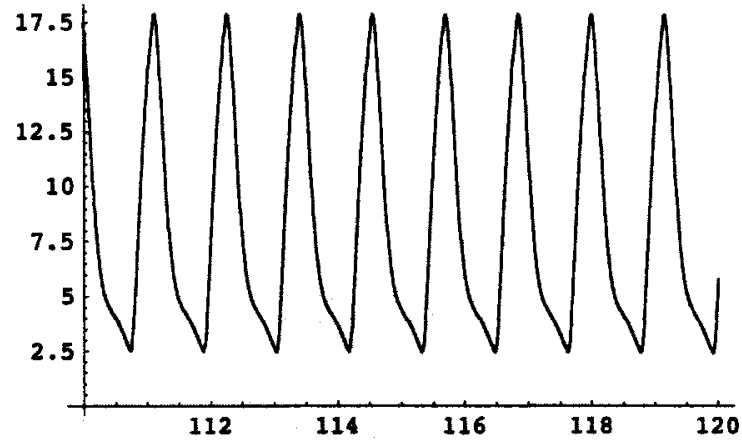


Internal VAD pressure versus time

Plot [PHeart [VHeart [t] ] * A2Bg / . sow, , [t, pstart, pstop \}]

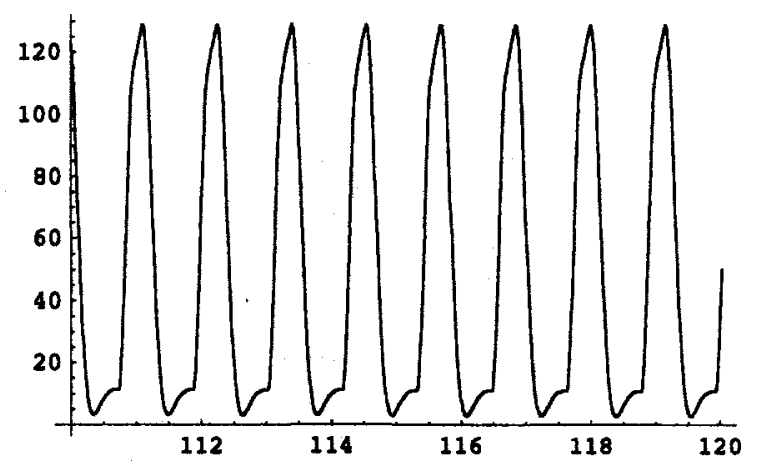

Internal VAD volume versus time

Plot [VHeart $[t]+100-8 P 1 s t o n[t]$ * Area /. souk, \{t, pstart, pstop\}];

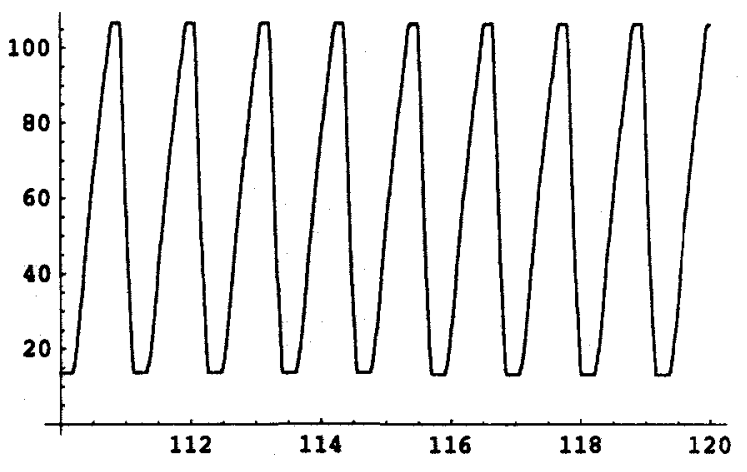




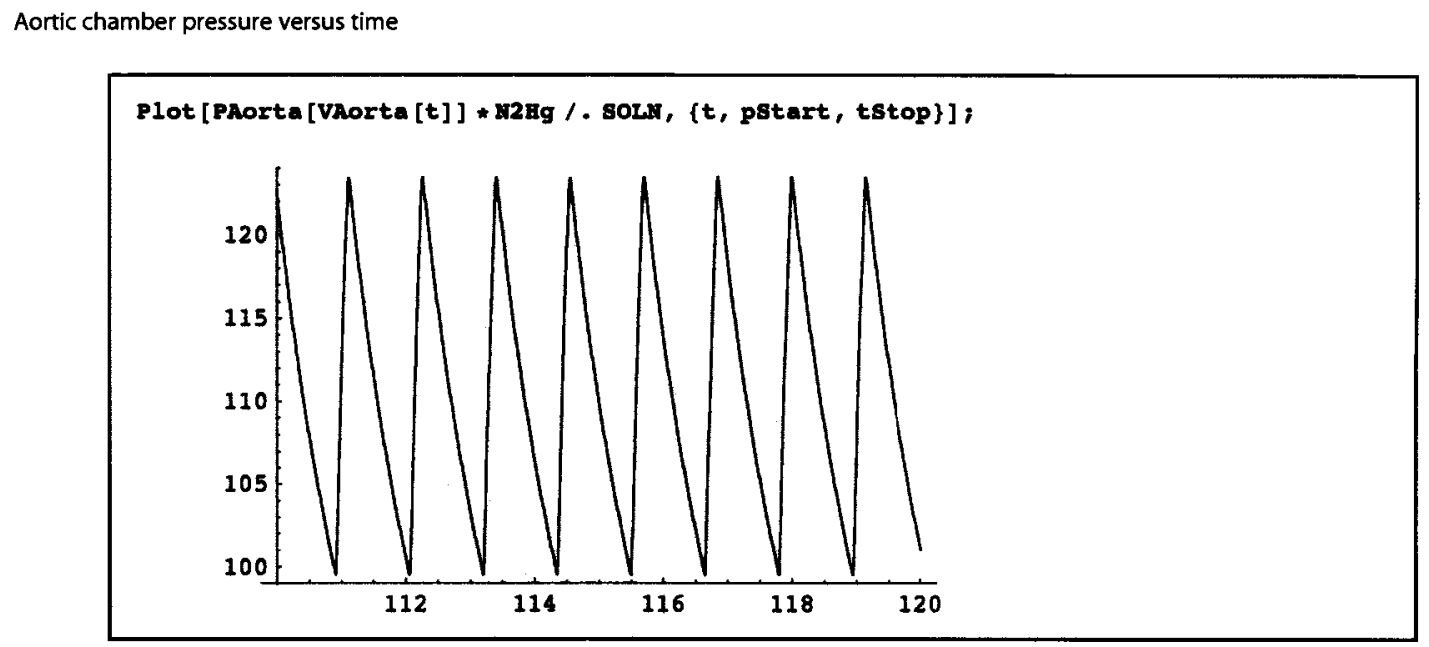

Venous chamber pressure versus time

Plot [PVeins [VVeins [t] ] * k2Bg/. soLn, $\{t$, pstart, tstop $]$; ;

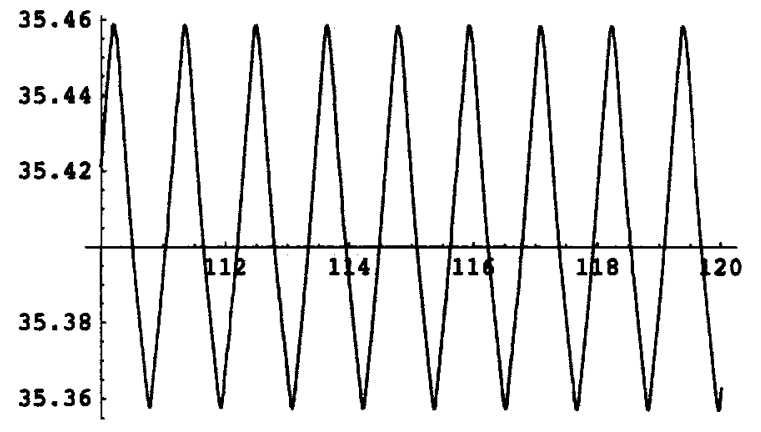




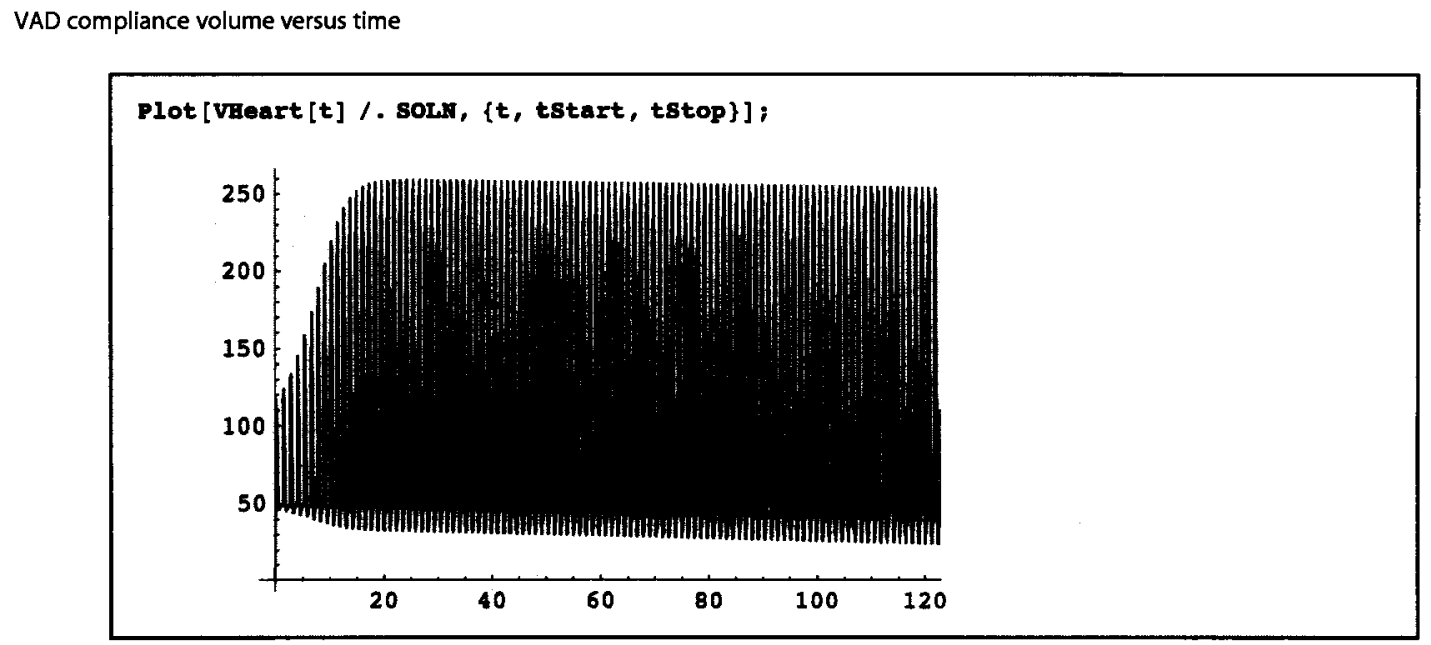

Aortic water height (in inches) versus time

Plot [VAorta [t] / CSecArea / 2.54/. sozk, \{t, tstart, tStop\}];

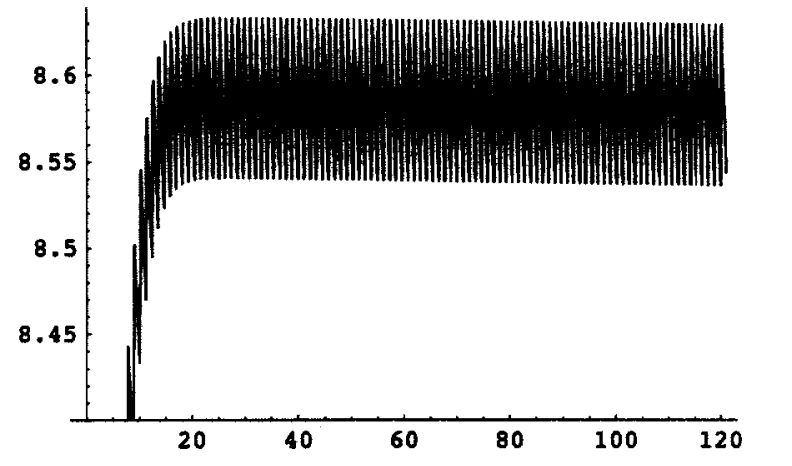


Venous water height (in inches) versus time

Plot [Vveins [t] / Csechrea / 2.54/. sour, \{t, tstart, tstop\}];

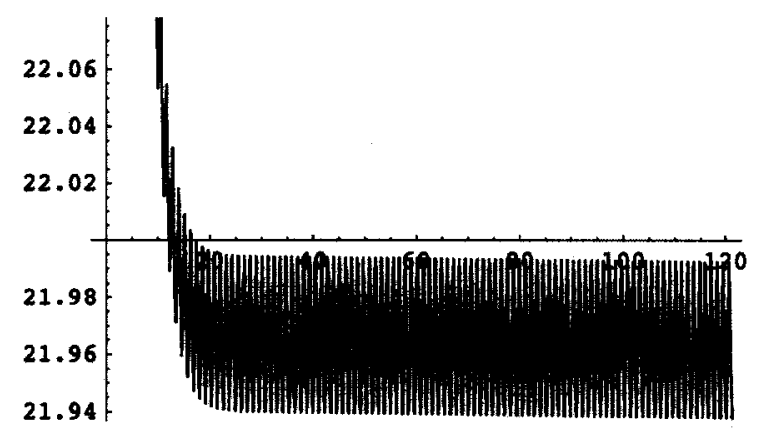

Total system volume versus time (check integrator error; should be constant)

Plot [Vveins [t] + VAorta [t] + VHeart $[t]+100-$ sPiston [t] *Area /. sols,

$\{t$, tstart, tstop $\}$, Plotrange $\rightarrow\{$ All,$\{0$, Automatic $\}\}$;

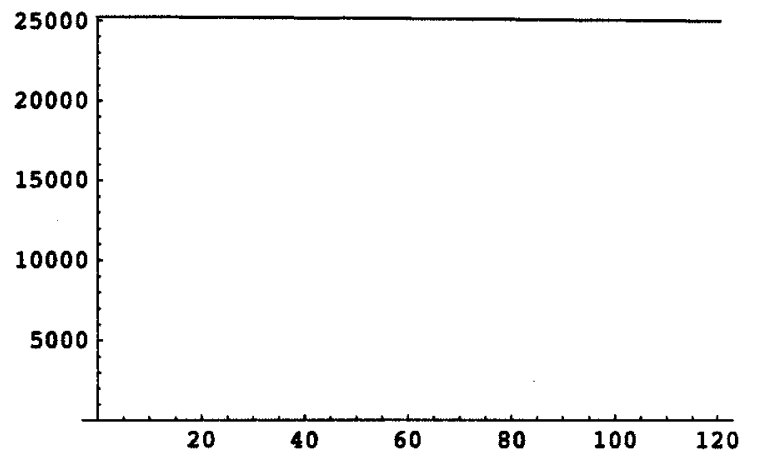


Outlet valve flow rate

Plot [ROutletValve [PHeart [VHeart [t]] - densityH20 * gravity *2*2.54/100^3pAorta [VAorta [t] ] ] /. som, \{t, pstart, pstop\}];

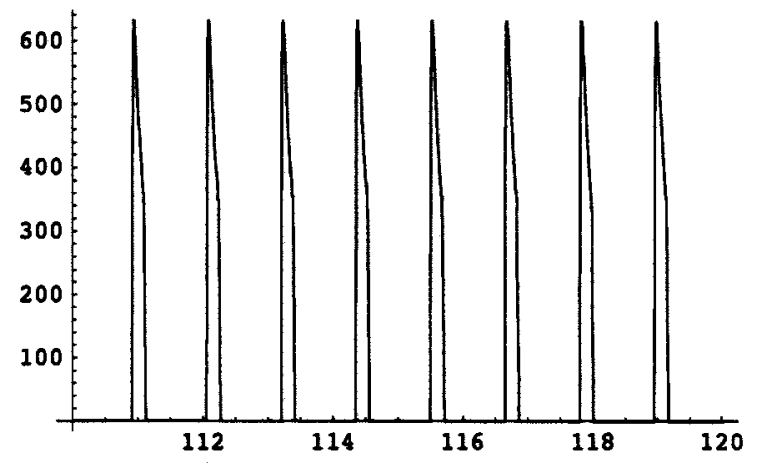

Check mean flow through outlet valve

Mean [Table [ROut] etValve [PHeart [VBeart [t]] - densityH20*gravity $2 * 2.54 / 100 \wedge 3-$ pAorta [VAorta [t] ] ] . sors, \{t, pstart, pstop, 0.001\}]]

$\{74.3858\}$ 


\section{Appendix D}

\section{MTS Simulation}

$\mathbf{W}$

HEN the rolling diaphragm chambers were added to the existing mock circulatory system, the viscous rolling friction became a concern. Although the air-water chambers have some fluid damping, the magnitude of the diaphragm's viscous rolling friction could be substantial in comparison.

The baseline human circulatory system model was revised to include viscous friction in the compliance chambers, but the inlet cannula dynamics were eliminated in order to reduce the complexity of the resultant model. In the pVAD setup the dynamics of the system between the venous compliance chamber and the pVAD pump are dominated by the pVAD cycle; the MTS machine is forceful enough that fluid will be drawn in during diastole, no matter the inflow valve setting. In a few instances the pVAD diaphragm inverted (due to high negative internal pressure) as the MTS attempted to draw in fluid which was simply not available.

The pVAD pumps into the arterial system through a 60 in long, 1 in internal diameter tube. The PVAD itself is elevated 18.5 in relative to the base of the MCS chambers. The arterial system consists of two rolling diaphragm chambers, modelled as compliance elements with viscous rolling friction. The arterial system and venous chamber are once again connected through tubing, and separated by the adjustable peripheral valve. The venous chamber remains a static water column, with its top open to atmosphere (through a small opening). The bond graph for the system is illustrated in Figure D.1. 


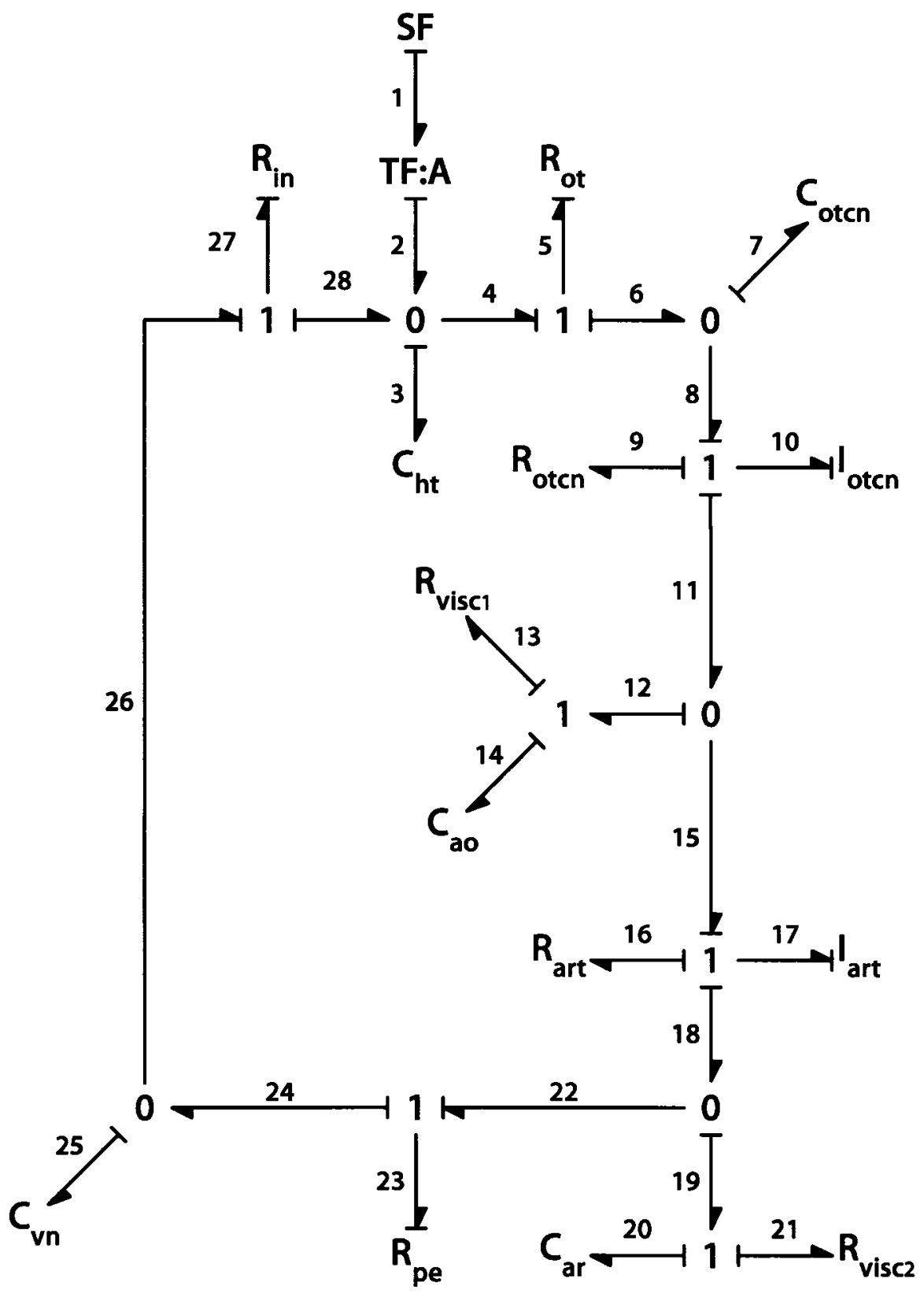

Figure D.1: Bond graph representation of the MTS (and pVAD) setup 


\section{MTS (and pVAD) Mock Circulatory System Simulation}

Setoptions [Plot, PlotPoints $\rightarrow$ 1000]

off [General : : spell, General : : spell1, solve : : ifun]

To convert from $\mathrm{mmHg}$ to $\frac{\mathrm{N}}{\mathrm{cm}^{2}}$, take the density of Mercury, $13579.04 \frac{\mathrm{kg}}{\mathrm{m}^{3}}$, and multiply it by the gravitational constant, $9.81 \frac{\mathrm{m}}{\mathrm{s}^{2}}$, and the height, $\mathrm{mm}$. Multiply the result by $\frac{1 \mathrm{~cm}}{10 \mathrm{~mm}}$ and $\left(\frac{1 \mathrm{~m}}{100 \mathrm{~cm}}\right)^{3}$ for unit conversion.

gravity $=9.81$

densityHg = 13579.04

Hg2n $=$ densityHg * gravity $*(1 / 10) *(1 / 100) \wedge 3$

F2Hg $=1 / \mathrm{Hg} 2 \mathrm{H}$

Fluid parameters for blood; density in $\frac{\mathrm{kg}}{\mathrm{m}^{3}}$ and viscosity in $\frac{\mathrm{kg}}{\mathrm{ms}}$.

density $120=998$

viscosityH $20=1 . * 10^{\wedge}-3$

Pipe inertia and volume equations

PipeflowInertla $=$ densityH20 $*(1 / 100)^{\wedge} 4 *$ Length $/(P i / 4 *$ Diameter`2);

PipeVolume = Length $* P i *$ Diameter^2/4;

Reynold's number; substitute for kinematic viscosity

$$
\begin{aligned}
& v * d / v / .\{v \rightarrow \mu / \rho\} \\
& \text { dv } \rho
\end{aligned}
$$


Use friction factor (from Moody chart, laminar flow) to compute head loss in tube

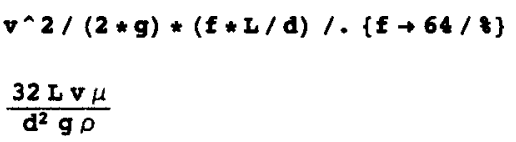

Substitute for velocity (flow rate by cross-sectional area)

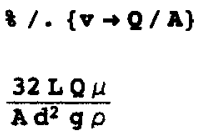

Substitute for cross-sectional area to get pipe-flow head loss equation

$$
\begin{aligned}
& \approx / . \mathrm{A} \rightarrow \pi * \mathrm{~d}^{\wedge} 2 / 4 \\
& \frac{128 \mathrm{~L} Q \mu}{d^{4} \mathrm{~g} \pi \rho}
\end{aligned}
$$

Pipe-flow equation (head loss multiplied by specific weight; change units from meters to centimeters)

PiperlowResistance $=\rho * g * \hbar / 100 \wedge 2 /, \mu \rightarrow$ viscosityH20

$$
\frac{4.07437 \times 10^{-6} \mathrm{LQ}}{\mathrm{d}^{4}}
$$

Form equation based on loss coefficient, $k$

$$
\begin{aligned}
& \rho * \nabla^{\wedge} 2 / 2 * x \\
& \frac{1}{2} v^{2} K \rho
\end{aligned}
$$

Substitute for velocity (flow rate by cross-sectional area)

$$
\begin{aligned}
& 8 / . v \rightarrow Q / A \\
& \frac{Q^{2} K \rho}{2 A^{2}}
\end{aligned}
$$


Substitute for cross-sectional area to get fitting head loss equation

$$
\begin{aligned}
& \text { \& } A \rightarrow \pi * d \wedge 2 / 4 \\
& \frac{8 Q^{2} \times p}{d^{4} \pi^{2}}
\end{aligned}
$$

Fitting loss equation (first-order Taylor series expansion about mean flow rate; change units from meters to centimeters)

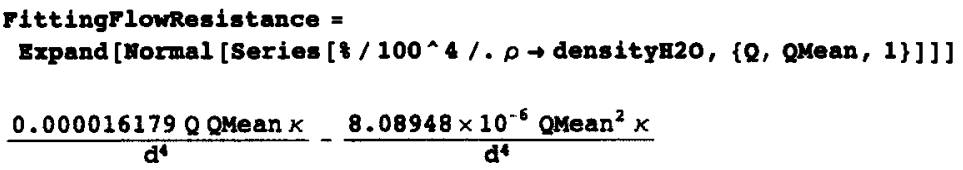

Plot both the original and linearized functions to make sure they look right

Plot $\left[\left\{8 * Q^{\wedge} 2 * x *\right.\right.$ density $20 /\left(d^{\wedge} 4 * \pi^{\wedge} 2 * 100^{\wedge} 4\right) / .\{x \rightarrow 3.45, d \rightarrow 2.54\}$, FittingFlowResistance $/ .\{x \rightarrow 3.45, d \rightarrow 2.54$, QMean $\rightarrow 80\}\}$,

$\{Q, 60,240\}$, Plotrange $\rightarrow\{$ All, $\{0$, Automatic $\}\}$;

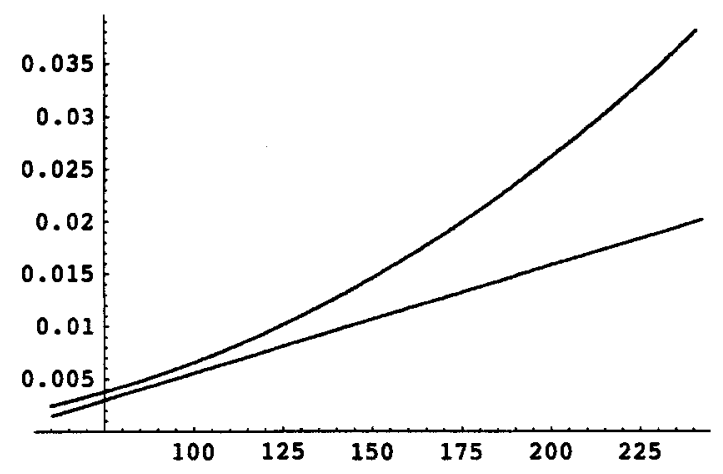

Pressure losses across the fully-open VAD outlet valve

Fittingrlowresistance $/ .\{x \rightarrow 2.45, d \rightarrow 2.54$, QMean $\rightarrow 80\}$

$-0.00304742+0.00007618540$

Pressure losses in the first segment of tubing (including fittings) between the VAD and the arterial pressure chamber

(PiperlowResistance $/ .\{I \rightarrow 12 * 2.54, d \rightarrow 2.54\})+$

(FittingFlowResistance $/ .\{x \rightarrow 1, d \rightarrow 2.54$, QMean $\rightarrow 80\}$ )

$-0.00124384+0.00003407970$

Reproduced with permission of the copyright owner. Further reproduction prohibited without permission. 
Cylinder cross-sectional area (in $\mathrm{cm}^{\wedge} 2$ )

CSecarea $=P 1 \star 8 \wedge 2 / 4 \star 2.54{ }^{\wedge}$

324.293

Pressure losses in the next segment of tubing (including fittings) between the arterial chamber and the peripheral resistance valve

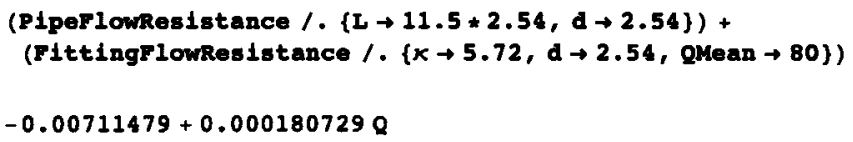

Pressure losses in the curved section of tubing (including fittings) between the peripheral resistance valve and the venous chamber

(PipeFlowResistance $/ .\{\mathrm{L} \rightarrow 12 * 2.54, \mathrm{~d} \rightarrow 2.54\})+$

(FittingFlowResistance $/ .\{x \rightarrow 2.172, d \rightarrow 2.54$, QMean $\rightarrow 80\}$ )

$-0.00270163+0.0000705243 Q$

The venous chamber is open to atmosphere, therefore the compliance is a function of the cross-sectional area

$\operatorname{VnEQ}=\operatorname{Expand}\left[\rho * g *(\mathrm{~V} / \mathrm{CsecArea}-3 * 2.54) / 100^{\wedge} 3 / .(\rho \rightarrow\right.$ density $[20, g \rightarrow$ gravity $\}]$

$-0.0746027+0.0000301899 \mathrm{v}$

Pressure losses in the segment of tubing (including fittings) between the venous chamber and the inflow control valve

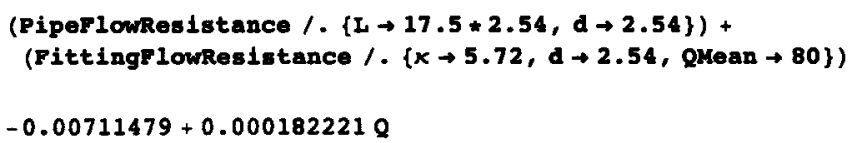

Pressure losses in the segment of tubing (including fittings) between the inflow control valve and the VAD inlet valve

(PlpeflowResistance $/ .\{\mathrm{L} \rightarrow 13 * 2.54, d \rightarrow 2.54\})+$

(FittingF lowresistance $/ .(x \rightarrow 4.72, d \rightarrow 2.54$, Quean $\rightarrow 80\}$ )

$-0.00587094+0.0001500060$ 
Pressure losses across the fully-open VAD inlet valve

Fittingrlownesistance $/ .\{x \rightarrow 2.45, d \rightarrow 2.54$, QMean $\rightarrow 80\}$

$-0.00304742+0.00007618540$

This is the value of Rot (open valve)

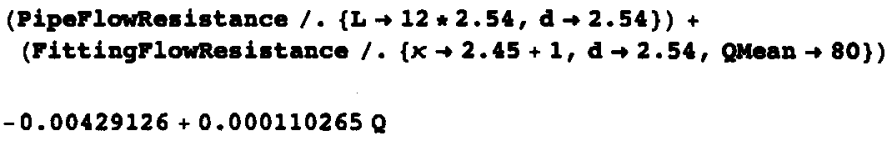

This is the value of Rpe, silver valve taken as 0.0085 nominal ( $8 \mathrm{~V}$ setting)

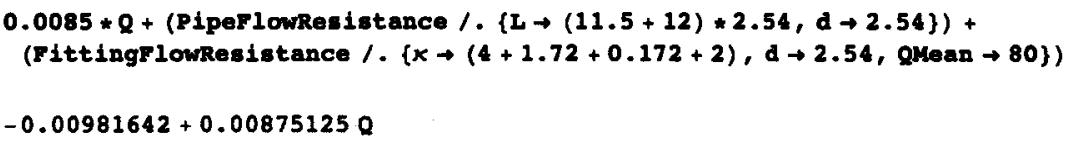

This is the value of Rin (open valve) with yellow valve taken as 0.01

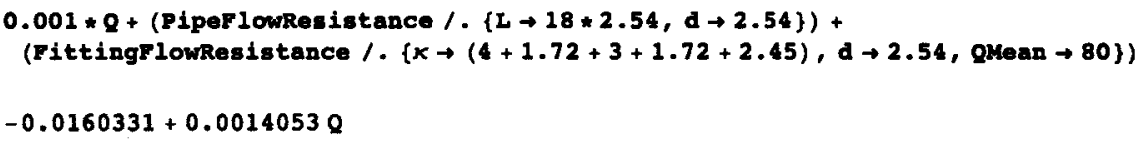


This is the outlet valve flow resistance function; it accepts a pressure (delta) input in $\frac{\mathrm{N}}{\mathrm{cm}^{2}}$ and returns a flow in $\frac{\mathrm{ml}}{\mathrm{s}}$

ROutletValve $\left[x_{-}\right]:=$If $\left[x>0, x /\right.$ RoutletValvekin, $\left.x /\left(8 . * 10^{\wedge} 2\right)\right]$

This is the inlet valve flow resistance function; it accepts a pressure (delta) input in $\frac{\mathrm{N}}{\mathrm{cm}^{2}}$ and returns a flow in $\frac{\mathrm{m}}{\mathrm{s}}$

RInletValve $\left[x_{-}\right]:=I f\left[x>0, x /\right.$ RInletValvexin, $\left.x /\left(8 . * 10^{\wedge} 2\right)\right]$

Piston area

Area $=10.15 * 2.54 \wedge 2$

This is the forcing frequency

Beat Freq $=60 / 60$

This is the piston position; sinusoidal fixed rate.

Pistonposition $\left[x x_{-}\right.$? KumberQ] : $=0.25 * 2.54 * 2 * f * P i * \operatorname{Cos}[2 * P i * f * x x] / . f \rightarrow$ Beatrreq

Plot $[$ Pistonposition $[t],\{t, 0,2\}]$

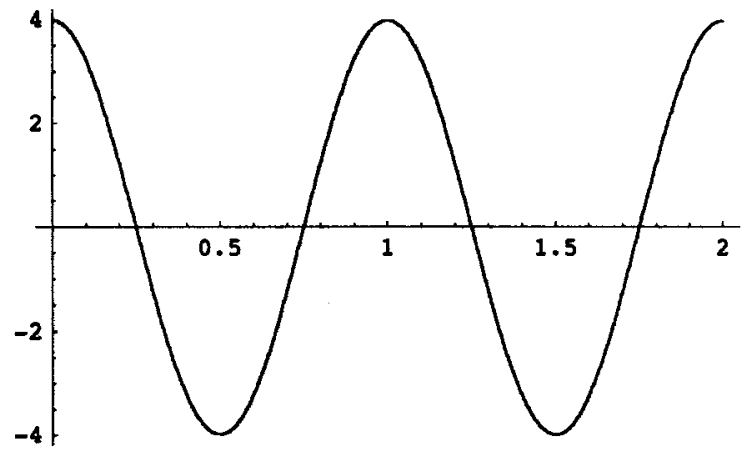

This is the VAD chamber's constitutive relation; it accepts a volume input in $\mathrm{ml}$ and returns a pressure in $\frac{N}{\mathrm{~cm}^{2}}$

PHeart $\left[x x_{3}\right]:=$ Module $[\{$ Mominalv, Mominalp, Compliance, offset $\}$,

Mominalv $=46.0 ;$ MominalP $=100 *$ Ig2n; Compliance $=0.01$;

Offset $=(-$ MominalV $/$ Compliance + MominalP $) ; \forall[x x /$ Compliance + Offset $]]$;

Reproduced with permission of the copyright owner. Further reproduction prohibited without permission. 
Outlet tube (cannula) constitutive relations

OutletCannulaLength $=5 * 12 * 2.54$

Outletcannuladiameter $=2.54$;

POutletCannula [xx_] : = Module [ Mominalv, MominalP, Compliance, offset $\}$, Hominalv =

(Pipevolume /. Diameter $\rightarrow$ OutletCannuladianeter $/$. Length $\rightarrow$ Out letCannulaLength);

NominalP $=100 *$ Hg2N; Compliance $=10 . ;$ Offset $=(-$ HominalV $/$ Compliance + HominalP $) ;$

I [xx / Compliance + Offset $]$;

IOutletCannula =

PipeplowInertia /. Diameter $\rightarrow$ OutletCannulabiameter /. Length $\rightarrow$ OutletCannulaLength;

RoutletCannula $=$

PiperlowResistance /. d $\rightarrow$ OutletCannulabiameter $/$. I $\rightarrow$ OutletCannularength $/ . Q \rightarrow 1$;

Aorta and connecting tube

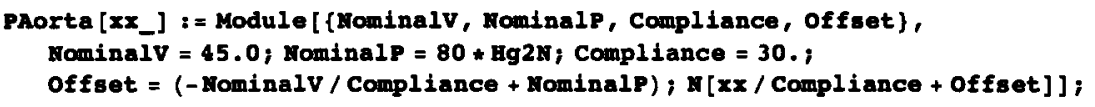

IArterialTube $=$ PipeplowInertia $/$. Diameter $\rightarrow 2.54 /$. Length $\rightarrow 12 * 2.54$;

Rarterialtube $=100 *$ PipeplowResistance $/ . d \rightarrow 2.54 / . \mathrm{I} \rightarrow 12 * 2.54 / . \mathrm{Q} \rightarrow 1$;

Viscous rolling friction values for diaphragms

RViscous $1=1 \cdot * 10^{\wedge}-4$;

RViscous $2=1 \cdot * 10^{\wedge}-4 ;$

This is the arterial chamber's constitutive relation; it accepts a volume input in $\mathrm{ml}$ and returns a pressure in $\frac{\mathrm{N}}{\mathrm{cm}^{2}}$

PArterial [xx_] := Module [ $\{$ Mominalv, Mominalp, Compliance, offset $\}$ MominalV $=650$; MominalP $=80 *$ Hg2N; Compliance $=110$;

offset $=(-$ Mominalv / Compliance + Mominalp); $\mathrm{H}[\mathrm{xx} /$ Compliance + Offset $]$; 
This is the venous chamber's constitutive relation; it accepts a volume input in $\mathrm{ml}$ and returns a pressure in $\frac{\mathrm{N}}{\mathrm{cm}^{2}}$

PVeins $\left[x_{-}\right]:=\operatorname{VnEQ} / . \mathrm{v} \rightarrow \mathrm{xx}$

VAD compliance volume

EQ1 = VHeart ' $[t]=$ Pistonposition $[t] *$ Area + RInletValve [

PVeins [VVeins [t]] - densityH20*gravity *18.5*2.54/100^3 - PHeart [VHeart [t] ] ] -

Rout letValve [PHeart [VHeart [t] ] + density 20 *gravity *18.5*2.54/100^3 -

PoutletCannula [VoutletCannula [t]] ];

Outlet tube (cannula) volume

$\mathbf{8 Q 2}=$ voutletCannula $\mathbf{a}^{\prime}[\mathrm{t}]=$

ROut letValve [PHeart [VHeart [t]] + densityH20 * gravity *18.5*2.54/100^3 -

pout letCannula [VoutletCannula [t] ] ] poutletCannula [t] / IOutletCannula ;

Outlet cannula momentum

EQ3 = poutletCannula $[t]=$ PoutletCannula [VoutletCannula $[t]$ ]

ROut letCannula * poutletCannula [t] / Iout letCannula -

(poutletCannula [t] / IoutletCannula - parterialTube [ $t$ ] / IArterialTube) * RViscous1 -

pAorta [VAorta [t]];

Aortic chamber volume

$\mathbf{8 0 4}=$

VAorta $[t]=$ poutletcannula [t] / IoutletCannula - parterialTube [ $t$ ] / IArterialTube;

Arterial tube momentum

BO5 = pArterialTube ' $[t]$ = PAorta [VAorta $[t]]+$

RViscous1 * (poutletCannula [t] / IOutletCannula - pArterialTube[t] / IArterialTube) -

parterlalTube [t] / IArterialTube * RArterialTube - (1/(RPeripheral + RViscous2)) *

(PArterial [VArterial [t]] * RPeripheral + PVeins [VVeins [t]] * RViscous2 +

pArterialTube [t] / IArterialTube * RPeripheral * RViscous2);

Arterial volume

EQ6 = Varterial $[t]=$

(1 / (RPeripheral + RViscous2)) * (parterialTube [t] / IarterialTube * RPeripheral * pVeins [VVeins [t] ] - PArterial [VArterial [t] ]); 
Venous volume

EQ7 $=$ vveins ' $[t]=$

(1/ (RPeripheral + RViscous2)) *(parterial Tube [t] / IArterialTube * RViscous2 +

PArterial [VArterial [t] ] - PVeins [VVeins [t] ]) - RInletValve [

PVeins [VVeins [ $t]$ ] - densityH20 * gravity * $18.5 * 2.54 / 100 \wedge 3$ - PHeart [VHeart [ $t]]$ ];

tStart $=0 ;$ tstop $=100 ;$ pstart $=85 ;$ pstop $=90 ;$

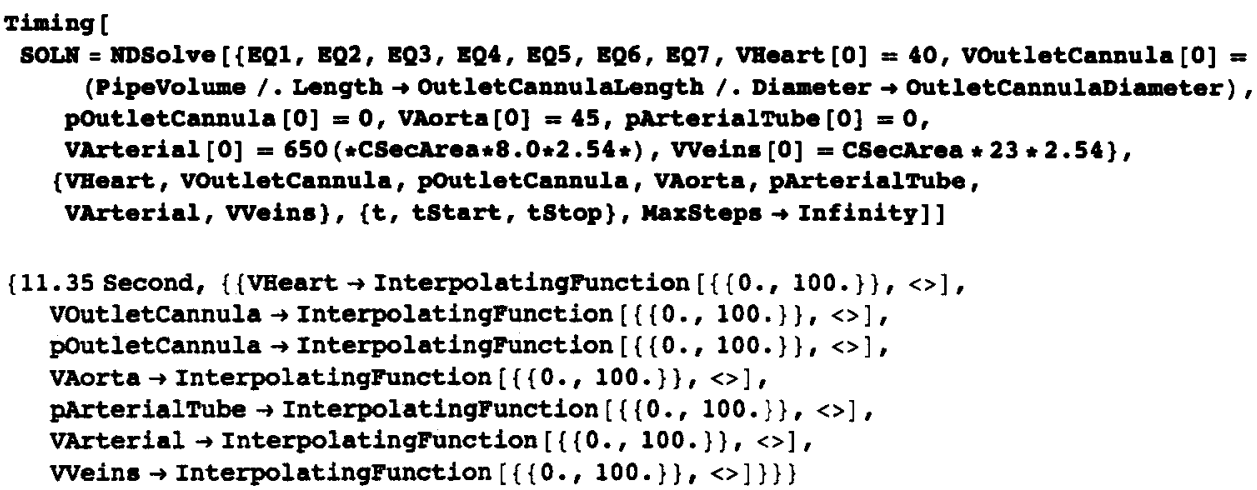

$\{11.35$ Second, $\{$ (VBeart $\rightarrow$ InterpolatingFunction $[\{\{0 ., 100\}\},.<>]$, vout letCannula $\rightarrow$ InterpolatingFunction $[\{\{0 ., 100\}\},.<>]$,

Plot the aortic pressure waveform over the course of a beat

Plot [PAorta [VAorta $[t]] *$ N2Hg /. SOLM, $\{t, 88.8,89.8\}]$

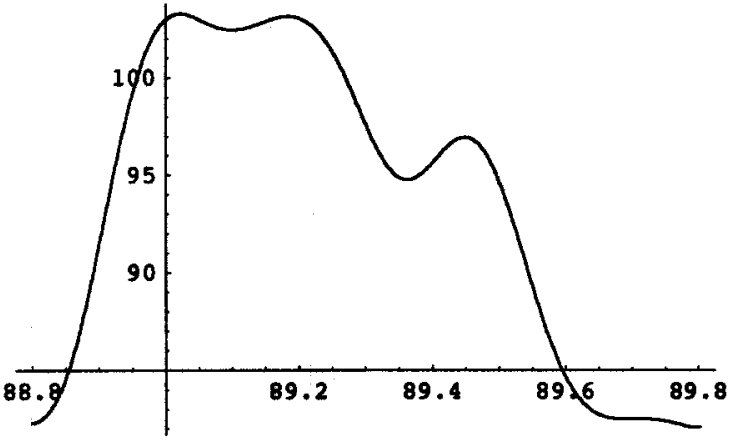


This is the aortic piston position (i.e., motion)

Plot [VAorta [t] / (3.64*2.54^2)/. sows

$\{t$, pstart, pstop\}, PlotRange $\rightarrow$ \{Automatic, All\}];

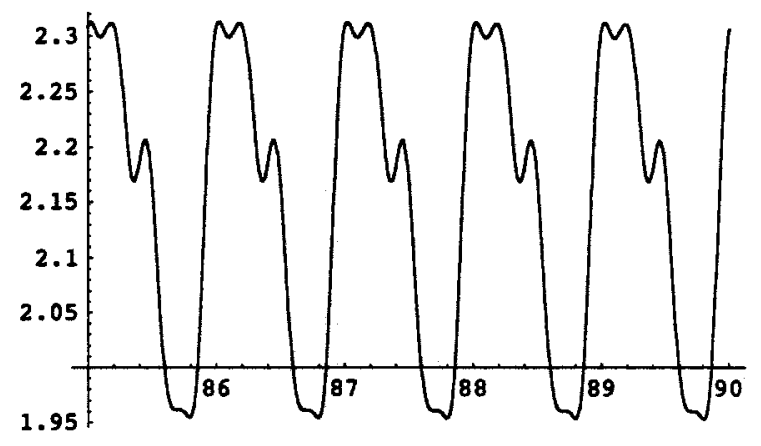

Arterial chamber pressure versus time

Plot [Parterial [VArterial [t] ] * k2Hg /. soLN,

$\{t$, pstart, pstop $\}$, PlotRange $\rightarrow$ (Automatic, Automatic $\}]$;

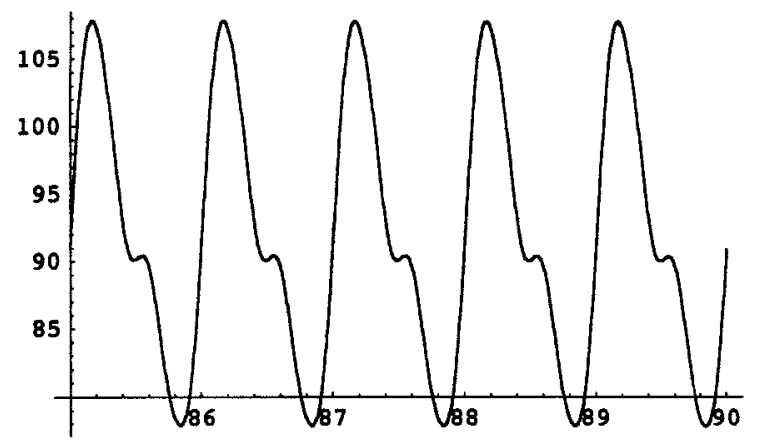


Arterial piston position

Plot [Varterial [t] / (10.15*2.54^2)/. sows,

$\{t$, pstart, pstop\}, PlotRange $\rightarrow$ \{Automatic, All $\}]$;

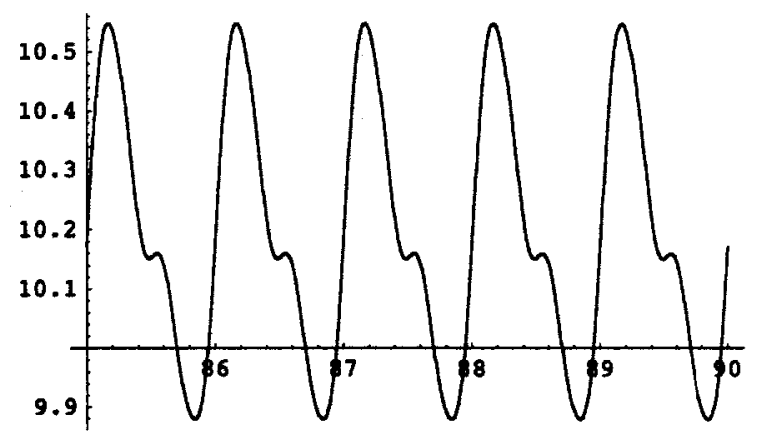

Venous chamber height versus time (in inches)

Plot [VVeins [t] / CSecArea / 2.54/. soLk, \{t, pstart, tStop $\}$ ];

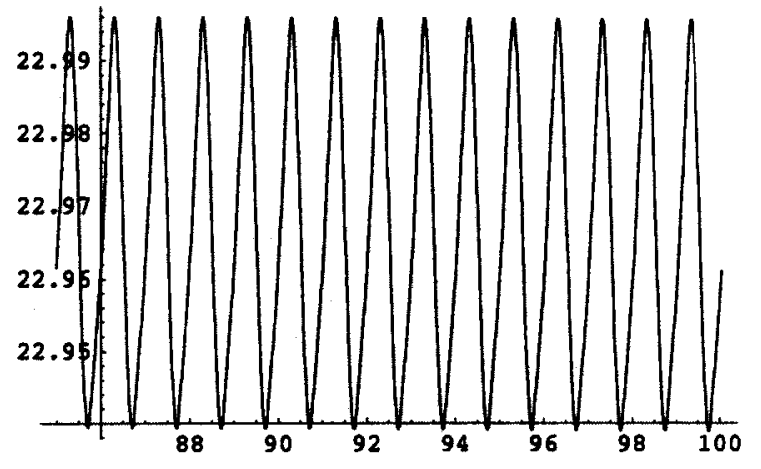


VAD compliance volume versus time

Plot [PHeart [VHeart [t] ] * M2Hg / sowM, \{t, pstart, pstop $\}$ )

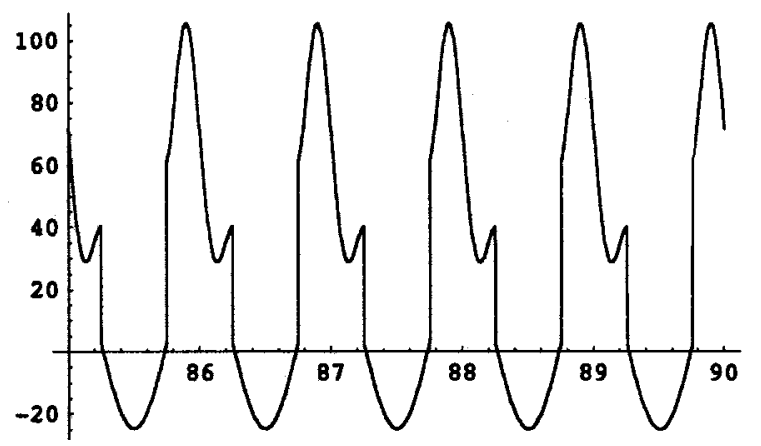

Total system volume versus time (check integrator error; should be constant)

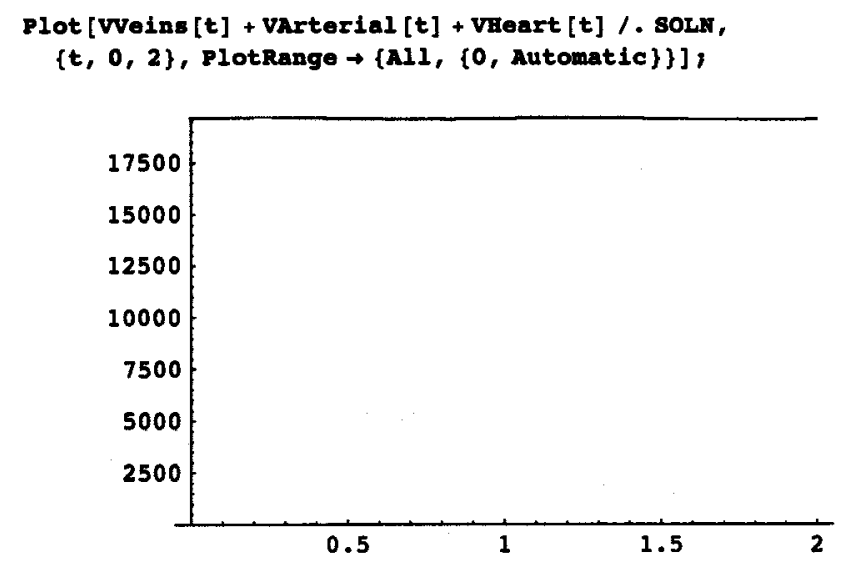


Outlet valve flow rate

Plot [ROutletValve [PHeart [VHeart [t]] + densityH20 * gravity *18.5*2.54/100^3 Pout letCannula [VoutletCannula $[t]]] /$ sows, $\{t$, pstart, pstop $\}]$;

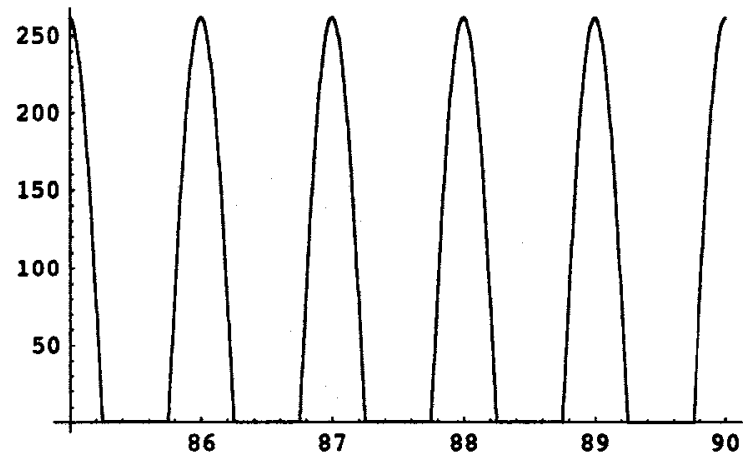

Check mean flow through outlet valve

Mean [Table [ROutletValve [PHeart [VHeart $[t]]+$ densityH20 * gravity $* 18.5 * 2.54 / 100 \wedge 3-$ poutletcannula [voutletcannula [t]]] /. sous, \{t, pstart, pstop, 0.001\}]]

$\{83.1916\}$

Inlet valve flow rate

Plot [RIn letValve [PVeins [VVeins [t] ] - densityH20 * gravity *18.5*2.54/100^3PHeart [VHeart [t] ] ] / sold, \{t, pstart, pstop\}, Plotrange $\rightarrow$ All] ;

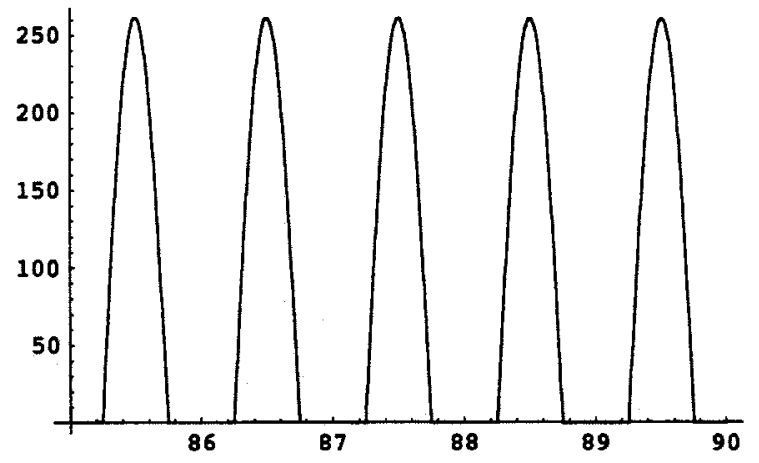




\section{Appendix E}

\section{Pressure Chamber Design}

WANSON and Clark (1977a) outline several germane requirements for mock circulatory systems, and their recommendations were taken into account during the development of the experimental apparatus for this work. Many of the suggestions focus on the actual pump itself, but Swanson and Clark also recommend the test sections be modular, observable, and photographable. The bond graph model of the circulatory system (first presented in Chapter 2) is intrinsically modular, and the experimental setup was designed to match the mathematical model as closely as possible.

Many mock circulatory systems use water columns as compliance elements and have chambers which are open to atmosphere, resulting in a linear constitutive relationship. In these chambers the compliance is governed by the cross-sectional area of the column, and is not easily varied; changing the effective cross-sectional area of a container is non-trivial. Either by closing the chamber to atmosphere or by tapering the walls of the container the chamber compliance can be made nonlinear. Nevertheless, this sort of chamber does not lend itself well to experimentation; changing a parameter often involves changing the entire geometry of the accumulator.

A spring-loaded piston assembly allows simpler parameter changes, but sealing and friction concerns come to the fore. The human circulatory system operates at relatively low pressure and the sliding contact found in a standard piston-cylinder arrangement will result in excessive losses. A solution to this sliding contact problem was developed in the $1950 \mathrm{~s}$ in the form of rolling diaphragm seals. These elastomer seals are molded in the shape of a truncated cone and are inverted prior to installation. As the piston moves along the cylinder the diaphragm rolls alternately between the piston and the cylinder wall; the gap between the two parts is sealed by the diaphragm convolution, and the internal pressure in 
the chamber ensures virtually friction-free motion. An assembly is illustrated in Figure E.1, showing the piston in three key positions: at bottom-dead-centre (BDC), at the neutral plane, and at top-dead-centre (TDC).

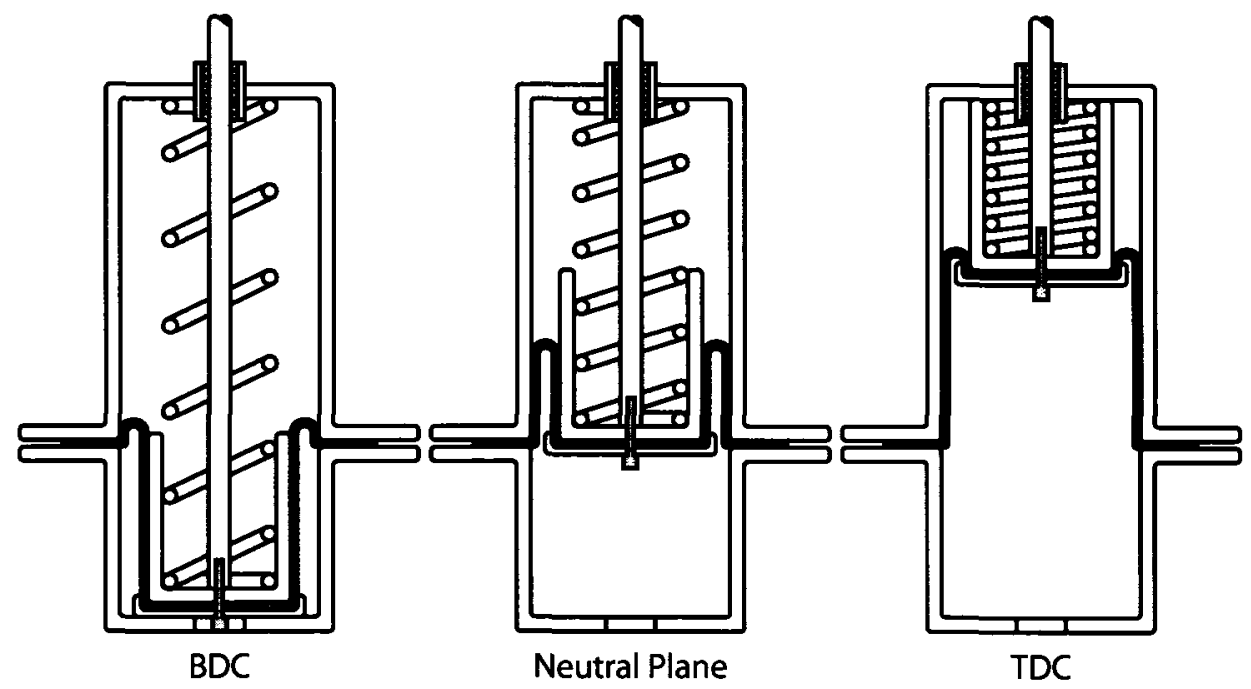

Figure E.1: Piston-cylinder assembly sealed using a rolling diaphragm

The maximum stroke length is limited by the height of the diaphragm, but diaphragms with up to 1:1 aspect ratios are available. There are a few companies which produce rolling diaphragm seals, and Marsh Bellofram* (the originators of the rolling diaphragm concept) were chosen as suppliers. This decision was based on their stock catalogue offerings, which (at first glance) appeared more substantial than the competition. Additionally, Bellofram's diaphragms have higher aspect ratios, and better stroke lengths than similar offerings from Diacom ${ }^{\dagger}$, for example. In hindsight the compliance chambers were designed with excess capacity, and supply issues ${ }^{\ddagger}$ complicated the project immensely. If the

*http://www. bellofram.com

thttp: //www.diacom.com

$\ddagger$ When Bellofram was first contacted, the "stock" catalogue sizes were not stocked; they were simply sizes for which molds were available. Individual diaphragm orders were apparently filled using leftovers from other production runs, and one of the diaphragms received in the first order had an inherent manufacturing weakness which eventually led to piston binding. By the time the diaphragm was replaced, Bellofram was actually stocking the "stock sizes" in their catalogue, and the ordering process was much simpler. Unfortu- 
project were to be re-done from the beginning, the piston stroke would be reduced and the diaphragms would be ordered from a different manufacturer.

This reduced piston stroke would mean slightly lower headroom in all of the chambers and limit the off-nominal operation of the loop, however the current chamber design allows for unnecessarily large volume variations in order to accommodate loop parameter studies; a mock circulatory system designed to run near nominal operating conditions would require substantially less volume variation in each of the chambers.

One benefit of reducing the diaphragm aspect ratio (and hence the piston stroke) is that the piston would operate above the neutral plane at nominal conditions. Piston travel through the neutral plane is the most critical phase of rolling diaphragm motion; ideally one would like to operate either below or above the neutral plane as much as possible.

In order to meet Swanson and Clark's "observable, photographable" requirement, the cylinders were designed in clear acrylic. The clear tubes were sourced from GE Polymershapes*, and the piston material from Canus Plastics ${ }^{\dagger}$. Both of these companies have Ottawa offices and excellent service.

An assembled chamber is shown in Figure E.2. A metric ruler with $1 \mathrm{~cm}$ markings is included for scale; shop drawings are included in Appendix F. The clear acrylic cylinder segments are clamped between two aluminum end flanges by way of five tapped steel rods. The ends of the cylinders are stepped to accommodate Nitrile O-Ring seals, which prevent leakage in between the cylinder and the flange. The end flanges are then bolted to each other (in the case of the mid-section) or to the top flange or to the bottom base flange. The rolling diaphragm's skirt acts as a gasket seal between the mid-flanges. The bottom base flange contains O-Ring grooves to ensure water-tight sealing between all surfaces.

All of the fluid interconnects in the University of Ottawa Heart Institute mock circulatory system are 1 in internal diameter Tygon tubing, and an internal sleeve was added

nately the replacement order was not filled properly, and only half of the lot arrived. Even though only one diaphragm (of the lot of four) was required to continue the experiment, the bungled shipment further eroded confidence in the supplier.

${ }^{*}$ http://www.gepolymershapes.com

${ }^{\dagger}$ http: //www. canusplastics.com 


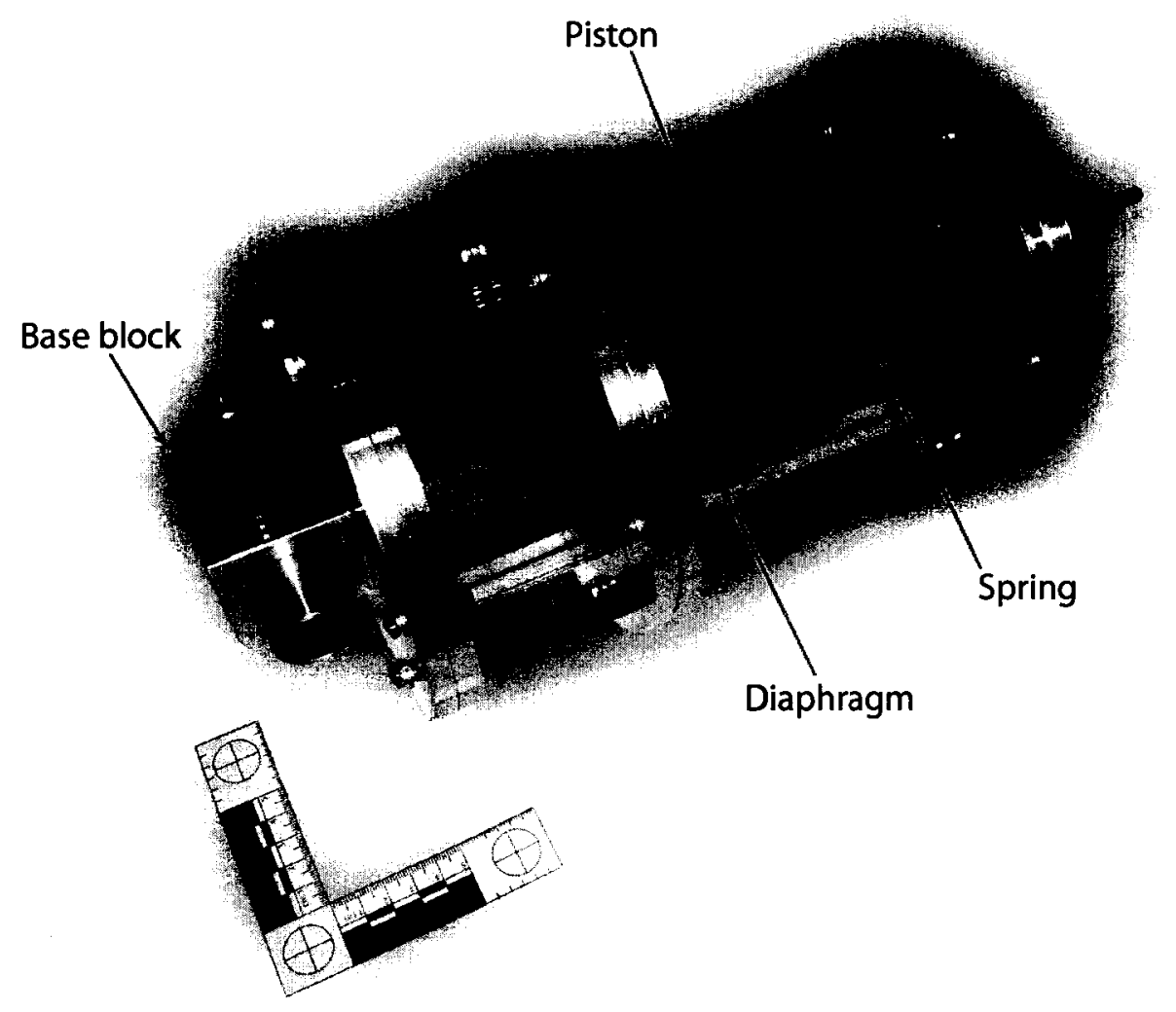

Figure E.2: Arterial pressure chamber 
to the cylinder base block. This allows the new pressure chambers to easily interconnect with existing loop components. The internal sleeve was held in place and sealed using Cyanoacrylate. Figure E. 3 shows a closeup of the bottom of the chamber; the O-Ring seal between the cylinder and the flange is visible. There is no seal between the mating surface of the top flanges as this is not a wetted surface and the entire purpose of the topmost flange is to ensure true motion of the piston rod.

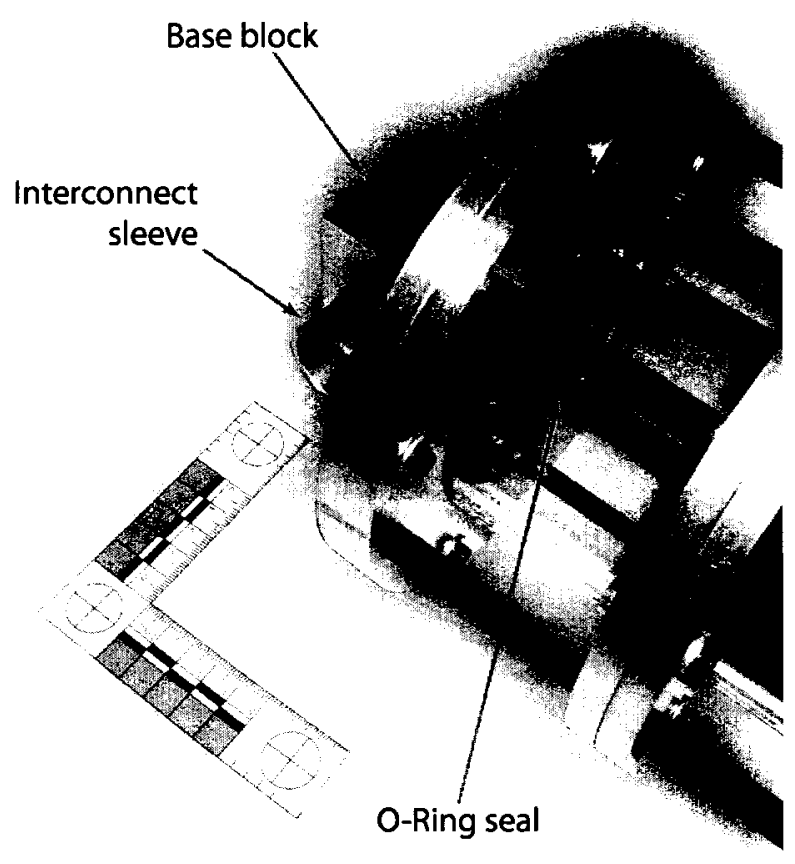

Figure E.3: Closeup of arterial pressure chamber base

The piston is machined from Ultra-High Molecular Weight Polyethylene (UHMWPE). The Bellofram design manual recommends tapering the piston to improve diaphragm rolling characteristics, but the recommended taper produces an interference fit at the top of the piston, at least for the small (aortic) chamber; after this problem was discovered all of the piston cups were squared rather than tapered. The piston shaft, which is made from 
0.25 in diameter drill rod, is tapped on one end and attached to the piston cup with a single stainless steel cap screw. The diaphragm (which is sandwiched between the piston and the retainer plate) seals the interface.

The initial chamber design called for an adjustable linear bushing assembly mounted at the top flange, which would provide a wider support for the piston rod (countering off-axis loads) and allow sideways adjustments of the piston rod (to guarantee the rod and cylinder axes were collinear). However, after assembly the cylinder walls were found to be nicely perpendicular to the flanges themselves. The top flange was redesigned to accommodate a single linear ball bearing instead of an adjustable bushing. A closed Thomson* Super-4 Ball Bushing, which is only 0.75 in long, provides the piston rod with less lateral support than the linear bushing assembly, but very smooth axial motion. The linear bearing is mounted in a 0.5 in diameter hole and held in place with spring steel retaining clips.

Truncated cones were used to centre the springs on the piston shaft; these were made out of brass or, alternatively, UHMWPE. A closeup of the top of the arterial pressure chamber is shown in Figure E.4.

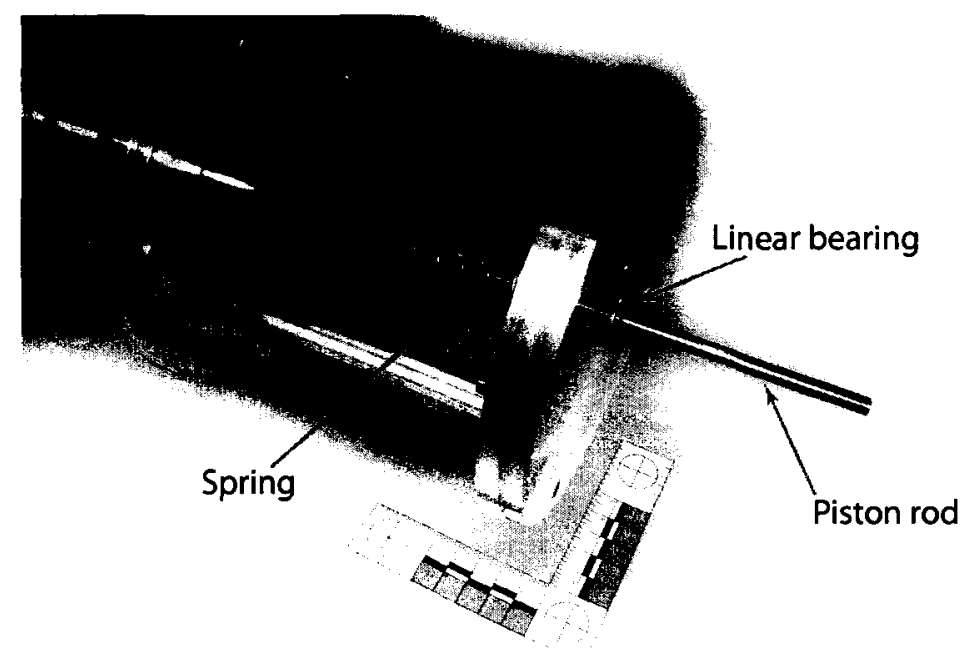

Figure E.4: Closeup of arterial pressure chamber top

\footnotetext{
*http://www.thomsonindustries.com
} 


\section{E.1 Volume Sizing}

The individual chambers were designed such that the nominal chamber volume was contained in the portion of cylinder below the piston's neutral plane. This, combined with the 1:1 diaphragm aspect ratio, means that the chamber volume can vary $\pm 100 \%$ from the nominal volume. This encompasses all of the behaviour seen in the simulations and allows us to run the system at off-nominal conditions for significant periods of time. (For example, if the peripheral resistance were to dramatically increase, blood would pool in the arterial system; we can simulate this in the mock circulatory system as both the aortic and arterial chambers can accommodate $2 \times$ their nominal volume. Obviously the arterial pressure would increase in this situation.)

The nominal chamber volumes were chosen based on the physiological data presented in Table 2.1, which is the same data used in the mathematical simulation. The nominal volume contained in the aorta is $100 \mathrm{ml}$ or $6 \mathrm{in}^{3}$ and the nominal volume contained in the arteries is $600 \mathrm{ml}$ or $36 \mathrm{in}^{3}$. The diaphragm selected for the aortic chamber was 4-225-225$\mathrm{CBJ}$ and for the arterial chamber was 4-375-375-DBJ. These were selected because acrylic tubing with internal diameters of $2.25 \mathrm{in}$ and 3.75 in (respectively) was readily available. The maximum half-stroke of the 4-225-225-CBJ is $1.87 \mathrm{in}$ and of the 4-375-375-DBJ is $3.20 \mathrm{in}$. With effective pressure areas of $3.64 \mathrm{in}^{2}$ and $10.15 \mathrm{in}^{2}$ (respectively), this means that the pistons can displace $6.81 \mathrm{in}^{3}$ and $32.48 \mathrm{in}^{3}$ (respectively) on their down-stroke.

\section{E.2 Pressure Sizing}

Rolling diaphragms fill the gap between the piston and the cylinder wall with a convolution. This means that they present a constant effective pressure area that is dependent on both the cylinder and piston sizing; each stock diaphragm in the catalogue has its effective area listed.

The internal chamber pressure in this apparatus is rather low, and we would expect a maximum axial force (in the larger of the two chambers) of roughly $40 \mathrm{lb}$. In the worstcase scenario (right before de-clamping, when the force sustained by the clamped members tends to zero) this force would be born as a tension load in the socket head cap screws hold- 
ing each pair of flanges together. This type of bolt can support a tension load of $120 \mathrm{kpsi}$ (minimum), and has a tensile stress area of $0.014 \mathrm{in}^{2}$; there are five bolts at each interface.

\section{E.3 Spring Sizing}

Recall that Equation (2.18) gave a vessel's change in internal volume for a given change in internal pressure:

$$
V(P)=C \cdot P
$$

In order to size the springs for the various pressure chambers, we need the inverse of this relationship:

$$
P(V)=\frac{V}{C}
$$

The spring applies a force to the piston, and this force varies with piston displacement. We need to relate the spring force and the piston position:

$$
F(V(x))=\frac{S A \cdot V(x)}{C}
$$

Writing the internal volume in terms of piston area gives:

$$
F(x)=\frac{S A^{2} \cdot x}{C}
$$

From this equation it is clear that the spring stiffness and the vessel compliance are related by:

$$
\left.k\right|_{\text {spring }}=\frac{1}{\mathrm{C}} \cdot S A^{2}
$$

\section{E.3.1 Linear Aorta}

For the linear aortic segment in the dual-compliance model (DCM), the nominal pressure, volume, and piston area are given in Table E.1.

Recall* that the linear aortic compliance is $29.3 \mathrm{~cm}^{5} \cdot \mathrm{N}^{-1}$. Converting from metric to imperial units gives:

$$
k_{a o}=0.7922 \quad\left[l b \cdot i^{-5}\right]
$$

*From Page 41. 


\begin{tabular}{|c|c|c|c|c|c|}
\hline \multicolumn{2}{|c|}{$P$} & \multicolumn{2}{c|}{$V$} & \multicolumn{2}{c|}{$S A$} \\
\hline$[m m H g]$ & {$[p s i]$} & {$[m l]$} & {$\left[\mathrm{in}^{3}\right]$} & {$\left[\mathrm{cm}^{2}\right]$} & {$\left[\mathrm{in}^{2}\right]$} \\
\hline \hline $90-110$ & $1.74-2.13$ & 98 & 5.98 & 23.4 & 3.64 \\
\hline
\end{tabular}

Table E.1: Nominal parameters for the linear aortic chamber

The aorta stiffness is scaled by the square of the piston cross-sectional area to produce the aortic spring stiffness:

$$
\begin{aligned}
\left.k_{a o}\right|_{\text {spring }} & =k_{a 0} \cdot S A_{a 0}^{2} \\
& =0.7922 \cdot 3.64^{2} \\
& =10.5 \quad\left[l b \cdot \mathrm{in}^{-1}\right]
\end{aligned}
$$

The minimum and maximum aortic volumes are $3.23 \mathrm{in}^{3}$ and $7.99 \mathrm{in}^{3}$ (respectively). Scaled by the inverse of the piston cross-sectional area, these correspond to minimum and maximum piston positions of $0.89 \mathrm{in}$ and $2.2 \mathrm{in}$ (respectively). We are therefore looking for a spring with a stiffness of $10.5 \mathrm{lb} / \mathrm{in}$, and a minimum travel (between free length and solid height) of $1.3 \mathrm{in.}$

Diamond Wire* has several springs which fit this profile, including those listed in Table E.2.

\begin{tabular}{|c|c|c|c|c|}
\hline Model Number & $\begin{array}{c}\text { Outside Diameter } \\
{[\text { in }]}\end{array}$ & $\begin{array}{c}\text { Solid Height } \\
{[\text { in }]}\end{array}$ & $\begin{array}{c}\text { Free Length } \\
{[\text { in }]}\end{array}$ & $\begin{array}{c}\text { Stiffness } \\
{[\mathrm{lb} / \mathrm{in}]}\end{array}$ \\
\hline \hline DWC-092K-16-SS & 0.975 & 1.286 & 4 & 10.83 \\
DWC-096L-16-SS & 1.1 & 1.17 & 4 & 10.41 \\
DWC-105LM-18-SS & 1.187 & 1.34 & 5 & 10.66 \\
DWC-105LM-17-SS & 1.187 & 1.22 & 4.5 & 11.91 \\
\hline
\end{tabular}

Table E.2: Springs suitable for the linear aortic chamber.

\footnotetext{
*Diamond Wire Spring Company (http: / /www . diamondwire.com) of Pittsburgh, PA.
} 


\section{E.3.2 Nonlinear Aorta}

For the nonlinear aortic compliance, the nominal pressure, volume, and piston area are given in Table E.3.

\begin{tabular}{|c|c|c|c|c|c|}
\hline \multicolumn{2}{|c|}{$P$} & \multicolumn{2}{c|}{$V$} & \multicolumn{2}{c|}{$S A$} \\
\hline$[\mathrm{mmHg}]$ & {$[p s i]$} & {$[\mathrm{ml}]$} & {$\left[\mathrm{in}^{3}\right]$} & {$\left[\mathrm{cm}^{2}\right]$} & {$\left[\mathrm{in}^{2}\right]$} \\
\hline \hline $90-100$ & $1.7-2.13$ & 98 & 5.98 & 23.4 & 3.64 \\
\hline
\end{tabular}

Table E.3: Nominal parameters for the nonlinear aortic chamber

A plot of aortic pressure versus volume* shows that there are three "zones" with different slopes. The medial zone has a nearly linear rise, and beyond a certain point in either direction the slope increases (either in the negative sense or the positive sense). The delineations in Figure E.5 are arbitrary; it is obvious from both the data points and the cubic curve of best-fit that there is no distinct transition zone between "nominal" and "high stiffness" behaviour.

Rather than model the low-pressure falloff of aortic stiffness, the focus was placed on the stiffening behaviour, which was intuited to be the most important nonlinear feature of the arterial system. This mitigated the spring design requirements somewhat; two springs in series can model this stiffening behaviour if the softer spring reaches solid height before the stiffer spring.

Looking at Figure E.5, we can see that the first data point corresponds to a volume of $3.23 \mathrm{in}^{3}$, or a piston displacement of $0.89 \mathrm{in}$. The eighth data point (at a pressure of $2 \mathrm{psi}$ ) has a corresponding volume of $6.35 \mathrm{in}^{3}$, or a piston displacement of $1.74 \mathrm{in}$. This means that the softer spring must go from rest length to solid height in under an inch of travel. After this point, we look for a spring stiffness of $19.7 \mathrm{lb} / \mathrm{in}$, or approximately $20 \mathrm{lb} / \mathrm{in}$. The combined stiffness of the two springs in series should be approximately $8 \mathrm{lb} / \mathrm{in}$, so we can

*This is a modified version of Figure 2.20 in imperial units this time (for consistency; the manufacturer's spring data are imperial) with the linear and exponential curves omitted. 


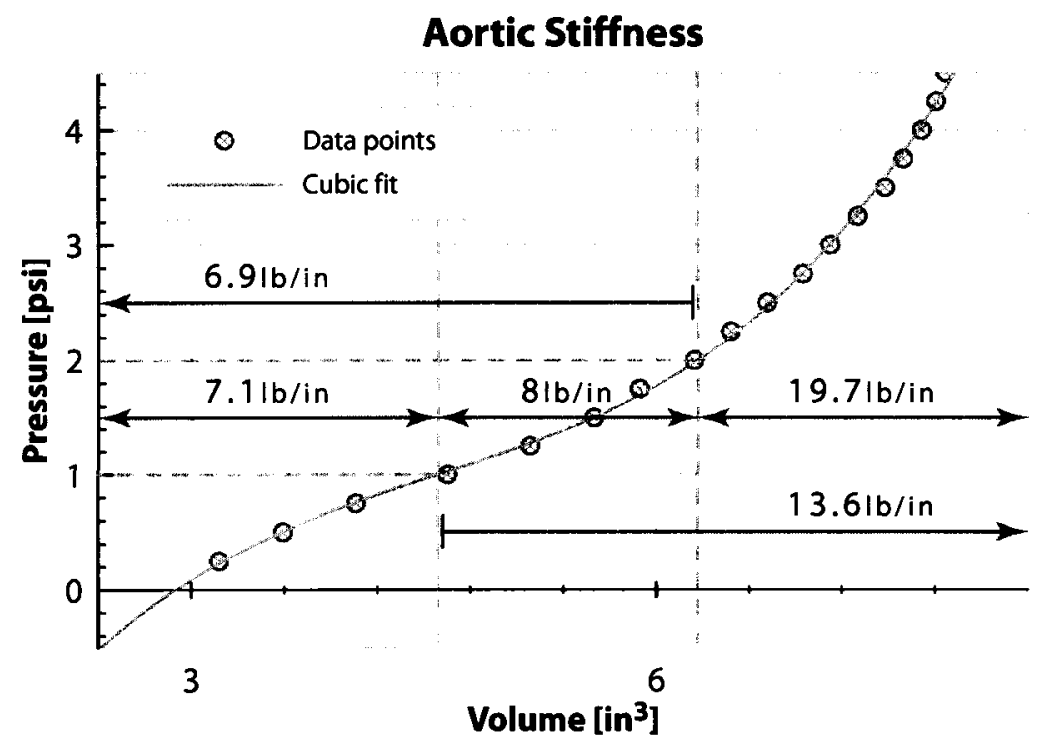

Figure E.5: Aortic spring stiffness graph

solve for the spring constant of the softer spring:

$$
k=\frac{1}{\frac{1}{8}-\frac{1}{20}}=\frac{40}{3} \quad\left[l b \cdot \mathrm{in}^{-1}\right]
$$

Therefore we are looking for a $13 \mathrm{lb} /$ in spring in series with a $20 \mathrm{lb} /$ in spring; the softer spring should reach solid height at $2 \mathrm{psi}$, or $7.25 \mathrm{lb}$ force. At an internal volume of $4 \mathrm{in}^{3}$, the combined spring force should be 3.53lb. Suitable springs are given in Table E.4.

\begin{tabular}{|c|c|c|c|c|}
\hline Model Number & $\begin{array}{c}\text { Outside Diameter } \\
{[\mathrm{in}]}\end{array}$ & $\begin{array}{c}\text { Solid Height } \\
{[\mathrm{in}]}\end{array}$ & $\begin{array}{c}\text { Free Length } \\
{[\mathrm{in}]}\end{array}$ & $\begin{array}{c}\text { Stiffness } \\
{[\mathrm{lb} / \mathrm{in}]}\end{array}$ \\
\hline \hline DWC-049HI-7-SS & 0.66 & 0.23 & 0.875 & 12.5 \\
DWC-085L-10-SS & 1.1 & 0.467 & 1.5 & 19.91 \\
DWC-085L-7-SS & 1.1 & 0.348 & 0.875 & 37.57 \\
DWC-105L-7-SS & 1.1 & 0.453 & 0.875 & 81.38 \\
\hline
\end{tabular}

Table E.4: Springs suitable for the nonlinear aortic chamber. 
As an example, take the DWC-049-HI7-SS: the spring has a free length of $0.875 \mathrm{in}$ and a solid height of $0.23 \mathrm{in}$. Its spring constant determines the total available travel as follows:

$$
0.875-0.23-\frac{3.53}{12.5}=0.3626 \quad[\text { in }]
$$

Table E.4 also contains some stiffer springs. These were ordered to see how the net arterial stiffness affected arterial behaviour; they allow for more combinations of springs.

\section{E.3.3 Arteries}

For the linear arterial segment in the dual-compliance model (DCM), the nominal pressure, volume, and piston area are given in Table E.5.

\begin{tabular}{|c|c|c|c|c|c|}
\hline \multicolumn{2}{|c|}{$P$} & \multicolumn{2}{c|}{$V$} & \multicolumn{2}{c|}{$S A$} \\
\hline$[\mathrm{mmHg}]$ & {$[p s i]$} & {$[\mathrm{ml}]$} & {$\left[\mathrm{in}^{3}\right]$} & {$\left[\mathrm{cm}^{2}\right]$} & {$\left[\mathrm{in}^{2}\right]$} \\
\hline \hline $80-100$ & $1.55-1.93$ & 600 & 36.6 & 65.5 & 10.15 \\
\hline
\end{tabular}

Table E.5: Nominal parameters for the linear arterial chamber

Recall that the arterial compliance in the dual-compliance model is $101 \mathrm{~cm}^{5} \cdot N^{-1}$. Converting from metric to imperial units gives:

$$
k_{a o}=0.2377 \quad\left[l b \cdot i n^{-5}\right]
$$

The arterial stiffness is scaled by the square of the piston cross-sectional area to produce the arterial spring stiffness:

$$
\begin{aligned}
\left.k_{a o}\right|_{\text {spring }} & =k_{a o} \cdot S A_{a o}^{2} \\
& =0.2377 \cdot 10.15^{2} \\
& =24.5 \quad\left[l b \cdot \mathrm{in}^{-1}\right]
\end{aligned}
$$

${ }^{*}$ The numerical simulation had shown that a stiffer artery could give rise to more interesting nonlinear behaviour. 
The nominal arterial volume is $600 \mathrm{ml}$ or $36.6 \mathrm{in}^{3}$, which results in a nominal piston position of 3.6 in above bottom dead centre. The total available piston travel is $7.74 \mathrm{in}$. Given that there is no maximum set volume for the arterial system (in the numerical simulation), the spring was sized based on the nominal chamber pressure and volume, and the total available travel.

The nominal arterial pressure ranges between $80-100 \mathrm{mmHg}$ or $1.55-1.93 \mathrm{psi}$. This implies a piston force of between $15.7-19.91 \mathrm{~b}$ at the nominal position, which in turn suggests a spring pre-compression of $0.64-0.81$ in at that volume. The free length of the spring is therefore either:

$$
L=7.74+0.64-3.6=4.78 \quad[i n]
$$

or

$$
L=7.74+0.81-3.6=4.95 \quad[i n]
$$

A slightly stiffer spring was also sized using similar calculations. Both selected springs are listed in Table E.6.

\begin{tabular}{|c|c|c|c|c|}
\hline Model Number & $\begin{array}{c}\text { Outside Diameter } \\
{[\mathrm{in}]}\end{array}$ & $\begin{array}{c}\text { Solid Height } \\
{[\mathrm{in}]}\end{array}$ & $\begin{array}{c}\text { Free Length } \\
{[\mathrm{in}]}\end{array}$ & $\begin{array}{c}\text { Stiffness } \\
{[\mathrm{lb} / \mathrm{in}]}\end{array}$ \\
\hline \hline DWC-135MN-18-MW & 1.343 & 1.765 & 5 & 24.5 \\
DWC-162NO-18-MW & 1.58 & 1.903 & 5 & 35.6 \\
\hline
\end{tabular}

Table E.6: Springs suitable for the linear arterial chamber. 


\section{Appendix F}

\section{Pressure Chamber Drawings}

RAWINGS for the various pressure chambers are included in this appendix. In Figure F.1 the assembled aortic chamber is shown sitting in its cradle. In this particular photo the aortic base block is not attached to the chamber. Instead, a smaller pressure attachment (which can be connected to a pressurized air source) is mounted on the bottom of the cylinder, and invisible in the photo.

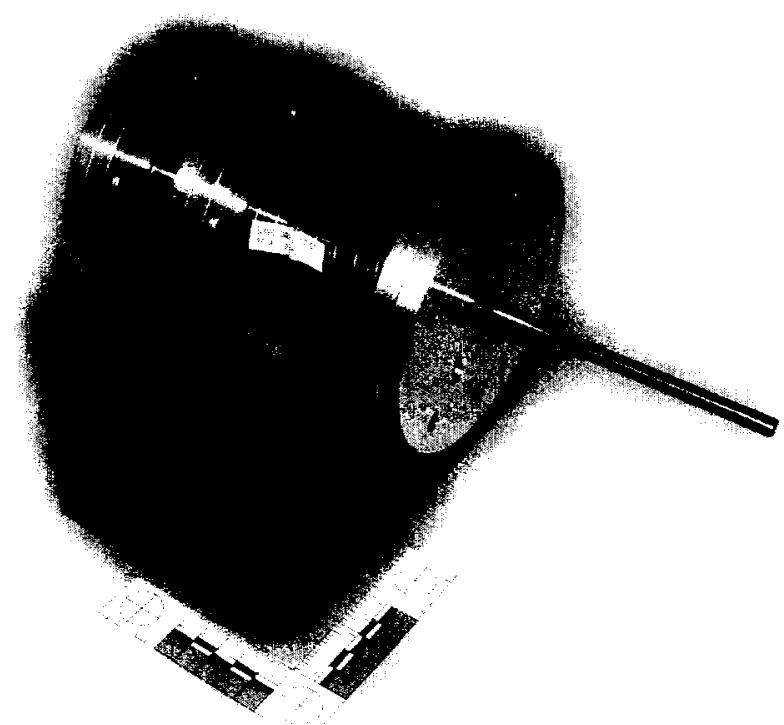

Figure F.1: The assembled aortic pressure chamber (sitting in its cradle) 


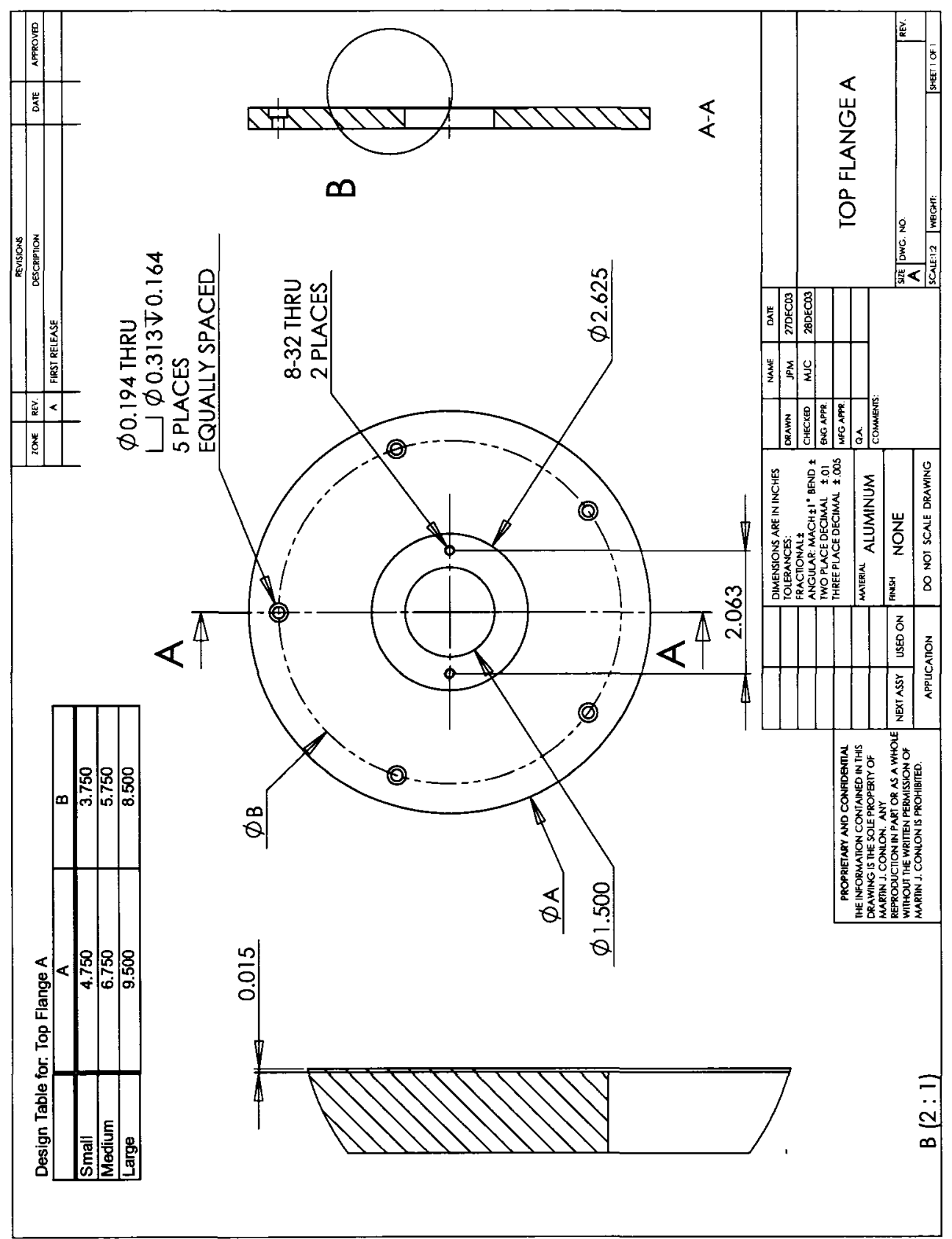




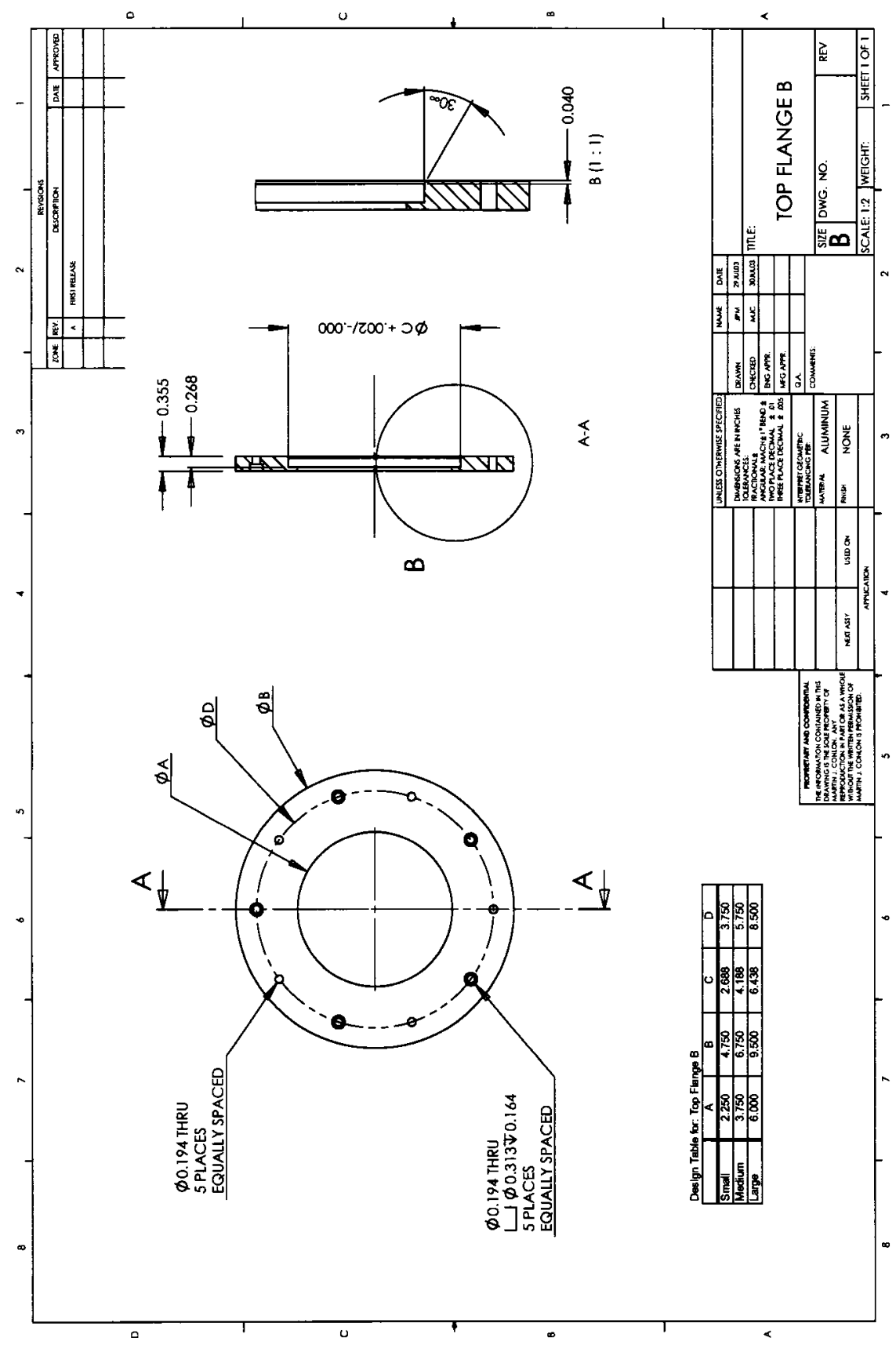




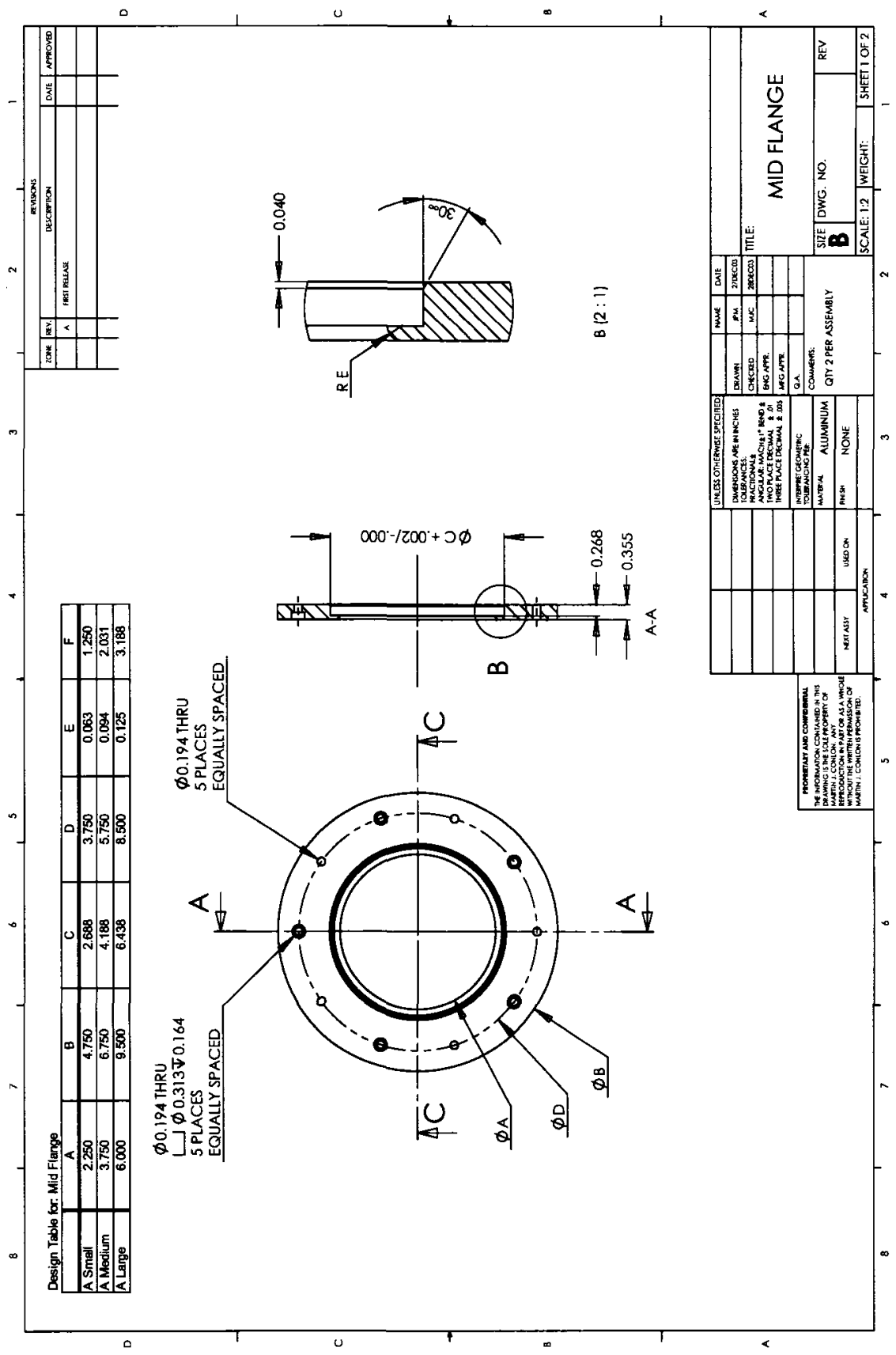




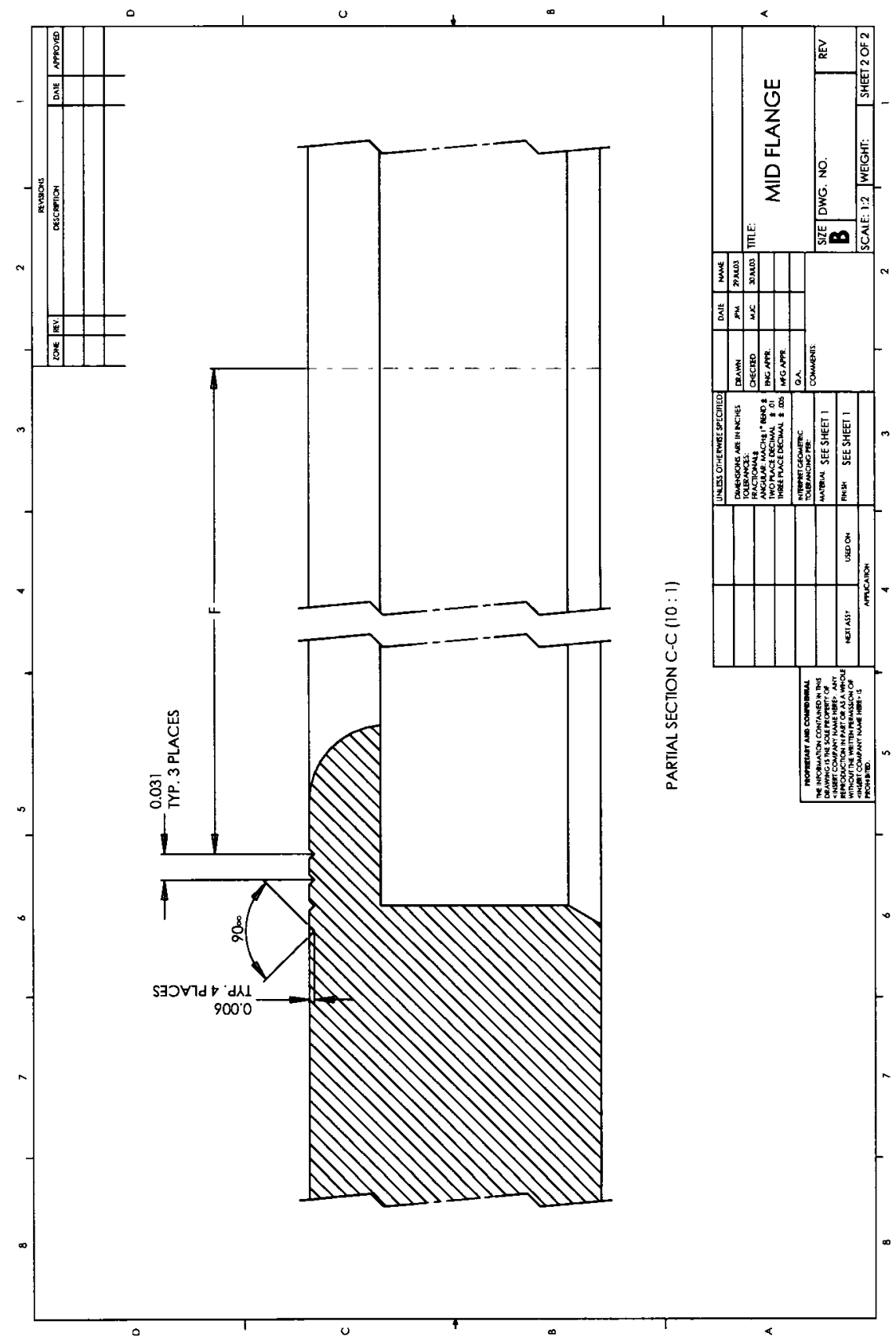




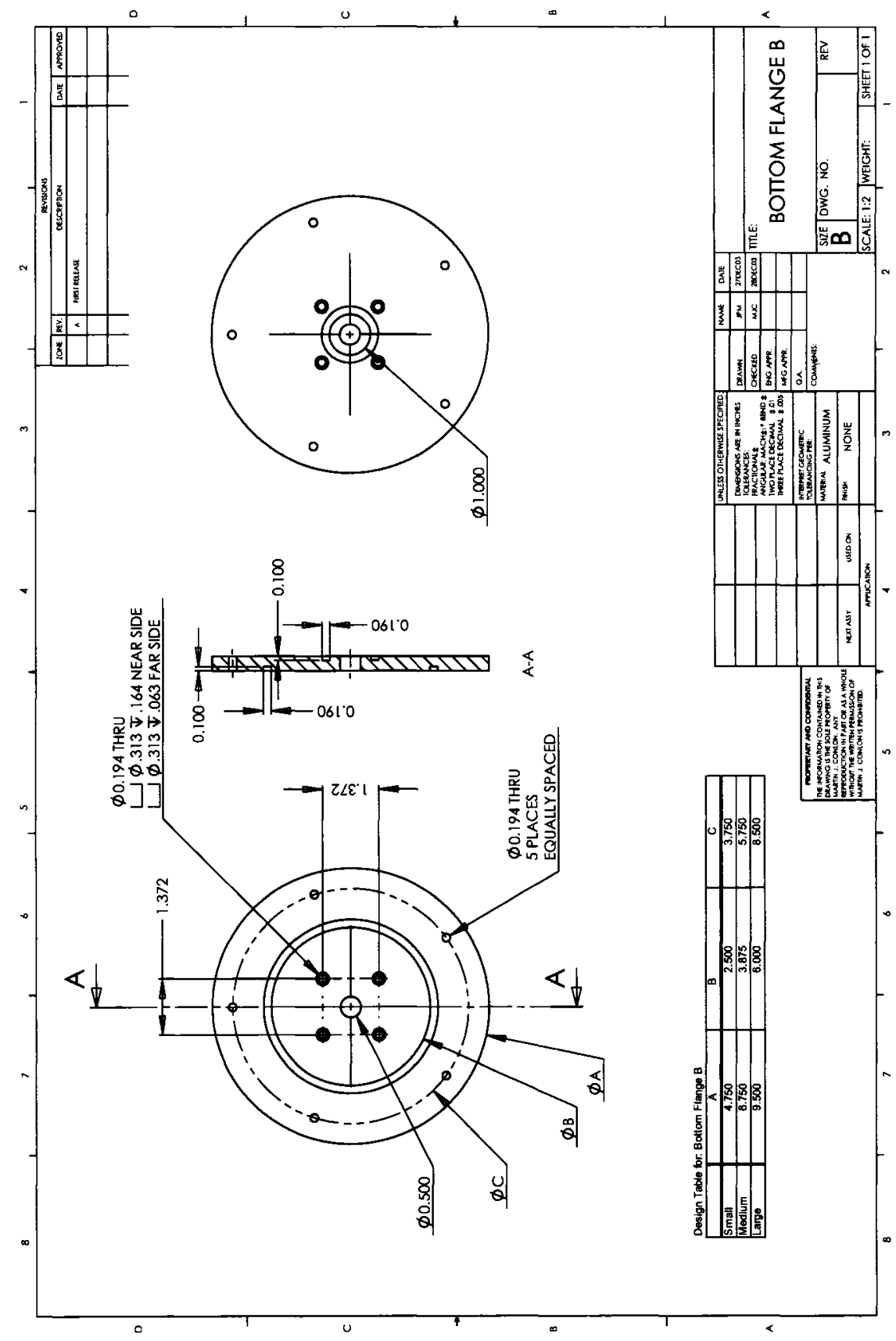


Design Table for. Cylinder

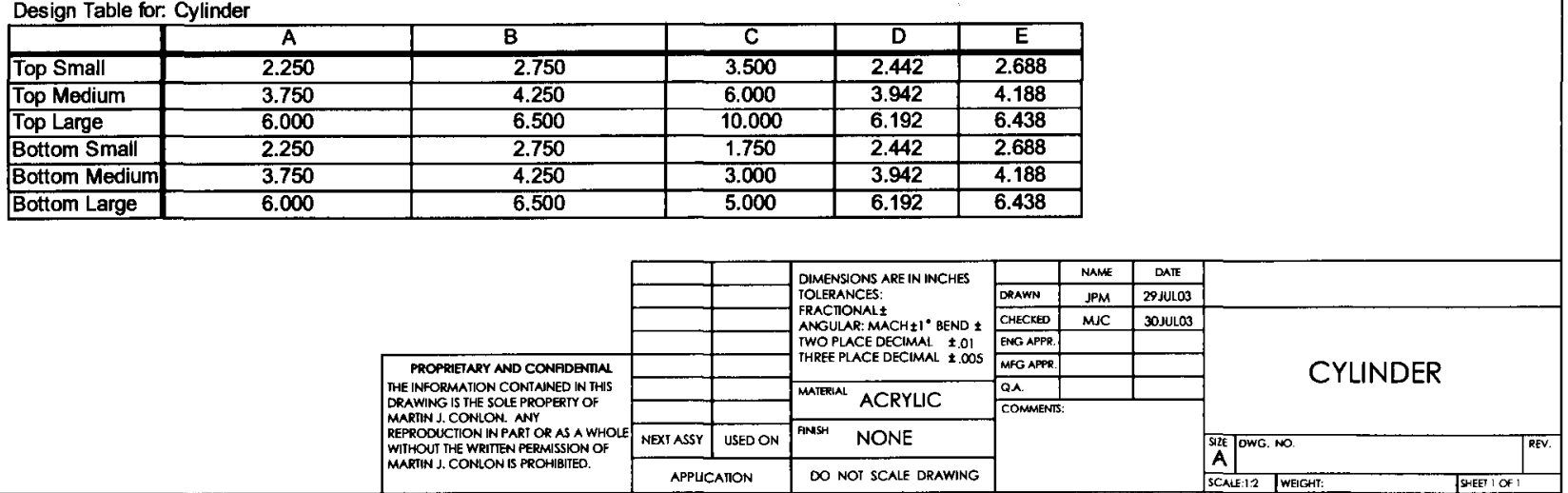




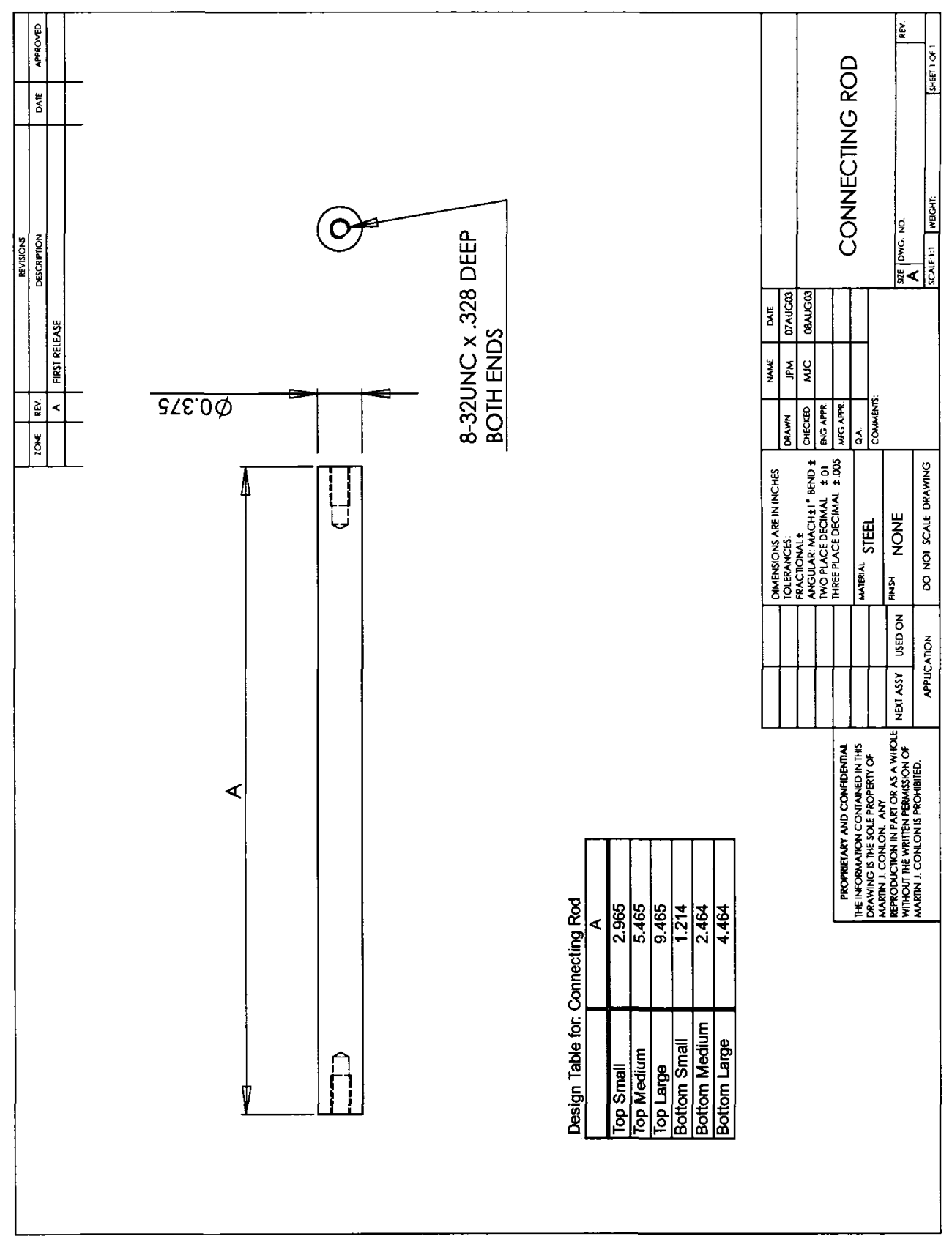




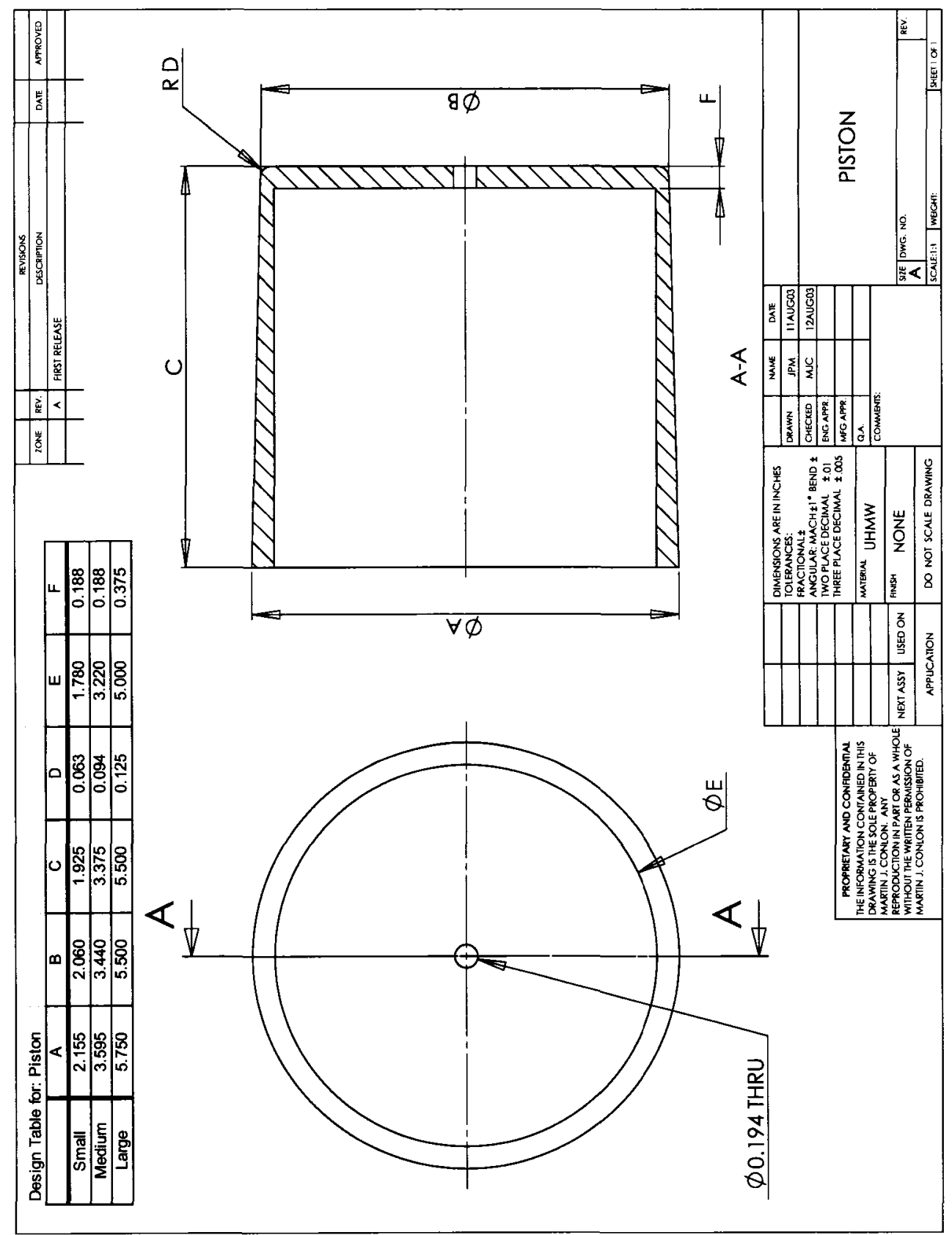




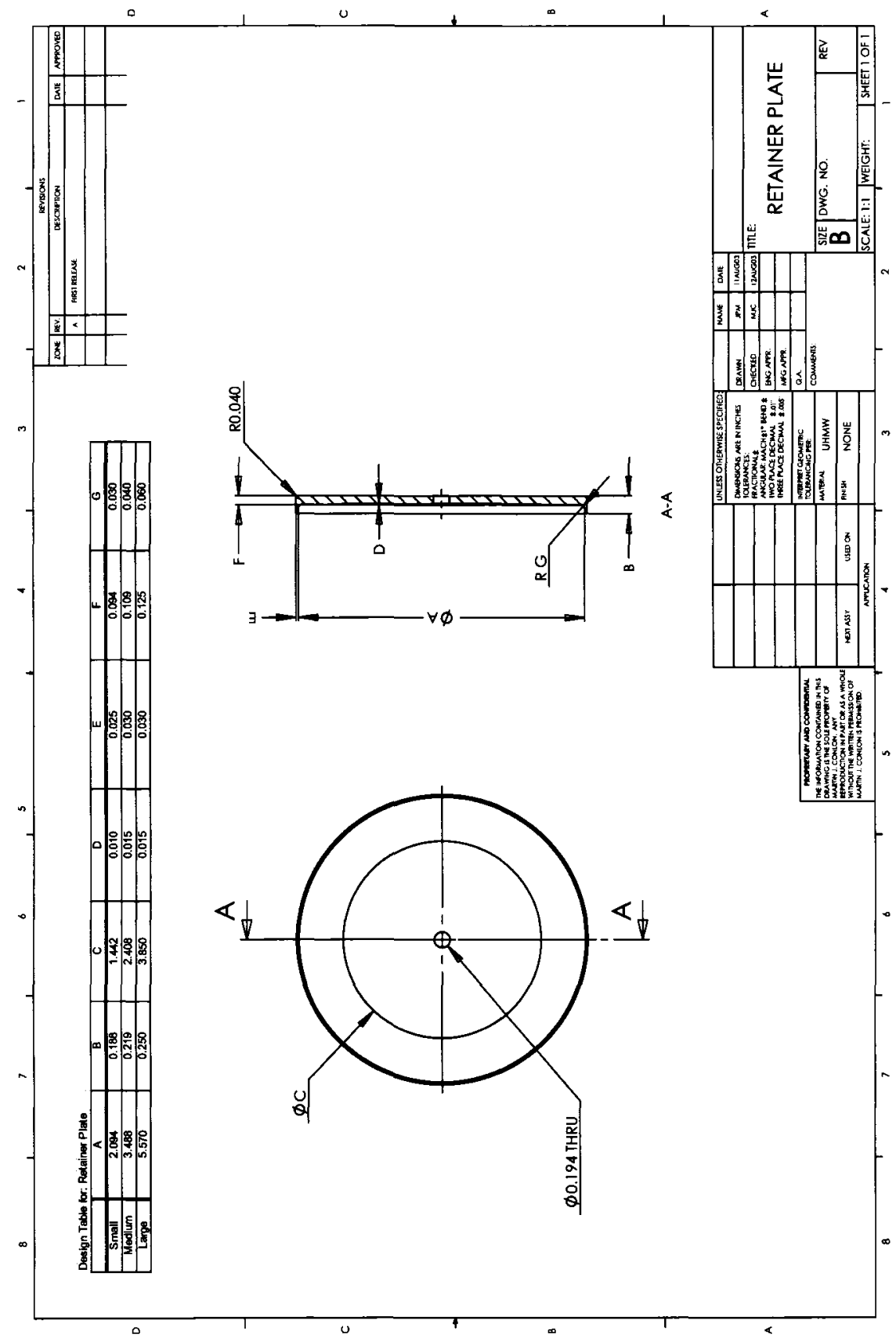




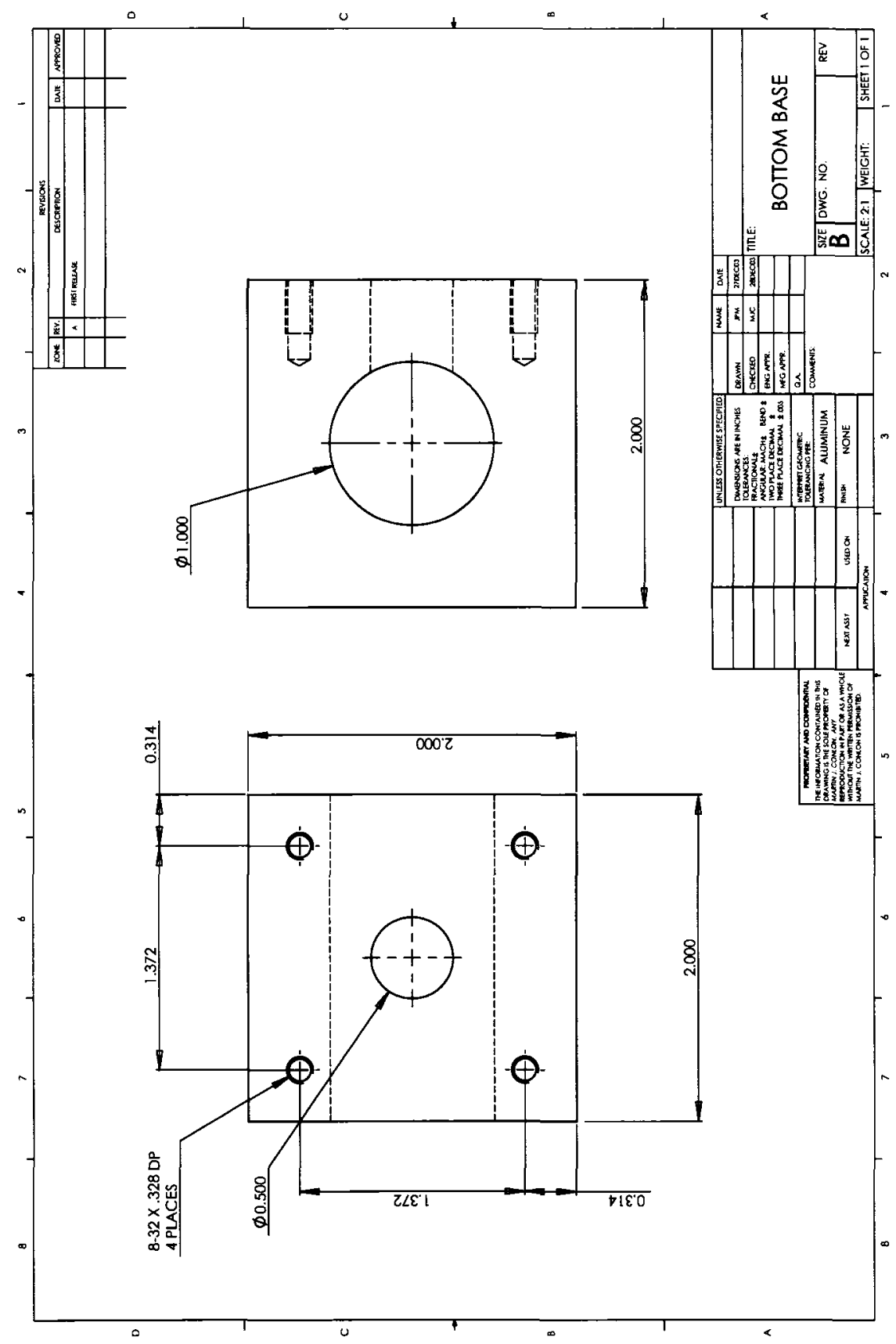




\section{Appendix G}

\section{Experimental Setup}

\section{G.1 Valve Calibration}

The peripheral resistance valve presents a variable flow resistance that depends on input (excitation) voltage. In order to determine this flow resistance, the venous tank was emptied through the valve into a bucket, and the liquid level in the venous tank was recorded at discrete time intervals. Using the steady-flow energy equation between the two liquid surfaces, neglecting the flow losses in tubing and the various fittings, we can calculate the valve flow resistance for the various fractional openings. Figure G.1 presents the valve flow resistances for the various input voltage settings; an exponential trend line is also included, and error bars corresponding to a $95 \%$ confidence interval appear with each data point.

The plot displays the behaviour we would expect from this sort of globe valve, with the nearly constant flow resistance (up until roughly $4 \mathrm{~V}$ ) followed by a sharp upswing at higher voltages (which correspond to higher occlusion, or lower fractional openings). White (1994) presents similar data for globe valves.

The nominal peripheral resistance (in the mathematical simulation) is $0.013 \mathrm{~N} \cdot \mathrm{s} \cdot \mathrm{cm}^{-5}$, which corresponds to a peripheral valve setting of roughly $8.5 \mathrm{~V}$.

\section{G.2 Pressure Transducer Calibration}

Pressures in the mock circulatory system were measured using a Druck PDCR 120/2WL transducer. This transducer accepts a DC voltage input and produces a DC voltage output. The output voltage is in the millivolt range (25 $\mathrm{mV}$ full-scale) and was amplified using an 


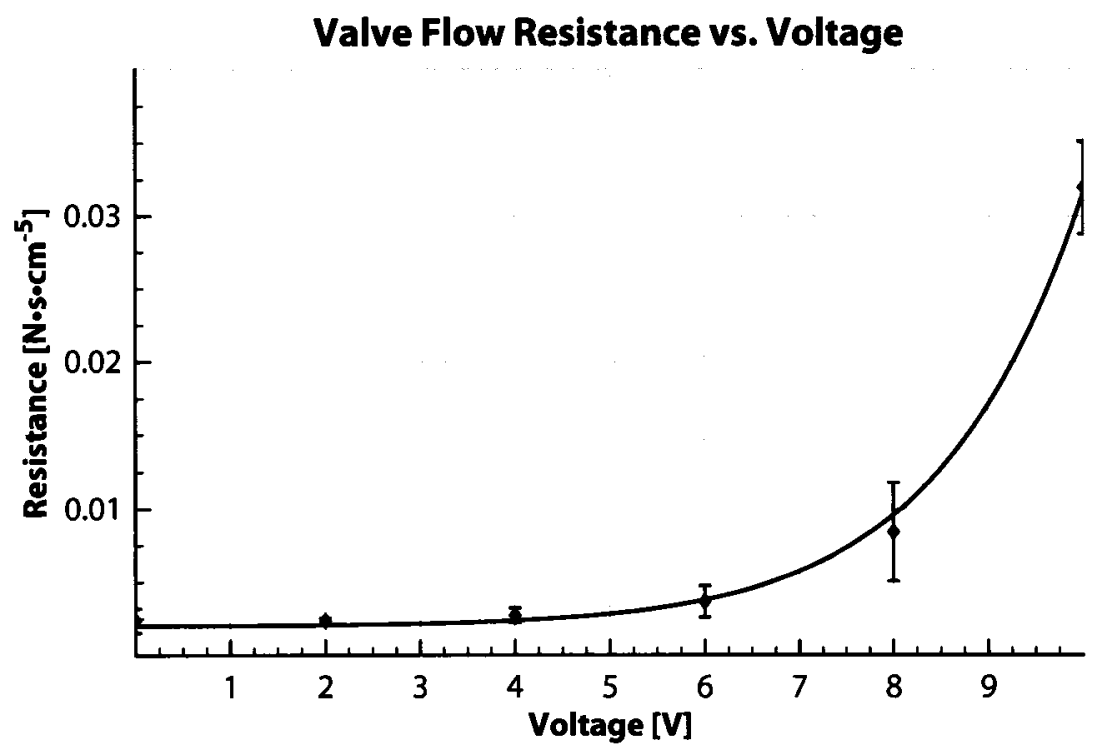

Figure G.1: Valve calibration curve for various voltage settings

Omega "Omni-Amp IV" transducer amplifier. Figure G.2 gives the amplifier output versus pressure differential for a range of input pressures. The solid curve presents the nominal voltage output (based on multiple static pressure tests) and the dashed curves bound the $95 \%$ confidence interval.

The Omega amplifier offset null was found to drift as the amplifier "warmed up." Table G.1 documents this offset change, which asymptotically approaches a fixed value. As the experiments consisted of multiple, short runs of approximately $5 \mathrm{~min}$ in length, amplifier drift over the course of an individual experimental run is negligible. (And the pressure transducer itself has a $\pm 10 \%$ variability in its null.)

\section{G.3 Position Sensor Calibration}

The piston position in the rolling diaphragm chambers was measured using a Celesco SP4 cable-extension transducer. This string encoder accepts a DC voltage input and produces a proportional DC voltage output (depending on position). Figure ?? gives the sensor output versus extension for a range of input positions. The solid curve presents the 


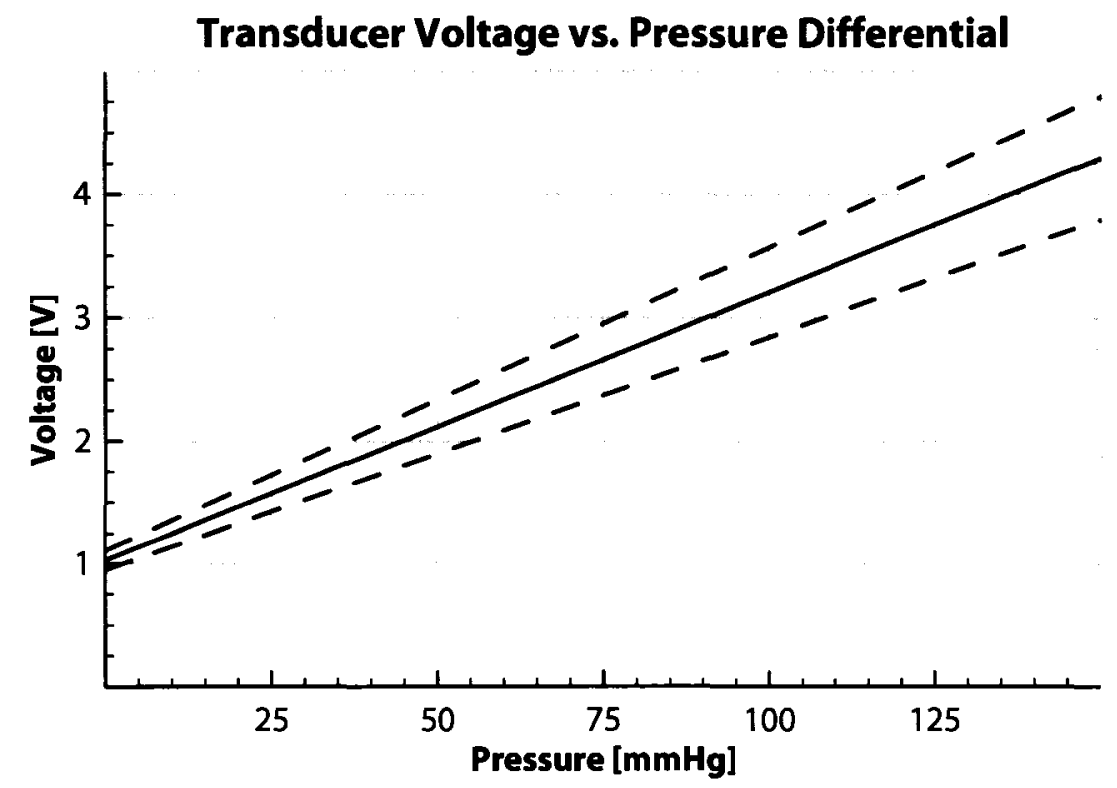

Figure G.2: Pressure transducer calibration curve

nominal voltage output (based on multiple static pressure tests) and the dashed curves bound the $95 \%$ confidence interval.

\section{G.4 Equipment Specifications}

This section details all the equipment (model number, serial number, identifying marks, etc.) used in the various experiments. Note that different experimental configurations make use of different subsets of this equipment.

\section{UOHI Arterial Compliance Chamber}

No identifying marks

\section{UOHI Venous Compliance Chamber}

No identifying marks

\section{Worcester Series 75 Actuator (Model No. 12755 R1)}

Serial No.: 10CPTMK44T 10-75 


\begin{tabular}{|r|c|}
\hline Time $(\mathrm{min})$ & Voltage $(\mathrm{V})$ \\
\hline \hline 0 & 0.71 \\
\hline 30 & 0.74 \\
\hline 60 & 0.78 \\
\hline 90 & 0.79 \\
\hline 210 & 0.82 \\
\hline$>300$ & 0.82 \\
\hline
\end{tabular}

Table G.1: Amplifier offset null drift

Valve Body:

Model CF8M

Ball 316

Seat TFE A120 E99

Calibrated 1999-07-21

Jordan Controls Actuator (Model No. MV1020)

Serial No.: 1538C97-60089-1

Valve Body:

Fisher Controls Valve (Model No. 90-24.688)

Serial No. 9703-201-1-11

Calibrated 1997-03-11 by JEC

HSVAD (Series No. 250)

Date of Manufacture 2003-02-26

HSVAD Controller

Identifying Marks:

HEC \#3

$1 / 6 \# 550$

C.M. 514

\section{External TET Coil (102262 Rev. 01)}

Serial No.: 001 


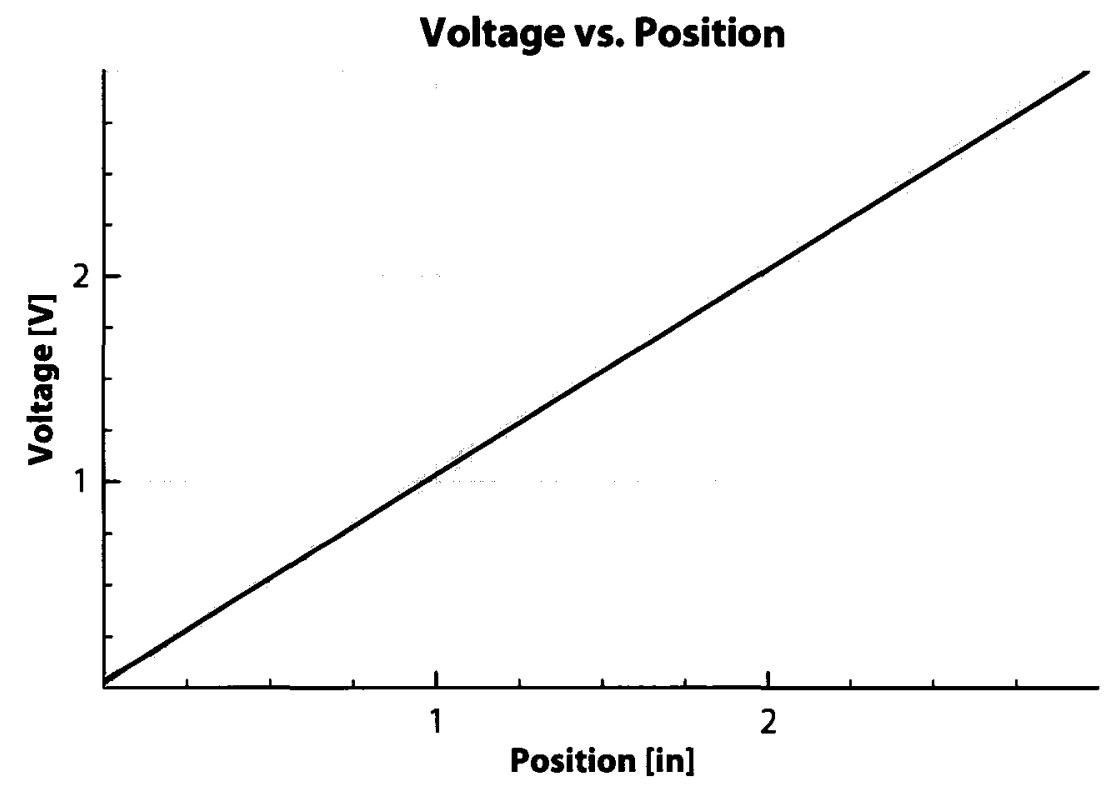

Figure G.3: Cable-extension transducer calibration curve

\section{Touchscreen Control Computer (CUI V033)}

Serial No.: 027

Identifying Marks:

AC10100234

TP0B003107

Date of Manufacture 2001-01-10

\section{GW Laboratory Power Supply (Model No. GPR-1810HD)}

Serial No.: A610791

Calibration:

Pylon Electronics, Inc.

Calibration Tracking No. 10041509

Calibrated 2002-01-28 by 11

Expires 2003-01-28

ID No. A610791/C95398 
US Power Switching Power Supply (Model No. RP2-4200F)

Serial No.: B0164483

Part No.: 11-0061

\section{UOHI VAD Power Supply}

Identifying Marks:

UOHI ID No. PS7

\section{Electronics for Medicine Monitor}

Serial No.: 83049

Identifying Marks:

UOHI ID No. 01-67-33

CVD Asset No. 400215

Property Tag 1-1432

Modules:

ECG (Model No. M1101A)

Property Tag 1-1425-1

Pressure Monitor (Model No. M2103B)

Property Tag 80478

Pressure Monitor (Model No. M2103B)

Property Tag 14243

CRT Display

Property Tag 1-1438-4

Baxter Pressure Transducers (4)

No identifying marks

Baxter TruWave Cables (Model No. PX-1800)

Serial No.: 896003021 E9826

Druck Pressure Sensor (Model No. PDCR 120/2WL)

Serial No.: 443453 
Celesco Linear Potentiometer (Model No. SP1-4)

Serial No.: 2205

Omega Omni-amp IV (Model No. 13-115)

Serial No.: 470-01-03-115

National Instruments DAQ (Model No. 6024E)

Serial No.: 1073DEF 187168D-02

IBM Thinkpad (Model No. T20, Type 2647-WGU)

Serial No.: 78-WMHC4 12/00

MTS Material Test System

Model No.: 810

Miscellaneous Cabling

HSVAD Cable (Designated CBL6; Burn-in 5)

VAD Controller Power Cable (Designated WR-837-003)

VAD Controller RS232 Data Cable (Designated WS028-03) 


\section{Appendix H}

\section{Experimental Data}

$\mathbf{T}$

HIS appendix contains a sample of the experimental data collected over the course of the work. Much of the data presented in the main matter has been ensemble averaged; the data presented here is not ensemble averaged, although it has been low-pass filtered to remove instrumentation noise.

\section{H.1 Fixed-rate VAD Tests}

Data collected during the fixed-rate VAD tests included the differential pressure between the arterial and venous chambers. The pressure transducer was connected to the chambers using lengths of 0.3125 in ID Tygon tubing. Figure H.1 presents a sample of the experimental data that went into producing Figure 5.3. During these three consecutive beats (at $60 \mathrm{bpm}$ ) the pressure trace has a characteristic skew, due to the shorter systolic (as compared with diastolic) portion of the beat. Recall that the VAD's ejection duty cycle is a fraction of the total beat length, typically less than 0.5. The waveform in Figure H.1 contains a high-frequency, low amplitude harmonic which may be due to outlet cannula oscillation. This higher frequency component disappears when the data is ensemble averaged, although it does affect the standard deviation of the dataset and therefore the shading in Figure 5.3.

Figure H.2 presents a sample of the experimental data that went into producing Figure 5.4, the data set which is most affected by sloshing in the arterial tank. The data in Figure H.2 corresponds to three consecutive beats at $60 \mathrm{bpm}$, and a comparison with Figure H.1 emphasizes the impact that the arterial sloshing has on the pressure trace. Due to the surface wave oscillations the pressure minimum no longer occurs at the start of the beat. 


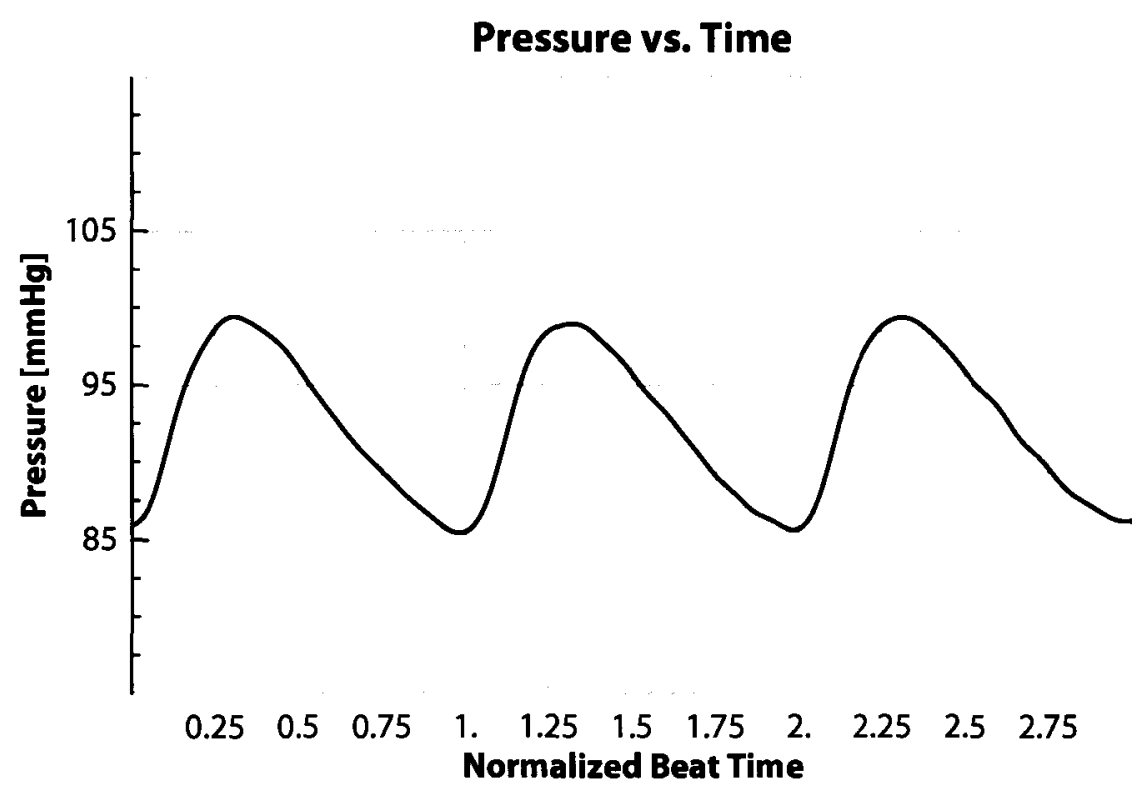

Figure H.1: Experimental data for fixed-rate VAD operation; the beat rate was $60 \mathrm{bpm}$ and the peripheral valve voltage was set to $8 \mathrm{~V}$

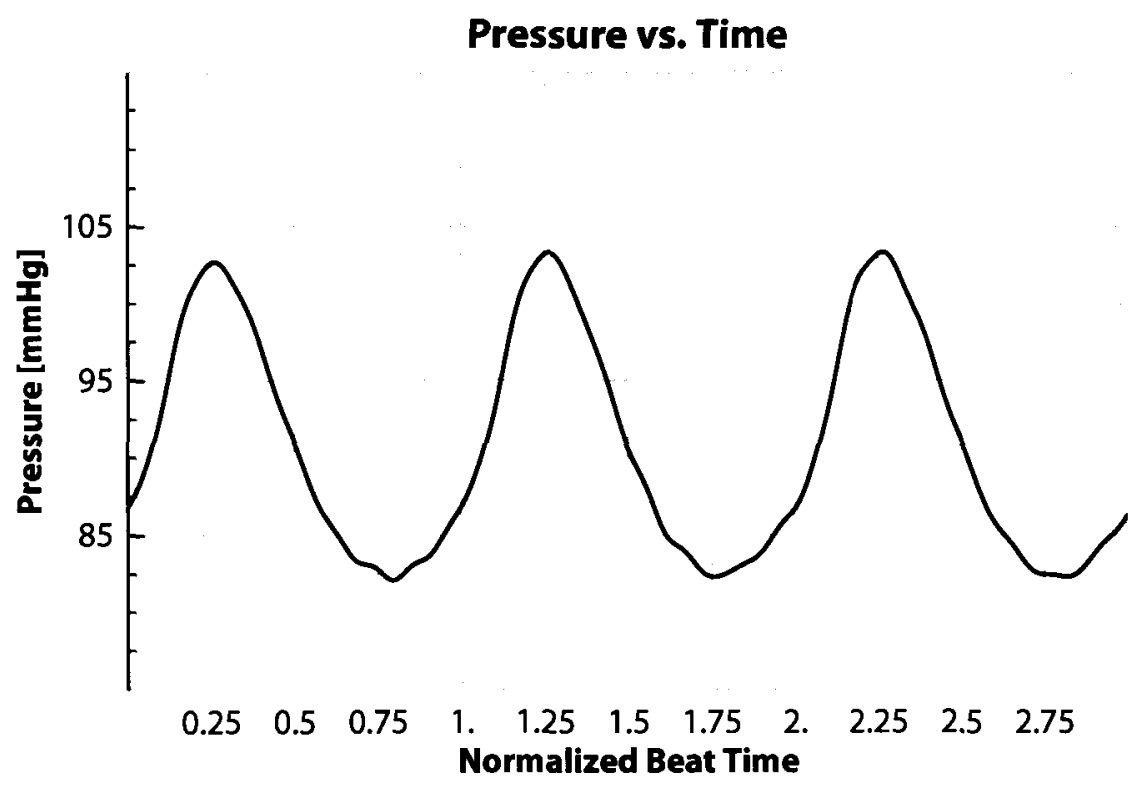

Figure H.2: Experimental data for fixed-rate VAD operation; the beat rate was $60 \mathrm{bpm}$ and the peripheral valve voltage was set to $6 \mathrm{~V}$ 


\section{H.2 FFFE VAD Tests}

During the full-fill, full-eject VAD experiments the peripheral and inlet valve settings were varied and data was collected for fifteen combinations of valve settings. As with the fixed-rate experiments, the differential pressure between the arterial and venous chambers was recorded using a transducer. The data in Figure H.3 were collected during FFFE op-

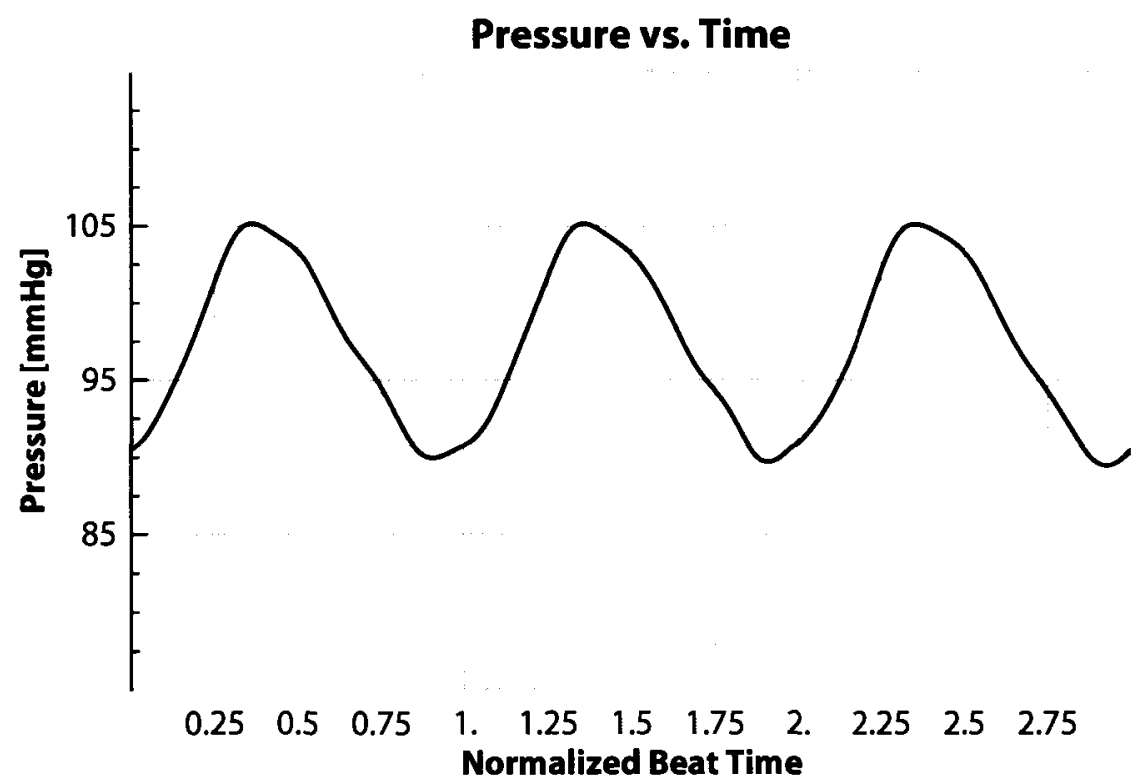

Figure H.3: Experimental data for FFFE VAD operation; peripheral and inlet valve voltages were $4 \mathrm{~V}$ and $1 \mathrm{~V}$ (respectively) and the beat rate was approximately $88 \mathrm{bpm}$ eration, with the peripheral and inlet valve voltages set to $4 \mathrm{~V}$ and $1 \mathrm{~V}$, respectively. This corresponds to the centre plot in Figure 5.6. We can see that FFFE VAD cycling produces a very repeatable waveform with almost no variation from one beat to the next; this results in the tight confidence intervals of Figure 5.6.

\section{H.3 MTS Tests}

The final phase of pVAD experiments saw the replacement of the arterial air-water chamber with a spring-loaded compliance chamber. This modification precluded the differential pressure measurements which had been the foundation of the initial VAD experiments. In order to provide measurement continuity, the mock circulatory system was up- 
dated in stages. The first (qualifying) pVAD experiments were performed with the MCS in its original configuration, with both the arterial and venous air-water chambers. Figure H.4

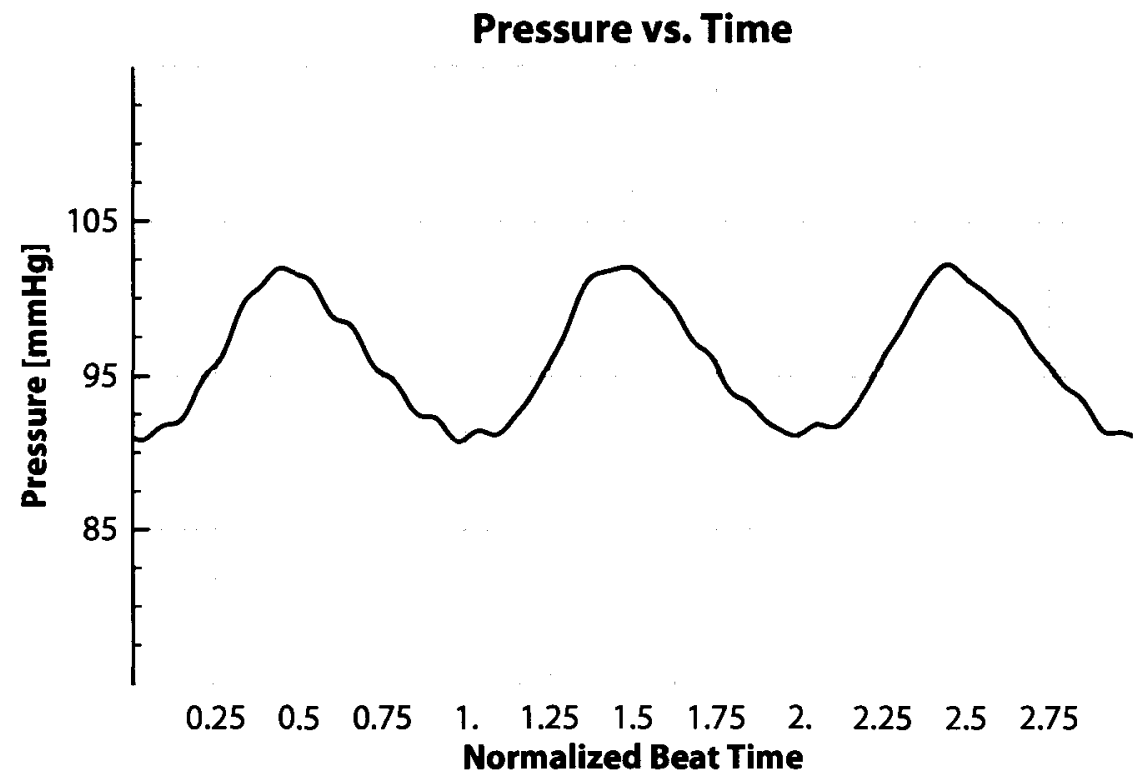

Figure H.4: Experimental data for the first set of MTS experiments; peripheral valve voltage was $8 \mathrm{~V}$

presents a sample of the experimental data that went into producing Figure 5.12. The data in Figure H.4 corresponds to three consecutive beats at $60 \mathrm{bpm}$. We recognize that, unlike the pressure trace seen during VAD operation, the MTS data lacks skew and the pressure peak is centred on the beat. This is due to the symmetric cycling of the MTS machine; recall that the material test machine was programmed to cycle sinusoidally. As with the earlier VAD tests, a higher frequency harmonic is evident in the pressure waveform. This harmonic is more noticeable in the MTS data, and at a slightly lower frequency when compared with VAD testing. Once again, the higher frequency component of the waveform disappears when the data is ensemble averaged, although it does affect the standard deviation of the dataset and therefore the shading in Figure 5.12.

Figure H.5 presents a sample of the aortic pressure data from the "dicrotic notch" set of MTS experiments. The data in Figure H.5 corresponds to a beat rate of $60 \mathrm{bpm}$, which is a lower beat rate than that which produced Figure 5.15. We can see by comparison with 


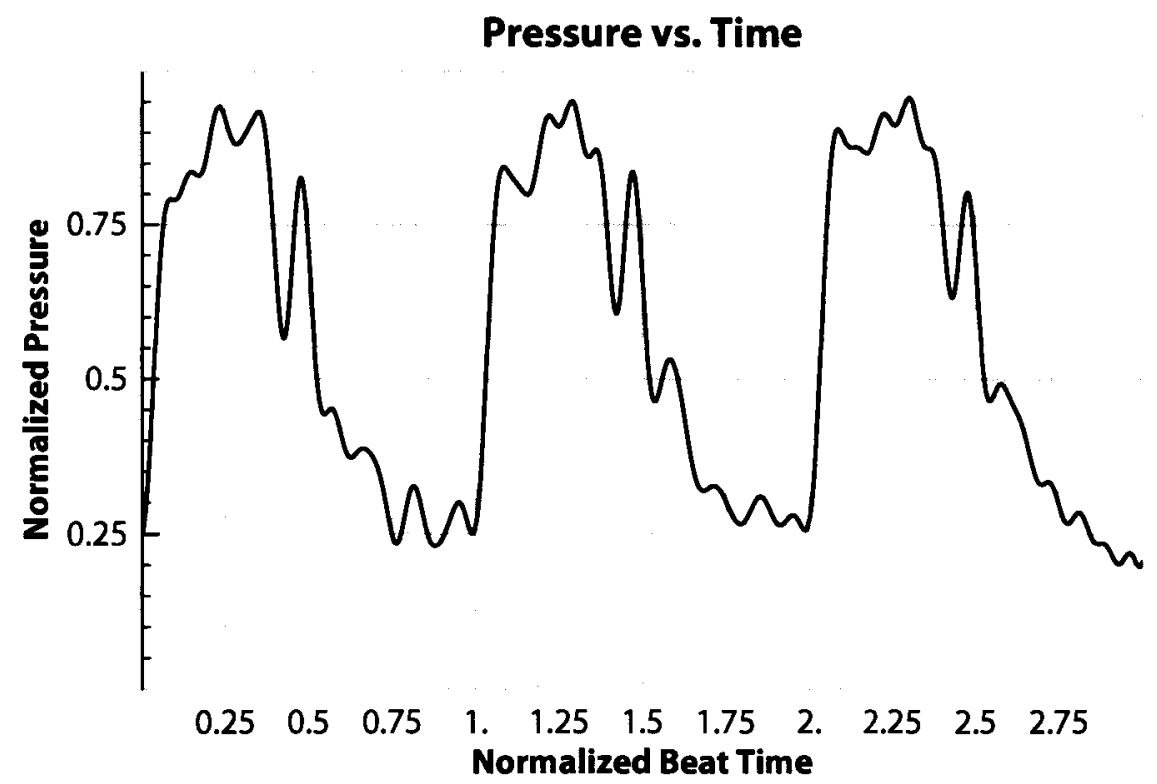

Figure H.5: Experimental data for the final set of tests; the MTS beat rate was $60 \mathrm{bpm}$ and the compliance ratio $\left(C_{a r}: C_{a 0}\right)$ is 3.3

Figure 5.15 that the overall arterial pressure affects the aortic pressure wave; the higher arterial pressures which result from high $(90 \mathrm{bpm})$ beat rates affect the slope of the postsystolic portion of the aortic pressure trace. In the human body a higher blood flow rate is normally accompanied by a drop in peripheral resistance.

As in the previous figures, the waveform in Figure H.5 displays a high-frequency oscillatory component. The amplitude of this oscillation is reduced by the ensemble averaging process, but the oscillation results in the wider confidence intervals which appear in Figure 5.15 .

Figure H.6 shows the arterial pressure waveform from the "dicrotic notch" set of MTS experiments. The high-frequency oscillation which is seen in the aorta is mostly absent in the arterial pressure trace. The pressure waveform shows little variation from one beat to the next, and the post-systolic portion of the curve has the (predicted) slight knee-like artifact which corresponds to the dicrotic notch in the aorta. 


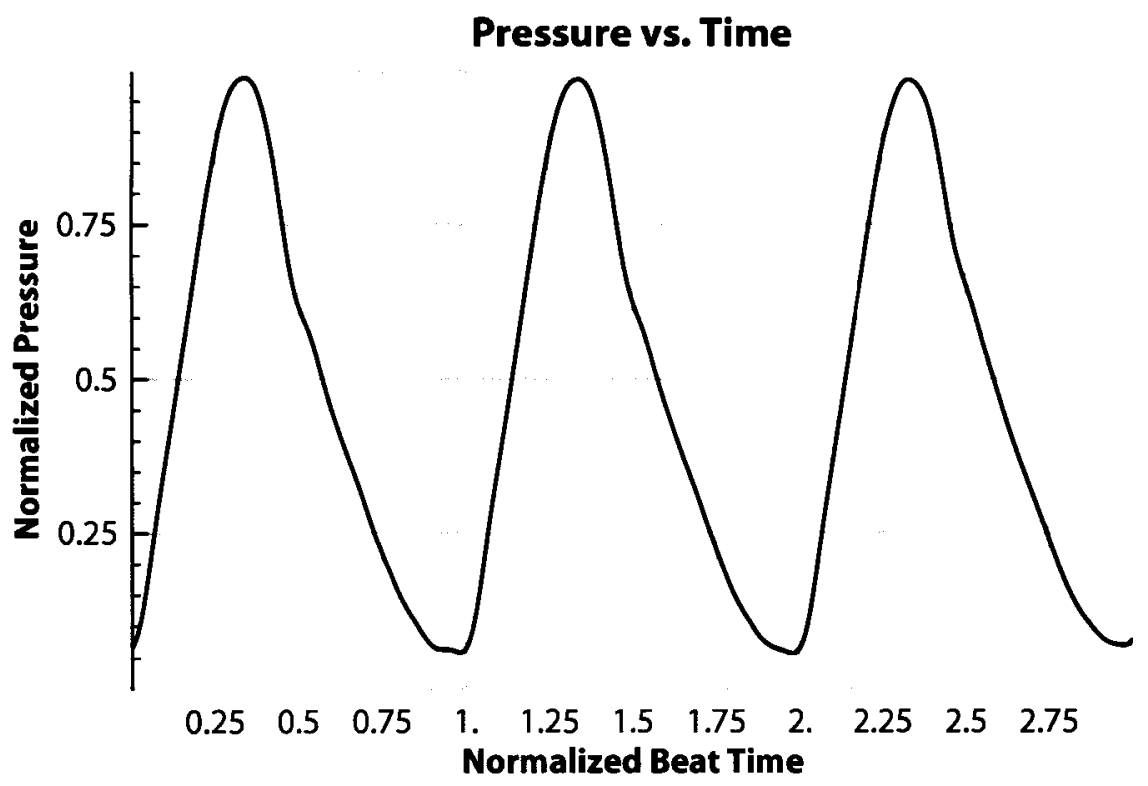

Figure H.6: Experimental data for the final set of tests; the MTS beat rate was $60 \mathrm{bpm}$ and the compliance ratio $\left(C_{a r}: C_{a o}\right)$ is 2.7 


\section{Appendix I}

\section{Schematics}

HIS appendix presents the schematics and printed circuit boards for all the electronic equipment designed for the experiment. These include:

- A current and voltage regulator to limit the excitation input to the pressure transducer.

- An amplified electret microphone to record the VAD cycle.

- A $2 \mathrm{~Hz}$ low-pass filter to condition the excitation signal for the pressure transducer.

- A $15 \mathrm{kHz}$ low-pass filter to condition the amplified pressure transducer output for input to the data acquisition card.

\section{I.1 Transducer Excitation}

The pressure transducer used in the experiments was a Druck Model 123. This particular transducer requires a 10VDC input, and a maximum input current of $15 \mathrm{~mA}$. To provide this excitation signal, a current-limited voltage regulator was designed, based around the LM317 adjustable voltage regulator. The LM317 requires only two external resistors to set the output voltage. The resistors, one connected between the output and the adjustment pin (which will be designated "R1"), and the other connected between the adjustment pin and ground (which will be designated "R2"), determine the output voltage as per

$$
V_{\text {OUT }}=1.25\left(1+\frac{R 2}{R 1}\right)+I_{A D J} \cdot R 2
$$

where the adjustment pin current $I_{A D J}$ is between $50-100 \mu A$, with $50 \mu A$ being typical. 


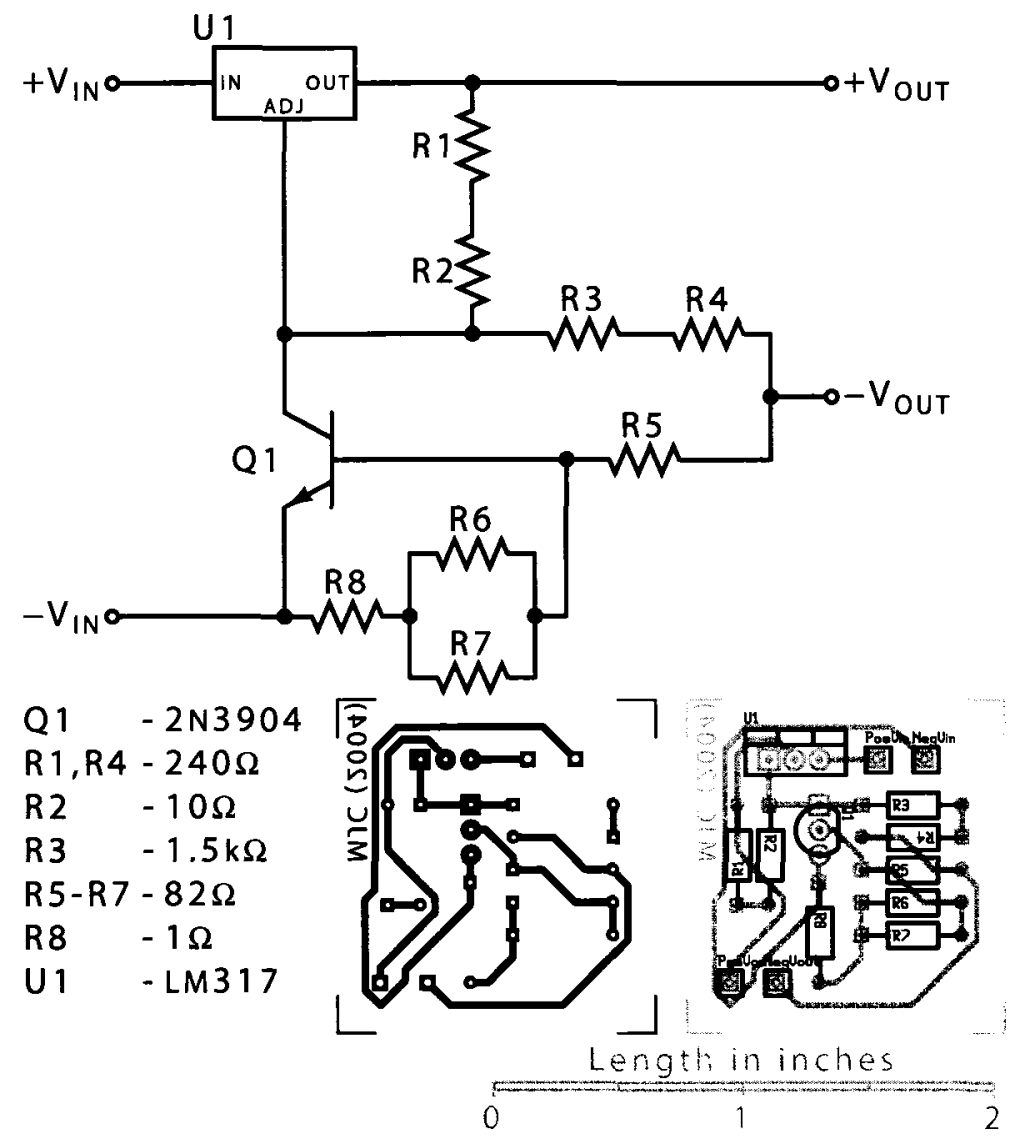

Figure I.1: Voltage regulator circuit; $10 \mathrm{~V}$ output, $15 \mathrm{~mA}$ maximum current

The problem with a straight-forward application of the LM317 voltage regulator is that the device can pass a lot of current. The transducer specifications call for a limited current; in the case of a short circuit somewhere beyond the regulator, we would like the device to shut down. As with the power supply circuitry, this can be achieved using a currentsense resistor across the base-emitter junction of an NPN transistor. The resultant circuit is shown in Figure I.1.

If the voltage drop across the base-emitter junction of $Q 1$ is sufficiently low, the transistor stays off, and the LM317 output voltage is set by the ratio of $R 1+R 2$ and $R 3+R 4$, as per Equation (I.1). The selected resistances give an output voltage of $9.99 \mathrm{~V}$. The 2N3904 used in the circuit had a base-emitter turn-on voltage of $0.63 V$, and R6-R8 were chosen to limit 
the output current to $15 \mathrm{~mA}$. If the $\mathrm{Q} 1$ transistor turns on it clamps the adjustment terminal of the LM317 to ground, which programs the output to $1.2 \mathrm{~V}$.

The PCB layout is also included (full-scale) in Figure I.1. The layout can be printed off on a transparency and used to expose a pre-sensitized board.* Note that this solderside image is reversed; the printed side of the transparency (i.e., the side with the toner) should be placed closest to the PCB surface; this limits UV leakage through the edges of the transparency and improves exposure. An additional component placement diagram is also included in Figure I.1 to aid assembly.

\section{I.2 Valve Mic}

Relating the acquired data to the VAD cycle requires some sort of a timing signal. The VAD control computer and the data acquisition computer are separate machines, and interfacing them was deemed problematic. Instead, an amplified electret microphone was used to record the VAD noise and this signal was analyzed to extract features such as valve action. The schematic and PCB layout are provided in Figure I.2.

\section{I.3 Filtering}

With the push towards digital data acquisition and analysis, proper signal filtering has become even more important. A digital sampling of an analog signal registers the original at a finite resolution and harmonics higher than the Nyquist frequency ${ }^{\dagger}$ are aliased, meaning that they appear as lower frequency signals in the resulting digital data. In fact it becomes impossible to distinguish between a high-frequency signal which has been aliased and a low-frequency signal after-the-fact, if care is not taken to do proper sampling.

The easiest way to prevent aliasing is to insure that when the signal is digitally sampled it contains no frequency components higher than the Nyquist frequency. This is

\footnotetext{
*Suitable boards are made by MG Chemicals (http: //www .mgchemicals . com) and available at most electronics supply stores.

${ }^{\dagger}$ In order to recover all Fourier components of a periodic waveform, it is necessary to sample more than twice as fast as the highest waveform frequency.
} 


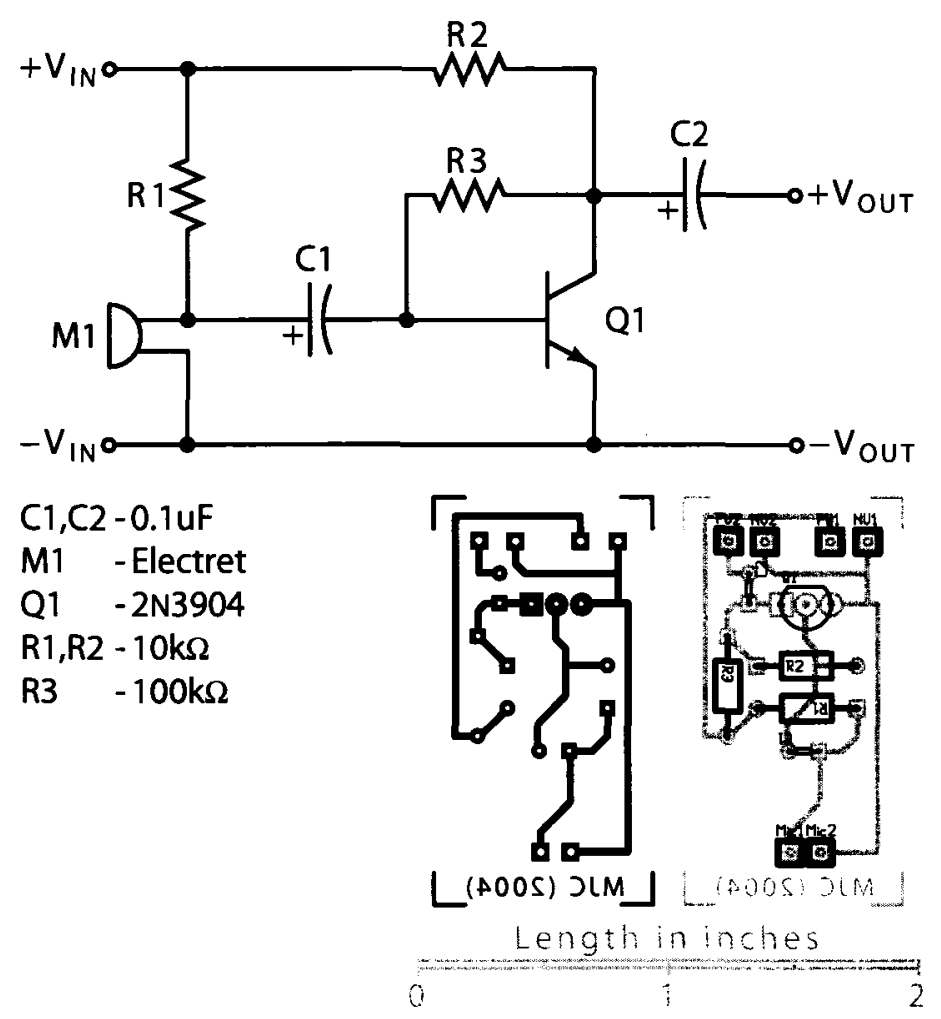

Figure 1.2: Electret microphone circuit

accomplished by routing the signal through a low-pass filter with an appropriate cutoff frequency.

The low-pass filter can be either passive or active; passive filters are networks of capacitors, inductors and resistors, while most active filters are based around operational amplifiers (op-amps). The filters are designed with a set goal in mind: normally the specifications call for a certain corner (or cutoff) frequency and a certain signal attenuation beyond that mark. These requirements determine the filter's transfer function, and this in turn can be translated into electronic circuit elements.

The required signal attenuation determines the filter order. Each pole can provide a $-6 d B /$ octave falloff slope beyond the cutoff frequency. The cutoff frequency itself is determined by pole-placement, which also affects the phase shift of the filtered output. Perhaps 
the most common low-pass filter is the Butterworth filter, which has equi-angular spaced poles.* The Butterworth filter has a maximally flat passband, but a non-uniform group delay. ${ }^{\dagger}$ This non-uniform group delay causes "ringing" as frequencies within the passband are delayed by different time constants (or given different phase shifts). It also means that temporal analysis of the filtered signal is less significant; each frequency component has been phase-shifted by a different amount, and the filtered signal no longer has the same topology as the original.

A filter which addresses this phase-shift problem is the Bessel or Thompson filter. The transfer function of the Bessel filter is based on a uniform time-delay, and it produces a near-uniform phase shift within the pass band. The cost of this improved temporal performance is a slightly droopy passband and a slow initial falloff (after the cut-off frequency).

Bessel low-pass filters were used to pre-filter the excitation signal for the Druck pressure transducer and condition its output (after amplification, before digital sampling). Both filters were fourth order, but the pre-filter had a $2 \mathrm{~Hz}$ cut-off frequency (to remove any high-frequency fluctuations in the supply voltage) while the signal conditioning filter had a $15 \mathrm{kHz}$ cutoff.

There are many filter design books, but two noteworthy ones are Lancaster's "Active Filter Cookbook" (Lancaster, 1996) and Winder's "Analog and Digital Filter Design" (Winder, 2002). Both contain information on dimensioning Bessel filter components. There is also an online component-selection tool available at:

$$
\text { http://www.beis.de/Elektronik/Filter/ActiveLPFilter.html }
$$

The filters were based around the NTE941 op-amp, which is a general purpose op-amp with an adjustable offset null. The chip is a standard DIP8 package, and two chips are required per filter. A mock-up of each filter was constructed on a breadboard and tested using an oscilloscope and function generator to insure the proper performance. The opamp offset null adjustment was also set at this time (before the components were soldered to the PCB). The final schematics and printed circuit board layouts for the $2 \mathrm{~Hz}$ and $15 \mathrm{kHz}$ filters are shown in Figure I.3 and Figure I.4 (respectively).

\footnotetext{
*In the case of an odd-order Butterworth filter, there is one pole on the real axis.

'The "group delay" is the derivative of the phase shift. A linear phase shift implies a constant group delay.
} 


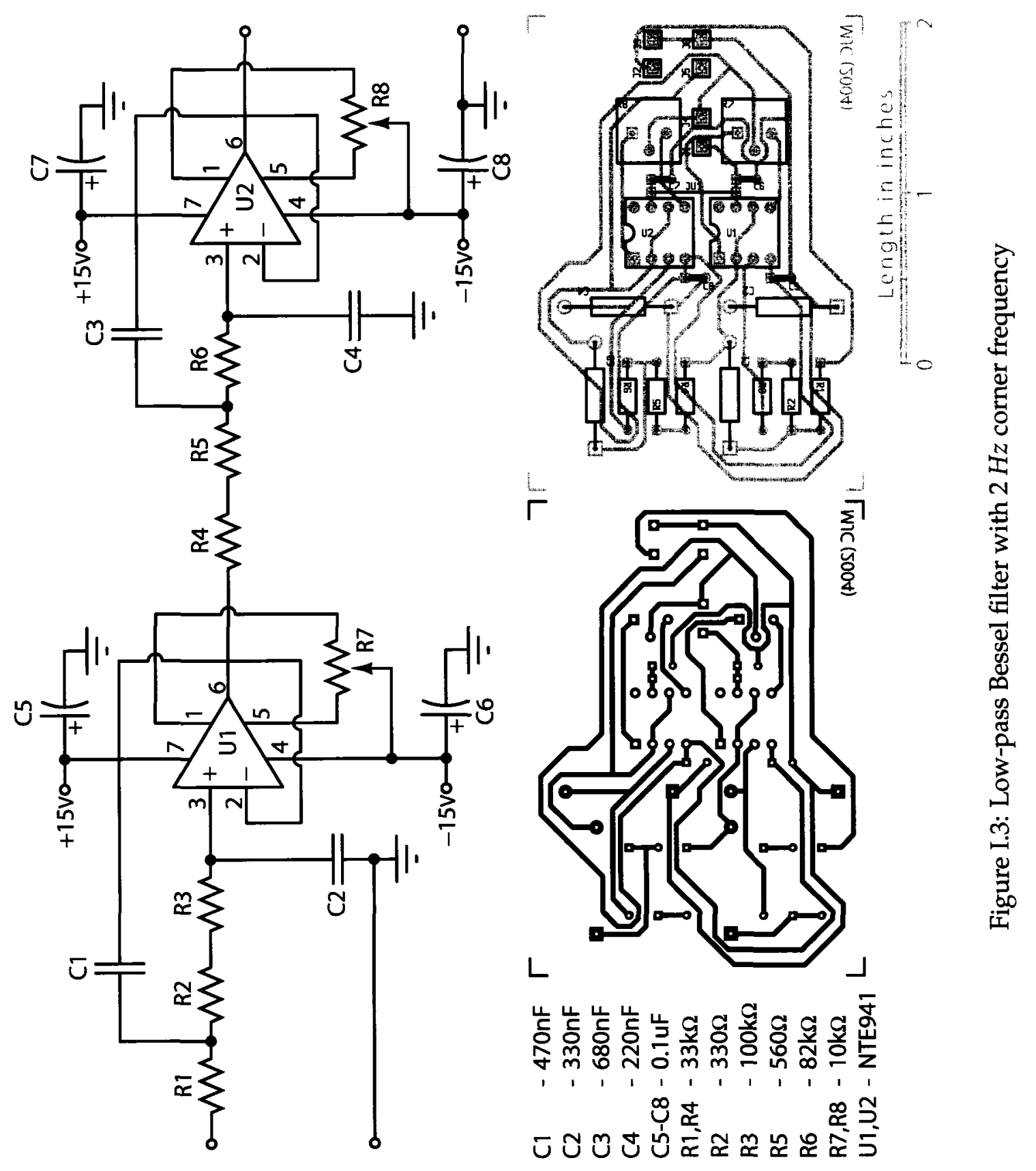




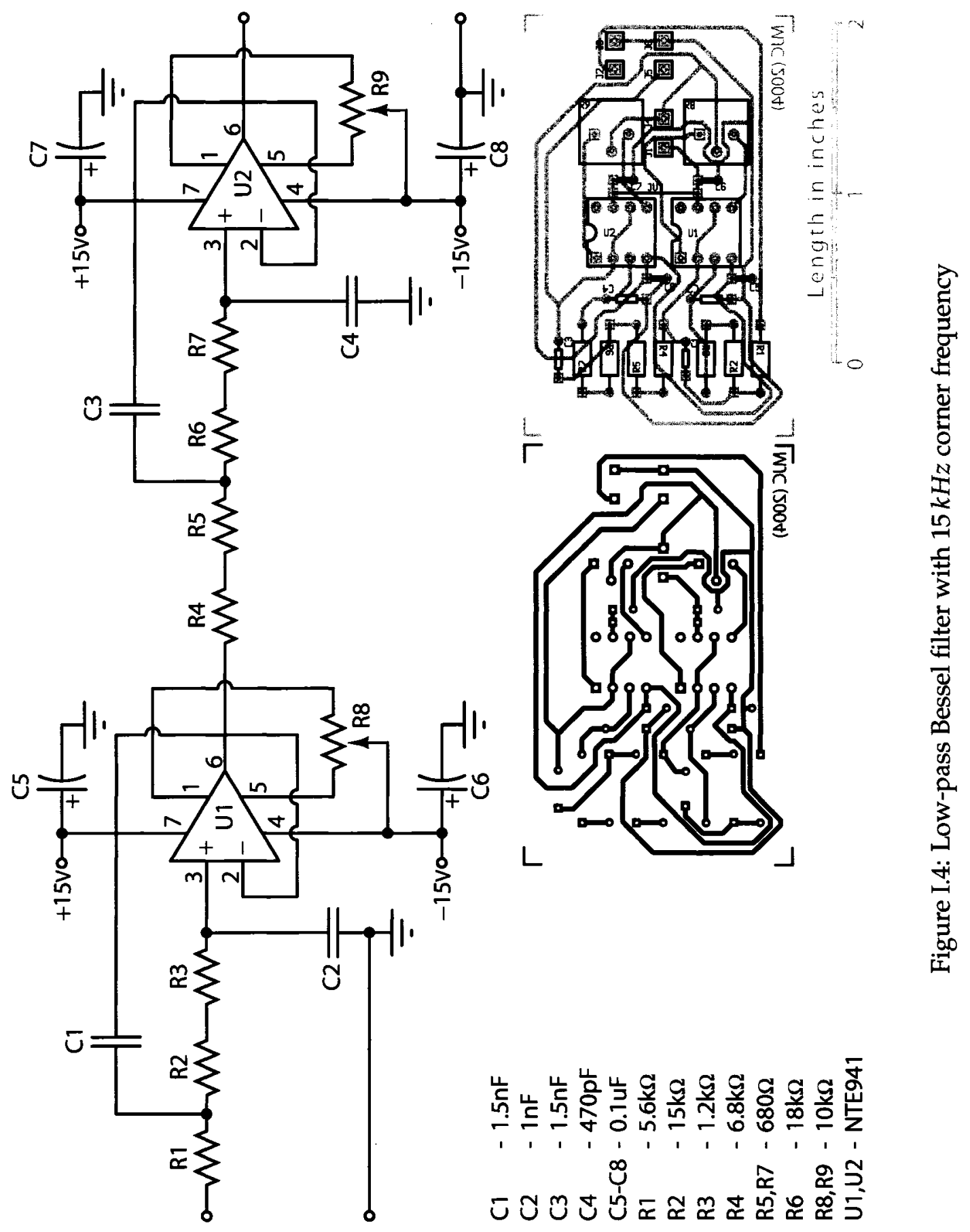




\section{Appendix J}

\section{XML Bond Graph Parsing}

The Mathematica routine presented in this appendix takes an XML description of a bond graph and converts it into a system of equations. The description of the bond graph should be formulated as follows:

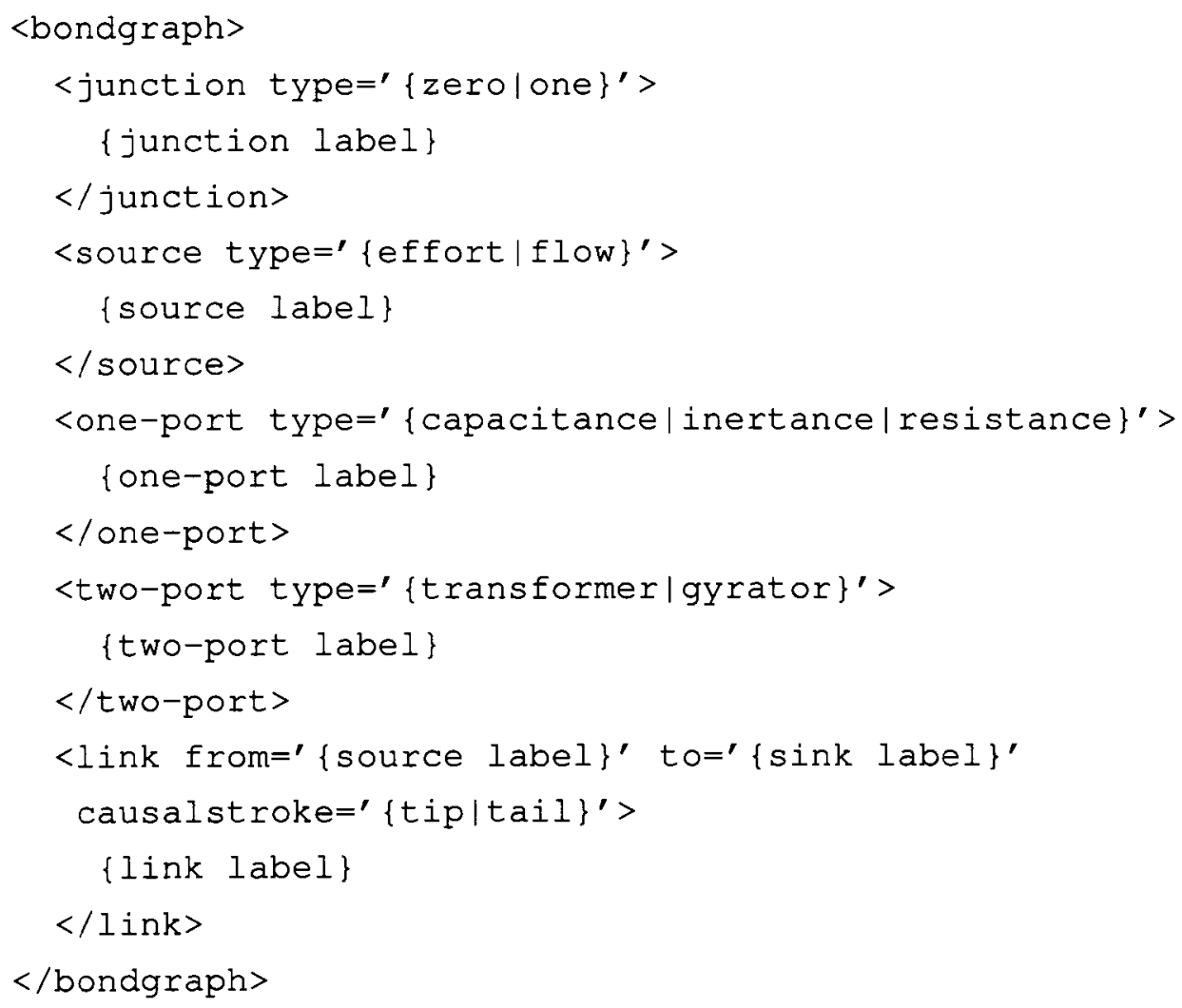




\section{XML Bond Graph Parsing Routine}

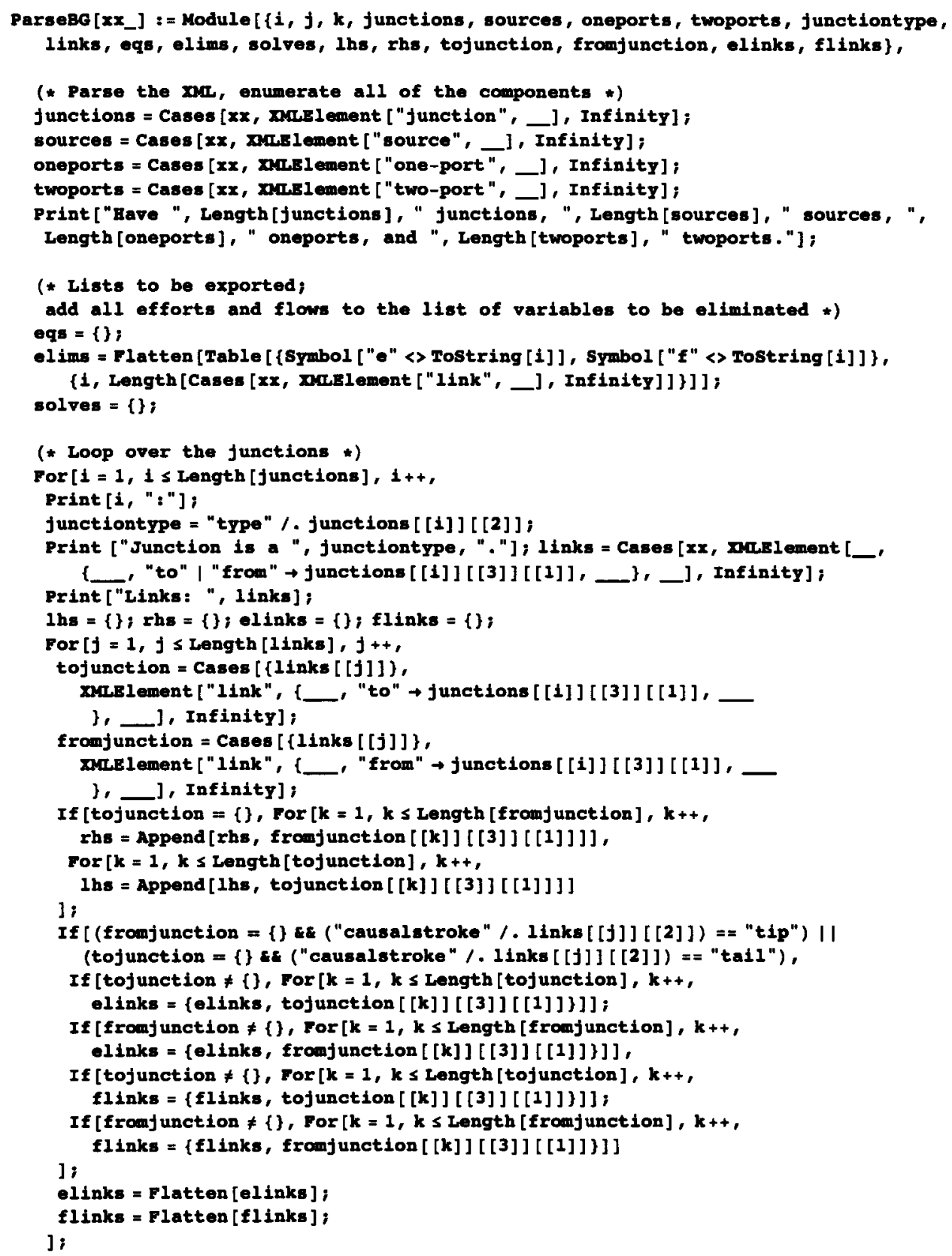




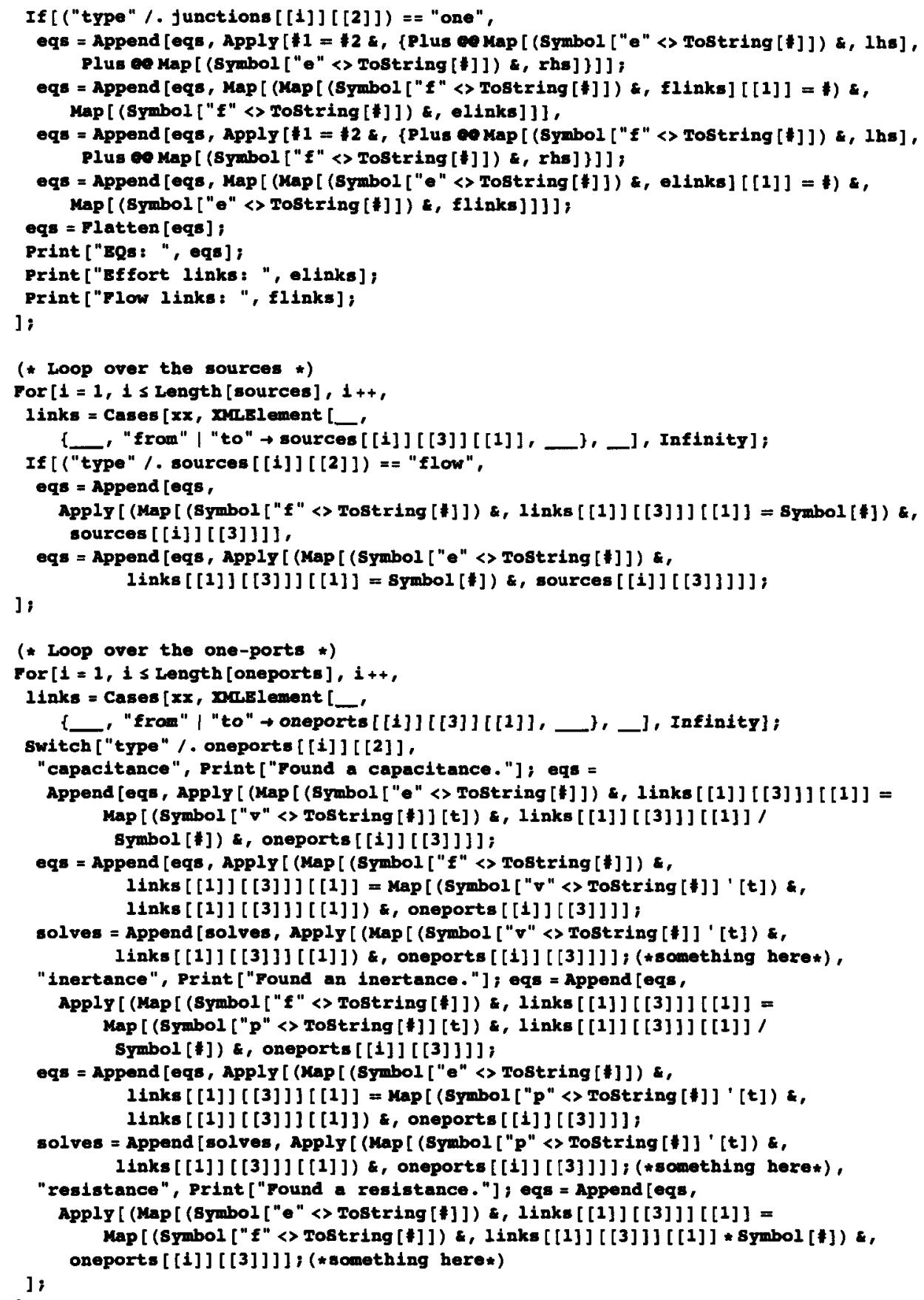




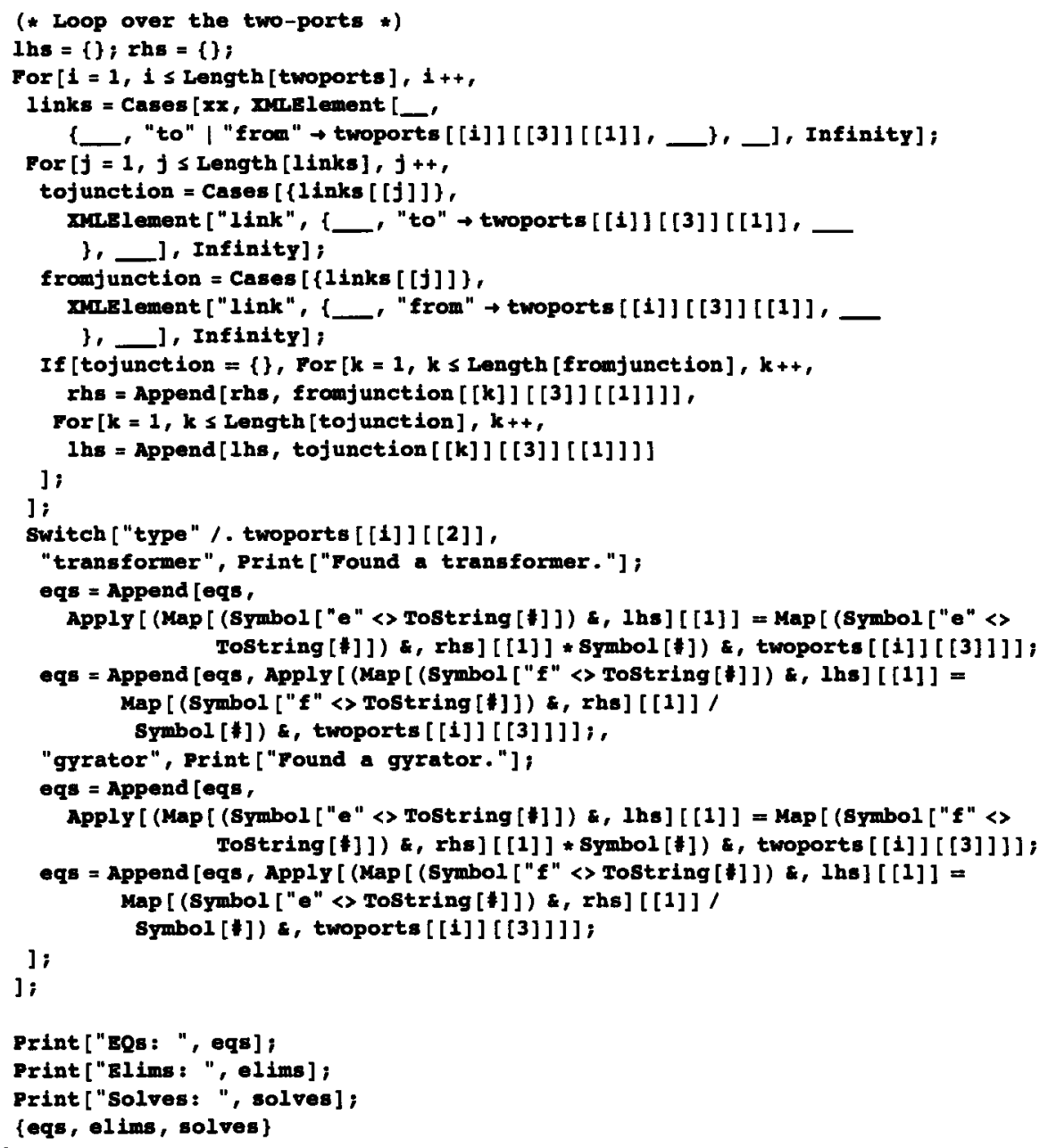




\section{Appendix K}

\section{Periodicity Determination}

Find periodicity by analyzing the peaks in the power spectrum

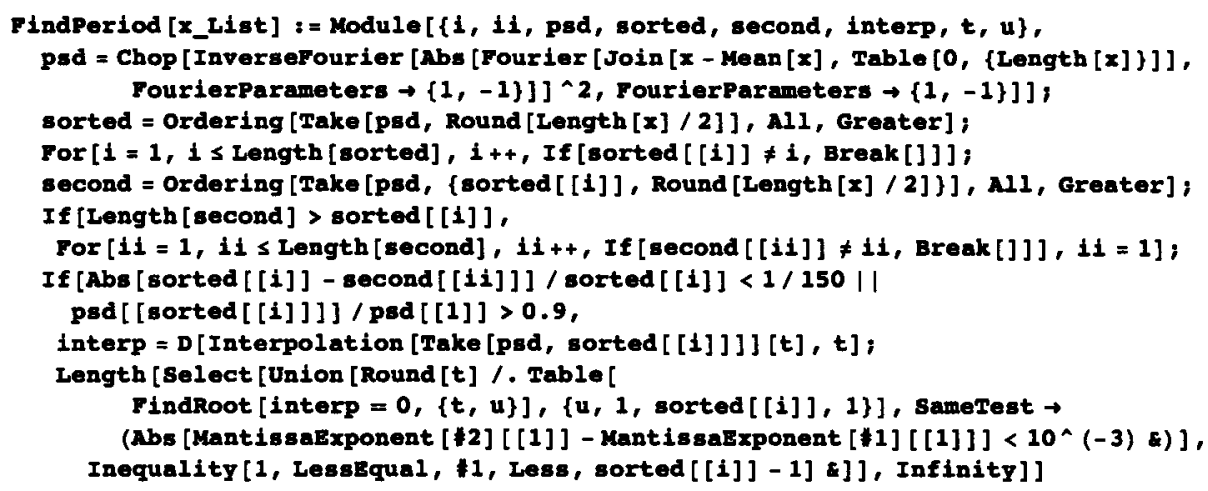

\section{Find periodicity by analyzing the pseudo-phase plot}

FindPeriod [x_List] : = Module $[\{\pi 4, u\}$,

Length [Union [Flatten [ $(\pi 3[\pi 4] / . x) /$. Partition [Map [ $(\pi 4 \rightarrow \&)$, select [

Union $[\pi 4 /$. Table $[$ FindRoot $[(\pi 3 \cdot[\pi 4] / . x)=0,\{\pi 4, u\}],\{u, 50,100,0.1\}]$,

SameTest $\rightarrow\left(\right.$ abs $\left.\left.\left.\left.\left.\left.[(* 2-1)]<10^{\wedge}-3 \&\right)\right], 50 \leq * 100 \&\right]\right], 1\right]\right]$,

] :

SameTest $\rightarrow\left(\right.$ Abs $[(12-1)]<10^{\wedge}-3$ \& $\left.\left.)\right]\right]-1$ 


\section{Find periodicity by correlating subsets of the waveform}

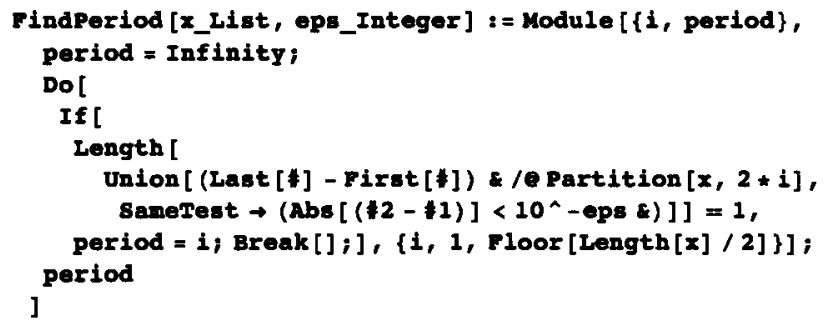




\section{Appendix L}

\section{Mathematica Bugs}

ATHEMATICA'S numerical routines contain a few bugs which were discovered over the course of this work. Wolfram (the company which produces Mathematica) have been made aware of the problems.

\section{L.1 NDSolve Memory Leaks}

Mathematica's numerical integration routine, NDSolve, contains an optional switch which is similar to MATLAB's Events functionality; whenever the StoppingTest condition evaluates to True the integration is halted and the intermediate results returned. Integration can then be resumed based on new initial conditions (or even the final conditions in the intermediate results, depending on what the user is trying to achieve). For simulations of impacting systems, such as a ball bouncing on a floor, this feature is very useful because the integration will halt on impact with the floor and can be resumed once the appropriate elastic (or inelastic) reflection has been applied to change the ball's direction.

Unfortunately, StoppingTest leaks memory, which is to say that memory allocated for the NDSolve integration becomes unrecoverable (i.e., cannot be deallocated) if the StoppingTest feature is used. In large simulations the Mathematica kernel will eventually use up all available system memory and need to be restarted.

Although StoppingTest is still supported, Wolfram recommends the use of a newer function, EventLocator, in its stead. The implication is that EventLocator avoids many of the pitfalls associated with StoppingTest. Alas, EventLocator suffers from 
the same memory leaks as StoppingTest; memory use grows without bound and eventually the Mathematica kernel must be restarted.

The only workaround available to-date is restarting the Mathematica kernel (and the simulation) manually at intervals, before the process consumes all system memory (at which point Mathematica crashes).

\section{L.2 Errors in the NumericalMath 'IntervalRoots ' Package}

The Mathematica Book introduces interval root finding methods as follows:

A nice feature of interval root-finding methods is that they find all roots of the given function on a given interval. More precisely, they start with the given interval and discard parts of it that cannot possibly contain any roots. What you end up with are some subintervals of the given interval that are guaranteed to contain all of the roots that are contained in the the given interval. If the roots of the given interval are well separated then the result consists of short subintervals, each of which contains exactly one root.

Unfortunately the interval root finding methods (such as IntervalBisection) will fail to return the smallest root if that root is close to zero. In this instance "close" may be a distance substantially larger than eps, the supposed tolerance of the methods. There is no workaround for this problem. 


\section{Index}

ПI-group, 31

adjustable flow resistor, 69

amplitude peak, 118

anatomy

circulatory system, 6

aorta, 9, 12

aortic

arch, 18

elastance, 78

elastic response, 96

expansion, 96

flow waveform, 115

inertia, 105

pressure, 128

aperiodic, 129

aperiodic behaviour, 150

arrhythmia, 148

arterial

chamber, 128

equivalence, 146

impedance, 63

pressure, 138, 144

pressure waveform, 92

reactance, 65

reservoir, 59 system, 37

tree, 68

arteries, 7,12

chaotic pressure oscillations, 54

conducting, 12

distributing, 13

proximal, 146

arterioles, 13

artery

pulmonary, 8

radial, 79

artificial heart, 14

asymmetric inputs, 117

atrioventricular valves, 7

atrium, 7

left, 8

right, 8

auricle, 7

autocorrelation, 104

autonomous, 21

baroreceptor feedback, 146

baroreflex, 56

barrier impacts, 118

base block, 132

bellows, 66 
Bender bouncer, 99

bifurcation, 56

bifurcations, 99, 102, 114

binomial coefficient, 31

blood, 16

density, 43

flow (pulsatile), 16

pressure (high), 9

bond graphs, 23

bonds

active, 23

causal stroke, 24

power, 23

signal, 23

boundary condition

inlet, 82

outlet, 83

boundary conditions, 82

broadband noise, 154

Buckingham's П-Theorem, 28

cannula, 17

inlet, 39

outlet, 39

cannulae, 17, 39

capacitance, 24

capillaries, 14

Cardano's Method, 89

cardiac cycle, 10

causality, 95

ceiling motion, 95

chamber compliance, 149

chaos

deterministic, 55 chaotic oscillations, 147

circulation

pulmonary, 10

systemic, 10

circulatory system dynamics, 52

complementary solution, 96

compliance

aortic, 41

arterial, 42,93

vena cava, 47

congestive heart failure, 9

constitutive relations, 51

control strategy, 93

correlation dimension, 55

cross-sectional area, 149

cubic

depressed, 90

discriminant, 91

roots, 91

cubic polynomial, 90

cycle

cardiac, 10

pVAD, 133

DCM, 41

arterial, 84

damping ratio, 89

flow-in, flow-out, 84

flow-in, pressure-out, 85

natural frequency, 89

stability, 87

state-space formulation, 86

damage

left ventricle, 10 
derivative terms, 35

deterministic chaos, 57

diaphragm

binding, 140

rolling, 68

chamber, 138

wear, 140, 154

wrinkling, 140

diastole, 12

dicrotic

notch, 77,92

pressure, 79

dimension matrix, 29

dimensional analysis, 28

dimensional reduction, 30

discharge paths, 76

discretization, 62

dissipative element, 24

disturbing force, 96

divergence, 99, 109

Donovan, 40

driving frequency, 99, 102

dual-compliance model

arterial, 41, 129

venous, 46

dynamic behaviour, 82

ECG, 10

effective area, 143

efficiency

artificial heart, 93

effort, 24

eigenvalues, 87,140

ejection duty cycle, 38
EKG, 10

elastic

collision, 96

recoil, 77, 129

reflection, 98

elastomer diaphragm, 140

electret microphone, 121

electrocardiograph, 10

embedding dimension, 55

endothelial denudation, 54

energetic efficiency, 118, 147

energy

kinetic, 24

potential, 24

energy storing elements, 87

entrainment, 56

entropies, 55

equivalence, 80

extrema

local, 90

FFFE, 37, 121, 125

falling mass, 30

Fermi oscillator, 99

fixed points, 108

fixed-rate, 38,122

flow, 24

laminar, 26

resistance, 27

turbulent, 26

force

gravitational, 20

Fourier Transform, 104

fractal, 109 
fractal anatomy, 55

full-fill, full-eject, 37

fundamental dimension, 28

fundamental objective, 3

General Theory of Relativity, 19

gravitational

field, 19

force, 20

gyrator, 25

HCS, 36, 144

half-sine wave, 96

heart

anatomy of, 6

attack, 9

disease, 1 coronary, 9

failure

congestive, 9

rate, 105

total artificial, 1

transplant, 1

hemodynamics, 57

human circulatory system, 82

model, 36

hydraulic

impedance, 72

schematic, 48

hypertension, 129

ideal physical model, 23

impact

intervals, 106

oscillator, $80,99,105$ soft-wall, 100, 111

inertance, 24

inertia, 24

peripheral, 95

inflection points, 90

initial

conditions

sensitive dependence on, 99, 107

disturbance, 109

position, 32

velocity, 32, 33

input matrix, 22

integrated findings, 144

internal pressure, 133, 154

interval

closed, 88

notation, 88

open, 88

intra-aortic balloon pumping, 53

junction

one, 25

zero, 25

junctions, 25

kernel, 29

knots, 25

left atrium, 8

left ventricle, 9

load cell, 150

local velocity, 100

loop

pulmonary, 12

systemic, 12 
Lyapunov exponents, 56, 109

MCS, 145

Arabia, 67

Baloa, 73

Cochrane, 75

Donovan, 65

Ferrari, 69

Knierbein, 68

Kolff, 59

modification, 93

Scotten, 65

Sharp, 72

Swanson, 63

Testa, 74

UOHI, 119

Vermette, 72

Wallner, 74

Withington, 74

Woodruff, 71

MTS, 129

mass position, 34

mass-spring-damper, 95

maxima

cubic, 90

maximum amplitude, 118

mean arterial pressure, 79,92

minima

cubic, 90

mitral valve, 8

mock circulatory system, 3

modulus

gyrator, 25

transformer, 25 natural frequency, 118

Navier-Stokes, 54

Newton's Second Law, 19

non-autonomous, 102

nonautonomous, 21

nonlinear

behaviour, 96

spring, 96

null space, 29

numerical

simulation, 72, 138

Ottawa Heart Institute, 132

output matrix, 22

PDH, 28

pVAD, 132

P-wave, 10

parabolic rate of fall, 32

parameter space, 112

particular solution, 96

period-doubling, 102, 114

period-n, 102

periodic peak, 118

periodicity, 102

periodicity determination, 104

peripheral

area, 44

flow rate, 135

inertia, 95

system, 37

valve, 146,150

phase-space, 99,108

physical

properties, 100 
quantity, 28

piston

motion, 151

rod, 132

plaque, 52

Poincaré section, 108

ports, 24

post-systolic, 145

power

law, 55

source, 17

variables, 23

predictability, 2

pressure

fluctuations, 83

sink, 83

workload, 63

Principle of Dimen-

sional Homogeneity, 28

proximal

capacitance, 63

vessels, 82

pseudo-phase plot, 102, 108

pseudo-VAD, 132

pulmonary

artery, 8

loop, 53

resistance, 66

veins, 8

pulsatile flow, 146

pump, 37

pumped volume, 132

pumping

mechanism, 17 mode, 117

QRS-complex, 10

$R-R$ intervals, 12, 55

rank

matrix, 29

unit matrix, 30

regurgitation, 75

relative compliance, 145

repeating variables, 29, 100

resistor, 24

resonance, 118

respiration entrainment, 149

response

periodic, 95

sympathetic, 9

transient, 95

right atrium, 8

rigid floor, 98

Routh-Hurwitz, 88

SCM, 41, 145

sawtooth

input, 150

waveform, 92

scaling parameters, 31

shadowing theory, 109

shear, 27

SI system, 28

single-compliance model

arterial, 41

venous, 46

sinusoidal piston motion, 133

sloshing, 122 
solution curve, 33

source

effort, 25

flow, 25

sources, 25

Starling's Law, 70

state

equations

HCS model, 48

matrix, 22

space, 21

stenosis, 75

stiffening behaviour, 100

stiffness threshold, 147

strange attractor, 109

stroke volume, 135

surge tank, 64

sympathetic

activity, 55

response, 9

system, 19

continuous time, 82

input, 116

mass-spring-damper, 20

response

chaotic, 107

systemic

loop, 53

resistance, 67

systole, 12

TAH, 1

tachyarrhythmia, 55

theoretical assertion, 153 time invariant, 21

total artificial heart, 16

tractable system, 82

trajectories, 32, 108

divergence, 108

transducer

cable-extension, 138

pressure, 121

transfer function, 22

transformer, 25

transients, 102

transmission matrix, 22

transmural pressure, 65

tunics, 12

unit matrix, 35

UOHI, 119

VAD, 2, 37

fill pressure, 46

stability, 148

Valsalva manoeuvre, 79

valve

aortic semilunar, 9

atrioventricular, 7

inlet, 37

mitral, 8

outlet, 37

passive, 132

pulmonary semilunar, 8

valves, 39

variable pool, 31

variables, 28

dependent, 36

independent, 36 
vasoconstriction, 13

vasomotion, 54

vector space, 29

vein

pulmonary, 8

veins, 7,14

vena cava

inferior, 7

vena cava

superior, 7

volume, 47

venae cavae, 14

venous

blood volume, 47

collapse, 45,47

system, 37

ventricle, 7

left, 9

right, 8

ventricular

assist device, 2, 16

output, 78

venules, 14

vessel

compliance, 27

geometry

changes, 102

inertia, 27

resistance, 27

vibrating flow pump, 57

viscous rolling, 140

volume render, 112

windowed analysis, 105 